\title{
Der Übergangsprozess westdeutscher Jugendlicher verschiedener Bildungsniveaus in die Ausbildung zwischen 1971 und 2012 vor dem Hintergrund der Zunahme hochqualifizierter Arbeitskräfte
}

\author{
Inauguraldissertation \\ zur Erlangung des Grades eines Doktors der Philosophie \\ im Fachbereich Gesellschaftswissenschaften \\ der Johann-Wolfgang Goethe-Universität \\ zu Frankfurt am Main \\ vorgelegt von \\ Bernd Bender \\ aus Bad Homburg
}

2021

1. Gutachter/-in: Prof. Dr. Birgit Becker

2. Gutachter/-in: Prof. Dr. Julian Garritzmann

Tag der mündlichen Prüfung: 


\section{Inhaltsverzeichnis}

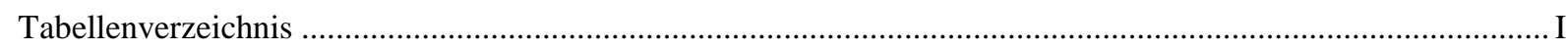

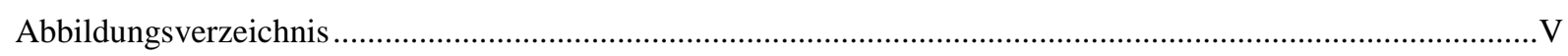

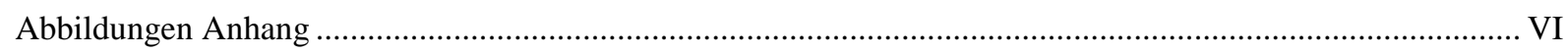

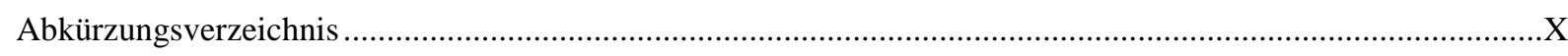

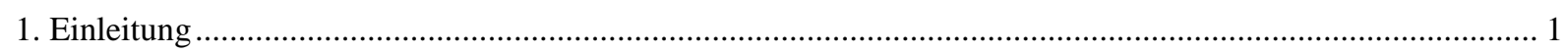

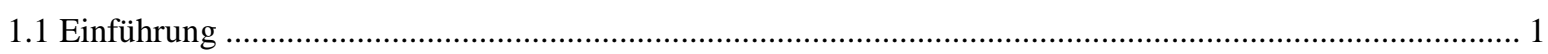

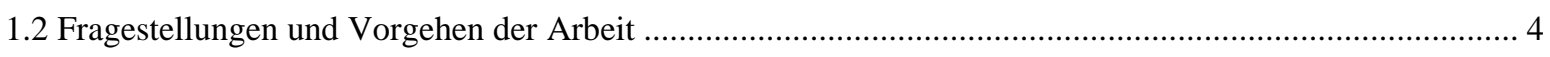

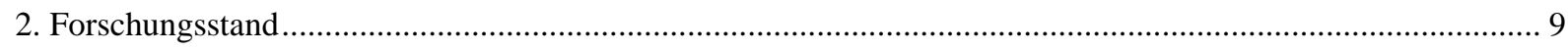

2.1 Studien zum Übergang von der Schule in eine vollqualifizierende berufliche oder akademische

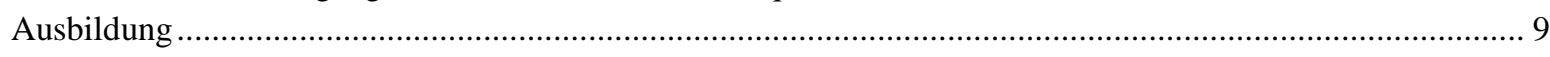

2.2 Studien zur Übergangsdauer von der Schule in eine vollqualifizierende berufliche oder akademische

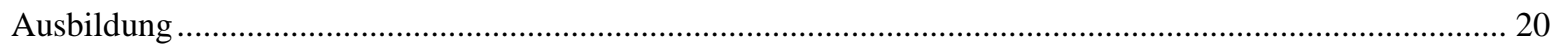

2.3 Studien zu Übergangsstationen von der Schule in eine vollqualifizierende berufliche oder akademische

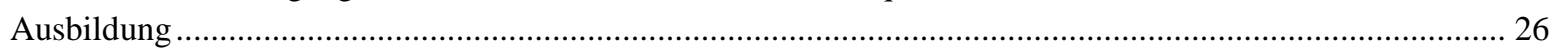

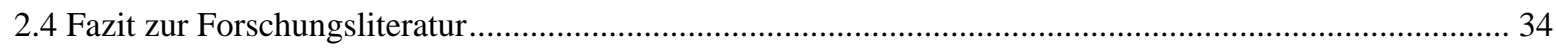

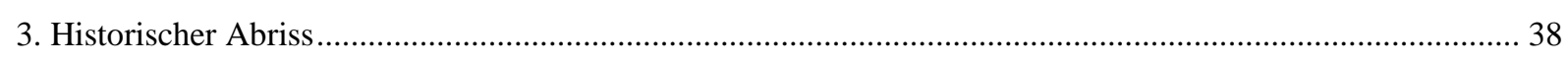

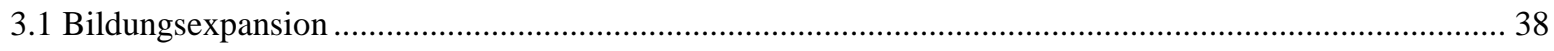

3.2 Gestiegener Anteil an hochqualifizierten Arbeitskräften ............................................................ 43

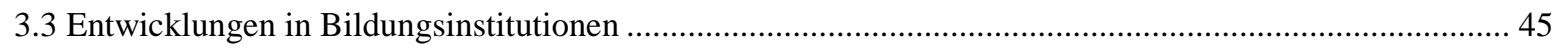

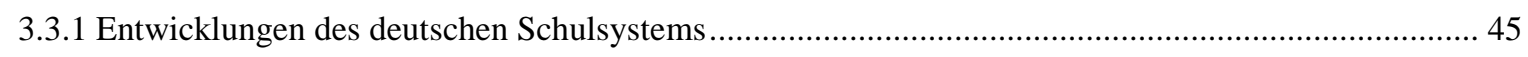

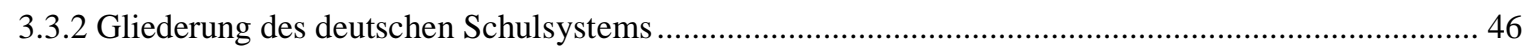

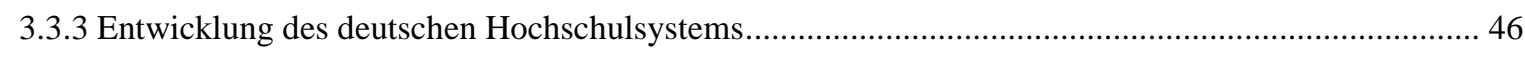

3.3.4 Gliederung und Entwicklung des beruflichen Ausbildungssystems .......................................... 47

3.4 Entwicklung der Übergangsverläufe von der Schule in die Ausbildung ........................................... 49

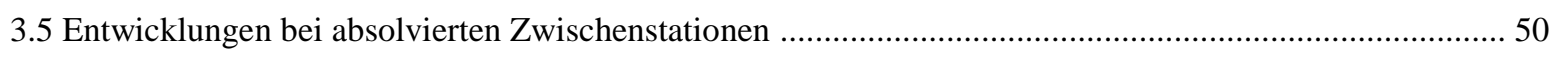

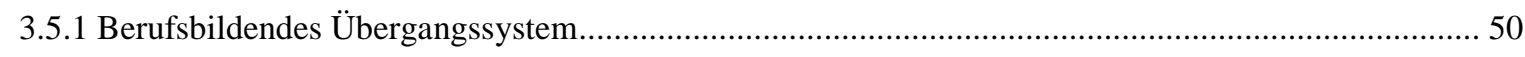

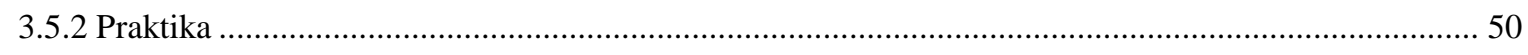

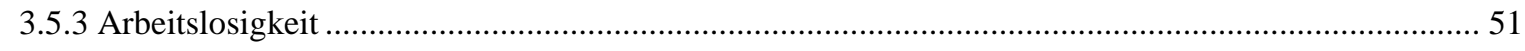

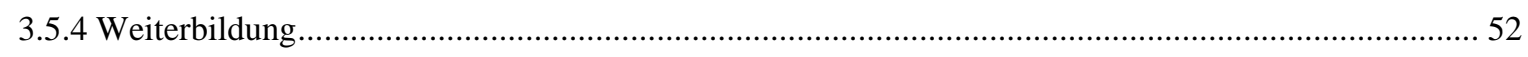

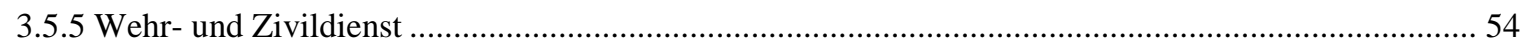




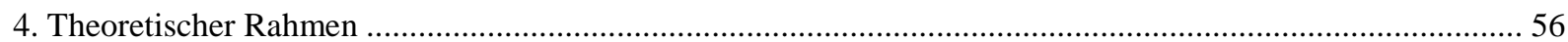

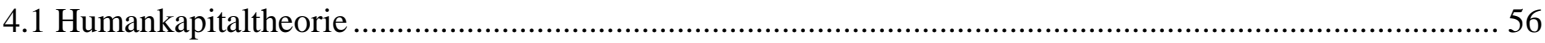

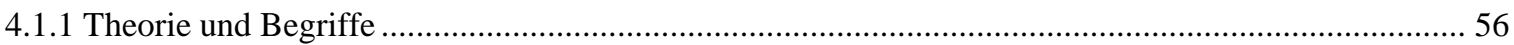

4.1.2 Übertragung der Humankapitaltheorie auf die vorliegende Untersuchung .................................... 62

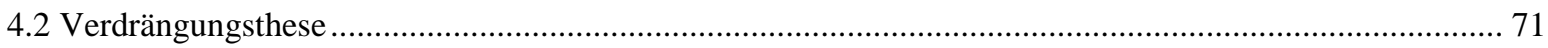

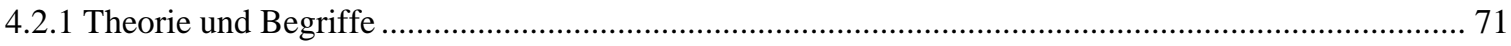

4.2.2 Übertragung der Verdrängungsthese auf die vorliegende Untersuchung ....................................... 72

4.3 Begründung für die Auswahl der theoretischen Ansätze ............................................................ 73

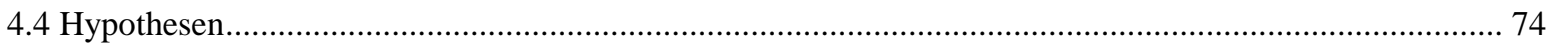

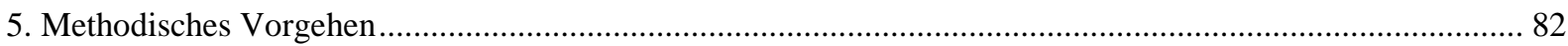

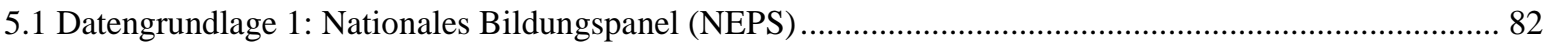

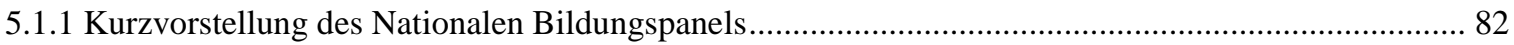

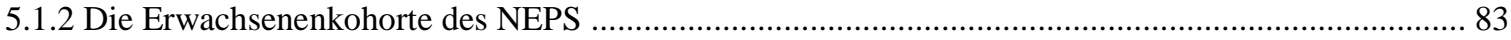

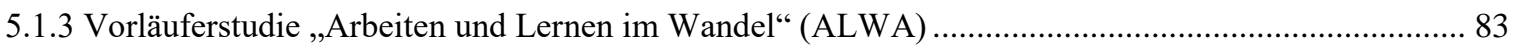

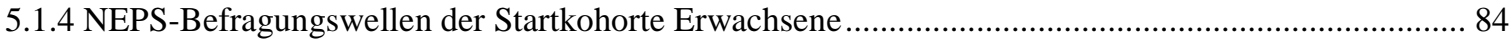

5.1.5 Begründung für die Auswahl der ALWA-/NEPS-Daten .......................................................... 85

5.1.6 Stichprobenzusammensetzung der einzelnen Befragungswellen …............................................ 86

5.1.7 Begründung für die Eingrenzung des Untersuchungszeitraums zwischen 1971 und 2012 ................89

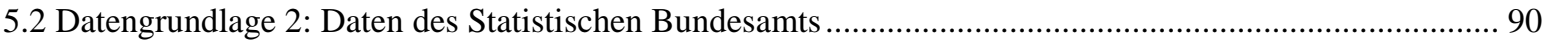

5.2.1 Datenvorlage des Statistischen Bundesamts zu hochqualifizierten Arbeitskräften .......................... 90

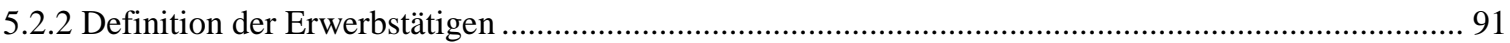

5.2.3 Implementieren der Daten des Statistischen Bundesamts in die NEPS-Daten............................... 92

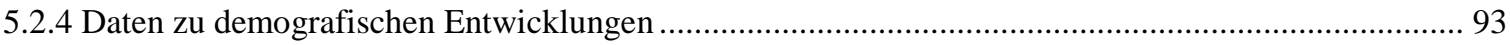

5.3 Datengrundlage 3: Daten der Bundesagentur für Arbeit zur Arbeitslosenquote .....................................94

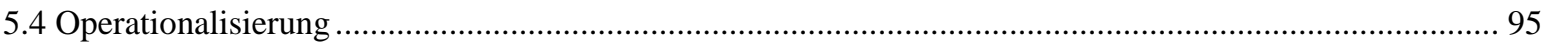

5.4.1 Abhängige Variablen Übergangsdauer und Übergangsstationen ................................................. 95

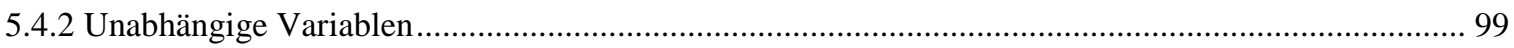

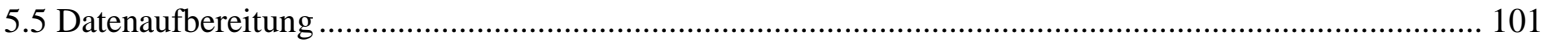


5.5.2 Rekonstruktion, Bereinigung und Sortierung von Datumsangaben zum Schulenddatum,

Erstausbildungsbeginn und Übergangsstationen

5.5.3 Aufbereitung von Schulabschlüssen, Erstausbildungstypen, Herkunftskategorien und

Praktikumskategorien

5.5.4 Limitationen des Stichprobendesigns und Ausschluss von Personen

5.5.5 Bereinigung von zeitlichen Dopplungen und Überschneidungen bei Übergangsstationen,

Schulenddatum und Erstausbildungsbeginn

5.5.6 Berechnung der Übergangsdauer und der Anzahl der Übergangsstationen

5.6 Methoden

5.6.1 Deskriptive Statistiken

5.6.2 Korrelationen und Mittelwerttests

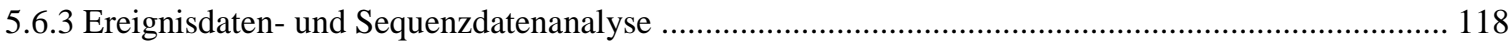

5.6.4 Ereignisdatenanalyse: Sterbetafeln und Cox-Regression

5.6.5 Sequenzdatenanalyse: Umstrukturierung der Daten und deskriptive Übersicht.

5.6.6 Sequenzdatenanalyse: Bildung von Stationskategorien

5.6.7 Sequenzdatenanalyse: Multinomial logistisches Regressionsmodell ....................................... 126

5.6.8 Sequenzdatenanalyse: Poisson- und negatives binomiales Regressionsmodell

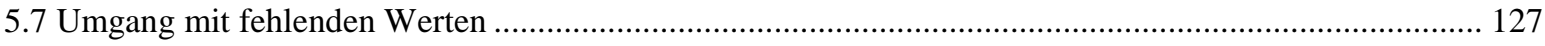

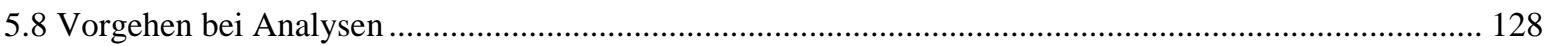

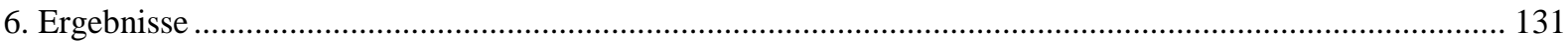

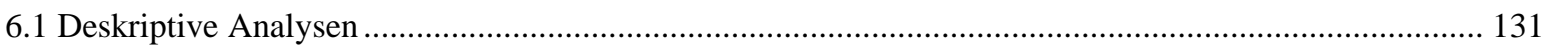

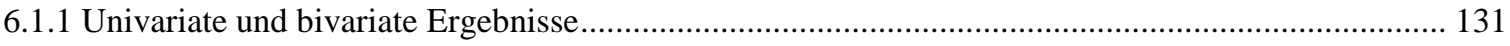

6.1.2 Zusammenfassung der deskriptiven Ergebnisse ............................................................... 151

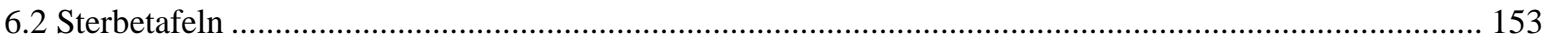

6.2.1 Ergebnisse der kumulierten Überlebenswahrscheinlichkeiten ................................................ 153

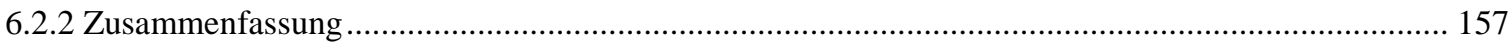

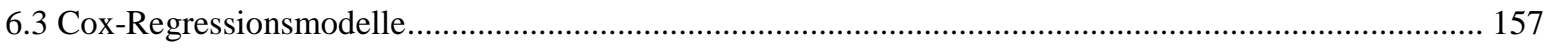

6.3.1 Ergebnisse der Cox-Regressionsmodelle zu Hypothesen 1a) und 1b) ....................................... 157

6.3.2 Ergebnisse der Cox-Regressionsmodelle zu Hypothese 2a) .................................................. 160

6.3.3 Ergebnisse der Cox-Regressionsmodelle zu Hypothese 2b) .................................................. 162 


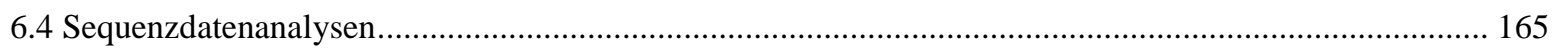

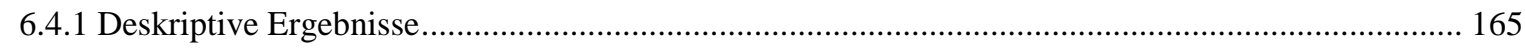

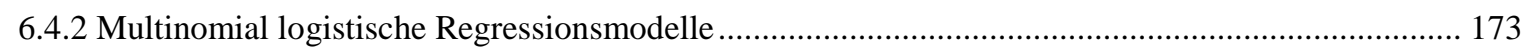

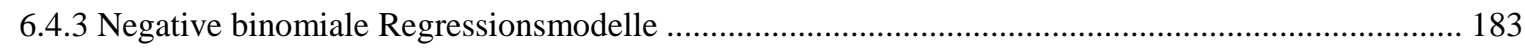

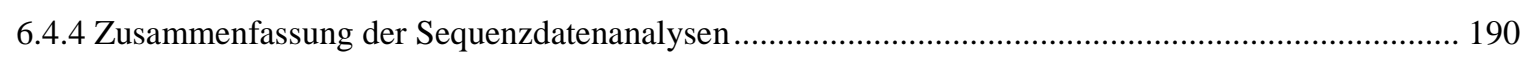

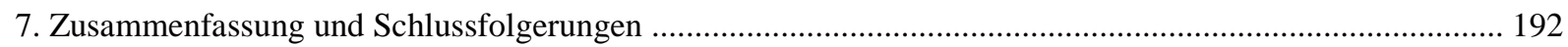

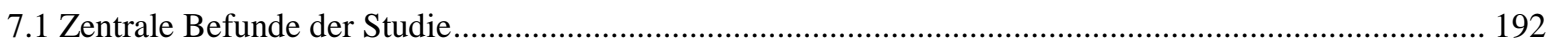

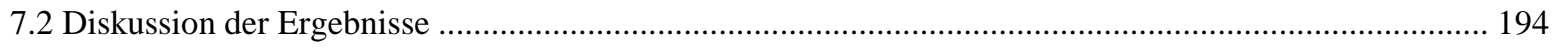

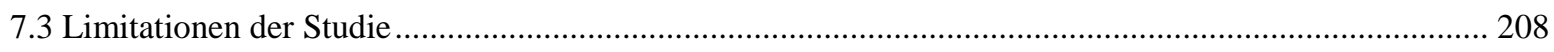

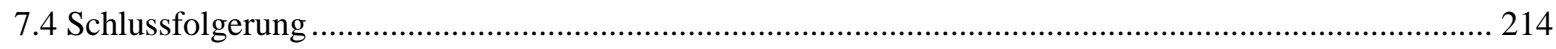

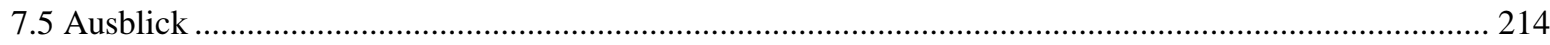

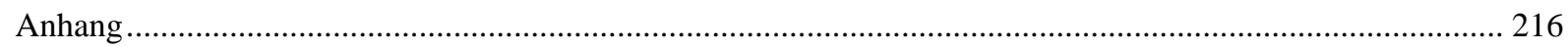

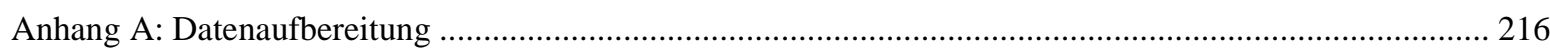

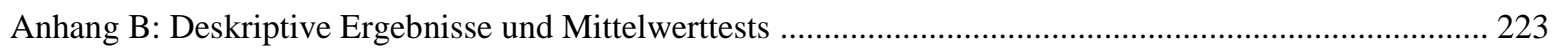

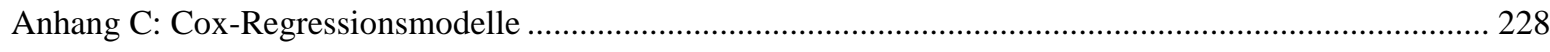

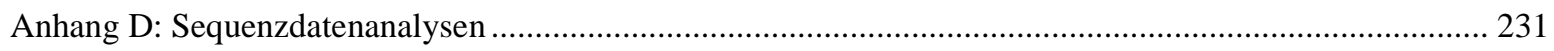

Anhang E: Abbildungen zu Überschneidungskonstellationen von Stationen ................................................ 254

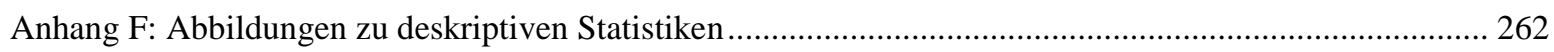

Anhang G: Abbildungen zu Sterbetafeln (Überlebenswahrscheinlichkeiten) …............................................ 268

Anhang H: Abbildungen zu Cox-Regressionsmodellen....................................................................... 271

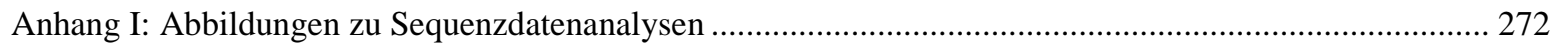

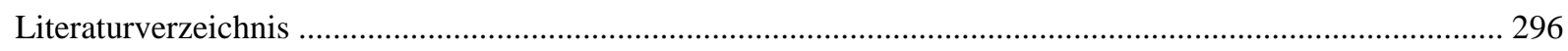

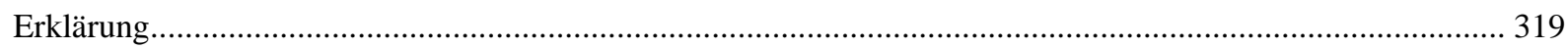




\section{Tabellenverzeichnis}

Tabelle 1: Zwei Stadien der jüngeren deutschen Bildungsgeschichte mit prozentualen Schüleranteilen der drei Schulformen.

Tabelle 2: Prozentuale Anteile der Abiturientenquoten und der Studienanfängerquoten.

Tabelle 3: Befragungswellen 1 bis 7 der Erwachsenenbefragung des NEPS mit Zeiträumen, Anzahl und Geburtskohorten der befragten Personen sowie der Erhebungsform.

Tabelle 4: Abhängige und unabhängige Variablen sowie Kontrollvariablen.

Tabelle 5: Einbezogene unabhängige Variablen der Regressionsmodelle.

Tabelle 6: Effekte des Schulenddatumsjahres und des Schulabschlusses auf die Übergangsdauer (ohne Wehrdienst, Elternzeit, FSJ/Urlaub/Krankheit).

Tabelle 7: Effekt des Anteils an Dienstleistungstätigen auf die Übergangsdauer (ohne Wehrdienst, Elternzeit, FSJ/Urlaub/Krankheit).

Tabelle 8: Effekt des Schulabschlusses auf die Übergangsdauer (ohne Wehrdienst, Elternzeit,

FSJ/Urlaub/Krankheit) mit Interaktion Schulabschluss und Anteil Dienstleistungstätige.

Tabelle 9: Häufigkeit der Stationen, die von den Personen durchlaufen wurden.

Tabelle 10: Verteilung der zehn häufigsten Übergangssequenzen beziehungsweise Übergangsmuster.

Tabelle 11: Deskriptive Statistiken zur Länge der Übergangssequenzen und zur Anzahl an unterschiedlichen Stationen pro Übergangsverlauf.....

Tabelle 12: Häufigkeitsverteilung der Anzahl unterschiedlicher Übergangsstationen in den Übergangssequenzen.

Tabelle 13: Deskriptive Statistik zur Anzahl an absolvierten Übergangsstationen während des Übergangsverlaufs nach Schulabschluss.

Tabelle 14: Deskriptive Statistik zur Anzahl an unterschiedlichen Übergangsstationen während des Übergangsverlaufs nach Schulabschluss.

Tabelle 15: Effekt des Schulabschlusses auf die Zugehörigkeit zur jeweiligen Stationskategorie.

Tabelle 16: Effekt des Schulabschlusses und des Anteil an Dienstleistungstätigen auf die Zugehörigkeit zur jeweiligen Stationskategorie.

Tabelle 17: Effekt des Schulenddatumsjahres und des Schulabschlusses auf die Anzahl der Übergangsstationen.

Tabelle 18: Effekt des gestiegenen Dienstleistungstätigenanteils auf die Anzahl der Übergangsstationen. 186

Tabelle 19: Effekt des gestiegenen Dienstleistungstätigenanteils auf die Anzahl der Übergangsstationen mit Interaktion zwischen Anteil Dienstleistungstätige und Schulabschluss. 


\section{Tabellen Anhang}

Tabelle 1: Übersicht zu Stichprobenziehung, Zielgruppe und Fallzahlen der ALWA-Studie....

Tabelle 2: Übersicht Ausprägungen Schulabschluss, Ausbildungstyp und soziale Herkunft sowie Zusammenfassung zu verwendeten Kategorien.

Tabelle 3: Aus ursprünglicher Stichprobe ausgeschlossene Gruppen und dazugehörige Fallzahlen.

Tabelle 4: Übersicht über Gewichtungen der Übergangsstation für alle Kombinationen.

Tabelle 5: Zuordnung und Zusammenfassung der Ausprägungen beider Monatsvariablen in Rohdaten zu gemeinsamer Monatsvariablen.

Tabelle 6: Abhängige und unabhängige Variablen und Fallzahl mit gültigen Werten.

Tabelle 7: Deskriptive Statistik zu Übergangsdauer, Übergangsstationen, Anteil Dienstleistungstätige, demografischer Anteil und Arbeitslosenquote.

Tabelle 8: Häufigkeiten und prozentuale Anteile zu Schulabschluss, Erstausbildungstyp, soziale Herkunft (Bildung der Eltern) und Geschlecht.

Tabelle 9: Mittelwerttest Übergangsdauer zwischen Schulabschlussgruppen.

Tabelle 10: Mittelwerttest Übergangsdauer zwischen Erstausbildungstypen.

Tabelle 11: Mittelwerttest Summe Übergangsstationen zwischen Schulabschlussgruppen

Tabelle 12: Mittelwerttest Summe Übergangsstationen zwischen Erstausbildungstypen.

Tabelle 13: Effekte des Schulenddatumsjahres und des Schulabschlusses auf die Übergangsdauer (ohne Wehrdienst und Elternzeit; mit FSJ/Urlaub/Krankheit).

Tabelle 14: Effekt des Anteils an Dienstleistungstätigen auf die Übergangsdauer (ohne Wehrdienst und Elternzeit; mit FSJ/Urlaub/Krankheit).

Tabelle 15: Effekt des Schulabschlusses auf die Übergangsdauer (ohne Wehrdienst und Elternzeit; mit FSJ/Urlaub/Krankheit) mit Interaktion Schulabschluss und Anteil Dienstleistungstätige.

Tabelle 16: Häufigkeit der Stationen, die von den Personen durchlaufen wurden (mit FSJ/Urlaub; ohne unbestimmte Lückenepisoden).

Tabelle 17: Verteilung der zehn häufigsten Übergangssequenzen beziehungsweise Übergangsmuster (mit FSJ/Urlaub; ohne unbestimmte Lückenepisoden).

Tabelle 18: Deskriptive Statistiken zur Länge der Übergangssequenzen (Anzahl der absolvierten Stationen insgesamt) und zur Anzahl an unterschiedlichen Stationen pro Übergangsverlauf (mit FSJ/Urlaub; ohne unbestimmte Lückenepisoden).

Tabelle 19: Häufigkeitsverteilung der Anzahl unterschiedlicher Übergangsstationen in den Übergangssequenzen (mit FSJ/Urlaub; ohne unbestimmte Lückenepisoden).

Tabelle 20: Deskriptive Statistik zur Anzahl an Übergangsstationen nach Schulabschluss (mit FSJ/Urlaub; ohne unbestimmte Lückenepisoden). 
Tabelle 21: Deskriptive Statistik zur Anzahl an unterschiedlichen Übergangsstationen nach Schulabschluss (mit FSJ/Urlaub; ohne unbestimmte Lückenepisoden).

Tabelle 22: Häufigkeit der Stationen, die von den Personen durchlaufen wurden (ohne FSJ/Urlaub; mit unbestimmten Lückenepisoden)

Tabelle 23: Verteilung der zehn häufigsten Übergangssequenzen beziehungsweise Übergangsmuster (ohne FSJ/Urlaub; mit unbestimmten Lückenepisoden).

Tabelle 24: Deskriptive Statistiken zur Länge der Übergangssequenzen (Anzahl der absolvierten Stationen insgesamt) und zur Anzahl an unterschiedlichen Stationen pro Übergangsverlauf (ohne FSJ/Urlaub; mit unbestimmten Lückenepisoden)

Tabelle 25: Häufigkeitsverteilung der Anzahl unterschiedlicher Übergangsstationen in den Übergangssequenzen (ohne FSJ/Urlaub; mit unbestimmten Lückenepisoden).

Tabelle 26: Deskriptive Statistik zur Anzahl an Übergangsstationen nach Schulabschluss (ohne FSJ/Urlaub; mit unbestimmten Lückenepisoden)

Tabelle 27: Deskriptive Statistik zur Anzahl an unterschiedlichen Übergangsstationen nach Schulabschluss (ohne FSJ/Urlaub; mit unbestimmten Lückenepisoden).

Tabelle 28: Häufigkeit der Stationen, die von den Personen durchlaufen wurden (mit FSJ/Urlaub; mit unbestimmten Lückenepisoden)

Tabelle 29: Verteilung der zehn häufigsten Übergangssequenzen beziehungsweise Übergangsmuster (mit FSJ/Urlaub; mit unbestimmten Lückenepisoden).

Tabelle 30: Deskriptive Statistiken zur Länge der Übergangssequenzen (Anzahl der absolvierten Stationen insgesamt) und zur Anzahl an unterschiedlichen Stationen pro Übergangsverlauf (mit FSJ/Urlaub; mit unbestimmten Lückenepisoden)

Tabelle 31: Häufigkeitsverteilung der Anzahl unterschiedlicher Übergangsstationen in den Übergangssequenzen (mit FSJ/Urlaub; mit unbestimmten Lückenepisoden)......

Tabelle 32: Deskriptive Statistik zur Anzahl an Übergangsstationen nach Schulabschluss (mit FSJ/Urlaub; mit unbestimmten Lückenepisoden)

Tabelle 33: Deskriptive Statistik zur Anzahl an unterschiedlichen Übergangsstationen nach Schulabschluss (mit FSJ/Urlaub; mit unbestimmten Lückenepisoden).

Tabelle 34: Effekt des Schulabschlusses auf die Zugehörigkeit zur jeweiligen Stationskategorie (mit FSJ/Urlaubsepisoden; ohne unbestimmte Lückenepisoden).

Tabelle 35: Effekt des Schulabschlusses und des Anteil an Dienstleistungstätigen auf die Zugehörigkeit zur jeweiligen Stationskategorie (mit FSJ/Urlaubsepisoden; ohne unbestimmte Lückenepisoden).

Tabelle 36: Effekt des Schulabschlusses auf die Zugehörigkeit zur jeweiligen Stationskategorie (ohne FSJ/Urlaubsepisoden; mit unbestimmten Lückenepisoden).

Tabelle 37: Effekt des Schulabschlusses und des Anteil an Dienstleistungstätigen auf die Zugehörigkeit zur jeweiligen Stationskategorie (ohne FSJ/Urlaubsepisoden; mit unbestimmten Lückenepisoden).

Tabelle 38: Effekt des Schulabschlusses auf die Zugehörigkeit zur jeweiligen Stationskategorie (mit FSJ/Urlaubsepisoden; mit unbestimmten Lückenepisoden). 
Tabelle 39: Effekt des Schulabschlusses und des Anteil an Dienstleistungstätigen auf die Zugehörigkeit zur jeweiligen Stationskategorie (mit FSJ/Urlaubsepisoden; mit unbestimmten Lückenepisoden).

Tabelle 40: Effekt des Schulenddatumsjahres und des Schulabschlusses auf die Anzahl der Übergangsstationen (mit FSJ- und Urlaubsepisoden; ohne unbestimmte Lückenepisoden).

Tabelle 41: Effekt des Schulenddatumsjahres und des Schulabschlusses auf die Anzahl der Übergangsstationen (ohne FSJ- und Urlaubsepisoden; mit unbestimmten Lückenepisoden).

Tabelle 42: Effekt des Schulenddatumsjahres und des Schulabschlusses auf die Anzahl der Übergangsstationen (mit FSJ- und Urlaubsepisoden; mit unbestimmten Lückenepisoden).

Tabelle 43: Effekt des gestiegenen Dienstleistungstätigenanteils auf die Anzahl der Übergangsstationen (mit FSJ- und Urlaubsepisoden; ohne unbestimmte Lückenepisoden).

Tabelle 44: Effekt des gestiegenen Dienstleistungstätigenanteils auf die Anzahl der Übergangsstationen mit Interaktion zwischen Anteil Dienstleistungstätige und Schulabschluss (mit FSJ- und Urlaubsepisoden; ohne unbestimmte Lückenepisoden).

Tabelle 45: Effekt des gestiegenen Dienstleistungstätigenanteils auf die Anzahl der Übergangsstationen (ohne FSJ- und Urlaubsepisoden; mit unbestimmten Lückenepisoden).

Tabelle 46: Effekt des gestiegenen Dienstleistungstätigenanteils auf die Anzahl der Übergangsstationen mit Interaktion zwischen Anteil Dienstleistungstätige und Schulabschluss (ohne FSJ- und Urlaubsepisoden; mit unbestimmten Lückenepisoden)

Tabelle 47: Effekt des gestiegenen Dienstleistungstätigenanteils auf die Anzahl der Übergangsstationen (mit FSJ- und Urlaubsepisoden; mit unbestimmten Lückenepisoden).

Tabelle 48: Effekt des gestiegenen Dienstleistungstätigenanteils auf die Anzahl der Übergangsstationen mit Interaktion zwischen Anteil Dienstleistungstätige und Schulabschluss (mit FSJ- und Urlaubsepisoden; mit unbestimmten Lückenepisoden) 


\section{Abbildungsverzeichnis}

Abbildung 1: Prozentuale Verteilung des ersten erreichten Schulabschlusses

Abbildung 2: Prozentuale Verteilung des ersten erreichten Schulabschlusses im Zeitverlauf.

Abbildung 3: Prozentuale Verteilung des Erstausbildungstyps.

Abbildung 4: Prozentuale Verteilung nach Erstausbildungstyp im Zeitverlauf.

Abbildung 5: Durchschnittliche Übergangsdauer nach Schulabschluss.

Abbildung 6: Durchschnittliche Übergangsdauer nach Erstausbildungstyp.

Abbildung 7: Durchschnittliche Übergangsdauer im Zeitverlauf.

Abbildung 8: Durchschnittliche Übergangsdauer ohne rechtszensierte Fälle im Zeitverlauf.

Abbildung 9: Durchschnittliche Übergangsdauer (ohne Wehrdienst, Elternzeit, FSJ/Urlaub/Krankheit) nach Schulabschluss im Zeitverlauf.

Abbildung 10: Durchschnittliche Übergangsdauer (ohne Wehrdienst und Elternzeit, mit FSJ/Urlaub/Krankheit) nach Schulabschluss im Zeitverlauf.

Abbildung 11: Durchschnittliche Anzahl Übergangsstationen nach Schulabschluss.

Abbildung 12: Durchschnittliche Anzahl Übergangsstationen nach Erstausbildungstyp.

Abbildung 13: Durchschnittliche Anzahl Übergangsstationen im Zeitverlauf.

Abbildung 14: Durchschnittliche Anzahl Übergangsstationen ohne rechtszensierte Fälle im Zeitverlauf. 146

Abbildung 15: Durchschnittliche Anzahl Übergangsstationen (ohne Wehrdienst, Elternzeit, FSJ/Urlaub/Krankheit) nach Schulabschluss im Zeitverlauf.

Abbildung 16: Durchschnittliche Anzahl Übergangsstationen (ohne Wehrdienst und Elternzeit; mit FSJ/Urlaub/Krankheit) nach Schulabschluss im Zeitverlauf.

Abbildung 17: Durchschnittliche Anzahl Übergangsstationen (ohne Wehrdienst, Elternzeit, FSJ/Urlaub/Krankheit, unbestimmte Phasen) nach Schulabschluss im Zeitverlauf.

Abbildung 18: Durchschnittliche Anzahl Übergangsstationen (ohne Wehrdienst, Elternzeit, unbestimmte Phasen; mit FSJ/Urlaub/Krankheit) nach Schulabschluss im Zeitverlauf.

Abbildung 19: Prozentuale Anteile zu Dienstleistungstätigen, demografischem Anteil und Arbeitslosenquote im Zeitverlauf nach fortlaufendem Schulenddatumsjahr.

Abbildung 20: Kumulierte Überlebenswahrscheinlichkeit insgesamt beim Übergang in die Ausbildung. 153

Abbildung 21: Kumulierte Überlebenswahrscheinlichkeit getrennt nach Schulabschluss

Abbildung 22: Kumulierte Überlebenswahrscheinlichkeit getrennt nach Erstausbildungstyp.

Abbildung 23: Geschätzte Hazard-Ratio des Schulabschlusses über Anteil der Dienstleistungstätigen auf die Übergangsdauer (ohne FSJ/Urlaub/Krankheit). 
Abbildung 25: Grafischer Verlauf aller Übergangsverläufe.

Abbildung 26: Grafischer Verlauf der zehn häufigsten Übergangsverläufe.

Abbildung 27: Geschätzte Wahrscheinlichkeiten, Stationen ohne Weiterbildungscharakter zu durchlaufen, nach

Schulabschluss in Abhängigkeit vom Anteil an Dienstleistungstätigen.

Abbildung 28: Geschätzte Wahrscheinlichkeiten, berufsvorbereitende Maßnahmen zu durchlaufen, nach Schulabschluss in Abhängigkeit vom Anteil an Dienstleistungstätigen.

Abbildung 29: Geschätzte Wahrscheinlichkeiten, Kurse, Weiterbildungen oder Praktika zu durchlaufen, nach Schulabschluss in Abhängigkeit vom Anteil an Dienstleistungstätigen.

Abbildung 30: Geschätzte Wahrscheinlichkeiten, zusätzliche Schulepisoden zu durchlaufen, nach Schulabschluss in Abhängigkeit vom Anteil an Dienstleistungstätigen.

Abbildung 31: Geschätzte Anzahl der Übergangsstationen (ohne FSJ- und Urlaubsepisoden und ohne unbestimmte Lückenepisoden) nach Schulabschluss unter Einfluss des Anteils an Dienstleistungstätigen. 189

\section{Abbildungen Anhang}

Abbildung 1: Mögliche Dauerkonstellationen einer Station während Übergangszeitraum.

Abbildung 2: Zeitliche Überschneidung einer Station mit Startdatum vor Schulenddatum.

Abbildung 3: Zeitliche Überschneidung einer Station mit Enddatum nach Startdatum Erstausbildung.

Abbildung 4: Zeitliche Überschneidung einer Station mit Startdatum vor Schulenddatum und Enddatum nach Startdatum Erstausbildung.

Abbildung 5: Zeitliche Überschneidung zweier identischer Stationen mit identischem Enddatum.................. 256

Abbildung 6: Zeitliche Überschneidung zweier identischer Stationen mit identischem Startdatum. ................ 256

Abbildung 7: Jeweils zeitliche Überschneidung des Start- und Enddatums zweier identischer Stationen. ........ 257

Abbildung 8: Stationsdauer innerhalb Dauer einer identischen Station.

Abbildung 9: Zeitliche Überschneidung Startdatum zweier verschiedener Stationen mit identischem Enddatum. 258

Abbildung 10: Zeitliche Überschneidung Enddatum zweier verschiedener Stationen mit identischem Startdatum und Ausschluss der gesamten Stationsdauer.

Abbildung 11: Zeitliche Überschneidung Enddatum zweier verschiedener Stationen mit identischem Startdatum.

Abbildung 12: Jeweils zeitliche Überschneidung des Start- und Enddatums zweier verschiedener Stationen... 260

Abbildung 13: Dauer einer stärker gewichteten Station während einer weniger gewichteten Station. 261

Abbildung 14: Durchschnittliche Übergangsdauer im Zeitverlauf mit zusammengefassten

Schulenddatumsjahren. 
Abbildung 15: Durchschnittliche Übergangsdauer ohne rechtszensierte Fälle im Zeitverlauf mit zusammengefassten Schulenddatumsjahren

Abbildung 16: Durchschnittliche Übergangsdauer nach Schulabschluss im Zeitverlauf mit zusammengefassten Schulenddatumsjahren.

Abbildung 17: Durchschnittliche Übergangsdauer (ohne Wehrdienst und Elternzeit, mit FSJ/Urlaub/Krankheit) nach Schulabschluss mit zusammengefassten Schulenddatumsjahren im Zeitverlauf.

Abbildung 18: Durchschnittliche Anzahl Übergangsstationen im Zeitverlauf mit zusammengefassten Schulenddatumsjahren.

Abbildung 19: Durchschnittliche Anzahl Übergangsstationen ohne rechtszensierte Fälle im Zeitverlauf mit zusammengefassten Schulenddatumsjahren.

Abbildung 20: Durchschnittliche Anzahl Übergangsstationen (ohne Wehrdienst, Elternzeit,

FSJ/Urlaub/Krankheit) nach Schulabschluss im Zeitverlauf mit zusammengefassten Schulenddatumsjahren. . 266

Abbildung 21: Durchschnittliche Anzahl Übergangsstationen (ohne Wehrdienst, Elternzeit; mit FSJ/Urlaub/Krankheit) nach Schulabschluss im Zeitverlauf mit zusammengefassten Schulenddatumsjahren. . 266

Abbildung 22: Durchschnittliche Anzahl Übergangsstationen (ohne Wehrdienst, Elternzeit,

FSJ/Urlaub/Krankheit, unbestimmte Phasen) nach Schulabschluss im Zeitverlauf mit zusammengefassten Schulenddatumsjahren.

Abbildung 23: Durchschnittliche Anzahl Übergangsstationen (ohne Wehrdienst, Elternzeit, unbestimmte Phasen; mit FSJ/Urlaub/Krankheit) nach Schulabschluss im Zeitverlauf mit zusammengefassten Schulenddatumsjahren.

Abbildung 24: Kumulierte Überlebenswahrscheinlichkeit insgesamt beim Übergang (ohne Wehrdienst und Elternzeit, mit FSJ/Urlaub/Krankheit).

Abbildung 25: Kumulierte Überlebenswahrscheinlichkeit getrennt nach Schulabschluss beim Übergang (ohne Wehrdienst und Elternzeit, mit FSJ/Urlaub/Krankheit).

Abbildung 26: Kumulierte Überlebenswahrscheinlichkeit getrennt nach Erstausbildungstyp beim Übergang (ohne Wehrdienst und Elternzeit, mit FSJ/Urlaub/Krankheit)....

Abbildung 27: Geschätzte Hazard-Ratio des Schulabschlusses über Anteil Dienstleistungstätige auf Übergangsdauer (mit FSJ/Urlaub/Krankheit).

Abbildung 28: Grafische Verteilung der Übergangsverläufe (mit FSJ/Urlaub, ohne unbestimmte Lückenepisoden).

Abbildung 29: Grafischer Verlauf aller Übergangsverläufe (mit FSJ/Urlaub, ohne unbestimmte Lückenepisoden).

Abbildung 30: Grafischer Verlauf der zehn häufigsten Übergangsverläufe (mit FSJ/Urlaub, ohne unbestimmte Lückenepisoden).

Abbildung 31: Grafische Verteilung der Übergangsverläufe (ohne FSJ/Urlaub, mit unbestimmten Lückenepisoden).

Abbildung 32: Grafischer Verlauf aller Übergangsverläufe (ohne FSJ/Urlaub, mit unbestimmten Lückenepisoden). 
Abbildung 33: Grafischer Verlauf der zehn häufigsten Übergangsverläufe (ohne FSJ/Urlaub, mit unbestimmten Lückenepisoden)

Abbildung 34: Grafische Verteilung der Übergangsverläufe (mit FSJ/Urlaub, mit unbestimmten Lückenepisoden).

Abbildung 35: Grafischer Verlauf aller Übergangsverläufe (mit FSJ/Urlaub, mit unbestimmten Lückenepisoden).

Abbildung 36: Grafischer Verlauf der zehn häufigsten Übergangsverläufe (mit FSJ/Urlaub, mit unbestimmten Lückenepisoden).

Abbildung 37: Geschätzte Wahrscheinlichkeiten, Stationen ohne Weiterbildungscharakter zu durchlaufen, nach Schulabschluss in Abhängigkeit vom Anteil an Dienstleistungstätigen (mit FSJ/Urlaub, ohne unbestimmte Lückenepisoden).

Abbildung 38: Geschätzte Wahrscheinlichkeiten, berufsvorbereitende Maßnahmen zu durchlaufen, nach Schulabschluss in Abhängigkeit vom Anteil an Dienstleistungstätigen (mit FSJ/Urlaub, ohne unbestimmte Lückenepisoden).

Abbildung 39: Geschätzte Wahrscheinlichkeiten, Kurse, Weiterbildungen oder Praktika zu durchlaufen, nach Schulabschluss in Abhängigkeit vom Anteil an Dienstleistungstätigen (mit FSJ/Urlaub, ohne unbestimmte Lückenepisoden).

Abbildung 40: Geschätzte Wahrscheinlichkeiten, zusätzliche Schulepisoden zu durchlaufen, nach Schulabschluss in Abhängigkeit vom Anteil an Dienstleistungstätigen (mit FSJ/Urlaub, ohne unbestimmte Lückenepisoden).

Abbildung 41: Geschätzte Wahrscheinlichkeiten, Stationen ohne Weiterbildungscharakter zu durchlaufen, nach Schulabschluss in Abhängigkeit vom Anteil an Dienstleistungstätigen (ohne FSJ/Urlaub, mit unbestimmten Lückenepisoden).

Abbildung 42: Geschätzte Wahrscheinlichkeiten, berufsvorbereitende Maßnahmen zu durchlaufen, nach Schulabschluss in Abhängigkeit vom Anteil an Dienstleistungstätigen (ohne FSJ/Urlaub, mit unbestimmten Lückenepisoden).

Abbildung 43: Geschätzte Wahrscheinlichkeiten, Kurse, Weiterbildungen oder Praktika zu durchlaufen, nach Schulabschluss in Abhängigkeit vom Anteil an Dienstleistungstätigen (ohne FSJ/Urlaub, mit unbestimmten Lückenepisoden).

Abbildung 44: Geschätzte Wahrscheinlichkeiten, zusätzliche Schulepisoden zu durchlaufen, nach Schulabschluss in Abhängigkeit vom Anteil an Dienstleistungstätigen (ohne FSJ/Urlaub, mit unbestimmten Lückenepisoden)

Abbildung 45: Geschätzte Wahrscheinlichkeiten, Stationen ohne Weiterbildungscharakter zu durchlaufen, nach Schulabschluss in Abhängigkeit vom Anteil an Dienstleistungstätigen (mit FSJ/Urlaub, mit unbestimmten Lückenepisoden).

Abbildung 46: Geschätzte Wahrscheinlichkeiten, berufsvorbereitende Maßnahmen zu durchlaufen, nach Schulabschluss in Abhängigkeit vom Anteil an Dienstleistungstätigen (mit FSJ/Urlaub, mit unbestimmten Lückenepisoden).

Abbildung 47: Geschätzte Wahrscheinlichkeiten, Kurse, Weiterbildungen, Praktika oder FSJ-Episoden zu durchlaufen, nach Schulabschluss in Abhängigkeit vom Anteil an Dienstleistungstätigen (mit FSJ/Urlaub, mit unbestimmten Lückenepisoden) 
Abbildung 48: Geschätzte Wahrscheinlichkeiten, zusätzliche Schulepisoden zu durchlaufen, nach Schulabschluss in Abhängigkeit vom Anteil an Dienstleistungstätigen (mit FSJ/Urlaub, mit unbestimmten Lückenepisoden)

Abbildung 49: Geschätzte Anzahl der Übergangsstationen (mit FSJ- und Urlaubsepisoden; ohne unbestimmte Lückenepisoden) nach Schulabschluss unter Einfluss des Anteils an Dienstleistungstätigen.

Abbildung 50: Geschätzte Anzahl der Übergangsstationen (ohne FSJ- und Urlaubsepisoden; mit unbestimmten Lückenepisoden) nach Schulabschluss unter Einfluss des Anteils an Dienstleistungstätigen.

Abbildung 51: Geschätzte Anzahl der Übergangsstationen (mit FSJ- und Urlaubsepisoden; mit unbestimmten Lückenepisoden) nach Schulabschluss unter Einfluss des Anteils an Dienstleistungstätigen. 


\section{Abkürzungsverzeichnis}

$\mathrm{ABM}$

Arbeitsbeschaffungsmaßnahme

ALWA Arbeiten und Lernen im Wandel

BIBB Bundesinstitut für Berufsbildung

BMBF .Bundesministerium für Bildung und Forschung

CAPI Computer Assisted Personal Interview

CASMIN Comparative Analysis of Social Mobility in Industrial Nations

CATI Computer Assisted Telephone Interview

DJI. Deutsches Jugendinstitut

EOS Erweiterte Oberschule

FEJ Freiwilliges Europäisches Jahr

FÖJ ..Freiwilliges Ökologisches Jahr FSJ Freiwilliges Soziales Jahr

IAB Institut für Arbeitsmarkt- und Berufsforschung

ID Identifikation Industrie- und Handelskammer IRR Incidence Rate Ratios KJS Katholische Jugendsozialarbeit LIfBi. Leibniz-Institut für Bildungsverläufe e. V.

LML .Log-minus-log NEPS .National Educational Panel Study NRW Nordrhein-Westfalen

OECD .Organisation for Economic Co-operation and Development PAPI Paper And Pencil Interview

POS ..Polytechnische Oberschule SOEP. Sozio-oekonomisches Panel VWA Verwaltungs- und Wirtschaftsakademie 


\section{Einleitung}

\subsection{Einführung}

Der Übergang von der Schule in den beruflichen oder akademischen Ausbildungsmarkt stellt eine sehr entscheidende Weiche für die weitere berufliche Perspektive junger Erwachsener dar und ist auch in gesellschaftspolitischer Hinsicht von Bedeutung. Ein gelungener Übergang bildet in der Regel die maßgebliche Grundlage für eine gute berufliche oder akademische Ausbildung und stellt damit automatisch auch eine Grundvoraussetzung für den Eintritt in die spätere Erwerbstätigkeit dar. Jedoch hat sich dieser Übergangsprozess im Laufe der vergangenen Jahrzehnte grundlegend verändert. Dieser Themenbereich war bereits Gegenstand zahlreicher Forschungsarbeiten in der empirischen Bildungsforschung. Verschiedene Autoren haben sich in erheblichem Umfang mit den Übergängen und Übergangschancen Jugendlicher auseinandergesetzt und dabei verschiedene wirtschaftliche und demografische Einflussfaktoren sowie unterschiedliche Schulabschlussformen bei den Jugendlichen, die die allgemeinbildende Schule verlassen und den Übergang in die Ausbildung absolvieren (u. a. Kleinert und Jacob 2012; Hillmert 2001; Konietzka 1999; Steinmann 2000), in den Fokus genommen. Bei diesen Arbeiten wurden auch mögliche Entwicklungen und Verläufe über mehrere Jahre und Jahrzehnte in einer Retrospektivbetrachtung untersucht und hierbei Jugendliche aller schulischen Bildungsniveaus sowie der Übergang in alle möglichen Ausbildungsformen mit einbezogen. Weitere Studien beschäftigten sich mit dem Gelingen des Übergangs von der Schule in die Ausbildung bei Jugendlichen oder jungen Erwachsenen mit einer bestimmten Schulabschlussform wie beispielsweise dem Abitur (u. a. Hillmert und Jacob 2003; Jacob und Weiss 2010; Maaz 2006) oder dem Hauptschulabschluss (u. a. Gaupp 2008; Protsch 2014; Carl et al. 2002) und fokussierten sich auch auf die Übergänge von Jugendlichen, welche keinen Schulabschluss erworben hatten (u. a. Gaupp et al. 2008; Solga 2004; Solga 2002). Des Weiteren wurden bislang die Übergänge und die Übergangsverteilungen in verschiedene Ausbildungsformen untersucht (u. a. Palamidis und Schwarze 1989; Seibert und Kleinert 2009; Eberhard und Ulrich 2009).

Dabei wurde deutlich, dass sich die Übergangschancen von Jugendlichen mit einem niedrigen oder mittleren Schulabschluss in eine vollqualifizierende Ausbildung zunehmend verschlechtert hatten, wozu auch die einbezogenen makrostrukturellen Einflussfaktoren beitrugen (u. a. Kleinert und Jacob 2012: 229; Steinmann 2000: 254). Vor diesem 
Hintergrund haben sich die Übergangschancen in eine Ausbildung zwischen den verschiedenen Schulabschlussformen im Laufe der Zeit deutlich auseinander entwickelt (Kleinert und Jacob 2012: 229). Jugendliche, welche keinen Schulabschluss erreicht hatten, kamen immer mehr von der Möglichkeit, in eine vollqualifizierende Berufsausbildung einzumünden, ab (Gaupp et al. 2008: 388) und gerieten immer häufiger in verschiedene berufsvorbereitende Maßnahmen des Übergangssystems, wodurch ihre Chancen auf dem beruflichen Ausbildungsmarkt sukzessive geringer wurden (Solga 2004: 99). Jugendliche, die einen Hauptschulabschluss erreicht hatten, benötigten zum Teil länger, um den Übergang in eine vollqualifizierende berufliche Ausbildung zu realisieren, wobei dieser Umstand unter anderem von soziodemografischen Gesichtspunkten abhängig war (Gaupp 2008: 26 f.). Außerdem hatte diese Schulabschlussgruppe insgesamt schlechtere Chancen beim Übergang in eine Berufsausbildung als die Gruppe von Schulabgängerinnen und Schulabgängern, welche einen Realschulabschluss erreicht hatten (Protsch 2014: 201).

Ebenso wurde der Prozess dieses Übergangs bereits in verschiedenen Arbeiten anhand der Übergangsdauer und der dabei zu absolvierenden Übergangsstationen untersucht und dabei für bestimmte Schulabschlussgruppen die Übergangsdauer (u. a. Beicht und Ulrich 2008b) und die Bedeutung von Übergangsstationen, welche während der Übergangsphase absolviert wurden, für den Übergangsprozess (u. a. Lex und Zimmermann 2011) in den Blick genommen. Hierbei stellte sich heraus, dass sich die Übergangsdauer von Jugendlichen mit einem niedrigen Schulabschluss in den vergangenen Jahrzehnten verlängert hatte, da sie nicht wie Jugendliche mit Abitur oder mit einem Realschulabschluss auf einen alternativen Ausbildungsgang ausweichen konnten (Kleinert und Jacob 2012: 225). Der Übergang von der Schule in eine berufliche Ausbildung wurde immer häufiger in mehreren Schritten und Übergangsstationen absolviert (Fink 2011: 92), welche unter anderem durch Umwege sowie Warteschleifen mit berufsvorbereitenden Maßnahmen verursacht wurden (Pätzold 2008: 603). Während es früher ein standardisiertes Übergangsmuster gab, das in die Stationen Schule, Ausbildung und Beruf unterteilt war, hat während der vergangenen Jahrzehnte eine sukzessive Abkehr von diesem standardisierten Übergangsverlauf stattgefunden. Dies ging mit einer permanenten Vervielfältigung und Individualisierung der Übergangsprozesse beim Übergang von der allgemeinbildenden Schule in eine berufliche oder akademische Ausbildung einher (Konietzka 1999: 315). Dieser Umstand führte zu der nachgewiesenen zeitlichen Ausdehnung des Übergangs, woraus resultierte, dass der Eintritt in die berufliche oder akademische Erstausbildung im Laufe der Jahrzehnte tendenziell immer später erfolgte. 
Jedoch gestaltete sich dieser Übergangsverlauf, auch unter den genannten Veränderungen und Entwicklungen, nicht für alle Schulabgängerinnen und Schulabgänger gleich, sondern ließ sich aufgrund der erwähnten Entwicklung hin zu individualisierten Übergangsverläufen vollkommen unterschiedlich und vielfältig charakterisieren. Diese unterschiedlichen Übergangsmuster ließen sich einerseits also individualisieren, andererseits auf einer übergeordneten Ebene aber auch durchaus nach soziodemografischen sowie bildungsbezogenen Merkmalen kategorisieren und unterscheiden, was ebenfalls die Vervielfältigung der Übergangsverläufe beim Übertritt von der Schule in die Ausbildung unterstrich. Hierbei fand lediglich eine Kategorisierung der vielfältigen Übergangsmuster nach soziodemografischen und bildungstechnischen Gesichtspunkten statt, um einen besseren Überblick über die Vielfalt dieser Übergangsmuster zu erhalten.

Zusammengefasst wurde bislang die Entwicklung der Übergangschancen in eine vollqualifizierende Ausbildung für Jugendliche differenziert nach Schulabschlussform im zeitlichen Verlauf untersucht, wobei die Rolle verschiedener wirtschaftlicher und demografischer Einflussfaktoren berücksichtigt wurde. Auch wurde der Übergangsprozess selbst anhand der Übergangsdauer und der absolvierten Stationen in den Fokus genommen. Hierdurch fand eine Vertiefung und Erweiterung statt, welche über das Betrachten der Übergangschancen und des bloßen Gelingens oder Nicht-Gelingens des Übergangs von der Schule in die Erstausbildung hinausging und die dabei zugrunde liegende Übergangsphase, charakterisiert durch die Dauer und die absolvierten Zwischenstationen, in den Fokus nahm. Allerdings wurde hierbei noch nicht eine systematische Verbindung mit verschiedenen makrostrukturellen Einflussfaktoren untersucht. Neben den geschilderten Entwicklungen der Übergangsverläufe in die Ausbildung fand gleichzeitig ein Wandel des Beschäftigungssystems statt. Dabei wurden durch personenbezogene Dienstleistungen aber auch Berufe im Wissens- und Informationsbereich neuartige sowie gestiegene Qualifikationen erforderlich und bezogen dadurch sukzessive einen immer größer werdenden Anteil der erwerbstätigen Personen mit ein (Autorengruppe Bildungsberichterstattung 2008: 6). Diese Entwicklung der Qualifikationssteigerung auf dem Arbeitsmarkt im Laufe der vergangenen Jahrzehnte war nicht von der Hand zu weisen. In der deutschen Volkswirtschaft kam es somit im Dienstleistungssektor $\mathrm{zu}$ einer kontinuierlichen Expansion (Autorengruppe Bildungsberichterstattung 2008: 22). Dadurch hat sich das Qualifikationsniveau auf dem deutschen Arbeitsmarkt, insbesondere im Bereich der Dienstleistungsberufe, grundlegend verändert und muss als möglicher Einflussfaktor auf den Übergangsprozess in die Ausbildung 
Berücksichtigung finden. Dies fand jedoch in der bisherigen Forschung nicht statt und soll im Rahmen der vorliegenden Studie aufgegriffen werden.

\subsection{Fragestellungen und Vorgehen der Arbeit}

Diese offen gelegte Forschungslücke soll nun im Folgenden aufgegriffen und der Frage nachgegangen werden, wie genau sich der Übergangsprozess in Form der Übergangsdauer und der absolvierten Übergangsstationen von Jugendlichen unterschiedlicher Schulabschlüsse im zeitlichen Verlauf vor dem Hintergrund eines gestiegenen Anteils an hochqualifizierten Arbeitskräften zwischen den Jahren 1971 und 2012 entwickelt hat. Auf die Begrenzung des Zeitraums zwischen 1971 und 2012 und die ausführliche Begründung hierzu wird im Kapitel 5 Methodisches Vorgehen detailliert eingegangen. Eine Neuerung in diesem Forschungsfeld stellt der gestiegene Anteil an hochqualifizierten Arbeitskräften auf dem Arbeitsmarkt dar, welcher erstmals als zentraler Einflussfaktor untersucht werden soll und als ein möglicher Einfluss bislang noch nicht in Betracht gezogen wurde.

Dadurch lässt sich feststellen, ob die Übergangsdauer und die absolvierten Übergangsstationen als Charakteristika des Übergangsprozesses von der Schule in die Ausbildung durch ein verändertes Qualifikationsniveau auf dem Arbeitsmarkt im Zeitraum zwischen 1971 und 2012 beeinträchtigt wurden. Darüber hinaus können durch eine differenzierte Betrachtung der Übergangsprozesse für die verschiedenen Schulabschlussgruppen Erkenntnisse für die Übergangsverläufe in Abhängigkeit zum schulischen Bildungsniveau gewonnen werden. Die Untersuchung eines möglichen Einflusses des gestiegenen Anteils von hochqualifizierten Arbeitskräften auf den Übergangsprozess in einem historischen Verlauf stellt einen interessanten und bedeutsamen Erkenntnisgewinn im fokussierten Forschungsfeld dar. Dieser lässt sich durch eine differenzierte und detaillierte Betrachtung des Übergangsverlaufs nach den einzelnen Schulabschlussgruppen einerseits, aber auch andererseits über sämtliche Schulabschlussgruppen hinweg, noch steigern. Darüber hinaus kann aus den ausführlichen Erkenntnissen zur Entwicklung des Übergangsprozesses von Jugendlichen unterschiedlicher schulischer Bildungsniveaus unter einem möglichen Einfluss eines gestiegenen Qualifikationsniveaus auf dem Arbeitsmarkt auch rückgeschlossen werden auf die Entwicklung der Übergangschancen in die Ausbildung. Dies ermöglicht ein Verständnis darüber, warum bestimmte Gruppen von Schulabgängerinnen und Schulabgängern im betrachteten Zeitraum zwischen 1971 und 2012 den Übergang in eine berufliche oder akademische Ausbildung besser bewältigen als andere Jugendliche. 
Die Fokussierung auf die Übergangsprozesse im Kontext des westdeutschen Bildungssystems empfiehlt sich, da in anderen Ländern andere Strukturen der Bildungssysteme vorliegen. Dies erfordert ein ausführliches Auseinandersetzen mit den Gegebenheiten im jeweiligen Land. Da die Rahmenbedingungen des westdeutschen Bildungssystems hinlänglich bekannt sind, konzentriert sich die vorliegende Studie auf Übergangsverläufe von Jugendlichen in Westdeutschland. Des Weiteren bieten die verwendeten Daten des Nationalen Bildungspanels sehr detaillierte und aufschlussreiche Informationen zu den Übergangsverläufen zwischen der Schule und der Erstausbildung. Diese Daten liegen jedoch nur für Deutschland vor, was ebenfalls für die Fokussierung auf westdeutsche Übergangsprozesse spricht. Die genauere Vorstellung des Nationalen Bildungspanels und die Eingrenzung auf Übergangsverläufe von westdeutschen Jugendlichen erfolgt im Kapitel 5 Methodisches Vorgehen.

Zum inhaltlichen Verständnis werden zunächst die Begriffe „Übergang“ beziehungsweise „Übergangsprozess“ erläutert und dargelegt, wie diese im Rahmen der Untersuchung zu verstehen sind. Mit dem „Übergang“ ist der Übergang von der allgemeinbildenden Schule in eine vollqualifizierende Erstausbildung gemeint. Diesem Übergang soll ein „Übergangsprozess“ zugrunde gelegt werden, welcher im Rahmen des Übergangs absolviert wird. Der Übergangsprozess kann unterschiedlich hinsichtlich seiner Dauer und der dabei absolvierten Zwischenstationen charakterisiert werden. Zum Beispiel kann der Übergangsprozess von der Schule in die Erstausbildung direkt ohne Zwischenstationen, aber auch mit einer längeren Dauer und einer Vielzahl von absolvierten Zwischenstationen vollzogen werden. Wichtig ist dabei, dass der Übergangsprozess im Rahmen dieser Untersuchung mit dem erstmaligen Verlassen der allgemeinbildenden Schule beginnt und mit dem Eintritt in eine vollwertige Erstausbildung abgeschlossen wird. Dabei ist zu betonen, dass unter der Kategorie „vollwertige vollqualifizierende Erstausbildung“ drei Ausbildungsformen verstanden werden sollen, nämlich eine duale Berufsausbildung, eine vollzeitschulische Berufsausbildung und eine akademische Ausbildung in Form eines Studiums. Der Übergang in das berufsvorbereitende Übergangssystem fällt nicht darunter und wird deswegen als Zwischenstation verstanden, welche während des Übergangsprozesses durchlaufen wird. Somit sollen die Übergangsprozesse in diese drei Ausbildungsformen sowie die vierte Variante, dass bis zum Ende des Untersuchungszeitraums keine Ausbildung begonnen wurde, betrachtet werden. Des Weiteren liegt der Fokus auf dem Übergangsprozess in eine Erstausbildung, wodurch Zweitausbildungen und weitere Übergangsverläufe im Anschluss an die Erstausbildung für diese Studie irrelevant sind. Das bedeutet, dass beispielsweise bei 
Übergangsverläufen, bei denen zunächst eine berufliche Ausbildung absolviert und im Anschluss daran ein Studium aufgenommen wird, der Zeitpunkt des Eintritts in die berufliche Ausbildung das Ende des betrachteten Übergangszeitraums markiert. Eine detaillierte Darstellung zu den Erstausbildungstypen wird im Methodenkapitel gegeben.

Auf der Grundlage des identifizierten Forschungsdesiderats und des geschilderten Erkenntnisinteresses werden zwei zentrale Fragestellungen formuliert, welche den Gegenstand der vorliegenden Studie bilden. Die erste in dieser Untersuchung zu behandelnde Fragestellung wird wie folgt formuliert: „Inwieweit hat ein erhöhter Anteil von hochqualifizierten Arbeitskräften auf dem Arbeitsmarkt die Dauer des Übergangsprozesses vom erstmaligen Verlassen der Schule bis zum Beginn einer dualen, schulischen oder akademischen Erstausbildung generell für alle Jugendlichen und jungen Erwachsenen sowie differenziert für die einzelnen Schulabschlussgruppen im Zeitraum zwischen 1971 und 2012 verändert?“. Diese Dauer inkludiert auch sämtliche Zwischenstationen, welche in diesem Zeitraum absolviert wurden.

Die zweite zu fokussierende Fragestellung lautet wie folgt: „Inwieweit hat ein erhöhter Anteil von hochqualifizierten Arbeitskräften auf dem Arbeitsmarkt das Absolvieren von Übergangsstationen, und dabei sowohl die Anzahl als auch die Kategorien der absolvierten Stationen generell für alle Jugendlichen und jungen Erwachsenen sowie differenziert für die einzelnen Schulabschlussgruppen im Zeitraum zwischen 1971 und 2012 verändert?". Zu diesen Übergangsstationen bis zum Einmünden in die berufliche oder akademische Erstausbildung können beispielsweise berufsvorbereitende Maßnahmen, Arbeitslosigkeitsepisoden, Weiterbildungsmaßnahmen, Praktika, Wehr- oder Zivildienst oder das Absolvieren eines freiwilligen sozialen Jahres sowie beim Übergang von jungen Frauen in die berufliche oder akademische Erstausbildung eine mögliche Schwanger- beziehungsweise Mutterschaft und eine darauf folgende Elternzeit gezählt werden. Einige der Stationen werden im Rahmen der vorliegenden Studie nicht weiter berücksichtigt. Auf die einzelnen Übergangsstationen sowie den Ausschluss einiger Stationen und die Begründung hierzu wird im Methodenkapitel detailliert eingegangen.

Im Rahmen der beiden Fragestellungen geht es um die Überprüfung, ob überhaupt ein Einfluss von qualifikationsbezogenen Veränderungen auf dem Arbeitsmarkt auf die Dauer und die Stationen des Übergangs besteht und wenn ja, wie stark dieser Einfluss ist, oder ob ein solch möglicher Einfluss durch veränderte wirtschaftliche oder demografische Gegebenheiten widerlegt wird. Bei beiden zu untersuchenden Fragestellungen soll einerseits 
eine Überprüfung über alle Schulabschlussgruppen hinweg stattfinden, um einen Gesamtüberblick zu erhalten, welcher unabhängig vom erreichten Bildungsabschluss ist. Andererseits soll aber auch ein differenzierter Blick für die einzelnen Schulabschlussgruppen erfolgen, um für jede einzelne Gruppe eine Erkenntnis zu den beiden formulierten Fragen zu erhalten und auch um einen Vergleich zwischen den einzelnen Bildungsabschlussgruppen anstellen zu können. Diese differenzierte Betrachtung stellt einen zusätzlichen Erkenntnisgewinn dar. Bezüglich der zu berücksichtigenden Schulabschlussformen sollen alle Sekundarabschlüsse, nämlich das Abitur, der Real- und der Hauptschulabschluss, aber auch das Nichtvorhandensein eines Schulabschlusses einbezogen werden.

Im folgenden Kapitel 2 wird ein Überblick über bisherige Studien und den Forschungsstand zu dem behandelten Themenfeld gegeben und daraus die der vorliegenden Studie zugrunde liegende Forschungslücke identifiziert. Das Kapitel 3 schließt mit einem historischen Abriss $\mathrm{zu}$ den bisherigen Entwicklungen und Veränderungen der Prozesse der Bildungsexpansion und des Arbeitsmarktes daran an. Auch die Entwicklungen des Bildungssystems, der Übergangsdauer und der dabei durchlaufenen Übergangsstationen werden berücksichtigt. Dabei werden Veränderungsprozesse aufgegriffen, welche für die untersuchten Fragestellungen und Hypothesen sowie das inhaltliche Verständnis der späteren Ergebnisse eine wichtige Rolle spielen. Im Kapitel 4 werden die beiden theoretischen Ansätze der Studie, die Humankapitaltheorie und die Verdrängungsthese, vorgestellt, deren Auswahl begründet und beide Ansätze auf die vorliegende Untersuchung übertragen. Die vollständige methodische Vorgehensweise wird im Kapitel 5 behandelt. Dabei wird zunächst das verwendete Datenmaterial vorgestellt und dessen Auswahl begründet. Des Weiteren wird auf die Eingrenzung der verwendeten Stichprobe für die späteren Analysen eingegangen und darauf folgend sämtliche Aufbereitungsschritte der Rohdaten beschrieben und begründet. Im Anschluss werden die verwendeten Analyseverfahren der Ereignisdaten- und der Sequenzdatenanalyse vorgestellt und deren Verwendung für die Untersuchung der vorliegenden Fragestellungen und Hypothesen begründet. Kapitel 6 stellt sämtliche Ergebnisse sowohl zu den deskriptiven Verteilungen und Übersichten als auch zu den ereignisdatenanalytischen und sequenzdatenanalytischen Auswertungen vor. Diese Resultate werden sowohl in tabellarischer als auch in grafischer Form dargestellt und ausführlich beschrieben und interpretiert. Abschließend werden im Kapitel 7 die zuvor vorgestellten Ergebnisse aufgegriffen und auf die zentralen Fragestellungen und Hypothesen zurückgeführt. Dabei geht es einerseits darum, anhand der vorliegenden Ergebnisse eine Antwort auf die 
Fragestellungen und Hypothesen zu geben und andererseits die Ergebnisse sowohl in den bisherigen Stand der Forschung zum Themenfeld als auch in die beiden theoretischen Ansätze einzuordnen. Darüber hinaus werden die sich aus den Analysen ergebenen Ergebnisse auch auf die historischen Entwicklungen und Veränderungen bezogen. Zusätzlich liefert der abschließende Anhang zusätzliche Tabellen und Grafiken für weitere Übersichten im Rahmen des methodischen Vorgehens sowie für weitere Analysevarianten im Rahmen der Datenauswertung. Bei den Analysen werden verschiedene Varianten des jeweils gleichen Analyseschritts durchgeführt und die verschiedenen Versionen miteinander verglichen. Bei der Vorstellung der Ergebnisse (Kapitel 6) wird eine einheitliche Variante vorgestellt und auf die anderen Varianten im Anhang verwiesen. 


\section{Forschungsstand}

Im Folgenden wird ein Studienüberblick des behandelten Themenfeldes gegeben. Dabei werden zunächst bisherige Studien und Erkenntnisse zu der Frage, wie sich der Übergang von der Schule in die berufliche oder akademische Ausbildung generell gestaltet hat, betrachtet (vgl. Abschnitt 2.1). Dabei liegt der Schwerpunkt darauf, wie sich dieser Übergang generell und getrennt nach unterschiedlichen schulischen Bildungsniveaus gestaltet, welche Ausbildungsformen von welchen Schulabschlussgruppen gewählt werden, wie lange dieser Übergang jeweils dauert und welche Stationen dabei durchlaufen werden sowie welche möglichen Einflussfaktoren auf den Übergang einwirken. Im Anschluss daran wird der Frage nachgegangen, inwieweit sich die Übergangsdauer vom Verlassen der Schule bis zum Beginn der Erstausbildung generell als auch differenziert nach Schulabschluss verändert hat (vgl. Abschnitt 2.2). Auch hierfür werden mögliche Einflussfaktoren in den Blick genommen. Anschließend soll ein Studienüberblick über die Entwicklung der Anzahl der dabei absolvierten Übergangsstationen generell sowie differenziert nach den einzelnen Schulabschlussgruppen erfolgen (vgl. Abschnitt 2.3). Hierbei soll auch beleuchtet werden, welche Faktoren die Anzahl der absolvierten Stationen tangieren sowie welche Übergangsstationen Jugendliche unterschiedlicher Schulabschlüsse unter dem Einfluss der veränderten Arbeitsmarktverhältnisse absolvieren. Abschließend wird noch eine zusammenfassende Einordnung des bisherigen Forschungsstands geliefert (vgl. Abschnitt 2.4).

\subsection{Studien zum Übergang von der Schule in eine vollqualifizierende berufliche oder akademische Ausbildung}

Betrachtet man den Übergang von der allgemeinbildenden Schule in eine Berufsausbildung, zeigen bisherige Forschungsarbeiten, dass sich für viele Jugendliche dieser Übergang langwieriger gestaltete (vgl. u. a. Rukwid 2011; Palamidis und Schwarze 1989; Konsortium Bildungsberichterstattung 2006). Insgesamt betrachtet stiegen zwar die Anzahlen der tatsächlich neu abgeschlossenen Ausbildungsverhältnisse sowie der Studienanfängerinnen und Studienanfänger zwischen Mitte der 1970er Jahre und Mitte der 1980er Jahre deutlich an (Schober 1985: 251). Jedoch zeigten sich in späteren Untersuchungen deutliche Verschiebungen bei den Übergängen. So ging der Anteil an Jugendlichen im dualen Ausbildungssystem zwischen 1995 und 2004 zurïck (Konsortium Bildungsberichterstattung 2006: 21). Diese Entwicklung setzte sich in den folgenden Jahren kontinuierlich fort, da sich 
die Zahl der Ausbildungsanfängerinnen und Ausbildungsanfänger in der Berufsbildung zwischen 2005 und 2013 verringerte (Bertelsmann Stiftung 2015: 22). Dagegen setzte sich der Trend des Anstiegs der Anzahl an Studienanfängerinnen und Studienanfängern aus den vorherigen Jahrzehnten zwischen 1995 und 2005 fort (Konsortium Bildungsberichterstattung 2006: 21). Andere Studien zeigten dagegen einen nichtlinearen Trend bezüglich der Ausbildungsquoten. Nachdem die Berufsausbildungsquote zwischen 1990 und 2002 gesunken war, stieg sie bei den folgenden Jahrgängen von 2005 bis 2006 leicht an (Heine et al. 2010: 4). Jedoch wurde auch deutlich, dass eine Teilgruppe vorübergehend oder dauerhaft von einer Berufsausbildung ausgeschlossen war, was sich kaum mit einem freiwilligen Ausbildungsverzicht begründen ließ (Carl et al. 2002: 18).

Auch bei Betrachtung des Übergangs von der Schule in die Ausbildung differenziert nach Schulabschluss lieferten bisherige Studien einige interessante Erkenntnisse. Beim Überblick über die Entwicklung über mehrere Jahrzehnte stellte sich heraus, dass sich die Übergangschancen in eine Ausbildung oder ein Studium zwischen den Bildungsniveaus im Laufe der Zeit auseinanderentwickelt haben. Insbesondere zu historisch späteren Zeitpunkten hatten Schulabgängerinnen und Schulabgänger mit einem niedrigen oder mittleren Bildungsniveau schlechtere Übergangschancen, als diejenigen mit einem höheren Bildungsniveau (Kleinert und Jacob 2012: 229). Bereits in älteren Studien wurde ersichtlich, dass vor allem Jugendliche ohne Schulabschluss große Schwierigkeiten hatten, in eine duale Berufsausbildung einzumünden (Stegmann und Holzbauer 1978: 148). Dagegen gestaltete sich der Übergang für diejenigen mit einer mittleren Reife in einen Ausbildungsplatz leichter (Schober 1985: 253).

Auch spätere Forschungsarbeiten konnten eine ähnliche Übergangscharakteristik für bestimmte Schulabschlussgruppen herausarbeiten. So ging der Anteil der Jugendlichen mit einem Hauptschulabschluss, welche eine duale Berufsausbildung aufgenommen hatten, zwischen 2000 und 2004 zurück. Nur wenige Jugendliche, welche keinen Schulabschluss erworben hatten, mündeten in eine Berufsausbildung ein (Konsortium Bildungsberichterstattung 2006: 17 f.). Trotz deutlicher Entspannung des dualen Ausbildungsstellenmarktes vor allem in Westdeutschland und einer gewährleisteten Integration von Schulabgängerinnen und Schulabgängern mit einem Hauptschulabschluss war eine Integration von Jugendlichen ohne Schulabschluss in das duale Ausbildungssystem nicht gegeben (Seibert und Kleinert 2009: 1). Wie bereits in früheren Jahrzehnten hatten Jugendliche mit einem Realschulabschluss insgesamt bessere Chancen beim Übergang in die 
Berufsausbildung als diejenigen mit einem Hauptschulabschluss (Protsch 2014: 201; Beicht und Ulrich 2008b: 21). Somit erhöhte ein guter Schulabschluss die Chance, in eine vollqualifizierende Berufsausbildung einzumünden, deutlich (Bertelsmann Stiftung 2015: 24 f.). Des Weiteren stellte sich heraus, dass die Verteilung der Schulabschlüsse im historischen Verlauf bei den westdeutschen Jugendlichen ohne eine berufliche Ausbildung heterogener und die Jugendlichen zu Beginn der 2000er Jahre schulisch besser ausgebildet waren als vor 40 Jahren (Wagner 2005: 219). Andererseits hatte sich der Anteil geringer gebildeter Jugendlicher, die in eine berufliche Ausbildung übergegangen waren, im Laufe der Geburtskohorten kontinuierlich erhöht (Solga 2004: 110). Des Weiteren mündete lediglich ein geringer Anteil an Frauen und Männern ohne Schulabschluss der Geburtsjahrgänge 1964 und 1971 nie in das berufliche Bildungssystem ein (Solga 2002: 9).

Bei Betrachtung eines möglichen Effekts einer deutlichen Verschiebung hin zu höheren Schulabschlüssen zeigten sich unterschiedliche Erkenntnisse. Zum einen haben sich die Chancen von Hauptschülerinnen und Hauptschülern, eine duale Ausbildung aufzunehmen, durch die verstärkte Konkurrenz Jugendlicher mit höheren Schulabschlüssen verschlechtert (Beicht und Walden 2018: 165; Steinmann 2000: 254). Zum anderen wurde jedoch herausgefunden, dass eine Verdoppelung der Abiturjahrgänge nicht zu einer Verdrängung von Jugendlichen mit maximal einem Hauptschulabschluss vom beruflichen Ausbildungsmarkt geführt hat. Im Gegensatz dazu haben sich deren Ausbildungschancen sogar verbessert. Dennoch hatten Hauptschülerinnen und Hauptschüler im Vergleich zu den anderen Schulabschlussgruppen die mit Abstand schlechtesten Chancen, einen betrieblichen Ausbildungsplatz zu ergattern (Holtmann et al. 2019: 380; Holtmann et al. 2018: 2; Baas und Philipps 2017: 31; KJS Katholische Jugendsozialarbeit 2014).

Betrachtet man den Einfluss weiterer potentieller Faktoren neben dem Schulabschluss über die Jahrzehnte, zeigt sich, dass vor Mitte der 1980er Jahre demografische Entwicklungen durch geburtenstarke Jahrgänge für eine Verringerung der Übergangschancen gesorgt hatten, wogegen hierfür ab Mitte der 1980er Jahre vorwiegend negative konjunkturelle Veränderungen in Form von hohen Arbeitslosigkeitsquoten die Ursache waren. Bei Betrachtung der Veränderungen innerhalb der Bildungsniveaus waren gestiegene Übergangsprobleme von Jugendlichen mit einem Haupt- oder Realschulabschluss in den untersuchten 30 Jahren $\mathrm{zu}$ verzeichnen, welche in einer Abhängigkeit zur gegebenen konjunkturellen und demografischen Situation standen. Die Übergangschancen von Abiturientinnen und Abiturienten gestalteten sich im Gegensatz dazu unabhängig von diesen 
Einflüssen (Kleinert und Jacob 2012: 229). Weiterhin wurde festgestellt, dass die generelle wirtschaftliche Situation einen größeren Einfluss auf kurzfristige Veränderungen der Übergangsraten in das duale Ausbildungssystem hatte als die Entwicklung der Geburtskohorten. Des Weiteren unterschied sich das Niveau der Übergangsquoten nach Geschlecht (Männer und Frauen), bei der Herkunft (Deutsche und Ausländer) und beim Vorhandensein einer allgemeinen Hochschulreife (Personen mit und ohne Abitur) über die Zeit (Hillmert 2001: 27). Bereits Mitte der 1980er Jahre zeichnete sich vor dem Hintergrund einer Diskrepanz zwischen einem Ausbildungsplatzmangel und einer hohen Ausbildungsplatznachfrage der Ausschluss einer bestimmten Gruppe von Ausbildungsplatzinteressierten beim Übergang von der Schule in eine betriebliche Berufsausbildung ab. Dies war an personengebundene Eigenschaften wie Geschlecht, Nationalität, Schulbildung und soziale Herkunft der Ausbildungsinteressierten gekoppelt (Palamidis und Schwarze 1989: 114). Frauen, Bewerberinnen und Bewerber mit Hauptschulabschluss, ausländische Bewerberinnen und Bewerber oder solche mit einer Fachhoch- oder einer Hochschulreife sowie ausländische oder behinderte Jugendliche zählten zwischen Mitte der 1970er Jahre und Mitte der 1980er Jahre als schwer vermittelbar für einen betrieblichen Ausbildungsplatz (Schober 1985: 253). Die Auswirkungen solcher Faktoren zeigten sich zum Teil auch in späteren Untersuchungen. Dabei hatten vor allem Jugendliche mit einem niedrigen Schulabschluss sowie mit Migrationshintergrund große Probleme, den Übergang von der Schule in eine duale oder vollzeitschulische Berufsausbildung auf direktem Weg zu bewerkstelligen (Rukwid 2011: 1). Junge Frauen, Jugendliche mit Migrationshintergrund, aus Familien mit wenig Möglichkeiten zur Unterstützung, mit einem schwierigen biografischen Bildungsverlauf oder mit einer unklaren beruflichen Perspektive waren am stärksten mit Risiken beim Übergang in die duale oder vollzeitschulische Ausbildung konfrontiert und hatten einen erhöhten Unterstützungsbedarf (Gaupp 2008: 27).

Der Anteil von Jugendlichen mit einem Migrationshintergrund stieg unter den Jugendlichen ohne eine berufliche Ausbildung in Westdeutschland zwischen den Geburtskohorten 1959 bis 1964 und 1971 bis 1975 deutlich an, wodurch Jugendliche mit einem Migrationshintergrund vor allem seit den 1990er Jahren eine wesentliche Teilpopulation unter den Jugendlichen ohne berufliche Ausbildung waren (Wagner 2005: 220). Demgegenüber ist im Vergleich zu früheren Jahrzehnten vor allem die Ausbildungspartizipation bei den Frauen im Zeitverlauf deutlich angestiegen (Steinmann 2000: 253). Andere Studien zeigen jedoch, dass junge Frauen mit einem Hauptschulabschluss weniger Chancen hatten als junge Männer, was sich 
mit einer deutlich geringeren Zahl von Übergängen in eine betriebliche Berufsausbildung und einer größeren Häufigkeit von weiterführenden Schulbesuchen belegen ließ (Carl et al. 2002: 17). Auch für die soziale Herkunft in Form des elterlichen Bildungsniveaus konnte ein Einfluss auf den Übergang in die Ausbildung nachgewiesen werden. Dabei zeigte sich erstaunlicherweise, dass Jugendliche aller Schulabschlussformen, deren Eltern maximal über einen Hauptschulabschluss verfügten, signifikant häufiger direkt im Anschluss an die Schulzeit in eine Berufsausbildung, und damit sowohl in eine duale als auch in eine vollzeitschulische Ausbildung, einmündeten als Jugendliche, deren Eltern einen Hochschulabschluss aufwiesen (Lex und Zimmermann 2011: 616).

Einerseits zeigte sich im Kohortenvergleich eine optimierte Ausbildungsintegration (Konietzka 1999: 315) in der Form, dass von den jährlichen potentiellen Ausbildungsanfängerinnen und Ausbildungsanfängern in der Berufsbildung in allen Bundesländern ein sukzessiv größer werdender Anteil in eine duale oder vollzeitschulische Ausbildung überging. Andererseits nahm jedoch die Anzahl an Schulabgängerinnen und Schulabgängern von allgemeinbildenden Schulen sukzessive ab, wobei diese immer weniger eine Berufsausbildung und immer mehr ein Hochschulstudium präferierten (Bertelsmann Stiftung 2015: 22).

Auch beim Vergleich zwischen Ost- und Westdeutschland fielen Unterschiede bei den Übergangsquoten in eine berufliche Ausbildung auf. Laut den Ergebnissen der Bewerberbefragung 2008 durch die Bundesagentur für Arbeit und das Bundesinstitut für Berufsbildung waren in den neuen Bundesländern mehr als die Hälfte aller Bewerberinnen und Bewerber in eine Berufsausbildung eingemündet. In den alten Bundesländern hatten weniger als die Hälfte der Befragten den Übergang in eine Berufsausbildung vollzogen (Eberhard und Ulrich 2009: 2 ff.). Die Unterschiede zwischen den alten und den neuen Bundesländern waren jedoch den unterschiedlichen gegebenen Möglichkeiten an Alternativen zur beruflichen Ausbildung geschuldet. Die aufgeführten Probleme vieler Jugendlicher beim Übergang von der Schule in die Ausbildung standen auch mit der Tatsache in Zusammenhang, dass im berufsvorbereitenden Übergangssystem statt des Erwerbs eines anerkannten Ausbildungsabschlusses lediglich eine Kompetenzaneignung hinsichtlich des Ausbildungsbeginns erfolgen konnte (Konsortium Bildungsberichterstattung 2006: 17 f.). Jedoch wurde festgestellt, dass Jugendliche durch das Absolvieren von betrieblichen Praxistagen an einzelnen Unterrichtstagen in der Hauptschule bessere Chancen hatten, den Übergang in die Ausbildung zu meistern, da sie dadurch ihre individuellen Interessen und 
Fähigkeiten besser herausfinden konnten und die ausbildenden Betriebe somit auch die Stärken der Jugendlichen besser kennen lernten (Solga et al. 2012: 5).

Bei detaillierterer Betrachtung der bisherigen Forschung zum Übergang von der Schule in die Ausbildung wurde unter anderem von Seibert und Kleinert die Anzahl der 2008 neu geschlossenen Ausbildungsverträge für eine duale Berufsausbildung im Verhältnis zu den Zahlen der Bewerberinnen und Bewerber, die bei der Bundesagentur für Arbeit gemeldet waren, untersucht. Dabei wurden diejenigen, die eine duale Ausbildung aufgenommen hatten, sowie diejenigen, die sich auf einen dualen Ausbildungsplatz beworben hatten, jedoch keinen Ausbildungsplatz bekamen, einbezogen. Studierende wurden dagegen nicht berücksichtigt. Ebenso wurden die Anzahlen der Schulabgängerinnen und Schulabgänger von allgemeinbildenden Schulen mit einem Hauptschulabschluss oder ohne Abschluss aus dem Vorjahr mit den Auszubildenden mit einem Hauptschulabschluss oder ohne Abschluss zwischen West- und Ostdeutschland verglichen. Das verwendete Datenmaterial bestand einerseits aus Erhebungen zu neu geschlossenen Ausbildungsverträgen und den bei der Bundesagentur für Arbeit gemeldeten Bewerberinnen und Bewerbern auf einen Ausbildungsplatz in West- und Ostdeutschland durch das Bundesinstitut für Berufliche Bildung in Kooperation mit der Bundesagentur für Arbeit und andererseits aus dem Datenbestand des Mikrozensus durch das Statistische Bundesamt (Seibert und Kleinert 2009: 1 ff.). Beim Mikrozensus handelt es sich um eine repräsentative Haushaltsbefragung von etwa 830.000 Personen in circa 370.000 privaten deutschen Haushalten und Gemeinschaftsunterkünften, welche seit 1957 besteht und Daten zur Bevölkerungsstruktur sowie zur wirtschaftlichen und sozialen Lage der Bevölkerung liefert (Statistisches Bundesamt 2015d). Seibert und Kleinert hoben bei ihren Ergebnissen hervor, dass sich der duale Ausbildungsstellenmarkt im Jahr 2008 vor allem in Westdeutschland aufgrund einer gestiegenen Anzahl an abgeschlossenen Ausbildungsverträgen deutlich stabilisierte, während in Ostdeutschland die Bewerberzahlen zurückgingen. Im Ausbildungsjahr 2008 wurden laut der Erhebung des Bundesinstituts für Berufsbildung bis zum 30. September etwa 502.500 neue Ausbildungsverträge abgeschlossen. Eine Integration von gering qualifizierten Schulabgängerinnen und Schulabgängern mit einem Hauptschulabschluss, jedoch nicht von denjenigen ohne Schulabschluss, war durch das duale Ausbildungssystem gewährleistet (Seibert und Kleinert 2009: 1). Dieser Befund unterstrich noch einmal die ungleichen Übergangschancen in die Ausbildung in Abhängigkeit vom schulischen Bildungsniveau. So verringerte sich der Anteil an Auszubildenden in Westdeutschland mit einem 
Hauptschulabschluss zwischen 1993 und 2005 um sieben Prozentpunkte (Seibert und Kleinert 2009: 3). Des Weiteren brachten Seibert und Kleinert die Entspannung auf dem dualen Ausbildungsmarkt im Jahr 2008 auch mit den deutlich gesunkenen Bewerberzahlen in Verbindung. So gingen die Bewerberzahlen 2008 im Vergleich zum Jahr 2007 in den neuen Bundesländern um 26 Prozentpunkte und in den alten Bundesländern um zwölf Prozentpunkte zurück (Seibert und Kleinert 2009: 2).

Der Ländermonitor berufliche Bildung 2015 beschäftigte sich mit dem vorhandenen Fachkräftepotenzial und den individuell betrachteten Zugangschancen in die Berufsbildung in allen 16 Bundesländern (Bertelsmann Stiftung 2015: 9). Im Rahmen dessen wurden die duale und vollzeitschulische Ausbildung sowie das unterhalb angesiedelte und nicht in einen berufsqualifizierenden Abschluss mündende Übergangssystem einbezogen (Bertelsmann Stiftung 2015: 8). Als Datengrundlage dienten diverse regelmäßig erhobene und für alle Bundesländer verfügbare amtliche Daten, Statistiken sowie repräsentative Surveys. In Bezug auf den Bereich der dualen Berufsausbildung kamen insbesondere die Statistiken des Bundesinstituts für Berufsbildung und der Bundesagentur für Arbeit zur Verwendung, während für das Schulberufs- und das Übergangssystem in erster Linie Daten der statistischen Ämter des Bundes und der Länder, insbesondere zur Integrierten Ausbildungsberichterstattung, genutzt wurden. Des Weiteren wurde der Einfluss von zusätzlichen Faktoren auf die Berufsbildung in den einzelnen Bundesländern überprüft wie beispielsweise der Einfluss der Wirtschaftskraft auf das Ausbildungsangebot der Betriebe oder die Auswirkungen der Schulabgangsquote auf die Ausbildungsnachfrage. Außerdem wurden Daten $\mathrm{zu}$ den öffentlichen Ausgaben für Berufsbildung herangezogen, um die berufsbildungspolitischen Aktivitäten in den einzelnen Bundesländern zu rekonstruieren (Bertelsmann Stiftung 2015: 10).

Im Rahmen der Ergebnisse stellte sich heraus, dass sowohl die Ausbildungsbetriebsquoten als auch die Ausbildungsquoten zwischen den Jahren 2007 und 2013 in allen 16 Bundesländern gesunken waren, wobei dies durch eine gestiegene Anzahl an sozialversicherungspflichtigen Beschäftigten und durch eine gesunkene Anzahl an Auszubildenden verursacht wurde (Bertelsmann Stiftung 2015: 13). Neben den gesunkenen Schülerzahlen auf allgemeinbildenden Schulen konnte als weiterer wesentlicher Trend ein verstärktes Interesse für ein Hochschulstudium ausgemacht werden (Bertelsmann Stiftung 2015: 22). Bei der Untersuchung des Verhältnisses zwischen Ausbildungsplatzangebot und -nachfrage wurde deutlich, dass das Angebot zwischen 2007 und 2013 bundesweit um etwa 13 Prozent 
gesunken war, während sich die Nachfrage im gleichen Zeitraum um etwa 19 Prozent verringerte. Aufgrund der Tatsache, dass die Nachfragequote stärker gesunken war als die Angebotsquote, konnten Veränderungen in Form der Angebots-Nachfrage-Relation auf dem dualen Ausbildungsmarkt als primäre Ursache sowohl auf Bundes- als auch auf Länderebene für die scheinbare Entlastung auf dem Ausbildungsmarkt identifiziert werden. Dies zeigte sich beispielsweise daran, dass die Nachfrage nach einem dualen Ausbildungsplatz stärker gesunken war wie das vorhandene Angebot an Ausbildungsplätzen (Bertelsmann Stiftung 2015: 15 f.).

Die Anzahl derjenigen, die in eine duale, in eine vollzeitschulische Berufsausbildung oder in das berufsvorbereitende Übergangssystem eintraten, verringerte sich bundesländerübergreifend zwischen 2005 und 2013 von 1,15 Millionen auf etwa 960.000 Personen. Hierfür konnte eine sukzessiv abnehmende Anzahl an Schulabgängerinnen und Schulabgängern von allgemeinbildenden Schulen verantwortlich gemacht werden, welche tendenziell mehr ein Hochschulstudium statt einer Berufsausbildung präferierten. Von den jährlichen Ausbildungsanfängerinnen und Ausbildungsanfängern in der Berufsbildung schaffte es bundesländerübergreifend ein immer größer werdender Anteil, den Übergang in eine duale oder vollzeitschulische Berufsausbildung zu realisieren. Insgesamt hatten sich die Chancen für alle Ausbildungsanfängerinnen und Ausbildungsanfänger, eine vollqualifizierende Berufsausbildung $\mathrm{zu}$ beginnen, seit dem Jahr 2005 erhöht. Im Bundesländervergleich lag die höchste Einmündungsquote in eine vollqualifizierende Berufsausbildung im Jahr 2005 bei 79 Prozent und 2013 bei 85 Prozent, während das Minimum von 53 auf 63 Prozent anstieg. Ein guter Schulabschluss führte zu einer deutlich erhöhten Einmündungswahrscheinlichkeit in eine vollqualifizierende Berufsausbildung. Fast alle Bewerberinnen und Bewerber mit Abitur oder Fachabitur erhielten einen dualen oder vollzeitschulischen Ausbildungsplatz oder nahmen ein Hochschulstudium auf, während der Anteil derjenigen mit einem Realschulabschluss, die in eine duale oder vollzeitschulische Berufsausbildung einmündeten, bundesweit bei 85 Prozent lag und von denjenigen, die maximal den Hauptschulabschluss erreicht hatten, 50 Prozent einen dualen oder vollzeitschulischen Ausbildungsplatz erhielten (Bertelsmann Stiftung 2015: 22 ff.).

Die Studie von Kleinert und Jacob untersuchte in einem historischen Rückblick die Entwicklung der Übergangschancen von Jugendlichen in eine Ausbildung oder ein Studium. Dabei wurden die Übergangschancen von Schulabgängerinnen und Schulabgängern aller Bildungsniveaus miteinbezogen und deren Übergangschancen unter dem Einfluss von 
demografischen und konjunkturellen Entwicklungen zwischen 1975 und 2005 untersucht. Konkret ging es darum, ob sich die Übergangschancen in Ausbildung und Studium durch den Einfluss der beiden genannten Entwicklungsfaktoren zwischen den verschiedenen Bildungsniveaus und innerhalb einer Schulabschlusskohorte zu verschiedenen Zeitpunkten verändert haben (Kleinert und Jacob 2012: 212). Dies wurde auf Basis des Datenmaterials der Retrospektivstudie „Arbeiten und Lernen im Wandel“" (ALWA) untersucht, welche vom Institut für Arbeitsmarkt- und Berufsforschung in den Jahren 2007 und 2008 repräsentativ durchgeführt wurde. Dabei wurden über 10.000 Personen in Deutschland der Geburtsjahrgänge 1956 bis 1988 retrospektiv zu ihren Bildungs- und Erwerbsverläufen sowie kognitiven Grundkompetenzen befragt (Kleinert et al. 2008: 4). Ein aus den ursprünglichen ALWA-Rohdaten neu erstellter Analysedatensatz bildete die empirische Datengrundlage, wobei alle Personen ausgeschlossen wurden, die ihre Schullaufbahn vor 1975 und nach 2005 beendet hatten. Dies lag darin begründet, dass es vor 1975 kaum und nach 2005 zu viele Abiturientinnen und Abiturienten gab und dies $\mathrm{zu}$ weitreichenden Verzerrungen in den Analysen hätte führen können. Des Weiteren wurden in der ALWA-Studie keine befragten Personen berücksichtigt, die die Schullaufbahn in der DDR oder in den neuen Bundesländern abgeschlossen haben, da die Übergangsbedingungen in der sozialistischen DDR andere waren als in der marktwirtschaftlich orientierten Bundesrepublik und nach der Wende auf dem Ausbildungsmarkt in den neuen Bundesländern eine besondere strukturelle Situation aufgrund des Wandels von einer Plan- zu einer Marktwirtschaft gegeben war (Kleinert und Jacob 2012: 218).

Der Übergangsprozess von der Schule in die Ausbildung wurde in zeitlicher Hinsicht mit einem festgelegten Übergangsbeginn durch den Abschluss der ersten ununterbrochenen Schullaufbahn operationalisiert. Hierzu zählte auch das Verlassen von beruflichen Schulen wie beispielsweise beruflichen Gymnasien, an denen ebenfalls allgemeinbildende Abschlüsse erworben werden können. Bei einer ununterbrochenen Schullaufbahn lagen zwischen sämtlichen Schulbesuchen während der Schullaufbahn maximal vier Monate. Der Abschluss des Übergangsprozesses wurde mit der Aufnahme einer vollqualifizierenden Ausbildung oder eines Studiums definiert, wozu jedoch keine berufsvorbereitenden Bildungsgänge zählten. Zeitliche Phasen während des Übergangsprozesses, in denen Wehr- oder Zivildienst geleistet worden war, wurden ebenfalls nicht berücksichtigt und aus der Übergangszeit herausgerechnet (Kleinert und Jacob 2012: 218). 
Im Rahmen der Ergebnisse kristallisierte sich ein Auseinanderentwickeln der Übergangschancen in eine Ausbildung oder ein Studium im Laufe der Zeit zwischen den Bildungsniveaus heraus. Insbesondere zu späteren Zeitpunkten hatten Schulabgängerinnen und Schulabgänger mit einem niedrigen oder mittleren Bildungsniveau schlechtere Übergangschancen als diejenigen mit einem höheren Bildungsniveau. Außerdem wurde festgestellt, dass vor Mitte der 1980er Jahre demografische Entwicklungen in Form eines Übertritts geburtenstarker Jahrgänge auf den Ausbildungsmarkt die Übergangschancen gemindert hatten, während für die Minderung der Übergangschancen ab Mitte der 1980er Jahre vorwiegend negative konjunkturelle Veränderungen in Form von hohen Arbeitslosigkeitsquoten verantwortlich waren. Bezüglich der Veränderungen innerhalb der Bildungsniveaus haben sich die Übergangsprobleme derer mit einem Haupt- oder Realschulabschluss in den untersuchten 30 Jahren deutlich vergrößert, wobei die Übergangschancen dieser beiden Bildungsgruppen in einer Abhängigkeit zur gegebenen konjunkturellen und demografischen Situation standen. Die Übergangschancen von Abiturientinnen und Abiturienten waren dagegen unberührt von diesen Einflüssen (Kleinert und Jacob 2012: 229).

Die Untersuchung von Protsch versuchte unter anderem Erkenntnisse zu den Wirkungen sozialer Mechanismen als mögliche Ursachen zu bildungsspezifischen Ungleichheiten beim Zugang zur beruflichen Ausbildung zu gewinnen (Protsch 2014: 99). Zunächst wurden die bildungsgruppenspezifischen Ausbildungschancen von verschiedenen Schulabgangskohorten zwischen 1954 und 2002 anhand des Vergleichs von deren Übergangsquoten und -mustern in die Berufsausbildung oder in das Studium untersucht. Außerdem wurde ein möglicher Einfluss von erhöhten kognitiven und nicht-kognitiven Voraussetzungen von Seiten des Ausbildungsmarktes auf eventuell veränderte berufsspezifische Ausbildungschancen überprüft (Protsch 2014: 105 f.). Auch die Bedeutung von zertifizierten Leistungen in Form von Schulnoten sowie kognitiven und nicht-kognitiven Potenzialen für die einzelnen Bildungsgruppen im Hinblick auf eine erfolgreiche Ausbildungsplatzsuche bildeten einen Gegenstand (Protsch 2014: 145). Außerdem ging es um die Frage, ob betriebliche Auswahlverfahren als soziale Mechanismen zum Ausschluss auf dem Ausbildungsmarkt Folgen für die Ausbildungschancen von Hauptschülerinnen und Hauptschülern haben konnten (Protsch 2014: 169). Als empirische Datenbasis dienten retrospektive Längsschnittdaten der Deutschen Lebensverlaufsstudien des Max-Planck-Instituts für Bildungsforschung sowie der Befragung „Arbeiten und Lernen im Wandel“ (ALWA) des Instituts für Arbeitsmarkt- und 
Berufsforschung (Protsch 2014: 21) zu Bildungs- und Erwerbsverläufen, wodurch diese Verläufe zwischen 1954 und 2002 sichtbar wurden. Durch Bildung verschiedener Schulabgangskohorten wurden bei der Untersuchung der Zugänge in die Berufsausbildung insgesamt etwas mehr als 12.000 Personen einbezogen. Die einzelnen Teilstudien der Deutschen Lebensverlaufsstudien umfassten die Geburtsjahrgänge 1939 bis 1941 und 1949 bis 1951, die Jahrgänge 1954 bis 1956 und 1959 bis 1961 sowie die Jahrgänge 1964 und 1971. Dagegen beinhaltete die ALWA-Studie die Jahrgänge von 1956 bis 1988 (Protsch 2014: 107 f.). Als weitere empirische Datenbasis dienten die Erhebungswellen zwischen 2004 und 2010 des Sozio-ökonomischen Panels (SOEP) des Deutschen Instituts für Wirtschaftsforschung als einer repräsentativen, jährlichen Befragung in privaten deutschen Haushalten. Aus dem SOEP wurde ein Sample mit 502 Jugendlichen der Geburtsjahrgänge 1987 bis 1992 verwendet (Protsch 2014: 146). Festgestellt wurde unter anderem, dass die Ausbildungschancen von Hauptschülerinnen und Hauptschülern im betrachteten Kohortenverlauf geringer wurden (Protsch 2014: 198). Deren Anteil in einer vollqualifizierenden Ausbildung verringerte sich von 71 Prozent bei den Schulabgangskohorten von 1954 bis 1972 auf 14 Prozent bei den Kohorten von 1990 bis 2002 (Protsch 2014: 114) unter der Berücksichtigung, dass der generelle Anteil der Hauptschülerinnen und Hauptschüler im Zeitverlauf gesunken war (Statista Research Department 2012). Jugendliche mit einem Realschulabschluss hatten insgesamt bessere Chancen beim Übergang in die Berufsausbildung als diejenigen mit einem Hauptschulabschluss (Protsch 2014: 201), da ihr Anteil in einer vollqualifizierenden Ausbildung von 22 Prozent bei den Schulabgangskohorten von 1954 bis 1972 auf 27 Prozent bei den Kohorten von 1990 bis 2002 anstieg. Bei denjenigen mit einer Fachhochschulreife konnte ein noch deutlicherer Anstieg verzeichnet werden, nämlich von sieben Prozent bei den Kohorten 1954 bis 1972 auf 59 Prozent bei den Kohorten 1990 bis 2002 (Protsch 2014: 114). Bei Hauptschülerinnen und Hauptschülern war eine gute Mathematiknote essentiell wichtig, um sich innerhalb ihrer Bildungsgruppe positiv abheben zu können, wogegen Realschülerinnen und Realschüler den Übergang in die Berufsausbildung durch eine gewissenhafte Persönlichkeitseigenschaft bei der Ausbildungsplatzsuche und -bewerbung positiv beeinflussen konnten. Das betriebliche Auswahlverhalten wurde bei der Besetzung von Ausbildungsplätzen maßgeblich von wahrgenommenen Veränderungen des Arbeitsmarktes und den damit verbundenen veränderten beruflichen Anforderungen beeinflusst und legitimiert. Hauptschülerinnen und Hauptschüler galten nicht als Zielgruppe 
der Betriebe, welche den erforderlichen Fachkräftenachwuchs sicherstellen sollte (Protsch 2014: $201 \mathrm{f}$.).

Fasst man den Studienüberblick zum Übergang von der Schule in die Ausbildung oder das Studium zusammen, lässt sich sagen, dass die Partizipation an einer dualen, vollzeitschulischen sowie akademischen Ausbildung zwar im Zeitverlauf anstieg, jedoch Schulabgängerinnen und Schulabgänger ohne Schulabschluss oder mit einem Hauptschulabschluss noch immer die mit Abstand schlechtesten Chancen hatten, in eine vollqualifizierende Ausbildung überzugehen. Die insgesamt gestiegene Ausbildungsbeteiligung musste wiederum damit relativiert werden, dass die Bewerberzahlen im Zeitverlauf vor allem im dualen Ausbildungssystem deutlich gesunken waren. Demgegenüber war das Interesse an der Aufnahme eines Hochschulstudiums im Zeitverlauf deutlich gestiegen. Somit tangierten demografische Entwicklungen die Übergangschancen der Schulabgängerinnen und Schulabgänger wesentlich. Darüber hinaus waren jedoch auch Jugendliche mit einem Migrationshintergrund beim Übergang in die Ausbildung oftmals im Nachteil. Auch wirtschaftliche Veränderungen oder die soziale Herkunft konnten in den bisherigen Forschungsarbeiten als bedeutende Prädiktoren für die Übergangschancen in die Ausbildung ausgemacht werden. Somit konnte zwar von einer gestiegenen Ausbildungsquote gesprochen werden, welche durch gesunkene Bewerberzahlen bei einer dualen Berufsausbildung $\mathrm{zu}$ relativieren war. Jedoch bestand weiterhin eine Teilgruppe, welche erhebliche Übergangsprobleme in die Ausbildung aufwies. Diese Gruppe ließ sich durch einen niedrigen oder gar keinen Schulabschluss, eine geringe soziale Herkunft in Form von mangelnden familiären Unterstützungsmöglichkeiten oder auch durch einen Migrationshintergrund charakterisieren.

\subsection{Studien zur Übergangsdauer von der Schule in eine vollqualifizierende berufliche oder akademische Ausbildung}

Betrachtet man im Rahmen des Übergangs von der Schule in die Ausbildung oder das Studium auch die Dauer des Übergangsprozesses, lassen sich anhand bisheriger Studien ebenfalls interessante Erkenntnisse gewinnen. Schon zu Beginn der 1990er Jahre wurde festgestellt, dass sich in Deutschland etwa ein Jahr nach dem Schulabschluss zwei Drittel der Schulabgängerinnen und Schulabgänger in einer schulischen oder dualen Berufsausbildung befanden, während zu diesem Zeitpunkt nur ein sehr geringer Anteil ein Hochschulstudium aufgenommen hatte (Büchtemann et al. 1993: 509). Auch in aktuelleren Untersuchungen 
bestätigten sich diese Übergangsquoten. Viele Jugendliche mit einem Hauptschulabschluss befanden sich bereits drei Monate nach Beendigung des neunten Schuljahres und dem Verlassen der Schule in einer beruflichen oder schulischen Ausbildung (Solga et al. 2012: 4). Bezüglich des Übergangs in eine Berufsausbildung begann knapp die Hälfte aller Jugendlichen während der ersten sechs Monate nach dem Verlassen der allgemeinbildenden Schule eine duale oder vollzeitschulische Ausbildung. Nach 18 Monaten beziehungsweise 30 Monaten hatte ein Großteil der Jugendlichen eine duale oder vollzeitschulische Ausbildung begonnen (Beicht und Ulrich 2008b: 6, 36). Dennoch war ein beträchtlicher Anteil der Jugendlichen ohne Studienberechtigung auch zwei Jahre nach Ende der Schulzeit noch nicht in eine betriebliche Berufsausbildung eingemündet (Eberhard et al. 2013: 8). Auch vier Jahre nach dem Verlassen der Hauptschule befand sich ein kleinerer Anteil an Jugendlichen weder in einer schulischen noch in einer beruflichen Bildung (Gaupp 2008: 28). Eine Abnahme von direkten Übergängen in die Ausbildung wurde somit durch eine Zunahme von zeitlich verzögerten Übergängen größtenteils kompensiert (Brandes et al. 1986: 287).

Bei Betrachtung der Übergangsdauer getrennt nach Schulabschluss offenbart der bisherige Forschungsstand unterschiedliche Erkenntnisse. Insgesamt zeigte sich, dass sich ein höherer Schulabschluss positiv auf einen häufigen und schnellen Eintritt in eine Berufsausbildung auswirkte (Beicht und Ulrich 2008b: 30 f.). Für Bewerberinnen und Bewerber mit schwächeren schulischen Leistungen wurden einerseits längere Übergangszeiträume in die duale Berufsausbildung festgestellt (Seibert und Kleinert 2009: 1). Andererseits zeigte sich jedoch, dass auch Jugendlichen, die keinen Schulabschluss erworben hatten, der Übergang mehrheitlich zwei bis drei Monate nach dem Verlassen der Schule (Solga 2002: 9) und etwa einem Drittel aller Hauptschulabsolventinnen und Hauptschulabsolventen ein direkter Übergang in eine betriebliche Berufsausbildung (Carl et al. 2002: 18) gelang. Dennoch hatten viele Schulabgängerinnen und Schulabgänger mit einem Hauptschulabschluss, Realschulabschluss oder mit einem Fachabitur oder Abitur nicht im Jahr des Erwerbs des Schulabschlusses eine direkte Einmündung in eine berufliche oder akademische Ausbildung vollzogen (Lex und Zimmermann 2011: 614 f.). In einer anderen Untersuchung zeigte sich im Gegensatz dazu, dass jede zweite Studienanfängerin beziehungsweise jeder zweite Studienanfänger das Studium noch im gleichen Jahr aufnahm, in dem er beziehungsweise sie die allgemeine Hochschulreife erworben hatte (Willich et al. 2011: 2). Sechs Monate nach Verlassen der allgemeinbildenden Schule befand sich etwa die Hälfte der Jugendlichen mit einem mittleren Schulabschluss oder einem höheren Schulabschluss in einer dualen oder 
vollzeitschulischen Ausbildung und hatte damit eine wesentlich höhere Ausbildungsquote als diejenigen mit maximal einem Hauptschulabschluss (Beicht und Ulrich 2008b: 8). 18 Monate nach dem Ende der Schulzeit war der Anteil der Jugendlichen mit einem niedrigen Schulabschluss in einer dualen oder vollzeitschulischen Ausbildung geringer als der Anteil derjenigen mit einem mittleren oder einem höheren Schulabschluss. Letztere Gruppe hatte dabei den höchsten Anteil beim Partizipieren an einer dualen oder vollzeitschulischen Ausbildung. 30 Monate nach dem Ende der Schulzeit blieben die Unterschiede zwischen den drei Schulabschlussgruppen in der Ausbildungspartizipation weiterbestehen beziehungsweise nahmen noch etwas zu, was auf weiterhin gravierende Unterschiede zwischen den Schulabschlussformen auch 30 Monate nach Beendigung der Schulzeit hindeutete (Beicht und Ulrich 2008b: 10).

Auch in Abhängigkeit zu diversen weiteren Faktoren neben dem erworbenen Schulabschluss zeigten sich unterschiedliche Entwicklungen der Übergangsdauer. Nichtstudienberechtigte Jugendliche, die nach 2006 die allgemeinbildende Schulzeit beendeten und auf der Suche nach einem betrieblichen Ausbildungsplatz waren, hatten im Vergleich zu Schulabgängerinnen und Schulabgängern des Jahres 2006 etwas bessere Chancen, zügig in eine Ausbildung überzugehen. Des Weiteren hing die Übergangsdauer von nichtstudienberechtigten Jugendlichen in eine nichtakademische Ausbildung neben den schulischen Leistungen wie dem Schulabschluss oder den Abschlussnoten von der sozialen oder ethnischen Herkunft der Jugendlichen ab. Bei Nicht-Vorhandensein eines Berufsausbildungsabschlusses oder keiner vorhandenen Erwerbstätigkeit beider Elternteile verringerten sich die Übergangschancen. Auch ein Migrationshintergrund wirkte sich negativ auf die Übergangschancen der Jugendlichen aus. Je höher der erreichte Schulabschluss und je besser die Abschlussnoten waren, desto zügiger gelang der Übergang in eine nichtakademische Berufsausbildung. Trotz vermehrter institutioneller Unterstützungsmaßnahmen hatte sich die Übergangsdauer bis zur Einmündung in eine berufliche Ausbildung nicht verkürzt, was dem Ausbildungsplatzmangel über die Jahre hinweg geschuldet war (Eberhard et al. 2013: 8 f.). Auch der elterliche Bildungsabschluss spielte bei der Übergangsdauer eine wichtige Rolle. Bei Jugendlichen, bei denen kein Elternteil über eine abgeschlossene Berufsausbildung verfügte, gestaltete sich der Übergang in die Ausbildung deutlich langwieriger (Solga et al. 2012: 5). Bei Betrachtung der Schulabschlussgruppe mit einer allgemeinen Hochschulreife stellte sich heraus, dass sich viele Studienberechtigte ein halbes Jahr nach dem Erwerb der Hochschulreife an einer 
Hochschule eingeschrieben hatten. Ein deutlich geringerer Anteil dieser Gruppe hatte zu diesem Zeitpunkt eine Berufsausbildung aufgenommen (Heine et al. 2010: 1). Darüber hinaus waren aber auch die Gegebenheiten der regionalen Arbeitsmärkte bei der Dauer des Übergangsprozesses maßgeblich, wobei es in diesem Zusammenhang deutliche Unterschiede gab (Gaupp et al. 2008: 388). Jugendliche aus Regionen mit einer höheren Arbeitslosenquote nahmen deutlich später eine Berufsausbildung auf als Jugendliche aus Regionen mit einer niedrigen Arbeitslosenquote (Gaupp et al. 2008: 399).

In Bezug auf die bisherige Forschung zur Übergangsdauer in die Ausbildung sollen zwei Forschungsarbeiten detaillierter betrachtet werden. Die Übergangsstudie des Bundesinstituts für Berufsbildung (BIBB) aus dem Jahr 2011 ging der Frage nach, welche Auswirkungen die Veränderung des Ausbildungsmarktes von einem angebotsorientierten zu einem nachfrageorientierten Ausbildungsmarkt auf die Bildungs- und Berufsverläufe von jungen Menschen in Deutschland hatte. Außerdem wurde untersucht, wie Unterstützungsangebote von Institutionen wie beispielsweise Angebote zur Berufsorientierung während der allgemeinbildenden Schulzeit den Übergang in eine berufliche Ausbildung beeinflusst haben. Dabei stellte die BIBB-Übergangsstudie eine Wiederholungsstudie der ersten Übergangsstudie aus dem Jahr 2006 dar, bei welcher insgesamt 5.579 junge Erwachsene zwischen 18 und 24 Jahren im Zeitraum zwischen Juli 2011 und September 2011 im Rahmen von Telefoninterviews per Mobilfunk befragt wurden. Bei den befragten jungen Erwachsenen handelte es sich um eine deutschlandweite und repräsentative Auswahl. Diese wurden zu ihren Bildungs- und Berufsverläufen seit der Grundschule, zu den dabei erhaltenen institutionellen Unterstützungen und zum erfolgreichen Abschluss oder vorzeitigen Abbruch der einzelnen Bildungsphasen befragt (Eberhard et al. 2013: 7). Bei den Ergebnissen stellte sich heraus, dass nichtstudienberechtigte Jugendliche, die nach 2006 die allgemeinbildende Schulzeit beendeten und auf der Suche nach einem betrieblichen Ausbildungsplatz waren, im Vergleich zur Schulabgangs-Kohorte von 2006 etwas bessere Chancen hatten, zügig eine Ausbildung aufzunehmen. Diese Erkenntnis untermauerte den Höhepunkt der Krise auf dem Ausbildungsmarkt im Jahr 2006. Dennoch waren 38 Prozent der Jugendlichen, die nach 2006 die Schulzeit beendeten und zu diesem Zeitpunkt nach einem Ausbildungsplatz suchten, auch zwei Jahre danach noch nicht in einer betrieblichen Berufsausbildung. Die Übergangsdauer von nichtstudienberechtigten Jugendlichen in eine nichtakademische Ausbildung wurde neben den schulischen Leistungen wie dem Schulabschluss oder den Abschlussnoten auch von der sozialen oder ethnischen Herkunft der Jugendlichen beeinflusst. Lag zum Beispiel bei beiden 
Elternteilen kein Berufsausbildungsabschluss vor oder gingen beide Elternteile keiner Erwerbstätigkeit nach, hatte dies schlechtere Übergangschancen für die jeweiligen Jugendlichen zur Folge. Jugendliche mit einem Migrationshintergrund hatten ebenfalls schlechtere Übergangschancen, um in eine Ausbildung zu gelangen. Je höher der erreichte Schulabschluss und je besser die Abschlussnoten waren, desto zügiger konnte der Übergang in eine nichtakademische Berufsausbildung realisiert werden. Trotz vermehrter institutioneller Unterstützungsmaßnahmen kam es zu keiner Verkürzung der Übergangsdauer bis zur Einmündung in eine Berufsausbildung, was durch den Mangel an Ausbildungsplätzen über die Jahre hinweg verursacht wurde (Eberhard et al. 2013: 8 f.).

In einer anderen Studie von Lex und Zimmermann wurden die nach dem Besuch der allgemeinbildenden Schule vollzogenen Übergänge in eine Berufsausbildung oder ein Studium bis zum Erwerb von anerkannten Zertifikaten bei Personen mit unterschiedlichen Schulabschlüssen im Zeitraum von Mitte der 1990er Jahre bis zum Jahr 2007 untersucht (Lex und Zimmermann 2011: 603). Konkret untersuchten Lex und Zimmermann die Fragestellungen, welcher Gruppe von Jugendlichen oder jungen Erwachsenen ein direktes unmittelbares Einmünden in die Berufsausbildung oder das Studium gelang, wer für dieses Einmünden Umwege, Warteschleifen oder Zwischenschritte in Kauf nehmen musste und wer die Berufsausbildung oder das Studium mit Erfolg absolvierte und wer nicht. Als Datengrundlage verwendeten sie Daten des Surveys „AID:A - Aufwachsen in Deutschland: Alltagswelten“ des Deutschen Jugendinstituts, bei dem es sich um eine im Herbst 2009 basierend auf einer repräsentativen Stichprobe von 25.000 Personen in Form eines computergestützten Telefoninterviews durchgeführten bundesweiten Untersuchung zur retrospektiven Erfassung der Lebenslagen von Kindern, Jugendlichen, Erwachsenen und Familien handelte. Aus diesen Daten bildeten 7.448 Personen der untersuchten Altersgruppe der 18- bis 32-Jährigen die verwendete Teilstichprobe, da sie bereits die Schule beendet hatten (Lex und Zimmermann 2011: 608 f.). Lex und Zimmermann kamen zu dem Ergebnis, dass der Schulabschluss, das Geschlecht sowie die soziale und ethnische Herkunft die Entwicklung des Übergangsprozesses beeinflusste (Lex und Zimmermann 2011: 603). Beispielsweise zeigte sich, dass 41,3 Prozent derjenigen mit einem Hauptschulabschluss, 29,6 Prozent derer mit einem Realschulabschluss und 30,0 Prozent derer mit einem Fachabitur oder Abitur nicht im Jahr des Erwerbs des Schulabschlusses eine direkte Einmündung in eine berufliche oder akademische Ausbildung vollzogen hatten. Von den Personen mit einer Studienberechtigung, die sich für eine berufliche Ausbildung entschieden hatten, begannen 
70,0 Prozent die Ausbildung im gleichen Jahr des Schulabschlusses, während dies bei den Studierenden 73,9 Prozent waren (Lex und Zimmermann 2011: 614 f.). Auch die Faktoren der Region Ost - West sowie der Situation auf dem Ausbildungsmarkt beeinflussten den Übergangsverlauf maßgeblich. Über alle Schulabschlussformen hinweg wurde deutlich, dass ein niedrigeres elterliches Bildungsniveau erstaunlicherweise mit einer größeren Wahrscheinlichkeit zu einem unmittelbaren Übergang in eine Berufsausbildung im Anschluss an die allgemeinbildende Schule führte. Jugendliche aller Schulabschlussformen, deren Eltern maximal über einen Hauptschulabschluss verfügten, gingen signifikant häufiger direkt im Anschluss an die Schulzeit in eine duale oder vollzeitschulische Berufsausbildung über als Jugendliche, deren Eltern einen Hochschulabschluss aufwiesen (Lex und Zimmermann 2011: $603,616)$.

Zusammenfassend betrachtet zeigten die bisherigen Studien zur Entwicklung der Übergangsdauer, dass ein Großteil der Schulabgängerinnen und Schulabgänger innerhalb eines überschaubaren Zeitraums in eine Ausbildung überging. Andererseits unterschied sich dies jedoch nochmals nach dem erreichten Schulabschluss und nach dem jeweiligen Ausbildungstyp. Je höher der erworbene Schulabschluss war, desto schneller gelang der Übergang. Auch gestalteten sich die Übergänge in ein Hochschulstudium manchmal schneller als in eine duale oder vollzeitschulische Berufsausbildung. Somit verzögerte sich für diejenigen Jugendlichen, welche lediglich einen Hauptschulabschluss oder keinen Schulabschluss erworben hatten, der Eintritt in eine vollqualifizierende Ausbildung zum Teil deutlich. Auch wenn sich in Studien gezeigt hat, dass ein Teil der Jugendlichen mit einem niedrigen schulischen Bildungsgrad dennoch einen sehr zügigen und schnellen Übergang in eine Ausbildung vollzog, war für diese Gruppe ein deutlich verlängerter Übergangszeitraum bis zum Ausbildungsbeginn zu diagnostizieren. Auf der Grundlage der betrachteten Studien war somit ein starker Zusammenhang zwischen dem erworbenen allgemeinbildenden Schulabschluss und der Übergangsdauer vom Verlassen der allgemeinbildenden Schule bis zum Einmünden in eine duale, vollzeitschulische oder akademische Erstausbildung festzustellen. Darüber hinaus standen auch soziale, ethnische oder geschlechtsbezogene Faktoren durchaus im Zusammenhang mit der Dauer des Übergangsprozesses. Jugendliche, bei denen die Eltern über keine abgeschlossene Ausbildung verfügten, und mit Migrationshintergrund waren tendenziell von einem verlängerten Übergangsprozess betroffen. 


\subsection{Studien zu Übergangsstationen von der Schule in eine vollqualifizierende berufliche oder akademische Ausbildung}

Betrachtet man bisherige Forschungsarbeiten $\mathrm{zu}$ den Stationen, welche während des Übergangsprozesses von der Schule in die Ausbildung oder in das Studium durchlaufen werden, treten ebenfalls einige interessante Erkenntnisse hervor. Insgesamt hat sich der Übergang von der Schule in eine Berufsausbildung dahingehend gewandelt, dass dieser Prozess sukzessive im Laufe der Zeit in immer mehr Zwischenschritten und Übergangsstationen absolviert wurde (Reißig und Gaupp 2018: 192; Fink 2011: 92). Dieser gestiegene Anteil an Übergangsstationen nach dem Verlassen der allgemeinbildenden Schule bestätigte sich durch diverse Studien. In diesen wurde deutlich, dass ein erheblicher Teil der Jugendlichen nach dem Verlassen der Schule nicht direkt in eine Berufsausbildung einmündete, sondern sich in Alternativen aufhielt (Eberhard und Ulrich 2009: 2 ff.). Somit gestaltete sich der Einmündungsprozess in eine berufliche Ausbildung als sehr komplex, was mit dem Absolvieren von diversen Zwischenstationen oder einem weiteren Schulbesuch verbunden war (Gaupp 2008: 27).

Betrachtet man die bisherige Forschungslage zu den absolvierten Übergangsstationen getrennt nach den einzelnen Schulabschlussgruppen, lassen sich unterschiedliche Erkenntnisse gewinnen. Für Bewerberinnen und Bewerber mit schwächeren schulischen Leistungen wurde beim Übergang in das duale Ausbildungssystem beispielsweise ein häufigeres Absolvieren von berufsvorbereitenden Maßnahmen festgestellt (Seibert und Kleinert 2009: 1). Ein Großteil der Hauptschulabsolventinnen und Hauptschulabsolventen bewältigte in den drei bis vier Jahren nach dem Erreichen des Hauptschulabschlusses zwei oder mehr Stationen (Carl et al. 2002: 18). Immer mehr Jugendliche ohne einen Schulabschluss landeten beim Übergang von der Schule in die berufliche Ausbildung in berufsvorbereitenden Maßnahmen. Durch diese Maßnahmen konnten sie ihre Chancen auf dem Ausbildungs- und Arbeitsmarkt jedoch kaum verbessern. (Solga 2004: 99). Seit dem Ausbau von berufsvorbereitenden Maßnahmen während der 1970er Jahre hatte ein beträchtlicher Anteil der schulabschlusslosen Jugendlichen, welche mindestens eine berufliche Bildungsepisode vorweisen konnten, zunächst oder ausschließlich an einer berufsvorbereitenden Maßnahme teilgenommen mit dem primären Ziel, einen Schulabschluss nachzuholen (Menze und Holtmann 2019: 521; Solga 2002: 10). Dagegen mündeten von den Jugendlichen mit einem Hauptschulabschluss oder einer mittleren Reife ein deutlich geringerer Anteil in eine berufsvorbereitende Maßnahme ein (Solga 2002: 10). Viele Jugendliche, die an einer berufsvorbereitenden 
Maßnahme des Übergangssystems partizipiert hatten, blieben langfristig wesentlich öfter ohne eine abgeschlossene Berufsausbildung als diejenigen ohne Schulabschluss, welche in ihrer ersten Ausbildungsepisode in eine Berufsausbildung einmündeten. Ein beträchtlicher Anteil hatte lediglich an berufs- und ausbildungsvorbereitenden Maßnahmen partizipiert oder ausschließlich aus Maßnahmen bestehende Ausbildungskarrieren absolviert (Solga 2002: 14 f.). Damit war ein deutlicher Anstieg des Anteils der Jugendlichen mit einem Hauptschulabschluss beim Einmünden in das Übergangssystem zu verzeichnen (Konsortium Bildungsberichterstattung 2006: 17 f.). Jugendliche mit maximal einem Hauptschulabschluss traten nach dem Verlassen der allgemeinbildenden Schule zunächst größtenteils in das Übergangssystem ein (Bertelsmann Stiftung 2015: 25; Baethge et al. 2007: 8), was den deutlichen Zuwachs am Übergangssystem unterstreicht. Diese Gruppe musste somit Umwege beziehungsweise Überbrückungsphasen in Kauf nehmen (Lex und Zimmermann 2011: 621 f.).

Ein Großteil der Hauptschülerinnen und Hauptschüler musste mehrere Zwischenstationen im Übergangssystem absolvieren (Lex und Geier 2010: 183). Jugendliche mit einem niedrigen oder ohne Schulabschluss waren die primäre Zielgruppe des berufsvorbereitenden Übergangssystems (Holtmann et al. 2019: 380), was sich in ihren nicht unerheblichen Anteilen in diesen Maßnahmen sechs und auch 18 Monate nach dem Verlassen der allgemeinbildenden Schule niederschlug (Beicht und Ulrich 2008b: 10, 19; Christe 2016: 15). Andere Untersuchungen kamen zu ähnlichen Erkenntnissen, da viele Jugendliche mit oder ohne Hauptschulabschluss erst nach einem Aufenthalt von mehreren Jahren im Übergangssystem eine vollqualifizierte Berufsausbildung begannen (Plicht 2016: 147; Krüger-Charlé 2010: 1). Viele Jugendliche mit maximal einem Hauptschulabschluss blieben dagegen sechs Monate nach Beendigung der Schulzeit aus verschiedensten Gründen zu Hause (Beicht und Ulrich 2008b: 10).

Im Gegensatz dazu zeigte die bisherige Forschung aber auch, dass viele Hauptschülerinnen und Hauptschüler im Herbst des ersten Übergangsjahres weiter an einer schulischen Bildung partizipierten, um in erster Linie den mittleren Schulabschluss zu erreichen. Vor allem Jugendliche mit guten Schulnoten entschieden sich für eine schulische Höherqualifikation (Gaupp und Geier 2008: 43). Viele Hauptschülerinnen und Hauptschüler besuchten vorzugsweise weiter die Schule mit der Zielsetzung, einen höheren Bildungsabschluss zu erwerben, um bessere Chancen auf eine Ausbildung zu haben (Lex und Geier 2010: 184). Jugendliche mit einem mittleren Schulabschluss besuchten die Berufsfachschule ohne 
abschließendes Erreichen eines Berufsabschlusses genauso häufig wie Jugendliche mit einem niedrigen Schulabschluss (Beicht und Ulrich 2008b: 10). Auch ein beträchtlicher Anteil der Jugendlichen mit einem mittleren Schulabschluss mündete in das berufsvorbereitende Übergangssystem ein (Baethge et al. 2007: 8). Jedoch gelang dieser Gruppe der Wechsel von den Maßnahmen des Übergangssystems in eine Berufsausbildung deutlich häufiger und schneller (Beicht und Ulrich 2008b: 19). In dieser Schulabschlussgruppe wurde auch entweder direkt im Anschluss an die mittlere Reife oder im späteren Verlauf das Abitur nachgeholt (Winkler 2017: 275).

Auch Jugendliche mit guten Schulleistungen entschieden sich meist für schulische Wege in Form von schulischen Zwischenschritten vor einem Ausbildungsbeginn oder für den Zugang zur tertiären Bildung (Gaupp et al. 2011: 182 f.). Viele Studienberechtigte hatten bereits an diversen Weiterbildungsveranstaltungen teilgenommen (Lewin und Schacher 1990: 1), absolvierten ein Praktikum, befanden sich in Übergangstätigkeiten wie Jobben, Au-PairAufenthalten oder einem freiwilligen sozialen Jahr oder leisteten Wehr- beziehungsweise Zivildienst (Heine et al. 2010: 1; Beicht und Ulrich 2008b: 8).

Im Rahmen der bisherigen Forschung fällt jedoch auch auf, dass neben dem erworbenen Schulabschluss noch weitere Faktoren in Zusammenhang mit den absolvierten Übergangsstationen standen. Bewerberinnen und Bewerber in den neuen Bundesländern, welche sich erfolglos um einen betrieblichen Ausbildungsplatz beworben hatten, wurden größtenteils durch außerbetriebliche vollqualifizierende oder schulische Berufsausbildungsformen aufgefangen, während in den alten Bundesländern diejenigen, die keinen betrieblichen Ausbildungsplatz ergattern konnten, in das Übergangssystem einmündeten. Vor allem Frauen sowie Jugendliche mit einem Migrationshintergrund landeten mit einer größeren Wahrscheinlichkeit in einer teilqualifizierenden Ausbildungsform oder Maßnahme (Eberhard und Ulrich 2009: 2 ff.). In einer anderen Studie wurde dagegen festgestellt, dass junge Frauen oftmals eine schulische Weiterqualifizierung mit der Entscheidung für einen über mehrere Jahre fortgesetzten Schulbesuch favorisierten (Gaupp et al. 2011: 182). Des Weiteren wurden zusätzliche relevante Faktoren deutlich, welche einen positiven Einfluss auf einen gelungenen Übergang in eine berufliche Ausbildung hatten. Hierzu zählten das Absolvieren von Betriebspraktika, die Unterstützung durch die Herkunftsfamilie (Gaupp et al. 2008: 388; Lex und Zimmermann 2011: 603) sowie eine professionelle Unterstützung im Rahmen des Berufswahlprozesses. Jedoch spielten zusätzlich auch die Gegebenheiten der regionalen Arbeitsmärkte beim Übergangsprozess eine relevante 
Rolle (Gaupp et al. 2008: 388). Bei Vorhandensein von Unterstützungsmöglichkeiten der Herkunftsfamilie durchliefen Jugendliche vor dem Beginn der Erstausbildung zusätzliche Bildungs- und Orientierungsphasen. Jugendliche ohne elterliche Unterstützungsmöglichkeiten mussten dagegen Umwege beziehungsweise Überbrückungsphasen durchlaufen (Lex und Zimmermann 2011: 621 f.). Jugendliche mit einem höheren elterlichen Sozialstatus hatten somit auch größere Chancen auf eine schulische Höherqualifikation in Form eines verlängerten Schulbesuchs (Gaupp et al. 2011: 182).

Dagegen war ein direkter Einstieg in eine Berufsausbildung für Jugendliche mit einem Migrationshintergrund nur schwer möglich (Gaupp et al. 2011: 180, 182). Diese Gruppe absolvierte vorwiegend berufsvorbereitende Maßnahmen, mündete jedoch seltener beziehungsweise später in eine Berufsausbildung ein als Jugendliche ohne Migrationshintergrund (Lex und Geier 2010: 184). Jugendliche mit Migrationshintergrund oder aus bildungsfernen Familien hatten auch nach einem dreijährigen Aufenthalt im Übergangssystem den Übergang in eine vollqualifizierende Berufsausbildung noch nicht vollzogen (Krüger-Charlé 2010: 1), wodurch sich ihre Übergangswahrscheinlichkeit wesentlich verringerte (Baethge et al. 2007: 43).

Bei Betrachtung der Stationen an sich, die während des Übergangsprozesses durchlaufen werden, fallen in den bisherigen Studien einige stark frequentierte Stationen auf. Insgesamt betrachtet wurden im Laufe der Zeit immer mehr Umwege sowie Warteschleifen mit berufsvorbereitenden Maßnahmen absolviert (Pätzold 2008: 603; Eberhard und Ulrich 2009: 2 ff.; Konsortium Bildungsberichterstattung 2006: 17 f.; Baethge et al. 2007: 7; Gaupp et al. 2005: 147 f.; Beicht und Ulrich 2008a: 11). In anderen Studien war von einer Abnahme des Anteils im berufsvorbereitenden Übergangssystem die Rede, wobei dieser Anteil immer noch recht hoch war (Bertelsmann Stiftung 2015: 22). Der Aufenthalt in diesen berufsvorbereitenden Maßnahmen gestaltete sich für einige Jugendliche zum Teil recht lange (Beicht und Ulrich 2008b: 6). Zum berufsvorbereitenden Übergangssystem gehören unter anderem auch das Berufsgrundbildungs- und das Berufsvorbereitungsjahr. Bereits in früheren Jahrzehnten konnte eine deutliche Zunahme bei diesen Maßnahmen verzeichnet werden (Schober 1985: 251). Diejenigen, die an einem Berufsvorbereitungsjahr teilgenommen hatten, konnten mit einer größeren Wahrscheinlichkeit einen erfolgreichen Berufsabschluss bewerkstelligen (Erban 2010: 345). Gleiches galt auch für die Teilnehmerinnen und Teilnehmer an einem Berufsgrundbildungsjahr (Mollwo 1980: 242). Auch der Besuch von teilqualifizierenden Berufsfachschulen wurde als Übergangsstation oftmals favorisiert, um 
einen höheren Schulabschluss zu erwerben (Beicht 2009: 14). Viele Jugendliche absolvierten alternative Bildungswege (Stegmann und Holzbauer 1978: 148), wobei einige eine Fachoberschule oder ein Fachgymnasium besuchten (Beicht und Ulrich 2008b: 6).

Herausgefunden wurde auch, dass es bei den Jugendlichen einerseits Bildungsverläufe gab, die direkt oder nach dem Absolvieren von Zwischenstationen während der Übergangsphase in einer Berufsausbildung mündeten, und andererseits Verläufe bestanden, bei denen die Jugendlichen immer stärker vom Bildungs- und Ausbildungssystem abkamen und gegebenenfalls in der Arbeitslosigkeit landeten (Gaupp et al. 2008: 388). Dabei konnten verschiedene Verlaufstypen hinsichtlich des Übergangsprozesses identifiziert werden. Während einige Jugendliche im direkten Anschluss an die Pflichtschulzeit oder nach einer Verlängerung der Schulzeit eine Ausbildung begannen, gelang anderen dieser Übergang erst über diverse berufsvorbereitende Maßnahmen oder andere Schritte. Andere Jugendliche entschieden sich für einen dauerhaften mehrjährigen Schulbesuch, während ein weiterer Teil in der Ausbildungslosigkeit landete (Gaupp et al. 2011: 176). Im Gegensatz dazu wurde festgestellt, dass sich nur ein sehr geringer Teil der Jugendlichen längere Zeit nach dem Verlassen der allgemeinbildenden Schule in einer ungelernten Erwerbsarbeit befand oder unversorgt war (Gaupp et al. 2005: 147 f.). Als weitere auftretende Übergangsstationen wurde in den bisherigen Studien auch eine Arbeitslosigkeitsphase oder die Suche nach einer Ausbildungsmöglichkeit angegeben. Andere Jugendliche blieben wiederum bedingt durch private Gründe zu Hause (Beicht und Ulrich 2008a: 11; Beicht und Ulrich 2008b: 6). Weitere vorhandene Zwischenstationen waren das Ableisten eines Wehrdienstes, Zivildienstes oder eines freiwilligen sozialen oder ökologischen Jahres (Beicht und Ulrich 2008b: 6).

Bei detaillierterer Betrachtung einiger ausgewählter Forschungsarbeiten zu den absolvierten Übergangsstationen lassen sich die geschilderten Erkenntnisse noch weiter vertiefen. Auch Jugendliche, die nach dem Verlassen der allgemeinbildenden Schule den Übergang in eine berufsqualifizierende Ausbildung nicht erfolgreich bewerkstelligt hatten, rückten dabei in den Fokus. Beicht und Ulrich untersuchten, wie sich die Gruppe der Jugendlichen charakterisieren ließ, die von einer beruflichen Ausbildung ausgeschlossen wurden. Dabei konzentrierten sie sich vor allem auf die Verläufe der ersten Phase der Jugendlichen nach dem Verlassen der allgemeinbildenden Schule (Beicht und Ulrich 2008a: 1) und überprüften, welche Einflussfaktoren für das Risiko maßgeblich waren, dass sie keinen beruflichen Abschluss erreichten (Beicht und Ulrich 2008a: 3). Als empirisches Datenmaterial verwendeten sie die BIBB-Übergangsstudie, bei welcher 7.230 Jugendliche der Geburtsjahrgänge 1982 bis 1988 
zwischen Juni und August 2006 mittels computergestützter Telefoninterviews zu ihren Bildungs- und Berufsverläufen befragt wurden. Die BIBB-Übergangsstudie war eine retrospektive Längsschnittdatenbefragung, bei welcher die erfragte Bildungs- und Berufsbiografie der Jugendlichen mit ihrer allgemeinbildenden Schulzeit begann (RohrbachSchmidt 2010: 4). Beicht und Ulrich verwendeten hieraus die Angaben von insgesamt 2.595 Jugendlichen, die beim ersten Verlassen der allgemeinbildenden Schule maximal einen Realschulabschluss besaßen (Beicht und Ulrich 2008a: 3 ff.). Dabei fanden sie heraus, dass zum Erhebungszeitpunkt im Sommer 200634 Prozent der Jugendlichen ohne eine berufliche Ausbildung erwerbstätig war, wogegen mehr als jeder zehnte Jugendliche an einer berufsvorbereitenden Maßnahme des Übergangssystems teilnahm und 55 Prozent der befragten Jugendlichen sich nicht innerhalb des Bildungs- oder Erwerbssystems aufhielten, sondern nach einer Ausbildungsmöglichkeit suchten, arbeitslos waren oder bedingt durch private Gründe zu Hause blieben. Im Vergleich dazu befanden sich von den Jugendlichen mit einer Ausbildung lediglich 13 Prozent außerhalb des Bildungs- oder des Erwerbssystems (Beicht und Ulrich 2008a: 11).

Lex und Geier versuchten Erkenntnisse zum Übergangsverlauf bildungsbenachteiligter Jugendlicher in die Berufsausbildung und die Erwerbsarbeit und zur Rolle des Übergangssystems im Rahmen der beruflichen Integration zu gewinnen (Lex und Geier 2010: 165 f.). Dabei gingen sie der Frage nach, ob der Übergang an der ersten Schwelle von der Schule in die Ausbildung aufgrund einer strukturellen Anbindung an das Bildungs- und Ausbildungssystem nicht als verengt betrachtet werden musste, während dem Übergangsverhalten der Jugendlichen andere Logiken zugrunde lagen. Dies versuchten sie anhand von Analysen mit Daten des Übergangspanels des Deutschen Jugendinstituts (DJI) herauszufinden, welches eine bundesweite Längsschnittuntersuchung zu Bildungs-, Ausbildungs- und Erwerbsverläufen von Hauptschülerinnen und Hauptschülern war. Beim DJI-Übergangspanel wurden bei der Basiserhebung im März 20043.922 Jugendliche aus 126 Schulen in Form einer Fragebogenerhebung im Klassenverband befragt. Im Anschluss daran wurden zunächst in einem halbjährigen Turnus und ab dem Jahr 2006 dann in einem jährlichen Abstand telefonische Folgebefragungen durchgeführt, wobei sich 2.933 Jugendliche für eine Folgebefragung zur Verfügung stellten und hiervon in der zweiten Befragungswelle 2.414 Interviews umgesetzt wurden. In den von Lex und Geier verwendeten Analysen waren alle Untersuchungspersonen berücksichtigt, die an der im November 2004 durchgeführten Befragung teilgenommen hatten (Lex und Geier 2010: 170 f.). Lex und Geier 
konnten nachweisen, dass Hauptschülerinnen und Hauptschüler nach der Vollendung des neunten oder zehnten Pflichtschuljahres primär nicht direkt in eine Ausbildung oder eine Berufsvorbereitung übergingen, sondern vorzugsweise weiter die Schule besuchten. Darin bestand für die Jugendlichen die Zielsetzung, einen höheren Bildungsabschluss zu erwerben, um bessere Chancen auf eine Ausbildung zu haben. Ein Großteil der Hauptschülerinnen und Hauptschüler trug mit diesem Bildungsverhalten dem Umstand Rechnung, dass in sehr vielen Berufsbranchen bei der Vergabe der Ausbildungsplätze ein mittlerer Schulabschluss zur Grundvoraussetzung durch die Ausbildungsbetriebe geworden war. Lex und Geier konnten zwar eine kompensatorische Funktion für bildungsbenachteiligte Jugendliche durch berufsvorbereitende Maßnahmen nachweisen, stellten jedoch anhand der durchgeführten Analysen auch fest, dass deutliche Nachteile für Jugendliche mit Migrationshintergrund in der Form bestanden, dass diese nach dem Absolvieren von berufsvorbereitenden Maßnahmen wesentlich seltener in eine Berufsausbildung einmündeten oder für dieses Einmünden deutlich mehr Zeit benötigten als Jugendliche ohne Migrationshintergrund. Nur etwa 40 Prozent aller Hauptschülerinnen und Hauptschüler schaffte direkt nach einer berufsvorbereitenden Maßnahme den Übergang in eine Berufsausbildung, wogegen der Großteil dafür mehr als ein Jahr benötigte und mehrere Zwischenstationen im Übergangssystem absolvieren musste, ehe der Übergang in die Ausbildung gelang (Lex und Geier 2010: 183 ff.).

Gaupp, Lex und Reißig rekonstruierten in einer Längsschnittanalyse die Bildungs- und Ausbildungsverläufe von Jugendlichen, die die allgemeinbildende Schule ohne einen Schulabschluss verlassen hatten (Gaupp et al. 2008: 388). Dafür verwendeten sie als Datengrundlage eine Teilstichprobe des DJI-Übergangspanels. Diese bis 2009 stattgefundene Untersuchung beinhaltete sowohl die Übergangsphase nach dem Verlassen der Schule als auch den Übergang von der Berufsausbildung in den Beruf. Aus diesem Panel nutzten Gaupp, Lex und Reißig eine Teilstichprobe von Jugendlichen ohne einen Schulabschluss, die bayerische Praxisklassen absolviert hatten. Diese Praxisklassen sind ein einjähriges freiwilliges Angebot für Jugendliche auf einer Hauptschule, welche keine Aussicht mehr auf das Erreichen des Hauptschulabschlusses haben, wobei diese Unterrichtsform insbesondere praxis- und berufsorientiert ist. Die Ausgangsstichprobe dieser Teilstichprobe bildete sich aus 477 Jugendlichen aus einer Praxisklasse von 36 Hauptschulen, wobei bei der letzten Befragungswelle noch 208 Jugendliche teilnahmen (Gaupp et al. 2008: 392 f.). Gaupp, Lex und Reißig kamen zu dem Ergebnis, dass es bei den untersuchten Jugendlichen einerseits Bildungsverläufe gab, die direkt oder nach dem Absolvieren von Zwischenstationen während 
der Übergangsphase in einer Berufsausbildung mündeten, und andererseits Verläufe bestanden, bei denen die Jugendlichen immer stärker vom Bildungs- und Ausbildungssystem abkamen und gegebenenfalls in der Arbeitslosigkeit landeten. Im Rahmen dieser Übergangsverläufe legten sie eine Reihe von relevanten Faktoren offen, welche einen positiven Einfluss auf einen gelungenen Übergang in eine berufliche Ausbildung hatten. $\mathrm{Zu}$ diesen Faktoren gehörten das Absolvieren von Betriebspraktika, um erste praktische Erfahrungen zu sammeln, vorhandene elterliche Unterstützungsmöglichkeiten durch die Herkunftsfamilie sowie eine professionelle Unterstützung beim Auswahlprozess des zukünftigen Berufs (Gaupp et al. 2008: 388).

Gaupp, Geier, Lex und Reißig überprüften mit Hilfe von Daten des DJI-Übergangspanels, welche soziodemografischen Merkmale ausbildungslose Jugendliche hatten, wie sie in die Ausbildungslosigkeit gelangten, welche schulischen Startbedingungen sie hatten und welche Schnittstellen in ihren Übergangswegen kritisch waren (Gaupp et al. 2011: 173). Auf Basis der Daten des bereits erwähnten und auch schon vorgestellten DJI-Übergangspanels wurden die Übergangswege der Jugendlichen in einem Längsschnitt als Sequenz von Stationen betrachtet (Gaupp et al. 2011: 183). Bei den Ergebnissen ergaben sich insgesamt fünf Verlaufstypen zu den Bildungs- und Ausbildungswegen. Eine Gruppe begann im direkten Anschluss an die Pflichtschulzeit eine Berufsausbildung (28 Prozent), eine zweite Gruppe mündete nach einer Verlängerung der Schulzeit in eine berufliche Ausbildung ein (20 Prozent) und eine Gruppe gelang über diverse berufsvorbereitende Maßnahmen oder andere Schritte in eine berufliche Ausbildung (24 Prozent). Eine weitere Gruppe entschied sich für einen dauerhaften mehrjährigen Schulbesuch (zehn Prozent), während die fünfte Gruppe in der Ausbildungslosigkeit landete (18 Prozent) (Gaupp et al. 2011: 176). Bezogen auf die soziodemografischen Merkmale der Verlaufstypen stellte sich unter anderem heraus, dass junge Frauen vor allem beim Verlauf einer „schulischen Höherqualifizierung“ überrepräsentiert waren. Dagegen war ein direkter Einstieg in eine Berufsausbildung für Jugendliche mit einem Migrationshintergrund nur schwer möglich, was sich daran belegen ließ, dass mit fast 25 Prozentpunkten Unterschied im Vergleich zum direkten Übergang in eine Berufsausbildung ihr Anteil im Verlaufstyp „Wege in Ausbildungslosigkeit“ wesentlich erhöht war (Gaupp et al. 2011: 180).

Junge Frauen wählten somit den über mehrere Jahre fortgesetzten Schulbesuch. Dagegen wirkte sich ein Migrationshintergrund negativ auf die Chancen eines direkten oder nach einer verlängerten Schulzeit erfolgreichen Einmündens in eine berufliche Ausbildung aus. 
Jugendliche mit einem höheren elterlichen Sozialstatus hatten größere Chancen auf eine schulische Höherqualifikation in Form eines verlängerten Schulbesuchs. Die Wahrscheinlichkeit einer Ausbildungslosigkeit wurde durch eine Arbeitslosigkeit beider Elternteile erhöht. Jugendliche mit guten Schulleistungen entschieden sich meist für schulische Wege in Form von schulischen Zwischenschritten vor einem Ausbildungsbeginn oder für den Zugang zur tertiären Bildung (Gaupp et al. 2011: 182 f.).

Zusammenfassend zum bisherigen Forschungsstand bezüglich der absolvierten Übergangsstationen lässt sich sagen, dass das Durchlaufen von weiteren Zwischenstationen sukzessive zugenommen hat. Sowohl das Absolvieren von berufsvorbereitenden Maßnahmen als auch von weiteren Schulepisoden hat eine gewichtige Bedeutung erlangt, wobei neben dem erworbenen Schulabschluss auch eine Reihe von soziodemografischen Faktoren einen Einfluss darauf hatte, welche Stationen durchlaufen wurden und welchen Effekt diese auf den Übergang in eine vollqualifizierende Berufsausbildung hatten. Vor allem Frauen und Jugendliche mit einem höheren elterlichen Sozialstatus absolvierten zusätzliche Übergangsstationen zur schulischen Weiterqualifizierung, um höhere Schulabschlüsse zu erwerben und ihre Ausbildungschancen $\mathrm{zu}$ verbessern. Jugendliche mit einem Migrationshintergrund dagegen hatten auch bei einer schulischen Höherqualifizierung schlechtere Chancen, in eine Berufsausbildung einzumünden.

\subsection{Fazit zur Forschungsliteratur}

Anhand der aufgelisteten empirischen Studien zum Themenfeld des Übergangs von der Schule in die berufliche oder akademische Ausbildung wird deutlich, dass sich die Übergangsquote und damit die Ausbildungspartizipation generell im Laufe der Jahrzehnte zwar erhöht hat, jedoch die Chancen für Schulabgängerinnen und Schulabgänger, welche maximal einen Hauptschulabschluss oder gar keinen Schulabschluss erworben haben, in eine vollqualifizierende Berufsausbildung einzumünden, geringer geworden sind. Diese Gruppe war stärker davon betroffen, in eine oder mehrere berufsvorbereitende Maßnahmen einzumünden, entschied sich aber auch verstärkt, über den erreichten Hauptschulabschluss hinaus, länger an schulischer Bildung $\mathrm{zu}$ partizipieren, um ihre individuellen Ausbildungschancen $\mathrm{zu}$ erhöhen. Die Übergangschancen von Abiturientinnen und Abiturienten waren dagegen unbeeinflusst von verschiedenen Faktoren über die Jahrzehnte konstant geblieben. Die Abiturientenquote hat sich im Laufe der Zeit zudem deutlich erhöht, wodurch sich wiederum die Übergangschancen insbesondere von Jugendlichen ohne 
Schulabschluss oder mit maximal einem Hauptschulabschluss verringert haben. Wirtschaftliche oder demografische Veränderungsprozesse haben hierzu im Laufe der Zeit beigetragen. Darüber hinaus ist der Anteil der Jugendlichen, welche nach dem Verlassen der allgemeinbildenden Schule in eine der berufsvorbereitenden Maßnahmen des Übergangssystems einmündeten, deutlich gestiegen. Zwar war der Anstieg der Studierendenquote nicht genauso hoch wie der Anstieg der Abiturientenquote, was daran lag, dass sich immer mehr Abiturientinnen und Abiturienten für eine Berufsausbildung entschieden. Dennoch haben auch die Studienanfängerquote und damit die Studierendenzahlen im Laufe der Jahre und Jahrzehnte zugenommen, was wiederum die erhöhte Ausbildungspartizipation, in diesem Fall die erhöhte Partizipation an einer akademischen Ausbildung, belegte.

Des Weiteren stellte sich in der bisherigen Forschung heraus, dass sich der Übergangsprozess vom erstmaligen Verlassen der allgemeinbildenden Schule bis zur Aufnahme einer beruflichen oder akademischen Erstausbildung im Laufe der Jahrzehnte deutlich verändert hat. Lag dieser Prozess in früheren Jahrzehnten noch relativ standardisiert in einem Dreischritt bestehend aus den Stationen Schule, Ausbildung und Beruf vor, hat sich diese Phase mittlerweile stark vervielfältigt und zum Teil individualisiert. Dabei gestaltete sich die Übergangsphase für die verschiedenen Schulabschlussgruppen unterschiedlich. Schulisch niedrig qualifizierte Jugendliche landeten oftmals in berufsvorbereitenden Maßnahmen des Übergangssystems, wodurch sich die Übergangsdauer bis zur Einmündung in eine vollqualifizierende Berufsausbildung bei ihnen deutlich verzögerte und diese Maßnahmen darüber hinaus ihre Chancen, den Übergang in die Ausbildung erfolgreich zu bewerkstelligen, nicht unbedingt erhöhten. Generell hat sich die Übergangsphase bis zum Einmünden in die Ausbildung verlängert, da viele Schulabgängerinnen und Schulabgänger bestrebt waren, sich zusätzliche Bildung und weiterführende Qualifikationen anzueignen und dadurch ihre Chancen auf dem Ausbildungs- und Arbeitsmarkt zu verbessern. Auch schulisch niedrig qualifizierte Jugendliche waren zu einem nicht unerheblichen Ausmaß gewillt, weiter die Schule zu besuchen, falls die direkte Einmündung in die Ausbildung nicht klappte, und allgemeinbildende Schulabschlüsse nachzuholen, um ihre Ausbildungsperspektiven zu verbessern.

In diesem Forschungsfeld wurde bislang lediglich untersucht, ob die Übergänge für einzelne Bildungsabschlussgruppen von der Schule in die Ausbildung gelingen oder nicht, wie sich die Chancen, diesen Übergang $\mathrm{zu}$ bewältigen, verändert haben und wie sich der 
Übergangsprozess, gemessen an der Übergangsdauer und den Zwischenstationen, entwickelt hat. Jedoch hat bislang keinerlei detaillierte Betrachtung von möglichen Einflüssen auf den Übergangsprozess stattgefunden. Verschiedene Einflussfaktoren wie demografische oder wirtschaftliche Veränderungen wurden bislang nur im Zusammenhang mit den Übergangschancen und den vollzogenen Übergängen in die Ausbildung überprüft. Jedoch bleibt unklar, ob die deutlich nachgewiesenen Veränderungen des Übergangsprozesses auch durch externe Einflüsse begründet sind. Bei diesen Einflussfaktoren fand in der bisherigen Forschung der im Laufe der Jahrzehnte deutlich gestiegene Anteil an hochqualifizierten Arbeitskräften (Statistisches Bundesamt 2015a) ebenfalls keine Berücksichtigung. Dieser hat seinen Ursprung in der starken Expansion der Dienstleistungsberufe und einem damit einhergehenden gestiegenen Qualifikationsniveau auf dem Arbeitsmarkt, welches im folgenden Kapitel 3 Historischer Abriss ausführlich thematisiert wird. Damit soll erstmals untersucht werden, ob die Entwicklung der Übergangsverläufe, nämlich die Übergangsdauer und die absolvierten Übergangsstationen vom Verlassen der Schule bis zur Aufnahme der akademischen oder beruflichen Erstausbildung in einer retrospektiven Betrachtung extern beeinflusst wurden. Eine weitere Neuerung in diesem Forschungsfeld stellt der Anteil an hochqualifizierten Arbeitskräften auf dem Arbeitsmarkt dar, welcher erstmals als zentraler Einflussfaktor untersucht werden soll und diesbezüglich bislang noch nicht in Betracht gezogen wurde, wodurch sich ein deutliches Forschungsdesiderat in diesem Themenfeld identifizieren lässt und dieses Gegenstand der vorliegenden Arbeit sein soll.

Somit soll einerseits untersucht werden, ob der gestiegene Anteil von hochqualifizierten Arbeitskräften auf dem Arbeitsmarkt im Zeitraum zwischen den Jahren 1971 und 2012 einen Einfluss auf die Übergangsdauer vom Verlassen der allgemeinbildenden Schule bis zum Einmünden in die berufliche oder akademische Erstausbildung hat. Andererseits soll der Frage nachgegangen werden, ob dieser gestiegene Anteil an hochqualifizierten Arbeitskräften im Untersuchungszeitraum einen Einfluss auf die in der Übergangsphase absolvierten Übergangsstationen, in Form der Anzahl als auch der Kategorien der absolvierten Stationen, hat. Beide Untersuchungsvorhaben sollen anhand der in der Einleitung formulierten Fragestellungen aufgegriffen und untersucht werden.

Dadurch lässt sich in einer retrospektiven Betrachtungsweise feststellen, ob die Übergangsdauer und die absolvierten Übergangsstationen als Charakteristika des Übergangsprozesses von der Schule in die Ausbildung durch ein verändertes Qualifikationsniveau auf dem Arbeitsmarkt im Zeitraum zwischen 1971 und 2012 
beeinträchtigt wurden und sich dadurch die Entwicklung auf dem Arbeitsmarkt in den Übergangsprozessen in den Ausbildungsmarkt oder in das Studium niedergeschlagen hat. Darüber hinaus können durch eine differenzierte Betrachtung der beiden Fragestellungen für die verschiedenen Schulabschlussgruppen Erkenntnisse für unterschiedliche schulische Bildungsniveaus gewonnen werden. 


\section{Historischer Abriss}

Beim Übergang in die Ausbildung sind die historischen Rahmenbedingungen verschiedener Bildungsinstitutionen bedeutsam. In diesem Kapitel wird ein kurzer Überblick über die historischen Entwicklungen gegeben, um die späteren Ergebnisse der Untersuchung dahingehend einordnen zu können. Diese Entwicklungen hängen stark mit den zentralen theoretischen Grundlagen des Forschungsbereiches zusammen. Der Fokus liegt zunächst auf dem Prozess und den Folgen der Bildungsexpansion (vgl. Abschnitt 3.1), der Entwicklung des Anteils an hochqualifizierten Arbeitskräften auf dem Arbeitsmarkt (vgl. Abschnitt 3.2) sowie den zentralen Bildungsinstitutionen Schule und Hochschule (vgl. Abschnitte 3.3.1, 3.3.2 und 3.3.3). Auch die Veränderungen des Ausbildungs- und Arbeitsmarktes (vgl. Abschnitt 3.3.4) gehen mit der Bildungsexpansion einher und stellen aufgrund der technischen und wirtschaftlichen Weiterentwicklung neue Herausforderungen beim Übergang in die Ausbildung dar. Zusätzlich wird die historische Entwicklung der Übergangsverläufe von der Schule in die Ausbildung (vgl. Abschnitt 3.4) betrachtet.

Da neben der Übergangsdauer auch die dabei absolvierten Zwischenstationen untersucht werden, wird die gestiegene Bedeutung von Stationen wie dem berufsvorbereitenden Übergangssystem, den Praktika, Arbeitslosigkeit oder Weiterbildungen aufgeworfen (vgl. Abschnitte 3.5.1, 3.5.2, 3.5.3 und 3.5.4). Darüber hinaus ist der von jungen Männern bis 2011 zu absolvierende Wehr- oder Zivildienst zu berücksichtigen (vgl. Abschnitt 3.5.5), dessen Dauer während des Untersuchungszeitraumes zwischen 1971 und 2012 aufgrund von politischen Vorgaben deutlich variierte. Auch auf Schwangerschafts- oder Elternzeitepisoden wird kurz eingegangen (vgl. Abschnitt 3.5.6).

\subsection{Bildungsexpansion}

Der Prozess der Bildungsexpansion hatte einen wesentlichen Einfluss auf die im Laufe der Jahrzehnte veränderte Schulbildung und das erreichte schulische Bildungsniveau, was das Übergangsverhalten von der Schule in die Ausbildung geprägt hat. Grundlegende Zielsetzungen der Bildungsexpansion waren der Abbau von Bildungsungleichheiten und das Erreichen eines allgemein höheren Bildungsniveaus durch einen verstärkten Ausbau des Bildungssystems und eine wesentliche Erhöhung der Bildungspartizipation für sämtliche Bevölkerungsteile (Hadjar und Becker 2006: 11).

Die Entwicklung des deutschen Bildungssystems war stark mit der Anfang des 19. Jahrhunderts entstehenden bürgerlichen Gesellschaft verknüpft. Das dabei entstandene neu- 
humanistische Bildungskonzept zeichnete sich durch mündige Staatsbürger, der Vorstellung einer Nation und der Öffentlichkeit aus. Trotz einer intendierten herkunftsunabhängigen Bildung für die gesamte Nation wurden bei den zu erwartenden Leistungen der Schülerschaft einheitliche Kriterien institutionalisiert. Unter den formal gleichen Bedingungen fanden die sozial extrem variierenden Herkunftsbedingungen und Voraussetzungen keine Berücksichtigung, woraus soziale Benachteiligungen resultierten. Eine anschließende materiale Standardisierung der Lehrpläne sowie die Verflechtung eines standardisierten Bildungsverlaufs von der Allgemeinbildung hin zum Erwerb der allgemeinen Hochschulreife führten zur Differenzierung des dreigliedrigen Schulsystems und zu einer klaren Unterscheidung zwischen allgemeiner und beruflicher Bildung. Hiervon profitierten insbesondere das Bildungsbürgertum und die akademischen Berufsbereiche. Die Struktur eines gegliederten Bildungssystems wurde über die historischen Epochen wie das Kaiserreich, die Weimarer Republik, den Nationalsozialismus und das Kriegsende im Jahr 1945 hinaus beibehalten und existierte auch nach der Gründung der Bundesrepublik Deutschland weiter. Anfang der 1960er Jahre wurde, insbesondere initiiert durch die 1961 gegründete „Organisation für ökonomische Kooperation und Entwicklung“ (OECD) der Zusammenhang zwischen der Entwicklung eines durchdachten Bildungssystems und eines Wirtschaftswachstums thematisiert. Diese verstärkten Bildungsinvestitionen dienten zur Absicherung einer internationalen Konkurrenzfähigkeit der Mitgliedsstaaten der OECD (Bauer et al. 2014: 10 f.).

Zu Beginn der 1960er Jahre wurde festgestellt, dass der Anteil der Schülerschaft, welche eine weiterführende Schule besuchte, zwischen den Bundesländern teilweise deutlich, vor allem nach Regierungsbezirk, Landkreis oder Gemeinde, variierte (Dahrendorf 1965: 47 f.). Als Anknüpfungspunkte für eine expansive Bildungspolitik galt beispielsweise die Diskrepanz zwischen dem Bevölkerungsanteil der Arbeiterkinder in der Bundesrepublik, welcher bei etwa 50 Prozent lag, und dem Anteil der Arbeiterkinder unter den Studierenden an einer Hochschule, welcher sechs Prozent betrug (Dahrendorf 1965: 50). Der 1965 vorgestellte Schulentwicklungsplan in Baden-Württemberg zielte auf einen verbesserten qualitativen Ausbau des Schulsystems ab (Dahrendorf 1965: 12). Bezüglich des eigentlichen Beginns der Bildungsexpansion galt die Forderung nach „Bildung als Bürgerrecht“, da bis zum Beginn der 1960er Jahre Chancenungleichheiten beim Zugang zu höherer Bildung bestanden, von welchen insbesondere Arbeiterkinder und Kinder von Landwirten, einfachen Angestellten oder einfachen Beamten nachteilig betroffen waren. Bildung sollte nicht nur unter 
ökonomischen Gesichtspunkten gesehen werden, sondern auch als zentrale Voraussetzung für das Erschaffen und Bestehen einer demokratischen Gesellschaft mit mündigen Bürgerinnen und Bürgern. Die Bildungsexpansion führte als Reform und Expansion des sekundären und tertiären Bildungswesens, als erhöhte Bildungspartizipation, als längere Bildungsdauer und als häufigerer Erwerb von höheren Bildungsabschlüssen $\mathrm{zu}$ einer gesellschaftlichen Modernisierung und zur Verringerung bestehender Bildungsungleichheiten (Becker 2006: 27 f.). Ein symbolisches Ziel bestand in der Anhebung der Abiturientenquoten aufgrund eines durch die expansive Bildungspolitik deutlich erhöhten Lehrkräftebedarfs an Schulen. Damit einhergehend wurde eine Verschiebung im gesamten Bildungsbereich mit einer gleichzeitigen Erhöhung der Schülerschaft mit einer mittleren Reife vermutet (Dahrendorf 1965: 30 f.).

Ausschlaggebend für die Bildungsexpansion war ebenfalls die Proklamation der ,Deutschen Bildungskatastrophe' durch Georg Picht 1964, welche mit einem prognostizierten Anstieg der Schülerzahlen von 1961 bis 1970 und der pensionierten Lehrkräfte bis 1970 begründet wurde (Eckert 2010: 589). Dabei war das Betreuungsverhältnis zwischen vorhandenem Lehrpersonal und der Schülerzahl bereits 1961 problematisch. Das Anwachsen der Schülerzahlen wurde vor allem durch eine stetige Bevölkerungsvermehrung und die Einführung eines neunten Volksschuljahres verursacht. Durch das gleichzeitig massenhafte Ausscheiden von Lehrkräften entstand ein sehr großer Lehrkräftebedarf. Dabei sollten nicht nur die ausgeschiedenen Lehrkräfte ersetzt, sondern auch der immer größer werdende Lehrkräftemangel kompensiert werden (Picht 1965: 12). Diese Diskrepanz war durch zu geringe Abiturientenzahlen und dadurch zu wenige Lehramtsstudierende verursacht, wodurch ein geringer Lehrkräftenachwuchs einer zu großen Schülerzahl gegenüber stand (Eckert 2010: 589). Darin wurde das zentrale Problem des Bildungsnotstands in Westdeutschland in den 1960er Jahren gesehen. Dies konnte nur durch eine deutliche Anhebung der Abiturientenquote behoben werden, um zukünftig genügend Lehrkräfte für die Unterrichtung einer wachsenden Schülerzahl zu haben (Picht 1965: 109).

Die Strukturen des nach erreichbaren Schulabschlüssen hierarchisch gegliederten und zwischen allgemeiner und beruflicher Bildung differenzierten Schulsystems wurden beispielsweise durch das „Hamburger Abkommen“ der einzelnen Bundesländer 1964 bestätigt. Dies ermöglichte diverse Reformen, welche eine größere Durchlässigkeit zwischen den einzelnen Schulformen und Schulstufen verfolgten. In den darauf folgenden Jahren wurde die zweiteilige Sekundarstufe etabliert. Mit der Gestaltung der Hauptschule als weiterführender Schulform erhöhten sich das Schulpflichtalter und das frühestmögliche 
Eintrittsalter in das Erwerbssystem vom 14. auf das 16. Lebensjahr. Trotz des Anstiegs der Bildungspartizipation und des durchschnittlichen Bildungsniveaus bestand das überlieferte Bildungssystem mit unterschiedlichen Zugangsberechtigungen weiterhin. Deshalb konnten die großen bildungspolitischen Reformvorhaben der 1960er und 1970er Jahre nicht vollständig vollzogen werden, so dass letzten Endes lediglich einzelne Teilreformen übrig blieben (Bauer et al. 2014: $11 \mathrm{f}$.).

In der jüngeren deutschen Bildungsgeschichte existierten zwei Stadien. Die erste Phase deckte den Zeitraum zwischen 1955 und 1995 mit einer starken Bildungsexpansion ab. Die Phase nach 1995 war durch eine bildungsbezogene Stagnation gekennzeichnet (Allmendinger et al. 2010: 52). Die Tabelle 1 gibt einen Überblick über beide Stadien sowie die prozentualen Anteile der Schülerzahlen der drei Schulformen Hauptschule, Realschule und Gymnasium.

Tabelle 1: Zwei Stadien der jüngeren deutschen Bildungsgeschichte mit prozentualen Schüleranteilen der drei Schulformen.

\begin{tabular}{lccc}
\hline \multirow{1}{*}{ Stadium } & $\begin{array}{c}\text { Anteil von } \\
\text { Hauptschülerinnen und } \\
\text { Hauptschülern }\end{array}$ & $\begin{array}{c}\text { Anteil von } \\
\text { Realschülerinnen und } \\
\text { Realschülern }\end{array}$ & $\begin{array}{c}\text { Anteil von } \\
\text { Gymnasiastinnen und } \\
\text { Gymnasiasten }\end{array}$ \\
\hline 1. Stadium: $1955-1995$ & $74 \%$ & $9 \%$ & $16 \%$ \\
2. Stadium: Nach 1995 & $25 \%$ & $27 \%$ & $31 \%$ \\
\hline
\end{tabular}

Quelle: Eigene Darstellung basierend auf Allmendinger et al. 2010: 52.

Durch die Bildungsexpansion verringerte sich der Anteil von Schülerinnen und Schülern auf Hauptschulen zwischen beiden Stadien deutlich, während er sich auf den Gymnasien fast verdoppelte und auf den Realschulen sogar verdreifachte (Allmendinger et al. 2010: 52). Dadurch hat das Gymnasium die Hauptschule Mitte der 1990er Jahre als die am häufigsten besuchte Schulform abgelöst (Lauterbach 2012: 580). Das formale Bildungsniveau der Schülerschaft erhöhte sich zwischen 1955 und 1995 ebenfalls deutlich, während es nach 1995 nahezu keine Veränderungen mehr zu verzeichnen gab (Allmendinger et al. 2010: 52).

Die Abiturienten- und die Studierendenquoten nahmen im Laufe der Jahrzehnte deutlich zu (Becker 2006: 32 f.). Die Tabelle 2 zeigt die Anstiege der Abiturienten- und der Studienanfängerquoten für ein Hochschulstudium für die Altersgruppe der 18- bis 22-Jährigen in den prozentualen Anteilen zwischen den Jahren 1960 und 2000 (Abiturientenquote) beziehungsweise zwischen 1980 und 2000 (Studienanfängerquote). 
Tabelle 2: Prozentuale Anteile der Abiturientenquoten und der Studienanfängerquoten.

\begin{tabular}{cc|cc}
\hline Jahr & $\begin{array}{c}\text { Anteil der } \\
\text { Abiturientenquote }\end{array}$ & Jahr & $\begin{array}{c}\text { Anteil der } \\
\text { Studienanfängerquoten }\end{array}$ \\
\hline 1960 & $6 \%$ & 1980 & $13 \%$ \\
2000 & $37 \%$ & 2000 & $24 \%$ \\
\hline
\end{tabular}

Anteile der Abiturientenquoten bezogen auf Zeitraum zwischen 1960 und 2000. Anteile der Studienanfängerquoten (Hochschulstudium) für Altersgruppe der 18- bis 22-jährigen bezogen auf Zeitraum zwischen 1980 und 2000. Quelle: Eigene Darstellung basierend auf Becker 2006: $32 \mathrm{f}$.

Der erwartete und erwünschte Anstieg der Bildungspartizipation hat jedoch zu weiter bestehenden Bildungsungleichheiten als unerwarteter und unerwünschter Folge geführt (Becker 2006: 52). Bei Betrachtung aller Erwerbsmöglichkeiten einer Studienberechtigung wurde deutlich, dass fast die Hälfte eines Jahrgangs eine Hochschulzugangsberechtigung besaß, wobei diese nicht nur an allgemeinbildenden Schulen, sondern auch zu circa 40 Prozent durch das berufliche Bildungssystem oder über den zweiten Bildungsweg erworben wurde (Schindler 2012: 2). Der Anstieg der Studierendenzahlen ließ sich nicht auf Studienberechtigte aus bildungsfernen Familien übertragen, da diese das Abitur vermehrt als formale Voraussetzung für die Aufnahme einer Berufsausbildung erwarben (Schindler 2012: 5 ff.). Im Vergleich entschieden sich mehr Studienberechtigte mit einer allgemeinen Hochschulreife für ein Studium als für eine berufliche Ausbildung, wogegen Studienberechtigte mit einer Fachhochschulreife eher auf ein Studium verzichteten und eine Berufsausbildung begannen (Schindler 2012: 5).

Daneben gab es unbeabsichtigte, wenngleich teilweise zu erwartende, Folgen. Durch das deutlich gestiegene Bildungsniveau in der Bevölkerung wurden niedrig Gebildete immer stärker aus dem Bildungssystem verdrängt, wodurch bestehende Bildungsungleichheiten nicht vollständig beseitigt werden konnten (Hadjar und Becker 2006: 14 f.). Wesentliche Veränderungen im Zuge der Bildungsexpansion, eines gestiegenen Wohlstandes und eines berufsstrukturellen Wandels zeigten sich laut Becker darin, dass in früheren Jahrzehnten ein markanter Einfluss des sozioökonomischen Status und der Klassenlage auf den Bildungserfolg bestand, wogegen im späteren Verlauf das elterliche Bildungsniveau in Form eines intergenerationalen Bildungstransfers den Bildungserfolg der Kinder maßgeblich beeinflusste (Becker 2006: 52).

Dabei schrumpfte die Schülerschaft einer Hauptschule, da sich der Anteil der 13-Jährigen auf dieser Schulform in Gesamtdeutschland auf lediglich etwa 20 Prozent verringerte. Dieser Trend stand in starkem Kontrast zu den Entwicklungen der anderen Schulformen (Solga und 
Wagner 2001: 108). So zeigte sich, dass sich der soziale Abstand zwischen Haupt- und Realschülerinnen und -schülern sukzessive vergrößert hat. Die Ursache für die Schrumpfung der Hauptschule war die Abwanderung von Mädchen, deren Eltern qualifizierte Berufstätigkeiten ausübten. Die auf den Hauptschulen verbliebene Schülerschaft setzte sich überproportional aus Schülerinnen und Schülern zusammen, deren Eltern einfache Berufstätigkeiten oder keine Erwerbstätigkeit ausübten. Diese Kinder wuchsen in schwierigen familiären Verhältnissen auf, was bei ihnen eine Verschlechterung der Bedingungen des Bildungserfolgs durch die Bildungsexpansion vermuten ließ. Dies konnte in erster Linie durch eine im zeitlichen Verlauf sozial homogenere, weil sozial schwächere Schülerschaft auf Hauptschulen verursacht worden sein (Solga und Wagner 2001: 123).

Im Rahmen der Bildungsexpansion hat das Bildungsniveau Jugendlicher, die an einer Berufsausbildung interessiert waren, durchschnittlich zugenommen, wogegen die Chancen für Hauptschülerinnen und Hauptschüler, in eine Berufsausbildung zu gelangen, gesunken sind (Protsch 2014: 71). Insgesamt war die Bildungsexpansion somit durch eine höhere Bildungspartizipation, einen längeren Aufenthalt im Bildungssystem und einen Anstieg von höheren Schulabschlüssen gekennzeichnet. Die Expansion des Bildungssystems lag aber auch im wirtschaftlichen Wachstum und im technischen Fortschritt begründet (Hadjar und Becker 2006: $11 \mathrm{ff}$.$) .$

\subsection{Gestiegener Anteil an hochqualifizierten Arbeitskräften}

Im Zuge der Entwicklungen durch die Bildungsexpansion ist der Anteil der sehr gut ausgebildeten Arbeitskräfte angestiegen. Während den Betrieben immer mehr Schulabgängerinnen und Schulabgänger mit höheren Schulabschlüssen zur Auswahl standen, hoben sie bei einem Ausbildungsplatzmangel aufgrund der schwachen konjunkturellen Lage die Zugangs- und Qualifikationsvoraussetzungen für eine Berufsausbildung an, wodurch Jugendliche mit einem Hauptschulabschluss oder ohne Schulabschluss sukzessive aus dem Ausbildungsmarkt herausgedrängt wurden (Protsch 2014: 74). Die Unternehmen hatten ein verstärktes Interesse an jungen Erwachsenen mit Abitur oder Fachhochschulreife für eine Berufsausbildung, da diese den erhöhten Qualifikationsanforderungen des Ausbildungs- und Arbeitsmarktes entsprachen und wählten aus den verbliebenen Ausbildungsinteressierten lediglich diejenigen für einen beruflichen Ausbildungsplatz aus, die diese Anforderungen ebenso erfüllten. Insbesondere Jugendliche mit Hauptschulabschluss oder ohne Schulabschluss taten dies aus Unternehmenssicht nicht, weshalb sie bei der 
Ausbildungsplatzvergabe die schlechtesten Chancen hatten. Hatten die Unternehmen bei der Besetzung ihrer vorhandenen Ausbildungsplätze nicht genügend ausreichend qualifizierte Schulabgängerinnen und Schulabgänger, strichen sie die übrigen Ausbildungsplatzkapazitäten und sahen von einer Besetzung der Plätze eher ab (Krone 2010: 30), als sie an geringer gebildete Schulabgängerinnen und Schulabgänger zu vergeben, welche aus ihrer Sicht nicht ausreichend dafür qualifiziert waren.

Ein Wandel des Beschäftigungssystems vollzog sich in den vergangenen Jahrzehnten vor allem in Form einer für die wirtschaftliche Dynamik gestiegenen Bedeutung der humanen Ressourcen in hochentwickelten Volkswirtschaften, welche im Gegensatz zum Sachkapital immer relevanter wurden. Personenbezogene Dienstleistungen aber auch Berufe im Wissensund Informationsbereich machten neuartige und gestiegene Qualifikationen erforderlich und bezogen dadurch einen immer größer werdenden Anteil der erwerbstätigen Personen mit ein. Im Gegensatz dazu sank die Nachfrage nach Arbeitskräften ohne Ausbildungsabschluss kontinuierlich (Autorengruppe Bildungsberichterstattung 2008: 6). Die deutsche Volkswirtschaft, traditionell bestehend aus einem primären Sektor der Land- und Forstwirtschaft sowie der Fischerei, einem sekundären Sektor des produzierenden Gewerbes und einem tertiären Sektor der Dienstleistungen, war in den vergangenen Jahrzehnten tiefgreifenden strukturellen Veränderungen unterworfen. Diese drei Wirtschaftssektoren unterlagen einer nahezu identischen Gliederungssystematik, welche darin bestand, jeden der Sektoren mehrstufig in einzelne Abteilungen und Unterabteilungen zu subsumieren. Die Gliederungssystematik der drei Wirtschaftssektoren bezog sich auf die Einteilung zwischen 1950 und 1969 nach den Kriterien der Klassifikation der Wirtschaftszweige von 1979, für die Einteilung zwischen 1970 und 1990 nach der Klassifikation der Wirtschaftszweige von 2003 und ab 1991 nach der Klassifikation der Wirtschaftszweige von 2008 (Statistisches Bundesamt 2017a, Statistisches Bundesamt 1992: 9 f., Statistisches Bundesamt 2003: 13, Statistisches Bundesamt 2008: 69).

Während es beim Dienstleistungssektor zu einer kontinuierlichen Expansion kam, verringerte sich die ökonomische Bedeutung der beiden anderen Sektoren. Vor allem bei unternehmensbezogenen Dienstleistungen, in Unternehmen der Informations- und Kommunikationstechnologie und bei öffentlichen und personenbezogenen Dienstleistungen wie beispielsweise im Pflegebereich kam es verstärkt zur Schaffung neuer Arbeitsplätze. Aufgrund dessen und auch des damit einhergehenden technischen Wandels erfolgte eine Modifikation von vielen Berufsbildern und Anforderungsprofilen, wodurch in einer 
Dienstleistungs- und Wissensgesellschaft insbesondere analytisches Denken sowie Kompetenzen im Bereich der Kommunikation und Problemlösung verstärkt gefragt waren (Autorengruppe Bildungsberichterstattung 2008: 22 f.). Dies führte zu deutlich gestiegenen Qualifikationsanforderungen beim Zugang zu einer Berufsausbildung. Gleichzeitig wurde für junge Erwachsene mit Abitur auch eine Berufsausbildung als Alternative zum klassischen Hochschulstudium lukrativ, was sich insbesondere auf Abiturientinnen und Abiturienten aus bildungsfernen Schichten bezog. Aufgrund des geschilderten Wandels stellt das Abitur in vielen Berufen mittlerweile die Grundvoraussetzung dar (Schindler 2012: 2).

In Bezug auf den für die vorliegende Arbeit zentralen tertiären Sektor der Dienstleistungsberufe und übrigen Wirtschaftsbereiche ist die Definition dieses Sektors durch die Bundeszentrale für politische Bildung von Relevanz. Nach dieser Definition wird der tertiäre Sektor auch als Dienstleistungssektor bezeichnet, da er sich durch das Bereitstellen von Dienstleistungen auszeichnet. Dabei handelt es sich um ein vielseitiges Dienstleistungsangebot, welches unter anderem die Bereiche Handel und Verkehr, Bank- und Versicherungsgewerbe, öffentlicher Dienst, Grundstücks- und Wohnungswesen, Gesundheitsund Sozialwesen, Information und Kommunikation, Kunst und Unterhaltung oder Tourismus abdeckt (Bundeszentrale für politische Bildung 2016).

\subsection{Entwicklungen in Bildungsinstitutionen}

\subsubsection{Entwicklungen des deutschen Schulsystems}

Der Bildungsexpansionsprozess beeinflusste sämtliche Bildungsinstitutionen wie die Schule, in der die bildungsbezogenen Grundlagen für spätere Bildungs- und Weiterbildungsepisoden sowie die Erwerbstätigkeit gelegt werden. Nachdem in Westdeutschland bis in die 1960er Jahre ausschließlich das dreigliedrige Schulsystem, bestehend aus Haupt-, Realschule und Gymnasium, existierte, wurde Ende der 1960er Jahre die Gesamtschule als zusätzliche Schulform, wenn auch nicht flächendeckend, eingeführt. Das klassische dreigliedrige Schulsystem existierte in späteren Jahrzehnten nur noch in Bayern und Baden-Württemberg (Lauterbach 2012: 576 f.). Während Anfang der 1990er Jahre die Hauptschule noch die am häufigsten besuchte Schulform war, befand sich seit Mitte der 1990er Jahre der größte Schüleranteil auf dem Gymnasium und hat sich seitdem kontinuierlich erhöht. Dadurch konnte das Gymnasium seine Stellung als die am häufigsten besuchte Schulform weiter ausbauen. Demgegenüber hat sich die Anzahl der Hauptschülerinnen und Hauptschüler konstant verringert, während die Schülerzahlen auf Realschulen in den letzten Jahrzehnten in 
etwa konstant geblieben sind. Des Weiteren entstanden in den 1990er Jahren weitere Schulformen, welche mehrere Bildungsgänge offerieren (Lauterbach 2012: 580).

\subsubsection{Gliederung des deutschen Schulsystems}

Die Schullaufbahn ist unterteilt in einen Primar- und Sekundarbereich. Der Bereich der sekundären Bildung, aufbauend auf der Elementarbildung in Form des Kindergartens und der Primärbildung in Form der Grundschule, lässt sich in die Sekundarstufe I bis zur Beendigung der 10. Schulklasse und in die Sekundarstufe II mit der Vollendung der Jahrgangsstufe zwölf beziehungsweise 13 differenzieren. Im Gegensatz zum einheitlichen Schulunterricht in der Primarstufe erfolgt beim Übergang in die Sekundarstufe I eine Differenzierung in unterschiedliche Schulformen. Beim späteren Übergang in die Sekundarstufe II erfolgt eine weitergehende Unterteilung in eine fortlaufende schulische Bildung in Form der gymnasialen Oberstufe oder in eine berufliche Bildung im dualen Ausbildungssystem (Lauterbach 2012: 573 f.). Junge Erwachsene, die die Schule mit dem Abitur verlassen, durchlaufen die Sekundarstufe II und damit die allgemeinbildende Schule länger als Jugendliche ohne Abitur. Im Bereich der Sekundarstufe I besteht in den alten Bundesländern ein dreigliedriges Schulsystem aus Hauptschule, Realschule und Gymnasium, wobei sich zusätzlich noch die Gesamtschule mit den Bildungsebenen aller drei vorgenannten Schulformen etabliert hat. Die Sekundarstufe II dagegen beinhaltet neben diversen Schulformen des Berufsschulwesens wie der Berufsschule oder der Fachoberschule die gymnasiale Oberstufe als höhere allgemeinbildende Schulform (Horstkemper und Tillmann 2008: 286 f.). Nach dem Abschluss der Sekundarstufe II einer allgemeinbildenden Schule mit dem Erwerb der allgemeinen Hochschulreife oder der Fachhochschulreife kann eine tertiäre Ausbildung an einer Universität oder Fachhochschule aufgenommen werden.

\subsubsection{Entwicklung des deutschen Hochschulsystems}

Die Hochschulen in Deutschland sind entweder staatlich oder nicht-staatlich wie beispielsweise bei kirchlichen Trägern finanziert. Unter diesen zweiten Typus fallen auch private Hochschulen, welche hauptsächlich staatlich anerkannte Fachhochschulen sind (Hochschulrektorenkonferenz: Hochschulkompass o.J.). Das hochschulpolitische Leitbild der vergangenen Jahrzehnte war maßgeblich durch das Bildungsideal Wilhelm von Humboldts und seiner Universitätsidee geprägt und besteht unter anderem in der Einheit und Freiheit von Forschung und Lehre, wobei die universitäre Lehre an der Forschung ausgerichtet sein muss, und der Universität als Einheit der philosophisch zusammenhängenden 
Wissenschaftsdisziplinen. Ab Ende der 1990er Jahre wurde Humboldts Universitätsidee zum Gegenstück staatlicher Hochschulreformen wie der Bologna-Reform (Druwe 2011). Der Globalisierungsprozess in Form der Liberalisierung des Handels und der Märkte, ein stärkerer Wettbewerb, die Entwicklung einer globalen Wissensgesellschaft sowie die drastische Unterfinanzierung der Hochschulen im Zuge gestiegener Studierendenzahlen, die Frage nach einer einheitlichen Ausbildungsqualität, einer eingeschränkten Mobilität, die Vielfalt nationaler Hochschulabschlüsse auf dem internationalen Arbeitsmarkt sowie die generell sehr heterogene und vielfältige europäische Hochschullandschaft galten als Ursachen der Reform (Walter 2006: 187 ff.). Ziele waren eine bessere Kompatibilität der europäischen Hochschulsysteme zueinander sowie eine stärkere Attraktivität und Wettbewerbsfähigkeit der europäische Hochschulbildung (Walter 2006: 194). Humboldts Bildungsideal ließ sich größtenteils mit der Bologna-Reform vereinbaren und wurde durch eine forschungs- und kompetenzorientierte Lehre sowie die Entwicklung differenzierter Studiengänge ergänzt (Druwe 2011). Die Reform trug einerseits den gestiegenen Studierendenzahlen und den Engpässen der Hochschulkapazitäten Rechnung und orientierte sich andererseits an der globalen Ausrichtung einer internationalen Wissensgesellschaft. Die Einführung der international ausgerichteten Bachelor- und Masterabschlüsse intendierte eine stärker praxisund berufsbezogene Lehre an den Hochschulen, wodurch die Differenzierung zwischen der klassischen Universität und der Fachhochschule aufgeweicht und das Lehrangebot beider Institutionen sukzessive angeglichen wurde (Kamm 2014: 128).

\subsubsection{Gliederung und Entwicklung des beruflichen Ausbildungssystems}

Neben der tertiären Ausbildung an einer Universität oder Fachhochschule können bereits nach der Phase der Sekundarstufe I oder nach der Sekundarstufe II zwei verschiedene Berufsbildungsformen absolviert werden, die duale Berufsausbildung in der Berufsschule und im Betrieb und die ausschließlich durch die Berufsschulen gewährleistete vollzeitschulische Berufsausbildung (Dobischat 2010: 102). Insbesondere die duale Berufsausbildung erwies sich aufgrund der Kombination aus Berufsschule und vor allem Betrieb als hervorragende Vorbereitung auf die Anforderungen des Arbeitsmarktes (Krone 2010: 35).

Im Zuge des Globalisierungsprozesses war trotz der demografisch bedingt rückläufigen Anzahl an Schulabgängerinnen und Schulabgängern weiterhin ein erheblicher Ausbildungsplatzmangel $\mathrm{zu}$ konstatieren. Viele Jugendliche haben sich bei der Ausbildungsplatzsuche nicht an die Bundesagentur für Arbeit gewandt oder befanden sich in 
berufsqualifizierenden Maßnahmen, um die fehlende Ausbildungsreife zu erwerben. Bei Berücksichtigung dieser Gruppe wurde das Ausmaß an mangelnden Ausbildungsplätzen deutlich, von dem vor allem diejenigen ohne Abschluss nachteilig betroffen waren (Krone 2010: 19 ff.). Über Jahre hinweg überstieg die Nachfrage von Jugendlichen insbesondere nach einem dualen Ausbildungsplatz das vorhandene Angebot an Ausbildungsstellen. Diese massiv erhöhte Diskrepanz zwischen Angebot und Nachfrage führte dazu, dass vor allem Jugendliche mit einem Hauptschulabschluss oder ohne Abschluss kaum zu einem berufsqualifizierenden Abschluss kommen konnten (Severing 2010: 93), was jedoch in Widerspruch zu einem in Deutschland in einigen Berufsbranchen offenkundig vorhandenen Fachkräftemangel stand. Dieser seit Jahren vorhandene Mangel an qualifizierten Arbeitskräften hing stark mit dem demografischen Wandel zusammen und fokussierte sich vorwiegend auf die naturwissenschaftlichen und technischen Branchen sowie den Bereich des Gesundheits- und Sozialwesens. Als Ursache ließ sich der Wandlungsprozess des Beschäftigungssystems in den vergangenen Jahren ausmachen, welcher mit deutlich gestiegenen qualitativen Anforderungen an die Qualifikationsprofile von Bewerberinnen und Bewerbern einherging. Da viele Schulabgängerinnen und Schulabgänger diese Anforderungen aus Sicht der Unternehmen nicht mehr erfüllten, wurden vorhandene Ausbildungsplätze tendenziell nicht besetzt oder sogar Ausbildungskapazitäten eingeschränkt. Für die kommenden Jahre ist eine Verstärkung des Trends der Höherqualifikation und damit einer verstärkten Nachfrage nach gut ausgebildeten Fachkräften der dualen Berufsausbildung sowie Hochschulabsolventinnen und Hochschulabsolventen vorhergesagt. Des Weiteren richteten Unternehmen ihre Personalpolitik nicht längerfristig aus und bezogen den vorhandenen Fachkräftebedarf nicht in die Ausbildungspolitik mit ein. Dies war jedoch nötig, um dem Mangel an qualifizierten Arbeitskräften gerecht zu werden (Krone 2010: 27 ff.). Anders als beim Zugang zur akademischen Ausbildung wird der Übergang in die Berufsausbildung über den Arbeitsmarkt durch die Wirtschaft gesteuert, welche von staatlicher Seite diese Funktion zugewiesen bekommen hat (Eberhard und Ulrich 2010: 133). Zu den Selektionsmechanismen beim Übergang in den beruflichen Ausbildungsmarkt spielten neben den schulischen Leistungen und Noten auch nicht-leistungsbezogene Kriterien wie die ethnische Herkunft oder der Wohnort der Jugendlichen eine maßgebliche Rolle (Eberhard und Ulrich 2010: 157 f.). 


\subsection{Entwicklung der Übergangsverläufe von der Schule in die Ausbildung}

Der Übergangsprozess in die Ausbildung hat sich in seinen Mustern im Laufe der vergangenen Jahrzehnte ebenfalls wesentlich verändert. Zunächst ließen sich typische Übergangsverläufe charakterisieren, welche statistisch betrachtet relativ häufig auftraten. In Bezug auf die gängigen Übergangsformen in den Ausbildungs- und Arbeitsmarkt konnten vier mögliche Verläufe für den beruflichen Werdegang extrahiert werden. Der Übergang konnte von der Haupt- oder der Realschule direkt in Ausbildungsgänge unterhalb der tertiären Bildung erfolgen. Alternativ konnte eine tertiäre Ausbildung an einer Hochschule im Anschluss an den Besuch eines Gymnasiums absolviert werden. Zwei weitere Übergangsmöglichkeiten waren durch die organisatorischen Strukturen des Bildungssystems nicht beabsichtigt und vorgesehen, tauchten aber dennoch empirisch in einem nicht geringen Umfang auf. Nach dem Besuch eines Gymnasiums konnte eine nicht-tertiäre Ausbildung absolviert werden. Ebenso wurde der Übergang in den Arbeitsmarkt auch ohne erworbenen Schulabschluss und ohne vollqualifizierende Berufsausbildung vollzogen oder dieser Übertritt zwar mit einem erworbenen Schulabschluss, aber ohne eine berufliche Ausbildung absolviert (Weil und Lauterbach 2011: 329).

Dennoch war eine Reduzierung der Übergangsverläufe auf solche Typen zu kurz gegriffen. In früheren Jahrzehnten hatte ein standardisiertes Übergangsmuster aus den drei zu durchlaufenden Stationen Schule, Ausbildung und Beruf festen Bestand. Diese Standardisierung des Übergangsverlaufes hat sich jedoch in den vergangenen Jahrzehnten sukzessive aufgelöst. Anstatt der ausschließlich fest vorgegebenen Abfolge Schule, Ausbildung und Beruf ergab sich eine permanente Vervielfältigung und Individualisierung der Übergangsprozesse (Konietzka 1999: 315), wodurch es zur Entstehung einer enormen Vielzahl an Übergangsverläufen in die Ausbildung und den Beruf kam. Dabei stellte sich im Laufe der Jahrzehnte heraus, dass der Übergang verstärkt in mehreren Zwischenschritten und Übergangsstationen absolviert wurde (Fink 2011: 92). Es ist $\mathrm{zu}$ vermuten, dass beim Übergang in die Erwerbstätigkeit heutzutage durchschnittlich mehr als die drei oben aufgezählten Stationen absolviert werden, wobei zusätzliche Zwischenstationen vor allem beim Übergang von der Schule in die Ausbildung durchlaufen werden. $\mathrm{Zu}$ diesen zählen Umwege sowie Warteschleifen mit berufsvorbereitenden Maßnahmen im Übergangssystem (Pätzold 2008: 603), Praktika, Weiterbildungen oder eine Arbeitslosigkeitsepisode. 


\subsection{Entwicklungen bei absolvierten Zwischenstationen}

\subsubsection{Berufsbildendes Übergangssystem}

Da viele Jugendliche mit einem Hauptschulabschluss oder ohne Schulabschluss Probleme beim Übergang in die Berufsausbildung hatten, absolvierten sie eine teilqualifizierende Berufsbildung im Übergangssystem. Dieses System stand in der Vergangenheit in der Kritik, da es einen raschen Übergang von Jugendlichen, die auf Anhieb keinen Ausbildungsplatz gefunden hatten, in eine Berufsausbildung nicht gewährleisten konnte (Beicht 2009: 1 ff.). Nur wenige schafften nach dem Durchlaufen des Übergangssystems einen schnellen Einstieg in eine Berufsausbildung (Beicht 2009: 10), weshalb der Übergangsprozess bei ihnen deutlich verlängert wurde. Benachteiligte Jugendliche hofften durch den Besuch des Übergangssystems auf eine Verbesserung der eigenen Ausbildungschancen, wogegen sich ihr Anteil im Übergangssystem im Laufe der Jahre verdoppelte und sie sich zudem länger darin aufhalten mussten als ursprünglich vorgesehen (Krone 2010: 23 ff.). Im Übergangssystem fanden sich insbesondere diejenigen Bewerberinnen und Bewerber wieder, die beim ersten Versuch oder auch bei mehreren Versuchen keinen Ausbildungsplatz gefunden hatten. Dieses System enthält viele schulische Bildungswege und Maßnahmen, wie das berufsvorbereitende Jahr, welche jedoch nicht $\mathrm{zu}$ einem berufsqualifizierenden Abschluss führen und sich zusätzlich intransparent und unübersichtlich für ausbildungsbezogen benachteiligte Jugendliche darstellen (Krone 2010: 23).

\subsubsection{Praktika}

Eine weitere bedeutsame Übergangsstation ist ein Praktikum, welches primär einem ersten Einblick in das Arbeits- und Berufsleben dienen soll. Die Bezeichnung „Praktikum“ kann nur mehrdeutig verstanden werden und verfügt über die unterschiedlichsten Bedeutungsfacetten, angefangen von einer „Schnupperlehre“ mit dem Ziel, einen Ausbildungsplatz zu erreichen, über Ferienjobs, welche der Berufsorientierung von Schülerinnen und Schülern sowie Studierenden dienen bis hin zu Pflichtpraktika bei Studierenden und einem Berufseinstieg im Rahmen von Trainee- oder Volontariatsprogrammen. Ein Praktikum stellte historisch betrachtet einen Zusatz zur theoretischen Ausbildung, der Berufsorientierung und dem Sammeln von ersten Berufserfahrungen dar und wurde von Studierenden und Hochschulabsolventinnen und -absolventen meist im Laufe oder nach dem Studium absolviert, wobei es mittlerweile in einigen Studienordnungen als Pflichtbestandteil festgeschrieben ist (Kock 2008: 30). Viele Jugendliche, die nach dem Verlassen der 
allgemeinbildenden Schule auf Anhieb keinen Ausbildungsplatz erhielten, absolvierten während der Übergangsphase ein Praktikum (BMBF 2005: 78), um dadurch ihre Ausbildungs- und Berufschancen zu verbessern.

\subsubsection{Arbeitslosigkeit}

Jedoch kann die Übergangsphase bei einigen Jugendlichen auch durch Episoden der Arbeitslosigkeit geprägt sein, da der direkte Übertritt in eine vollqualifizierende Ausbildung nicht gelingt oder sich keine alternativen Möglichkeiten, wie beispielsweise eine Weiterbildung, ergeben. In der durch die Marktwirtschaft geprägten alten Bundesrepublik Deutschland kam nach dem Ende des zweiten Weltkriegs eine hohe Arbeitslosigkeit zustande, welche als große politische Herausforderung betrachtet wurde. In den folgenden Jahren konnte zwar vorübergehend eine Vollbeschäftigung hergestellt werden. Das in der alten Bundesrepublik bestehende marktwirtschaftliche Modell war jedoch besonders empfindlich für konjunkturelle Schwankungen und dadurch bedingte Krisen. Nach dem Abflauen der Stabilisierungskrise durch die Währungsreform aus dem Juni 1948 konnte die anfängliche Arbeitslosenquote zunächst abgebaut werden. Die sehr geringe Quote erhöhte sich erst zu Beginn der 1970er Jahre wieder. Die in der Folge steigenden Arbeitslosenzahlen hingen stark mit den aus beiden Ölpreiskrisen der Jahre 1973 und 1980/1981 hervorgehenden Rezessionen in der alten Bundesrepublik zusammen. Die hohe Arbeitslosigkeitsquote verfestigte sich auch durch den starken Anstieg der Langzeitarbeitslosenzahlen. Trotz eines leichten Rückgangs der Arbeitslosigkeit ab 1985 durch eine verstärkte Beschäftigungsrate waren zur Wiedervereinigung 1990 in Westdeutschland in etwa zwei Millionen Menschen arbeitslos. Nach der deutschen Wiedervereinigung stieg in den alten Bundesländern die Konjunktur durch den Wiederaufbau in den neuen Bundesländern an und verringerte die Arbeitslosigkeit. Vor allem Frauen, ältere Arbeitskräfte und gering Qualifizierte waren dem Risiko, arbeitslos $\mathrm{zu}$ werden, besonders stark ausgesetzt. Auch die Langzeitarbeitslosigkeit war trotz einer gesunkenen Arbeitslosigkeitsdauer ein zentrales Problem. Ältere Arbeitslose waren über viele Jahre eine zentrale Zielgruppe für die öffentliche Arbeitsmarktpolitik. In den alten Bundesländern bestand lediglich ein marginaler Unterschied zwischen der Arbeitslosenquote von älteren Menschen und der gesamtdurchschnittlichen Quote. Personen ohne einen Berufsoder Schulabschluss waren stark gefährdet, aus dem Arbeitsmarkt heraus gedrängt zu werden. Seit der Wiedervereinigung war der Anteil der Langzeitarbeitslosen stark angestiegen (Bundeszentrale für politische Bildung 2010). 


\subsubsection{Weiterbildung}

In den vergangenen Jahrzehnten wurde die Weiterbildung immer bedeutsamer mit zahlreichen Formen und Ausprägungen. Die absoluten Ursprünge der Weiterbildung in Deutschland lassen sich in der Aufklärung verorten mit dem heute noch verfolgten Ziel, eine organisierte Bildung zu etablieren. Frühzeitig kam es zu einer Differenzierung der Arbeiterbildung von der bürgerlichen Bildung. Bildung wurde instrumentalisiert, um über die Arbeiterklasse zu herrschen, wodurch sich ein durch die ab Mitte des 19. Jahrhunderts entstehende Arbeiterschaft klassenspezifisch geprägtes Bildungsbedürfnis herausbildete. Bildung wurde als Mittel im Klassenkampf für die Arbeiterklasse bis zum Ersten Weltkrieg immer bedeutsamer. Ein dritter Ursprung besteht schon im 19. Jahrhundert mit der Entstehung der betrieblichen und beruflichen Weiterbildung. Durch das Entstehen immer größerer Unternehmen aufgrund kapitalistischer Produktionsweisen wurde das Anlernen am Arbeitsplatz, Bildungsveranstaltungen mit dem Ziel eines betrieblichen Aufstiegs oder Fortbildungsmaßnahmen für betriebliche Führungskräfte immer wichtiger.

In der Weimarer Republik (1919 bis 1933) wurde die deutsche Erwachsenenbildung insgesamt bedeutsamer. Zwischen 1918 und 1920 wurden sehr viele Volkshochschulen als eigenständige Institutionen eines freien Volksbildungswesens gegründet. Dabei gab es unterschiedliche Bewegungen in Form der bürgerlich-liberalen Volksbildungsbewegung und der vom Menschen ausgehenden individuellen Bildungsarbeit. Diesbezüglich kam es $1931 \mathrm{zu}$ einer Einigung mit dem Fokus auf eine Berufsbildung. Von der Arbeiterbewegung ging in der Weimarer Republik eine eigenständige institutionelle und theoretische Errichtung der Erwachsenenbildung aus. Die betriebliche Weiterbildung verblieb außerhalb der Wahrnehmung der öffentlichen Diskussion, wurde jedoch in vielen Betrieben in Relation zur Erstausbildung relevanter. Dadurch ist die betriebliche Weiterbildung heutzutage eine der zentralen Säulen von sämtlichen Weiterbildungsaktivitäten. Während der Herrschaft der Nationalsozialisten (1933 bis 1945) versuchten diese eine nationalsozialistische Umgestaltung der Erwachsenenbildung.

Die Gleichstellung der Erwachsenenbildung wurde in der Folge vorangetrieben. Weiterbildung hatte nach dem Zweiten Weltkrieg im Bereich der westlichen Besatzungszonen die Funktion einer demokratischen Re-Education durch die alliierten Siegermächte, was sich vor allem auf die Volkshochschulen, auf Bundes- und Landeszentralen für die politische Bildung und auf meist parteibezogenen Stiftungen und Heimvolkshochschulen bezog. Die Organisationen der betrieblichen, konfessionellen und gewerkschaftlichen 
Erwachsenenbildung übernahmen im Gegensatz dazu die Konzepte aus der Weimarer Republik. Erst 1960 wurde die Erwachsenenbildung wieder in die öffentliche Wahrnehmung gerückt, wobei sie als wesentlicher Baustein der öffentlichen Bildung hervorgehoben wurde. Der im Jahr 1970 durch den Deutschen Bildungsrat beschlossene „Strukturplan“ sowie der „Bildungsgesamtplan“ der Bund-Länder-Kommission aus dem Jahr 1973 können als Meilensteine zur Entwicklung der Erwachsenenbildung als eigenständigen Bildungsbereich betrachtet werden.

Die Zuweisung des Terminus „Weiterbildung“ für den Begriff „Erwachsenenbildung“ indizierte eine höhere staatliche Verantwortung, was in den meisten deutschen Bundesländern gesetzlich verankert wurde. Dabei wurde die Erwachsenenbildung vor allem in den Bereich der Berufsbildung und der allgemeinen Schulbildung aufgenommen. Bis 1983 wurde auf Bemühen des westdeutschen Staates die Erwachsenenbildung systematisch geregelt und gefördert, wogegen ein Engagement von staatlicher Seite in der Weiterbildung teilweise zurückgefahren wurde. $\mathrm{Zu}$ Beginn der 1990er Jahre setzte eine wesentliche Ausdifferenzierung bei betrieblichen Fortbildungseinrichtungen ein. Offene Einrichtungen, vor allem Volkshochschulen, fokussierten sich von da an auf das Nachholen von allgemeinen Bildungsabschlüssen, welche heutzutage flächendeckend institutionalisiert sind. Bundesweit verbreitet sind mittlerweile auch Einrichtungen von gesellschaftlichen Organisationen wie beispielsweise Kirchen, Gewerkschaften oder Wirtschafts- und Wohlfahrtsverbänden ebenso wie Institutionen im Bereich der beruflichen Weiterbildung, wobei letztere Einrichtungen aufgrund der Hartz-Gesetze zwischen 2004 und 2007 tiefgreifenden Umbrüchen unterworfen waren. Dabei richtete sich die Finanzierung nicht mehr an die verschiedenen Einrichtungen, sondern an die Nachfragerinnen und Nachfrager an diesen Angeboten.

Während früher der Begriff „Volksbildung“ für das Lernen von Erwachsenen gebraucht wurde, galt nach dem Zweiten Weltkrieg hierfür der Begriff der „Erwachsenenbildung“. Die gestiegene politische und ökonomische Bedeutung in der Erwachsenenbildung in den westlichen Ländern führte nachhaltig zu einem expansiven Gebrauch des Begriffs „Weiterbildung“. Dieser wurde als vierter in der staatlichen Verantwortung stehender Bildungsbereich eingeführt und deckte die Bereiche Erwachsenenbildung, Umschulung, Fortbildung und Berufsbildung im Erwachsenenalter ab. Von da an fungierte Weiterbildung als Oberbegriff für das gesamte Lernen Erwachsener. Während Weiterbildung in den vergangenen 25 Jahren verstärkt mit einer berufsbezogenen Ausrichtung versehen wurde, behielt der Begriff der Erwachsenenbildung weiterhin die Bedeutung einer 
gesellschaftsorientierten, allgemeinbezogenen und politisch ausgerichteten Bildung. Heutzutage werden in Deutschland die Begriffe Weiterbildung und Erwachsenenbildung überwiegend synonym verwendet, wobei die Weiterbildung einen stärkeren Berufsbezug hat als die Erwachsenenbildung. In den letzten 20 Jahren des 20. Jahrhunderts stieg die Partizipation an Weiterbildung und die Anzahl der dafür notwendigen Einrichtungen und Organisationen rapide an. Obwohl diese Entwicklung zu Beginn der 2000er Jahre nicht in dem gleichen Ausmaß anhielt, waren die finanziellen Subventionen in die Weiterbildung wesentlich größer als die der Schulen und Hochschulen. Die Expansion der Weiterbildung wurde jedoch im Wesentlichen ohne staatliche Unterstützung vorangetrieben und nur in einem sehr geringen Umfang staatlich finanziert und strukturiert. Dieser geringe Umfang bestand beispielsweise in der Analyse, der Informationsverbreitung oder der Qualitätssicherung der Weiterbildung. Das staatliche Engagement nahm sukzessive ab, wobei sich die Weiterbildung zu einem marktorientierten Dienstleistungsbereich wandelte. Im Laufe der vergangenen Jahre wurde die Erwachsenenbildung zunehmend an europäischen Standards ausgerichtet und bezog sich auch auf Ost- und Südosteuropa (Nuissl und Brandt 2009: 15 ff.).

\subsubsection{Wehr- und Zivildienst}

Die Dauer des gesetzlich vorgeschriebenen Grundwehrdienstes variierte im Laufe der Jahre zwischen 18 Monaten von 1962 und 1972 und sechs Monaten vom 01.12.2010 bis zur Aussetzung der Wehrpflicht am 01.07.2011 (Bundeswehr.de 2013). Daneben bestand die Möglichkeit zum Zivildienst und zur Verweigerung des Kriegsdienstes aus Gewissensgründen. Dieser wurde zum 10.04.1961 eingeführt und in der alten Bundesrepublik in den 1970er und 1980er Jahren mit dem Wehrdienst gleichgestellt (Bundeszentrale für politische Bildung 2011). Der Zivildienst hatte eine maximale Dauer von bis zu 20 Monaten, wobei er teilweise die gleiche Dauer wie der Grundwehrdienst hatte. Nachdem sich in den folgenden Jahrzehnten die Dauer auf bis zu neun Monate verkürzt hatte, betrug sie ab dem 01.12.2010 bis zur Aussetzung zum 01.07.2011 lediglich sechs Monate (Bundesamt für Familie und zivilgesellschaftliche Aufgaben o.J.). Die Wehrdienst- und Zivildienstepisoden finden im Rahmen der Analysen der vorliegenden Studie keine Berücksichtigung und werden daher ausgeschlossen, worauf im Kapitel 5 Methodisches Vorgehen noch ausführlich eingegangen wird. 


\subsubsection{Elternzeit}

Der Übergangsprozess kann bei Frauen durch eine Schwangerschaft und Elternzeit verlängert werden. Trotz eines sich wandelnden Familienlebens mit Schwangerschafts- und Elternphasen stieg durch die Bildungsexpansion die Partizipation von Frauen in weiterführenden Schulen und Hochschulen deutlich an. Diese verstärkte Berufsorientierung wurde durch verstärkte Investitionen in das eigene Humankapital und verbesserte berufliche und finanzielle Perspektiven sowie einen wesentlichen Wandel der Arbeitswelt in Form der Expansion der Dienstleistungsberufe begünstigt, wobei es von da an zu einem Konkurrieren zwischen einer Berufsorientierung und der Familienorientierung bei den Frauen kam. Im Zuge dessen wurde eine Erwerbstätigkeit für Frauen attraktiver als die häusliche Erziehung von Kindern (Gerlach und Laß 2012: 35). Eine Geburt des ersten Kindes und Elternschaft wurde oftmals als zeitlich aufschiebbar betrachtet, wogegen eine Ausbildung und eine anschließende berufliche Positionierung zunächst präferiert wurden (Gerlach und Laß 2012: 42). Auch Elternzeitepisoden finden bei den Analysen dieser Studie keine Berücksichtigung und werden deswegen ausgeschlossen, worauf ebenfalls im Rahmen des methodischen Vorgehens ausführlich eingegangen wird. 


\section{Theoretischer Rahmen}

Im Folgenden soll nun das theoretische Gerüst dieser Untersuchung als Grundlage für die formulierten Fragestellungen ausführlich dargestellt werden. Im Rahmen der vorliegenden Arbeit sind zwei theoretische Argumentationslinien für den betrachteten Übergangsprozess von der allgemeinbildenden Schule in die duale, vollzeitschulische oder akademische Erstausbildung, und für die beiden eingangs formulierten Fragestellungen von zentraler Bedeutung: die Humankapitaltheorie und die Verdrängungsthese. Die beiden theoretischen Ansätze werden zunächst ausführlich vorgestellt (vgl. Abschnitte 4.1.1 und 4.2.1) und auf die Fragestellungen übertragen (vgl. Abschnitte 4.1.2 und 4.2.2). Im Anschluss daran wird die Auswahl der beiden Ansätze begründet (vgl. Abschnitt 4.3) sowie aus den theoretischen Ansätzen abgeleitete Hypothesen formuliert (vgl. Abschnitt 4.4).

\subsection{Humankapitaltheorie}

\subsubsection{Theorie und Begriffe}

Die erste bedeutsame theoretische Grundlage ist die in der Bildungssoziologie sehr bedeutende Humankapitaltheorie, zu deren bekanntesten Vertretern der Ökonom Gary Stanley Becker gehört. In seinem Werk „Der ökonomische Ansatz zur Erklärung menschlichen Verhaltens“ beschreibt er diese Theorie damit, dass jedes Individuum einen gewissen Teil der eigenen Zeit und der Marktgüter dafür verwendet, um eine Schule zu besuchen, sich weiter zu bilden oder sich berufsrelevante Qualifikationen anzueignen. Diese Bildung beziehungsweise Aus- oder Weiterbildung wird als eine Ausprägung von Humankapital betrachtet (Becker 1982: 137), wobei ein Vermehren dieses Humankapitals die individuellen Chancen auf dem Ausbildungs- und Arbeitsmarkt verbessert.

Im Folgenden soll auf das Grundprinzip der Humankapitaltheorie eingegangen werden. Nach der zentralen humankapitaltheoretischen Annahme führt eine erhöhte Investition sowohl in die schulische als auch in die berufliche Bildung sowie zusätzlich in Weiterbildung zu einer erhöhten Produktivität und damit einhergehend $\mathrm{zu}$ einem erhöhten Einkommen auf dem Arbeitsmarkt. Mit dem langfristigen Ziel der Einkommensmaximierung investieren Personen solange in ihre Bildung, wie der erwartete Nutzen, in Form des späteren Einkommens, die mit den Investitionen verbundenen Kosten übersteigt (Becker 2011: 27). Ein höherer zu erwartender Nutzen gegenüber den anfallenden Kosten stellt somit die Voraussetzung für die Realisierung von Humankapitalinvestitionen dar. Bestehen bei der Entscheidung, in Humankapital zu investieren, jedoch mehrere mögliche Alternativen, kann eine Rangfolge 
dieser Entscheidungsalternativen gebildet werden. Im Zuge dessen wird dann bei der Entscheidung diejenige Alternative präferiert, bei welcher die Differenz zwischen den anfallenden Investitionskosten und dem zu erwartenden Nutzen am größten ist und der Nutzen die Kosten am meisten übersteigt. Bei dieser Entscheidungsalternative kann das betreffende Individuum dann den größten Vorteil für sich aus der Investition in Humankapital ziehen (Rissiek 1998: 34). Nach den Annahmen des Humankapitalmodells treffen rationale Akteure Bildungsentscheidungen mit dem Ziel, ihren Nutzen zu maximieren (Becker und Hecken 2011: 374). Dem Humankapitalansatz liegt ein ökonomischer Ansatz zugrunde (Rissiek 1998: 26), welcher unter anderem die Grundannahme des Bestehens einer individuellen Rationalität beinhaltet (Becker 1978: 5).

Im Folgenden werden die zentralen Begriffe Humankapital, Kosten und Nutzen näher beleuchtet. Zunächst soll der Begriff des Humankapitals theoretisch genauer definiert werden. Es besteht keine klare und präzise Einordnung, was unter die Kategorie „Humankapital“ fällt und wie weit der Humankapitalbegriff reicht. Der Begriff des Humankapitals kann generell in Bezug auf seine inhaltliche Abgrenzung und seinen Umfang hin unterschiedlich weit gefasst und ausgelegt werden. Theodore William Schultz fasste im Jahr 1961 den Humankapitalbegriff sehr weit und subsumierte unter ihn fünf unterschiedliche Kategorien, die sich wie folgt benennen lassen: „Formally Organized Education“, „On-the-job Training“, „Health“, „Study Programs for Adults“ und „Migration“ (Schultz 1961: 1; 8 ff.; zit. nach: Rissiek 1998: 23). Gary S. Becker bezeichnet die formale Bildung im Bereich des Schul- und Hochschulsystems und die praktische Berufsausbildung in einem Unternehmen als die zwei zentralsten und bedeutsamsten Kategorien des Humankapitals (Becker 1993: 17).

Die von Schultz verwendete Bezeichnung „Schooling“ kann verstanden werden als das formalisierte und vollzeitige Aneignen von individuellem Humankapital innerhalb von Bildungsinstitutionen. Unter der Kategorie „On-the-job Training“ wird das Aneignen von Humankapital in Form einer Berufsausbildung und damit einhergehend dem berufsrelevanten Erwerb von praktischen Fertigkeiten und umfassenden Kenntnissen verstanden. Dies umfasst unter anderem sämtliche berufliche Maßnahmen zur Aus- und Weiterbildung, welche in den Betrieben durchgeführt werden. Das Humankapital soll als Zusammenfassung aller arbeitsmarktrelevanten Kenntnisse und Fertigkeiten dienen, welche durch individuelle Investitionen gebildet werden (Rissiek 1998: 24 ff.). Dabei ist insbesondere das „On-the-job Training“ hinsichtlich von Weiterbildungen und Qualifizierungen während des Übergangs 
von der Schule in die Erstausbildung von Interesse. Hierzu können beispielsweise Praktika oder berufsvorbereitende Maßnahmen zählen.

Unter der Bezeichnung „Humankapital“ können verschiedene Kapitalformen wie Wissen und Fertigkeiten, der gesundheitliche Zustand, das äußere persönliche Erscheinungsbild, das soziale Prestige des Individuums, der Arbeitsethos oder persönliche Gewohnheiten im Alltag zusammengefasst werden. Bei diesen Eigenschaften handelt es sich in erster Linie um Facetten, die die persönlichen Chancen und die Produktivität auf dem Arbeitsmarkt beeinflussen. Dabei wird davon ausgegangen, dass es sich bei den aufgezählten Merkmalen um knappe und begrenzte Ressourcen handelt. Diese Kapitalien müssen jedoch zunächst erst einmal aufgebaut, erhalten und, wenn möglich, gesteigert werden, wozu wiederum Investitionen in das Humankapital erforderlich sind (Bröckling 2003: 18). Insofern wird ersichtlich, dass aufwendige Investitionen nicht nur notwendig sind, um Humankapital zu akkumulieren, sondern auch, um es überhaupt erhalten zu können und den individuellen Bestand an Humankapital zu sichern.

Der Begriff des Humankapitals kann aus zwei verschiedenen Perspektiven heraus verstanden werden: aus einer Individualperspektive und aus einer Unternehmensperspektive (Persch 2008: 37), wobei im Rahmen der Studie die individuelle Perspektive von Bedeutung ist. Individuell betrachtet wird das Humankapital deshalb als human bezeichnet, weil es unmittelbar und untrennbar an ein menschliches Individuum gebunden ist und nicht losgelöst von diesem betrachtet werden kann. Eine weitere zentrale Eigenschaft des Humankapitals ist, dass es eine temporär befristete Lebensdauer hat. Durch die Einbettung in ein menschliches Individuum hat das entsprechende Humankapital die identische Lebensdauer wie das Individuum, mit dem es untrennbar verknüpft ist (Rissiek 1998: 17).

Das individuelle Humankapital ist somit generell abhängig von einem einzelnen Menschen. Eine Verfügbarkeit und Nutzbarkeit des Humankapitals ist für Unternehmen als potentielle Nachfrager auf dem Arbeitsmarkt nur dann möglich, wenn das Humankapital von dem jeweiligen Individuum, mit dem es verwoben ist, dem Unternehmen auf dem Arbeitsmarkt angeboten und gegen eine angemessene Entlohnung seitens des Unternehmens zur Verfügung gestellt wird. Dieses zugänglich gemachte Humankapital muss durch das Unternehmen auch produktiv eingesetzt werden, um einen entsprechenden Profit daraus zu erzielen. Jedoch kann das individuell gebundene Humankapital nicht immer in ein allgemein zugängliches technisches Wissen transformiert werden. Der Bestand an individuellem Humankapital besitzt darüber hinaus zu einem bestimmten Zeitpunkt bei jedem Menschen einen klar definierten 
Umfang. Diesen Bestand kann jedes Individuum nun im Produktionsprozess den Unternehmen auf dem Arbeitsmarkt anbieten und es anschließend einsetzen (Rissiek 1998: 17 f.). Dies ist jedoch einzig und allein davon abhängig, ob das entsprechende Individuum die Bereitschaft und den Willen dazu hat, den Zugang und die Bereitstellung seines individuellen Humankapitals in Form seines Wissens und seiner Fertigkeiten externen Institutionen wie beispielsweise einem Unternehmen in der Wirtschaft zur Verfügung zu stellen (Straubhaar 1994: 115). Dieses individuell gebundene menschliche Kapital, welches in der Regel in der Güterproduktion einer Wirtschaft zum Einsatz kommt, ist klar zu unterscheiden von Sachkapital. Unter den Begriff des Sachkapitals lassen sich sämtliche in sachlichen und finanziellen Werten gebundene Kapitalien subsumieren, welche zur Produktion von materiellen und immateriellen Gütern wie beispielsweise Betriebsgrundstücken, Produktionsmaschinen, Verwaltungsgebäuden, Wertpapieren oder Patenten eingesetzt werden (Rissiek 1998: 19).

Von sehr großer Bedeutung sind auch die für die Investition in Humankapital anfallenden Kosten. Diese Kosten sind neben dem zeitlichen Aufwand für das Absolvieren weiterer Schulepisoden und das Nachholen von Bildungsabschlüssen in erster Linie so genannte Opportunitätskosten, welche dadurch entstehen, dass in der Phase der Partizipation an Weiterbildung und Zusatzqualifizierung unter Umständen kein eigenes Einkommen erwirtschaftet werden kann (Becker und Hecken 2011: 376). Dies ist für die betroffenen Personen unmöglich, da sie in dieser Phase in der Regel ihre Zeit nahezu ausschließlich in den Erwerb von zusätzlicher Bildung oder ausbildungs- beziehungsweise arbeitsmarktrelevanter Qualifikationen investieren. Diese nicht-monetären Kosten können als indirekte Kosten bei Humankapitalinvestitionen angesehen werden (Elliott 1991: 156, zit. nach: Rissiek 1998: 38). Die aufgewendeten Opportunitätskosten in Form eines entgangenen Einkommens während des Aneignens von Humankapital müssen im späteren biografischen Verlauf durch das berufliche Einkommen ausgeglichen werden (Becker und Hecken 2011: 376). Individuen, die die Bereitschaft dazu aufbringen, nehmen diese Kosten in Kauf, um ihre zukünftigen Ausbildungs- und Erwerbstätigkeitsperspektiven aufzubessern. Zusammenfassend sind die aufzuwendenden Kosten in allererster Linie als Zeitaufwand zu betrachten, da auch vergütete Weiterbildungsprogramme bestehen wie beispielsweise bezahlte Praktika.

Die Bereitschaft, Opportunitätskosten aufzuwenden, ist im Sinne der Humankapitaltheorie abhängig von dem anvisierten Nutzen. Dabei dient die berufliche Weiterqualifizierung nicht dem Selbstzweck. Die Individuen partizipieren an beruflicher Weiterbildung mit dem Ziel der 
Verbesserung ihrer zukünftigen Arbeitsmarkt- und Einkommenschancen (Becker und Hecken 2011: 373). Ein konkreter Nutzen aus der Sicht der Teilnehmenden an Weiterbildungsveranstaltungen kann zum Beispiel in der Auffrischung oder Erweiterung beruflicher Kenntnisse, dem Erzielen von zusätzlichen Einkommen oder von besseren Arbeitsbedingungen, der Realisierung beruflicher Aufstiege oder Neuorientierungen, einer erhöhten Arbeitsmarktflexibilität oder der Vermeidung oder Beendigung einer Arbeitslosigkeit bestehen (Becker und Hecken 2011: 369). Diese unterschiedlichen zukünftigen Anreize haben jedoch nicht bei jeder Person die gleiche Investitionsbereitschaft zur Folge. Dies und die unterschiedlichen vorhandenen Möglichkeiten führen dazu, dass jedes Individuum einen unterschiedlichen Umfang an Humankapital akkumuliert und über dieses verfügt. In einer individuellen Perspektive bemisst sich in diesem Zusammenhang der Wert der Arbeitnehmerin beziehungsweise des Arbeitnehmers auf dem Arbeitsmarkt aufgrund des zur Verfügung stehenden Humankapitals in Form seiner individuellen Produktivität (Becker 2011: 26 f.). Die Produktivität ist genauso wie der Umfang an verfügbarem Humankapital und die Bereitschaft, in dieses $\mathrm{zu}$ investieren, individuell unterschiedlich ausgeprägt. Diesbezüglich sind die Maßnahmen und zugrundeliegenden Mechanismen für die Bereitschaft, in Humankapital zu investieren, und zur Erreichung der aufgelisteten Ziele von Interesse. Betrachtet man das Investieren in Humankapital als Handlung an sich genauer, muss vor allem der rationale Hintergrund dieses Handelns in den Vordergrund rücken. Das individuelle Handeln kann dann als rational bezeichnet werden, wenn es auf eine Maximierung des beabsichtigten individuellen Nutzens im Eigeninteresse der Individuen abzielt. Dies kann beispielsweise durch die Investition in eine Berufsausbildung oder in ein universitäres Studium vollzogen werden. Die Intention für diese nach rationalen Gesichtspunkten handelnden Individuen besteht darin, bei der Bildung von Humankapital mit einem vorgegebenen Einsatz von Ressourcen das bestmögliche Resultat zu erstreben. Das grundlegende Ziel besteht dabei in der Maximierung der meist in monetärer Form vorliegenden Nettoerträge im Zeitverlauf (Rissiek 1998: 29). Konkret besteht die Zielsetzung für die rational handelnden Individuen darin, ihr Einkommen über den gesamten Lebenslauf hinweg zu maximieren.

Die Entscheidung, ob in Humankapital investiert wird oder nicht, hängt maßgeblich davon ab, wie die jeweilige individuelle Kosten-Nutzen-Abwägung bezüglich einer Investition in Humankapital ausfällt (Persch 2008: 29). Diesbezüglich trifft das Individuum eigenständig eine Wahl darüber, ob und welches Humankapital es sich aneignet. Entscheidend ist dabei, 
dass die Bildung von Humankapital insbesondere auf der Basis von selbständigen Wahlentscheidungen der einzelnen Personen bezüglich der verschiedenen und in Frage kommenden Investitionsalternativen erfolgt. Dies lässt sich daran verdeutlichen, dass jedes einzelne Individuum für sich selbst entscheidet, welche Investitionsalternative es auswählt, in welchem Umfang und in welche Form von Humankapital es investiert und welche berufliche Erwerbstätigkeit es im späteren Verlauf zur Erzielung von finanziellen Erträgen im Produktionsprozess einer Volkswirtschaft praktizieren möchte. Dies geschieht unter den jeweiligen Gegebenheiten der bereits erwähnten elterlichen Unterstützungsmöglichkeiten in finanzieller und intellektueller Hinsicht. Das rationale Verhalten von jedem Individuum ist somit durch eine Wahlentscheidung zur Investition in Humankapital gekennzeichnet. Darauf aufbauend soll dieses Humankapital mit dem Ziel eines späteren Erwerbsberufs verwendet werden (Arrow 1994: 1). Damit investieren viele Menschen individuell in ihre eigenen Qualifikationen und in ihr eigenes Humankapital, indem sie vor allem zeitliche Kosten aufwenden, um Wissen, Fertigkeiten oder Fähigkeiten erwerben zu können. Darauf aufbauend wird ersichtlich, dass das Aneignen von Humankapital als aktives Handeln bewertet werden kann, welches immer mit der Entscheidung verbunden ist, ob man in Bildung investiert oder nicht. Bei dieser Abwägung spielen neben den angesprochenen Kosten auch die individuell zur Verfügung stehenden finanziellen und intellektuellen Ressourcen der Personen eine maßgebliche Rolle (Becker 2011: 27).

Für das erwähnte rationale Kalkül und das daran orientierte Entscheidungsverhalten für die Investitionen in Humankapital sind weitere Faktoren bedeutsam, welche mit den individuell wahrgenommenen Kosten und Erträgen zusammenhängen. Zu diesen Faktoren gehört die soziale Herkunft, welche einen Einfluss darauf hat, ob und inwieweit in Humankapital investiert ist. Dies lässt sich sowohl beim Erwerb allgemeinbildender Bildungsabschlüsse als auch bei der Auswahl einer weiterführenden Ausbildung belegen. Beispielsweise hat die soziale Herkunft einen Einfluss auf das Erreichen der gymnasialen Oberstufe oder auf das Aufnehmen eines Hochschulstudiums (Allmendinger et al. 2010: 55 ff.). Basierend auf dieser Erkenntnis ist anzunehmen, dass die soziale Herkunft nicht nur einen Einfluss auf den Schulabschluss hat, welcher erworben wird, sondern auch auf den Umfang an Humankapital, welches aufgeholt werden muss, um die Chancen für den Übergang in die Erstausbildung zu verbessern. Eine niedrige soziale Herkunft kann demnach nicht nur zu einem niedrigen Schulabschluss führen, sondern auch dazu, dass beispielsweise Bildungsabschlüsse vor dem Beginn der Erstausbildung nachgeholt werden müssen, um bessere Zugangschancen in den 
Ausbildungsmarkt zu haben. Eine niedrige soziale Herkunft und ein niedriger Schulabschluss können somit die Notwendigkeit von Humankapitalinvestitionen vor Beginn der Erstausbildung erforderlich im Hinblick auf die Ausbildungschancen machen.

Unterschiede bei der Kosten-Nutzen-Abwägung bei Humankapitalinvestitionen werden vor allem in Abhängigkeit von der jeweiligen Kostenbelastung gesehen. In Familien mit einem niedrigen Einkommen werden die Kosten von höherer und zusätzlicher Bildung als schwerwiegender empfunden. Dies führt wiederum zu einer geringeren Nachfrage nach dem Humankapital Bildung. Des Weiteren werden die Erfolgsaussichten in schulischer Hinsicht von dieser Gruppe als geringer eingeschätzt, was mit einem erhöhten Risiko im Rahmen von Bildungsinvestitionen einhergeht. Im Vergleich zu den unteren Herkunftsschichten erscheint der Nutzen für die mittleren und höheren Schichten dagegen deutlich wahrscheinlicher. Dies ist mit einer erhöhten Risikobereitschaft und einer größeren Nachfrage nach Bildung bei diesen Gruppen verbunden (Kristen 1999: 21).

\subsection{2 Übertragung der Humankapitaltheorie auf die vorliegende Untersuchung}

In den folgenden Ausführungen soll die konkrete Anwendung der vorgestellten Humankapitaltheorie auf die vorliegende Untersuchung dargestellt werden. Im Rahmen dieser Studie ist das Humankapital in der individuellen Perspektive relevant, da es um die Untersuchung der individuellen Übergangsprozesse von Schulabgängerinnen und Schulabgängern mit unterschiedlichen Schulabschlussformen geht. Dabei sollen weder die einbezogenen Personen noch die einzelnen Schulabschlussgruppen in einer Unternehmensperspektive betrachtet werden, was beispielsweise analog bei der Betrachtung der Akkumulation von Humankapital eines Landes oder einer Gesellschaft der Fall wäre. Stattdessen stehen die individuellen Verläufe bei der Aneignung von Humankapital während der Übergangsphase von der Schule in die Ausbildung im Fokus.

Dabei ist die Betrachtung des Humankapitals sowie dessen Entwicklung und Bedeutung speziell auf dem Arbeitsmarkt in der Bundesrepublik Deutschland interessant. Der Erwerb von Humankapital in Form von zusätzlichen Qualifikationen und Bildungsbeständen wird auch auf dem deutschen Arbeitsmarkt sukzessive immer wichtiger. Dabei ist das Humankapital mittlerweile in der Bundesrepublik Deutschland der Produktionsfaktor Nummer eins, wobei in Zukunft von einer weiter kontinuierlich steigenden Bedeutung des Humankapitals ausgegangen werden muss. Dies hat im Wesentlichen seinen Ursprung darin, dass sich auch der Arbeitsmarkt und damit einhergehend viele Berufsbilder gewandelt haben. 
Diese Entwicklung in den vergangenen Jahren und Jahrzehnten lässt sich vor allem in Form einer für die ökonomische Dynamik gestiegenen Bedeutung der Humanressourcen in hochentwickelten Volkswirtschaften charakterisieren. Diese wurden im Zeitverlauf immer relevanter, wogegen die Bedeutung des Sachkapitals sukzessive zurückging. Beispielsweise werden durch personenbezogene Dienstleistungen oder durch Berufe im Wissens- und Informationsbereich neuartige und gestiegene Qualifikationen notwendig, von welchen ein immer größer werdender Anteil von erwerbstätigen Personen tangiert wird (Autorengruppe Bildungsberichterstattung 2008: 6). Die deutsche Volkswirtschaft besteht traditionell aus drei verschiedenen Bereichen, aus einem primären Sektor im Bereich der Land- und Forstwirtschaft sowie der Fischerei, einem sekundären Sektor im Bereich des produzierenden Gewerbes und einem tertiären Sektor der Dienstleistungen. Die Volkswirtschaft war im Laufe der vergangenen Jahrzehnte von tiefgreifenden strukturellen Veränderungen betroffen, was sich auf ihre drei Sektoren und insbesondere auf deren einzelnen Bedeutungen und Entwicklungen auswirkte. Der Dienstleistungssektor expandierte kontinuierlich und gewann immer stärker an Bedeutung in der deutschen Volkswirtschaft. Dagegen wurden die beiden anderen Sektoren im Laufe der Zeit ökonomisch unbedeutender. Im expandierenden Dienstleistungssektor werden insbesondere bei unternehmensbezogenen Dienstleistungen, in Unternehmen mit dem Schwerpunkt Informations- und Kommunikationstechnologie sowie bei öffentlichen und personenbezogenen Dienstleistungen wie etwa im Pflegebereich verstärkt neue Arbeitsplätze geschaffen. Dadurch und auch durch den damit verbundenen technischen Wandel wurden sehr viele Berufsbilder und berufliche Anforderungsprofile grundlegend modifiziert, was sich auch mit der verstärkten Nachfrage vor allem nach analytischem Denken und Kompetenzen im Bereich der Kommunikation und Problemlösung in einer Dienstleistungs- und Wissensgesellschaft belegen lässt (Autorengruppe Bildungsberichterstattung 2008: 22 f.). Ein Ende dieser Entwicklung ist derzeit nicht absehbar, wodurch sich diejenigen Personen, die vor der Entscheidung stehen, in zusätzliche Weiterbildung $\mathrm{zu}$ investieren, auf ständig veränderte Rahmenbedingungen und Einflussfaktoren einstellen müssen.

Insofern können Berufsbranchen aus dem Dienstleistungsbereich als die bedeutendsten Wachstumsbranchen im Zeitraum der 1980er Jahre bezeichnet werden. Im Zuge dessen ist auch eine zunehmende Dienstleistungsorientierung des Arbeitstätigkeitsprofils in vielen Berufsbereichen zu verzeichnen, was sich darüber hinaus auch auf den produzierenden Sektor übertragen lässt. Dies kann damit belegt werden, dass zu Beginn der 1990er Jahre im Bereich 
des produzierenden Gewerbes nahezu 40 Prozent aller Arbeitsplätze über einen Dienstleistungscharakter verfügten. Viele Routinetätigkeiten werden vor allem durch den Gebrauch neuer Techniken beziehungsweise technischer Hilfsmittel immer weiter abgelöst und durch Arbeitsinhalte mit einer prozessorientierten und themenübergreifenden Ausrichtung ersetzt. Diese neuartigen Arbeitsinhalte inkludieren ein erhöhtes Anforderungsprofil in Bezug auf Handlungskompetenzen und vorhandene Qualifikationen auf sämtlichen Ebenen (Buttler und Tessaring 1993: 468). Somit ist die Weiterbildung als Humankapitalform im Zuge der Expansion von dienstleistungsorientierten Berufen immer zentraler und bedeutsamer geworden. Dies kann sowohl auf die Weiterbildung vor Beginn der Ausbildung beziehungsweise vor Beginn einer Erwerbstätigkeit als auch auf die betriebliche Weiterbildung parallel zur ausgeübten Erwerbstätigkeit bezogen werden.

Des Weiteren kommen bei der gestiegenen Bedeutung von Humankapital zusätzlich noch die beiden Gegebenheiten zum Tragen, dass einerseits eine Verknappung von physischen Ressourcen und von Rohstoffen konstatiert werden muss und dass andererseits die internationale Konkurrenz durch andere Länder den Arbeitsmarkt- und Wirtschaftsstandort Deutschland vor große Herausforderungen stellt (Buttler und Tessaring 1993: 467). Der verstärkte Bedarf an Humankapital und damit einhergehend dessen verstärkte Aneignung führen damit zu einem insgesamt erhöhten Qualifikationsniveau auf dem Arbeitsmarkt, was weitreichende Konsequenzen insbesondere für die Arbeitnehmerinnen und Arbeitnehmer mit sich bringt. Somit ist für die vorliegende Untersuchung das Humankapital als erworbene Weiterbildung und Zusatzqualifikation beim Absolvieren von Übergangsstationen während des Übergangsprozesses in die Ausbildung von Bedeutung. Hierzu gehört auch der bereits erläuterte Begriff des „Schooling“ im Sinne eines Nachholens von Bildungsabschlüssen im Rahmen von zusätzlichen Schulepisoden.

Beim Übergangsprozess in die Ausbildung spielt die Humankapitaltheorie in kostenbezogener Hinsicht eine entscheidende Rolle. Das Investieren in zusätzliche Weiterbildung und Zusatzqualifikation, aber auch in das Nachholen von Schulabschlüssen im Anschluss an die allgemeinbildende Schulzeit bedeuten Kosten aufgrund des entsprechenden Zeitaufwands für das Absolvieren zusätzlicher Übergangsstationen, was verbunden ist mit einer längeren Übergangsdauer. In diesem Fall wird eine Verzögerung des Eintritts in den Ausbildungsmarkt in Kauf genommen, um sich durch das Investieren in zusätzliche, über die allgemeine Schulbildung hinausgehende, Qualifikationen oder das Nachholen von Schulabschlüssen eine bessere Ausgangsposition für den Zugang zum Ausbildungs- und Arbeitsmarkt zu 
verschaffen. In dieser Phase ergeben sich zusätzlich Opportunitätskosten, da während einer Weiterbildungsphase kein Einkommen erworben wird. Die Kosten werden in der vorliegenden Studie, auch im Hinblick auf die untersuchte Übergangsdauer, als Zeitaufwand betrachtet.

Um diesen Zeitaufwand zu erbringen, spielen bessere Einkommenschancen und verbesserte berufliche Aufstiegschancen als Zielsetzungen bei einer Weiterbildungsmaßnahme die zentrale Rolle (Beicht et al. 2004: 6). Der Nutzen durch das Investieren in Humankapital wird im Rahmen dieser Arbeit lediglich in Form von besseren Ausbildungschancen berücksichtigt. Nur diese Zielsetzung fällt in den zu untersuchenden Übergangszeitraum, da sich ein mögliches höheres Berufsprestige oder ein höheres Einkommen erst im späteren Berufsverlauf ergibt.

Wird der Entscheidungsprozess, ob in Humankapital investiert wird oder nicht, auf die vorliegende Untersuchung übertragen, ist ein wesentlicher Aspekt zu berücksichtigen. Die Individuen, die von diesen Investitionen und deren Folgen betroffen sind, sind teilweise, wenn sie die allgemeinbildende Schule nicht mit einer allgemeinen Hochschulreife verlassen, noch nicht volljährig. Dies führt dazu, dass die Entscheidung, ob in Humankapital investiert wird und wenn ja, in welchem Umfang, auch von den Eltern getroffen wird. Zumindest haben die Eltern hierbei ein Mitspracherecht, da sie die Investitionen oftmals erst ermöglichen, indem sie die Bereitschaft aufbringen, die damit verbundenen Kosten aufzuwenden, sofern ihnen dies möglich ist. Schulabgängerinnen und Schulabgänger, die die Schule mit der allgemeinen Hochschulreife verlassen und aufgrund dessen einen längeren Aufenthalt in der allgemeinbildenden Schule vorweisen als diejenigen mit einem Realschulabschluss, Hauptschulabschluss oder ohne Schulabschluss, sind in der Regel volljährig. Deshalb ist zu vermuten, dass sie meist selbst über ihren eigenen Ausbildungs- und Berufsverlauf sowie über das Investieren in Weiterbildung entscheiden. Dies hängt jedoch maßgeblich davon ab, inwieweit sie selbst in der Lage sind, die Investitionskosten zu tragen oder ob ihre Eltern bereit sind, sie hierbei zu unterstützen. Somit haben die Eltern auch bei dieser Gruppe unter Umständen noch ein indirektes Mitspracherecht bei der Entscheidung, in Humankapital zu investieren oder nicht. Zumindest dienen die Eltern bei Abiturientinnen und Abiturienten als die wichtigsten Ansprechpartner in Bezug auf den Berufswahlprozess beziehungsweise die Berufsorientierung (Knauf und Rosowski 2009: 305). In diesem Zusammenhang hängt die jeweilige Entscheidung außerdem davon ab, welche elterlichen Unterstützungsmöglichkeiten im jeweiligen Haushalt gegeben sind. Eltern mit einem höheren Einkommen können ihren 
Kindern eher den Zugang zu Bildungsangeboten ermöglichen als Eltern mit einem geringen Einkommen. Darüber hinaus spielt auch eine Rolle, wie gut die Eltern über die verschiedenen Bildungsangebote und deren Zugangsmöglichkeiten informiert sind.

Im Folgenden soll eine differenzierte Betrachtung der vier einbezogenen Schulabschlussgruppen hinsichtlich der Investition in Humankapital und der damit verbundenen Dauer und der absolvierten Stationen während des Übergangs von der Schule in die berufliche oder akademische Erstausbildung stattfinden. Zentral ist dabei, dass es bei dem Investieren in Humankapital nur um das Humankapital im Rahmen von Übergangsstationen geht, welches zwischen dem erstmaligen Verlassen der allgemeinbildenden Schule und der Aufnahme einer beruflichen oder akademischen Erstausbildung akkumuliert wird. Grundsätzlich ist davon auszugehen, dass jede der vier Schulabschlussgruppen rational betrachtet versuchen wird, möglichst schnell die Erstausbildung zu beginnen. Der Ausbildungs- und Berufswunsch lässt sich größtenteils neben den gegebenen wirtschaftlichen Rahmenbedingungen auch aus dem $\mathrm{zu}$ erwartenden allgemeinbildenden Schulabschluss ableiten (Pätzold 2008: 604). Bei Schulabgängerinnen und Schulabgängern mit Abitur ist zu vermuten, dass sie Investitionen in Humankapital im Rahmen von Zwischenstationen vor Beginn der beruflichen oder akademischen Erstausbildung eigentlich nicht benötigen. Dennoch ergibt sich bei dieser Gruppe oftmals die Konstellation, dass sie die Zeit vor Beginn der Erstausbildung sinnvoll überbrücken möchte. Dadurch bringen sie die Bereitschaft auf, im Anschluss an die allgemeinbildende Schulzeit Bildungsinvestitionen $\mathrm{zu}$ tätigen und entsprechende Kosten aufzuwenden, um den daraus folgenden Nutzen, verbesserte Chancen auf dem Ausbildungs- und Arbeitsmarkt sowie höhere Einkommenschancen, zu realisieren. Bei einer Kosten-Nutzen-Abwägung im Sinne der Humankapitaltheorie wird der zu erwartende Nutzen möglicherweise groß genug sein, um in Humankapital in Form von zusätzlichen Qualifikationen vor der Aufnahme der Erstausbildung zu investieren. Der Nutzen besteht dabei vor allem in höheren beruflichen Positionen, Aufstiegschancen oder einem höheren Einkommen. Dieses Humankapital in Form der Zwischenstationen vor Aufnahme einer beruflichen oder akademischen Erstausbildung können bei dieser Schulabschlussgruppe vor allem Praktika, Kurse oder sonstige Weiterbildungsmöglichkeiten sein, welche über die allgemeine Schulbildung hinausgehen oder diese ergänzen. Dies wird auch daran deutlich, dass junge Erwachsene nach dem Erwerb des Abiturs Übergangstätigkeiten vor allem deswegen ausüben, da sie die eigenen Chancen auf dem Ausbildungsmarkt verbessern möchten (Knauf und Rosowski 2009: 291 f.). Anzumerken ist, 
dass diejenigen mit Abitur bereits über den höchsten allgemeinbildenden Schulabschluss verfügen und die von ihnen möglicherweise erworbenen zusätzlichen Qualifikationen als Ergänzung dienen, um den Übergang in die Berufsausbildung oder in das Studium zu erleichtern.

Jugendliche, die an einer allgemeinbildenden Schule die mittlere Reife erworben haben, vollziehen beim Übergangsprozess in die berufliche Erstausbildung ebenfalls eine Abwägung zwischen Kosten und Nutzen für die Investition in Humankapital. Hierbei kann angenommen werden, dass viele Personen dieser Schulabschlussgruppe den Nutzen als hoch genug betrachten, um vor Beginn der Erstausbildung in Humankapital in Form eines Nachholens von Schulabschlüssen oder anderer Weiterbildungen zu investieren. Damit sind auch sie bereit, in zusätzliche Bildung $\mathrm{zu}$ investieren und Kosten in Form von Zeit hierfür aufzuwenden. Insbesondere ist für diese Gruppe das Nachholen des Abiturs besonders attraktiv (Jacob und Tieben 2010: 169 f.), um die eigenen Ausbildungs- und Arbeitsmarktchancen zu verbessern. Durch das Nachholen von Bildungsabschlüssen erhoffen sie sich beispielsweise bessere Ausbildungs- und weitergehend bessere Arbeitsmarktchancen als angestrebten Nutzen. Dadurch ist davon auszugehen, dass ein erheblicher Anteil dieser Gruppe das Abitur nachholen wird, um einerseits verbesserte Chancen auf dem Ausbildungsmarkt $\mathrm{zu}$ haben und andererseits die Zugangsmöglichkeit $\mathrm{zu}$ einem Hochschulstudium zu erlangen. Dieses Bestreben ist jedoch auch gekoppelt an die schulischen Leistungen dieser Gruppe. Für diesen Nutzen werden viele Schulabgängerinnen und Schulabgänger mit einem Realschulabschluss bereit sein, Kosten in Form von Zeit aufzuwenden.

Bei Betrachtung der Optionen bezüglich der Zwischenstationen vor Beginn der Erstausbildung wird deutlich, dass viele Jugendliche versuchen, sich so lange wie möglich viele Bildungs- und Berufsalternativen offen zu halten. Diese Tendenz hat sich im Laufe der Zeit sukzessive verstärkt (Pätzold 2008: 594). Beispielsweise mündet etwa ein Viertel aller Absolventinnen und Absolventen einer Realschule nach dem Verlassen der allgemeinbildenden Schule vorerst in das Übergangssystem ein (Baethge et al. 2007: 8). Viele Jugendliche, die sich eine duale Berufsausbildung zum Ziel gesetzt hatten, absolvieren den Wehr- oder Zivildienst, ein freiwilliges soziales beziehungsweise ökologisches Jahr, ein Praktikum, sind arbeitslos oder befinden sich in anderweitigen Übergangsstationen (BMBF 2005: 78). Während der bis zum Jahr 2011 gesetzlich vorgeschriebene Wehr- oder Zivildienst unabhängig vom erreichten Schulabschluss von den jungen Männern geleistet werden musste, 
kann auch ein freiwilliges soziales oder ökologisches Jahr sowohl von Abiturientinnen und Abiturienten als auch von Jugendlichen mit einem Realschulabschluss absolviert werden. Gleiches gilt für Praktika, wobei anzunehmen ist, dass diejenigen mit Abitur, welche ein Hochschulstudium aufnehmen, Praktika sowohl vor als auch während des Studiums absolvieren. Eine Arbeitslosigkeit ist bei Schulabgängerinnen und Schulabgängern mit Abitur eher nicht anzunehmen. Dennoch soll an dieser Stelle nicht die Gruppe derjenigen mit Abitur oder Realschulabschluss vergessen werden, welche direkt nach dem Verlassen der allgemeinbildenden Schule in eine berufliche oder akademische Ausbildung einmündet und keine Zwischenstationen während des Übergangs absolviert.

Bei der Untersuchung der Entwicklung im Zeitverlauf ist zu erwarten, dass sich für diejenigen mit einem Realschulabschluss durch das Investieren in Humankapital im Laufe der Jahrzehnte die Übergangsdauer bis zum Eintritt in die duale oder vollzeitschulische berufliche Erstausbildung verlängert und die Anzahl der Übergangsstationen erhöht hat. Dies ist damit zu begründen, dass diese Gruppe verstärkt in Humankapital in Form des Nachholens des Abiturs investiert hat. Des Weiteren ist anzunehmen, dass diese Gruppe vor Beginn der Erstausbildung zusätzlich von Weiterbildungsmöglichkeiten oder Kursen Gebrauch gemacht hat. Für Schulabgängerinnen und Schulabgänger mit Abitur ist dagegen zu erwarten, dass sich die Übergangsdauer und die Anzahl der Übergangsstationen im Zeitverlauf nicht verändert haben. Für diese Gruppe erscheint ein Aufenthalt im Übergangssystem oder eine unfreiwillige Arbeitslosigkeit als eher unwahrscheinlich. Dennoch muss betont werden, dass bei einer im Laufe der Jahrzehnte angenommenen konstanten Übergangsdauer sowie einer konstant gleich gebliebenen Anzahl an Zwischenstationen während der Übergangsphase die Studienanfängerinnen und Studienanfänger vielfältige Zwischenstationen und Tätigkeiten auch zwischen dem Verlassen der allgemeinbildenden Schule und dem Beginn des Studiums absolvieren. Diese Stationen verzögern auch bei ihnen die Aufnahme des Studiums (Friebertshäuser 2008: 617), wobei angenommen wird, dass dieser Umstand über die Jahrzehnte gleichgeblieben ist und hierbei keine Veränderung stattgefunden hat. Der Wehrbeziehungsweise Zivildienst beispielsweise wurde bereits in früheren Jahrzehnten von jungen Männern absolviert, da er zu dieser Zeit bereits gesetzlich verankert war und sich lediglich die Dienstdauer aufgrund von gesetzlichen Modifizierungen teilweise veränderte (Bundeswehr.de 2013; Bundesamt für Familie und zivilgesellschaftliche Aufgaben o.J.). Auch weitere Zwischenstationen wurden bereits in früheren Jahren und Jahrzehnten beim Übergang von der Schule in das Studium durchlaufen. Zum Beispiel wurden bereits Praktika auch schon vor 
Beginn eines Studiums absolviert, um erste Praxiseinblicke zu erhalten. Dies galt beispielsweise damals bereits für Hochschulzugangsberechtigte, die im Anschluss ein Studium im Bereich der Natur- oder Ingenieurwissenschaften oder in der Medizin begannen (Lewin et al. 1996: 432). Da das Durchlaufen solcher Zwischenstationen auch bis in die Gegenwart der Fall ist, liegt die Vermutung nahe, dass für diese Gruppe eine über Jahrzehnte hinweg konstante Übergangsdauer und eine unveränderte Anzahl an Übergangsstationen vorliegen könnte.

Bei den Zwischenstationen, die von Abiturientinnen und Abiturienten vor Beginn des Studiums ausgeübt werden, handelt es sich beispielsweise um Erwerbstätigkeiten, Praktika, ein freiwilliges soziales Jahr oder Au-pair-Aufenthalte im Ausland (Friebertshäuser 2008: 617). Auch bei denjenigen Abiturientinnen und Abiturienten, welche sich für eine duale oder vollzeitschulische Berufsausbildung entscheiden, ist $\mathrm{zu}$ vermuten, dass sie vor dem Ausbildungsbeginn diverse Zwischenstationen wie Praktika oder Weiterbildungsmöglichkeiten nutzen. Diese Etappen dienen ihnen als berufsrelevante Qualifizierung, wobei bei dieser Gruppe nicht von einem Aufenthalt im berufsvorbereitenden Übergangssystem, zu welchem unter anderem das Berufsvorbereitungsjahr gehört, oder einer Arbeitslosigkeit während der Übergangsphase auszugehen ist. Bei dieser Gruppe kann somit von einer konstanten Übergangsdauer und einer gleichgebliebenen Anzahl von Übergangsstationen zwischen 1971 und 2012 ausgegangen werden. Dabei werden vor allem weiterbildende sowie berufsorientierende Stationen vermutet.

Für Schulabgängerinnen und Schulabgänger mit einem Hauptschulabschluss oder einem fehlenden Schulabschluss ist zu vermuten, dass diese im Sinne der Humankapitaltheorie ebenfalls Investitionen in zusätzliche Bildung tätigen. Jugendliche mit einem niedrigen Schulabschluss setzen sich vorwiegend das Ziel, eine duale Berufsausbildung beginnen zu können (Beicht und Ulrich 2008b: 8). Hierbei ist zu vermuten, dass sie in der Regel zusätzliche Bildung in Form des Nachholens von Schulabschlüssen anstreben, um bessere Chancen beim Einstieg in die Ausbildung haben. Jedoch hat sich gezeigt, dass vielen Jugendlichen aus dieser Gruppe der Eintritt in den beruflichen Ausbildungsmarkt nicht sofort, sondern oftmals mit deutlicher Verzögerung gelingt. Da sie einen niedrigeren Schulabschluss besitzen als diejenigen mit Abitur oder einem Realschulabschluss, müssen sie deutlich mehr Humankapital aufholen, um ihre Ausbildungschancen zu verbessern. Dies ist insbesondere mit dem Nachholen von allgemeinbildenden Bildungsabschlüssen verbunden. Für den Nutzen in Form von verbesserten Ausbildungs- und Erwerbschancen und besseren 
Einkommenschancen müssen sie deutlich mehr Kosten in Form von Zeit aufwenden. Deshalb vollziehen viele Jugendliche mit einem Hauptschulabschluss nicht im Jahr des Erwerbs des Schulabschlusses den direkten Übergang in eine berufliche Ausbildung (Lex und Zimmermann 2011: 614). Jedoch befinden sie sich sechs Monate nach dem erstmaligen Verlassen der allgemeinbildenden Schule oftmals im berufsvorbereitenden Übergangssystem und sind dadurch die primäre Zielgruppe von berufsvorbereitenden Maßnahmen (Beicht und Ulrich 2008b: 10). Durch diese Maßnahmen erhoffen sie sich eine bessere Unterstützung und Vorbereitung für den Zugang in den Ausbildungsmarkt. Durch das Partizipieren an berufsvorbereitenden Maßnahmen investieren sie somit auch in Humankapital. Bei den während der Übergangsphase absolvierten Stationen dieser Gruppe handelt es sich demnach neben dem Nachholen von Bildungsabschlüssen oftmals um berufsvorbereitende Maßnahmen. Im berufsvorbereitenden Übergangssystem werden sie mit einer Vielzahl von ausbildungs- und berufsvorbereitenden Maßnahmen konfrontiert. Diese vielfältigen Bildungswege und Maßnahmen sind bezogen auf die Voraussetzungen, Inhalte und Abschlüsse sehr unterschiedlich, münden jedoch allesamt nicht in einem anerkannten beruflichen Abschluss. Zu diesen zählen berufsfachschulische Bildungsgänge sowie viele verschiedene kompensatorisch ausgerichtete und ganztägig organisierte berufsvorbereitende Bildungsangebote (Krone 2010: 23).

Einige dieser Maßnahmen dienen jedoch auch der Weiterbildung und zielen darauf ab, allgemeinbildende Schulabschlüsse nachholen zu können. Neben Maßnahmen wie dem schulischen Berufsvorbereitungsjahr, dem Berufsgrundschuljahr oder diversen von der Agentur für Arbeit angebotenen berufsvorbereitenden Bildungsmaßnahmen, die primär der Vorbereitung auf eine berufliche Ausbildung oder auf eine darauffolgende Erwerbstätigkeit dienen, werden im Übergangssystem auch verschiedene Bildungsgänge an Berufsfachschulen angeboten. Diese führen zwar nicht zu einem berufsqualifizierenden Abschluss, ermöglichen aber den nachträglichen Erwerb eines mittleren Schulabschlusses oder des Fachabiturs (Lex und Geier 2010: 167). Aus diesem Grund muss berücksichtigt werden, dass es auch unter den Schulabgängerinnen und Schulabgängern mit einem Hauptschulabschluss oder ohne Schulabschluss Jugendliche gibt, die unter anderem im Rahmen der vielfältigen Angebote des Übergangssystems an Weiterbildung partizipieren und sich dadurch während des Übergangsprozesses im Sinne der Humankapitaltheorie weiterqualifizieren. Neben den vielfältigen berufsvorbereitenden Maßnahmen können als Übergangsstationen für diese Gruppe vor allem zusätzliche Schulepisoden zum Nachholen von Schulabschlüssen im Sinne 
der Akkumulation von Humankapital vermutet werden. Dadurch versuchen diejenigen ohne Abschluss oder mit einem Hauptschulabschluss ihre Ausgangsposition vor dem Übergang in eine vollqualifizierende Ausbildung zu verbessern. Hierfür sind sie bereit, zusätzliche Zeit aufzuwenden, wobei zu vermuten ist, dass dies auch aus Mangel an Alternativen geschieht.

Da in der Regel keine strukturierten Wege aus dem Übergangssystem hinausführen, kommt es beim Durchlaufen der Maßnahmen zu deutlichen Verzögerungen und damit zu langen Warteschleifen (Krone 2010: 25). Aufgrund dieser Aspekte kann davon ausgegangen werden, dass sich für Jugendliche mit einem Hauptschulabschluss oder ohne einen Schulabschluss die Übergangsdauer bis zum Eintritt in die Erstausbildung im Zeitverlauf eklatant verlängert hat. Auch zusätzliche Schulepisoden, die dem Erwerb weiterer Schulabschlüsse dienen, tragen zu einer Verlängerung der Übergangsphase bis zum Eintritt in eine vollqualifizierende Berufsausbildung bei, wodurch bei beiden Gruppen die Anzahl der Übergangsstationen gestiegen sein dürfte.

\subsection{Verdrängungsthese}

\subsubsection{Theorie und Begriffe}

Die zweite für diese Untersuchung zentrale theoretische Leitlinie, die sogenannte Verdrängungsthese, wurde von verschiedenen Autoren formuliert und behandelt (u. a. Blossfeld 1983; 1990; Fürstenberg 1978) und hat ihren Ursprung in dem durch die Bildungsexpansion seit den 1970er Jahren gestiegenen Anteil an qualifizierten und gut ausgebildeten Arbeitskräften auf dem Arbeitsmarkt. Dieser Verlauf führte zu unterschiedlichen Entwicklungen der Arbeitsmarktchancen in Abhängigkeit vom erreichten Schulabschluss und der erworbenen beruflichen Qualifizierung. Im Zuge dessen haben sich die Arbeitsmarktchancen für gering qualifizierte Arbeitskräfte im Laufe der Zeit deutlich verschlechtert. Je geringer deren erreichter allgemeinbildender Schulabschluss sowie deren Berufsqualifikation sind, desto schwieriger gestaltet sich der Zugang in den Arbeitsmarkt und in eine geregelte Erwerbstätigkeit. Im Zuge des Prozesses der Bildungsexpansion und des damit verbundenen Trends der Höherqualifizierung kam es zu einer deutlichen Verringerung der Anzahl an Schulabgängerinnen und Schulabgängern, welche keinen allgemeinbildenden Schulabschluss erreicht haben. Einhergehend mit der in quantitativer Hinsicht gesunkenen Bedeutung dieser Gruppe erhielt diese auch sukzessive eine randständige Position in Bezug auf den Übergangsprozess vom Bildungs- in das Erwerbs- beziehungsweise 
Beschäftigungssystem (Hanesch 1990: 188). Im Rahmen dessen zeichnete sich bereits eine Verdrängung der gering Gebildeten beziehungsweise der gering Qualifizierten ab.

Aufbauend auf dieser Entwicklung wurde die Verdrängungsthese aufgestellt, welche besagt, dass sich auf der einen Seite die Zugangsmöglichkeiten von gering qualifizierten Personen zu lukrativen Arbeitsplätzen verringern, je höher der Anteil und das Angebot an gut qualifizierten Bewerberinnen und Bewerbern ist. Dadurch kommt es zu einer sukzessiven Verdrängung von gering qualifizierten Arbeitskräften durch höher qualifizierte Arbeitskräfte (Blossfeld 1990: 165). Hochqualifizierte und gut ausgebildete Arbeitskräfte nehmen einen immer größeren Teil der vorhandenen Plätze auf dem Arbeitsmarkt ein. Dadurch werden gering qualifizierte Bewerberinnen und Bewerber auf einfache Arbeitsplätze verdrängt. Andererseits sind auch viele der hochqualifizierten Bewerberinnen und Bewerber aufgrund ihres stark gestiegenen Anteils dazu gezwungen, einfache Arbeitsplätze anzunehmen, welche unter ihrer Qualifikation liegen. Dies ist dem Umstand geschuldet, dass es auf dem Arbeitsmarkt Arbeitsplätze, welche adäquat zum Qualifikationsniveau von sehr gut ausgebildeten Arbeitskräften sind, nicht in ausreichendem Umfang gibt. Aufgrund dessen müssen viele hochqualifizierte Bewerberinnen und Bewerber auf die übrigen Arbeitsplätze ausweichen, welche unter ihrem eigentlichen Qualifikationsniveau liegen. Dieser Prozess wiederum führt dazu, dass die gering Qualifizierten, für welche diese einfachen Arbeitsplätze ursprünglich vorgesehen waren, vom Arbeitsmarkt verdrängt werden und sich oft in der Arbeitslosigkeit wiederfinden, da sie auf dem Arbeitsmarkt gegenüber den hochqualifizierten Arbeitskräften das Nachsehen haben (Solga 2011: 419 f.).

\subsection{2 Übertragung der Verdrängungsthese auf die vorliegende Untersuchung}

Das Konkurrenzverhalten zwischen hoch und niedrig qualifizierten Bewerberinnen und Bewerbern auf dem Arbeitsmarkt lässt sich auf den Übergang in den Ausbildungsmarkt übertragen. Die Zugangschancen in eine vollqualifizierende Berufsausbildung haben sich ebenfalls im Laufe der Jahrzehnte grundlegend verändert. Da es im Zuge der Bildungsexpansion immer mehr Schulabgängerinnen und Schulabgänger mit Abitur gab und die Anzahl derjenigen mit einem Hauptschulabschluss kontinuierlich zurückgegangen ist, hat ein gestiegener Anteil an schulisch höher Qualifizierten die Übergangschancen der schrumpfenden Gruppe der schulisch niedrig Qualifizierten in den Ausbildungsmarkt sukzessive verringert. Es ist $\mathrm{zu}$ vermuten, dass vor allem Jugendliche mit einem Hauptschulabschluss oder ohne Schulabschluss gegenüber denjenigen mit Abitur oder einem 
Realschulabschluss kaum Chancen hatten, den Zugang in den beruflichen Ausbildungsmarkt $\mathrm{zu}$ realisieren. Diese Vermutung spricht für ein sukzessives Herausdrängen aus dem Ausbildungsmarkt.

Diese deutlich erschwerten Zugangsmöglichkeiten führen zu der Erwartung, dass schulisch gering qualifizierte Jugendliche über die Jahrzehnte betrachtet eine deutlich verlängerte Übergangsdauer bis zum Eintritt in die berufliche Erstausbildung gehabt haben müssen. Diese Gruppe musste gezwungenermaßen häufiger Umwege in Kauf nehmen wie zusätzliche Schulepisoden oder den Aufenthalt im berufsvorbereitenden Übergangssystem. Ein relativ hoher Anteil aus dieser Gruppe ist sechs Monate nach dem Ende der allgemeinbildenden Schulzeit noch auf der Suche nach einem vollqualifizierenden dualen oder vollzeitschulischen Ausbildungsplatz (Beicht und Ulrich 2008b: 10). Dieser Umstand kann zu einer deutlichen Verzögerung des Eintritts in den beruflichen Ausbildungsmarkt vor allem bei Jugendlichen mit einem Hauptschulabschluss oder ohne Schulabschluss geführt haben, woraus sich die wesentliche Verlängerung der Übergangsdauer ableiten lässt.

\subsection{Begründung für die Auswahl der theoretischen Ansätze}

Die Humankapitaltheorie erscheint für die Studie geeignet, da die Aneignung von Humankapital als Wissen beziehungsweise Bildung unter der Voraussetzung eines die Kosten übersteigenden Nutzens auch während des Übergangsprozesses von der Schule in eine Berufsausbildung oder ein Hochschulstudium vollzogen werden kann. Dies hat hinsichtlich der formulierten Fragestellungen zur Folge, dass sich durch das Aneignen von Humankapital der Übergangsprozess und damit die Übergangsdauer verlängert und im Rahmen dessen Übergangsstationen durchlaufen werden. Diese Stationen haben, sofern während des Übergangs Humankapital angeeignet wird, einen Weiterbildungs- oder Qualifizierungscharakter.

Die Verdrängungsthese als zweiter theoretischer Ansatz nimmt den möglichen zentralen Einflussfaktor auf den Übergangsprozess, das erhöhte Qualifikationsniveau auf dem Arbeitsmarkt, in den Fokus. Durch einen im Laufe der Jahrzehnte deutlich gestiegenen Anteil an hochqualifizierten Arbeitskräften und einer damit verbundenen Verdrängung von gering qualifizierten Arbeitskräften hat sich das Qualifikationsniveau auf dem Arbeitsmarkt sukzessive erhöht. Des Weiteren lässt sich dies nicht nur auf den Übergang vom Ausbildungsmarkt in das Erwerbssystem, sondern bereits auf den Übergang von der allgemeinbildenden Schule in den Ausbildungsmarkt beziehen. Schulisch gering qualifizierte 
Jugendliche hatten es im Laufe der Jahrzehnte immer schwerer, den Übergang von der Schule in eine vollqualifizierende Ausbildung zu vollziehen (Steinmann 2000: 253 ff.). Stattdessen haben sie eine immer längere Übergangsdauer sowie eine erhöhte Anzahl an zu durchlaufenden Übergangsstationen, welche neben dem Nachholen von Bildungsabschlüssen zum Teil nicht der Weiterqualifikation dienen, vorzuweisen (Lex und Zimmermann 2011: 621 f.). Hierbei ist zu vermuten, dass der Prozess der Bildungsexpansion in Form eines deutlich gestiegenen Anteils von höheren Schulabschlüssen, ein erhöhter Anteil fachlich gut ausgebildeter Arbeitskräfte und damit ein deutlich erhöhtes Qualifikationsniveau auf dem Arbeitsmarkt zu dieser Entwicklung beigetragen haben.

Im Folgenden werden aus beiden theoretischen Ansätzen diverse Hypothesen abgeleitet, welche als Grundlage für die durchzuführenden Analysen dienen.

\subsection{Hypothesen}

H1a) Generell verlängert sich die Dauer vom Verlassen der Schule bis zum Beginn der Erstausbildung zwischen 1971 und 2012.

Zunächst wird mit der Hypothese 1a) untersucht, ob sich die Übergangsdauer vom Verlassen der allgemeinbildenden Schule bis zum Beginn der Erstausbildung im Laufe des Zeitraums zwischen 1971 und 2012 generell verlängert. Dadurch wird eingangs ein genereller Zeittrend untersucht, welcher gegebenenfalls zu einer längeren Übergangsdauer führen könnte. Dies ist aufgrund der beschriebenen Individualisierung der Übergangsverläufe sowie der hohen Bedeutung des Erwerbs von Humankapital und dem damit verbundenen Zeitaufwand im Rahmen des Übergangs zu vermuten.

H1b) Die Übergangsdauer von Schulabgängerinnen und Schulabgängern mit einem Hauptschulabschluss oder ohne einen Schulabschluss ist länger als bei denjenigen mit Abitur oder einem Realschulabschluss.

Nach der Untersuchung eines allgemein zeitlichen Trends bei der Entwicklung der Übergangsdauer im Rahmen der Hypothese 1a) wird mit der Hypothese 1b) ein Vergleich zwischen Schulabschlussgruppen bezüglich der Übergangsdauer angestellt. Dabei wird die Übergangsdauer nun differenziert nach Schulabschlussgruppen untersucht. Der Gruppenvergleich der Dauer erfolgt unabhängig von einem zeitlichen Trend. Die Vermutung, dass diejenigen ohne Schulabschluss oder mit einem Hauptschulabschluss einen längeren Übergang absolvieren als diejenigen mit Realschulabschluss oder Abitur, wird auf die Verdrängungsthese zurückgeführt. Im Zuge der Verdrängung von schulisch gering 
qualifizierten Jugendlichen aus dem Ausbildungs- und Arbeitsmarkt ist anzunehmen, dass Jugendliche mit einem geringeren schulischen Bildungsgrad während der Übergangsphase häufig Stationen durchlaufen, welche nicht der Weiterbildung oder Zusatzqualifikation dienen (wie zum Beispiel Arbeitslosigkeit, Jobben oder Maßnahmen des Übergangssystems). Dadurch akkumuliert diese Gruppe weniger Humankapital als diejenigen mit einem höheren schulischen Bildungsgrad. Dies kann insbesondere an der unübersichtlichen Vielzahl von berufsvorbereitenden Maßnahmen des Übergangssystems liegen. Nicht jede dieser Maßnahmen ermöglicht zwangsläufig den Zugang in eine vollqualifizierende Ausbildung (Krone 2010: 23). Des Weiteren ist der Verbleib in solchen Maßnahmen häufig oftmals länger als ursprünglich vorgesehen, was den Übergangsprozess ungeplant verlängert. Ein weiterer Grund ist sicherlich, dass die Gruppe der schulisch gering Qualifizierten mehr Humankapital aufholen muss, als schulisch besser qualifizierte Jugendliche und junge Erwachsene. Dies kann in Form des Nachholens von allgemeinbildenden Schulabschlüssen erfolgen sowie in der Aneignung anderer Qualifikationen. Aufgrund dessen müssen sie für den Erwerb von mehr Humankapital mehr Kosten in Form von Zeit aufwenden. Dementsprechend ist von einer längeren Übergangsdauer bei Jugendlichen mit niedrigem schulischem Bildungsgrad im Vergleich zu denjenigen mit höherem schulischem Bildungsgrad auszugehen.

H2a) Vor dem Hintergrund eines gestiegenen Dienstleistungstätigenanteils ist eine längere Dauer vom Verlassen der Schule bis zum Beginn der Erstausbildung zu beobachten.

Im Gegensatz zu den Hypothesen 1a) und 1b) wird bei der Hypothese 2a) nun der gestiegene Anteil an Dienstleistungstätigen im tertiären Sektor einbezogen. Dabei wird untersucht, ob dieser zentrale Prädiktor zu einer Verlängerung der Übergangsdauer beiträgt. Ein im Laufe der Jahrzehnte deutlich gestiegenes Qualifikationsniveau auf dem Arbeitsmarkt in Form von erhöhten Qualifikationsanforderungen und einer deutlich gestiegenen Konkurrenz durch gut qualifizierte Arbeitskräfte erfordert es, unabhängig vom erreichten Schulabschluss, immer stärker, diesen Qualifikationen gerecht zu werden, um in den Ausbildungsmarkt einmünden zu können. Im Sinne der Humankapitaltheorie muss für den Erwerb dieser notwendigen zusätzlichen Qualifikationen vor dem Beginn der Erstausbildung meist mehr Zeit eingeplant werden, wodurch sich die Übergangsdauer erhöht. Diese Zeit wird bewusst aufgewendet, um sich während der Übergangsphase weiteres Humankapital anzueignen. Dieses erhöht die Chancen, den Übergang in eine vollqualifizierende Ausbildung und später in eine Erwerbstätigkeit zu schaffen. Der zu erzielende Nutzen, bessere Ausbildungschancen durch 
zusätzliche Qualifikationen als Humankapital, erfordert jedoch das In-Kauf-Nehmen von Kosten in Form von Zeit.

H2b) Vor dem Hintergrund eines gestiegenen Dienstleistungstätigenanteils ist eine längere Übergangsdauer derjenigen mit einem Hauptschulabschluss oder ohne Schulabschluss als bei denjenigen mit Abitur oder einem Realschulabschluss zu beobachten.

Während in Hypothese 2a) der generelle Effekt des gestiegenen Anteils an Dienstleistungstätigen auf die Übergangsdauer untersucht wird, fokussiert Hypothese 2b) darauf, ob der gestiegene Anteil im tertiären Sektor einen Einfluss auf die Effekte der Schulabschlüsse auf die Übergangsdauer hat. Damit werden im Rahmen der Untersuchung der Übergangsdauer bei dieser Hypothese schlussendlich sowohl der zentrale Prädiktor des gestiegenen Anteils an Dienstleistungstätigen als auch die differenzierte Betrachtung nach Schulabschluss einbezogen. Wie bereits bei Hypothese 1b) ausgeführt, ist zu vermuten, dass sich Jugendliche ohne Schulabschluss oder mit Hauptschulabschluss oft in berufsvorbereitenden Maßnahmen befinden, welche den Übergangsprozess verzögern. Des Weiteren müssen sie für den Zugang in den Ausbildungs- und Arbeitsmarkt deutlich mehr Humankapital erwerben als diejenigen mit Realschulabschluss oder Abitur. Ein gestiegener Dienstleistungstätigenanteil im tertiären Sektor geht einher mit einem erhöhten Qualifikationsniveau auf dem Arbeitsmarkt. Im Sinne der Verdrängungsthese erschweren die gestiegenen Anforderungen auf dem Arbeitsmarkt den Jugendlichen mit einem niedrigen schulischen Bildungsgrad den direkten Übergang von der allgemeinbildenden Schule in eine vollqualifizierende Berufsausbildung zunehmend und stellen erhöhte Anforderungen an sie. Hierbei ist von einer Verdrängung schulisch gering qualifizierter Jugendlicher vom Ausbildungsmarkt auszugehen. Vor dem Hintergrund dieser Rahmenbedingungen ist zu vermuten, dass diejenigen mit niedrigem schulischem Bildungsgrad noch mehr Humankapital erwerben müssen. Im Zuge der oben beschriebenen Humankapitaltheorie ist dieses Humankapital erforderlich, um beim Zugang in den Ausbildungs- und Arbeitsmarkt bestehen zu können. Je mehr Humankapital erworben werden muss, desto mehr Zeit muss dafür während der Übergangsphase investiert werden. Fehlt dieses Humankapital, droht der Ausschluss beim Zugang in die Ausbildung oder zumindest eine deutliche Verringerung bei den Zugangschancen. Die Notwendigkeit bestimmter Qualifikationen ist auch einem veränderten und sich ständig wandelnden Arbeitsmarkt geschuldet, auf welchem neuartige Qualifikationen und Fertigkeiten erforderlich geworden sind. Daher ist zu vermuten, dass ein gestiegener Anteil an Dienstleistungstätigen die Übergangsdauer derjenigen mit niedrigem 
schulischem Bildungsgrad im Vergleich zu denjenigen mit hohem schulischem Bildungsgrad zusätzlich verlängert.

H3a) Jugendliche mit Abitur absolvieren häufiger Weiterbildungsstationen wie Kurse oder Praktika als Jugendliche mit Haupt- oder ohne Schulabschluss.

Im Folgenden werden die inhaltlichen Kategorien der Stationen in den Fokus genommen, welche während des Übergangs durchlaufen werden. Im Rahmen von Hypothese 3a) wird zunächst untersucht, ob diejenigen mit Abitur eher Weiterbildungsstationen durchlaufen als Jugendliche ohne Schulabschluss oder mit Hauptschulabschluss. Abiturientinnen und Abiturienten verfügen bereits über den höchsten allgemeinbildenden Schulabschluss und somit über das schulisch maximale Humankapital. Aufgrund der erhöhten Individualisierung von Übergangsverläufen und gestiegenen Qualifikationsanforderungen auf dem Arbeitsmarkt ist zu vermuten, dass diese Schulabschlussgruppe über die schulische Bildung hinaus bestrebt ist, sich im Sinne der Humankapitaltheorie weiteres Humankapital anzueignen, um den gestiegenen Anforderungen noch besser gerecht $\mathrm{zu}$ werden. Hiermit verfolgen sie einen erhöhten Nutzen in Form von verbesserten Zugangsmöglichkeiten in eine Ausbildung oder ein Studium.

H3b) Jugendliche mit Realschulabschluss absolvieren häufiger Weiterbildungsstationen wie Kurse oder Praktika als Jugendliche mit Haupt- oder ohne Schulabschluss.

Die Hypothese 3b) nimmt die Gruppe der Personen mit Realschulabschluss in den Fokus. Dabei wird untersucht, ob diejenigen mit einer mittleren Reife häufiger Weiterbildungsstationen durchlaufen als Jugendliche ohne Schulabschluss oder mit Hauptschulabschluss. Jugendliche mit Realschulabschluss könnten ebenfalls danach bestrebt sein, den erhöhten Qualifikationsanforderungen auf dem Ausbildungs- und Arbeitsmarkt gerecht $\mathrm{zu}$ werden. Daher ist $\mathrm{zu}$ vermuten, dass sie sich im Anschluss an die allgemeinbildende Schulzeit im Rahmen von Kursen oder Praktika weiterbilden und hierbei erste ausbildungs- und berufsrelevante Fertigkeiten und Kenntnisse erwerben können.

H3c) Jugendliche mit Haupt- oder ohne Schulabschluss holen im Vergleich zu denjenigen mit Realschulabschluss häufiger Bildungsabschlüsse nach.

Bei denjenigen mit Haupt- oder ohne Schulabschluss haben die absolvierten Übergangsstationen einen etwas anderen Charakter. Diese Übergangsstationen dienen ebenfalls der Zusatzqualifizierung. Diese Gruppe muss im Zuge der Humankapitaltheorie sehr viel Humankapital aufholen, welches diejenigen mit einem Realschulabschluss und mit Abitur bereits haben. Das Nachholen von Bildungsabschlüssen und das damit einhergehende 
Durchlaufen zusätzlicher Schulepisoden erfordert ein hohes Maß an Zeit, was dem Kostenfaktor der Humankapitaltheorie entspricht. Das Bestreben dieser Gruppe, Bildungsabschlüsse nachzuholen, hängt jedoch von unterschiedlichen Einflüssen ab wie beispielsweise der persönlichen Motivation oder der beruflichen Zielsetzung. Dementsprechend ist davon auszugehen, dass diejenigen ohne Abschluss oder mit einem Hauptschulabschluss weiter die Schule besuchen werden, um zusätzliche Schulabschlüsse zu erwerben. Durch diesen nachgeholten Erwerb von Humankapital erhoffen sie sich bessere Chancen beim Übergang in die Ausbildung.

H3d) Jugendliche mit Realschulabschluss absolvieren vor dem Hintergrund eines gestiegenen Dienstleistungstätigenanteils häufiger Weiterbildungsstationen wie Kurse oder Praktika als Jugendliche mit Haupt- oder ohne Schulabschluss.

Während in den Hypothesen 3a) bis 3c) die absolvierten Stationskategorien für die einzelnen Schulabschlussgruppen untersucht werden, wird nun der gestiegene Anteil an Dienstleistungstätigen einbezogen. Im Rahmen von Hypothese 3d) wird untersucht, ob der gestiegene Anteil an Dienstleistungstätigen dazu führt, dass diejenigen mit Realschulabschluss häufiger Weiterbildungsstationen während des Übergangs durchlaufen als diejenigen mit Haupt- oder ohne Schulabschluss. Wie bereits bei Hypothese $3 b$ ) ausgeführt ist $\mathrm{zu}$ vermuten, dass die Gruppe derer mit Realschulabschluss ebenfalls bestrebt ist, weiteres Humankapital in Form von Kursen oder Praktika zu erwerben, um ihre Chancen auf dem Ausbildungs- und Arbeitsmarkt zu verbessern. Dieses Bestreben wird durch das gestiegene Qualifikationsniveau auf dem Arbeitsmarkt in Form eines gestiegenen Anteils von hochqualifizierten Arbeitskräften in Dienstleistungstätigkeiten maßgeblich verstärkt. Ein erhöhtes Qualifikationsniveau auf dem Ausbildungs- und Arbeitsmarkt erfordert zusätzliches Humankapital. Jugendliche mit mittlerer Reife versuchen diesem Umstand durch das Absolvieren von Weiterbildungen Rechnung zu tragen.

H3e) Jugendliche mit Haupt- oder ohne Schulabschluss holen vor dem Hintergrund eines gestiegenen Dienstleistungstätigenanteils im Vergleich zu denjenigen mit Realschulabschluss häufiger Bildungsabschlüsse nach.

Die Hypothese 3e) nimmt die Jugendlichen mit Haupt- oder ohne Schulabschluss in den Fokus. Dabei wird untersucht, ob ein gestiegener Dienstleistungstätigenanteil dazu beiträgt, dass diese Gruppe häufiger Bildungsabschlüsse nachholt als diejenigen mit Realschulabschluss. Wie bei Hypothese 3c) beschrieben muss diese Gruppe deutlich mehr Humankapital nachholen als diejenigen mit Realschulabschluss oder Abitur. Vor dem 
Hintergrund der Verdrängungsthese werden diese Jugendlichen, zumindest vorübergehend, vom Ausbildungsmarkt verdrängt und müssen zunächst Schulabschlüsse zum Erwerb von notwendigem Humankapital nachholen. Durch das Nachholen von Schulabschlüssen versuchen diese Jugendlichen ihre Ausbildungschancen deutlich zu verbessern. Dabei ist zu vermuten, dass ein gestiegener Dienstleistungstätigenanteil und damit ein erhöhtes Qualifikationsniveau auf dem Arbeitsmarkt das Nachholen von Bildungsabschlüssen zusätzlich verstärkt. Damit ist die Hoffnung verbunden, durch zusätzlich angeeignetes Humankapital dem erhöhten Qualifikationsniveau auf dem Ausbildungs- und Arbeitsmarkt besser gerecht werden zu können. Die Kosten in Form von Zeit werden bei dieser Gruppe für den Nutzen aufgewendet, bessere Ausbildungschancen zu erlangen.

H4a) Generell erhöht sich die Anzahl der Stationen vom Verlassen der Schule bis zum Beginn der Erstausbildung zwischen 1971 und 2012.

Im Folgenden wird die Anzahl der absolvierten Übergangsstationen untersucht. Die Hypothese 4a) untersucht zunächst eingangs, ob sich die Anzahl der Stationen im Untersuchungszeitraum zwischen 1971 und 2012 generell erhöht. Dabei wird ein genereller zeitlicher Effekt auf die Anzahl der Stationen überprüft. Aufgrund der zunehmenden Individualisierung der Übergangsverläufe und einer gestiegenen Bedeutung des Erwerbs von Humankapital kann eine generelle Erhöhung der Anzahl der Stationen im Untersuchungszeitraum vermutet werden.

H4b) Die Anzahl der Stationen ist bei denjenigen ohne Schulabschluss oder mit Hauptschulabschluss höher als bei denjenigen mit Abitur oder Realschulabschluss.

Während es bei Hypothese 4a) um die Überprüfung der zeitlichen Entwicklung der Stationsanzahl geht, setzt sich die Hypothese 4b) mit einem Vergleich von Schulabschlussgruppen bei der Anzahl der Stationen auseinander. Dieser Gruppenvergleich bei der Anzahl der Stationen wird unabhängig von einem zeitlichen Trend untersucht. Dabei wird überprüft, ob diejenigen mit Haupt- oder ohne Abschluss eine höhere Stationsanzahl aufweisen als diejenigen mit Realschulabschluss oder Abitur. Diese Vermutung ist auf die Verdrängungsthese zurückzuführen. Schulisch niedrig qualifizierte Jugendliche werden eher aus dem Ausbildungsmarkt verdrängt und befinden sich in verschiedenen Übergangsstationen. Hierbei kann es sich um berufsvorbereitende Maßnahmen, Weiterbildungen oder das Nachholen von Bildungsabschlüssen handeln. Ein längerer Aufenthalt in verschiedenen berufsvorbereitenden Maßnahmen oder das Nachholen mehrerer Bildungsabschlüsse kann bei schulisch niedrig qualifizierten Jugendlichen die Stationsanzahl 
deutlich erhöhen. Vor dem Hintergrund der Humankapitaltheorie muss diese Gruppe deutlich mehr Humankapital aufholen als diejenigen mit Realschulabschluss oder Abitur. Dieser Umstand begünstigt ebenfalls eine erhöhte Stationsanzahl.

H4c) Vor dem Hintergrund eines gestiegenen Dienstleistungstätigenanteils ist eine höhere Anzahl der Stationen vom Verlassen der Schule bis zum Beginn der Erstausbildung zu beobachten.

Gegenüber den Hypothesen 4a) und 4b) wird nun der gestiegene Anteil an Dienstleistungstätigen als zentraler Prädiktor mit einbezogen. Die Hypothese 4c) untersucht, ob der gestiegene Dienstleistungstätigenanteil im tertiären Sektor die Anzahl an absolvierten Stationen generell erhöht. Das deutlich erhöhte Qualifikationsniveau auf dem Arbeitsmarkt erfordert den verstärkten Erwerb von Humankapital in Form von Qualifikationen für den Zugang in den Ausbildungs- und Arbeitsmarkt. Dieser Erwerb lässt sich neben dem damit verbundenen Zeitaufwand auch mit zusätzlich absolvierten Stationen charakterisieren. Somit ist davon auszugehen, dass der Erwerb notwendiger Qualifikationen vor dem Hintergrund eines gestiegenen Qualifikationsniveaus auf dem Arbeitsmarkt die Anzahl der absolvierten Stationen generell erhöht.

H4d) Vor dem Hintergrund eines gestiegenen Dienstleistungstätigenanteils ist eine höhere Anzahl der Stationen bei denjenigen ohne Schulabschluss oder mit Hauptschulabschluss als bei denjenigen mit Abitur oder Realschulabschluss zu beobachten.

Im Rahmen der Hypothese 4d) wird sowohl der gestiegene Anteil an Dienstleistungstätigen als auch ein differenzierter Vergleich zwischen Schulabschlussgruppen in die Untersuchung der Stationsanzahl einbezogen. Dabei wird untersucht, ob der gestiegene Dienstleistungstätigenanteil zu einer höheren Stationsanzahl bei denjenigen mit Haupt- oder ohne Schulabschluss im Vergleich zu denjenigen mit mittlerer Reife oder Abitur führt. Hiervon ist sowohl aufgrund der Verdrängungsthese als auch der Humankapitaltheorie auszugehen. Jugendliche mit Hauptschulabschluss oder ohne Schulabschluss werden aufgrund des gestiegenen Qualifikationsniveaus eher aus dem Ausbildungsmarkt verdrängt und verbleiben somit länger im Übergangsprozess. Dies führt bei dieser Gruppe durchaus auch zu einer höheren Anzahl an Übergangsstationen. Des Weiteren muss die Gruppe derer mit Haupt- oder ohne Schulabschluss weitaus mehr Humankapital aufholen und erwerben, um den gestiegenen Qualifikationsanforderungen gerecht $\mathrm{zu}$ werden als diejenigen mit mittlerer Reife oder Abitur. Dieser Umstand führt ebenfalls zu einer Erhöhung der Stationsanzahl. Der gestiegene Anteil an Dienstleistungstätigen im tertiären Sektor und das damit einhergehende 
gestiegene Qualifikationsniveau auf dem Arbeitsmarkt erhöhen somit die Anzahl an absolvierten Stationen vor allem bei schulisch niedrig qualifizierten Jugendlichen.

Nach der Vorstellung der Hypothesen wird im folgenden Kapitel die methodische Vorgehensweise dargestellt und erläutert, wie genau die einzelnen in den Fragestellungen und Hypothesen enthaltenen Variablen operationalisiert werden. Des Weiteren werden die angewendeten Daten und Methoden für die Untersuchung vorgestellt. 


\section{Methodisches Vorgehen}

Im Folgenden wird das verwendete Datenmaterial für die empirische Durchführung der Studie vorgestellt und die Auswahl begründet (vgl. Abschnitte 5.1, 5.2 und 5.3). Im Anschluss werden sämtliche Variablen der formulierten Fragestellungen und Hypothesen anhand des verwendeten Datensatzes operationalisiert. Dabei wird erläutert, welche Variablen im zugrunde liegenden Datensatz die in den Fragestellungen und Hypothesen enthaltenen theoretischen Konstrukte gut abbilden und daher verwendet werden sollen (vgl. Abschnitt 5.4). Im nächsten Schritt wird der Aufbereitungsprozess der verwendeten Rohdaten beschrieben (vgl. Abschnitt 5.5). Dabei wird zunächst das Zusammenführen der einzelnen Rohdatensätze dargelegt (vgl. Abschnitt 5.5.1). Des Weiteren wird auf das Bereinigen, Rekonstruieren und Sortieren von Datumsangaben sowohl zum Schulenddatum und zum Erstausbildungsbeginn als auch zu den Übergangsstationen eingegangen (vgl. Abschnitt 5.5.2). Im Anschluss daran wird die Aufbereitung und Zusammenfassung von verschiedenen Gruppierungsvariablen wie dem Schulabschluss oder dem Erstausbildungstyp dargelegt (vgl. Abschnitt 5.5.3) und auf den Ausschluss bestimmter Personengruppen basierend auf Limitationen der Daten eingegangen (vgl. Abschnitt 5.5.4). Darauf folgend wird die Bereinigung von zeitlichen Dopplungen und Überschneidungen zwischen Schulenddatum, Erstausbildungsbeginn und Übergangsstationen beschrieben (vgl. Abschnitt 5.5.5). Im Anschluss daran wird die Berechnung der Übergangsdauer und der Anzahl der Übergangsstationen thematisiert (vgl. Abschnitt 5.5.6). Abgerundet wird das Kapitel mit der Vorstellung der ausgewählten Analysemethoden und der Begründung für deren Auswahl in der vorliegenden Arbeit (vgl. Abschnitt 5.6).

\subsection{Datengrundlage 1: Nationales Bildungspanel (NEPS) ${ }^{1}$}

\subsubsection{Kurzvorstellung des Nationalen Bildungspanels}

Im Rahmen dieser Studie werden in allererster Linie die Daten des Nationalen Bildungspanels (NEPS) verwendet. Das NEPS ist eine vom Leibniz-Institut für Bildungsverläufe e. V. (LIfBi)

\footnotetext{
${ }^{1}$ Folgende durch das Leibniz-Institut für Bildungsverläufe vorgegebene Referenz bezüglich der Verwendung der NEPS-Daten ist im Rahmen dieser Untersuchung aufzuführen: „Diese Arbeit nutzt Daten des Nationalen Bildungspanels (NEPS) Startkohorte 6 (Erwachsene), doi:10.5157/NEPS:SC6:5.1.0. Die Daten des NEPS wurden von 2008 bis 2013 als Teil des Rahmenprogramms zur Förderung der empirischen Bildungsforschung erhoben, welches vom Bundesministerium für Bildung und Forschung (BMBF) finanziert wurde. Seit 2014 wird NEPS vom Leibniz-Institut für Bildungsverläufe e.V. (LIfBi) an der Otto-Friedrich-Universität Bamberg in Kooperation mit einem deutschlandweiten Netzwerk weitergeführt. “(NEPS-Datenzentrum o.J. c).
} 
der Otto-Friedrich-Universität in Bamberg durchgeführte Untersuchung. Die Zielsetzung des NEPS besteht in der Erhebung von Längsschnittdaten zu Kompetenzentwicklungen, Bildungsprozessen und -entscheidungen sowie zu Bildungsrenditen in sowohl formalen als auch nicht-formalen oder informellen Kontexten über die gesamte Lebensdauer für verschiedene Alterskohorten wie beispielsweise die Erwachsenenkohorte (NEPS Nationales Bildungspanel 2020). Die Abbildung dieser Bildungsprozesse und Kompetenzentwicklungen reicht somit von der frühesten Kindheit bis hin zum höheren Erwachsenenalter (Blossfeld et al. 2011: 1 f.). Im Rahmen des NEPS bestehen verschiedene Teilstudien zu verschiedenen Kohorten: zu den Neugeborenen, zum Kindergarten, zur Klasse fünf beziehungsweise neun, zu Studierenden und zu Erwachsenen (NEPS-Datenzentrum o.J. d).

\subsubsection{Die Erwachsenenkohorte des NEPS}

Die letzte aufgezählte Startkohorte der Erwachsenen ist für die vorliegende Untersuchung entscheidend. In dieser Teilstudie des NEPS steht die Erhebung von Bildungs- und Erwerbsverläufen sowie der Entwicklung von Kompetenzen im Lebensverlauf von Erwachsenen im Fokus. Hierbei sind sämtliche formale, nicht-formale und informelle Bildungsaktivitäten und Lernprozesse sowie damit in Zusammenhang stehende Entscheidungen inkludiert. Ebenso erfolgt eine detaillierte Dokumentation des bisherigen Lebensverlaufs hinsichtlich der Bildungs- und Erwerbsbiografie, Partnerschaften und Kinder. Des Weiteren werden im Rahmen der Teilstudie diverse Kompetenzen in den Bereichen Lesen, Mathematik oder Naturwissenschaften sowie nicht-kognitive Kompetenzen wie Persönlichkeit, Motivation oder Sozialkompetenzen erfasst (NEPS-Datenzentrum o.J. a). Im Rahmen der $\mathrm{zu}$ untersuchenden Fragestellungen und Hypothesen ist insbesondere die bisherige Bildungsbiografie von zentraler Bedeutung. Da die NEPS-Daten in regelmäßigen Abständen aktualisiert werden, gibt es verschiedene Datenversionen (NEPS-Datenzentrum o.J. b). In der vorliegenden Studie wurde die Datenversion 5.1.0 der Startkohorte 6: Erwachsene verwendet. Diese steht seit dem 16.07.2015 im Datenzentrum des Nationalen Bildungspanels für die akademische Forschung zur Verfügung (NEPS-Datenzentrum o.J. c).

\subsubsection{Vorläuferstudie „Arbeiten und Lernen im Wandel“ (ALWA)}

Die Teilstudie zur Startkohorte Erwachsene begann im Jahr 2007 noch vor der Gründung des NEPS. Diese als Welle 1 notierte Erwachsenenbefragung wurde in den Jahren 2007 und 2008 vom Institut für Arbeitsmarkt- und Berufsforschung (IAB) unter dem Namen „Arbeiten und Lernen im Wandel“" (ALWA) durchgeführt. Die in die NEPS-Panelstudie eingebettete 
ALWA-Befragung umfasste die Erhebung und Dokumentation der gesamten Bildungsbiografie beginnend mit Episoden auf allgemeinbildenden Schulen, welche besucht wurden, über diverse Ausbildungsepisoden und weitere Episoden zum Nachholen von allgemeinbildenden Schulabschlüssen bis hin $\mathrm{zu}$ formellen Fortbildungen oder Weiterbildungskursen. Letzte wurden unabhängig von der Dauer und einem erfolgreichen Abschluss erhoben. Des Weiteren wurde auch die Erwerbsbiografie ausführlich dokumentiert, wobei alle Erwerbstätigkeiten, auch wenn sie parallel verliefen, Berücksichtigung fanden. Im Zuge dessen wurden sämtliche Arbeitslosigkeitsepisoden, unabhängig davon, ob Leistungen bezogen wurden oder nicht, Elternzeit-, Wehr- und Zivildienstepisoden sowie übrige Aktivitäten in diesem Zeitraum erhoben (Kleinert et al. 2008: 34 f.).

In verschiedenen Fragebogenmodulen waren die einzelnen Themengebiete der ALWABefragung aufgeführt. Hierzu gehörten die Erhebung von soziodemografischen Daten der befragten Personen, die Dokumentation der Wohngeschichte mit allen Wohnorten, in denen die Personen von Geburt an gelebt haben, die Erhebung der Schulzeit mit sämtlichen Schulepisoden auf verschiedenen Schulformen und deren Datierungen sowie den dabei jeweils erreichten Abschlüssen oder auch die Dokumentation sämtlicher Ausbildungsepisoden. Zusätzlich wurden umfassend Wehrdienst-, Erwerbstätigkeits-, Arbeitslosigkeits-, Lücken-, Berufsvorbereitungs- und Partnerschaftsepisoden sowie Daten zu Kindern im Haushalt und Eltern- beziehungsweise Erziehungszeiten erhoben. Des Weiteren lagen Informationen zu den Einkommensverhältnissen und zur Haushaltszusammensetzung sowie zum Bildungs- und Berufsstatus der Eltern vor (Matthes und Trahms 2012: 5 ff.).

\subsubsection{NEPS-Befragungswellen der Startkohorte Erwachsene}

Auf der ALWA-Befragung bauten die folgenden durchgeführten Haupterhebungen zur Startkohorte Erwachsene im Rahmen des NEPS auf. So schloss sich die erste Haupterhebung der Erwachsenenbefragung, welche als Welle 2 zwischen November 2009 und Juni 2010 durchgeführt wurde, direkt an die ALWA-Befragung von 2007/2008 an. Daraufhin wurden weitere Haupterhebungen in Form von Wiederholungsbefragungen in einem jährlichen Turnus durchgeführt. Im Rahmen der vierten Befragungswelle in den Jahren 2011 und 2012 wurden neue befragte Personen für die Stichprobe akquiriert (NEPS-Datenzentrum o.J. a). Im Rahmen der folgenden NEPS-Befragungswellen wurden die identischen Informationen zu den verschiedenen Episoden im Lebensverlauf erhoben wie bei der ALWA-Befragung (NEPS National Education Panel Study 2020: 11). 


\subsubsection{Begründung für die Auswahl der ALWA-/NEPS-Daten}

In den NEPS-Daten wurden die schulischen und beruflichen Bildungs- sowie die späteren Erwerbsverläufe erhoben, was bereits auf die ALWA-Befragung 2007/2008 zutraf. Diese biografischen Verläufe sind für die Untersuchung des Übergangsprozesses von der Schule in eine Erstausbildung besonders relevant, wobei hierfür der Zeitraum vom Verlassen der Schule bis zum Eintritt in die Erstausbildung bedeutsam ist. Die Studie enthält Zeitangaben zur Beendigung der allgemeinbildenden Schulzeit und zum Beginn einer beruflichen oder akademischen Erstausbildung. Die ausführliche Auflistung der im Übergangszeitraum absolvierten Zwischenstationen sowie der auf den Monat genau dokumentierten Eintritte und Austritte in beziehungsweise aus diesen Stationen (Matthes und Trahms 2012: 51, 59 ff.) liefert einen Aufschluss über die Aufenthaltsdauer in den Übergangsstationen, die Form der absolvierten Stationen selbst sowie deren Anzahl. Diese Informationen liefern erste Hinweise zur Beantwortung der vorgestellten Fragestellungen und Hypothesen. Da auch eine differenzierte Betrachtung der Übergangsdauer und der Übergangsstationen nach Schulabschluss beabsichtigt ist, bieten sich die NEPS-Daten mit der Dokumentation sämtlicher Schulabschlüsse der befragten Personen zur Verwendung an.

Auch die Tatsache, dass im Rahmen der Untersuchung ein historischer Verlauf in Form der Entwicklung der Übergangsdauer und der absolvierten Übergangsstationen von Bedeutung ist, spricht für die Auswahl der NEPS-Daten. Diese wurden als Retrospektivdaten von Personen der Geburtsjahrgänge zwischen 1956 und 1986 erhoben. Die Übergangsphasen von der Schule in die Ausbildung fanden bei den einbezogenen Geburtskohorten von den 1970er Jahren bis in die 2000er Jahre statt, was die Rekonstruktion eines historischen Verlaufs ermöglicht. Auf Basis der einbezogenen Geburtsjahrgänge kann der in dieser Untersuchung fokussierte Zeitraum zwischen 1971 und 2012 abgedeckt werden, da die betrachteten Übergangsprozesse somit definitiv in den NEPS-Daten ausführlich dokumentiert sind.

Aus der Startkohorte Erwachsene des NEPS werden lediglich Daten der ersten Befragungswelle, der 2007 und 2008 durchgeführten ALWA-Befragung, sowie der zweiten Befragungswelle 2009/2010 und der vierten Befragungswelle 2011/2012 verwendet. Diese Selektion innerhalb des NEPS-Datenbestands soll im Folgenden begründet werden. Die Übergangsverläufe von der allgemeinbildenden Schule in eine berufliche Ausbildung oder in ein Hochschulstudium der Personen durch die zeitliche Eingrenzung zwischen 1971 und 2012 waren zum Zeitpunkt der vierten NEPS-Befragungswelle von 2011/2012 als chronologisch letzter einbezogener Welle bereits abgeschlossen oder zumindest angelaufen und dauerten 
noch gegebenenfalls an. Aufgrund dessen ist das Hinzuziehen von Daten jüngerer Befragungsteilnehmer, welche nach 1986 geboren wurden und gegebenenfalls im Rahmen von weiteren Studien zu ähnlichen Daten im Lebens- und Bildungsverlauf befragt wurden, im Rahmen dieser Untersuchung nicht möglich. Selbst Personen, die 1986 geboren sind und damit die jüngste einbezogene Kohorte darstellen, haben in der Regel spätestens 2005 oder 2006 die allgemeinbildende Schule verlassen. Auch für diese Kohorte lagen zum Zeitpunkt der letzten einbezogenen Befragungswelle 2011/2012 umfassend Daten zu den Übergangsverläufen von der Schule in die Ausbildung vor. Dabei kann angenommen werden, dass ein Großteil dieser Kohorte spätestens zu diesem Zeitpunkt den Übergang abgeschlossen hat und sich bereits in einer Ausbildung oder in einem Studium befindet. Für die jüngeren Geburtskohorten nach 1986 kann davon nicht zwangsläufig ausgegangen werden, weshalb diese aus den Analysen ausgeschlossen werden.

Darüber hinaus führt ein Einbezug der beiden NEPS-Befragungswellen 2009/2010 und 2011/2012 zusätzlich zu den in das NEPS-Panel integrierten Daten der ALWA-Studie zu einer deutlichen Vergrößerung der für die Analysen verwendeten Stichprobe. Dadurch besteht der Vorteil einer größeren Repräsentativität sowie einer besseren statistischen Teststärke im Rahmen der Analysen. Des Weiteren wurden die dabei befragten Personen, und damit auch die 2009/2010 beziehungsweise 2011/2012 für das NEPS neu akquirierten Personen mit dem identischen Erhebungsinstrumentarium und dadurch auch $\mathrm{zu}$ den gleichen Lebens- und Bildungsverlaufsdaten befragt wie die Teilnehmenden der ALWA-Studie. Hinzu kommt, dass diejenigen, die zum Zeitpunkt der ALWA-Befragung den Übergangsprozess gegebenenfalls noch nicht abgeschlossen hatten, den Übergang in eine Ausbildung oder ein Studium zum Zeitpunkt der NEPS-Befragungswelle in den Jahren 2011 und 2012 vollendet haben. Dies hat den Vorteil, mehr Informationen zu vollständig abgeschlossenen Übergangsprozessen zu erhalten. Die Vergleichbarkeit, die deutliche Vergrößerung der Stichprobe und eine Vervollständigung der Informationen zu den Übergangsverläufen stellen zentrale Argumente für eine zusätzliche Berücksichtigung der NEPS-Befragungswellen der Jahre 2009/2010 und 2011/2012 zu den in das NEPS-Panel integrierten Daten der ALWA-Befragung dar.

\subsubsection{Stichprobenzusammensetzung der einzelnen Befragungswellen}

Bei der ALWA-Studie zwischen September 2007 und März 2008 wurde als Zielgruppe die deutsche Wohnbevölkerung der Geburtsjahrgänge von 1956 bis 1988 befragt, welche damit zum Befragungszeitpunkt zwischen 18 und 51 Jahre alt war (Kleinert et al. 2008: 6). Im 
Rahmen des NEPS wurden aus der ursprünglichen Stichprobe der ALWA-Befragung jedoch nur Personen der Geburtsjahrgänge 1956 bis 1986 übernommen (NEPS Nationales Bildungspanel 2016a: 2). Einen Überblick über die Stichprobenzusammensetzung der ALWA-Studie, die Verfahren der Stichprobenselektion, die Zielgruppe sowie der Fallzahlen liefert die Tabelle $1 \mathrm{im}$ Anhang. Dabei wird auch auf die veränderte Stichprobengröße im Rahmen der Einbettung der ALWA-Studie in das NEPS eingegangen.

Die Teilnehmerinnen und Teilnehmer der ALWA-Studie wurden anhand von Daten des Statistischen Bundesamtes und der Statistischen Landesämter sowie der Einwohnermeldeämter recherchiert und randomisiert ausgewählt (Forschungsdatenzentrum der Bundesagentur für Arbeit im Institut für Arbeitsmarkt- und Berufsforschung o.J.). Diese Personen wurden mit telefonischen und teilweise auch persönlichen Interviews befragt (Kleinert et al. 2008: 6). Für die Einbettung der ALWA-Studie in das NEPS wurden nur diejenigen beibehalten, welche auch an einer oder mehreren der späteren NEPS-Erhebungen teilgenommen haben (Skopek 2013: 16 f.). Da die Teilnehmerinnen und Teilnehmer der ALWA-Studie der Jahrgänge 1987 und 1988 an keiner der weiteren NEPS-Befragungen teilgenommen hatten, wurden diese ausgeschlossen (Aust et al. 2011: 18), wodurch von den Befragten der ALWA-Studie lediglich Personen der Geburtsjahrgänge 1956 bis 1986 im NEPS berücksichtigt wurden (NEPS Nationales Bildungspanel 2016a: 2).

Eine Übersicht aller in das NEPS einbezogenen Befragungswellen mit dem dazugehörigen Befragungszeitraum, der Anzahl und der Geburtsjahrgänge der befragten Personen sowie der jeweiligen Erhebungsform ist in Tabelle 3 aufgeführt. Zur sprachlichen Vereinheitlichung werden im folgenden Verlauf der Arbeit die einzelnen Befragungswellen mit ihrer entsprechenden Nummer benannt, also zum Beispiel Welle 1 für die ALWA-Studie 2007 und 2008 oder Welle 2 für die erste NEPS-Haupterhebung 2009 / 2010. 
Tabelle 3: Befragungswellen 1 bis 7 der Erwachsenenbefragung des NEPS mit Zeiträumen, Anzahl und Geburtskohorten der befragten Personen sowie der Erhebungsform.

\begin{tabular}{|c|c|c|c|c|c|}
\hline $\begin{array}{l}\text { Befragungs- } \\
\text { wellen-Nr. }\end{array}$ & $\begin{array}{c}\text { Teilstudie Erwachsenen- } \\
\text { befragung }\end{array}$ & Zeitraum & $\begin{array}{l}\text { Anzahl } \\
\text { befragte } \\
\text { Personen }\end{array}$ & Geburtsjahrgänge & Erhebungsform \\
\hline Welle 1 & $\begin{array}{c}\text { „Arbeiten und Lernen im } \\
\text { Wandel“" (ALWA) }\end{array}$ & 2007 / 2008 & 10.404 & 1956 bis 1986 & CATI \\
\hline Welle 2 & 1. NEPS-Haupterhebung & $2009 / 2010$ & 11.649 & 1944 bis 1986 & CATI, CAPI \\
\hline Welle 3 & 2. NEPS-Haupterhebung & $2010 / 2011$ & 9.322 & 1944 bis 1986 & $\begin{array}{c}\text { CATI, CAPI, } \\
\text { PAPI }\end{array}$ \\
\hline Welle 4 & 3. NEPS-Haupterhebung & $2011 / 2012$ & 14.112 & 1944 bis 1986 & CATI, CAPI \\
\hline Welle 5 & 4. NEPS-Haupterhebung & $2012 / 2013$ & ca. 10.500 & 1944 bis 1986 & $\begin{array}{c}\text { CATI, CAPI, } \\
\text { PAPI }\end{array}$ \\
\hline Welle 6 & 5. NEPS-Haupterhebung & 2013 / 2014 & 10.639 & 1944 bis 1986 & CATI, CAPI \\
\hline Welle 7 & 6. NEPS-Haupterhebung & 2014 / 2015 & 9.131 & 1944 bis 1986 & $\begin{array}{l}\text { CATI, CAPI, } \\
\text { PAPI }\end{array}$ \\
\hline
\end{tabular}

CATI $=$ Computergestützte Telefoninterviews, CAPI $=$ Computergestützte persönliche Interviews, PAPI $=$ Kompetenztests. Quelle: Eigene Darstellung basierend auf NEPS Nationales Bildungspanel 2016a; Skopek 2013.

Nach der ersten Befragungswelle zur Erwachsenenbefragung in den Jahren 2007 und 2008 wurde im Rahmen der zweiten Welle der Panelstudie 2009 und 2010 die Stichprobe deutlich vergrößert, indem auch Personen aus älteren Geburtsjahrgängen vor 1956 einbezogen wurden. Während bei der dritten Welle im Vergleich zu den ersten beiden Befragungswellen keine neuen Personen für die Stichprobe akquiriert wurden, wurden im Rahmen der vierten Welle zusätzlich neue Befragungspersonen für die Studie akquiriert und die Stichprobengröße erneut erhöht. Bei den Wellen fünf und sechs wurden keine neuen Befragungspersonen zur Stichprobe hinzugezogen (Skopek 2013: 16). Bei der siebten Befragungswelle handelte es sich um eine Wiederholungsbefragung von panelbereiten Personen, welche bereits an der ersten Welle oder ab der vierten Welle teilgenommen hatten.

Aus den vorgestellten Befragungswellen des NEPS sollen die Daten der ersten, der zweiten und der vierten Welle verwendet werden. Im Rahmen der zweiten und vierten Befragungswelle wurde die Ausgangsstichprobe wie beschrieben durch Akquirieren zusätzlicher Teilnehmerinnen und Teilnehmer des NEPS deutlich erhöht. Die in diesen beiden Befragungswellen zusätzlich akquirierten Personen der Geburtsjahrgänge 1944 bis 1955 
finden in der vorliegenden Studie keine Berücksichtigung, da der Übergangsprozess zwischen den Jahren 1971 und 2012 betrachtet wird und hierfür die befragten Personen der Geburtskohorten 1956 bis 1986 von Interesse sind. Die Geburtsjahrgangskohorten bis 1955 haben zum Untersuchungszeitraum ab 1971 den Übergangsprozess zumindest begonnen oder in der Regel längst abgeschlossen und sich zu dieser Zeit bereits in einer Ausbildung oder einem Erwerbstätigenverhältnis befunden. Da im Rahmen dieser Studie nur das Datenmaterial des NEPS zur Verfügung steht, wird für die Analysen auf das Subsample der ALWABefragung im Rahmen des NEPS zugegriffen. Diese Daten sind im Rahmen des NEPS unter der Startkohorte 6, den Daten zu den Bildungsverläufen der Erwachsenen, subsumiert.

Jedoch wird in den NEPS-Daten nicht der gestiegene Anteil an hochqualifizierten Arbeitskräften auf dem Arbeitsmarkt als zentrale unabhängige Variable der vorliegenden Untersuchung abgebildet. Des Weiteren lassen sich keine Kontrollvariablen zur demografischen und wirtschaftlichen Entwicklung aus dem NEPS-Datenbestand heranziehen, wobei eine Berücksichtigung dieser Entwicklungen als unerlässlich $\mathrm{zu}$ betrachten ist. Aufgrund dessen ist es notwendig, zusätzlich zu den NEPS-Daten weitere Daten zu den genannten Makrovariablen hinzuzuziehen.

\subsubsection{Begründung für die Eingrenzung des Untersuchungszeitraums zwischen 1971 und 2012}

Die Beschränkung auf den Zeitraum zwischen den Jahren 1971 und 2012 ist vor allem mit dem Anstieg des Anteils des tertiären Sektors der Dienstleistungstätigen zu begründen. Dieser Anteil stieg besonders im betrachteten Zeitraum an (Statistisches Bundesamt 2017a), wodurch sich eine Berücksichtigung dieser Entwicklung durch den gewählten Zeitraum realisieren lässt. Nach einem vergleichsweise moderaten Anstieg in den 1970er und 1980er Jahren gestaltete sich die Zunahme im tertiären Sektor vor allem in den 1990er Jahren sehr stark (ebd.). Einhergehend mit dem Anstieg des Anteils an Dienstleistungstätigen nahm auch der Anteil der schulisch höher Qualifizierten deutlich zu.

Des Weiteren soll mit dem Beginn des Untersuchungszeitraums ab 1971 auch dem Umstand Rechnung getragen werden, dass im Rahmen der ersten Welle, der ALWA-Befragung, Personen ab dem Geburtsjahrgang 1956 oder jünger befragt wurden und im Jahr 1971 mindestens 15 Jahre alt waren. Damit kann davon ausgegangen werden, dass diese Personen frühestens im Jahr 1971 bei Erreichen des Hauptschulabschlusses die allgemeinbildende Schule zum ersten Mal verlassen konnten und deren Übergangsprozess begann. Dabei ist das Jahr des Schulenddatums, also des ersten Verlassens der allgemeinbildenden Schule, 
maßgeblich für die Zuweisung des Anteils an Dienstleistungstätigen aus dem gleichen Jahr. Bei den Personen der Geburtsjahrgänge vor 1956 handelt es sich im Rahmen des NEPS um eine Stichprobenauffrischung, wobei bei diesen zusätzlichen Rekrutierungen auch weiterhin Personen der Jahrgänge 1956 oder jünger enthalten waren. Damit ist zu vermuten, dass auch den darauf folgenden NEPS-Befragungen der deutlich größere Anteil der Befragten den Geburtsjahrgängen ab 1956 entsprach. Dies kann damit belegt werden, dass insgesamt 8.997 der 10.404 Befragten der Geburtsjahrgänge 1956 bis 1986, die an der ersten Welle teilnahmen, auch an der zweiten Welle partizipierten (Hammon et al. 2016: 6). Damit fällt der Anteil der Befragten der Jahrgänge 1944 bis 1955 in den folgenden NEPS-Wellen deutlich geringer aus. Um mögliche Verzerrungen durch $\mathrm{zu}$ gering frequentierte ältere Geburtskohorten in den Analysen auszuschließen, werden die Befragten der Geburtsjahrgänge 1944 bis 1955 aus der vorliegenden Studie ausgeschlossen.

Die Begrenzung des Untersuchungszeitraums bis zum Jahr 2012 wiederum ist damit zu begründen, dass in den Jahren 2011 und 2012 die vierte Welle im Rahmen des NEPS stattfand, welche in die Studie einbezogen wird. Damit kann sichergestellt werden, dass bei vielen Personen, deren Übergangsverlauf $\mathrm{zu}$ einem vorherigen Zeitpunkt noch nicht abgeschlossen war, zu diesem letzten einbezogenen Befragungszeitpunkt der Übergang in die Erstausbildung vollzogen ist. Somit können viele Übergangsprozesse in der Retrospektivbetrachtung vollständig rekonstruiert werden.

\subsection{Datengrundlage 2: Daten des Statistischen Bundesamts}

\subsubsection{Datenvorlage des Statistischen Bundesamts zu hochqualifizierten Arbeitskräften}

Die Daten zur Entwicklung des Anteils von hochqualifizierten Arbeitskräften in den alten Bundesländern werden vom Statistischen Bundesamt öffentlich zur Verfügung gestellt (Statistisches Bundesamt 2017a) und stellen die zentrale unabhängige Variable dar. Dabei wird der Anteil der Erwerbstätigen im tertiären Dienstleistungssektor als Indikator für den gestiegenen Anteil der Hochqualifizierten auf dem Arbeitsmarkt dargestellt.

In der Tabelle des Statistischen Bundesamts sind einerseits die jährlichen absoluten Häufigkeiten der Erwerbstätigen in Deutschland ab 1950 bis in die Gegenwart und andererseits ab 1950 die jährlichen prozentualen Anteile der Erwerbstätigen in Deutschland für drei zentrale Wirtschaftssektoren aufgelistet. Dabei handelt es sich um den primären Sektor (Fischerei, Land- und Forstwirtschaft), den sekundären Sektor (produzierendes Gewerbe) und den tertiären Sektor, welcher die übrigen Wirtschaftsbereiche und den 
Dienstleistungsbereich umfasst. Im Rahmen dessen wird jährlich die prozentuale Verteilung der Erwerbstätigen in Deutschland in den drei Wirtschaftssektoren dargestellt. Dabei beziehen sich die Anteile bis zum Jahr 1990 auf das frühere Bundesgebiet und damit Westdeutschland. Die Daten ab 1991 berücksichtigen zusätzlich die neuen Bundesländer und beziehen sich damit auf ganz Deutschland (Statistisches Bundesamt 2017a).

Der tertiäre Sektor kann als repräsentativ für den Anteil an hochqualifizierten Arbeitskräften auf dem Arbeitsmarkt betrachtet werden (Matheus 1995: 224). Der Einbezug der neuen Bundesländer ab dem Jahr 1991 in den Daten des Statistischen Bundesamts wird daran deutlich, dass sich die Anzahl an erwerbstätigen Personen in Deutschland zwischen 1990 und 1991 sprunghaft von 30.406.000 auf 38.851.000 erhöhte. Dieser Anstieg war außergewöhnlich groß, da sich diese Anzahl in den Jahren zuvor und in den Jahren danach nur sehr gering erhöhte oder sich sogar verringerte. Diese Veränderungen waren jedoch im Vergleich zur Anstiegsrate zwischen 1990 und 1991 sehr geringfügig (Statistisches Bundesamt 2017a). Dies wird in einer weiteren vom Statistischen Bundesamt öffentlich zur Verfügung gestellten Tabelle deutlich, in welcher sowohl die Bevölkerungsentwicklung in Deutschland als auch der Anteil an Erwerbstätigen im Inland für jedes Jahr aufgelistet sind (Statistisches Bundesamt 2017b). Die Angaben zu den Erwerbstätigen stimmen mit den Angaben zu den absoluten Häufigkeiten der Erwerbstätigen weitgehend überein, bei welchen zusätzlich die prozentualen Anteile zwischen den drei Wirtschaftssektoren aufgelistet sind (Statistisches Bundesamt 2017a). In dieser zusätzlichen Tabelle wird deutlich, dass sich die Bevölkerungsentwicklung, welche im Jahr 1990, auf Basis der laufenden Bevölkerungsstatistik, 63.254.000 Einwohner betrug und sich auf das alte Bundesgebiet bezog, im Jahr 1991 auf 79.973.000 anstieg (Statistisches Bundesamt 2017b). Dies kann nur mit der Hinzunahme der Einwohner aus den neuen Bundesländern erklärt werden. Da der prozentuale Anteil der Erwerbstätigen im tertiären Sektor bis 1990 kontinuierlich angestiegen ist und sich auch ab 1991 und damit nach Berücksichtigung der Erwerbstätigen aus den neuen Bundesländern weiter erhöht hat (Statistisches Bundesamt 2017a), kann dieser Anteil für den tertiären Sektor verwendet werden.

\subsubsection{Definition der Erwerbstätigen}

Des Weiteren werden Daten zu Erwerbstätigen verwendet und nicht Daten zu den Arbeitnehmerinnen und Arbeitnehmern, da beim Statistischen Bundesamt unter die Kategorie „Erwerbstätige“ auch Selbstständige beziehungsweise mithelfende Familienangehörige 
gefasst werden (Statistisches Bundesamt 2017b). Beim gestiegenen Anteil an hochqualifizierten Arbeitskräften auf dem Arbeitsmarkt soll keine isolierte Fokussierung auf die Arbeitnehmerinnen und Arbeitnehmer stattfinden, weshalb die breiter gefasste Kategorie „Erwerbstätige“ berücksichtigt wird. Die Erwerbstätigenzahlen des Statistischen Bundesamts basieren auf der Definition von „Erwerbstätigen“ resultierend aus den Volkswirtschaftlichen Gesamtrechnungen. Dabei werden unter dem Begriff „Erwerbstätige“ alle Personen subsumiert, welche als Arbeitnehmerinnen und Arbeitnehmer, also als Arbeiterinnen und Arbeiter, Angestellte, Beamtinnen und Beamte, geringfügig Beschäftigte, Soldatinnen und Soldaten oder Selbstständige sowie als mithelfende Familienangehörige mit der Intention auf einen wirtschaftlichen Erwerb tätig sind. Die aufgeführte Definition als Erwerbstätige ist dabei unabhängig vom Umfang der ausgeübten Tätigkeit (Statistisches Bundesamt 2016b).

\subsubsection{Implementieren der Daten des Statistischen Bundesamts in die NEPS-Daten}

Die prozentualen Anteile des dritten, tertiären Sektors pro Jahr werden als zentrale unabhängige Variable für den Zeitraum zwischen 1971 und 2007 herangezogen. Dabei stellt das Jahr 2007 das chronologisch letzte einbezogene Schulenddatumsjahr, also das Jahr, in dem die allgemeinbildende Schule das erste Mal verlassen wurde, dar. Dieser Zeitraum gilt auch für die Zuweisung der anderen externen Daten $\mathrm{zu}$ demografischen und konjunkturellen Indikatoren. Die prozentualen Anteile pro Jahr werden generell in Abhängigkeit vom Jahr des Schulenddatums, dem Jahr des ersten Verlassens der Schule, zugewiesen. Diese Vorgehensweise betrifft sämtliche externe Daten, welche in die Daten des NEPS implementiert werden. Die Zuordnung zum Jahr des Schulenddatums ist damit zu begründen, dass die Personen nach dem Erwerb des ersten allgemeinbildenden Schulabschlusses und dem erstmaligen Verlassen der Schule zu Beginn des Übergangs den Bedingungen des Ausbildungs- und Arbeitsmarkts ausgesetzt sind und diese einen starken Einfluss auf den Übergangsprozess haben können. Wichtig ist zu erwähnen, dass es sich bei den Daten des Statistischen Bundesamts, den beschriebenen prozentualen Anteilen, um sogenannte Makrodaten handelt, welche den Anteil an der Gesamtbevölkerung darstellen. Diese Makrodaten, welche auf die Gesamtheit abzielen, werden zu Individualdaten, dem Jahr des Verlassens der Schule der einzelnen Person, zugeordnet. Dies ist in Bezug auf die Datenstruktur zu berücksichtigen. Des Weiteren ist zu beachten, dass durch diese Zuweisung ein starker Zusammenhang zwischen dem Schulenddatumsjahr selbst und den nach dem 
Schulenddatumsjahr zugewiesenen externen Daten entstehen kann, welcher im Rahmen der Analysen und Ergebnisse nicht zu vernachlässigen ist.

\subsubsection{Daten zu demografischen Entwicklungen}

Zusätzlich werden Daten zur demografischen und zur wirtschaftlichen Entwicklung bei der vorliegenden Studie berücksichtigt. Die Informationen hierzu dienen als Kontrollvariablen bei der Überprüfung, ob mögliche Effekte und Zusammenhänge beim Übergangsprozess tatsächlich vom Anteil hochqualifizierter Erwerbstätiger im Dienstleistungsbereich ausgehen. Dabei wäre möglich, dass solche Effekte durch demografische oder wirtschaftliche Veränderungen tangiert sind. Hinter einem möglichen Effekt des Anteils von Erwerbstätigen in Dienstleistungsberufen auf die Übergangsdauer und die dabei absolvierten Zwischenstationen könnte gegebenenfalls auch ein Konjunktureffekt oder ein DemografieEffekt bestehen. Um solche Scheinkorrelationen und den möglichen Einfluss von Drittvariablen zu berücksichtigen, ist der Einbezug von Kontrollvariablen wie beispielsweise der demografischen und der konjunkturellen Entwicklung zwingend erforderlich. Da zu diesen Kenngrößen in den NEPS-Daten keine Informationen enthalten sind, wird in Bezug auf demografische Informationen ebenfalls auf das Datenmaterial des Statistischen Bundesamts zurückgegriffen.

Im Hinblick auf die demografische Entwicklung werden Daten zur Bevölkerungsstatistik nach Altersgruppen (Statistisches Bundesamt 2017c) vom Statistischen Bundesamt zur Verfügung gestellt. Die Daten zur Bevölkerungsstatistik sind nach Altersgruppen unterteilt, wobei die Daten der Altersgruppe der unter 20-Jährigen für die Analysen herangezogen werden, da zum Ende dieser Altersphase hauptsächlich der Übergang in die berufliche oder akademische Erstausbildung vollzogen wird. Hierbei handelt es sich um die prozentualen Anteile der Altersgruppe der unter 20-Jährigen an der Gesamtbevölkerung. Dabei ist zu beobachten, dass diese Kohorte seit dem Jahr 2006 anteilsmäßig weniger als 20 Prozent an der Gesamtbevölkerung in Deutschland ausmacht (Statistisches Bundesamt 2017c). Es ist zu beachten, dass das Statistische Bundesamt die jährlichen prozentualen Anteile der einzelnen Altersgruppen gesondert für das frühere Bundesgebiet bis zum Jahr 1989 erhoben hat (Statistisches Bundesamt 2016a). Da der Übergangsprozess Jugendlicher in den alten Bundesländern betrachtet wird, werden bis 1989 die prozentualen Anteile für das frühere Bundesgebiet verwendet. Ab 1990, dem Jahr der deutschen Wiedervereinigung, werden die Anteile an der gesamtdeutschen Bevölkerung, also auch an der Bevölkerung der neuen 
Bundesländer, hinzugezogen. Diese Gesamtangaben und Anteile beziehen sich bis zum Jahr 1989 auf die Gesamtbevölkerung der alten Bundesrepublik und der DDR, was an der im Gegensatz zur alten Bundesrepublik deutlich höheren Bevölkerungsanzahl insgesamt ersichtlich wird (Statistisches Bundesamt 2017c). Somit dienen bis 1989 die Angaben der alten Bundesländer zum jährlichen prozentualen Anteil der Altersgruppe der unter 20Jährigen. Die Angaben ab dem Jahr 1990 für die gesamte Bundesrepublik werden deshalb verwendet, da davon auszugehen ist, dass sich die Bevölkerungsstruktur nach der Wiedervereinigung durch das Hinzukommen der Bevölkerung aus den neuen Bundesländern verändert hat. Dies kann gegebenenfalls einen Einfluss auf den Arbeitsmarkt und damit auch auf den Übergang in die Ausbildung gehabt haben. Auch die Angaben zu den jährlichen Anteilen der Altersgruppe der unter 20-Jährigen an der Gesamtbevölkerung liegen durch das Statistische Bundesamt prozentual vor. Damit werden bei den Daten des Statistischen Bundesamtes, welche zu den NEPS-Daten hinzugezogen werden, ausschließlich prozentuale Anteile verwendet. Die prozentualen Anteile zur demografischen Entwicklung pro Jahr werden ebenfalls in Abhängigkeit vom Jahr des Schulenddatums zugewiesen.

Generell sind die Daten zur demografischen Entwicklung der Alterskohorte, welche den Übergang in die Ausbildung oder in das Studium vollzieht, geeignet, um mögliche latente Effekte, welche nicht in der Untersuchung thematisiert werden, aufzudecken. Gleiches gilt für Daten zur konjunkturellen Entwicklung, welche im folgenden Abschnitt vorgestellt werden.

\subsection{Datengrundlage 3: Daten der Bundesagentur für Arbeit zur Arbeitslosenquote}

$\mathrm{Zu}$ den konjunkturellen Entwicklungen werden Daten der Bundesagentur für Arbeit zur Arbeitslosenquote zwischen 1971 und 2007 verwendet (Statistik der Bundesagentur für Arbeit, Arbeitslosigkeit im Zeitverlauf 2018), wobei davon ausgegangen werden kann, dass diese Quote bei einer positiven Konjunkturlage sinkt und bei einer negativen Lage ansteigt. Bei der Arbeitslosenquote handelt es sich um den Anteil der Arbeitslosen an allen abhängigen zivilen Erwerbspersonen. Die prozentualen Anteile zur Arbeitslosenquote beziehen sich bis zum Jahr 1990 auf das alte Bundesgebiet einschließlich West-Berlin und ab dem Jahr 1991 ebenfalls auf das frühere Bundesgebiet ohne Berlin (Statistisches Bundesamt 2017d). Bei der Entwicklung der Arbeitslosenquote an allen abhängigen zivilen Erwerbstätigen in Westdeutschland zeigt sich seit 1970 ein über Jahrzehnte hinweg andauernder Anstieg des Arbeitslosenanteils bis in das neue Jahrtausend. So stieg die Quote der Arbeitslosen von 0,7 Prozent im Jahr 1970 auf elf Prozent im Jahr 2005, wobei es sich hierbei um den Anteil an 
allen abhängig zivilen Erwerbspersonen handelt (Statistik der Bundesagentur für Arbeit, Arbeitslosigkeit im Zeitverlauf 2018). Dadurch ist bei den abhängig beschäftigten Erwerbspersonen in Westdeutschland ein klarer Anstieg der Arbeitslosenquote festzustellen. Die Arbeitslosenquote eignet sich gut als Indikator für die wirtschaftliche Entwicklung, da die Arbeitslosenzahlen die konjunkturelle Situation besser widerspiegeln als die Erwerbstätigenzahlen. Des Weiteren ist der Arbeitslosenanteil an allen abhängig beschäftigten Erwerbspersonen aussagekräftiger als der Anteil an generell allen Erwerbstätigen. Vor allem abhängig Beschäftigte sind von konjunkturellen Umbrüchen und Dynamiken betroffen, beispielsweise in Form von betriebsbedingten Kündigungen aufgrund einer schlechten Auftragslage, welche wiederum einer schwierigen konjunkturellen Situation geschuldet sein kann. Die Anteile zur Arbeitslosenquote werden ebenfalls nach dem Schulenddatumsjahr für den Zeitraum zwischen 1971 und 2007 in den Datenbestand des NEPS implementiert.

\subsection{Operationalisierung}

Im Folgenden werden die zentralen abhängigen und unabhängigen Variablen der vorliegenden Untersuchung vorgestellt. Die Abbildungen der Variablen und deren Ausprägungen in den Daten werden grob skizziert. Die erforderlichen Umformungen und Aufbereitungen für die Analysen werden anschließend dargelegt. Eine vollständige Übersicht der deskriptiven Statistiken, Häufigkeitsverteilungen und prozentualen Anteile aller verwendeten Variablen ist im Anhang in den Tabellen 7 und 8 aufgeführt.

\subsubsection{Abhängige Variablen Übergangsdauer und Übergangsstationen}

Die Tabelle 4 liefert einen Überblick über alle abhängigen und unabhängigen Variablen sowie die Kontrollvariablen der späteren Analysen. Deren Bedeutung wird nachfolgend ausführlich erläutert. 
Tabelle 4: Abhängige und unabhängige Variablen sowie Kontrollvariablen.

\begin{tabular}{lll} 
Variablentyp & \multicolumn{1}{c}{ Variablen } \\
\hline \multirow{2}{*}{ Abhängige Variablen } & Übergangsdauer vom erstmaligen Verlassen der Schule bis zum Beginn \\
& der Erstausbildung (in Monaten) \\
& - & Anzahl und Kategorien der absolvierten Übergangsstationen vom \\
& erstmaligen Verlassen der Schule bis zum Beginn der Erstausbildung \\
\hline Unabhängige Variablen & - & Erster erreichter Schulabschluss am Ende der allgemeinbildenden \\
& & Schulzeit \\
& Prozentualer Anteil von Erwerbstätigen im Dienstleistungsbereich \\
& (tertiärer Sektor) \\
& Schulenddatumsjahr (1971 - 2007) \\
\hline Kontrollvariablen & Arbeitslosenquote in Bezug auf alle abhängig beschäftigten \\
& - & Erwerbstätigen für jedes Jahr \\
& - & Soziale Herkunft (gebildet auf Basis der CASMIN-Bildungsklassifikation) \\
& Geschlecht
\end{tabular}

Quelle: Eigene Darstellung.

Die beiden zentralen abhängigen Variablen sind die Übergangsdauer vom erstmaligen Verlassen der Schule bis zum Eintritt in eine berufliche oder akademische Erstausbildung und die dabei absolvierten Zwischenstationen. Die Übergangsdauer liegt als Variable selbst noch nicht in den Ausgangsdaten vor. Diese wird als Datumsdifferenz zwischen dem Ende der Schulzeit und dem Beginn der Erstausbildung in Monaten gebildet. Sowohl die einzelnen Schulepisoden, wenn unterschiedliche Schulen beziehungsweise Schulformen besucht wurden, als auch die verschiedenen Ausbildungsepisoden sind mit einem monatsgenauen Start- und Enddatum dokumentiert. Während bei den Ausbildungsepisoden die Episode mit dem frühesten Startdatum maßgeblich ist und dieses als Erstausbildungsstartdatum gewertet wird, wird das Enddatum der Schulepisode, bei welcher der erste allgemeinbildende Schulabschluss erworben wurde, als Schulenddatum festgelegt. Die darauf folgenden Schulepisoden werden als Übergangsstationen während des Übergangszeitraums betrachtet, bei welchen eine Weiterqualifizierung durch den Erwerb weiterer Schulabschlüsse stattfindet. Auch wenn diese Schulepisoden zeitlich direkt im Anschluss an den Erwerb des ersten Schulabschlusses begonnen werden, werden sie als Übergangsstationen betrachtet. Der Erwerb von weiteren Schulabschlüssen im Rahmen von darauf folgenden Schulepisoden ist im Zusammenhang mit dem Humankapitalansatz als Erwerb von Humankapital mit dem Ziel besserer Ausbildungschancen zu verstehen. Bei der Erfassung der einzelnen Schulepisoden in 
den verwendeten Daten ist entscheidend, dass eine eigenständige Schulepisode nur dann erfasst wird, wenn ein Wechsel zwischen zwei unterschiedlichen Schulformen stattgefunden hat. Das bedeutet, dass nicht zwei Schulepisoden, sondern lediglich eine Episode angelegt wird, wenn ein Wechsel von einem zum anderen Gymnasium erfolgt. Hierbei spielt auch der Ort des Gymnasiums keine Rolle. Bei einem Wechsel zwischen zwei verschiedenen Schulformen, zum Beispiel von einem Gymnasium auf eine Realschule, werden dagegen zwei einzelne Schulepisoden angelegt (Matthes und Trahms 2012: 6). Dies wird bei der Datenerhebung im Rahmen des NEPS konsistent beibehalten (NEPS Nationales Bildungspanel 2009: 37). Bei den Schulepisoden finden auch Episoden Berücksichtigung, welche auf einer beruflichen Schule stattgefunden haben, dabei jedoch ein allgemeinbildender Schulabschluss erworben wurde.

Die andere zentrale abhängige Variable sind die während der Übergangsdauer absolvierten Übergangsstationen. Diese sind wie die Schul- und Ausbildungsepisoden in den vorliegenden Ausgangsdaten jeweils mit einem Start- und einem Enddatum angegeben, wobei der entsprechende Monat und das Jahr vorliegen. Mit der Monatsdifferenz zwischen diesen beiden Datumsangaben kann jeweils die Dauer der jeweiligen Übergangsstation berechnet werden. In den Ausgangsdaten liegen verschiedene Stationen vor. Dies sind berufsvorbereitende Maßnahmen, Arbeitslosigkeitsepisoden, Kursbesuche, Wehrdienstepisoden, Praktika, Elternzeitepisoden, Lückenepisoden und zusätzliche Schulbesuche. Zur Kategorie Lückenepisoden zählen unter anderem auch Urlaubsphasen. Hinzu kommen noch diverse Lehrgänge oder Kurse zum Erwerb eines Zertifikats, welche unter den Begriff „Weiterbildungen“ gefasst werden. Diese Episoden sind in den Rohdaten als Ausbildungsepisoden angegeben, werden aber für die vorliegende Untersuchung als zusätzliche Übergangsstationen behandelt.

Im Kontext der vorliegenden Studie sind lediglich arbeitsmarktrelevante Übergangsstationen von Relevanz, weshalb nicht alle der aufgezählten Stationen berücksichtigt werden. Als relevante Stationen werden berufsvorbereitende Maßnahmen, Arbeitslosigkeitsepisoden, Kursbesuche, Praktika, Weiterbildungen sowie zusätzliche Schulbesuche verwendet. Bei den zusätzlichen Schulepisoden ist es erforderlich, dass diese nach dem Schulenddatum und damit nach dem Erwerb des ersten Schulabschlusses sowie vor dem Startdatum der beruflichen oder akademischen Erstausbildung beginnen, wobei sie zeitlich auch direkt im Anschluss an das vergebene Schulenddatum erfolgen können. Zusätzlich werden noch ein freiwilliges soziales, ökologisches oder europäisches Jahr, welches im Rahmen der Wehrdienstepisoden mit 
aufgeführt ist, sowie ein Urlaub, welcher eine der Kategorien der Lückenepisoden darstellt, berücksichtigt. Ein freiwilliges soziales, ökologisches oder europäisches Jahr kann qualifikationsrelevant für den späteren Ausbildungs- und Arbeitsmarktverlauf sein. Bei einem Urlaub kann es sich eventuell um einen Bildungs- oder Sprachurlaub handeln. Diese beiden Stationen werden bei den späteren Analysen in einer Variante mit einbezogen und in einer anderen Variante ausgeschlossen. Ebenfalls im Rahmen der Lückenepisoden sind die Kategorien „Haustätigkeit“ und „etwas anderes gemacht“ enthalten. Auch diese beiden Ausprägungen werden als jeweils eine Stationskategorie optional bei den Analysen in einer Variante mit einbezogen. Bei den Analysen zu den Übergangsstationen werden die Tätigkeit als Hausfrau oder -mann sowie die Angabe, etwas anderes gemacht zu haben als zwei getrennte Stationskategorien aus der Lückenepisode extrahiert, um diese bei den Stationskategorien getrennt betrachten zu können. Die Kategorie „Krankheit“ wird dagegen bei den Analysen zu den Stationen ausgeschlossen. Des Weiteren werden die nicht durch Stationen definierten Zeiträume während der Übergangszeit als Lücken behandelt. Bei diesen Zeiträumen kann nicht bestimmt werden, welchen Aktivitäten die Befragten nachgegangen sind. Neben den ersten beiden Analysevarianten (mit/ohne Berücksichtigung der Stationen „Freiwilliges soziales Jahr“ (FSJ), Urlaub, Haustätigkeit, „etwas anderes gemacht“) zu den Stationen werden bei beiden Varianten diese unbestimmten Lücken in einer dritten und vierten Variante ausgeschlossen. Diese insgesamt vier Analysevarianten zu den Übergangsstationen dienen einem Vergleich der Ergebnisse.

Bei Vorliegen eines Start- und Enddatums wird für die jeweilige Station in der dafür gebildeten Stationsvariable der Wert 1 vergeben. Wenn die Station während des Übergangszeitraums beginnt, jedoch erst nach dem Beginn der Erstausbildung endet, ist der Beginn der Erstausbildung als Enddatum der Übergangsstation maßgeblich. Wenn der Beginn der Station vor dem Schulenddatum liegt, ist das Startdatum der Station mit dem Schulenddatum gleichzusetzen. Bei zeitlichen Überschneidungen oder Dopplungen zwischen den Übergangsstationen ist die inhaltliche Gewichtung der Stationen für die Bereinigung entscheidend, worauf im Abschnitt 5.5.5 detailliert eingegangen wird. Ziel ist dabei, dass keine zeitlichen Überschneidungen von Stationen existieren, sondern eine Sequenz von aufeinander folgenden Übergangsstationen besteht. Dies ist eine Grundvoraussetzung für das Verfahren der Sequenzdatenanalyse. In diesem Zusammenhang muss für den Fall des Vorliegens von mehreren Zuständen bei einem Individuum entschieden werden, welcher dieser Zustände dominierend ist. Es besteht zwar die Möglichkeit der Konstruktion von 
„Doppelzuständen“, welche zwei gleichzeitige Zustände abbilden. Diese Doppelzustände führen jedoch zu einer deutlichen Erhöhung der Anzahl an Sequenzen beziehungsweise Sequenztypen, welche durch die Sequenzdatenanalyse dokumentiert werden (Scherer und Brüderl 2010: 1035). Auch im Rahmen der in dieser Studie verwendeten Ereignisdatenanalyse ist die Wahl eines einzelnen Zustands ratsam. In der vorliegenden Untersuchung wird eine nach eigenem Ermessen überschaubare Anzahl an möglichen Sequenztypen angestrebt, weshalb eine Gewichtung der Stationen bevorzugt wird.

Als zentrale abhängige Variable sind die Übergangsstationen in zweierlei Hinsicht von Relevanz. Zum einen ist die Summe der Übergangsstationen von Interesse. Zum anderen sind die einzelnen Typen der Übergangsstationen und deren Abfolge bedeutsam. Hierbei geht es darum, welche Stationen während des Übergangs durchlaufen werden und $\mathrm{zu}$ welcher Kategorie sie sich vorwiegend zuordnen lassen.

\subsubsection{Unabhängige Variablen}

Die erste zentrale unabhängige Variable ist der erreichte Schulabschluss. Zu jeder Schulepisode ist der dabei erreichte Schulabschluss dokumentiert. Der erste erworbene Schulabschluss ist als zugewiesener Schulabschluss maßgeblich. Die anschließend erworbenen Schulabschlüsse werden im Rahmen von weiteren Schulepisoden und damit in Übergangsstationen erworben. Im Rahmen der vorliegenden Untersuchung werden vier Abschlussvarianten verwendet, nämlich das Nicht-Vorliegen eines Schulabschlusses, der Hauptschulabschluss, der Realschulabschluss sowie das Abitur. Diese vier Kategorien werden aus den deutlich ausdifferenzierteren Schulabschlusskategorien in den Rohdaten gebildet (siehe Anhang Tabelle 2). Eine weitere entscheidende unabhängige Variable ist der Anteil von Erwerbstätigen im Dienstleistungsbereich, welcher sukzessive zugenommen hat. Dieser Anteil ist in der vorliegenden Studie der zentrale Prädiktor, dessen Einfluss auf den Übergangsprozess untersucht werden soll. Weiterhin dient das Schulenddatumsjahr als das Jahr des erstmaligen Verlassens der Schule als wichtige unabhängige Variable, um einen Zeittrend der Übergangsdauer und der Stationen zu untersuchen.

Die weiteren unabhängigen Variablen dienen als Kontrollvariablen, um zu überprüfen, ob neben den untersuchten Zusammenhängen andere Effekte existieren und herausgefundene Effekte dadurch verzerrt sind. Eine erste einbezogene Kontrollvariable deckt die konjunkturelle Entwicklung im Laufe der Jahre und Jahrzehnte ab: die Arbeitslosenquote in Bezug auf alle abhängig beschäftigten Erwerbstätigen für jedes Jahr (Statistik der 
Bundesagentur für Arbeit, Arbeitslosigkeit im Zeitverlauf 2018). Des Weiteren wird die demografische Entwicklung hinzugezogen. Diese wird vom Statistischen Bundesamt in Form der prozentualen Anteile der Altersgruppe der unter 20-Jährigen für jedes Jahr angegeben (Statistisches Bundesamt 2017c). Diese Altersgruppe durchläuft in der Regel den Übergang von der Schule in die Ausbildung oder in das Studium. Eine weitere Kontrollvariable ist die soziale Herkunft. Zu dieser liegen in den Ausgangsdaten des NEPS mehrere Variablen vor. Dabei wird die Variable der CASMIN-Bildungsklassifikation in die Analysen einbezogen. Diese Bildungsklassifikation resultiert aus dem Projekt Comparative Analysis of Social Mobility in Industrial Nations (CASMIN), welches von 1983 bis 1988 von Sozialwissenschaftlern der Universität Mannheim durchgeführt wurde. Im Rahmen dieses Projekts wurde die Einteilung für das deutsche Bildungssystem sowie Bildungssysteme diverser anderer europäischer Länder entwickelt. Diese Zuordnung verfolgt die Intention, eine im internationalen Maßstab vergleichbare Messbarkeit von Bildung und Bildungsabschlüssen zu ermöglichen. Dies geschieht unter Berücksichtigung der nationalen Eigenheiten der Bildungssysteme in den einzelnen Ländern. Im Rahmen dieser Kategorisierung wird einerseits hierarchisch zwischen den Bildungsniveaus, wie beispielsweise der Sekundar- und Tertiärbildung, unterschieden und andererseits eine Differenzierung in allgemeine und berufsbezogene Bildung vorgenommen (CESifo Group Munich 2017). Die CASMINBildungsklassifikation gliedert sich dabei in neun verschiedene Kategorien (Brauns et al. 2003: 223). Da die Klassifikation sowohl für den Vater als auch für die Mutter vorliegt, wird das Maximum aus den Bildungsklassifikationen beider Elternteile gebildet und als Kontrollvariable für die soziale Herkunft verwendet. Aufgrund der Vielzahl der Kategorien werden diese zu wenigen Kategorien zusammengefasst (siehe Anhang Tabelle 2).

Zusätzlich wird das Geschlecht der einbezogenen Personen berücksichtigt. Die Variable „Geschlecht“ liegt in den Ausgangsdaten des NEPS in dichotomer Form mit den Kategorien „männlich“ und „weiblich“ vor. Dadurch kann überprüft werden, ob hinter einem möglichen Effekt des gestiegenen Anteils an Dienstleistungstätigen auf den Übergangsprozess in Wirklichkeit ein Geschlechtseffekt besteht. Genauso gut könnte auch ein direkter Effekt des Geschlechts auf den Übergangsprozess bestehen und durch den Einbezug des Geschlechts der Effekt des gestiegenen Anteils an Dienstleistungstätigen nivelliert oder abgeschwächt werden. Der Typ der chronologisch ersten Ausbildung dient einem differenzierten deskriptiven Überblick der Übergangsdauer und der Stationssumme. Dabei sind drei verschiedene Ausbildungsformen von Bedeutung: die duale Berufsausbildung, die vollzeitschulische 
Berufsausbildung und das Studium. Als zusätzliche vierte Kategorie wird „Keine Ausbildung“ vergeben, um Personen $\mathrm{zu}$ identifizieren, die bis zum Ende des Befragungszeitraums keine Ausbildung aufgenommen haben. Die ursprünglichen Ausprägungen des Erstausbildungstyps werden zu den vier Kategorien zusammengefasst (siehe Anhang Tabelle 2).

\subsection{Datenaufbereitung}

\subsubsection{Zusammenführen der einzelnen Rohdatensätze}

Die Rohdaten des NEPS sind in mehrere Datensätze mit einem jeweiligen Schwerpunkt wie Schule, Ausbildungen, Wehrdienst oder Arbeitslosigkeitsepisoden aufgeteilt und werden anhand einer einheitlichen Variable zur Identifikation (ID) zu einem Gesamtdatensatz zusammengefasst. Hierbei werden sämtliche für die Untersuchung irrelevanten oder nicht brauchbaren Variablen der Übersichtlichkeit halber ausgeschlossen. Des Weiteren werden lediglich Personen im Datensatz behalten, welche an der ALWA-Studie 2007/2008 sowie an den NEPS-Befragungswellen 2009/2010 und 2011/2012 beziehungsweise an mindestens einer dieser drei Befragungswellen teilgenommen haben.

\subsubsection{Rekonstruktion, Bereinigung und Sortierung von Datumsangaben zum Schulenddatum, Erstausbildungsbeginn und Übergangsstationen}

Des Weiteren liegen die Start- und Enddatumsangaben der Schulepisoden, Ausbildungsepisoden sowie sämtlicher Übergangsstationen in den Rohdaten getrennt in Monats- und Jahresvariablen vor. Dabei ist sowohl eine edierte als auch nicht-edierte Monatsvariable gegeben, wobei letztere neben den zwölf Monaten noch fünf verschiedene Kategorien zu Jahreszeiten enthält. Diesen Jahreszeiten wird jeweils ein Monat zugeordnet und aus beiden Variablen eine gemeinsame Monatsvariable gebildet, um einen zu hohen Informationsverlust bei den Datumsangaben zu vermeiden (siehe Anhang Tabelle 5). Aus der jeweiligen Monats- und Jahresvariablen wird anschließend eine einheitliche Datumsvariable gebildet, welche sowohl den Monat als auch das Jahr enthält. Die Übergangsstationen und Ausbildungsepisoden werden untereinander chronologisch nach dem Startdatum unter Berücksichtigung des Monats sortiert, da das Startdatum von Episoden eventuell im gleichen Jahr liegt.

Bei den Schulepisoden erfolgt die chronologische Sortierung nach dem Enddatum. Die Schulepisode mit dem chronologisch letzten Enddatum ist nach der angewendeten Sortierlogik auch die letzte Schulepisode. Hierbei ist das Enddatum der Schulepisode, bei 
welcher der erste Schulabschluss erworben wurde, auch gleichzeitig das Schulenddatum, wogegen die darauf folgenden Schulepisoden als Übergangsstationen geführt werden. Bei fehlendem Enddatum einer vorherigen Schulepisode wird das Startdatum der darauffolgenden Episode als Enddatum und bei fehlendem Startdatum das Enddatum der vorherigen Episode zugewiesen. Hierbei ist jedoch zu berücksichtigen, dass dies teilweise spekulativ ist, da bei der Ersetzung dieser fehlenden Start- und Enddatumsangaben davon ausgegangen werden muss, dass die Schulepisoden zeitlich direkt aneinander anschließen.

Darüber hinaus wird das Schulenddatum in Abhängigkeit zum Geburtsjahr der Personen angepasst. Ist die Person beim ersten Schulabschluss deutlich $\mathrm{zu}$ jung, wird unter Berücksichtigung der Schulform und des ersten Schulabschlusses das Schulenddatum entsprechend korrigiert. Das Schulenddatum und der Schulabschluss sollen dabei nicht unter einem Alter von 13 Jahren und nicht über einem Alter von 23 Jahren liegen. Diese Altersgrenzen sind unter anderem damit zu begründen, dass ein Alter von 13 Jahren beim Schulenddatum beispielsweise im Falle eines vorzeitigen Schulabbruchs vorkommen kann. Zwar kann ein vorzeitiger Schulabbruch theoretisch auch zu einem früheren oder zu einem späteren Zeitpunkt auftreten. Jedoch wird ein Mindestalter von 13 Jahren festgelegt, um nicht ein zu junges und dazu noch deutlich implausibles Alter für das Schulenddatum zu ermöglichen. Für den Fall, dass die Einschulung bereits im Alter von fünf Jahren erfolgt, kann je nach Konstellation des Geburtsmonats und des Monats des Schulenddatums auch der Erwerb eines Hauptschulabschlusses durchaus mit 13 oder 14 Jahren erfolgen. Ein Maximalalter von 23 Jahren beim Schulenddatum kann mit einer verspäteten Einschulung, dem Wiederholen eines Schuljahres oder einer längeren Krankheit während der Schulzeit begründet werden. Treten diese Faktoren gegebenenfalls in Kombination miteinander auf, ist in Ausnahmefällen ein solches Alter beim Erwerb des ersten Schulabschlusses denkbar. Liegt das vergebene Schulenddatum und der dabei zugewiesene Schulabschluss unter einem Alter von 13 Jahren, wird, sofern vorhanden, das Enddatum der chronologisch nächsten Schulepisode als Schulenddatum und dabei der höchste bis dahin erreichte Schulabschluss zugewiesen. Sind die Personen beim vergebenen Schulenddatum älter als 23 Jahre, wird das Enddatum der chronologisch vorherigen Schulepisode als Schulenddatum mit dem bis dahin höchsten Schulabschluss vergeben.

Wenn grundsätzlich keine Datierungen zu den Schulepisoden vorliegen, wird auf Basis des Geburtsjahres und des angegebenen Schulabschlusses ein hierfür plausibles Schulenddatum zugewiesen wie zum Beispiel bei einem Hauptschulabschluss ein Schulenddatum 15 Jahre 
nach dem Geburtsjahr. Als Monat wird hierbei der Monat Juli verwendet, welcher als üblicher Zeitpunkt für das Ende des Schuljahres angesehen wird. Liegen gänzlich überhaupt keine Informationen zu Schulabschlüssen oder Datierungen von Schulepisoden beziehungsweise Informationen zur besuchten Schulform vor, kann weder ein Schulabschluss noch ein Schulenddatum vergeben werden, da dies aufgrund der fehlenden Informationen als zu spekulativ erachtet wird.

Für die Berechnung der Übergangsdauer ist nach dem zugewiesenen Schulenddatum das Startdatum der ersten Ausbildungsepisode maßgeblich. Dabei wird das Startdatum der ersten Ausbildungsepisode als Erstausbildungsstartdatum verwendet. Wenn es sich bei den aufgezählten Ausbildungsepisoden jedoch um eine Weiterbildung oder Zusatzqualifikation handelt, welche ebenfalls als Kategorien unter den Ausbildungsepisoden angelegt sind, werden diese als Weiterbildungsepisoden und damit als Übergangsstationen betrachtet. Das bedeutet, dass für das Erstausbildungsstartdatum das Startdatum der ersten Ausbildungsepisode verwendet wird, deren Ausbildungstyp einer vollwertigen berufsqualifizierenden Ausbildung entspricht.

Im Vorfeld der Berechnung der Übergangsdauer ist ein weiterer Aspekt zu beachten. Personen, welche kein Erstausbildungsstartdatum vorliegen haben, da sie bis zum Ende des Beobachtungszeitraums keine Ausbildung begonnen haben, bekommen als Referenzzeitpunkt den letzten Befragungszeitpunkt zugewiesen, welcher als das Ende des Beobachtungszeitraums verstanden wird. Die Übergangsdauer selbst wird als Monatsdifferenz zwischen dem Startdatum der Erstausbildung und dem Enddatum der Schulepisode mit dem ersten Schulabschluss berechnet. Bei den Fällen ohne begonnene Ausbildung bis zum Ende des Untersuchungszeitraums wird die Übergangsdauer als Monatsdifferenz zwischen dem letzten Interviewzeitpunkt und dem Schulenddatum errechnet. Bei negativen Monatsdifferenzen aufgrund von Überschneidungen des Startdatums der Erstausbildung und des Schulenddatums wird nach der bereits erfolgten Korrektur des Schulenddatums eine Korrektur des Erstausbildungsbeginns vorgenommen. Dabei wird das Startdatum der darauf folgenden Ausbildungsepisode als Erstausbildungsbeginn zugewiesen, sofern es sich um eine duale, vollzeitschulische oder akademische Ausbildung handelt.

Bei Fällen, bei denen kein Datum für einen Erstausbildungsbeginn vorhanden ist, liegt als Ausbildungstyp „keine Ausbildung“ vor. Personen mit einem Erstausbildungsbeginn weisen eine duale, vollzeitschulische oder akademische Erstausbildung auf. Bei Personen, welche bis zum Ende des Beobachtungszeitraums keine Ausbildung begonnen haben, wird als 
Referenzzeitpunkt für die Berechnung der Übergangsdauer der letzte Interviewzeitpunkt der drei einbezogenen Befragungswellen zugewiesen. Das heißt, wenn eine Person an allen drei Befragungswellen teilgenommen hat, wird als Referenzzeitpunkt der Interviewzeitpunkt der letzten Befragungswelle (NEPS-Befragungswelle 2011/2012) vergeben. Hat die Person jedoch beispielweise nur an der ALWA-Befragung teilgenommen, wird der Interviewzeitpunkt der ALWA-Befragung als Referenzzeitpunkt zugewiesen. Maßgeblich ist hierbei immer der Interviewzeitpunkt der letzten Befragungswelle, an der die Person teilgenommen hat.

\subsubsection{Aufbereitung von Schulabschlïssen, Erstausbildungstypen, Herkunftskategorien und Praktikumskategorien}

Im Rahmen der Aufbereitung des Schulenddatums ist auch der erreichte Schulabschluss zentral. Zum Schulabschluss liegen in den Daten des NEPS verschiedene Ausprägungen vor, welche zu vier Kategorien zusammengefasst werden (siehe Anhang Tabelle 2). Wenn grundsätzlich kein Abschluss vorgesehen war, kann es sich um eine Grundschulepisode handeln. Des Weiteren kann bei der entsprechenden Schulepisode von vornherein keine Absicht bestanden haben, einen Abschluss zu erwerben. Dieser Umstand ist davon abzugrenzen, dass kein Abschluss erreicht wurde, weshalb die Ausprägung „Kein Abschluss vorgesehen“ ausgeschlossen und die Ausprägung „Ohne Abschluss“ beibehalten wird. Bei letzterer Kategorie ist anzunehmen, dass die Möglichkeit bestand, einen Schulabschluss zu erwerben, dieser jedoch nicht erreicht wurde. Die beiden in den Rohdaten vorhandenen Ausprägungen „Sonder-/ Förderschulabschluss“ und „Anderer Abschluss“ werden nicht unter eine der vier Schulabschlussformen subsumiert, sondern ausgeschlossen. Für diese Abschlusskategorien gibt es nur sehr wenige Fälle. Des Weiteren lassen sich beide Kategorien inhaltlich nicht $\mathrm{zu}$ einer der vier Schulabschlussformen zuordnen. Ein Sonderbeziehungsweise Förderschulabschluss stellt einen spezifischen Abschluss dar, welcher sich nicht ohne Weiteres mit einem der einbezogenen Abschlüsse vergleichen lässt. Zudem sind beide Abschlussformen nicht mit der Variante gleichzusetzen, dass kein Abschluss erworben wurde. Die Kategorie „Anderer Abschluss“ ist als Restkategorie zu betrachten, in welche alle übrigen Abschlüsse fallen, die nicht von den bisher ausdifferenzierten Abschlussausprägungen abgedeckt wurden.

Der jeweils erste erreichte Schulabschluss wird in Abhängigkeit zum zugewiesenen Schulenddatum zugeordnet. Beispielsweise kann das Enddatum der dritten Schulepisode gleichzeitig das Schulenddatum sein, da während der ersten beiden Schulepisoden kein 
Schulabschluss erworben beziehungsweise bei der Befragung angegeben wurde oder die befragte Person bei den vorherigen Schulepisoden zu jung für ein plausibles Schulenddatum war. In diesem Fall wird der Schulabschluss, welcher am Ende der dritten Schulepisode erworben wurde, beim Schulenddatum zugewiesen. Ist jedoch bei der ersten angegeben Schulepisode als Abschluss „Kein Abschluss“ angegeben und hat diese erste Schulepisode eine Dauer von vier Jahren, so wird hier von einer Grundschulepisode ausgegangen und das Schulenddatum sowie der Abschluss der darauf folgenden Schulepisode verwendet. Handelt es sich bei der ersten Schulepisode um eine vierjährige Schuldauer, also um eine angenommene Grundschulepisode sowie bei der dazugehörigen Schulabschlussausprägung um „Kein Abschluss“ und liegen keine weiteren Datierungen und Informationen zu den weiteren Schulepisoden und Schulabschlüssen vor, so wird bei diesen Fällen kein Schulenddatum und kein Schulabschluss vergeben. Das reguläre Alter am Ende der Grundschulzeit unterschreitet die oben genannte Altersgrenze für das Schulenddatum.

Weiterhin wird der Schulabschluss, sofern fehlend, auf Basis des zugewiesenen Schulenddatums, des Geburtsjahres und, falls vorhanden, der angegebenen Schulform zugeteilt. Bei einem Alter unter 16 Jahren beim Schulenddatum und der Hauptschule als Schulform wird der Hauptschulabschluss zugewiesen, mit 16 Jahren und der Realschule der Realschulabschluss und mit über 17 Jahren und dem Gymnasium das Abitur. Das Alter und die Schulform als wichtige Parameter bei dieser Rekonstruktion beziehen sich dabei immer auf die Schulepisode des zugewiesenen Schulenddatums. Bei der Gesamtschule erweist sich eine eindeutige Zuordnung als schwierig, da auf dieser Schulform verschiedene Abschlüsse erworben werden können. Deshalb wird bei der Gesamtschule beim Schulenddatum keine Rekonstruktion des Schulabschlusses vorgenommen. Die Zuweisung von Schulabschlüssen auf Basis der Schulform und des Alters ist streng genommen spekulativ und es ist nicht hundertprozentig gesichert, dass der jeweilige Abschluss auch wirklich erreicht wurde. Diese Rekonstruktion dient der Informationsgewinnung, um mehr Fälle mit zugewiesenen Schulabschlüssen für die Analysen nutzen zu können. Auf Basis vor allem der Schulform sind die rekonstruierten Abschlüsse jedoch in den meisten Fällen relativ naheliegend.

Der Ausbildungstyp der ersten Ausbildungsepisode wird als Erstausbildungsform zugewiesen. In den Ausgangsdaten liegen zum Ausbildungstyp verschiedene Ausprägungen vor, welche teilweise zu den verwendeten Kategorien zusammengefasst, teilweise aber auch ausgeschlossen werden (siehe Anhang Tabelle 2). Die ursprünglichen Ausprägungen werden zu den drei Ausbildungsformen „Duale Berufsausbildung“, „Vollzeitschulische 
Berufsausbildung“ und „Studium“ zusammengefasst. Bei den Ausbildungstypen liegen in den NEPS-Daten zwei Variablen vor (NEPS Nationales Bildungspanel 2016b: 2422 f.), wobei bei den beiden NEPS-Befragungswellen 2009/2010 und 2011/2012 bei einer der beiden Ausbildungstypvariablen kaum gültige Werte vorliegen und diese designbedingt fehlen. Um für den Erstausbildungstyp keinen Informationsverlust in Kauf zu nehmen, werden die Ausbildungstypen beider Variablen aufeinander angepasst und bei entsprechender Übereinstimmung in einer einheitlichen Erstausbildungstypvariablen zugeordnet. Bei einer der beiden Variablen liegt die Ausprägung „Andere Ausbildung“ vor (NEPS Nationales Bildungspanel 2016b: 2422), worauf bei der Zusammenfassung der Ausbildungskategorien näher eingegangen wird. Bestimmte Ausprägungen sind bei einer Variablen zu einer Kategorie zusammengefasst und bei der anderen Variablen in zwei unterschiedlichen Ausprägungen differenziert (NEPS Nationales Bildungspanel 2016b: 2422 f.).

Der Ausschluss einiger Ausprägungen erfolgt aufgrund der Tatsache, dass diese Ausbildungsformen als Weiterbildungs- beziehungsweise Zusatzqualifikationsmaßnahmen zu betrachten sind, welche eine grundlegende Erstausbildung und gegebenenfalls auch erste berufliche Erfahrungen voraussetzen. Eine Fachschulausbildung ist eine Form der beruflichen Weiterbildung, in der fachliches Vertiefungswissen vermittelt wird. Eine zentrale Voraussetzung hierfür ist eine einschlägige Berufsausbildung sowie vorhandene berufliche Erfahrung im entsprechenden Tätigkeitsgebiet (Kultusministerkonferenz o.J.). Das Gleiche gilt für eine berufliche Weiterbildung an sich sowie für eine Meister- oder Technikerausbildung und für eine Promotion, Habilitation sowie eine Laufbahnprüfung im öffentlichen Dienst, welche auf einer vorherigen beruflichen Ausbildung oder einem Erststudium sowie einer anschließenden Berufstätigkeit aufbauen. Hierunter ist auch die Kategorie „Studium an einer Verwaltungs- und Wirtschaftsakademie (VWA)“ zu fassen (NEPS Nationales Bildungspanel 2016b: 2422). Diese Studienform wird berufsbegleitend angeboten beziehungsweise setzt bereits praktische Berufserfahrung und zum Teil eine abgeschlossene Berufs- oder Hochschulausbildung voraus (VWA 2018). Bei einer der beiden ursprünglichen Ausbildungstypvariablen ist die ausgeschlossene Kategorie „Studium an einer Verwaltungs- oder Wirtschaftsakademie“ mit dem Studium an einer Berufsakademie zur gemeinsamen Ausprägung ,Studium an einer Berufsakademie (auch Verwaltungs- und Wirtschaftsakademie)“ zusammengefasst, was dadurch nicht voneinander getrennt werden kann und in dieser Form berücksichtigt werden muss. 
Beim Studium an einer Berufsakademie handelt es sich um die Kombination aus einer wissenschaftlichen Ausbildung an einer akademischen Einrichtung und einer praxisbezogenen Ausbildung in einem Betrieb. Diese Studienvariante wird auch als „duales Studium“ bezeichnet (Duales Studium Hessen o.J.). Da diese Ausbildungsform jedoch im Gegensatz zur dualen oder vollzeitschulischen Berufsausbildung auch akademische Inhalte beinhaltet, wird sie unter die Kategorie „Studium“ subsumiert. Das Studium an einer Verwaltungsfachhochschule wird ebenfalls der Ausprägung „Studium“ zugeordnet. Hierbei handelt es sich um eine duale Studienform. Die dreijährigen Bachelorstudiengänge an den Fachhochschulen der öffentlichen Verwaltung in Nordrhein-Westfalen beispielsweise setzen sich aus einem fachtheoretischen Ausbildungsteil an der Hochschule und einem berufspraktischen Ausbildungsteil zusammen (Fachhochschule für öffentliche Verwaltung NRW 2018a). Der Zugang zu einem Studium an einer Fachhochschule der öffentlichen Verwaltung ist für das Beispiel Nordrhein-Westfalen gebunden an eine zuvor begonnene Ausbildung an einer der vorgegebenen Einstellungsbehörden des Landes (Fachhochschule für öffentliche Verwaltung NRW 2018b).

Die übrigen Ausprägungen „ein Lehrgang bei einem Verband oder einer Kammer (Industrieund Handelskammer- (IHK) Lehrgang)“, „ein Kurs zum Erwerb einer Lizenz (Schweißer-, Stapler- oder Taxifahrerschein, Netzwerkadministrator)“ sowie „eine andere Ausbildung“ beziehungsweise ,anderer Kurs / Lehrgang“ lassen sich nicht zu einer der drei zentralen Ausbildungsformen zuordnen. Diese Kategorien stellen den Erwerb einer zusätzlichen Qualifikation beziehungsweise Lizenz dar, welche die Voraussetzung für den Beginn einer Ausbildung sein oder den Zugang zu einer Ausbildung erleichtern können. Aufgrund dessen werden diese Episoden als Weiterbildungsstation und damit als Übergangsstationen verwendet, sofern sie zeitlich während der Übergangsphase vollzogen wurden. Die Ausprägung ,weiß nicht“" wird ausgeschlossen.

Daneben wird die Kategorie „Keine Ausbildung“ den Personen zugewiesen, welche keine Angaben sowie kein Datum für den Erstausbildungsbeginn oder keinen beruflich vollqualifizierenden Ausbildungstyp vorliegen haben. Bei Personen, welche ein Erstausbildungsstartdatum vorliegen haben, deren Ausbildungskategorie jedoch nicht einer der drei definierten Ausbildungstypen zugeordnet werden kann, wird das Startdatum der darauf folgenden Ausbildungsepisode als Beginn vergeben, sofern bei dieser Episode eine den drei Ausbildungstypen entsprechende Kategorie vorliegt. Ist dies nicht der Fall, wird wiederum auf das Startdatum der darauf folgenden Episode referenziert, sofern hierzu eine 
vollqualifizierende Ausbildung vorliegt. Liegen ausschließlich Ausbildungen vor, welche sich vom Typ her nicht zu einer der drei zentralen Ausbildungskategorien zuordnen lassen, wird als Ausprägung „Keine Ausbildung“ vergeben. Dies ist beispielweise der Fall, wenn lediglich Episoden der Lizenzerwerbungen oder von IHK-Lehrgängen vorliegen, welche als Zusatzqualifikation in der Übergangsphase betrachtet werden. Auch das ausschließliche Vorliegen von Ausbildungsepisoden, welche vom Ausbildungstyp her beruflichen Weiterbildungen oder Zusatzqualifikationen entsprechen und eine vorherige berufliche oder akademische Erstausbildung erfordern wie beispielsweise eine Promotion oder eine Ausbildung zur Fachärztin beziehungsweise zum Facharzt, führt dazu, dass als Kategorie „Keine Ausbildung“ vergeben wird. Diese Konstellation ist ohne dokumentierte vorherige Erstausbildungen als unrealistisch zu betrachten.

Abschließend werden noch die ausdifferenzierten Kategorien der Bildungsklassifikation des CASMIN zu einigen wenigen Kategorien zusammengefasst, um die Komplexität der vielen Kategorien $\mathrm{zu}$ reduzieren (siehe Anhang Tabelle 2). Die Ausprägung „[1c] Hauptschulabschluss mit beruflicher Ausbildung" wird als separate Kategorie vom Hauptschulabschluss ohne eine berufliche Ausbildung geführt, da das Vorliegen beziehungsweise Nicht-Vorliegen einer beruflichen Ausbildung als ein wesentlicher Indikator für die soziale Herkunft gesehen wird. Bei den Kategorien der mittleren Reife mit und ohne Berufsausbildung erfolgt dagegen eine Zusammenfassung, da der Realschulabschluss im Gegensatz zum Hauptschulabschluss bessere Arbeits- und Berufschancen beinhaltet.

Auch bei den Praktikums- und Erwerbstätigkeitsepisoden werden die Kategorien Volontariat, ein anderes Praktikum, eine Arbeitsbeschaffungsmaßnahme (ABM), ein Ein-Euro-Job oder eine andere befristete Beschäftigung berücksichtigt. Ein Volontariat wird vorwiegend im Medien-/Journalistenbereich nach einem Studium absolviert, wobei hierfür praktische Erfahrungen von Vorteil sind. Jedoch kann ein Volontariat auch ohne besondere Vorbildung ab dem 18. Lebensjahr begonnen werden, wobei die Voraussetzung hierfür das Abitur ist (Trainee-Geflüster 2017). Auch wenn solche Fälle als extrem selten anzunehmen sind und die allermeisten Volontariate im Medien- oder Journalistenbereich nach dem Abschluss eines Hochschulstudiums absolviert werden, ist die theoretische Möglichkeit, ein Volontariat in einem bestimmten Bereich auch direkt nach dem Erwerb der allgemeinen Hochschulreife zu beginnen, bei den möglichen Übergangsstationen zu berücksichtigen. Die Ausprägung „,anderes“ Praktikum ist ebenfalls eine denkbare Übergangsstation. Die Ausprägungen ABMMaßnahme, Ein-Euro-Job und eine andere befristete Beschäftigung kommen bei keiner der 
einbezogenen Personen vor, weshalb hierzu keine Monatsdauern berechnet werden. Die übrigen Ausprägungen der Erwerbstätigkeitsepisoden finden keine Berücksichtigung, da es sich um weiterführende Stationen handelt, welche eine vorherige Berufsausbildung oder ein Hochschulstudium voraussetzen. Ein Referendariat wird am Ende eines Lehramtsstudiums, ein Vikariat nach einem Theologiestudium absolviert. Gleiches trifft auch auf ein Traineeprogramm zu, welches nach dem Studium als Einstieg in ein Unternehmen durchlaufen wird. Ein Anerkennungsjahr wird im pädagogischen Bereich durchlaufen und erfolgt im Anschluss an eine schulische Ausbildung zur staatlich anerkannten Erzieherin beziehungsweise zum staatlich anerkannten Erzieher (erzieherin-ausbildung.de o.J.). Ein pharmazeutisches Praktikum ist ein praktischer Ausbildungsbestandteil für angehende Pharmazeutinnen und Pharmazeuten (Landesapothekerkammer Hessen 2017). Die Station als „Arzt im Praktikum“ wurde im Rahmen des Medizinstudiums geleistet und zum 01. Oktober 2004 abgeschafft (Spiegel Online 2004). Eine Tätigkeit als studentische Hilfskraft kann nur im Rahmen eines Hochschulstudiums absolviert werden.

\subsubsection{Limitationen des Stichprobendesigns und Ausschluss von Personen}

In der verwendeten Stichprobe ist unter anderem der Stichprobenanteil der Personen, welche bereits an der ALWA-Befragung 2007/2008 teilgenommen haben und an einer oder mehreren der späteren NEPS-Befragungswellen teilnahmen, enthalten (Skopek 2013: 16 f.). Hinzu kommen noch die Personen der Geburtsjahrgänge 1956 bis 1986, welche im Rahmen der NEPS-Befragungswellen 2009/2010 und 2011/2012 neu akquiriert und in das NEPS aufgenommen wurden. Hierdurch erhöht sich die Stichprobengröße noch einmal deutlich. Jedoch werden diverse Fälle aus dem verwendeten Gesamtdatensatz ausgeschlossen (siehe Anhang Tabelle 3).

Da es in der vorliegenden Untersuchung um die Übergangsprozesse von westdeutschen Jugendlichen geht, werden Personen aus Ostdeutschland aus dem Datensatz ausgeschlossen. Idealerweise müssten hierbei Personen ausgeschlossen werden, welche in Ostdeutschland beziehungsweise in der DDR ihre Schulausbildung sowie den Übergang in die Erstausbildung absolviert haben. Im NEPS liegt hierzu auch eine Erhebung vor, ob die Gemeinde der besuchten Schule in Westdeutschland oder in Ostdeutschland inklusive Berlin liegt (NEPS Nationales Bildungspanel 2016b: 2266). Jedoch ist diese Information nicht im Rahmen der ALWA-Befragung erhoben worden. Hier wurde lediglich erfragt, ob die Schule in Deutschland oder im Ausland besucht wurde (Matthes und Trahms 2012: 49). Auch anhand 
des erhobenen Schultyps oder des erworbenen Schulabschlusses kann dies nicht rekonstruiert werden. Beim Schultyp sind westdeutsche mit ostdeutschen Schulformen und jenen der DDR zusammengefasst. Beispielsweise ist die Realschule mit der Polytechnischen Oberschule (POS) der DDR, als auch der Regel- und Mittelschule als Schulformen der neuen Bundesländer zu einer Kategorie zusammengefasst. Gleiches gilt auch für das Gymnasium und die Erweiterte Oberschule (EOS) als einer gemeinsamen Kategorie (Matthes und Trahms 2012: 49). Eine analoge Zusammenfassung des Hauptschulabschlusses mit einem Abschluss am Ende der achten Klasse der POS zu einer Kategorie findet sich bei der ALWA-Befragung auch bei der Erhebung des erreichten Schulabschlusses. Gleiche Zusammenfassungen zu einer gemeinsamen Kategorie liegen für die anderen Schulabschlüsse vor (Matthes und Trahms 2012: 53). Dementsprechend fehlt die Information, ob der Ort des Schulbesuchs in West- oder Ostdeutschland liegt, bei den Personen, die an der ALWA-Befragung teilgenommen haben, designbedingt. Auch bezüglich der Gemeinde der Ausbildungsstätte ist im Rahmen des NEPS erhoben, ob diese in West- oder in Ostdeutschland liegt (NEPS Nationales Bildungspanel 2016b: 2454). Im Rahmen der ALWA-Befragung ist jedoch ebenfalls lediglich erhoben, ob die Ausbildung in Deutschland oder im Ausland absolviert wurde (Matthes und Trahms 2012: 69). Daher fehlt diese Information auch für die Ausbildungsepisoden bei den Teilnehmerinnen und Teilnehmern der ALWA-Befragung designbedingt. Dadurch ergibt sich die Konstellation, dass Personen ihre Schulzeit in Ostdeutschland beziehungsweise der DDR verbracht haben können, jedoch die Ausbildung in Westdeutschland absolvierten. Darüber hinaus kann auch nicht festgestellt werden, ob der Übergangszeitraum in West- oder in Ostdeutschland stattgefunden hat. Die einzelnen absolvierten Übergangsstationen können theoretisch an unterschiedlichen Orten absolviert worden sein. Im Rahmen der ALWA-Befragung ist lediglich für Erwerbstätigkeiten beziehungsweise Praktika erhoben, in welchem Bundesland diese geleistet wurden (Matthes und Trahms 2012: 99). Ebenso ist es denkbar, dass Personen sich auf dem westdeutschen Arbeitsmarkt beworben haben, dort jedoch nicht erfolgreich waren und anschließend eine Ausbildung in Ostdeutschland begonnen haben.

Aufgrund der designbedingten Limitationen werden alle Personen ausgeschlossen, welche in Ostdeutschland geboren wurden. Dies führt dazu, dass nur Jugendliche einbezogen werden, welche auch in Westdeutschland geboren wurden. In der Regel haben diese Jugendlichen auch in Westdeutschland die Schule besucht. Zu Zeiten der DDR werden diese Personen mit großer Wahrscheinlichkeit keine Übergangsstationen in der DDR durchlaufen haben und auch 
nicht in eine vollqualifizierende Erstausbildung in der DDR eingemündet sein (von Ausnahmen und Einzelfällen abgesehen). Auch im Zeitraum nach der deutschen Wiedervereinigung ist davon auszugehen, dass westdeutsche Jugendliche keinen Übergang in den Ausbildungs- und Arbeitsmarkt der neuen Bundesländer absolviert haben, welcher im Zeitraum nach 1990 langwierigen Umstrukturierungen und damit verbundenen Problemen wie erhöhten Arbeitslosigkeitsquoten unterworfen war. Im betrachteten Untersuchungszeitraum sind solche Fälle durchaus möglich. Jedoch wird dieser Anteil eher als gering angesehen. Bei der Information zur Geburt in West-, Ostdeutschland oder im Ausland liegen im Rahmen des NEPS insgesamt vier Kategorien vor: „Westdeutschland/Westberlin“, „Ostdeutschland/Ostberlin“, „Ausland“ und „frühere deutsche Ostgebiete“, bei denjenigen, die vor 1950 geboren wurden (NEPS Nationales Bildungspanel 2016b: 11). Von den 6.778 Personen, welche sowohl an der ALWA-Befragung als auch an einer oder mehreren der folgenden NEPS-Befragungswellen teilgenommen haben, wurde niemand in den früheren deutschen Ostgebieten geboren, weshalb diese Kategorie entfällt. Dies gilt auch für Personen der Geburtskohorten 1956 bis 1986, welche im Rahmen der NEPS-Wellen 2009/2010 und 2011/2012 neu in das Panel aufgenommen wurden. Personen, die in Ostdeutschland oder Ostberlin geboren wurden, werden ausgeschlossen.

Von den im Ausland geborenen Personen werden lediglich diejenigen beibehalten, welche bei der Migration nach Deutschland nicht älter als 14 Jahre waren. Dabei ist davon auszugehen, dass Jugendliche, die im Ausland geboren wurden, im Alter von 14 Jahren mindestens noch ein Jahr die allgemeinbildende Schule besuchen müssten, sofern es sich hierbei um die Hauptschule handelt. Der Übergang in die Ausbildung würde dann in Deutschland durchlaufen werden. Dadurch ist zumindest theoretisch sichergestellt, dass die jugendlichen Migrantinnen und Migranten zumindest ein Jahr oder länger das deutsche Schulsystem durchlaufen, bevor der Übergang in den Ausbildungsmarkt erfolgt. Diejenigen im Ausland geborenen Personen, welche ihre gesamte Schulzeit im Ausland verbracht haben und dann im Anschluss den Übergang in das deutsche Ausbildungssystem absolvierten, können aufgrund der schwierigen Vergleichbarkeit keine Berücksichtigung finden. Bei den meisten im Ausland geborenen Personen im Datensatz liegt jedoch kein Alter bei der Migration nach Deutschland vor. Um einen Ausschluss eines Großteils der Fälle und damit einen großen Informationsverlust zu vermeiden, werden im Ausland geborene Personen ohne angegebenes Alter bei der Migration nach Deutschland beibehalten. Somit wird davon ausgegangen, dass diese Personen ihre Schulzeit zumindest teilweise in Deutschland durchlaufen haben. 
Personen, welche 1987 oder später geboren sind, werden ebenfalls ausgeschlossen, da zu diesen Geburtskohorten kaum Fälle vorhanden sind. Da der Übergang zwischen 1971 und 2012 betrachtet wird, sollten die Personen im Jahr 2005 mindestens 19 Jahre alt sein. Dabei kann davon ausgegangen werden, dass im Alter von 19 Jahren die allgemeinbildende Schulzeit in der Regel beendet ist und sich die Personen zumindest im Übergangsprozess von der Schule in die Ausbildung oder das Studium befinden. Dies gewährleistet, dass Personen, welche 1986 geboren wurden, die Schule 2005 mit dem Abitur verlassen haben und lediglich an der ersten Befragungswelle 2007/2008 teilgenommen haben, einen ausreichenden Übergangszeitraum aufweisen und mehrheitlich zum Zeitpunkt der ersten Befragungswelle in eine Erstausbildung übergegangen sein sollten.

Des Weiteren werden Personen ausgeschlossen, deren Schulenddatumsjahr, also das Jahr des ersten Schulabschlusses, vor 1971 oder nach 2007 liegt. Dies ist damit zu begründen, dass bei den Schulenddatumsjahren vor 1971 und nach 2007 relativ geringe Fallzahlen vorliegen, deren Werte für die Übergangsdauer und die Anzahl an absolvierten Übergangsstationen unter Umständen verzerrt sind. Während die wenigen Personen mit einem Schulenddatumsjahr vor 1971 durchschnittlich eine deutlich längere Übergangsdauer aufweisen als die darauffolgenden Jahreskohorten, sinkt die durchschnittliche Dauer bei den Personen, deren Schulenddatumsjahr nach 2007 liegt, deutlich. Bei letzterer Gruppe kann dies mit dem Umstand begründet werden, dass die Personen die Schule das erste Mal erst spät verlassen. Haben sie beispielsweise die Schule erst im Jahr 2008 verlassen und lediglich an der ALWABefragung in den Jahren 2007 und 2008, jedoch nicht an den weiteren NEPSBefragungswellen teilgenommen, haben sie zum Befragungszeitpunkt erst wenige Monate im Übergangsprozess verbracht, sofern kein direkter Übergang in die Erstausbildung erfolgte, oder die Schule unter Umständen noch nicht verlassen. Aufgrund der sehr geringen Fallzahl, der verzerrten deskriptiven Ergebnisse für die abhängigen Variablen und der zeitlichen Begrenzung des Untersuchungszeitraums werden diese Fälle ebenfalls nicht berücksichtigt. Im für die Analysen verwendeten Datensatz sind abschließend N = 9.254 Personen enthalten. Hierbei handelt es sich um in Westdeutschland beziehungsweise Westberlin geborene Personen der Geburtsjahrgänge 1956 bis 1986 sowie um im Ausland geborene Personen, welche bei der Migration nach Deutschland höchstens 14 Jahre alt waren oder bei denen keine Angabe zum Alter bei der Migration nach Deutschland vorliegt. 


\subsubsection{Bereinigung von zeitlichen Dopplungen und Überschneidungen bei Übergangsstationen, Schulenddatum und Erstausbildungsbeginn}

Als nächsten Schritt werden die Monatsdauern der einzelnen Übergangsstationen berechnet.

Dabei sind als zeitliche Referenzpunkte das Schulenddatum und das Erstausbildungsstartdatum beziehungsweise der letzte Befragungszeitpunkt relevant. Die Stationsdauern werden nur berechnet, wenn sie zwischen diesen beiden Zeitpunkten liegen. Die Monatsdauern für die einzelnen Stationen werden anhand der Angaben zum Start- und Enddatum berechnet. Fehlen diese, ist es schwierig, eine Monatsdauer für die jeweilige Station zu rekonstruieren. Dies trifft insbesondere auf die Stationen Elternzeit, Lücken, Arbeitslosigkeit, Praktikum oder auch Kurse zu, da diese beliebig lange dauern können. Beim Wehrdienst in Form der jeweiligen gesetzlichen Dauer und bei berufsvorbereitenden Maßnahmen in Form des Berufsvorbereitungs- oder Berufsgrundbildungsjahres wäre eine fehlende Datierung theoretisch rekonstruierbar. In den vorhandenen Daten ist dies aber kaum notwendig, da es bei den Übergangsstationen neben den verwendeten edierten Start- und Enddatumsvariablen zusätzlich nicht-edierte Start- und Enddatumsvariablen gibt, welche meist mehr Datumsinformationen zum Monat, Jahr und der Jahreszeit enthalten. Fehlende Datumsangaben werden bei den Übergangsstationen durch die Angaben der nicht-edierten Datumsvariablen ersetzt.

Bei der Berechnung der Stationsdauern manifestieren sich bei Durchsicht des verwendeten Datenmaterials verschiedene Varianten von zeitlichen Überschneidungen von identischen oder verschiedenen Stationen. Bei Überschneidung zweier unterschiedlicher Stationen ist die inhaltliche Gewichtung der Stationen entscheidend (siehe Anhang Tabelle 4). Die Bereinigung der vielfältigen Überschneidungen wird für alle einbezogenen Stationen durchgeführt. Im Zuge der Bereinigung von negativen Werten für die Übergangsdauer wird jeweils das Startdatum der darauf folgenden Ausbildungsepisode als Erstausbildungsstartdatum verwendet. Bei einer Überschneidung einer Übergangsstation mit dem Erstausbildungsbeginn wird lediglich die Stationsdauer berechnet, welche zeitlich vor dem Erstausbildungsstartdatum und damit im Übergangszeitraum liegt. Durch die Bereinigung und Berechnung kann es unter Umständen zu Stationsdauern von null Monaten kommen. Wenn beispielsweise im August 1994 die Erstausbildung begonnen hat und von August 1994 bis März 1995 eine Lückenepisode erfolgte, wird dementsprechend die Monatsdifferenz zwischen dem Erstausbildungsbeginn und dem Lückenbeginn gebildet. Da beide Zeitpunkte im August 1994 liegen, kommt eine Dauer von null Monaten für die 
Lückenepisode zustande. Dies wird zu einem Monat korrigiert, da keine Station null Monate dauern kann und Monate in den verwendeten Daten die kleinste verwendete zeitliche Maßeinheit sind (u. a. NEPS Nationales Bildungspanel 2016b: 2032, 2058, 2281, 2442). Als problematisch ist der Fall anzusehen, wenn eine Übergangsdauer von null Monaten vom Schulenddatum bis zum Erstausbildungsbeginn vorliegt, da beide Zeitpunkte im gleichen Monat liegen, und für Übergangsstationen eine Monatsdifferenz von null Monaten angegeben wird, da diese Station im gleichen Monat wie das Schulenddatum und das Erstausbildungsstartdatum liegt. Somit kann bei einer Übergangsdauer von null Monaten keine Station bestehen, welche einen Monat gedauert hat. Deshalb werden bei einer Übergangsdauer von null Monaten die Monatsdauern für angegebene Stationen ausgeschlossen.

Außerdem werden Dopplungen von jeweils gleichen Übergangsstationen, beispielsweise zwei oder mehr Arbeitslosigkeitsepisoden, mit identischem Start- und Enddatum bereinigt. Dabei wird die Stationsdauer einer Episode ausgeschlossen. Die gleiche Vorgehensweise erfolgt bei identischem Start- und Enddatum zweier unterschiedlicher Übergangsstationen, wobei hierbei die inhaltliche Gewichtung der Stationen maßgeblich ist (siehe Anhang Tabelle 4). Für die Gewichtung spielt die Relevanz für den Arbeitsmarkt eine wesentliche Rolle. Beispielsweise ist eine berufsvorbereitende Maßnahme in Bezug auf den Arbeitsmarkt von größerer Relevanz als eine Lückenepisode, da die berufsvorbereitende Maßnahme als Unterstützung zum Übergang in die Berufsausbildung dienen kann, wogegen es sich bei einer Lückenepisode um einen unbestimmten Zeitraum im Übergangsprozess handelt. Nachfolgend wird für jede Stationskombination die jeweilige Gewichtung begründet.

Kurse können beispielsweise im Rahmen des Wehrdienstes oder der Elternzeit absolviert werden. Im Gegensatz zu einer Arbeitslosigkeitsepisode wird die Elternzeit in der Regel dafür genutzt, um sich den im Haushalt lebenden Kindern zu widmen. Eine berufsvorbereitende Maßnahme kann im Rahmen einer Arbeitslosigkeit absolviert werden und stellt keine Arbeitslosigkeit im Sinne einer fehlenden Erwerbs- oder Qualifikationsphase dar, sondern dient der Berufsvorbereitung. Der Wehrdienst als gesetzlich vorgeschriebener Dienst stellt im Gegensatz zur Arbeitslosigkeit keine fehlende Tätigkeit dar. Dagegen ist die Arbeitslosigkeit als inhaltlich bedeutsamer einzustufen als die Lückenstation. Ein Praktikum und auch zusätzliche Schulepisoden haben im Gegensatz zu einer Arbeitslosigkeit jeweils einen deutlichen Qualifikationscharakter. Eine berufsvorbereitende Maßnahme ist im Vergleich zur Elternzeitepisode arbeitsmarktrelevant. Im Gegensatz zur Elternzeit sind bei den 
Wehrdiensttypen in den vorhandenen Daten unter anderem die Ausprägung „FSJ/FÖJ/FEJ“ für ein freiwilliges soziales, ökologisches oder europäisches Jahr (NEPS Nationales Bildungspanel 2016b: 2057) als durchaus wichtige Zusatzqualifikation enthalten. Elternzeitepisoden sind inhaltlich aussagekräftiger als eine Lücke. Praktika oder Volontariate sowie zusätzliche Schulepisoden sind arbeitsmarktrelevant im Gegensatz zu einer Elternzeit. Eine berufsvorbereitende Maßnahme ist arbeitsmarktrelevant gegenüber dem Wehrdienst oder einer Lücke. Praktika können gegebenenfalls im Rahmen von berufsvorbereitenden Maßnahmen im Bereich der Grundstufe absolviert werden (Rehadat Institut der deutschen Wirtschaft Köln e. V. 2017). Auch zusätzliche Schulepisoden können Bestandteil dieser Maßnahmen sein und finden bei entsprechenden Bildungsanbietern oder an einer Berufsschule statt (Rehadat Institut der deutschen Wirtschaft Köln e. V. 2017). Der Wehrdienst ist im Vergleich zu Lückenepisoden relevanter, jedoch im Gegensatz zu Praktika-, Volontariatsepisoden oder zusätzlichen Schulepisoden kaum arbeitsmarktrelevant. Lücken sind im Gegensatz zu Praktika, Volontariaten oder zusätzlichen Schulepisoden kaum aussagekräftig. Das Nachholen von Schulabschlüssen hat auch eine größere Bedeutung für den Übergang in die Ausbildung als Praktika. Praktika können als Einstiegsmöglichkeit in die Ausbildung dienen, jedoch genauso gut eine Überbrückungsstation sein. Kurse können als Bestandteile von Weiterbildungen betrachtet werden oder sich teilweise auch mit der Kategorie Weiterbildung doppeln. Weiterbildungen stellen im Vergleich mit Arbeitslosigkeits-, Lücken-, Elternzeit- und Wehrdienstepisoden eine bildungs- und berufsbezogene Aktivität dar und können Bestandteil von berufsvorbereitenden Maßnahmen sein. Praktika können in Weiterbildungsmaßnahmen integriert und Weiterbildungen ein Teil von Schulbesuchen sein. Generell gilt für die beschriebenen Gewichtungen der Stationen, dass die Dauer der weniger gewichteten Übergangsstation ausgeschlossen wird, sofern diese sich zeitlich mit einer anderen Station überschneidet, welche inhaltlich stärker gewichtet wird. Wenn sich grundsätzlich eine Stationsepisode nicht mit einer anderen Episode überschneidet, bleibt die Stationsdauer dieser Episode unberührt davon bestehen. Die einzelnen Varianten und Bereinigungen von zeitlichen Überschneidungen von Episoden sind im Anhang in den Abbildungen 1 bis 13 vollständig dargestellt. Im Anschluss daran werden aus den vorangegangenen Bereinigungsschritten entstandene Überschneidungen mit dem Schulenddatum und dem Erstausbildungsstartdatum bereinigt. Sämtliche Datenaufbereitungsprozeduren werden auch für Personen, welche bis zum Ende des Untersuchungszeitraums in keine Ausbildung eingemündet sind und dadurch den letzten 
Befragungszeitpunkt als Parameter für die Berechnung der Übergangsdauer zugewiesen bekommen, durchgeführt.

\subsubsection{Berechnung der Übergangsdauer und der Anzahl der Übergangsstationen}

Die Übergangsdauer errechnet sich als Monatsdifferenz zwischen dem Enddatum der Schulzeit mit dem ersten Schulabschluss und dem Erstausbildungsstartdatum, sofern eine Ausbildung begonnen wurde, beziehungsweise dem letzten Befragungszeitpunkt, sofern während des Untersuchungszeitraums keine Ausbildung begonnen wurde. Jedoch werden diverse Übergangsstationen nicht für den Übergangszeitraum berücksichtigt, da sie nicht als primär ausbildungs- und arbeitsmarktrelevant betrachtet werden. Hierzu zählen die Stationen Lücken, Elternzeit und Wehrdienst, deren Monatsdauern aus der Übergangsdauer herausgerechnet werden. Die Anzahl der im Übergangszeitraum durchlaufenen Stationen wird zu einer Summe addiert. Auch hierbei finden die Stationen Lücken, Elternzeit und Wehrdienst keine Berücksichtigung. Eine Ausnahme bilden die Ausprägungen „Freiwilliges Soziales, Ökologisches oder Europäisches Jahr“ sowie „Bundesfreiwilligendienst“ bei den Wehrdienstepisoden (NEPS Nationales Bildungspanel 2016b: 2057) und „in Ferien/Urlaub“, „krank/arbeitsunfähig“, „Hausfrau/Hausmann“ und „etwas anderes gemacht“ bei den Lückenepisoden (NEPS Nationales Bildungspanel 2016b: 2029). Treffen diese Ausprägungen zu, werden die Wehrdienst- beziehungsweise Lückenepisoden in der Übergangsdauer und bei der Anzahl der Stationen belassen. Neben einem FSJ kann auch der Bundesfreiwilligendienst einen positiven Einfluss auf den Übergang in die Ausbildung haben, welcher jedoch erst seit der Aussetzung der Wehr- und Zivildienstpflicht zum 1. Juli 2011 besteht (Bundesamt für Familie und zivilgesellschaftliche Aufgaben o.J.). Bei einem Urlaub kann es sich um einen Sprachurlaub handeln. Während eine Haustätigkeit als Auszeit einen Einfluss auf den weiteren Übergangsverlauf haben kann, ist die Kategorie „etwas anderes gemacht“ nicht direkt inhaltlich zu qualifizieren, sondern kann für verschiedene Tätigkeiten während des Übergangszeitraums stehen. Die Kategorie „Krankheit“ im Rahmen der Lückenepisoden wird dagegen bei den absolvierten Stationen nicht berücksichtigt.

Um einen Vergleich zu ermöglichen, ob sich die Resultate der Analysen durch den Einbezug bestimmter Stationen gegebenenfalls ändern, werden zwei Übergangsdauern und insgesamt vier Summenscores für die Anzahl an Übergangsstationen gebildet. Dabei wird eine Übergangsdauervariable erstellt, welche auch die Episoden FSJ und Urlaub erhält. Bei der zweiten gebildeten Dauervariablen werden diese beiden Stationen nicht berücksichtigt. Bei 
den Summenscores wird zunächst eine Variable aller absolvierten Übergangsstationen gebildet, in welcher die Stationen FSJ und Urlaub nicht berücksichtigt werden. Bei der zweiten Variablen werden diese beiden Stationen dagegen einbezogen. Bei diesen beiden Summenvariablen ist auch die Station „unbestimmte Phasen“ als Lücken mit enthalten. Diese Station wird gebildet, wenn die Dauer aller absolvierten Übergangsstationen geringer ausfällt als die Übergangsdauer zwischen dem Schulenddatum und dem Erstausbildungsstartdatum beziehungsweise dem letzten Befragungszeitpunkt. Diese Monate innerhalb des Übergangszeitraums sind unbestimmt, da hier von den befragten Personen keine Stationsepisoden angegeben wurden. Diese Zeitpunkte können sich über mehrere Phasen während des Übergangszeitraums verteilen oder nur $\mathrm{zu}$ einem bestimmten Zeitpunkt vorliegen. Die beiden Summenvariablen zu den Übergangsstationen werden noch einmal gebildet, allerdings ohne die Station „unbestimmte Phasen“. Generell werden die unbestimmten Phasen bei den beiden Übergangsdauervariablen mit berücksichtigt, da bei diesen Phasen unklar bleibt, welche Episode oder Tätigkeit vollzogen wurde. Die Episoden „Haustätigkeit“ und „etwas anderes gemacht“ sind generell in der Übergangsdauer und den Übergangsstationen enthalten. Dies gilt bei der Dauer auch für die Kategorie „Krankheit“, welche bei den Analysen zu den Stationen jedoch generell keine Berücksichtigung findet.

\subsection{Methoden}

\subsubsection{Deskriptive Statistiken}

Sämtliche im Folgenden vorgestellte Analyseverfahren werden, mit Ausnahme der abschließenden Sequenzdatenanalysen und dazugehörigen Regressionsmodelle, mit Hilfe der Statistiksoftware IBM SPSS Statistics 24 und 25 durchgeführt. Es werden zunächst deskriptive Statistiken und Diagramme erstellt. Dabei werden Mittelwerte und Standardabweichungen der abhängigen Variablen Übergangsdauer und Anzahl an Übergangsstationen ausgegeben und mit Hilfe von Liniendiagrammen die Entwicklung beider Variablen über den Untersuchungszeitraum hinweg betrachtet. Für den Zeitraum sind die einbezogenen Schulenddatumsjahre maßgeblich. Des Weiteren werden die deskriptiven Übersichten getrennt nach Schulabschluss sowie für die weiteren einbezogenen Variablen ausgegeben. Die deskriptiven Statistiken werden sowohl insgesamt als auch im Verlauf des Untersuchungszeitraums betrachtet. 


\subsubsection{Korrelationen und Mittelwerttests}

Darüber hinaus werden Korrelationen mit der Pearsons Produktmomentkorrelation $r$ als Koeffizient für die Überprüfung von linearen Zusammenhängen zwischen zwei metrisch skalierten Variablen berechnet (Völkl und Korb 2018: 207). Da die Richtung des jeweils untersuchten bivariaten Zusammenhangs vorab nicht bekannt ist, wird eine zweiseitige Signifikanztestung durchgeführt (IBM Knowledge Center o.J.). Weiterhin wird getestet, ob hinsichtlich der Übergangsdauer und der Anzahl der Stationen signifikante Mittelwertunterschiede zwischen den Schulabschlussformen und den Erstausbildungstypen sowie den sozialen Herkunftsformen und zwischen Männern und Frauen bestehen. Die Ergebnisse der Tests und Analyseverfahren werden als p-Werte dargestellt. Dabei wird das konventionelle 5\%-Signifikanzniveau verwendet (Döring und Bortz 2016: 665 f.). Für die Tests auf signifikante Mittelwertunterschiede wird die einfaktorielle univariate Varianzanalyse verwendet, welche jedoch keine konkreten Ergebnisse darüber liefert, zwischen welchen Gruppen exakt sich diese Mittelwertunterschiede lokalisieren lassen. Dies ermöglichen Post-Hoc-Tests, welche alle theoretisch möglichen Mittelwertvergleiche erlauben (Bühner und Ziegler 2017: 372 f.). Bei Vorliegen von Varianzhomogenität wird als Post-Hoc-Test der Tukey-Test (Universität Zürich 2018) und bei Vorliegen von Varianzheterogenität der Games-Howell-Test verwendet (Schulze-Bentrop 2014: 201). Des Weiteren wird der $t$-Test bei unabhängigen Stichproben verwendet (Bühner und Ziegler 2017: 295).

\subsubsection{Ereignisdaten- und Sequenzdatenanalyse}

Bei der Ereignis- oder auch Survivalanalyse geht es um die Betrachtung der Zeit nach einem dokumentierten Ereignis sowie um den Verlauf bis zum Eintritt eines Ereignisses und einer damit verbundenen Zustandsveränderung (Blossfeld 2010: 997). Dabei dient ein Setting an Verfahren dazu, die Zeitintervalle zwischen aufeinander folgenden Ereignissen beziehungsweise Zustandsveränderungen zu untersuchen. Die Ereignisdatenanalyse dient der Beschreibung der Zeitdauern zwischen den Ereignissen und der Aufklärung von Zustandsänderungen. Zentral ist dabei die prinzipiell jederzeit mögliche Veränderung und prozessuale Dynamik sowie deren Erklärung (Blossfeld 2010: 995).

Die Sequenzdatenanalyse wiederum umfasst diverse Verfahren zur Beschreibung und Analyse von Sequenzen beziehungsweise Lebensverläufen. Ereignisse sind als Übergänge zwischen unterschiedlichen Statuszuständen, wie beispielsweise der Übergang von der 
Erwerbstätigkeit in die Arbeitslosigkeit, definiert, wobei der Lebensverlauf in einzelne Etappen zerteilt wird. Die Sequenzdatenanalyse fokussiert den Lebensverlauf als komplexe, sortierte, sequenzielle Abfolge von diversen Statuszuständen mit dem Ziel, eine Typisierung von Lebensverläufen herbeizuführen sowie kontextuelle Einflüsse auf diese zu untersuchen (Scherer und Brüderl 2010: 1031 f.). Während die Ereignisdatenanalyse sinnvoll zur Untersuchung der Dauer bis zum Eintritt in die Ausbildung ist, erweist sich die Sequenzdatenanalyse als nützlich, um die gesamte Übergangssequenz inklusive Anzahl und Muster der absolvierten Übergangsstationen zu untersuchen.

\subsubsection{Ereignisdatenanalyse: Sterbetafeln und Cox-Regression}

Die Ereignisdatenanalyse beinhaltet drei zentrale Verfahren: Sterbetafeln, Kaplan-MeierSchätzungen und Cox-Regressionen. Durch diese Analyseverfahren wird die Zeit bis zum Eintritt eines bestimmten Ereignisses untersucht. Dies kann beispielsweise die Anstellung in einer beruflichen Position, die Heilung einer Krankheit oder der Tod sein (Schendera 2014: 233). Das in der vorliegenden Studie zentrale Ereignis ist der Beginn der Erstausbildung, wogegen die Übergangsdauer vom definierten Schulenddatum bis zum Erstausbildungsbeginn das zentrale Zeitintervall darstellt. Der Startpunkt des Zeitintervalls ist im definierten Schulenddatum zu verorten. Neben der Analyse der Dauer bis zum Ereigniseintritt untersucht die Ereignisdatenanalyse, ob das erwartete Ereignis während des Beobachtungszeitraums tatsächlich eingetreten ist. Im Fokus steht die Beschreibung der Überlebenswahrscheinlichkeit bis zum Eintritt des Ereignisses (Schendera 2014: 234). Jedoch tritt nicht bei jedem Fall das erwartete Ereignis ein, was durch einen Teilnahmeabbruch, eine Abwanderung beziehungsweise einen Verlust des entsprechenden Falles oder das Ende des Beobachtungszeitraumes verursacht werden kann (Schendera 2014: 243). Bei der Untersuchung, mit welcher Wahrscheinlichkeit bestimmte Ereignisse im Zeitverlauf auftreten, werden auch Fälle einbezogen, bei denen das Ereignis bis zum Ende des Untersuchungszeitraums nicht eintritt. Diese werden als „rechtszensierte“ Fälle bezeichnet. Grundsätzlich sind bei Ereignisdatenanalysen zwei Variablen zentral: Zum einen die „Überlebenszeit“" in Monaten für das Zeitintervall und zum anderen der Status, ob das betrachtete Ereignis bis zum Ende des Beobachtungszeitraums eingetreten ist oder nicht (Bühl 2014: 815). Während die Überlebenszeit als Übergangsdauer vom Schulenddatum bis zum Beginn der Erstausbildung in Monaten vorliegt, wird zusätzlich noch eine Statusvariable 
erstellt. Liegt ein Erstausbildungsbeginn während des Übergangszeitraums vor, gibt diese an, dass das definierte Ereignis eingetreten ist.

Beim Verfahren der Sterbetafel wird der Beobachtungszeitraum in gleich große Zeitintervalle zerlegt (Bühl 2014: 816) und für jedes einzelne Intervall die Wahrscheinlichkeiten für das Eintreten des Ereignisses berechnet. Dies geschieht pro Intervall für Fälle, welche das Ende des jeweiligen Intervalls erreicht haben. Danach wird aus den geschätzten Wahrscheinlichkeiten aller Intervalle eine Gesamtwahrscheinlichkeit für das Eintreten des definierten Ereignisses zu unterschiedlichen Zeitpunkten berechnet (Schendera 2014: 247). Bei der Analyse anhand von Sterbetafeln sind insbesondere die geschätzten kumulativen Überlebenswahrscheinlichkeiten bis zum Ende des Intervalls von Bedeutung. Bei diesen handelt es sich um die Wahrscheinlichkeit, das Ende des letzten vorhandenen Intervalls zu erleben unter der Voraussetzung, dass das erste Intervall erreicht wurde (Bühl 2014: 818; Schendera 2014: 279). Neben der „Anfangszeit des Intervalls“ als dem jeweiligen Startpunkt des Intervalls (Schendera 2014: 279) ist die „Anzahl der zur Anfangszeit Überlebenden“ relevant als Anzahl der zur Anfangszeit des jeweiligen Intervalls noch beobachteten Fälle. Dabei werden verstorbene beziehungsweise rechtszensierte Fälle nicht berücksichtigt (Bühl 2014: 818). Neben der „Anzahl der Ausgeschiedenen“ als denjenigen, die im jeweiligen Intervall aufgrund einer Zensierung durch Beendigung des Untersuchungszeitraums und Nicht-Eintretens des Ereignisses ausscheiden (Bühl 2014: 818; Schendera 2014: 279), gibt die „Anzahl terminaler Ereignisse“ an, für wie viele Fälle im jeweiligen Intervall das Zielereignis eingetreten ist. Der „Anteil der Terminierenden“ als die bedingte geschätzte Wahrscheinlichkeit für das Eintreten des Ereignisses im jeweiligen Intervall (Bühl 2014: 818; Schendera 2014: 279) sowie der „Anteil der Überlebenden“ als geschätzte Wahrscheinlichkeit, das jeweilige Intervall zu überleben, jeweils vorbehaltlich des Erreichens des Intervallanfangs (Bühl 2014: 818) sind weitere Kenngrößen. Außerdem wird der Zeitpunkt einer kumulierten Überlebenswahrscheinlichkeit von 0,5 ausgegeben. Zu diesem Zeitpunkt ist genau für die Hälfte aller Fälle das Zielereignis eingetreten (Bühl 2014: 819 f.). Damit haben im letzten Intervall noch mindestens 50 Prozent der Fälle überlebt (Schendera 2014: 279). Zum Medianzeitpunkt ist für 50 Prozent der Fälle das Zielereignis noch nicht eigetreten. Eine getrennt nach Untergruppen durchgeführte Analyse mit Gruppenvergleichen der Überlebensverläufe (Bühl 2014: 819 f.) wird mit Hilfe des Wilcoxon-Tests (Gehan-Tests) ermöglicht (SPSS Inc. 2007: 123). Das Verfahren der Sterbetafel-Methode gilt als nichtparametrisch (Fahrmeir 2007: 26) ohne weitergehende Voraussetzungen bis auf den 
Einbezug der rechtszensierten Fälle und kann im Gegensatz zur Kaplan-Meier-Methode auch bei sehr großen Fallzahlen angewendet werden (Bühl 2014: 837).

Die Regressionsanalyse nach Cox untersucht den Einfluss unabhängiger Variablen auf die Überlebenswahrscheinlichkeit bis zum Eintritt des Ereignisses. Hierbei werden die rechtszensierten Fälle mit einbezogen. Bei der Interpretation der Ergebnisse stellen positive Koeffizienten eine Verringerung der Überlebenswahrscheinlichkeit dar, wogegen negative Koeffizienten für eine Erhöhung der Überlebenswahrscheinlichkeit stehen (Bühl 2014: 837). In der vorliegenden Studie bedeutet eine Verringerung der Überlebenswahrscheinlichkeit eine kürzere und eine Erhöhung eine längere Übergangsdauer. Zentral bei Cox-Regressionen sind Hazard-Funktionen. Diese geben das Risiko an, dass für eine bestimmte Person zu einem bestimmten Zeitpunkt ein Zielereignis eintritt unter der Voraussetzung, dass dieses Ereignis bis zu diesem Zeitpunkt noch nicht eingetreten ist. Je größer der Hazard-Wert ist, desto größer ist das Risiko, dass das Zielereignis zum nächstmöglichen Zeitpunkt eintritt (Schendera 2014: 289). Daraus folgt, dass ein großer Hazard-Wert mit einer geringen Überlebensdauer einhergeht, was in der vorliegenden Studie als eine kürzere Übergangsdauer zu interpretieren ist. Insgesamt zeigt das Cox-Regressionsmodell den Effekt einer bestimmten Kovariate auf die Überlebenszeit unter Konstanthaltung aller anderen einbezogenen Kovariaten im Modell. Geschätzt wird der Hazard, also das „Risiko“, dass für eine bestimmte Person das Zielereignis eintritt. Eine Voraussetzung hierfür ist, dass für die Person die Werte für alle Einflussvariablen gleichzeitig gegeben sein müssen und keine fehlenden Werte vorliegen. Während das Vorliegen einer bestimmten Verteilung der Überlebenszeit nicht von Bedeutung ist, stellt die Annahme proportionaler Hazards, dass die Effekte der unterschiedlichen Kovariaten im Modell auf die Überlebenszeit über die Zeit hinweg konstant sind, eine zentrale Anforderung dar. Entscheidend ist des Weiteren, dass das definierte Zielereignis im Rahmen eines Cox-Regressionsmodells nicht auf einen bestimmten Beobachtungszeitraum fixiert werden kann. Die Hazard-Funktion wird als Risiko pro Zeiteinheit für den Eintritt des Zielereignisses zu einer bestimmten Zeit interpretiert. Dabei geht es um die gleichzeitige Schätzung des Einflusses von unterschiedlichen Einflussvariablen, wobei die HazardFunktion in Abhängigkeit zur jeweiligen Variable betrachtet wird (Ziegler et al. 2007: e42 f.). Bei der Berechnung des Modells werden so genannte Hazard-Ratios ausgegeben, welche Werte zwischen null und ,plus unendlich' annehmen können. Ein Hazard-Ratio-Wert größer Eins gibt an, dass das Risiko, dass das Zielereignis eintritt, sich bei der Erhöhung beziehungsweise Veränderung des Wertes der Einflussvariable vergrößert. Bei einem Hazard- 
Ratio-Wert kleiner Eins verringert sich das Risiko dagegen und bei einem Wert von genau Eins bleibt das Risiko unverändert (Ziegler et al. 2007: e43 f.). Durch die Untersuchung des Effekts des Anteils an Dienstleistungstätigen zwischen den Schulabschlussgruppen ist der Einbezug sogenannter Interaktionseffekte notwendig. Diese ermöglichen die Überprüfung, ob der Effekt einer bestimmten Einflussvariable in Abhängigkeit von der konkreten Ausprägung einer weiteren unabhängigen Variable abnimmt oder sich vergrößert (Lohmann 2010: 677 ff.). Außerdem werden die Grundwerte für die kumulative Hazard-Rate über die Zeit (Bühl 2008: 737) als Schätzer für die Chance, dass das Zielereignis eintritt, unter der Bedingung, dass alle einbezogenen Kovariaten für ein hypothetisches Individuum den Wert null erhalten, ausgegeben. Diese Raten werden für die einzelnen Zeitpunkte geschätzt und sind unabhängig von Kovariateneffekten zu interpretieren. In der Regel erhöhen sich die Hazard-Rate und damit das Risiko des Eintretens des Zielereignisses im Laufe der Zeit. Weiterhin werden Schätzungen für ein hypothetisches Individuum mit durchschnittlichen Werten für die Kovariaten angegeben, was den Vergleich einer Person mit durchschnittlichen Ausprägungen mit Personen mit realen Werten ermöglicht. Unter „Überlebensanalyse“ wird der geschätzte Anteil an Personen angegeben, für den zu einem Zeitpunkt das Zielereignis noch nicht eingetreten ist. Die „Kovariaten-Mittelwerte“ sind die Grundlage für die Schätzungen, da sie auf Basis dieser Mittelwerte berechnet werden (Schendera 2014: 301).

Die Cox-Regressionsanalyse setzt die Proportionalität der Hazards voraus, was bedeutet, dass das Hazard-Ratio für zwei unabhängige Fälle über die Zeit hinweg konstant bleibt (Schendera 2014: 343). Der Hazard kann als Ereignisrisiko zwar zu jedem Zeitpunkt unterschiedlich sein. Jedoch sollten die Unterschiede in den durch die Ausprägungen der unabhängigen Variablen definierten beiden Gruppen über die Zeit hinweg gleich sein (Zwiener et al. 2011: 167). Dies lässt sich anhand von annähernd parallel verlaufenden Linien in einem Log-minus-logDiagramm (LML) nachweisen. Die Linien stehen jeweils für die Ausprägungen der kategorialen Variablen im Modell. Dabei wird der LML-Plot für jede kategoriale Variable im Cox-Modell separat erstellt (Schendera 2014: 332 f.). Darüber hinaus wird zur Überprüfung der Proportionalitätsannahme das Kaplan-Meier-Verfahren mit einer Schätzung der Überlebenszeiten durch zweifaches Logarithmieren angewendet. Diese werden in einem gruppierten Streudiagramm grafisch visualisiert, wobei zur Erfüllung der Proportionalitätsannahme ein konstanter beziehungsweise paralleler Abstand zwischen den Punktelinien bestehen sollte (Schendera 2014: 333 f.). Dieses Verfahren eignet sich insbesondere bei Cox-Modellen mit zeitunabhängigen Kovariaten, also Variablen, welche 
sich innerhalb einer Person über die Zeit nicht verändern, mit wenigen Kategorien. Als weiteren Schritt werden die skalierten Schoenfeld-Residuen ermittelt und anhand des Verfahrens von Therneau und Grambsch unter anderem grafisch auf das Vorliegen einer Steigung ungleich null getestet (idre Institute for Digital Research and Education 2021). Dabei sollte die in den Plot der skalierten Schoenfeld-Residuen eingezeichnete geglättete Kurve zur Erfüllung der Proportionalität in etwa einem horizontalen Verlauf entsprechen (Wenzelburger et al. 2014: 194). Die Testung der Proportionalitätsannahme wird für alle im Modell einbezogenen kategorialen oder metrisch skalierten Variablen, welche nach Bereichen in Kategorien gruppiert werden, durchgeführt (Machin et al. 2006: 146).

Als Analyseverfahren sind Sterbetafeln dazu geeignet, eine Beschreibung der Übergangsdauer durch geschätzte Überlebenswahrscheinlichkeiten bis zum Eintritt der Erstausbildung als Ereignis zu ermöglichen. Durch die Einteilung des Beobachtungszeitraums in gleich große Intervalle eignen sich Sterbetafeln aufgrund der überschaubaren Berechnungsschritte für die vorliegende Studie. Cox-Regressionsmodelle ermöglichen den Einbezug von unabhängigen Variablen, deren Einfluss auf die Überlebenswahrscheinlichkeit beziehungsweise Übergangsdauer überprüft wird. Während beim Sterbetafel-Verfahren die Beschreibung der Übergangsdauer getrennt nach Schulabschluss ausgegeben werden kann, kann bei CoxRegressionen der Effekt des gestiegenen Anteils von Dienstleistungstätigen auf die Übergangsdauer generell sowie getrennt nach Schulabschluss untersucht, der Schulabschluss selbst aber auch als Kovariate in das Modell miteinbezogen werden. Außerdem wird ein Moderatoreffekt des Schulabschlusses auf den Effekt des gestiegenen Anteils der Dienstleistungstätigen auf die Übergangsdauer untersucht.

\subsubsection{Sequenzdatenanalyse: Umstrukturierung der Daten und deskriptive Übersicht}

Die Sequenzdatenanalyse fokussiert die gesamte sequenzielle Abfolge von Zwischenstationen, welche während der Dauer bis zum Eintritt eines Ereignisses durchlaufen werden. Für die Anwendung werden die einzelnen Stationsvariablen im Datensatz in eine einzelne Variable umstrukturiert, wobei die chronologische Sortierung dieser Stationen in Abhängigkeit vom Startdatum der jeweiligen Station erforderlich ist. Dies erscheint sinnvoll, da dadurch rekonstruiert werden kann, welche Stationen in der sequenziellen Abfolge nacheinander begonnen wurden. Nach der Umformung der Daten werden diese in die Statistiksoftware Stata geladen. In Stata stehen durch das zusätzliche $s q$-Package verschiedene Funktionen für die einzelnen Verfahren der Sequenzdatenanalyse zur 
Verfügung. Dazu zählen insgesamt fünf Kategorien: die Deskription, die Visualisierung, der Vergleich, die Gruppierung und die Anwendung. Während es bei der Deskription um die einfache Ausgabe von Häufigkeitstabellen der Sequenzen oder spezifischen Verteilungen zu bestimmten Charakteristika der Sequenzen geht, ermöglichen die Funktionen von Stata im Rahmen der Visualisierung die grafische Darstellung der Sequenzen (Brzinsky-Fay et al. 2006: 436). Die Schritte des Vergleichs und des Gruppierens werden in der vorliegenden Studie nicht verwendet. Diese Schritte wurden im Zuge einer Clusteranalyse durchgeführt, wobei sich hierzu keine inhaltlich trennscharfen Cluster und damit keine verwertbaren Ergebnisse ergaben. Deskriptive Funktionen dienen zur Betrachtung, welche Sequenzen beziehungsweise Stationskombinationen am häufigsten auftreten. Dabei werden auch vorhandene Lücken, also Positionen innerhalb der Sequenz, welche nicht definiert sind, miteinbezogen. Bei der Sequenzkonzentration werden Häufigkeitstabellen ausgegeben, welche anzeigen, welche Sequenzen von wie vielen Fällen belegt werden. Weiterhin werden deskriptive Statistiken für die Anzahl der Stationen sowie die Anzahl an unterschiedlichen Stationen in einer Sequenz ausgegeben. Eine zusätzliche deskriptive Übersicht erfolgt darüber, wie viele unterschiedliche Sequenzen mit wie vielen unterschiedlichen Stationen vorliegen (Brzinsky-Fay et al. 2006: 438 ff.). Visualisierungen veranschaulichen die Verteilungen der Stationen in den Sequenzen grafisch. Anhand von Sequenz-Index-Plots wird für jede einzelne Sequenz eine horizontale Linie mit individueller Farbe für jede Station erstellt, um die Elemente innerhalb der Sequenzen optisch zu verdeutlichen. Mit Hilfe weiterer visueller Ausgaben kann der grafische Verlauf der Sequenzen und die entsprechende Stationsfrequentierung in den Sequenzen optisch veranschaulicht werden. Durch diese Visualisierungsmöglichkeit können beispielsweise häufig vorkommende Wechsel zwischen unterschiedlichen Stationen gezeigt werden (Brzinsky-Fay et al. 2006: 444 ff.).

Für die weitere Anwendung ist eine zusätzliche Umstrukturierung der Daten notwendig. Vor dieser liegen die Daten in der Form vor, dass jede einzelne Übergangsstation einen Fall darstellt und die einzelnen Befragungsteilnehmer dadurch mehrfach im Datensatz auftreten, sofern sie mehr als eine Übergangsstation durchlaufen haben. Durch die Umstrukturierung wird der Datensatz so transponiert, dass ein Fall nun eine Person beziehungsweise eine Sequenz abbildet, da jede Person eine Sequenz von Übergangsstationen aufweist. Die absolvierten Stationen sind nach der Umstrukturierung als Variablen aufgeführt. Wichtig ist, dass nur Personen mit einer Sequenz von Übergangsstationen, die also mindestens eine Station durchlaufen haben, in den Daten und Analysen berücksichtigt werden. 


\subsubsection{Sequenzdatenanalyse: Bildung von Stationskategorien}

Nach der Aufbereitung der Stationssequenzen werden Stationskategorien gebildet, zu welchen die einzelnen Personen zugeordnet werden. Personen ohne Station und mit direktem Übergang von der Schule in die Erstausbildung sind ausgeschlossen. Es wird im Folgenden eine kategoriale Variable mit vier Stationskategorien gebildet. Dabei handelt es sich um Stationen ohne Weiterbildungscharakter, berufsvorbereitende Maßnahmen, Kurs/Weiterbildung/Praktikum sowie Schulepisoden. Die Stationen Kurs, Weiterbildung und Praktikum sind dabei zu einer Kategorie zusammengefasst, da sie separat voneinander betrachtet sehr selten auftreten. Da die Personen während des Übergangszeitraums theoretisch Stationen aus mehreren Kategorien durchlaufen können, wie beispielsweise eine Arbeitslosigkeit aus der Kategorie der Stationen ohne Weiterbildungscharakter und dann eine Schulepisode, erscheint eine inhaltliche Gewichtung notwendig. Diese wird in der Form vorgenommen, dass die Kategorie „Stationen ohne Weiterbildungscharakter" als erste Kategorie und damit als Referenzkategorie verwendet wird. Absolviert jemand neben einer Station ohne Weiterbildungscharakter noch zusätzlich eine berufsvorbereitende Maßnahme, einen Kurs/Praktikum beziehungsweise eine Weiterbildung oder eine Schulepisode, fällt er aus der Kategorie „Stationen ohne Weiterbildungscharakter" heraus, da die anderen drei Kategorien inhaltlich in Bezug auf die Hypothesen stärker gewichtet werden. In dieser Kategorie verbleiben diejenigen, die ausschließlich eine oder mehrere Stationen ohne Weiterbildungscharakter durchlaufen haben. Hat jemand neben einer berufsvorbereitenden Maßnahme noch einen Kurs/ein Praktikum/eine Weiterbildung absolviert, wird diejenige der beiden Kategorien stärker gewichtet, welche länger angedauert. Wenn bei gleichzeitigem Auftreten die absolvierten Kurse/Praktika/Weiterbildungen die identische Dauer haben wie die berufsvorbereitenden Maßnahmen, wird die Kategorie „Kurs/Weiterbildung/Praktikum“ stärker gewichtet. Bei dieser Kategorie kann eher von einem Weiterbildungsbezug ausgegangen werden. Bei berufsvorbereitenden Maßnahmen ist dies nicht immer der Fall, was mit der Vielzahl an unterschiedlichen Maßnahmen zusammenhängt, welche nicht alle einen weiterbildenden Charakter haben. Hat jemand neben Stationen aus den ersten drei Kategorien auch eine Schulepisode absolviert, wird diese Kategorie am stärksten gewichtet. Dies ist inhaltlich in erster Linie in Anlehnung an die Hypothesen sowie in Bezug auf die Humankapitaltheorie zu begründen. Der Erwerb weiterer Schulabschlüsse kann als stärkste Ausprägung von akkumuliertem Humankapital betrachtet werden, weshalb zusätzliche Schulepisoden die stärkste inhaltliche Gewichtung erhalten. 


\subsubsection{Sequenzdatenanalyse: Multinomial logistisches Regressionsmodell}

Die gebildeten Kategorien werden in einer kategorialen Variable in einem multinomial logistischen Regressionsmodell verwendet, um den Einfluss des Anteils an Arbeitskräften im Dienstleistungssektor und des Schulabschlusses auf die Frequentierung der Stationskategorien zu untersuchen. Da die abhängige Variable der Stationskategorien nominal beziehungsweise kategorial skaliert ist, ist die Anwendung eines logistischen Regressionsmodells (Best und Wolf 2010: 827, 829 ff., 839 f.) erforderlich. Bei der Regressionsdiagnostik werden die Modelle auf das Vorliegen von Multikollinearität (Kühnel und Krebs 2010: 883), auf die Linearität der Logits (Best und Wolf 2010: 830), auf Ausreißer der standardisierten Residuen (Best und Wolf 2010: 834 ff.) und auf extreme Datenpunkte getestet.

\subsubsection{Sequenzdatenanalyse: Poisson- und negatives binomiales Regressionsmodell}

Bei der Untersuchung der Anzahl an Übergangsstationen kommt das Verfahren der PoissonRegression zur Anwendung, da es sich um eine Zählvariable handelt (Diaz-Bone und Weischer 2015: 344). Die Koeffizienten der Poisson-Regression werden als „Incidence Rate Ratios“ (IRR) ausgegeben. Das bedeutet, dass bei Ansteigen einer unabhängigen metrischen Variablen um eine Einheit oder dem Vorliegen einer bestimmten Kategorie bei der abhängigen Zählvariable eine höhere oder niedrigere Rate vorliegt im Vergleich zur Referenzkategorie (idre Institute for Digital Research and Education 2020). Ist der Koeffizient für den IRR kleiner Eins, liegt eine niedrigere Rate im Vergleich zur Referenzkategorie vor. Bei einem IRR-Koeffizient von größer Eins ist von einer höheren Rate zu sprechen und bei einem Koeffizienten von gleich Eins von einer gleichen Rate wie bei der Referenzkategorie (Voos 2014). Eine Eigenschaft dieser Methode ist, dass Erwartungswert und Varianz als gleich angenommen werden (Bundesversicherungsamt 2015: 21).

Bei einer im Vergleich zum Mittelwert größeren Varianz der abhängigen Zählvariable, auch als „Overdispersion“ bezeichnet, wird die negative binomiale Regression verwendet. Diese stellt eine Korrektur der Poisson-Regression durch Berücksichtigung der Overdispersion dar. Bei Vorliegen einer Overdispersion sind die Ergebnisse des negativen binomialen Modells als präziser zu betrachten. Darüber hinaus muss im Poisson-Modell und im negativen binomialen Modell eine Variable berücksichtigt werden, welche den Zeitraum abbildet, in dem die gezählten Ereignisse stattgefunden haben können. Diese Variable wird als „ExposureVariable“ bezeichnet (idre Institute for Digital Research and Education 2020) und ist im vorliegenden Fall die Dauer des Übergangszeitraums, in dem die Stationen durchlaufen 
werden. Hierbei geht es darum, dass die gezählten Ereignisse, die Stationen, bei den einzelnen Personen in unterschiedlichen Zeiträumen durchlaufen werden. Beispielsweise hat jemand, der in zwölf Monaten zehn Übergangsstationen durchläuft, ein deutlich häufigeres Auftreten von Stationen als jemand, der in 120 Monaten zehn Stationen absolviert. Die ExposureVariable standardisiert diese individuell unterschiedlichen Zeiträume, wodurch einheitliche Raten für das Auftreten von Übergangsstationen berechnet werden können. Da die ExposureVariable unter anderem durch Logarithmieren gebildet wird (Grace-Martin 2019a) und die dafür verwendete Übergangsdauervariable aufgrund der Möglichkeit eines direkten Übergangs auch Nullen enthält, ist ein Fallausschluss notwendig. Der Logarithmus des Wertes Null ist mathematisch nicht definiert (Universität Innsbruck 2017: 19). Daher müssen bei Bildung der Exposure-Variablen und den Modellen alle Fälle mit einer Übergangsdauer von null Monaten sowie einer Anzahl von null Stationen ausgeschlossen werden. Die Poisson- und die negative binomiale Regression modellieren eine lineare Beziehung zwischen der logarithmierten Stationsanzahl und den unabhängigen Variablen. Im Sinne einer anschaulicheren Interpretation sind die Koeffizienten als IRR aufgeführt. Bei Vorliegen sehr vieler Fälle mit Nullen wäre eine Anpassung des Modells erforderlich, was aufgrund des Ausschlusses dieser Fälle nicht notwendig ist (Grace-Martin 2019b). Des Weiteren werden Stationsanzahlen aus den Analysen ausgeschlossen, welche nur von wenigen Fällen frequentiert werden. Dies führt zu Problemen bei der Maximum-Likelihood-Schätzung, da kein Maximum und damit auch keine optimale Schätzung des Modells gewährleistet werden kann.

\subsection{Umgang mit fehlenden Werten}

Bei den durchgeführten Analysen werden anhand eines listenweisen Fallausschlusses diejenigen Fälle ausgeschlossen, welche bei mindestens einer der einbezogenen Variablen einen fehlenden Wert aufweisen. Dies gilt für bivariate Analysen wie für die angewendeten Regressionsmodelle. Die abhängigen Variablen Übergangsdauer und Übergangsstationen werden für alle 9.254 einbezogenen Fälle gebildet. Bei den unabhängigen Variablen liegen lediglich für den Schulabschluss und die soziale Herkunft einige Fälle mit fehlenden Werten vor, weshalb sich die Stichprobe bei Modellen mit diesen Variablen etwas verringert. Bei den Regressionsmodellen zu den Kategorien und der Anzahl der absolvierten Übergangsstationen werden nur diejenigen Personen einbezogen, die mindestens eine oder mehr 
Übergangsstationen absolvieren. Eine detaillierte Übersicht über die verwendeten Variablen und die jeweilige Fallzahl mit gültigen Werten ist im Anhang in Tabelle 6 aufgelistet.

\subsection{Vorgehen bei Analysen}

Nach einem deskriptiven Überblick über die abhängigen und unabhängigen Variablen sowie der Präsentation verschiedener Sterbetafelmodelle als Überblick einer allgemeinen Tendenz werden verschiedene Regressionsanalysen durchgeführt. Diese Modelle enthalten grundsätzlich die unabhängigen Variablen Schulabschluss, Schulenddatumsjahr, soziale Herkunft und Geschlecht. In einigen Modellen werden zusätzlich aufgrund der formulierten Hypothesen diverse unabhängige Makrovariablen aufgenommen. Sämtliche in den Regressionsmodellen verwendeten Variablen sind in Tabelle 5 aufgeführt:

Tabelle 5: Einbezogene unabhängige Variablen der Regressionsmodelle.

Unabhängige Variablen

Schulabschluss

Mikrovariablen in allen

Schulenddatumsjahr (fortlaufend)

Regressionsmodellen

Soziale Herkunft

Geschlecht

Anteil Dienstleistungstätige

Zusätzliche Makrovariablen Demografischer Anteil der unter 20-Jährigen

Arbeitslosenquote

Quelle: Eigene Darstellung.

Zur Überprüfung der Hypothese 1a) „,Generell verlängert sich die Dauer vom Verlassen der Schule bis zum Beginn der Erstausbildung zwischen 1971 und 2012" wird ein CoxRegressionsmodell mit den oben aufgeführten unabhängigen Variablen und der abhängigen Variablen Übergangsdauer, jedoch ohne die zusätzlichen unabhängigen Makrovariablen berechnet. Dabei wird eine mögliche Verlängerung der Dauer im Laufe der Zeit (nach Schulenddatumsjahr) untersucht. Bei der Hypothese 1b) „Die Übergangsdauer von Schulabgängerinnen und Schulabgängern mit einem Hauptschulabschluss oder ohne einen Schulabschluss ist länger als bei denjenigen mit Abitur oder einem Realschulabschluss " wird das gleiche Modell wie für Hypothese 1a) verwendet und dabei der Effekt des 
Schulabschlusses auf die Übergangsdauer untersucht. Für die Untersuchung der Hypothese 2a) „Vor dem Hintergrund eines gestiegenen Dienstleistungstätigenanteils ist eine längere Dauer vom Verlassen der Schule bis zum Beginn der Erstausbildung zu beobachten “werden zusätzlich noch die unabhängigen Makrovariablen und damit sämtliche unabhängige Variablen aus der Tabelle 5 in das Modell aufgenommen. Dies dient der Überprüfung, ob der gestiegene Dienstleistungstätigenanteil die Übergangsdauer verlängert. Bei der Hypothese 2b) „Vor dem Hintergrund eines gestiegenen Dienstleistungstätigenanteils ist eine längere Übergangsdauer derjenigen mit einem Hauptschulabschluss oder ohne Schulabschluss als bei denjenigen mit Abitur oder einem Realschulabschluss zu beobachten “ wird zusätzlich zum Modell für die Hypothese 2a) noch eine Interaktion zwischen dem Anteil der Dienstleistungstätigen und dem Schulabschluss aufgenommen. Dies dient der Untersuchung, ob ein gestiegener Dienstleistungstätigenanteil die Effekte der Schulabschlüsse auf die Übergangsdauer verstärkt. Diese Interaktion wird grafisch veranschaulicht. Bei diesen ersten vier Hypothesen dient die Übergangsdauer als abhängige Variable.

Bei den Sequenzdatenanalysen werden zunächst deskriptive Statistiken und Grafiken ausgegeben, um einen ersten Eindruck über häufig frequentierte Stationssequenzen zu erhalten. Bezüglich der folgenden Hypothesen werden die Kategorien der Übergangsstationen als abhängige Variablen im Rahmen von multinomialen logistischen Regressionsmodellen verwendet. Für die Hypothesen 3a) „Jugendliche mit Abitur absolvieren häufiger Weiterbildungsstationen wie Kurse oder Praktika als Jugendliche mit Haupt- oder ohne Schulabschluss“ und 3b) „Jugendliche mit Realschulabschluss absolvieren häufiger Weiterbildungsstationen wie Kurse oder Praktika als Jugendliche mit Haupt- oder ohne Schulabschluss “ wird ein gemeinsames Modell verwendet. Dieses Modell findet auch für die Hypothese 3c) „Jugendliche mit Haupt- oder ohne Schulabschluss holen im Vergleich zu denjenigen mit Realschulabschluss häufiger Bildungsabschlüsse nach" Anwendung und beinhaltet die in Tabelle 5 aufgeführten unabhängigen Variablen ohne die zusätzlichen unabhängigen Makrovariablen. Dabei soll untersucht werden, ob eine Schulabschlussgruppe sich in einer bestimmten Stationskategorie tendenziell eher befindet als andere Schulabschlussgruppen.

Für die Hypothesen 3d) „Jugendliche mit Realschulabschluss absolvieren vor dem Hintergrund eines gestiegenen Dienstleistungstätigenanteils häufiger Weiterbildungsstationen wie Kurse oder Praktika als Jugendliche mit Haupt- oder ohne Schulabschluss“ und 3e) „Jugendliche mit Haupt- oder ohne Schulabschluss holen vor dem 
Hintergrund eines gestiegenen Dienstleistungstätigenanteils im Vergleich zu denjenigen mit Realschulabschluss häufiger Bildungsabschlüsse nach" werden zusätzlich die unabhängigen Makrovariablen in das Modell einbezogen. Damit werden alle in Tabelle 5 aufgeführten Variablen verwendet. Des Weiteren wird eine Interaktion zwischen dem Anteil der Dienstleistungstätigen und dem Schulabschluss in das Modell aufgenommen. Dadurch kann ein möglicher Einfluss des Anteils an Dienstleistungstätigen auf die Effekte der Schulabschlüsse auf die Stationskategorien untersucht werden. Die Interaktionen der Hypothesen 3d) und 3e) werden grafisch in Form von geschätzten Wahrscheinlichkeiten veranschaulicht.

Bei den Hypothesen 4a) bis 4d) dient die Anzahl an Übergangsstationen als abhängige Variable. Für diese Hypothesen werden negative binomiale Regressionsmodelle verwendet. Für die Hypothese 4a) „Generell erhöht sich die Anzahl der Stationen vom Verlassen der Schule bis zum Beginn der Erstausbildung zwischen 1971 und 2012 “ wird ein Modell mit den in Tabelle 5 aufgeführten Variablen, jedoch ohne die zusätzlichen unabhängigen Makrovariablen berechnet. Dabei wird ein möglicher Anstieg der Stationsanzahl im Zeitverlauf (nach Schulenddatumsjahr) untersucht. Das gleiche Modell dient auch zur Überprüfung von Hypothese 4b) „Die Anzahl der Stationen ist bei denjenigen ohne Schulabschluss oder mit Hauptschulabschluss höher als bei denjenigen mit Abitur oder Realschulabschluss“. Dabei wird ein möglicher Effekt des Schulabschlusses auf die Stationsanzahl untersucht. Zur Überprüfung der Hypothese 4c) „, Vor dem Hintergrund eines gestiegenen Dienstleistungstätigenanteils ist eine höhere Anzahl der Stationen vom Verlassen der Schule bis zum Beginn der Erstausbildung zu beobachten “ werden zusätzlich die unabhängigen Makrovariablen in das Modell einbezogen und dadurch alle in Tabelle 5 aufgeführten Variablen verwendet. Dies dient der Untersuchung eines möglichen Effekts des Dienstleistungstätigenanteils auf die Stationsanzahl. Abschließend wird bei Hypothese 4d) „,Vor dem Hintergrund eines gestiegenen Dienstleistungstätigenanteils ist eine höhere Anzahl der Stationen bei denjenigen ohne Schulabschluss oder mit Hauptschulabschluss als bei denjenigen mit Abitur oder Realschulabschluss zu beobachten" zusätzlich zum Modell für Hypothese 4c) noch eine Interaktion zwischen dem Anteil der Dienstleistungstätigen und dem Schulabschluss einbezogen. Dies ermöglicht die Überprüfung, ob ein gestiegener Anteil an Dienstleistungstätigen einen Einfluss auf die Effekte der Schulabschlüsse auf die Stationsanzahl hat. 


\section{Ergebnisse}

\subsection{Deskriptive Analysen}

\subsubsection{Univariate und bivariate Ergebnisse}

Zunächst wird auf die deskriptiven Resultate für einen ersten Überblick eingegangen (vgl. Abschnitt 6.1). Darauf folgend werden die Ergebnisse der Ereignisdatenanalysen in Form von Sterbetafeln (vgl. Abschnitt 6.2) und Cox-Regressionsmodellen (vgl. Abschnitt 6.3) zur Überprüfung der Hypothesen zur Übergangsdauer dargestellt. Abschließend werden die Ergebnisse der Sequenzdatenanalysen vorgestellt (vgl. Abschnitt 6.4). Diese basieren auf multinomial logistischen Regressionsanalysen und Poisson-Regressionsanalysen, deren Resultate eine Einschätzung zu den Hypothesen der absolvierten Übergangsstationen in Form der Anzahl und der absolvierten Stationskategorien liefern.

Insgesamt war der Realschulabschluss mit durchschnittlich 33,4 Prozent der am häufigsten erreichte Schulabschluss. Der Hauptschulabschluss mit 27,5 Prozent und das Abitur mit 27,8 Prozent wurden gleich häufig frequentiert. 11,4 Prozent der Befragten gaben als ersten erreichten Schulabschluss „Kein Abschluss“ an (siehe Abbildung 1).

Abbildung 1: Prozentuale Verteilung des ersten erreichten Schulabschlusses.

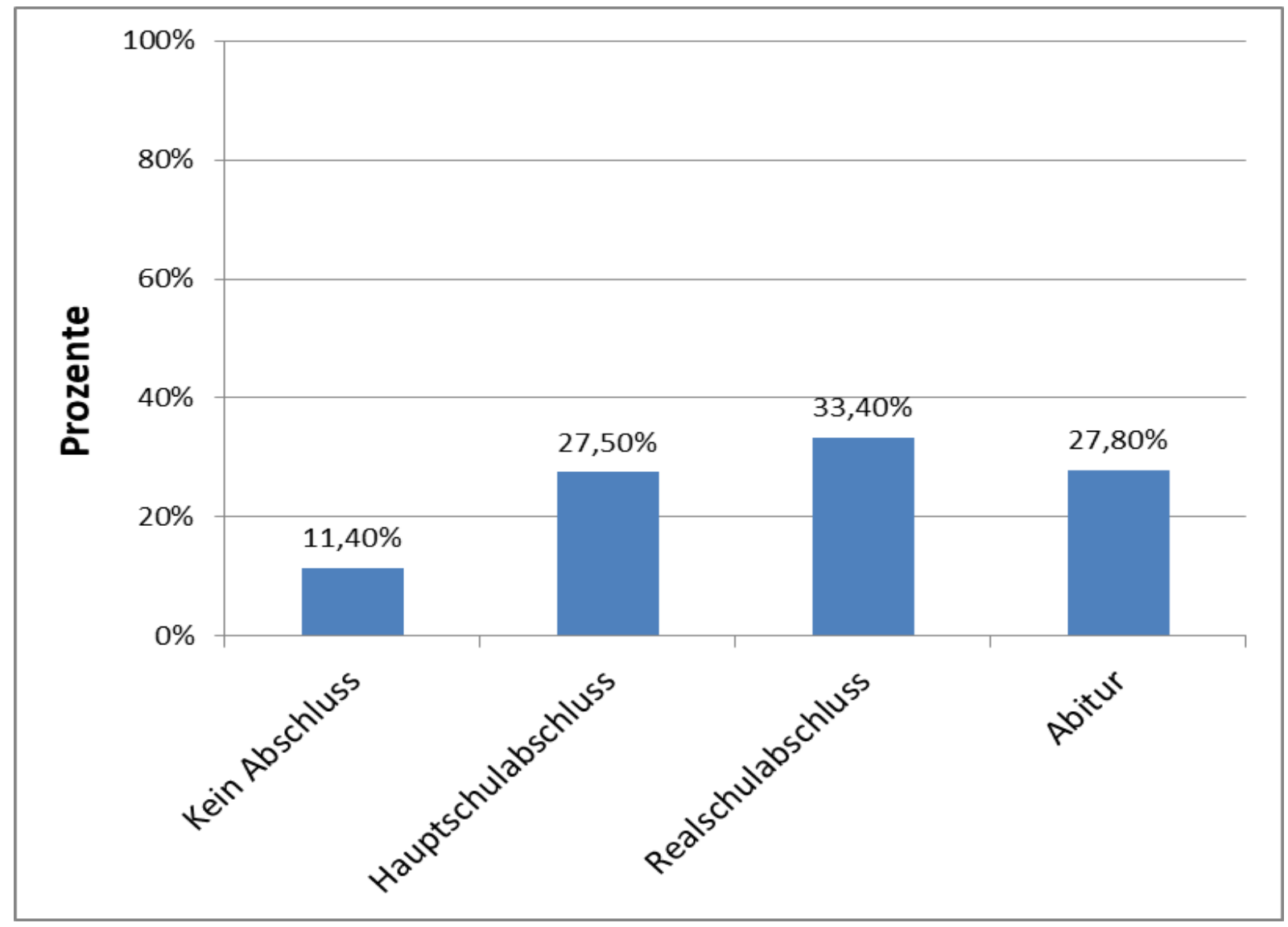

Quelle: Eigene Berechnungen basierend auf dem NEPS. N = 9.189. 
Die ungewöhnlich hohe Quote derjenigen ohne Abschluss ist damit zu erklären, dass es sich hierbei um den ersten angegebenen Schulabschluss handelte und ein nicht geringer Anteil dieser Personengruppe in einer darauffolgenden Schulepisode einen höheren Schulabschluss erwarb, um die Chancen beim Zugang in den Ausbildungsmarkt zu verbessern. Insofern ist bei diesen Resultaten von einer Ausgangssituation zu sprechen, welche auch die Möglichkeit einer Zusatzqualifikation in Form von weiteren Schulepisoden und dem Erwerb von höheren Schulabschlüssen beinhaltet. Insgesamt 9.189 der in die Analysen einbezogenen 9.254 Personen hatte einen ersten allgemeinbildenden Schulabschluss angegeben. Die fehlenden 65 Personen hatten auf Basis der zur Verfügung stehenden Daten keine Angaben zu Schulabschlüssen gemacht, weshalb ihnen kein Schulabschluss zugewiesen werden konnte.

Bei Betrachtung des ersten Schulabschlusses im Zeitverlauf wurden einige interessante Unterschiede deutlich (siehe Abbildung 2).

Abbildung 2: Prozentuale Verteilung des ersten erreichten Schulabschlusses im Zeitverlauf.

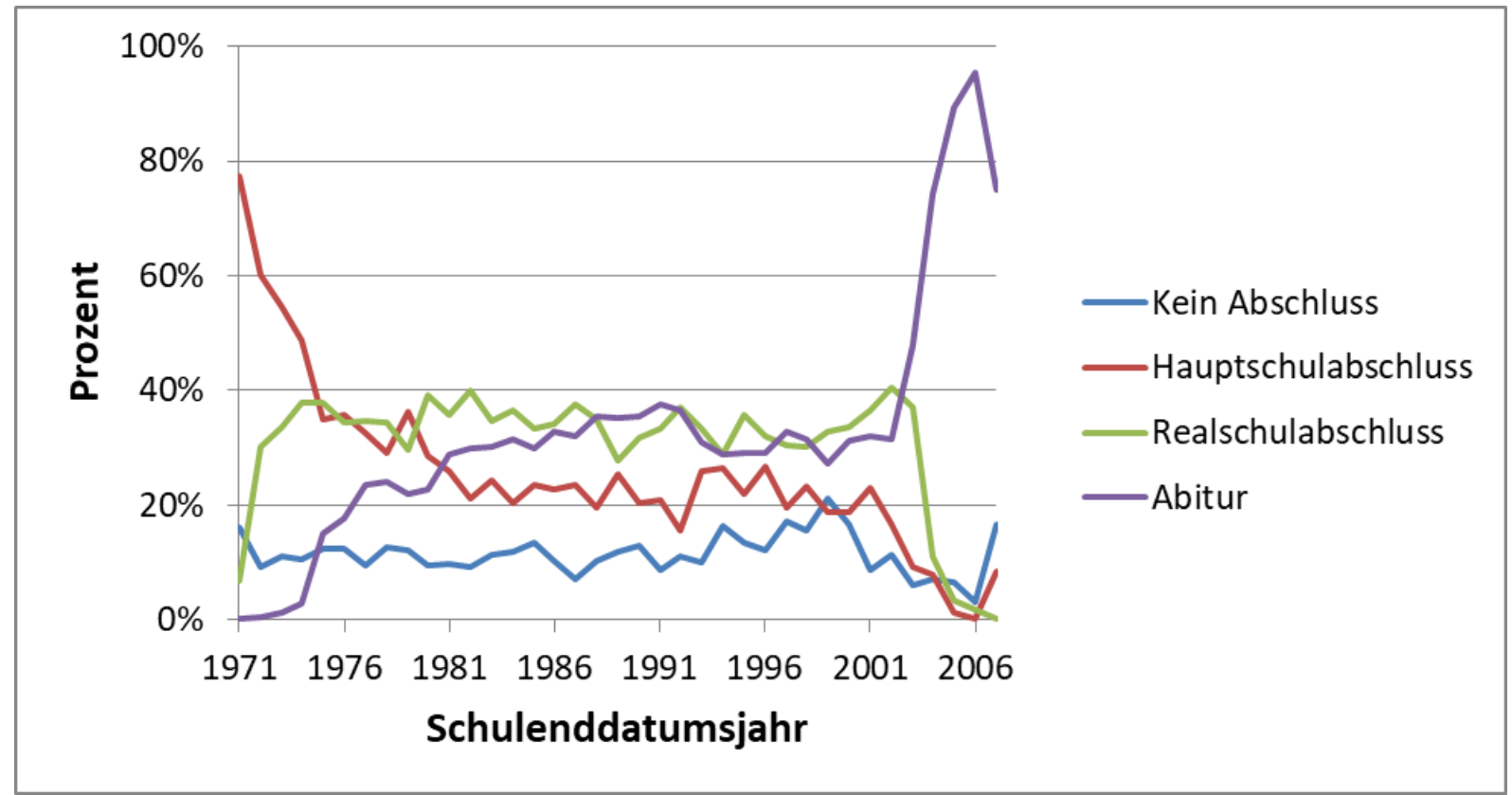

Quelle: Eigene Berechnungen basierend auf dem NEPS. N = 9.189.

Die Zeitpunkte stellen die einzelnen Schulenddatumsjahre dar. War der Hauptschulabschluss zu Beginn der 1970er Jahre noch der am häufigsten frequentierte Abschluss, konnte im neuen Jahrtausend das Abitur als der am häufigsten genannte erste Schulabschluss hervorgehoben werden. Zu Beginn der 1970er Jahre gaben dagegen nur sehr wenige der Befragten das Abitur als ersten Abschluss an. Lag der Anteil derer mit Abitur zu Beginn der 1970er Jahre noch unter fünf Prozent, stieg dieser Anteil ab 1976 auf über 20 Prozent an und erhöhte sich bis 
Anfang der 1990er Jahre auf knapp 40 Prozent. Ab dem Jahr 2003 wurde das Abitur der mit Abstand am häufigsten frequentierte Schulabschluss mit einem prozentualen Anteil von über 50 Prozent. Der Hauptschulabschluss hatte Mitte der 1970er Jahre deutlich an Bedeutung verloren, blieb jedoch in den darauf folgenden Jahren auf einem relativ konstanten Niveau. Zwischen etwa 15 und 35 Prozent der Befragten gaben zwischen den Schulenddatumsjahren 1975 und 2001 den Hauptschulabschluss als ersten Schulabschluss an. Ab dem Jahr 2002 wurde der Hauptschulabschluss als erster Schulabschluss deutlich seltener frequentiert als in den beiden Jahrzehnten zuvor. Die mittlere Reife gewann als erster Schulabschluss ab Beginn der 1970er deutlich an Bedeutung und wurde in den folgenden Jahrzehnten auf einem relativ konstanten Niveau frequentiert. Dies konnte daran verdeutlicht werden, dass zwischen den Schulenddatumsjahren 1972 und 2003 kontinuierlich zwischen 30 und 40 Prozent der befragten Personen den Realschulabschluss als ersten Schulabschluss angaben. Der Anteil derjenigen, die als ersten Schulabschluss „keinen Abschluss“ aufwiesen, lag nahezu über den gesamten Beobachtungszeitraum hinweg unter 20 Prozent.

$\mathrm{Zu}$ den teilweise sehr deutlichen Entwicklungen der prozentualen Anteile bei den einzelnen Schulabschlüssen zu Beginn und zu Ende des Beobachtungszeitraums muss angemerkt werden, dass die Fallzahlen der befragten Personen gerade in den ersten (1971, 1972, 1973) und in den letzten (2003, 2004, 2005, 2006, 2007) beobachteten Schulenddatumsjahren überwiegend bei $\mathrm{N}<10$ Personen lagen. In den Jahren dazwischen waren meist Fallzahlen von $\mathrm{N}>100$ oder teilweise deutlich höher $\mathrm{zu}$ konstatieren. Diese erheblichen Fallzahlunterschiede sind zusätzlich noch nach Schulabschlüssen zu differenzieren. Insofern sind insbesondere die sehr deutlichen Entwicklungen $\mathrm{zu}$ Beginn und $\mathrm{zu}$ Ende des Beobachtungszeitraums mit Vorsicht $\mathrm{zu}$ interpretieren. So war beispielsweise der Anteil derjenigen mit Hauptschulabschluss mit fast 80 Prozent (1971) und mit etwa 60 Prozent (1972) nur sehr eingeschränkt aussagekräftig, da in den ersten beiden Jahren abschlussübergreifend insgesamt nur sehr wenige Fälle vorlagen. Gleiches gilt für die sehr geringen Anteile derer mit mittlerer Reife im Jahr 1971 und derer mit Abitur in den Jahren 1971 bis 1974 sowie die sehr hohe Abiturientenquote und die sehr geringen Anteile derer mit Haupt- oder Realschulabschluss zwischen 2004 und 2007.

Bei der Verteilung des Erstausbildungstyps fiel auf, dass die duale Berufsausbildung als Kombination aus Betrieb und Berufsschule mit 59 Prozent die mit Abstand am häufigsten frequentierte Ausbildung war, die von den Befragten als erste Ausbildungsform vermerkt wurde. Während der Anteil derjenigen mit einem Studium als Erstausbildung bei 24 Prozent 
lag, waren die Anteile derer mit einer vollzeitschulischen Ausbildung oder ohne Ausbildung mit 8,6 Prozent beziehungsweise 8,4 Prozent deutlich geringer (siehe Abbildung 3).

Abbildung 3: Prozentuale Verteilung des Erstausbildungstyps.

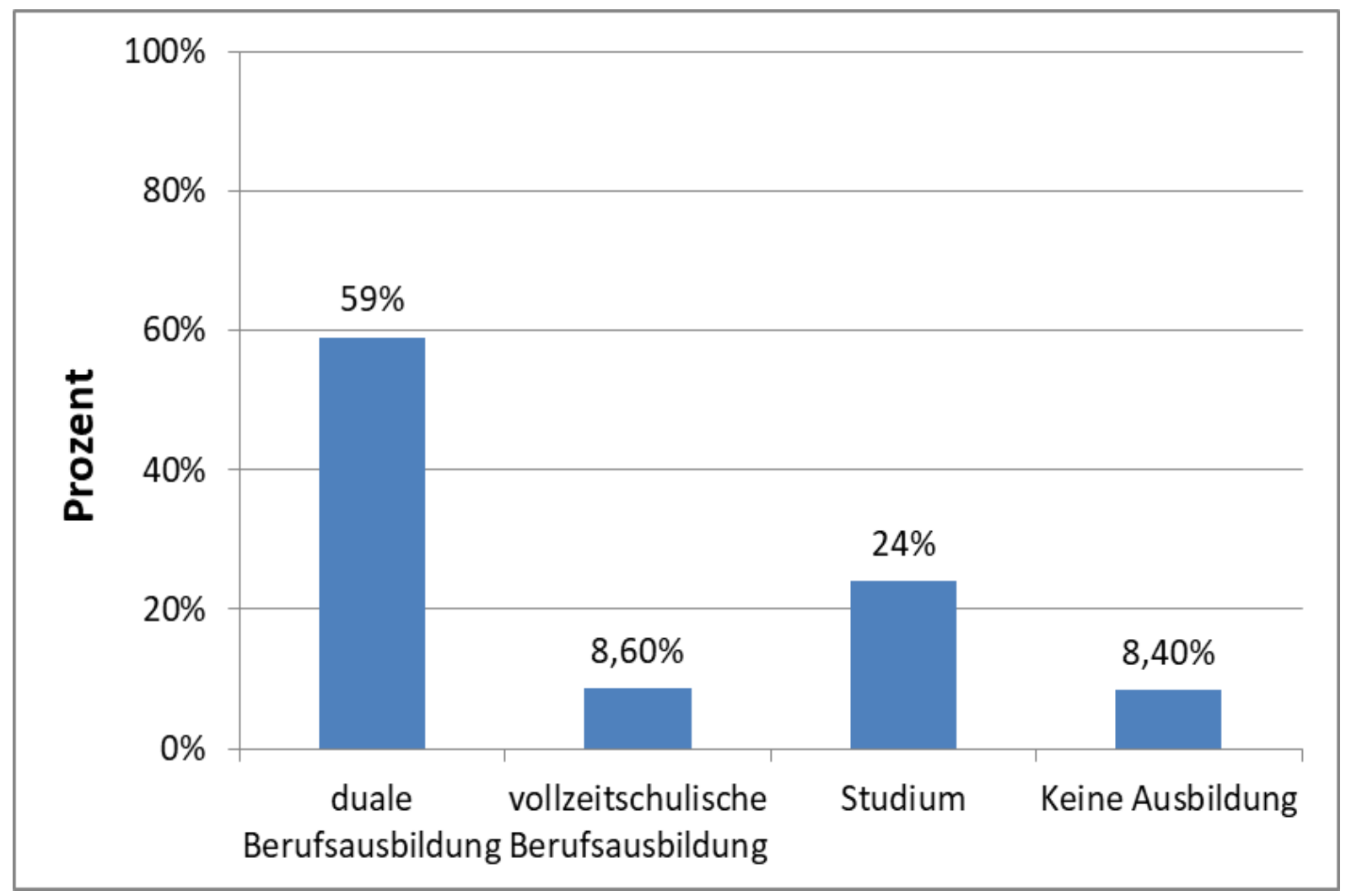

Quelle: Eigene Berechnungen basierend auf dem NEPS. N = 9.254.

Die überwiegend starke Frequentierung der dualen Berufsausbildung als erster Ausbildung ließ sich insgesamt auch im zeitlichen Verlauf verdeutlichen (siehe Abbildung 4). 


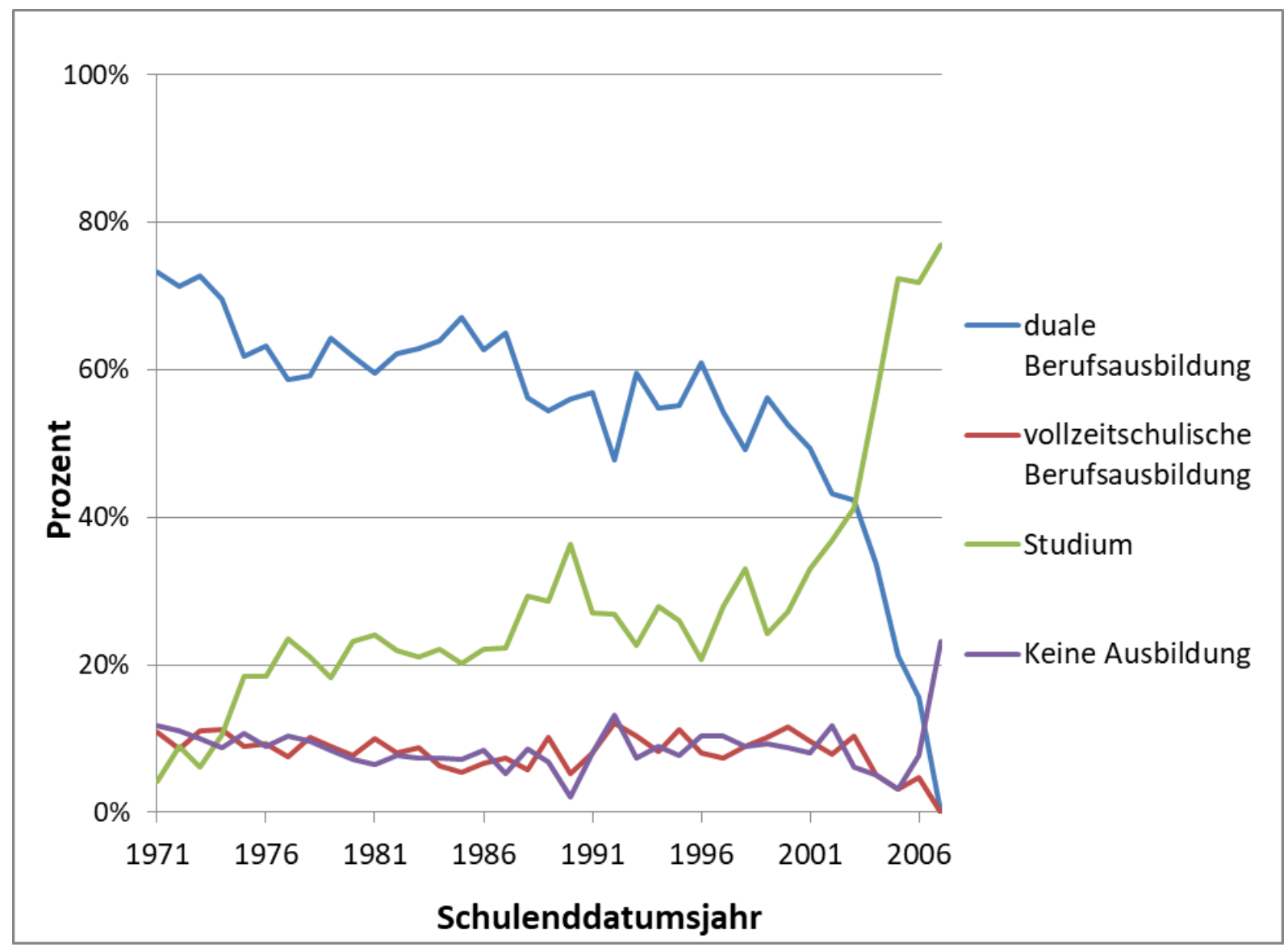

Quelle: Eigene Berechnungen basierend auf dem NEPS. N = 9.254.

Dennoch hatte diese Ausbildungsform im Zeitverlauf an Bedeutung eingebüßt. Dies konnte damit belegt werden, dass zu Beginn der 1970er Jahre noch bei über 70 Prozent der befragten Personen dieser Ausbildungstyp die erste Ausbildung war, wogegen dies im Jahr 2002 nur noch für knapp über 40 Prozent der Befragten zutraf. Von da an sank die Frequentierung der dualen Berufsausbildung als Erstausbildungstyp rapide bis zum letzten einbezogenen Schulenddatumsjahr 2007. Gleichzeitig erhöhte sich die Bedeutung des Studiums hinsichtlich der Nennung als Erstausbildung bei den Befragten im Zeitverlauf erheblich. War zu Beginn der 1970er Jahre bei etwa fünf Prozent der Befragten das Studium die erste absolvierte Ausbildung, erhöhte sich dieser Anteil auf über 40 Prozent im Jahr 2003. Im Anschluss daran stieg dieser Anteil weiter rapide auf über 75 Prozent an.

Diese beiden extremen Entwicklungen in Form des stark gestiegenen Anteils derer mit Studium und des stark gesunkenen Anteils derer mit dualer Berufsausbildung ab 2004 sind ebenfalls auf die sehr geringen Fallzahlen in den letzten Schulenddatumsjahren von 2004 bis 2007 zurückzuführen. Von daher sind die sehr starken Entwicklungen bei diesen beiden 
Gruppen ab dem Jahr 2004 nur mit Vorsicht zu interpretieren und auch nur sehr eingeschränkt aussagekräftig.

Die Anteile derer mit einer vollzeitschulischen Ausbildung oder ohne Ausbildung lagen über nahezu dem gesamten Beobachtungszeitraum deutlich unter 20 Prozent. Erst ab dem Schulenddatumsjahr 2005 stieg der Anteil derer, die bis zum Ende des Beobachtungszeitraums keine Ausbildung begonnen hatten, rapide an, wogegen der Anteil derjenigen mit einer vollzeitschulischen Ausbildung deutlich herab sank. Auch diese beiden Entwicklungen sind auf die bereits thematisierte Fallzahlproblematik zurückzuführen, weshalb hierbei die gleichen Beschränkungen hinsichtlich der Interpretation gelten.

Bei der deskriptiven Betrachtung der zentralen Outcomes wurde hinsichtlich der Übergangsdauer deutlich, dass diese über den gesamten Beobachtungszeitraum und über alle Gruppen hinweg durchschnittlich 20,8 Monate betrug. Dieser Mittelwert bezieht sich auf die Übergangsdauer, aus der Wehrdienst- und Elternzeitepisoden sowie FSJ-, Urlaubs- und Krankheitsepisoden rausgerechnet wurden. Die zweite Variante der Übergangsdauer, welche FSJ-, Urlaubs- und Krankheitsepisoden berücksichtigte und Wehrdienst- und Elternzeitepisoden ebenfalls ausschloss, diente einem Vergleich, um einen möglichen Unterschied offen zu legen. Bei der Übergangsdauer mit FSJ-, Urlaubs- und Krankheitsepisoden ergab sich über den gesamten Beobachtungszeitraum hinweg ein Mittelwert von 21,7 Monaten (siehe Anhang - Tabelle 7). Der Mittelwertunterschied um knapp einen Monat ist auf die Berücksichtigung der benannten Episoden in der zweiten Übergangsdauervariable zurückzuführen. Im Rahmen der deskriptiven Ergebnisse werden die Resultate beider Varianten zur Übergangsdauer dargestellt. Insgesamt gab es dabei keine markanten Unterschiede hinsichtlich der Verläufe und Muster. Die Wertigkeit eines FSJs oder eines Urlaubs im Hinblick auf die Vorbereitung für den Ausbildungs- und Arbeitsmarkt bleiben spekulativ. Auch aufgrund dessen werden im Rahmen der Ereignisdatenanalysen die Ergebnisse der Übergangsdauer ohne Wehrdienst- und Elternzeitepisoden sowie ohne FSJ-, Urlaubs- und Krankheitsepisoden berichtet. Die Resultate der Übergangsdauervariante mit FSJ-, Urlaubs- und Krankheitsphasen werden mit Verweis auf den Anhang kurz erwähnt.

Bei Betrachtung der Übergangsdauer ohne Wehrdienst-, Elternzeit-, FSJ-, Urlaubs- und Krankheitsepisoden getrennt nach Schulabschluss wurde deutlich, dass über den gesamten Beobachtungszeitraum hinweg diejenigen Personen ohne Schulabschluss die mit Abstand längste Übergangsdauer aufwiesen (siehe Abbildung 5). 
Abbildung 5: Durchschnittliche Übergangsdauer nach Schulabschluss.

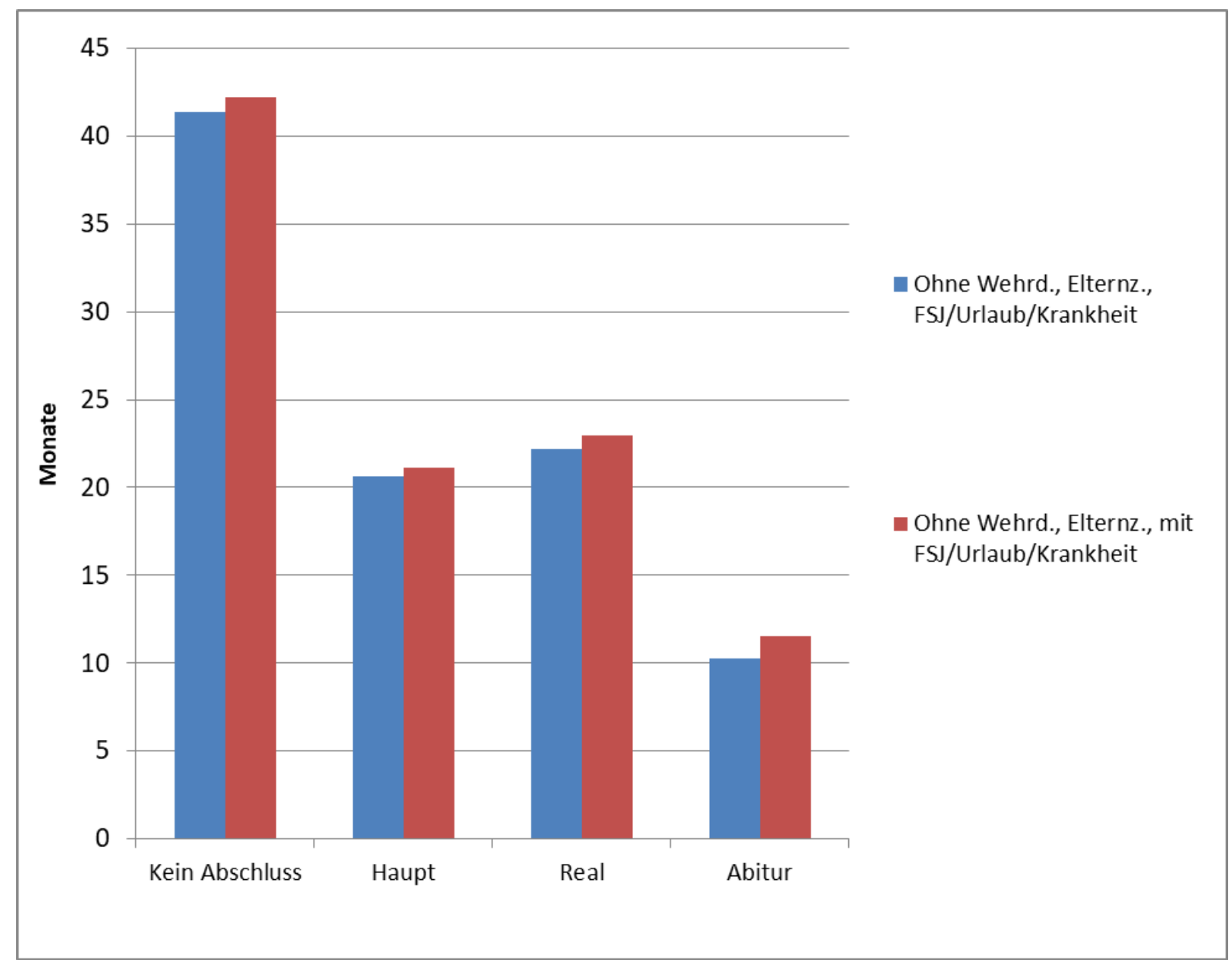

Quelle: Eigene Berechnungen basierend auf dem NEPS. Abkürzungen: Wehrd.: Wehrdienst, Elternz.: Elternzeit. $\mathrm{N}=9.189$.

Bei denjenigen ohne Abschluss ergab sich eine durchschnittliche Dauer von 41,4 Monaten. Personen mit einer mittleren Reife hatten mit 22,2 Monaten eine etwas längere Übergangsdauer als diejenigen mit einem Hauptschulabschluss mit 20,6 Monaten. Personen mit Abitur als ersten erreichten Schulabschluss hatten mit 10,2 Monaten die mit Abstand kürzeste Übergangsdauer. Bezüglich der Übergangsdauervariante ohne Wehrdienst- und Elternzeitepisoden und mit FSJ-, Urlaubs- und Krankheitsphasen ergaben sich die gleichen Relationen mit lediglich geringfügig erhöhten Mittelwerten, die sich durch die zusätzlich berücksichtigten Episoden erschlossen. Beim Vergleich der durchschnittlichen Übergangsdauer zeigten sich zwischen den Schulabschlussgruppen überwiegend signifikante Unterschiede. Die gleichen Resultate ergaben sich auch bei der Übergangsdauervariable mit FSJ-, Urlaubs- und Krankheitsphasen (siehe Anhang - Tabelle 9).

Auch bei Betrachtung der Übergangsdauer nach Erstausbildungstyp zeigten sich deutliche Unterschiede. Diejenigen, welche bis zum Ende des Beobachtungszeitraums keine 
Ausbildung aufgenommen hatten, wiesen die mit Abstand längste Übergangsdauer auf. Diese betrug über den gesamten Beobachtungszeitraum hinweg durchschnittlich 92,1 Monate. Bei den drei anderen Erstausbildungstypen war die durchschnittliche Übergangsdauer im Vergleich dazu weitaus geringer, was sich bei einer dualen (12,2 Monate), vollzeitschulischen (19,6 Monate) und akademischen Erstausbildung (17,5 Monate) verdeutlichen ließ. Die gleichen Relationen fanden sich mit sehr geringfügig erhöhten Mittelwerten bei der Übergangsdauervariable mit FSJ-, Urlaubs- und Krankheitsphasen (siehe Abbildung 6).

\section{Abbildung 6: Durchschnittliche Übergangsdauer nach Erstausbildungstyp.}

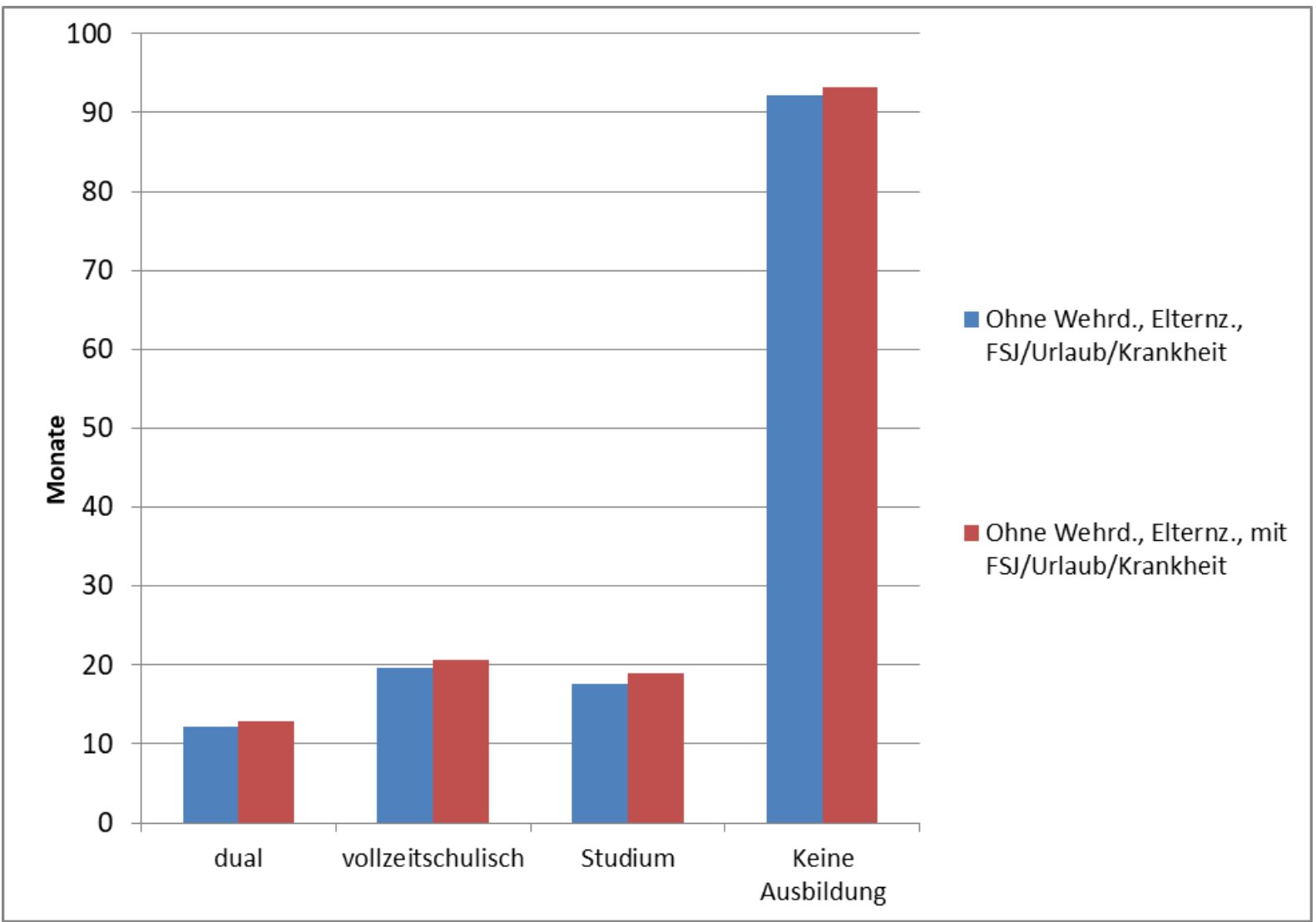

Quelle: Eigene Berechnungen basierend auf dem NEPS. Abkürzungen: Wehrd.: Wehrdienst, Elternz.: Elternzeit. Die Übergangsdauer derjenigen ohne Ausbildung entsprach dem Beobachtungszeitraum vom Verlassen der Schule an. $\mathrm{N}=9.254$.

Die einzelnen Erstausbildungstypen unterschieden sich bezüglich ihrer durchschnittlichen Übergangsdauer fast vollständig signifikant voneinander. Diese Ergebnisse deckten sich mit den Ergebnissen der Übergangsdauervariablen mit FSJ-, Urlaubs- und Krankheitsphasen (siehe Anhang - Tabelle 10).

Bei Betrachtung der Übergangsdauer im Zeitverlauf wurde ersichtlich, dass die durchschnittliche Dauer insgesamt über alle Schulabschlüsse und Erstausbildungstypen hinweg von über 30 Monaten zu Beginn der 1970er Jahre auf einen Bereich zwischen 15 und 
20 Monaten zum Ende der 1980er Jahre herab sank. Im Anschluss erfolgte ein leichter Anstieg der durchschnittlichen Übergangsdauer auf über 25 Monate bis zur Jahrtausendwende. Im Folgenden sank die Dauer bis 2005 auf durchschnittlich unter zehn Monate herab. Für die Dauervariante mit FSJ-, Urlaubs- und Krankheitsphasen ergab sich eine nahezu identische Entwicklung im Zeitverlauf (siehe Abbildung 7).

\section{Abbildung 7: Durchschnittliche Übergangsdauer im Zeitverlauf.}

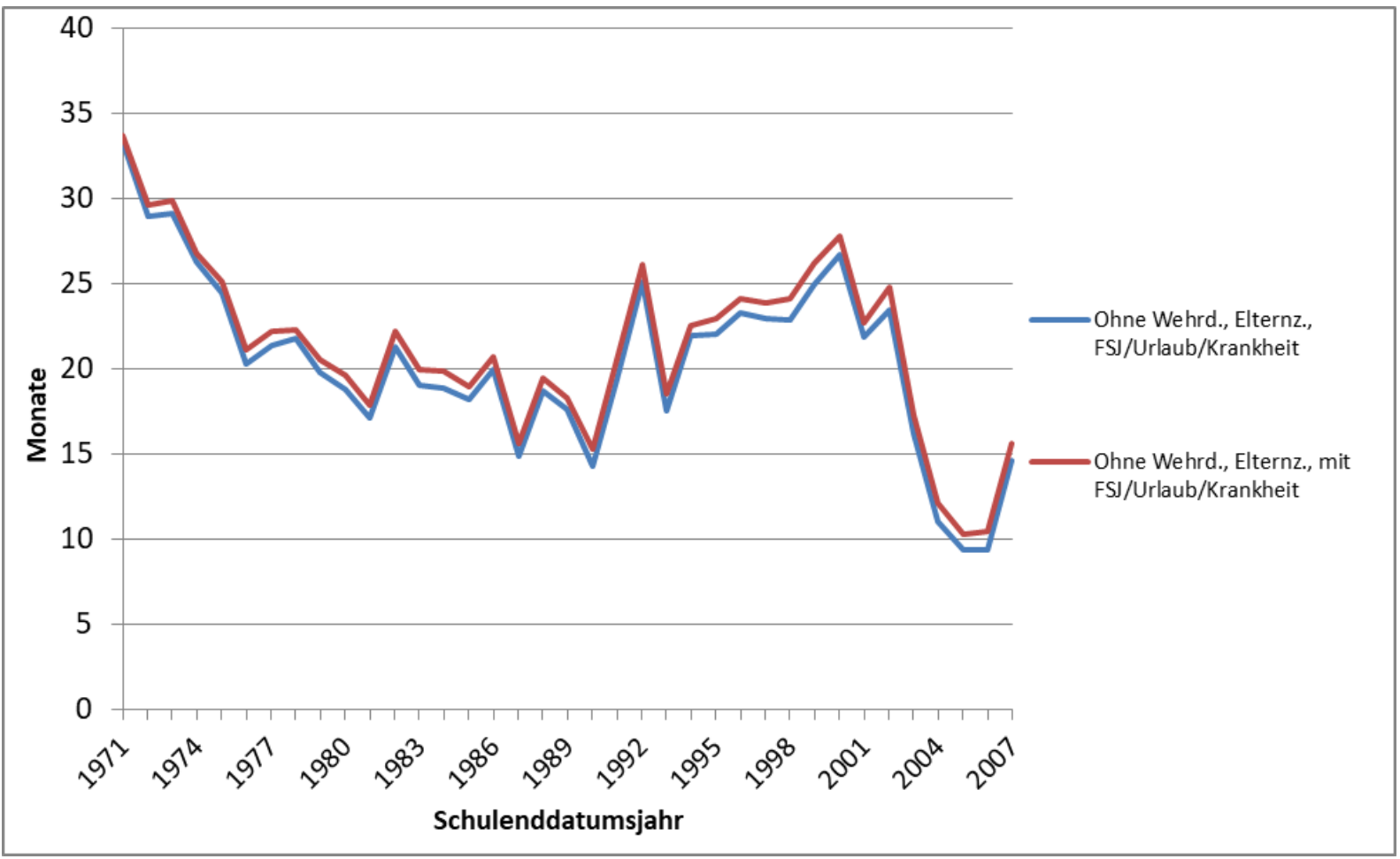

Quelle: Eigene Berechnungen basierend auf dem NEPS. Abkürzungen: Wehrd.: Wehrdienst, Elternz.: Elternzeit. $\mathrm{N}=9.254$.

Das sehr deutliche Herabsinken der durchschnittlichen Übergangsdauer zu Beginn der 1970er Jahre und nach dem Jahr 2000 ist ebenfalls mit den eventuellen Verzerrungen aufgrund geringer Fallzahlen in den jeweiligen Jahren zu begründen. Auch eine Betrachtung der Dauer in Fünf-Jahres-Schritten beim Zeitverlauf visualisiert die gleiche Entwicklung (siehe Anhang - Abbildung 14). Bei Ausschluss der rechtszensierten Fälle, die bis zum Ende des Beobachtungszeitraums keine Ausbildung aufgenommen haben, zeichnete sich eine sehr ähnliche Entwicklung der durchschnittlichen Übergangsdauer im zeitlichen Verlauf ab. Der einzige Unterschied bestand darin, dass sich die Durchschnittsdauer auf einem deutlich niedrigeren Level bewegte im Vergleich zur Berücksichtigung der rechtszensierten Fälle, welche deutlich längere Übergangsdauern bis zum Ende des Beobachtungszeitraums aufwiesen. Der gleiche Befund galt auch für die Übergangsdauer mit FSJ-, Urlaubs- und 
Krankheitsphasen (siehe Abbildung 8) sowie mit zusammengefassten Schulenddatumsjahren in Fünf-Jahres-Kategorien (siehe Anhang - Abbildung 15).

Abbildung 8: Durchschnittliche Übergangsdauer ohne rechtszensierte Fälle im Zeitverlauf.

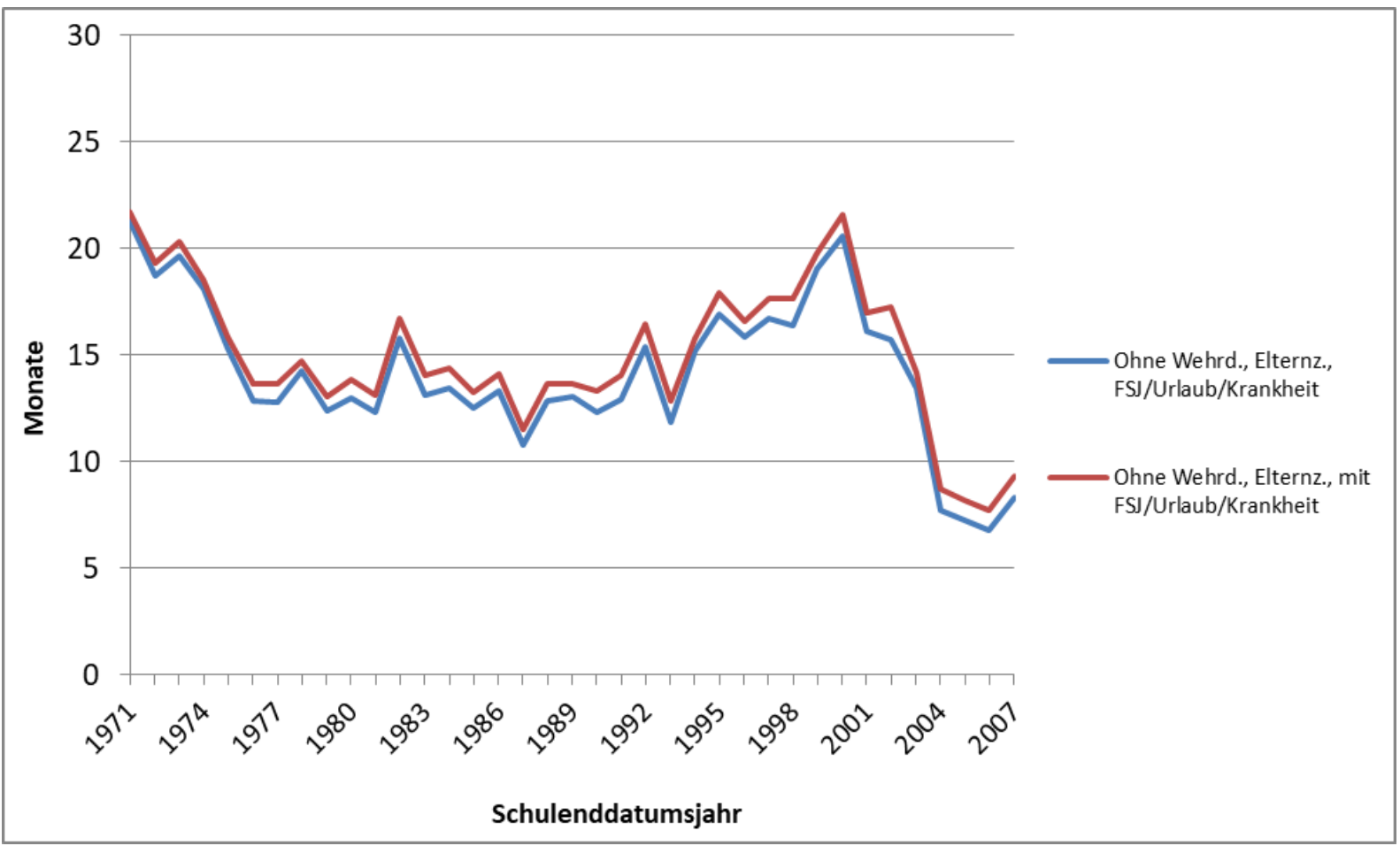

Quelle: Eigene Berechnungen basierend auf dem NEPS. Ohne rechtszensierte Fälle, die bis zum Ende des Untersuchungszeitraums keine Ausbildung begonnen haben. Abkürzungen: Wehrd.: Wehrdienst, Elternz.: Elternzeit. $\mathrm{N}=8.479$.

Bei Betrachtung der Entwicklung der durchschnittlichen Übergangsdauer im zeitlichen Verlauf getrennt nach Schulabschluss wurde deutlich, dass diejenigen ohne Schulabschluss bis zum Jahr 2000 durchschnittlich die längste Übergangsdauer hatten (siehe Abbildung 9). 
Abbildung 9: Durchschnittliche Übergangsdauer (ohne Wehrdienst, Elternzeit, FSJ/Urlaub/Krankheit) nach Schulabschluss im Zeitverlauf.

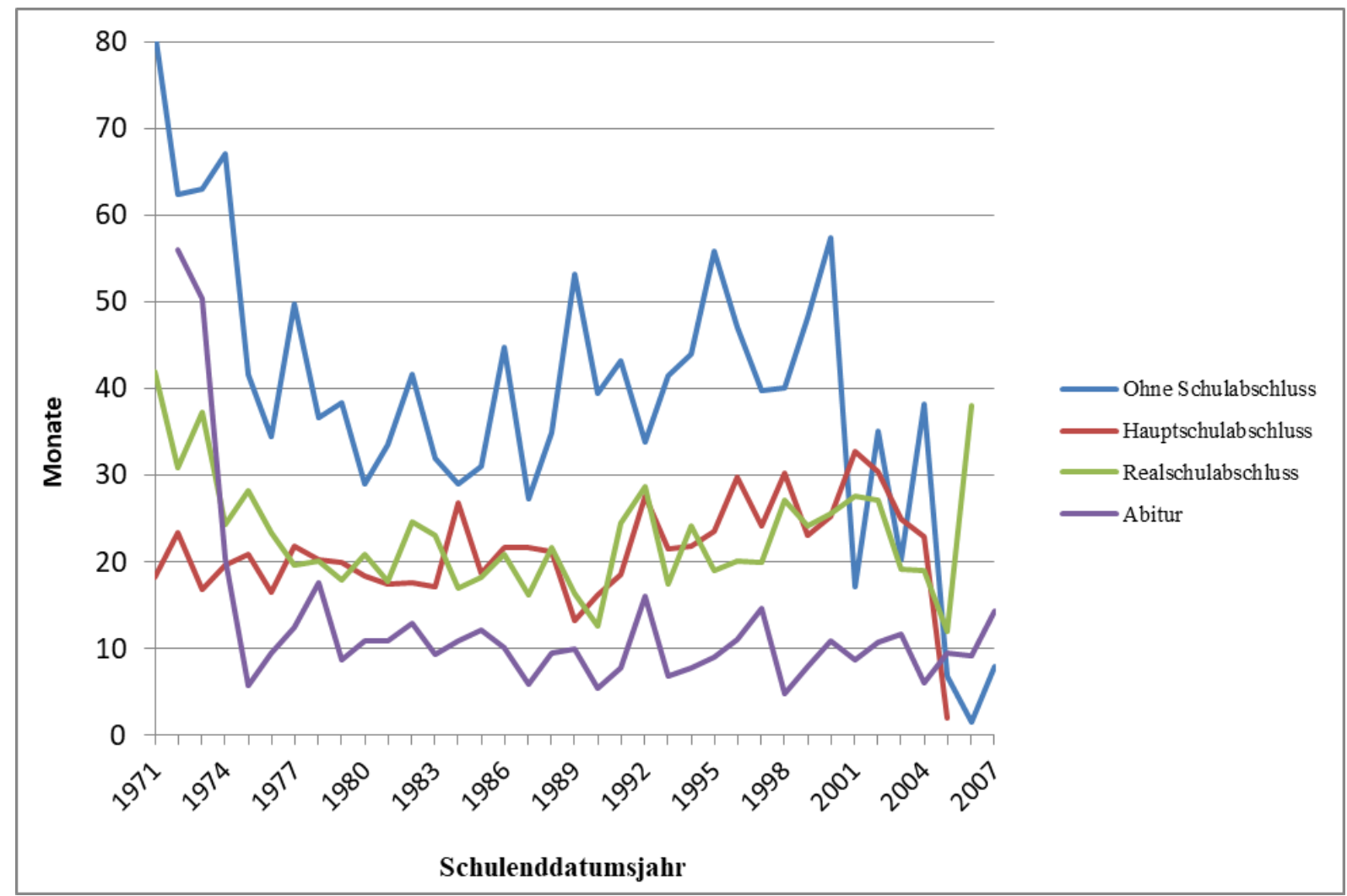

Quelle: Eigene Berechnungen basierend auf dem NEPS. N = 9.189.

Lag die Dauer derjenigen ohne Abschluss zu Beginn des Beobachtungszeitraums zwischen 1971 und 1975 durchschnittlich noch bei etwa 60 Monaten, sank sie bis Mitte der 1980er Jahre auf etwa 30 Monate herab. Nach einem kontinuierlichen Anstieg auf rund 47 Monate durchschnittlich bis zum Jahr 2000 war für die letzten einbezogenen Schulenddatumsjahre ein deutliches Herabsinken der durchschnittlichen Übergangsdauer auf teilweise unter 20 Monate $\mathrm{zu}$ beobachten. Dies ist damit zu erklären, dass viele derjenigen ohne Schulabschluss der Gruppe der rechtszensierten Fälle ohne begonnene Ausbildung im Beobachtungszeitraum zuzuordnen waren. Für diese Gruppe endete der Übergangszeitraum automatisch mit dem Ende des Beobachtungszeitraums, wobei viele dieser Personen gegebenenfalls eine längere Übergangsphase benötigten, um in die erste Ausbildung einmünden zu können. Aufgrund der Beendigung des Untersuchungszeitraums wurde die durchschnittliche Übergangsdauer automatisch verkürzt. Für die im Vergleich zu den späteren Jahren und Jahrzehnten durchschnittlich sehr lange Übergangsdauer zu Beginn des Beobachtungszeitraums Anfang der 1970er Jahre sind die sehr geringen Fallzahlen in den ersten einbezogenen Schulenddatumsjahren verantwortlich, welche eine derartig lange Dauer aufwiesen. 
Die durchschnittliche Übergangsdauer bei Personen mit einem Haupt- oder Realschulabschluss als erstem erreichten Schulabschluss blieb insgesamt über den gesamten Beobachtungszeitraum hinweg relativ konstant zwischen 20 und 30 Monaten, wobei sie bei diesen beiden Gruppen zum Ende des Beobachtungszeitraums leicht anstieg. Abiturientinnen und Abiturienten hatten im beobachteten Zeitverlauf die mit Abstand durchschnittlich kürzeste Übergangsdauer, welche durchgehend in etwa bei zehn Monaten lag. Somit hatten diejenigen ohne Schulabschluss im Beobachtungszeitraum die durchschnittlich längste und diejenigen mit Abitur die durchschnittlich kürzeste Übergangsdauer. Ein fast identisches Ergebnismuster ergab sich bei der Übergangsdauer mit FSJ-, Urlaubs- und Krankheitsphasen (Abbildung 10). Der gleiche Trend zeigte sich auch bei zusammengefassten Schulenddatumsjahren (siehe Anhang - Abbildungen 16 und 17).

Abbildung 10: Durchschnittliche Übergangsdauer (ohne Wehrdienst und Elternzeit, mit FSJ/Urlaub/Krankheit) nach Schulabschluss im Zeitverlauf.

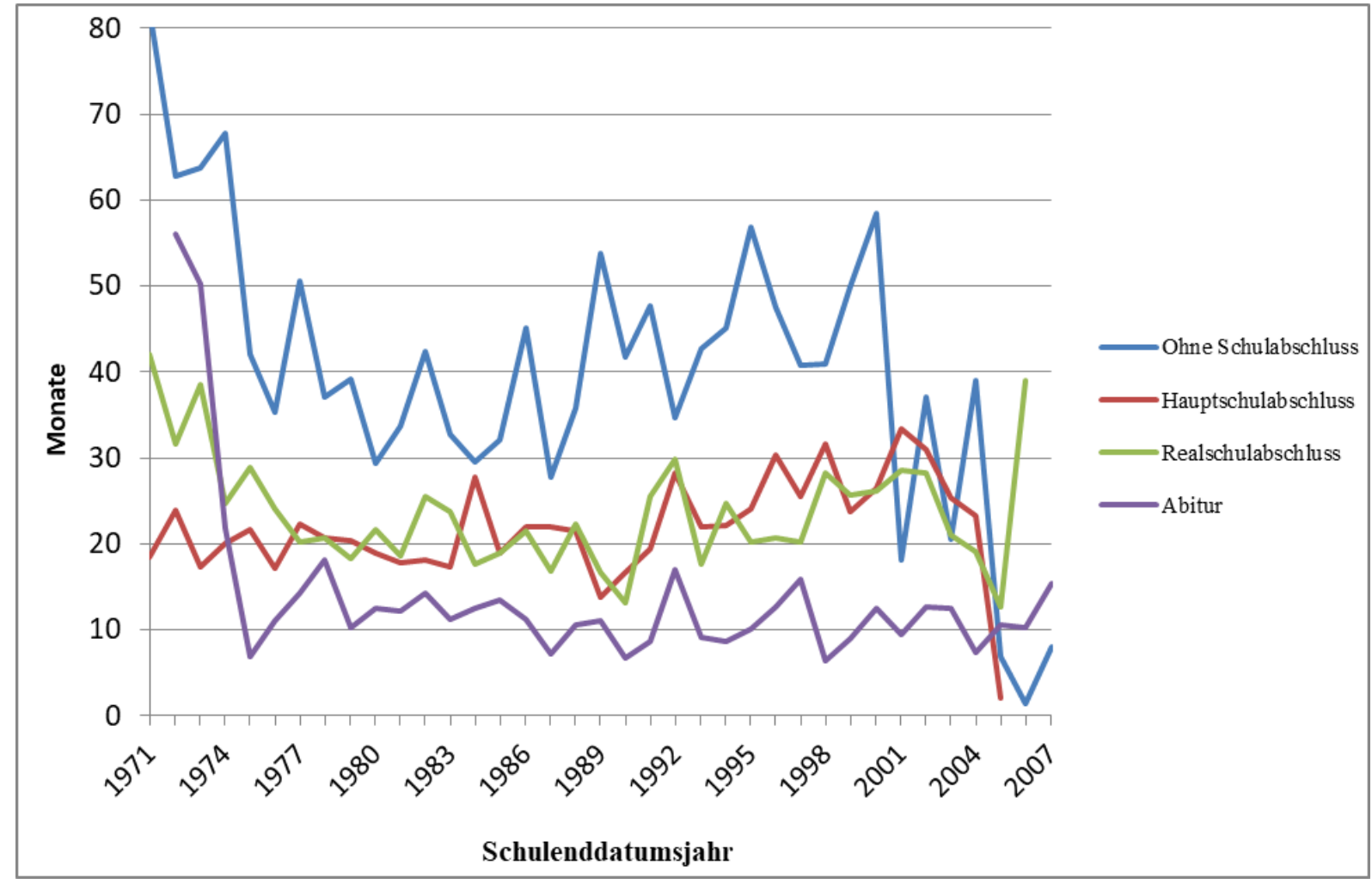

Quelle: Eigene Berechnungen basierend auf dem NEPS. $\mathrm{N}=9.189$.

Bei den absolvierten Übergangsstationen als zweites zentrales Outcome der vorliegenden Untersuchung wurden verschiedene Varianten der Summen berechnet, welche im Rahmen des methodischen Vorgehens bereits vorgestellt wurden. Die verschiedenen Varianten der Stationssumme dienten einem Vergleich. Dabei zeigte sich, dass sich die Ergebnisse in ihrem 
Muster kaum voneinander unterschieden. Aufgrund dessen wird die Variante ohne Wehrdienst-, Elternzeit-, FSJ-, Urlaubs- und Krankheitsepisoden und mit unbestimmten Phasen bei den folgenden Ergebnissen berichtet.

Beim Vergleich der durchschnittlichen Stationsanzahl nach Schulabschluss wurde deutlich, dass diejenigen ohne Schulabschluss mit durchschnittlich 1,82 absolvierten Stationen die mit Abstand höchste Anzahl an Stationen hatten. Die Befragten mit einer mittleren Reife verzeichneten mit durchschnittlich 1,08 absolvierten Stationen eine deutlich geringere Anzahl, während die Anzahl bei denjenigen mit Hauptschulabschluss $(0,99)$ und mit Abitur $(0,86)$ noch geringer war. Das gleiche Muster der Verteilungen, wenn auch mit höheren und niedrigeren Anteilen aufgrund der Berücksichtigung oder dem Ausschluss bestimmter Stationen, zeigte sich bei den anderen drei Stationsvariablen (siehe Abbildung 11).

\section{Abbildung 11: Durchschnittliche Anzahl Übergangsstationen nach Schulabschluss.}

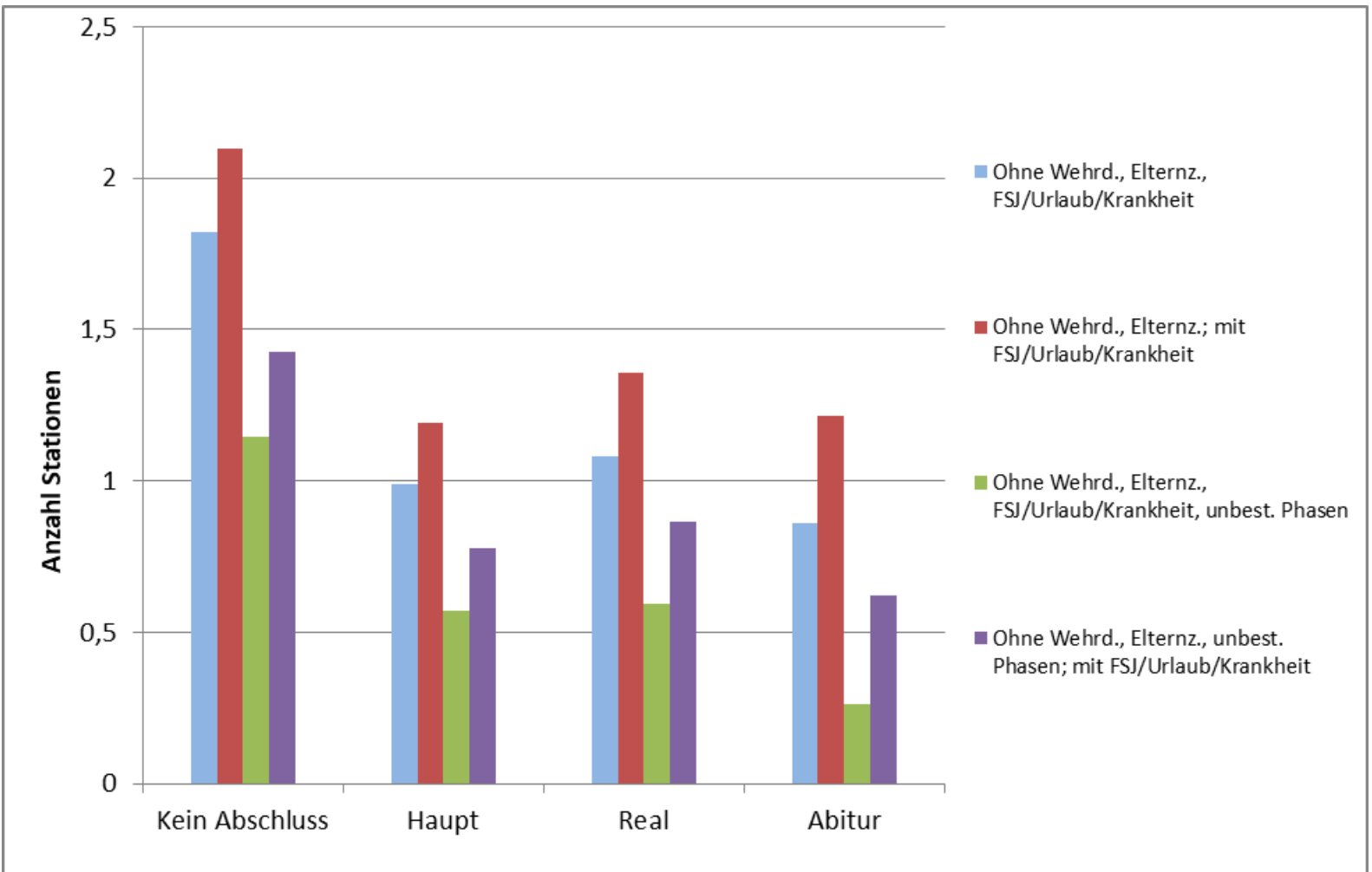

Quelle: Eigene Berechnungen basierend auf dem NEPS. Abkürzungen: Wehrd.: Wehrdienst, Elternz.: Elternzeit, unbest. Phasen: unbestimmte Lückenepisoden, bei denen keine Station dokumentiert wurde. N = 9.189.

Bei einem direkten Vergleich der Anzahl an absolvierten Übergangsstationen zwischen den einzelnen Schulabschlussgruppen stellte sich heraus, dass sich alle vier Schulabschlussgruppen hinsichtlich der Summe an Stationen jeweils signifikant voneinander unterschieden. Bezogen auf den direkten Vergleich und die Testung auf signifikante 
Unterschiede ergaben sich bei den drei anderen Stationsvariablen relativ ähnliche Ergebnisse hinsichtlich der berechneten p-Werte (siehe Anhang - Tabelle 11).

Beim deskriptiven Überblick der Stationsanzahl getrennt nach Erstausbildungstyp fiel auf, dass die Gruppe derer, die bis zum Ende des Untersuchungszeitraums keine Ausbildung begonnen hatte, mit durchschnittlich 2,17 Stationen die mit Abstand meisten Stationen absolvierte. Personen, die dagegen als Erstausbildung ein Studium (1,18), eine vollzeitschulische $(1,08)$ oder eine duale Berufsausbildung $(0,88)$ aufgenommen hatten, wiesen durchschnittlich eine deutlich kleinere Summe an durchlaufenen Übergangsstationen auf. Die identischen Resultate fanden sich fast exakt, wenn auch aufgrund der andersartigen Konstellationen mit leicht abweichenden Werten, bei den drei anderen Varianten (siehe Abbildung 12).

\section{Abbildung 12: Durchschnittliche Anzahl Übergangsstationen nach Erstausbildungstyp.}

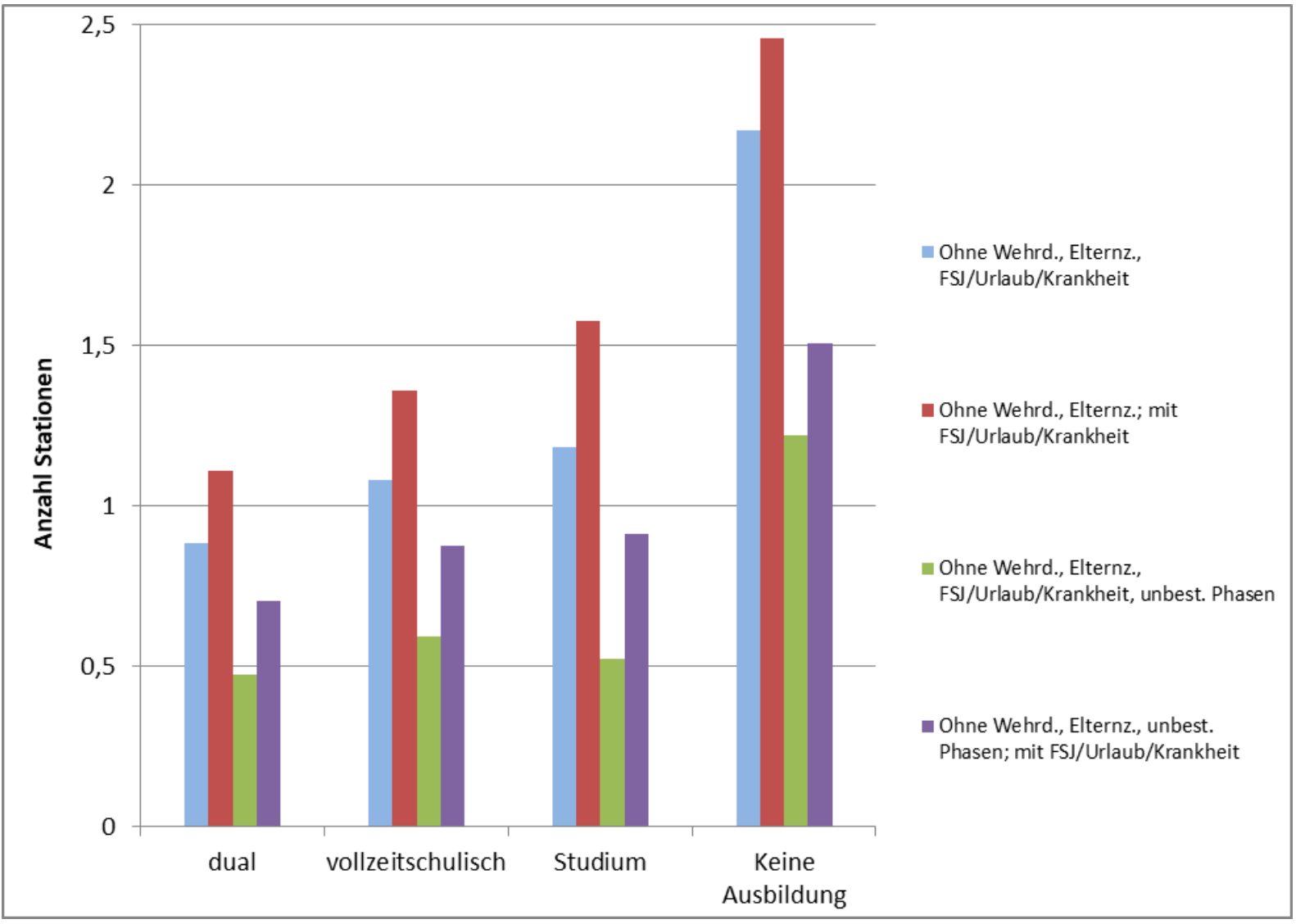

Quelle: Eigene Berechnungen basierend auf dem NEPS. Abkürzungen: Wehrd.: Wehrdienst, Elternz.: Elternzeit, unbest. Phasen: unbestimmte Lückenepisoden, bei denen keine Station dokumentiert wurde. Die Übergangsdauer derjenigen ohne Ausbildung entsprach dem Beobachtungszeitraum vom Verlassen der Schule an. $\mathrm{N}=9.254$.

Bei der Testung auf signifikante Unterschiede der Stationsanzahl wurde ersichtlich, dass sich fast alle Erstausbildungstypen signifikant voneinander unterschieden. Sehr ähnliche Resultate 
zu den Mittelwerttests fanden sich auch bei den drei weiteren Varianten der Stationsvariablen (siehe Anhang - Tabelle 12).

Bezüglich der Entwicklung der Stationsanzahl im Zeitverlauf zeigte sich, dass die Anzahl bis etwa 1990 relativ konstant blieb, von Beginn der 1990er Jahre jedoch leicht und kontinuierlich bis zum Jahr 2000 anstieg und im Anschluss daran wieder leicht sank. Diese Beobachtung des Absinkens nach dem Jahr 2000 kann mit der Beendigung des Beobachtungszeitraums einige Jahre darauf und der Nichtberücksichtigung von möglichen weiteren Stationen bei Personen ohne begonnene Ausbildung sowie den vergleichsweise geringen Fallzahlen in den letzten Schulenddatumsjahren begründet werden. Der gleiche Entwicklungsverlauf zeigte sich mit etwas veränderten Werten für die drei anderen erstellten Variablen zur Stationsanzahl (siehe Abbildung 13).

\section{Abbildung 13: Durchschnittliche Anzahl Übergangsstationen im Zeitverlauf.}

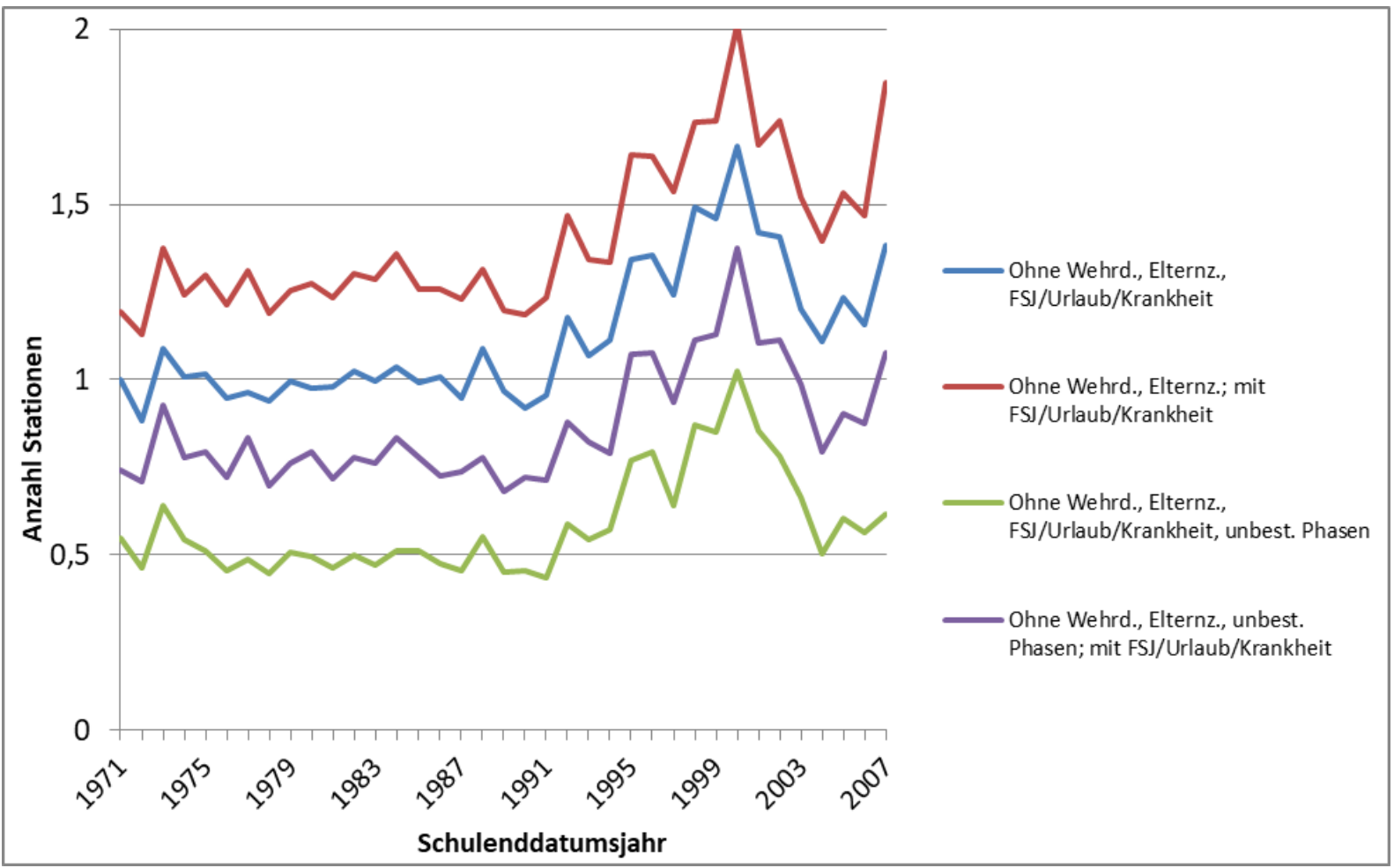

Quelle: Eigene Berechnungen basierend auf dem NEPS. Abkürzungen: Wehrd.: Wehrdienst, Elternz.: Elternzeit, unbest. Phasen: unbestimmte Lückenepisoden, bei denen keine Station dokumentiert wurde. N = 9.254.

Der gleiche Verlauf über den Beobachtungszeitraum wurde bei einer Zusammenfassung der Schulenddatumsjahre zu Fünf-Jahres-Kategorien festgestellt (siehe Anhang - Abbildung 18). Bei Nichtberücksichtigung der rechtszensierten Fälle stellte sich im Zeitverlauf die gleiche Entwicklung der durchschnittlichen Anzahl an Stationen heraus. Einziger Unterschied zu den Ergebnissen mit Einbezug der rechtszensierten Fälle war, dass sich die durchschnittliche 
Stationsanzahl im Zeitverlauf auf einem geringfügig niedrigeren Niveau bewegte. Personen ohne begonnene Ausbildung im Beobachtungszeitraum hatten die mit Abstand höchste durchschnittliche Stationsanzahl und trugen über den gesamten Zeitverlauf zu einem höheren Wert bei. Nahezu gleiche Tendenzen zeigten sich bei den drei anderen Stationsvariablen mit entsprechend angepassten Werten (siehe Abbildung 14) sowie mit zusammengefassten Schulenddatumsjahren in Fünf-Jahres-Kategorien (siehe Anhang - Abbildung 19).

Abbildung 14: Durchschnittliche Anzahl Übergangsstationen ohne rechtszensierte Fälle im Zeitverlauf.

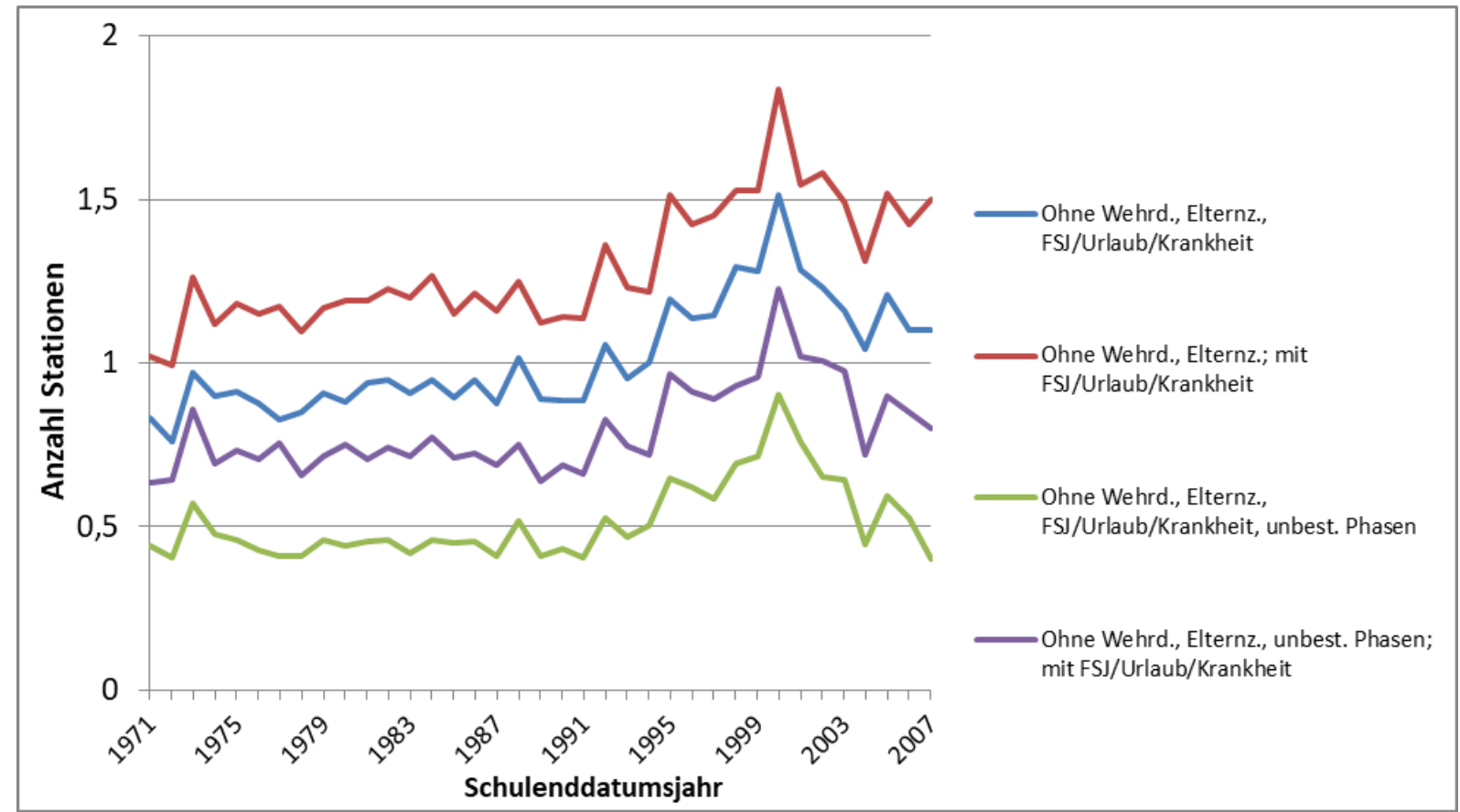

Quelle: Eigene Berechnungen basierend auf dem NEPS. Ohne rechtszensierte Fälle, die bis zum Ende des Untersuchungszeitraums keine Ausbildung begonnen haben. Abkürzungen: Wehrd.: Wehrdienst, Elternz.: Elternzeit, unbest. Phasen: unbestimmte Lückenepisoden, bei denen keine Station dokumentiert wurde. $\mathrm{N}=$ 8.479 .

Beim Überblick über die durchschnittliche Stationsanzahl im Zeitverlauf getrennt nach Schulabschluss fiel auf, dass diejenigen ohne Schulabschluss über nahezu den gesamten Beobachtungszeitraum die höchste Stationsanzahl hatten (siehe Abbildung 15). 


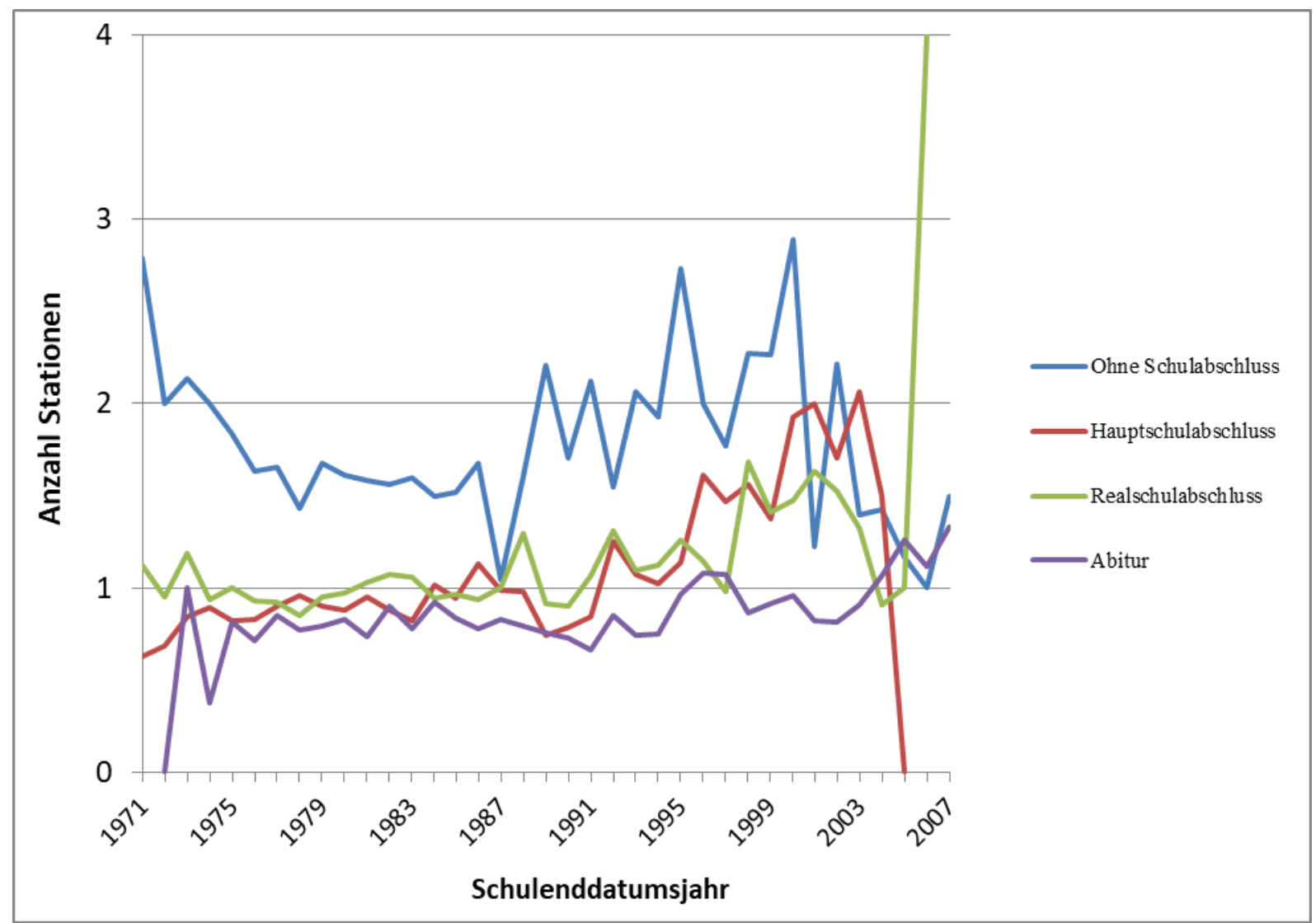

Quelle: Eigene Berechnungen basierend auf dem NEPS. N = 9.189.

Nach einem Absinken bei denjenigen ohne Abschluss von zwei absolvierten Stationen zu Beginn der 1970er Jahre auf etwa 1,5 Stationen in den 1980er Jahren ließ sich ein deutlicher Anstieg auf durchschnittlich etwa 2,3 Stationen bis zum Jahr 2000 ausmachen. Im Anschluss daran war für diejenigen ohne Schulabschluss eine Verringerung der Stationen nach $2000 \mathrm{zu}$ beobachten, welche jedoch der Problematik der $\mathrm{zu}$ geringen Fallzahlen in den letzten Schulenddatumsjahren geschuldet sein kann. Diejenigen mit einem Haupt- oder einem Realschulabschluss hatten dagegen über den Zeitverlauf hinweg einen Anstieg an Stationen zu verzeichnen. Interessant war dabei, dass von Anfang der 1970er Jahre bis in die 1990er Jahre diejenigen mit einem Hauptschulabschluss durchschnittlich etwas weniger Stationen vorzuweisen hatten als diejenigen mit einer mittleren Reife. Dieser Unterschied stellte sich jedoch als nicht allzu groß dar, wie sich in der grafischen Darstellung zeigt. Ab Ende der 1990er Jahre kehrte sich dieses Verhältnis jedoch deutlich um in der Form, dass Personen mit einem Hauptschulabschluss von da an einen deutlich stärkeren Anstieg an absolvierten Stationen und damit durchschnittlich mehr Stationen zu verzeichnen hatten als diejenigen mit einem Realschulabschluss. Ganz zum Ende des Beobachtungszeitraums überholte die Gruppe 
derer mit einem Hauptschulabschluss hinsichtlich der durchschnittlichen Stationsanzahl sogar die Gruppe derjenigen, die keinen Schulabschluss erworben hatten. Dieser Umstand ist hinsichtlich einer inhaltlichen und aussagekräftigen Interpretation jedoch dahingehend mit Vorsicht $\mathrm{zu}$ interpretieren, dass in den letzten Schulenddatumsjahren lediglich geringe Fallzahlen vorliegen und dies zu Verzerrungen bei den Ergebnissen führen kann. Diejenigen mit Abitur wiesen über den gesamten Zeitverlauf hinweg die konstanteste durchschnittliche Stationsanzahl auf, wenngleich sich auch für diese Gruppe ein leichter Anstieg bis zum Ende des Beobachtungszeitraums auf durchschnittlich eine absolvierte Station nachweisen lassen konnte. Nahezu adäquat stellte sich die Entwicklung für die drei anderen Stationsvariablen dar (siehe Abbildungen 16, 17 und 18), wobei bei einer Berücksichtigung von FSJ- und Urlaubsepisoden diejenigen mit einem Hauptschulabschluss bis etwa 1990 durchschnittlich eine etwas geringere Stationsanzahl aufwiesen als diejenigen mit Abitur. Dieser Unterschied war jedoch als sehr marginal zu betrachten und wurde auch ab den 1990er Jahren aufgehoben.

Abbildung 16: Durchschnittliche Anzahl Übergangsstationen (ohne Wehrdienst und Elternzeit; mit FSJ/Urlaub/Krankheit) nach Schulabschluss im Zeitverlauf.

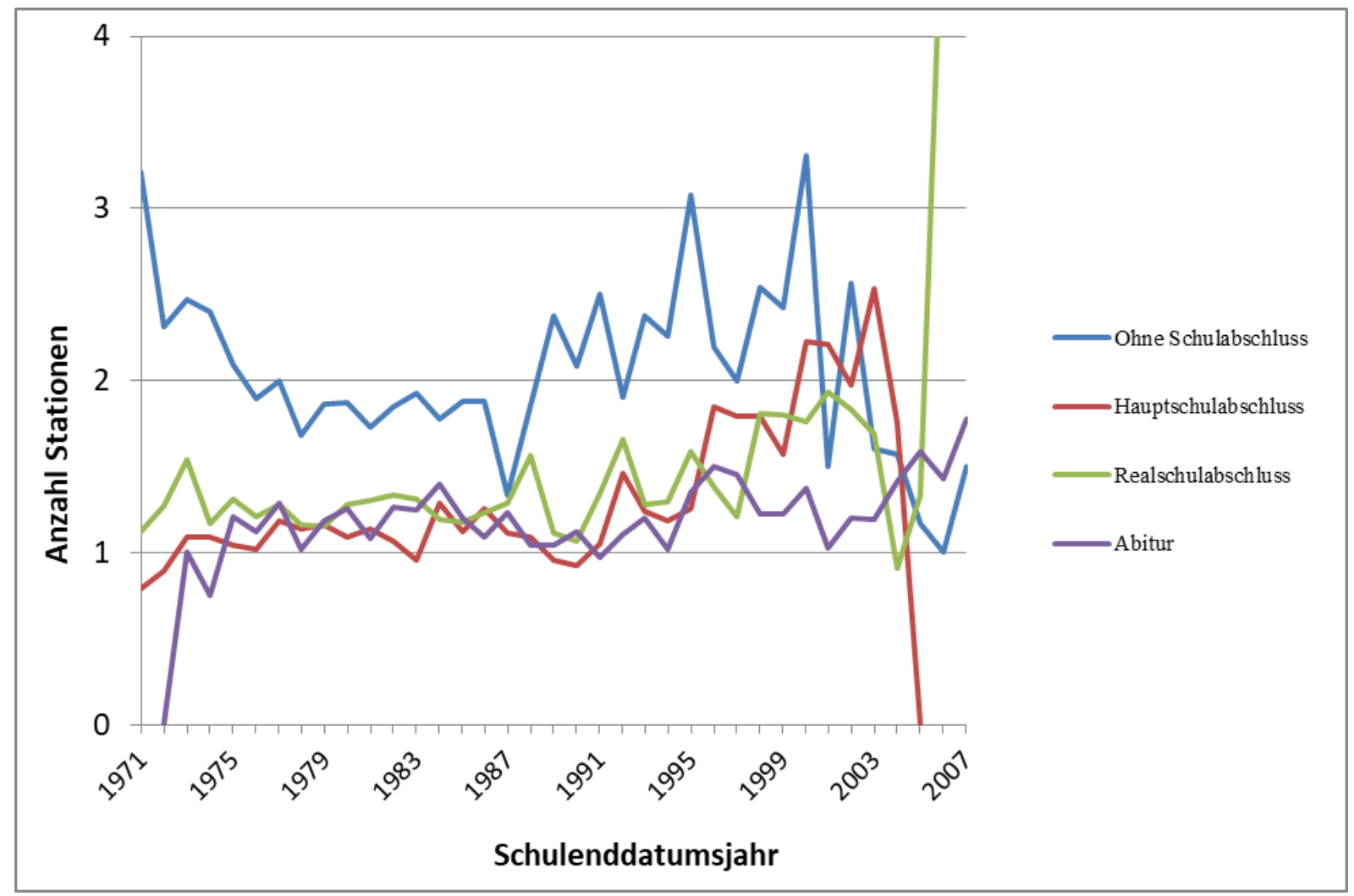

Quelle: Eigene Berechnungen basierend auf dem NEPS. N = 9.189. 
Abbildung 17: Durchschnittliche Anzahl Übergangsstationen (ohne Wehrdienst, Elternzeit, FSJ/Urlaub/Krankheit, unbestimmte Phasen) nach Schulabschluss im Zeitverlauf.

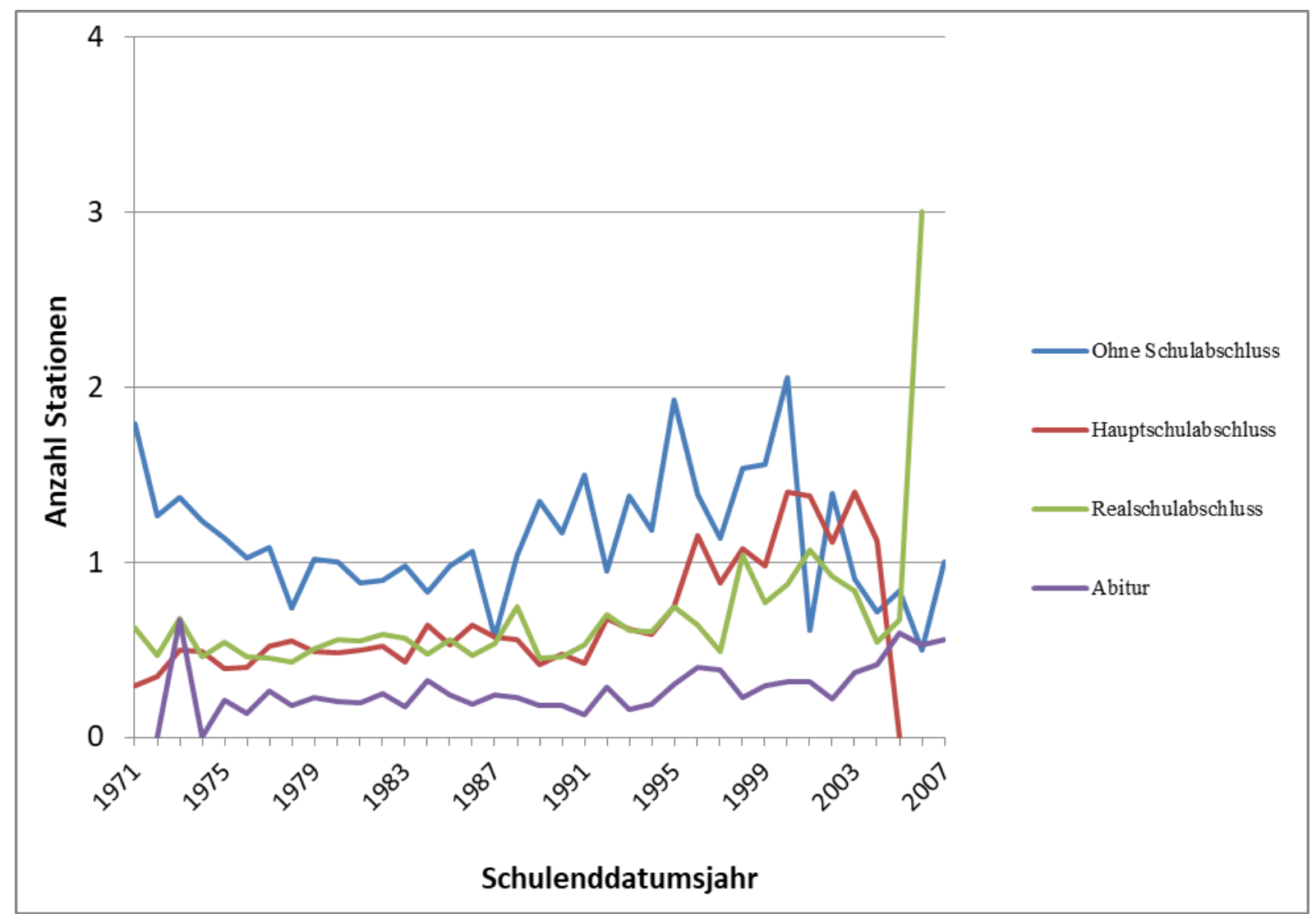

Quelle: Eigene Berechnungen basierend auf dem NEPS. N = 9.189. 
Abbildung 18: Durchschnittliche Anzahl Übergangsstationen (ohne Wehrdienst, Elternzeit, unbestimmte Phasen; mit FSJ/Urlaub/Krankheit) nach Schulabschluss im Zeitverlauf.

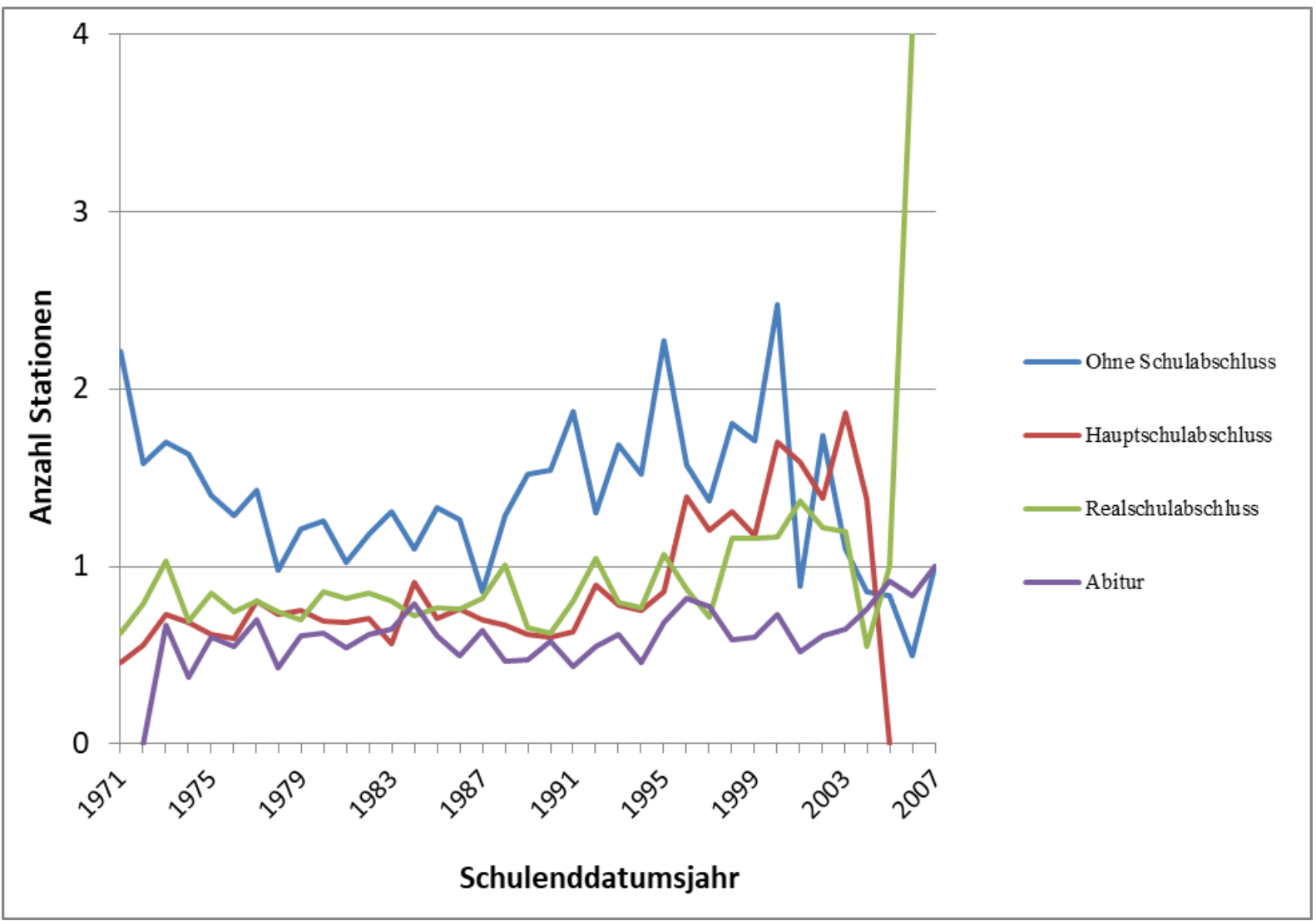

Quelle: Eigene Berechnungen basierend auf dem NEPS. $\mathrm{N}=9.189$.

Bei einer Zusammenfassung der Schulenddatumsjahre zu Fünf-Jahres-Kategorien konnten die geschilderten Entwicklungsverläufe in etwa nachgewiesen werden (siehe Anhang Abbildungen 20, 21, 22 und 23).

Abschließend wird noch ein grafischer Überblick zur Entwicklung der einbezogenen Makrovariablen gegeben (siehe Abbildung 19). 
Abbildung 19: Prozentuale Anteile zu Dienstleistungstätigen, demografischem Anteil und Arbeitslosenquote im Zeitverlauf nach fortlaufendem Schulenddatumsjahr.

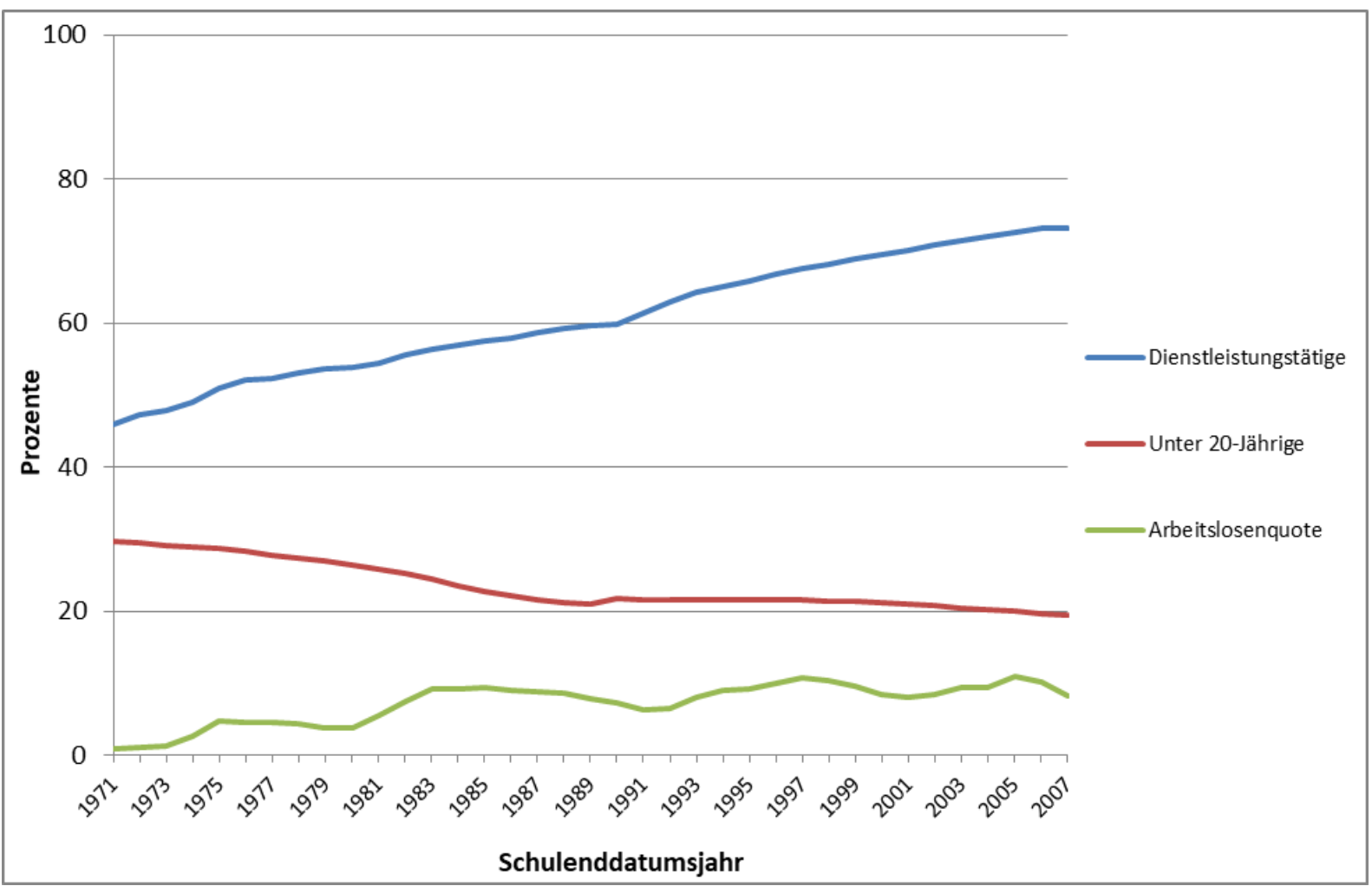

Demografischer Anteil bezieht sich auf die unter 20-Jährigen. N = 9.254. Quelle: Eigene Darstellung basierend auf Statistisches Bundesamt 2017a, Statistisches Bundesamt 2017c, Statistisches Bundesamt 2017d.

Während sich der Anteil der Dienstleistungstätigen im tertiären Sektor kontinuierlich von etwa 45 Prozent zu Beginn der 1970er Jahre auf über 70 Prozent bis 2007 erhöhte, sank der demografische Anteil der unter 20-Jährigen bis Ende der 1980er von etwa 30 Prozent auf etwa 20 Prozent ab und verblieb bis 2007 relativ konstant auf diesem Niveau. Die Arbeitslosenquote erhöhte sich von unter einem Prozent zu Beginn der 1970er Jahre auf circa zehn Prozent ab Mitte der 1980er Jahre. Nach einem leichten Herabsinken stieg dieser Anteil bis Ende der 1990er Jahre und nach 2000 nochmals auf etwa zehn Prozent an (Statistisches Bundesamt 2017a, 2017c, 2017d).

\subsubsection{Zusammenfassung der deskriptiven Ergebnisse}

Zusammenfassend konnte festgestellt werden, dass der Realschulabschluss im einbezogenen Untersuchungszeitraum am häufigsten erworben wurde. Das Abitur sowie der Hauptschulabschluss wurden fast gleich häufig erworben, jedoch nicht ganz so häufig wie die mittlere Reife. Im Zeitverlauf betrachtet wurde das Abitur immer häufiger erreicht, wogegen der Hauptschulabschluss im Laufe der Zeit immer seltener den ersten Schulabschluss darstellte. Des Weiteren wurde im Beobachtungszeitraum eine duale Berufsausbildung am 
häufigsten begonnen. Im Zeitverlauf wurde jedoch immer häufiger ein Studium als Erstausbildung aufgenommen, während die Aufnahmequote einer dualen Berufsausbildung relativ konstant blieb. Das Nichtaufnehmen einer Ausbildung wurde dagegen im Laufe der einbezogenen Jahrzehnte immer seltener.

Personen ohne Schulabschluss benötigten über den gesamten Untersuchungszeitraum hinweg die durchschnittlich längste Übergangsdauer, wogegen diejenigen mit Abitur am kürzesten brauchten, um von der allgemeinbildenden Schule in eine berufliche oder akademische Erstausbildung einzumünden. Die Dauer derjenigen mit einem Haupt- oder Realschulabschluss war in etwa gleich und lag zwischen der durchschnittlichen Dauer der beiden anderen Schulabschlussgruppen. Während diejenigen, die eine duale Berufsausbildung begannen, am kürzesten für den Übergang benötigten, war die Dauer derjenigen, welche eine vollzeitschulische oder akademische Erstausbildung aufnahmen, in etwa gleich. Generell hatte die durchschnittliche Übergangsdauer über alle Schulabschluss- und Erstausbildungsgruppen hinweg von Anfang der 1970er Jahre bis Ende der 1980er Jahre abgenommen und stieg dann wieder etwas an. Differenziert betrachtet blieb die Übergangsdauer bei denjenigen mit Abitur im Zeitverlauf konstant, während sie bei denjenigen mit einem Hauptschulabschluss leicht anstieg. Bei Personen ohne Abschluss verringerte sich die durchschnittliche Dauer zunächst, stieg dann jedoch wieder an.

Diejenigen ohne Schulabschluss hatten neben der längsten Übergangsdauer auch die höchste Anzahl an Übergangsstationen, während diejenigen mit Abitur die geringste Anzahl an Stationen durchliefen. Personen, die keine Ausbildung aufnahmen, hatten die höchste Anzahl an Übergangsstationen, während diejenigen mit einer dualen Erstausbildung die wenigsten Stationen durchliefen. Während die durchschnittliche Anzahl an Übergangsstationen generell in den 1970er und 1980er Jahren konstant blieb, stieg sie ab Anfang der 1990er Jahre leicht an, um etwa ab dem Jahr 2000 wieder zu sinken. Insgesamt stieg die durchschnittliche Stationsanzahl im Zeitverlauf bei allen Schulabschlussgruppen an. Jedoch verzeichneten diejenigen mit Abitur den deutlich geringsten Anstieg. Dagegen absolvierten Personen mit einem Hauptschulabschluss im Zeitverlauf immer mehr Stationen. Den deutlich größten Zuwachs bei der durchschnittlichen Stationsanzahl verzeichneten diejenigen ohne Schulabschluss, bei denen die Anzahl ab Mitte der 1980er Jahre kontinuierlich anstieg. 


\subsection{Sterbetafeln}

\subsubsection{Ergebnisse der kumulierten Überlebenswahrscheinlichkeiten}

Im Laufe der Monate nahm die kumulierte Überlebenswahrscheinlichkeit sukzessive ab, was damit zu begründen ist, dass im Laufe der Zeit immer mehr Personen in eine Erstausbildung einmündeten und sich dementsprechend nicht mehr im Übergangsprozess befanden. Dies zeigt sich in der folgenden Abbildung 20.

\section{Abbildung 20: Kumulierte Überlebenswahrscheinlichkeit insgesamt beim Übergang in die Ausbildung.}

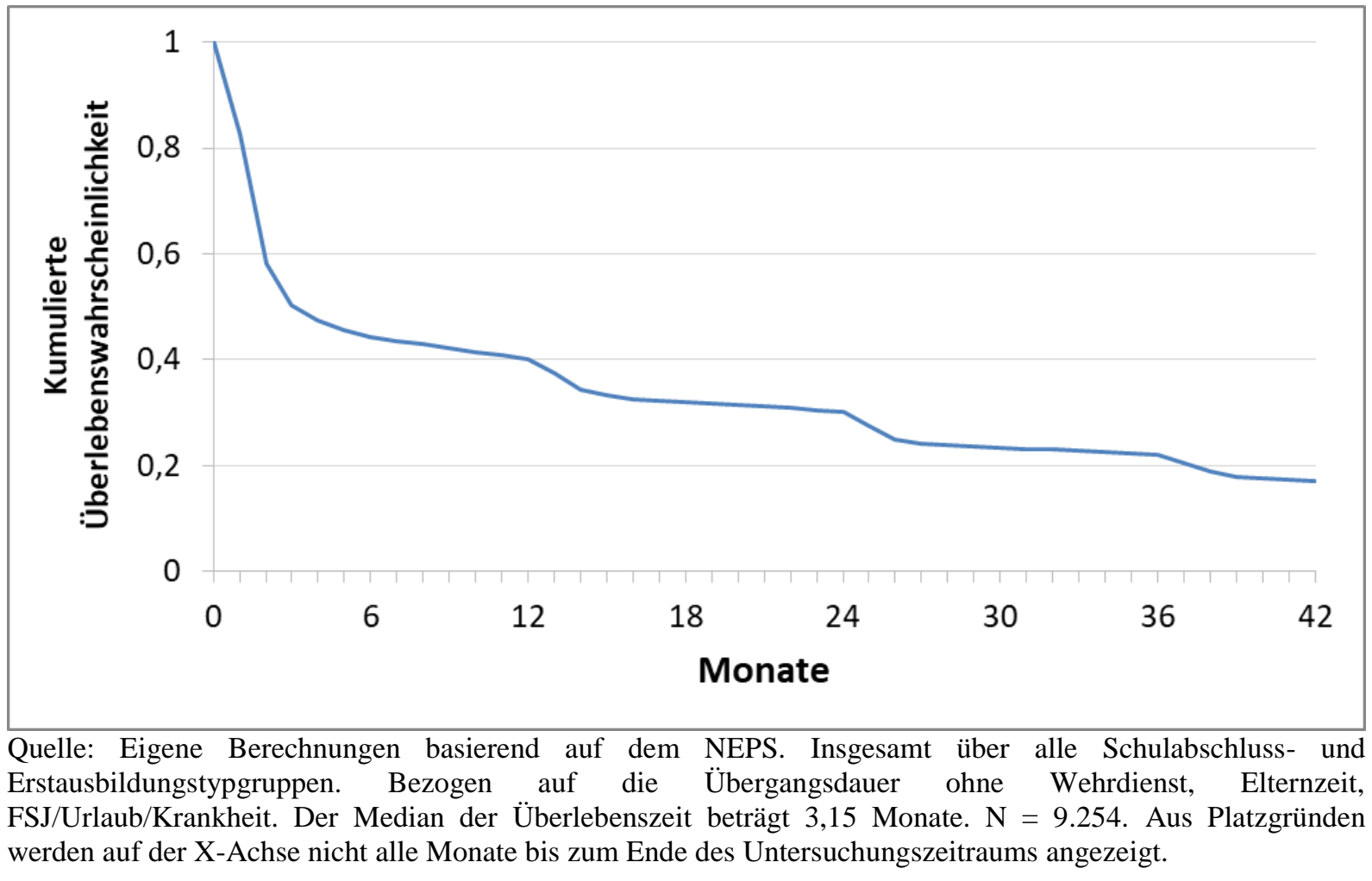

In den ersten Monaten war die kumulierte Überlebenswahrscheinlichkeit recht hoch, wodurch sich ein Großteil der einbezogenen Personen noch im Übergangsprozess befand und für diese Gruppe der Beginn der Erstausbildung noch nicht eingetreten war. Im Laufe der Monate verringerte sich die kumulierte Überlebenswahrscheinlichkeit, da sukzessive mehr Personen in die Erstausbildung einmündeten. Hierbei waren alle Schulabschlüsse und Erstausbildungstypen, und damit auch rechtszensierte Fälle, berücksichtigt. Die kumulierte Überlebenswahrscheinlichkeit stabilisierte sich nach einem schnellen Absinken nach einigen Monaten etwas und nahm in der Folge moderat ab. Die Übergangsdauer einiger Personen gestaltete sich somit deutlich länger. Die rechtszensierten Fälle hatten auch zum Ende der Beobachtungsphase eine kumulierte Überlebenswahrscheinlichkeit von > 0. Der Median der 
Überlebenszeit betrug über alle Gruppen hinweg 3,15 Monate. Das bedeutet, dass nach genau 3,15 Monaten die kumulierte Überlebenswahrscheinlichkeit genau 0,5 betrug und somit zu diesem Zeitpunkt exakt die Hälfte der einbezogenen Personen in die Erstausbildung eingemündet war. Die andere Hälfte benötigte für den Übergang dagegen weitaus länger, wobei die rechtszensierten Fälle bis zum Ende des Beobachtungszeitraums keine Ausbildung begannen. Dementsprechend ist der Median der Überlebenszeit nicht gleichzusetzen mit dem Mittelwert, welcher für beide Gruppenhälften eine identische Spannweite an Monaten für den Übergang in die Ausbildung voraussetzt. Bei der Übergangsdauer mit FSJ-, Urlaubs- und Krankheitsepisoden zeigte sich eine nahezu identische Entwicklung (siehe Anhang Abbildung 24). Durch Einbezug weiterer Stationen war der Median der Überlebenszeit mit 4,5 Monaten etwas höher.

Bei der kumulierten Überlebenswahrscheinlichkeit über die Zeit getrennt nach Schulabschluss fiel auf, dass die Überlebenswahrscheinlichkeit bei der Gruppe derer ohne Schulabschluss am wenigsten sank (siehe Abbildung 21).

\section{Abbildung 21: Kumulierte Überlebenswahrscheinlichkeit getrennt nach Schulabschluss.}

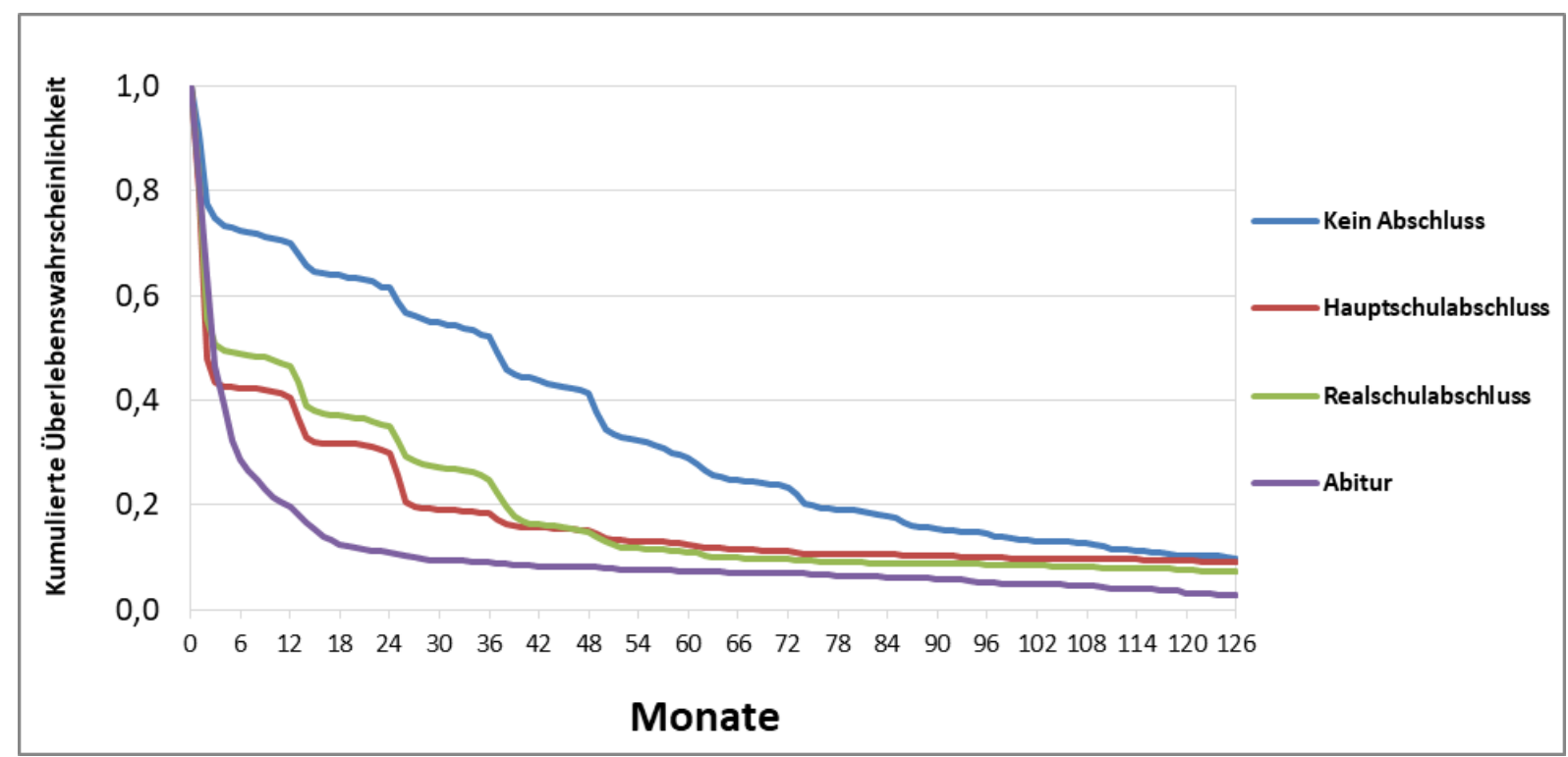

Quelle: Eigene Berechnungen basierend auf dem NEPS. Bezogen auf die Übergangsdauer ohne Wehrdienst, Elternzeit, FSJ/Urlaub/Krankheit. Die Medianwerte der Überlebenszeit betragen für die einzelnen Schulabschlussgruppen: Kein Abschluss: 36,77 Monate, Hauptschulabschluss: 1,93 Monate, Realschulabschluss: 3,59 Monate und Abitur: 2,8 Monate. $\mathrm{N}=9.189$. Aus Platzgründen werden auf der X-Achse nicht alle Monate bis zum Ende des Untersuchungszeitraums angezeigt.

Das bedeutet, dass diejenigen ohne Abschluss am längsten für den Übergang in die Erstausbildung brauchten. Der Median der Überlebenszeit war bei dieser Gruppe mit 36,77 Monaten mit Abstand am höchsten im Vergleich zu den drei anderen Gruppen. Nach drei 
Jahren war die Hälfte derjenigen ohne Schulabschluss in die Ausbildung eingemündet. Bei den Personen mit einem Hauptschulabschluss (Median der Überlebenszeit: 1,93 Monate), mit einer mittleren Reife (3,59 Monate) und mit Abitur (2,8 Monate) vollzog dagegen die Hälfte der jeweiligen Gruppe innerhalb der ersten Monate den Übergang in die Erstausbildung. Die Gruppe derer mit Hauptschulabschluss wiesen in den ersten drei Jahren eine etwas geringere kumulierte Überlebenswahrscheinlichkeit auf als diejenigen mit einem Realschulabschluss. Somit schafften es diejenigen mit Hauptschulabschluss in den ersten drei Jahren etwas schneller, in die Erstausbildung einzumünden als diejenigen mit einer mittleren Reife. Dieser Unterschied hob sich nach spätestens vier Jahren wieder auf und kehrte sich in der Folgezeit dann leicht um in der Form, dass diejenigen mit Hauptschulabschluss eine etwas höhere Überlebenswahrscheinlichkeit hatten als diejenigen mit einer mittleren Reife. Auch ein Großteil der anderen Hälfte der jeweiligen Gruppe schaffte in den folgenden Monaten den Einstieg in den Ausbildungsmarkt deutlich schneller als diejenigen, welche keinen Schulabschluss erreicht hatten. Erst nach etwa acht Jahren (96 Monaten) glichen sich die kumulierten Überlebenswahrscheinlichkeiten aller vier Schulabschlussgruppen an. Dies ist damit zu begründen, dass in jeder der vier Gruppen rechtszensierte Fälle vorhanden waren oder in jeder Gruppe einige wenige Fälle sehr lange Übergangsdauern bis zum Beginn der Erstausbildung aufwiesen.

Auch bei Betrachtung der kumulierten Überlebenswahrscheinlichkeit der Übergangsdauer mit FSJ-, Urlaubs- und Krankheitsepisoden getrennt nach Schulabschluss zeigte sich eine identische Entwicklung, wenngleich die Überlebenswahrscheinlichkeitswerte und die Medianwerte der Überlebenszeit für die einzelnen Gruppen aufgrund der Berücksichtigung weiterer Stationen etwas höher lagen (siehe Anhang - Abbildung 25).

Beim Überblick über die kumulierte Überlebenswahrscheinlichkeit getrennt nach Erstausbildungstypen zeigte sich, dass die Überlebenswahrscheinlichkeit der rechtszensierten Fälle konstant bei einem Wert von 1,0 verblieb (siehe Abbildung 22). 


\section{Abbildung 22: Kumulierte Überlebenswahrscheinlichkeit getrennt nach Erstausbildungstyp.}

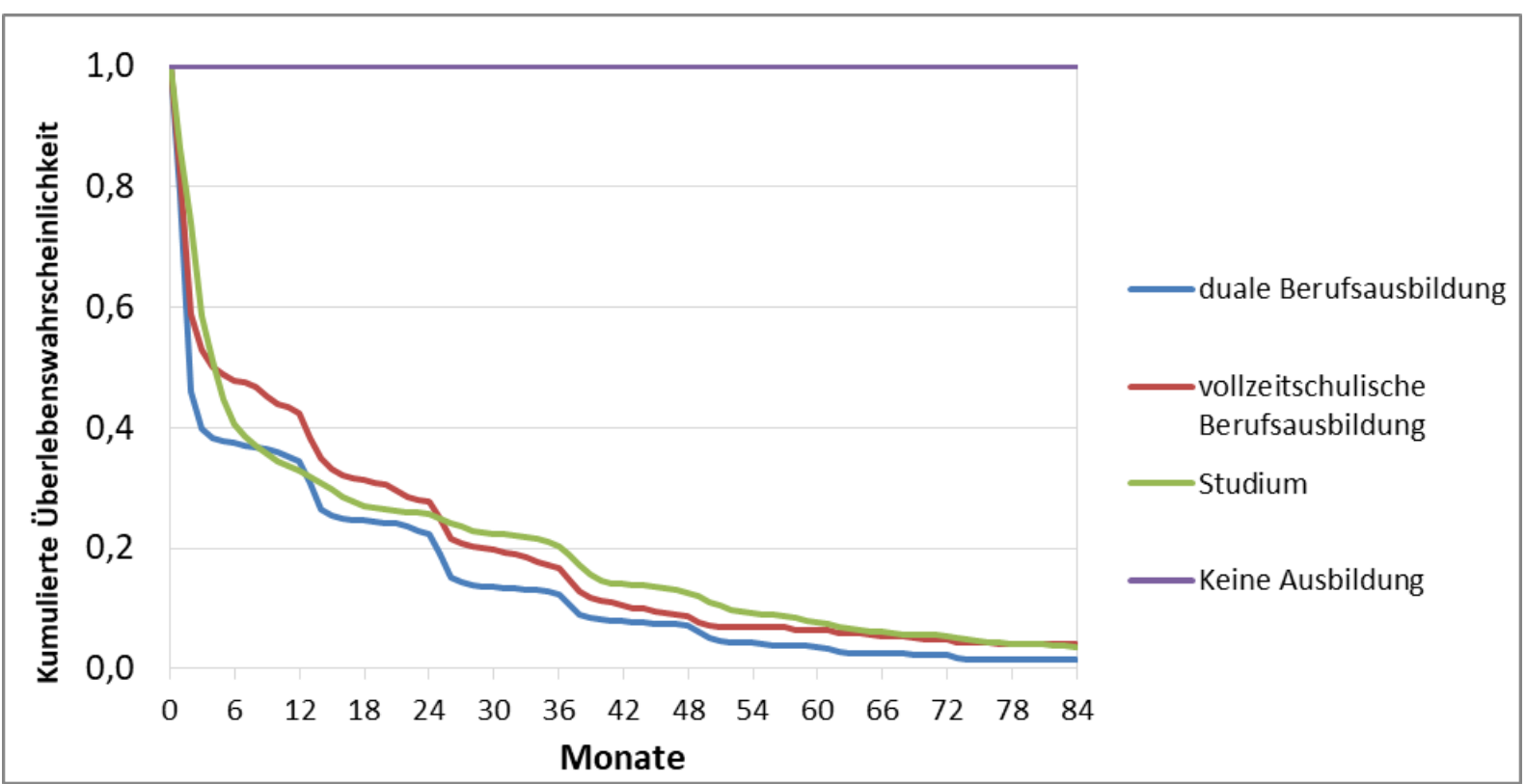

Quelle: Eigene Berechnungen basierend auf dem NEPS. Bezogen auf die Übergangsdauer ohne Wehrdienst, Elternzeit, FSJ/Urlaub/Krankheit. Die Medianwerte der Überlebenszeit betragen für die einzelnen Erstausbildungstypen: Duale Berufsausbildung: 1,87 Monate, Vollzeitschulische Berufsausbildung: 4,1 Monate, Studium: 4,17 Monate und Keine Ausbildung: 154,0 Monate. $\mathrm{N}=9.254$. Aus Platzgründen werden auf der XAchse nicht alle Monate bis zum Ende des Untersuchungszeitraums angezeigt.

Dies erklärt sich daraus, dass diese Gruppe während des gesamten Untersuchungszeitraums keine Ausbildung begonnen hatte und im Übergangsprozess verblieb. Bei den drei anderen Erstausbildungstypen sank die Überlebenswahrscheinlichkeit schon in den ersten Monaten rapide, was belegt, dass bei diesen Gruppen ein erheblicher Anteil zeitnah den Übergang in eine vollqualifizierende Ausbildung realisierte. Diejenigen, die eine duale Berufsausbildung als Erstausbildung aufnahmen, hatten mit leichtem Vorsprung fast durchgehend die geringste kumulierte Überlebenswahrscheinlichkeit. Diese Personen schafften es damit am schnellsten, in die Erstausbildung überzugehen, während diejenigen, die eine vollzeitschulische oder eine akademische Ausbildung aufnahmen, nur unwesentlich länger bis zum Erstausbildungsbeginn benötigten.

Dies stimmte mit den Medianwerten der Überlebenszeit für die drei Erstausbildungstypen überein. Während Personen mit einer dualen Berufsausbildung einen Median der Überlebenszeit von lediglich 1,87 Monaten aufwiesen und damit aus dieser Gruppe die Hälfte der Personen nach 1,87 Monaten den Übergang in die duale Berufsausbildung vollzogen hatte, lagen die Medianwerte für die vollzeitschulische Berufsausbildung (4,1 Monate) und das Studium (4,17 Monate) etwas höher. Die Personengruppe der rechtszensierten Fälle wies für die Überlebenszeit einen Medianwert von 154 Monaten auf. Dies hing damit zusammen, 
dass nach spätestens 154 Monaten alle rechtszensierten Fälle aus der Beobachtung ausgeschieden waren, da sie an einer der darauf folgenden einbezogenen Befragungswellen nicht mehr teilgenommen hatten oder der Untersuchungszeitraum endete. Die gleiche Entwicklung für die kumulierte Überlebenswahrscheinlichkeit zwischen den einzelnen Erstausbildungstypen zeigte sich auch bei der Übergangsdauer mit FSJ-, Urlaubs- und Krankheitsepisoden, bei welcher aufgrund der Berücksichtigung zusätzlicher Stationen die Überlebenswahrscheinlichkeiten und die Medianwerte der Überlebenszeit für die Erstausbildungstypen geringfügig höher waren (siehe Anhang - Abbildung 26).

\subsubsection{Zusammenfassung}

Bei einer zusammenfassenden Betrachtung der Sterbetafel-Analysen konnte festgestellt werden, dass nach etwa drei Monaten die Hälfte aller einbezogenen Personen in eine berufliche oder akademische Erstausbildung übergegangen war. Bei einer nach Schulabschluss differenzierten Betrachtung stellte sich heraus, dass die Hälfe derjenigen mit einem Hauptschulabschluss bereits nach etwa zwei Monaten eine Ausbildung aufgenommen hatte. Die Hälfte aller einbezogenen Personen mit Abitur benötigte hierfür etwa drei Monate und die Hälfte derjenigen mit einem Hauptschulabschluss war nach dreieinhalb Monaten in eine Ausbildung eingemündet. Diejenigen ohne Schulabschluss benötigten deutlich am längsten für den Übergang in die Erstausbildung. Die Hälfte dieser Gruppe hatte erst nach drei Jahren den Übergang vollzogen. Von denjenigen, die in eine duale Berufsausbildung einmündeten, benötigte die Hälfte etwa zwei Monate für den Übergang, während die Hälfte der Personen, die eine vollzeitschulische Ausbildung oder ein Studium aufnahmen, jeweils nach etwa vier Monaten übergegangen war.

\subsection{Cox-Regressionsmodelle}

\subsubsection{Ergebnisse der Cox-Regressionsmodelle zu Hypothesen 1a) und 1b)}

Zunächst war zu beachten, dass zur Verifizierung der aufgestellten Hypothesen bei einem Vergleich der Schulabschlussgruppen eine Differenz mit einer der beiden Gruppen genügte. In den Hypothesen wurden diejenigen mit Abitur und mit Realschulabschluss einerseits und diejenigen mit Hauptschulabschluss und ohne Abschluss andererseits gegenüber gestellt. In den Hypothesen wurde bewusst die Formulierung „mit Hauptschulabschluss oder ohne Abschluss“ gewählt, wodurch es für eine Verifizierung der Hypothese genügte, dass beispielsweise die Übergangsdauer derjenigen mit Abitur kürzer war als bei denjenigen ohne 
Abschluss. Dieses Prinzip galt auch bei den Hypothesen zu den Stationskategorien und den Stationssummen, weshalb vorab darauf hingewiesen wird, um Missverständnisse bei der Interpretation der Ergebnisse zu vermeiden. Sämtliche Cox-Regressionsmodelle wurden nochmals mit dem Schulenddatumsjahr als Kovariate in kategorisierter Form (5-JahresKategorien) berechnet. Dabei ergaben sich nahezu identische Koeffizienten und auch die gleichen Ergebnisse im Hinblick auf die untersuchten Hypothesen, weshalb auf eine gesonderte Darstellung dieser Modelle in dieser Arbeit verzichtet wird.

Das Vorliegen der Proportionalität der Hazards als zentrale Voraussetzung wurde wie im Methodenteil beschrieben mit Hilfe von LML-Plots, Kaplan-Meier-Kurven sowie Plots zu den Schoenfeld-Residuen und dem Test von Therneau and Grambsch grafisch und statistisch überprüft. Dabei konnte insbesondere anhand der grafischen Darstellungen vom Vorliegen einer Proportionalität der Hazards ausgegangen werden. Im Folgenden werden die CoxRegressionsmodelle bezogen auf die Hypothesen der vorliegenden Untersuchung vorgestellt. Den Anfang hierzu macht das Modell, welches die Hypothesen 1a) und 1b) untersucht (siehe Tabelle 6):

1a) Generell verlängert sich die Dauer vom Verlassen der Schule bis zum Beginn der Erstausbildung zwischen 1971 und 2012.

1b) Die Übergangsdauer von Schulabgängerinnen und Schulabgängern mit einem Hauptschulabschluss oder ohne einen Schulabschluss ist länger als bei denjenigen mit Abitur oder einem Realschulabschluss. 


\begin{tabular}{lcccc}
\hline & Koeffizient & $\begin{array}{c}\text { Hazard- } \\
\text { Ratio }\end{array}$ & $\begin{array}{c}\text { Standard- } \\
\text { fehler }\end{array}$ & p-Wert \\
\hline $\begin{array}{l}\text { Schulabschluss } \\
\text { (Referenz: Kein Abschluss) }\end{array}$ & & & $<0,001^{\text {a }}$ \\
$\quad$ Hauptschulabschluss & $\mathbf{0 , 5 1}$ & $\mathbf{1 , 6 6}$ & 0,04 & $<0,001$ \\
$\quad$ Realschulabschluss & $\mathbf{0 , 4 5}$ & $\mathbf{1 , 5 7}$ & 0,04 & $<0,001$ \\
$\quad$ Abitur & $\mathbf{0 , 8 0}$ & $\mathbf{2 , 2 3}$ & 0,04 & $<0,001$ \\
Schulenddatumsjahr & 0,00 & 1,00 & 0,00 & 0,10 \\
Soziale Herkunft - Bildung der Eltern & & & & \\
(Referenzkategorie: kein Abschluss / & & & & $<0,001^{\text {a }}$ \\
Hauptschulabschluss ohne berufliche Ausbildung & & & & \\
(b. A.)) & & & & 0,001 \\
$\quad$ Hauptschulabschluss mit b. A. & $\mathbf{0 , 1 4}$ & $\mathbf{1 , 1 5}$ & 0,04 & 0,359 \\
Mittlere Reife ohne / mit b. A. & 0,04 & 1,05 & 0,05 & 0,774 \\
Hochschulreife ohne / mit b. A. & 0,02 & 1,02 & 0,06 & 0,642 \\
FH-/ Universitätsabschluss & 0,02 & 1,02 & 0,05 & $<0,001$ \\
\hline Geschlecht (weiblich) & $\mathbf{- 0 , 1 3}$ & $\mathbf{0 , 8 8}$ & 0,02 & \\
\hline
\end{tabular}

Quelle: Eigene Berechnungen basierend auf dem NEPS. ${ }^{a}=$ Omnibus-Tests für die Schulabschlüsse und die soziale Herkunft (Nullhypothese: Alle Koeffizienten sind gleich null). Statistisch signifikante Koeffizienten (p $\leq 0,05)$ sind fett gedruckt. $\mathrm{N}=9.040$.

Anhand des in Tabelle 6 dargestellten Cox-Modells wurde deutlich, dass Hypothese 1a) bei Ausschluss von FSJ-, Urlaubs- und Krankheitsepisoden aus der Übergangsdauer falsifiziert werden musste. Durch ein fortlaufendes Schulenddatumsjahr und unter Konstanthaltung aller anderen einbezogenen Kovariaten änderte sich die Übergangsdauer nicht (Hazard-Ratio: 1,00), was in der konstanten Überlebenswahrscheinlichkeit deutlich wurde. Der Effekt war des Weiteren nicht signifikant. Somit veränderte sich durch ein Fortschreiten des Schulenddatumsjahres die Chance der einbezogenen Personen, den Übergangsprozess abzuschließen und in die Erstausbildung überzugehen, zum nächstmöglichen Zeitpunkt nicht, sofern der Erstausbildungsbeginn für diese Personen noch nicht eingetreten war (siehe Tabelle 6). Das identische Ergebnis verbunden mit der Falsifizierung von Hypothese 1a) zeigte sich auch bei Einbezug von FSJ-, Urlaubs- und Krankheitsepisoden in die Übergangsdauer (siehe Anhang - Tabelle 13).

Das in Tabelle 6 vorgestellte Cox-Modell konnte darüber hinaus nachweisen, dass Hypothese 1b) bestätigt wurde. Bei Personen mit Realschulabschluss (Hazard-Ratio: 1,57) und mit 
Abitur (Hazard-Ratio: 2,23) wurde jeweils eine signifikant geringere Überlebenswahrscheinlichkeit festgestellt als bei denjenigen ohne Abschluss. Damit hatten diejenigen mit mittlerer Reife und mit Abitur im Vergleich zur Gruppe ohne Schulabschluss jeweils eine signifikant größere Chance, zum nächstmöglichen Zeitpunkt in die Erstausbildung einzumünden, sofern bei ihnen der Erstausbildungsbeginn als Zielereignis zu diesem Zeitpunkt noch nicht eingetreten war. Das gleiche Ergebnis ergab sich auch bei Einbezug von FSJ-, Urlaubs- und Krankheitsepisoden in die Übergangsdauer, wenngleich marginale Unterschiede der Hazard-Ratio-Koeffizienten gegenüber dem Modell mit der Übergangsdauer ohne FSJ-, Urlaubs- und Krankheitsepisoden bestanden (siehe Anhang Tabelle 13).

\subsubsection{Ergebnisse der Cox-Regressionsmodelle zu Hypothese 2a)}

Das folgende Cox-Regressionsmodell untersucht die Hypothese 2a). In diesem Modell sind der Anteil an Dienstleistungstätigen, der demografische Anteil der unter 20-Jährigen und die Arbeitslosenquote zusätzlich zu den Kovariaten des vorherigen Modells einbezogen (siehe Tabelle 7). Hypothese 2a) ist wie folgt formuliert:

2a) Vor dem Hintergrund eines gestiegenen Dienstleistungstätigenanteils ist eine längere Dauer vom Verlassen der Schule bis zum Beginn der Erstausbildung zu beobachten. 
Tabelle 7: Effekt des Anteils an Dienstleistungstätigen auf die Übergangsdauer (ohne Wehrdienst, Elternzeit, FSJ/Urlaub/Krankheit).

\begin{tabular}{|c|c|c|c|c|}
\hline & Koeffizient & $\begin{array}{c}\text { Hazard- } \\
\text { Ratio }\end{array}$ & $\begin{array}{l}\text { Standard- } \\
\text { fehler }\end{array}$ & p-Wert \\
\hline Prozentualer Anteil der Dienstleistungstätigen & $-0,03$ & 0,97 & 0,03 & 0,317 \\
\hline Prozentualer Anteil der unter 20-Jährigen & $-0,01$ & 0,99 & 0,02 & 0,538 \\
\hline $\begin{array}{l}\text { Arbeitslosenquote am Anteil aller abhängigen } \\
\text { zivilen Erwerbspersonen }\end{array}$ & 0,02 & 1,02 & 0,01 & 0,182 \\
\hline $\begin{array}{l}\text { Schulabschluss } \\
\text { (Referenz: Kein Abschluss) }\end{array}$ & & & & $<0,001^{\mathrm{a}}$ \\
\hline Hauptschulabschluss & $\mathbf{0 , 5 1}$ & 1,67 & 0,04 & $<0,001$ \\
\hline Realschulabschluss & $\mathbf{0 , 4 5}$ & 1,57 & 0,04 & $<0,001$ \\
\hline Abitur & $\mathbf{0 , 8 0}$ & 2,22 & 0,04 & $<0,001$ \\
\hline Schulenddatumsjahr & 0,01 & 1,01 & 0,02 & 0,610 \\
\hline $\begin{array}{l}\text { Soziale Herkunft - Bildung der Eltern } \\
\text { (Referenzkategorie: kein Abschluss / } \\
\text { Hauptschulabschluss ohne berufliche Ausbildung } \\
\text { (b. A.)) }\end{array}$ & & & & $<0,001^{\mathrm{a}}$ \\
\hline Hauptschulabschluss mit b. A. & 0,14 & 1,15 & 0,04 & 0,001 \\
\hline Mittlere Reife ohne / mit b. A. & 0,04 & 1,04 & 0,05 & 0,386 \\
\hline Hochschulreife ohne / mit b. A. & 0,02 & 1,02 & 0,06 & 0,768 \\
\hline FH-/ Universitätsabschluss & 0,02 & 1,02 & 0,05 & 0,658 \\
\hline Geschlecht (weiblich) & $-0,13$ & $\mathbf{0 , 8 8}$ & 0,02 & $<0,001$ \\
\hline
\end{tabular}

Quelle: Eigene Berechnungen basierend auf dem NEPS. ${ }^{a}=$ Omnibus-Tests für die Schulabschlüsse und die soziale Herkunft (Nullhypothese: Alle Koeffizienten sind gleich null). Statistisch signifikante Koeffizienten (p $\leq 0,05)$ sind fett gedruckt. $\mathrm{N}=9.040$.

Basierend auf dem in Tabelle 7 dargestellten Cox-Modell zeigte sich, dass Hypothese 2a) abgelehnt werden musste. Durch einen steigenden Anteil an Dienstleistungstätigen im tertiären Sektor ergab sich unter Konstanthaltung aller anderen einbezogenen Kovariaten keine wesentliche Veränderung der Überlebenswahrscheinlichkeit (Hazard-Ratio: 0,97). Das bedeutete, dass sich durch einen Anstieg des Anteils an Dienstleistungstätigen die Chance, zum nächstmöglichen Zeitpunkt in die Erstausbildung überzugehen, kaum veränderte unter der Voraussetzung, dass für die Personen der Erstausbildungsbeginn noch nicht eingetreten war. Dies ging automatisch mit einer unveränderten Übergangsdauer für alle einbezogenen Personen einher. Dieser Effekt war nicht signifikant und konnte somit auch rein zufällig zustande gekommen sein (siehe Tabelle 7). Das exakte Ergebnis verbunden mit der Ablehnung von Hypothese 2a) zeigte sich bei Berücksichtigung von FSJ-, Urlaubs- und Krankheitsepisoden bei der Übergangsdauer (siehe Anhang - Tabelle 14). 


\subsubsection{Ergebnisse der Cox-Regressionsmodelle zu Hypothese 2b)}

Das dritte Cox-Regressionsmodell (siehe Tabelle 8) beschäftigt sich mit der Untersuchung von Hypothese 2b):

2b) Vor dem Hintergrund eines gestiegenen Dienstleistungstätigenanteils ist eine längere Übergangsdauer derjenigen mit einem Hauptschulabschluss oder ohne Schulabschluss als bei denjenigen mit Abitur oder einem Realschulabschluss zu beobachten.

Tabelle 8: Effekt des Schulabschlusses auf die Übergangsdauer (ohne Wehrdienst, Elternzeit, FSJ/Urlaub/Krankheit) mit Interaktion Schulabschluss und Anteil Dienstleistungstätige.

\begin{tabular}{|c|c|c|c|c|}
\hline & Koeffizient & $\begin{array}{c}\text { Hazard- } \\
\text { Ratio }\end{array}$ & $\begin{array}{l}\text { Standard- } \\
\text { fehler }\end{array}$ & p-Wert \\
\hline Prozentualer Anteil der Dienstleistungstätigen & 0,01 & 1,01 & 0,03 & 0,770 \\
\hline Prozentualer Anteil der unter 20-Jährigen & $-0,03$ & 0,97 & 0,02 & 0,100 \\
\hline $\begin{array}{l}\text { Arbeitslosenquote am Anteil aller abhängigen } \\
\text { zivilen Erwerbspersonen }\end{array}$ & 0,01 & 1,01 & 0,01 & 0,299 \\
\hline $\begin{array}{l}\text { Schulabschluss } \\
\text { (Referenz: Kein Abschluss) }\end{array}$ & & & & $<0,001^{\mathrm{a}}$ \\
\hline Hauptschulabschluss & $\mathbf{0 , 4 9}$ & 1,63 & 0,04 & $<0,001$ \\
\hline Realschulabschluss & $\mathbf{0 , 4 5}$ & $\mathbf{1 , 5 7}$ & 0,04 & $<0,001$ \\
\hline Abitur & $\mathbf{0 , 7 8}$ & 2,18 & 0,04 & $<0,001$ \\
\hline Schulenddatumsjahr & $-0,01$ & 0,98 & 0,03 & 0,528 \\
\hline $\begin{array}{l}\text { Soziale Herkunft - Bildung der Eltern } \\
\text { (Referenzkategorie: kein Abschluss / } \\
\text { Hauptschulabschluss ohne berufliche Ausbildung } \\
\text { (b. A.)) }\end{array}$ & & & & $<0,001^{\mathrm{a}}$ \\
\hline Hauptschulabschluss mit b. A. & 0,14 & 1,15 & 0,04 & 0,001 \\
\hline Mittlere Reife ohne / mit b. A. & 0,05 & 1,05 & 0,05 & 0,344 \\
\hline Hochschulreife ohne / mit b. A. & 0,02 & 1,02 & 0,06 & 0,754 \\
\hline FH-/ Universitätsabschluss & 0,02 & 1,02 & 0,05 & 0,688 \\
\hline Geschlecht (weiblich) & $-0,14$ & $\mathbf{0 , 8 7}$ & 0,02 & $<0,001$ \\
\hline $\begin{array}{l}\text { Hauptschulabschluss } \times \text { Anteil } \\
\text { Dienstleistungstätige }\end{array}$ & $-0,02$ & $\mathbf{0 , 9 8}$ & 0,01 & 0,001 \\
\hline $\begin{array}{l}\text { Realschulabschluss } \times \text { Anteil } \\
\text { Dienstleistungstätige }\end{array}$ & $-0,01$ & 1,00 & 0,01 & 0,323 \\
\hline Abitur $\times$ Anteil Dienstleistungstätige & 0,00 & 1,00 & 0,01 & 0,842 \\
\hline
\end{tabular}

Quelle: Eigene Berechnungen basierend auf dem NEPS. ${ }^{a}=$ Omnibus-Tests für die Schulabschlüsse und die soziale Herkunft (Nullhypothese: Alle Koeffizienten sind gleich null). Die metrisch skalierten unabhängigen Variablen im Modell wurden mittelwertzentriert. Das Schulenddatumsjahr wurde auf das Minimum gesetzt (1971). Statistisch signifikante Koeffizienten $(\mathrm{p} \leq 0,05)$ sind fett gedruckt. $\mathrm{N}=9.040$. 
Das in Tabelle 8 vorgestellte Cox-Modell konnte nachweisen, dass Hypothese 2b) falsifiziert werden musste. Personen mit Realschulabschluss (Hazard-Ratio: 1,57) und mit Abitur (Hazard-Ratio: 2,18) hatten unter Konstanthaltung aller anderen einbezogenen Kovariaten jeweils eine signifikant größere Chance, zum nächstmöglichen Zeitpunkt in die Erstausbildung überzugehen als diejenigen ohne Schulabschluss. Dies galt für diejenigen Personen mit Realschulabschluss und mit Abitur, welche zum jeweiligen Zeitpunkt noch nicht in die Erstausbildung übergegangen waren. Dadurch wiesen diese beiden Schulabschlussgruppen eine signifikant kürzere Übergangsdauer auf als die Gruppe derer ohne Schulabschluss. Der gestiegene Anteil hatte sowohl bei denjenigen mit Realschulabschluss (Hazard-Ratio: 1,00) als auch bei denjenigen mit Abitur (Hazard-Ratio: 1,00) keinen Einfluss auf den Effekt des jeweiligen Schulabschlusses. Des Weiteren waren die Interaktionsterme beider Schulabschlussgruppen nicht signifikant (siehe Tabelle 8). Somit hatte der gestiegene Anteil an Dienstleistungstätigen keinen Einfluss auf die Übergangsdauer derjenigen mit mittlerer Reife und mit Abitur. Auch bei Einbezug von FSJ-, Urlaubs- und Krankheitsepisoden bei der Übergangsdauer zeigte sich, dass diejenigen mit Realschulabschluss und mit Abitur jeweils eine signifikant kürzere Übergangsdauer hatten als die Gruppe derjenigen ohne Abschluss. Jedoch hatte auch bei dieser Analysevariante der Anteil an Dienstleistungstätigen keinen Einfluss auf die Dauer derjenigen mit mittlerer Reife und mit Abitur (siehe Anhang - Tabelle 15). Dadurch musste auch bei Berücksichtigung von FSJ-, Urlaubs- und Krankheitsepisoden bei der Übergangsdauer die Hypothese 2b) abgelehnt werden.

Grafisch konnte nachgewiesen werden, dass Abiturientinnen und Abiturienten mit einem steigenden Anteil an Dienstleistungstätigen eine steigende geschätzte Chance hatten, zum nächstmöglichen Zeitpunkt in die Erstausbildung überzugehen, sofern dies noch nicht eingetreten war (siehe Abbildung 23). 
Abbildung 23: Geschätzte Hazard-Ratio des Schulabschlusses über Anteil der Dienstleistungstätigen auf die Übergangsdauer (ohne FSJ/Urlaub/Krankheit).

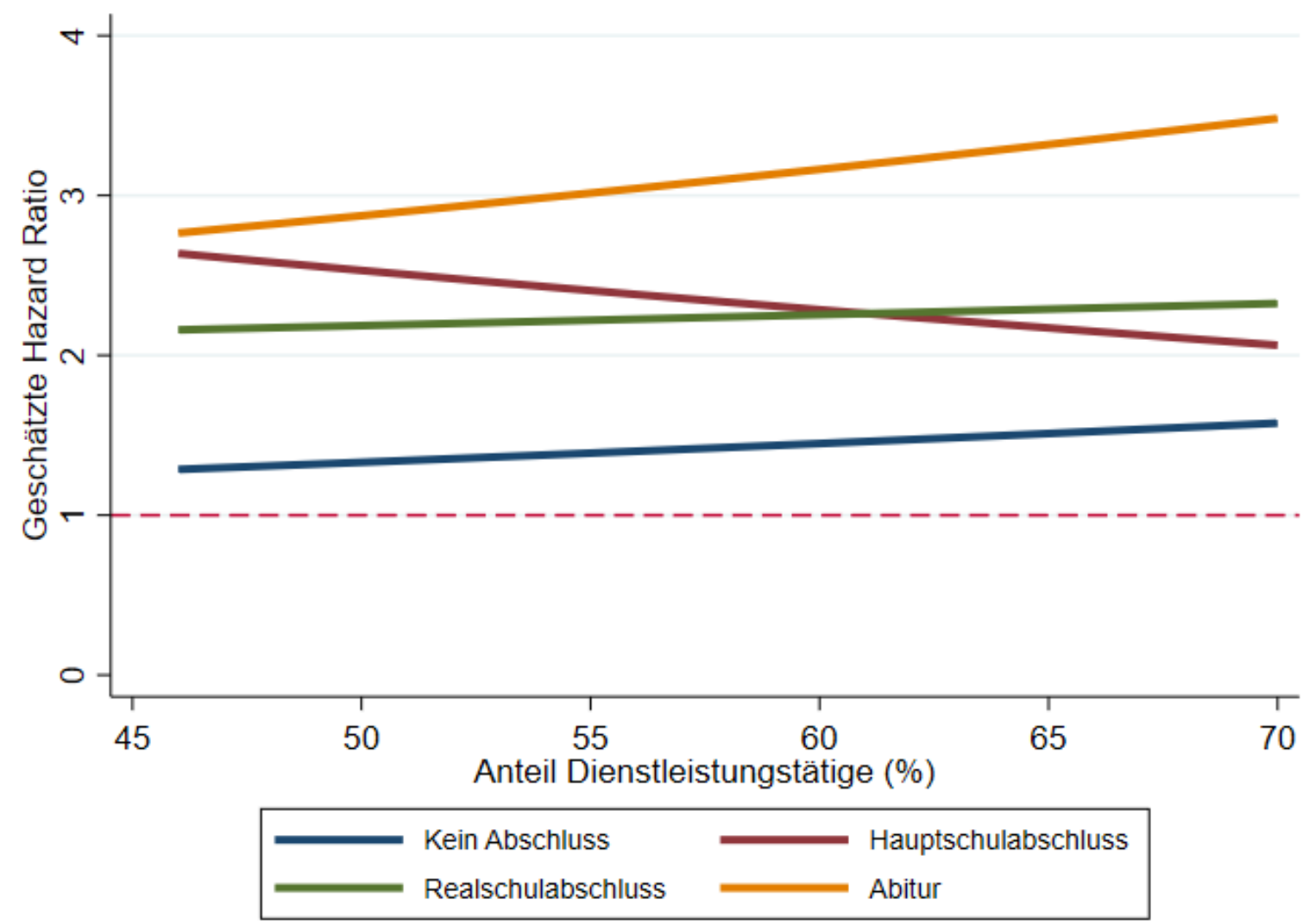

Quelle: Eigene Berechnungen basierend auf dem NEPS. Interaktionseffekt Anteil Dienstleistungstätige und Schulabschluss. $\mathrm{N}=9.040$.

Dagegen sank bei denjenigen mit Hauptschulabschluss die geschätzte Chance, zum nächstmöglichen Zeitpunkt in eine Erstausbildung einzumünden, mit einem steigenden Anteil an Dienstleistungstätigen. Bei Personen mit Realschulabschluss und ohne Schulabschluss führte ein Anstieg des Dienstleistungstätigenanteils zu einer leichten Erhöhung der geschätzten Chance, zum nächstmöglichen Zeitpunkt eine Erstausbildung aufzunehmen, sofern diese bis dahin noch nicht begonnen wurde. Dabei waren die geschätzten Chancen für den Beginn einer Erstausbildung bei beiden Schulabschlussgruppen unabhängig vom Anteil der Dienstleistungstätigen deutlich niedriger als bei Personen mit Abitur. Bei Einbezug von FSJ-, Urlaubs- und Krankheitsepisoden bei der Übergangsdauer zeigte sich grafisch bei den geschätzten Chancen für die Aufnahme einer Erstausbildung ein identisches Ergebnis (siehe Anhang - Abbildung 27). 


\subsubsection{Zusammenfassung zu Ergebnissen der Cox-Regressionsmodelle}

Insgesamt konnten aus den Ergebnissen der Cox-Regressionsmodelle verschiedene Erkenntnisse gewonnen werden. Zunächst zeigte sich, dass ein Fortschreiten des Schulenddatumsjahres keine Veränderung der Übergangsdauer herbeiführte. Somit konnte kein Zeittrend bei der Entwicklung der Übergangsdauer nachgewiesen werden. Dagegen hatten Personen mit Realschulabschluss und mit Abitur eine signifikant kürzere Übergangsdauer als diejenigen ohne Schulabschluss. Ein gestiegener Anteil an Dienstleistungstätigen hatte keinen signifikanten Effekt auf die Übergangsdauer und tangierte auch nicht die Übergangsdauer derjenigen mit mittlerer Reife und mit Abitur. Diese Ergebnisse ergaben sich sowohl bei Ausschluss als auch bei Einbezug von FSJ-, Urlaubs- und Krankheitsepisoden.

\subsection{Sequenzdatenanalysen}

\subsubsection{Deskriptive Ergebnisse}

In Bezug auf die Analysen zu den während des Übergangszeitraums absolvierten Stationen wurden Sequenzdatenanalysen durchgeführt. Dabei wurden die verwendeten Daten so strukturiert, dass jede Person eine Sequenz von absolvierten Stationen vorliegen hatte. Hierbei wurde die Reihenfolge der durchlaufenen Stationen für jede Person anhand der Datierung und der damit einhergehenden Anordnung der jeweiligen Stationen aufbereitet. Personen, welche direkt von der allgemeinbildenden Schule in eine berufliche, vollzeitschulische oder akademische Erstausbildung übergingen, hatten einen Übergangszeitraum von null Monaten und dementsprechend auch keine absolvierten Übergangsstationen vorliegen. Diese Personen wurden im Rahmen dieser Analysen ausgeschlossen. Die übrigen Personen, welche einen Übergangszeitraum von länger als null Monaten und mindestens eine Station aufwiesen, bildeten die Stichprobengrundlage dieser Analysen. Jede einbezogene Person beinhaltete eine Sequenz, was dazu führte, dass die Anzahl der einbezogenen Personen und Sequenzen identisch war.

Bei den Übergangsstationen wurde die Station „Krankheit“ ausgeschlossen, da sie inhaltlich nicht bedeutsam für den Übergang in den Ausbildungs- und Arbeitsmarkt war. Eine Krankheitsepisode trifft die Personen unverschuldet und ist in keinem Fall von diesen geplant. Dies führt in der Regel lediglich zu einer zeitlichen Verzögerung des Übergangs in Abhängigkeit vom individuellen Krankheitsverlauf. Des Weiteren spielte diese Kategorie auf Basis ihrer sehr geringen Häufigkeiten eine marginale Rolle. Aufgrund des Ausschlusses der 
Station „Krankheit“ aus den Übergangsstationen wurde die Summe der Übergangsstationen nochmals neu berechnet. Des Weiteren handelte es sich hierbei um eine Teilstichprobe aus Personen mit einer Übergangssequenz. Die hier ausführlich berichtete Analysevariante berücksichtigte bei den fokussierten Stationskategorien und bei der Stationssumme weder FSJ- und Urlaubsepisoden noch unbestimmte Lückenepisoden. Weiterhin wurden im Rahmen der Sequenzdatenanalysen drei weitere Varianten zu den Stationen berechnet, welche bereits bei der Beschreibung des methodischen Vorgehens vorgestellt wurden. Auf die Ergebnisse der drei anderen Varianten wird am Ende jedes Analyseschrittes zum Vergleich kurz eingegangen. Die dazugehörigen Tabellen und Grafiken werden im Anhang dargestellt.

Zunächst wurde deskriptiv betrachtet, wie oft die einbezogenen Stationen bei den Personen mit einem Übergangszeitraum zwischen dem Verlassen der allgemeinbildenden Schule und der Aufnahme einer dualen, vollzeitschulischen oder akademischen Erstausbildung auftraten. Diese Häufigkeiten der Stationen beinhalteten auch, dass eine Station bei einer Person mehr als einmal auftreten konnte. Deutlich wurde dabei, dass vor allem weitere Schulepisoden sehr häufig während des Übergangs absolviert wurden (47,96 Prozent aller Stationsepisoden). Hierbei handelte es sich oftmals um nacheinander absolvierte Schulepisoden, bei denen Bildungsabschlüsse nacheinander erworben wurden. 23,23 Prozent aller absolvierten Stationen waren dagegen Arbeitslosigkeitsphasen (siehe Tabelle 9).

Tabelle 9: Häufigkeit der Stationen, die von den Personen durchlaufen wurden.

\begin{tabular}{lcc}
\hline $\begin{array}{l}\text { Übergangsstationen }- \text { Sequenzen (ohne FSJ/Urlaub } \\
\text { und ohne Lücken) }\end{array}$ & Häufigkeit & Prozent \\
\hline Arbeitslosigkeit & 1.401 & 23,23 \\
Berufsvorbereitende Maßnahmen & 716 & 11,87 \\
Kurse/Weiterbildungen/Praktika & 603 & 10,00 \\
Hausfrau/-mann & 101 & 1,67 \\
Weitere Schulepisoden & 2.892 & 47,96 \\
Etwas Anderes gemacht & 317 & 5,26 \\
\hline Gesamt & 6.030 & 100,00 \\
\hline
\end{tabular}

Quelle: Eigene Berechnungen basierend auf dem NEPS. N = 6.030 absolvierte Stationen insgesamt.

Bei Betrachtung der Muster der verschiedenen Übergangsverläufe zeigte sich deutlich, dass ein Großteil der Übergangssequenzen durch das Absolvieren einer Schulepisode gekennzeichnet war. Bei einigen Verläufen wurden zwei Schulepisoden nacheinander absolviert. Ein geringerer Anteil an Übergangsverläufen ließ sich durch eine 
Arbeitslosigkeitsepisode oder durch eine berufsvorbereitende Maßnahme charakterisieren (siehe Abbildungen 24 und 25). Damit wurde deutlich, dass die allermeisten Übergangsverläufe der einbezogenen Personen lediglich ein Muster mit einer einzigen absolvierten Station aufwiesen und es sich hierbei am häufigsten um eine weitere, auf die erste allgemeinbildende Schulzeit aufbauende, Schulepisode handelte.

\section{Abbildung 24: Grafische Verteilung der Übergangsverläufe.}
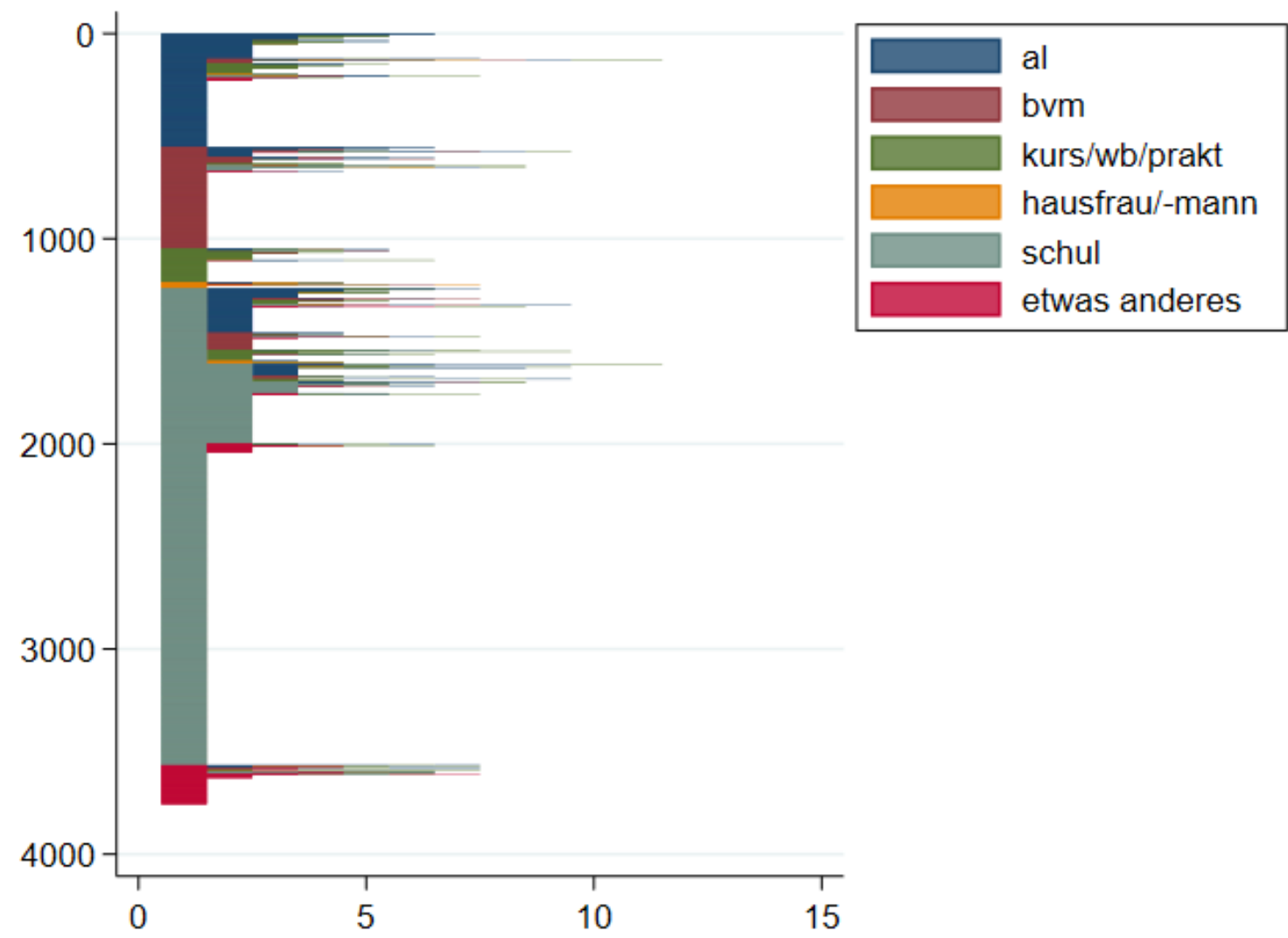

Quelle: Eigene Berechnungen basierend auf dem NEPS. Die Übergangsstationen sind in der Legende den Farben zugewiesen. Abkürzungen: al = Arbeitslosigkeit, bvm $=$ Berufsvorbereitende Maßnahme, kurs/wb/prakt = Kurs/Weiterbildung/Praktikum, hausfrau/-mann = Tätigkeit als Hausfrau/-mann, schul = Schulepisode, etwas anderes = Etwas anderes gemacht. X-Achse: Anzahl der absolvierten Stationen. Y-Achse: Häufigkeit der Übergangssequenzen, insgesamt: $\mathrm{N}=3.755$ Personen mit Übergangsverläufen. 


\section{Abbildung 25: Grafischer Verlauf aller Übergangsverläufe.}

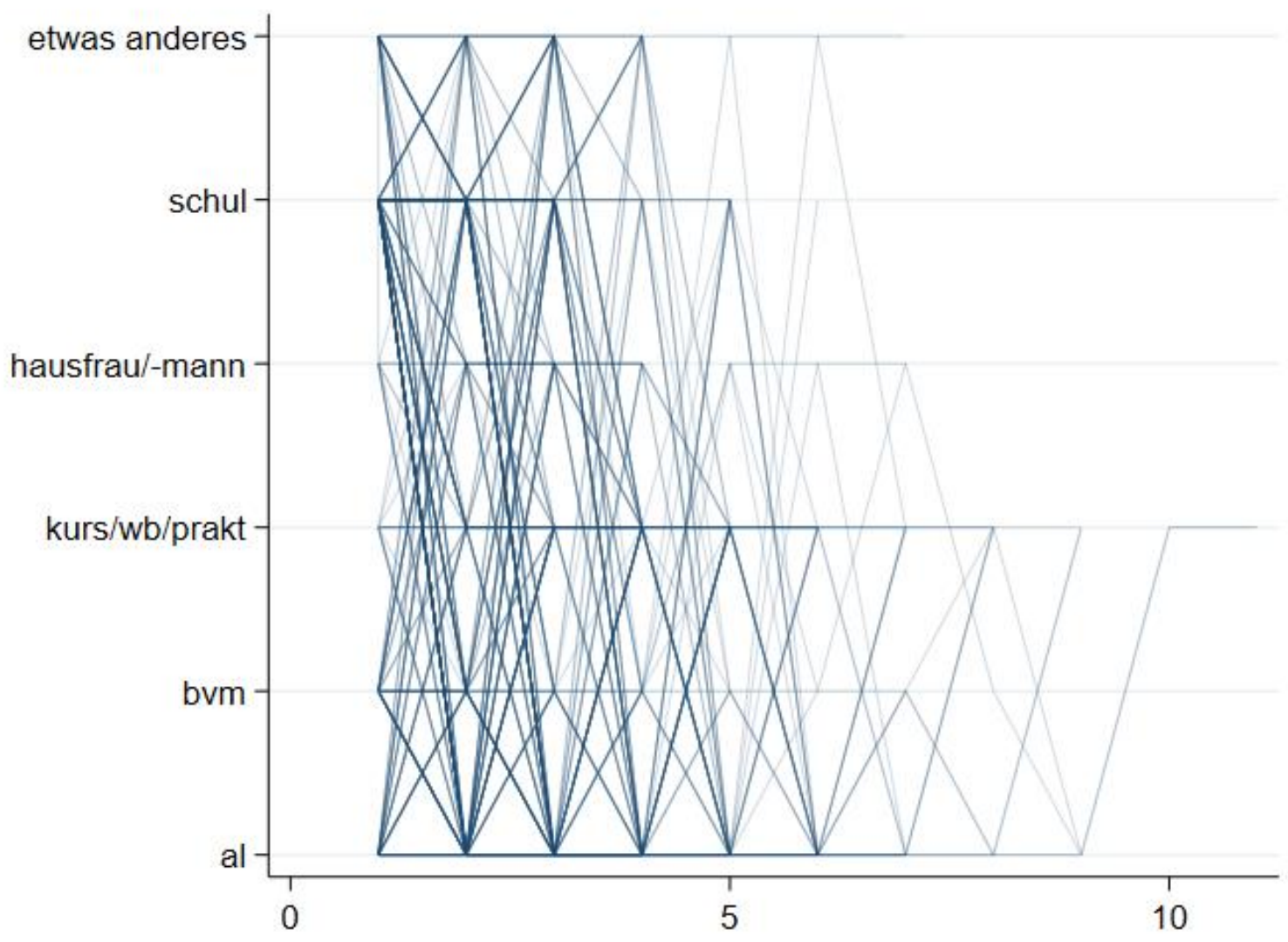

Quelle: Eigene Berechnungen basierend auf dem NEPS. Abkürzungen der Übergangsstationen: al = Arbeitslosigkeit, bvm = Berufsvorbereitende Maßnahme, kurs/wb/prakt $=$ Kurs/Weiterbildung/Praktikum, hausfrau/-mann = Tätigkeit als Hausfrau/-mann, schul = Schulepisode, etwas anderes = Etwas anderes gemacht. X-Achse: Anzahl der absolvierten Übergangsstationen. N = 3.755 Personen mit Übergangsverläufen.

Des Weiteren wurde bei den deskriptiven Auswertungen im Rahmen der Sequenzdatenanalysen ein Blick auf die zehn häufigsten Übergangssequenzen beziehungsweise Übergangsverläufe geworfen. Hierbei ist $\mathrm{zu}$ beachten, dass jede der einbezogenen Personen eine Übergangssequenz aufwies, deren häufigste Muster dadurch identifiziert werden sollten. 50,99 Prozent der zehn häufigsten Übergangssequenzen bestanden aus dem Absolvieren einer weiteren Schulepisode. 12,56 Prozent der zehn häufigsten Übergangssequenzen beinhalteten das Durchlaufen einer berufsvorbereitenden Maßnahme und 10,86 Prozent bestanden aus einer Arbeitslosigkeitsepisode. Bei 7,95 Prozent der zehn häufigsten Übergangsverläufe wurden zwei weitere Schulepisoden nacheinander absolviert (siehe Tabelle 10; Abbildung 26). 
Tabelle 10: Verteilung der zehn häufigsten Übergangssequenzen beziehungsweise Übergangsmuster.

\begin{tabular}{lcc}
\hline Sequenzmuster & Häufigkeit & Prozent \\
\hline Schulepisode & 1.526 & 50,99 \\
Berufsvorbereitende Maßnahme & 376 & 12,56 \\
Arbeitslosigkeit & 325 & 10,86 \\
Zwei Schulepisoden nacheinander & 238 & 7,95 \\
Schulepisode, dann Arbeitslosigkeit & 129 & 4,31 \\
Etwas Anderes & 128 & 4,28 \\
Kurs/Weiterbildung/Praktikum & 106 & 3,54 \\
Zwei Arbeitslosigkeiten nacheinander & 69 & 2,31 \\
Schulepisode, dann berufsvorbereitende Maßnahme & 59 & 1,97 \\
Zwei Schulepisoden nacheinander, dann Arbeitslosigkeit & 37 & 1,24 \\
\hline Gesamt & 2.993 & 100,00
\end{tabular}

Quelle: Eigene Berechnungen basierend auf dem NEPS. Die zehn häufigsten Übergangsverläufe wurden von N $=2.993$ Personen durchlaufen.

\section{Abbildung 26: Grafischer Verlauf der zehn häufigsten Übergangsverläufe.}

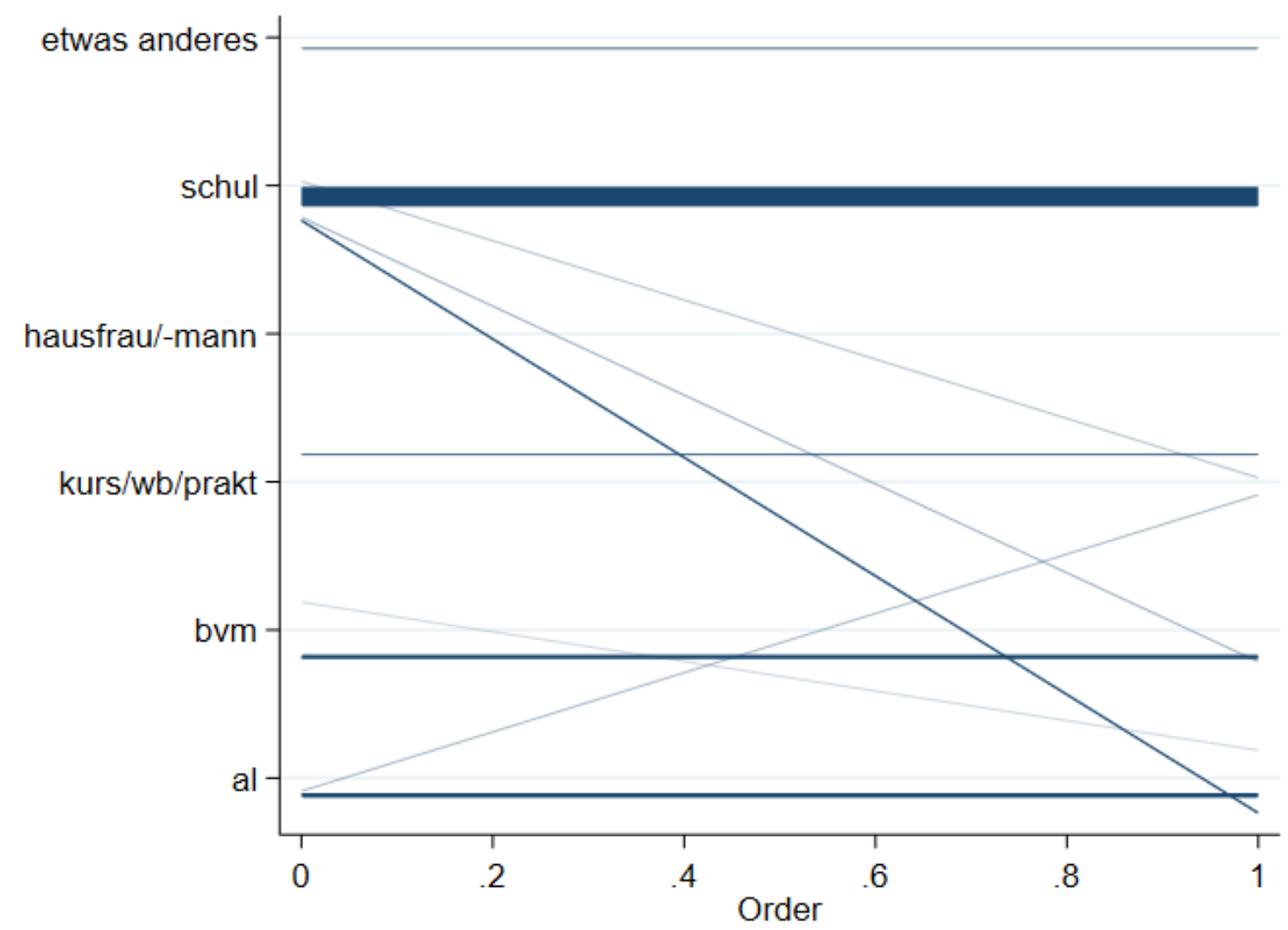

Quelle: Eigene Berechnungen basierend auf dem NEPS. Abkürzungen der Übergangsstationen: al = Arbeitslosigkeit, bvm = Berufsvorbereitende Maßnahme, kurs/wb/prakt $=$ Kurs/Weiterbildung/Praktikum, hausfrau/-mann = Tätigkeit als Hausfrau/-mann, schul $=$ Schulepisode, etwas anderes = Etwas anderes gemacht. Die zehn häufigsten Übergangsverläufe wurden von $\mathrm{N}=2.993$ Personen durchlaufen. 
Bei Betrachtung der Sequenzeigenschaften aller 3.755 einbezogenen Personen, bei denen eine Übergangssequenz vorlag, fiel auf, dass diese durchschnittlich 1,61 Stationen während des Übergangs absolvierten. Dabei wurden mindestens eine und maximal bis zu elf Stationen während des Übergangs durchlaufen. Hierbei bestand auch die Möglichkeit, dass Stationen mehrfach innerhalb einer Übergangssequenz auftreten konnten. Im Hinblick auf die Anzahl an unterschiedlichen Stationen, die absolviert wurden, zeigte sich, dass durchschnittlich 1,28 unterschiedliche Stationen durchlaufen wurden. Hierbei handelte es sich innerhalb der Übergangssequenzen um mindestens eine und maximal um fünf unterschiedliche Stationen pro Übergangsverlauf (siehe Tabelle 11). Das mehrfache Auftreten der gleichen Station innerhalb einer Übergangssequenz wurde in dieser Variante nicht mit einbezogen.

Tabelle 11: Deskriptive Statistiken zur Länge der Übergangssequenzen und zur Anzahl an unterschiedlichen Stationen pro Übergangsverlauf.

\begin{tabular}{lccccc}
\hline & Mittelwert & $\begin{array}{c}\text { Standard- } \\
\text { abweichung }\end{array}$ & Minimum & Maximum \\
\hline $\begin{array}{l}\text { Länge der Sequenz } \\
\text { Anzahl der Elemente }\end{array}$ & 1,61 & 1,11 & 1 & 11 \\
$\begin{array}{l}\text { (unterschiedliche Stationen) } \\
\text { Sequenz }\end{array}$ & 1,28 & 0,58 & 1 & 5 \\
\hline
\end{tabular}

Quelle: Eigene Berechnungen basierend auf dem NEPS. Länge der Übergangssequenzen: Anzahl der absolvierten Stationen insgesamt. N = 3.755 Personen mit Übergangsverläufen.

Bezüglich der deskriptiven Verteilung der Anzahl an unterschiedlichen Stationen innerhalb der Übergangssequenzen wurde deutlich, dass 77,9 Prozent aller Übergangsverläufe aus einer Station bestanden. 17,28 Prozent der Übergangsverläufe aller einbezogenen Personen beinhalteten zwei unterschiedliche Stationen, während bei lediglich 4,02 Prozent drei unterschiedliche Übergangsstationen durchlaufen wurden (siehe Tabelle 12). 
Tabelle 12: Häufigkeitsverteilung der Anzahl unterschiedlicher Übergangsstationen in den Übergangssequenzen.

\begin{tabular}{lccc}
\hline $\begin{array}{l}\text { Anzahl unterschiedlicher } \\
\text { Stationen in Sequenzen }\end{array}$ & Häufigkeit & Prozent & Kumulierte Prozent \\
\hline 1 & 2.925 & 77,90 & 77,90 \\
2 & 649 & 17,28 & 95,18 \\
3 & 151 & 4,02 & 99,20 \\
4 & 26 & 0,69 & 99,89 \\
5 & 4 & 0,11 & 100,00 \\
\hline Gesamt & 3.755 & 100,00 & \\
\hline
\end{tabular}

Quelle: Eigene Berechnungen basierend auf dem NEPS. N = 3.755 Personen mit Übergangsverläufen.

Bei Betrachtung der durchschnittlichen Länge der Übergangsverläufe der Personen gemessen an der Anzahl an absolvierten Stationen getrennt nach dem ersten erreichten Schulabschluss fiel auf, dass Personen ohne erworbenen Schulabschluss mit durchschnittlich 1,88 absolvierten Stationen die meisten Stationen während des Übergangs durchliefen. Diejenigen mit Hauptschulabschluss durchliefen durchschnittlich 1,66 Stationen, während die Personen mit Abitur mit 1,45 Übergangsstationen die durchschnittlich geringste Anzahl an absolvierten Stationen während des Übergangsprozesses aufwiesen (siehe Tabelle 13). Hierbei ist zu beachten, dass Stationen in dieser Konstellation auch mehrfach während eines Übergangsverlaufs auftreten konnten. Beispielsweise konnte eine Person während ihres Übergangs mehrere zusätzliche Schulepisoden oder mehrere Arbeitslosigkeitsphasen durchlaufen.

Tabelle 13: Deskriptive Statistik zur Anzahl an absolvierten Übergangsstationen während des Übergangsverlaufs nach Schulabschluss.

\begin{tabular}{lccc}
\hline $\begin{array}{l}\text { Erster erreichter } \\
\text { Schulabschluss }\end{array}$ & Mittelwert & Standardabweichung & Häufigkeit \\
\hline Kein Abschluss & 1,88 & 1,34 & 718 \\
Hauptschulabschluss & 1,66 & 1,19 & 1.011 \\
Realschulabschluss & 1,49 & 0,98 & 1.407 \\
Abitur & 1,45 & 0,85 & 588 \\
\hline Gesamt & 1,60 & 1,11 & 3.724
\end{tabular}

Quelle: Eigene Berechnungen basierend auf dem NEPS. $\mathrm{N}=3.724$ Personen, die einen Übergangsverlauf vorliegen hatten und für die ein Schulabschluss erhoben wurde. 
Bei der Anzahl an unterschiedlichen Stationen, die während des Übergangs absolviert wurden, zeigte sich dagegen ein leicht verändertes Bild. Diejenigen, die als ersten Schulabschluss einen Hauptschulabschluss erreicht hatten, absolvierten durchschnittlich 1,32 unterschiedliche Übergangsstationen und damit geringfügig mehr als diejenigen ohne erreichten Schulabschluss (Mittelwert: 1,30 unterschiedliche Stationen). Personen mit Abitur durchliefen mit durchschnittlich 1,18 unterschiedlichen Übergangsstationen die geringste Anzahl an unterschiedlichen Stationen unter allen Schulabschlussgruppen (siehe Tabelle 14). In dieser Konstellation wurde das mehrfache Auftreten von ein und derselben Station herausgerechnet.

Tabelle 14: Deskriptive Statistik zur Anzahl an unterschiedlichen Übergangsstationen während des Übergangsverlaufs nach Schulabschluss.

\begin{tabular}{lccc}
\hline $\begin{array}{l}\text { Erster erreichter } \\
\text { Schulabschluss }\end{array}$ & Mittelwert & Standardabweichung & Häufigkeit \\
\hline Kein Abschluss & 1,30 & 0,59 & 718 \\
Hauptschulabschluss & 1,32 & 0,62 & 1.011 \\
Realschulabschluss & 1,28 & 0,59 & 1.407 \\
Abitur & 1,18 & 0,44 & 588 \\
\hline Gesamt & 1,28 & 0,58 & 3.724 \\
\hline
\end{tabular}

Quelle: Eigene Berechnungen basierend auf dem NEPS. $\mathrm{N}=3.724$ Personen, die einen Übergangsverlauf vorliegen hatten und für die ein Schulabschluss erhoben wurde.

Bei Betrachtung der deskriptiven Ergebnisse zu den drei weiteren Varianten zeigte sich, dass je nach Variante neben Arbeitslosigkeitsphasen und zusätzlichen Schulepisoden noch Urlaubsepisoden oder Lücken (unbestimmte Übergangsphasen) sehr häufig während des Übergangszeitraums auftraten (siehe Anhang - Tabellen 16, 22 und 28). Bei den Sequenzmustern zeigte sich, dass in Abhängigkeit der jeweiligen Variante nur Schulepisoden, nur Urlaubsphasen, nur Lückenepisoden, Schulepisoden und anschließend Lückenepisoden oder Urlaubsphasen und anschließend Lücken durchlaufen wurden (siehe Anhang Abbildung 28, 29, 31, 32, 34 und 35). Auch bei den zehn häufigsten Verlaufsmustern der drei anderen Analysevarianten wurde deutlich, dass vor allem das Absolvieren einer weiteren Schulepisode, einer Lückenphase oder einer Urlaubsphase besonders häufig frequentiert war (siehe Anhang - Tabellen 17, 23 und 29, Abbildungen 30, 33 und 36). Durch die Berücksichtigung von Urlaubs- sowie FSJ-Episoden und beziehungsweise oder unbestimmten Lückenphasen wiesen die drei anderen Ergebnisvarianten eine durchschnittlich höhere Anzahl 
an absolvierten Stationen generell als auch bei der Anzahl an unterschiedlichen Stationen während des Übergangszeitraums auf (siehe Anhang - Tabellen 18, 24 und 30). Ebenso bestanden die meisten Übergangsverläufe aus einer oder zwei Übergangsstationen (siehe Anhang - Tabellen 19, 25 und 31). Während diejenigen ohne erreichten Schulabschluss durchschnittlich die meisten absolvierten Übergangsstationen generell als auch die meisten unterschiedlichen Stationen aufwiesen, hatten diejenigen Befragten mit Abitur durchschnittlich die geringste Anzahl an absolvierten Stationen generell sowie an unterschiedlichen Stationen während des Übergangs (siehe Anhang - Tabellen 20, 21, 26, 27 , 32 und 33). Somit zeigten sich viele Übereinstimmungen, aber auch einige Unterschiede zu den bereits ausführlich berichteten Ergebnissen der Konstellation ohne Berücksichtigung von Urlaubsphasen und FSJ-Episoden sowie ohne Einbezug von unbestimmten Lückenphasen. Dies war dem Umstand geschuldet, dass durch Berücksichtigung von zusätzlichen und stark frequentierten Stationen zum Teil etwas veränderte Übergangsverlaufsmuster auftraten.

\subsubsection{Multinomial logistische Regressionsmodelle}

In Bezug auf die Analysen zu den Übergangsstationen wurden multinomial logistische Regressionsmodelle verwendet. Dabei wurden die jeweiligen Chancenverhältnisse (Odds Ratios), zu einer der vier gebildeten Stationskategorien zu gehören, für die einzelnen Schulabschlüsse sowie für den Anteil an Dienstleistungstätigen unter Einbezug der im methodischen Vorgehen aufgelisteten Kontrollvariablen berechnet. Im Rahmen der Regressionsdiagnostik zeigte sich eine sehr starke Multikollinearität zwischen dem Schulenddatumsjahr, dem Anteil der Dienstleistungstätigen, dem demografischen Anteil der unter 20-Jährigen und der Arbeitslosenquote. Diese kam dadurch zustande, dass die prozentualen Anteile der drei Makrovariablen nach dem Schulenddatumsjahr zugeordnet wurden. Auch die Voraussetzung der Linearität der Logits war nicht durchgehend erfüllt. Bei einem Ausschluss der Ausreißer der standardisierten Residuen zeigte sich, dass einige wenige Effekte stärker und signifikanter wurden. Des Weiteren stellte sich heraus, dass mehr als $75 \%$ der einbezogenen Fälle außerhalb des berechneten Hebelwerts lagen und damit als extreme Datenpunkte galten. Da ein exorbitanter Informationsverlust durch Ausschluss dieser Fälle vermieden werden sollte, wurden diese beibehalten. Die im Rahmen der Regressionsdiagnostik gefundenen Eigenschaften waren bei der Interpretation der Regressionsmodelle zu berücksichtigen. Die Hypothesen 3a), 3b) und 3c) wurden im Rahmen des Modells in Tabelle 15 untersucht: 
3a) Jugendliche mit Abitur absolvieren häufiger Weiterbildungsstationen wie Kurse oder Praktika als Jugendliche mit Haupt- oder ohne Schulabschluss.

3b) Jugendliche mit Realschulabschluss absolvieren häufiger Weiterbildungsstationen wie Kurse oder Praktika als Jugendliche mit Haupt- oder ohne Schulabschluss.

3c) Jugendliche mit Haupt- oder ohne Schulabschluss holen im Vergleich zu denjenigen mit Realschulabschluss häufiger Bildungsabschlüsse nach.

Tabelle 15: Effekt des Schulabschlusses auf die Zugehörigkeit zur jeweiligen Stationskategorie.

\begin{tabular}{|c|c|c|c|}
\hline & $\begin{array}{l}\text { Berufsvorb. vs. } \\
\text { Stationen ohne } \\
\text { Weiterbildung }\end{array}$ & $\begin{array}{l}\text { Kurs/Weiterb./ } \\
\text { Praktikum vs. Stationen } \\
\text { ohne Weiterbildung }\end{array}$ & $\begin{array}{l}\text { Schulepisode vs. } \\
\text { Stationen ohne } \\
\text { Weiterbildung }\end{array}$ \\
\hline \multicolumn{4}{|l|}{$\begin{array}{l}\text { Schulabschluss } \\
\text { (Referenz: Kein Abschluss) }\end{array}$} \\
\hline Hauptschulabschluss & $2,68(0,73) * * *$ & $0,32(0,09) * * *$ & $0,51(0,11) * *$ \\
\hline Realschulabschluss & $2,01(0,54) *$ & $0,87(0,25)$ & $0,67(0,13) *$ \\
\hline Abitur & $0,12(0,04) * * *$ & $0,56(0,15) *$ & $0,01(0,00) * * *$ \\
\hline Schulenddatumsjahr & $1,01(0,01)$ & $0,96(0,01) * * *$ & $0,98(0,01) * *$ \\
\hline \multicolumn{4}{|l|}{ Soziale Herkunft - Bildung der } \\
\hline \multicolumn{4}{|l|}{ Eltern (Referenz: kein Abschluss } \\
\hline \multirow{2}{*}{\multicolumn{4}{|c|}{$\begin{array}{l}\text { / Hauptschulabschluss } \\
\text { berufl. Ausbildung (b. A.)) }\end{array}$}} \\
\hline & & & \\
\hline Hauptschulabschluss mit b. A. & $1,67(0,40) *$ & $0,96(0,28)$ & $1,81(0,37) * *$ \\
\hline Mittlere Reife ohne / mit b. A. & $1,13(0,31)$ & $0,92(0,29)$ & $2,04(0,47) * *$ \\
\hline Hochschulreife ohne/mit b. A. & $1,02(0,35)$ & $0,77(0,29)$ & $2,01(0,56) *$ \\
\hline FH-/ Universitätsabschluss & $0,67(0,21)$ & $0,76(0,25)$ & $2,43(0,60) * * *$ \\
\hline Geschlecht (weiblich) & $0,90(0,13)$ & $1,24(0,19)$ & $1,05(0,13)$ \\
\hline Konstante & $1,34 \mathrm{e}^{-08}\left(2,08 \mathrm{e}^{-07}\right)$ & $1,28 \mathrm{e}^{+31}\left(2,15 \mathrm{e}^{+32}\right) * * *$ & $6,55 \mathrm{e}^{+16}\left(8,47 \mathrm{e}^{+17}\right) * *$ \\
\hline $\mathrm{N}$ & & 3.651 & \\
\hline Log-Likelihood & & $-2958,79$ & \\
\hline McFadden-Pseudo- $\mathrm{R}^{2}$ & & 0,21 & \\
\hline
\end{tabular}

Quelle: Eigene Berechnungen basierend auf dem NEPS. Multinomiale logistische Regression. Die angegebenen Koeffizienten sind Odds Ratios sowie die dazugehörigen Standardfehler (in Klammern). Referenzkategorie bei

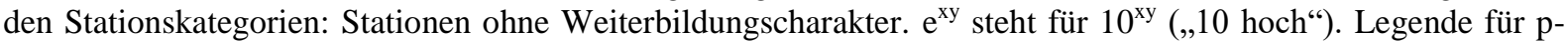
Werte: $* * *<0,001 ; * *<0,01 ; * \leq 0,05$.

Basierend auf dem berechneten Modell zeigte sich, dass Hypothese 3a) verworfen werden musste. Diejenigen mit Abitur absolvierten unter Kontrolle des Schulenddatumsjahres, der 
sozialen Herkunft und des Geschlechts im Vergleich zu denjenigen ohne Abschluss signifikant eher Stationen ohne Weiterbildungscharakter als Kurse, Weiterbildungen oder Praktika (Odds Ratio: 0,56). Dementsprechend befanden sich diejenigen ohne Abschluss eher in Kursen, Weiterbildungen oder Praktika. Dagegen durchliefen Jugendliche mit Hauptschulabschluss signifikant häufiger berufsvorbereitende Maßnahmen als Stationen ohne Weiterbildung (Odds Ratio: 2,68) (siehe Tabelle 15).

Bei den Stationskategorien ohne FSJ- und Urlaubsepisoden, aber mit unbestimmten Lückenepisoden wurde Hypothese 3a) ebenfalls verworfen. Diejenigen mit Abitur absolvierten bei dieser Variante ebenfalls signifikant eher Stationen ohne Weiterbildungscharakter als Kurse, Weiterbildungen oder Praktika. Damit hielten sich diejenigen ohne Abschluss eher in Kursen, Weiterbildungen oder Praktika auf (siehe Anhang Tabelle 36). Bei Einschluss von FSJ- und Urlaubsepisoden und gleichzeitigem Ausschluss von unbestimmten Lückenepisoden aus den Stationskategorien wurde Hypothese 3a) verworfen. Die Gruppe derjenigen mit Abitur befand sich signifikant seltener in Kursen, Weiterbildungen oder Praktika als Personen ohne Schulabschluss (siehe Anhang Tabelle 34). Bei Einbezug sowohl von FSJ- und Urlaubsepisoden als auch von unbestimmten Lückenepisoden bei den Stationskategorien musste Hypothese 3a) verworfen werden. Auch bei dieser Stationsvariante befanden sich Abiturientinnen und Abiturienten signifikant seltener in Kursen, Weiterbildungen oder Praktika als diejenigen ohne Schulabschluss (siehe Anhang Tabelle 38).

Bei der ausführlich vorgestellten Stationsvariante ohne FSJ-, Urlaubs- und unbestimmte Lückenepisoden wurde deutlich, dass Hypothese 3b) nicht eindeutig verworfen werden konnte. Personen mit Realschulabschluss befanden sich eher in Stationen ohne Weiterbildungscharakter und absolvierten damit seltener Kurse, Weiterbildungen oder Praktika als die Gruppe derjenigen ohne Abschluss (Odds Ratio: 0,87). Jedoch war dieser Effekt nicht signifikant und konnte damit auch rein zufälliger Natur sein (siehe Tabelle 15). Bei Ausschluss von FSJ- und Urlaubsepisoden und dem Einbezug von unbestimmten Lückenepisoden bei den Stationskategorien konnte Hypothese 3b) ebenfalls nicht eindeutig verworfen werden. Auch bei dieser Variante befanden sich diejenigen mit Realschulabschluss seltener in Kursen, Weiterbildungen oder Praktika als diejenigen ohne Abschluss, wobei der Effekt nicht signifikant war (siehe Anhang Tabelle 36). Bei den Modellen mit Stationskategorien, welche FSJ- und Urlaubsepisoden enthielten und unbestimmte Lückenepisoden ausgeschlossen hatten, sowie bei Einbezug sowohl von FSJ- und 
Urlaubsepisoden als auch von unbestimmten Lückenepisoden konnte Hypothese 3b) verworfen werden. Bei beiden Stationsvarianten befanden sich Schulabgängerinnen und Schulabgänger mit Realschulabschluss signifikant seltener in Kursen, Weiterbildungen oder Praktika als diejenigen ohne Schulabschluss (siehe Anhang Tabellen 34 und 38).

Bei der hauptsächlich berichteten Variante der Stationskategorien ohne FSJ- und Urlaubsepisoden und ohne unbestimmte Lückenepisoden konnte Hypothese 3c) bestätigt werden. Personen mit Realschulabschluss absolvierten signifikant seltener zusätzliche Schulepisoden als diejenigen ohne Abschluss (Odds Ratio: 0,67). Damit holten diejenigen ohne Abschluss häufiger Bildungsabschlüsse nach als diejenigen mit Realschulabschluss (siehe Tabelle 15). Das identische Ergebnis zeigte sich jeweils auch bei den drei anderen Analysevarianten (siehe Anhang Tabellen 34, 36 und 38).

Im folgenden Regressionsmodell wurden zusätzlich noch die Makrovariablen Anteil der Dienstleistungstätigen im tertiären Sektor, demografischer Anteil der unter 20-Jährigen und die Arbeitslosenquote sowie eine Interaktion zwischen dem Anteil an Dienstleistungstätigen und dem Schulabschluss einbezogen (siehe Tabelle 16). Dieses Modell diente zur Überprüfung folgender Hypothesen:

3d) Jugendliche mit Realschulabschluss absolvieren vor dem Hintergrund eines gestiegenen Dienstleistungstätigenanteils häufiger Weiterbildungsstationen wie Kurse oder Praktika als Jugendliche mit Haupt- oder ohne Schulabschluss.

3e) Jugendliche mit Haupt- oder ohne Schulabschluss holen vor dem Hintergrund eines gestiegenen Dienstleistungstätigenanteils im Vergleich zu denjenigen mit Realschulabschluss häufiger Bildungsabschlüsse nach. 
Tabelle 16: Effekt des Schulabschlusses und des Anteil an Dienstleistungstätigen auf die Zugehörigkeit zur jeweiligen Stationskategorie.

\begin{tabular}{|c|c|c|c|}
\hline & $\begin{array}{l}\text { Berufsvorb. vs. } \\
\text { Stationen ohne } \\
\text { Weiterbildung }\end{array}$ & $\begin{array}{l}\text { Kurs/Weiterb./Prak- } \\
\text { tikum vs. Stationen } \\
\text { ohne Weiterbildung }\end{array}$ & $\begin{array}{l}\text { Schulepisode vs. } \\
\text { Stationen ohne } \\
\text { Weiterbildung }\end{array}$ \\
\hline \multicolumn{4}{|l|}{ Schulabschluss } \\
\hline \multicolumn{4}{|l|}{ (Referenz: Kein Abschluss) } \\
\hline Hauptschulabschluss & $3,10(0,89) * * *$ & $0,37(0,12) * *$ & $0,53(0,11) * *$ \\
\hline Realschulabschluss & $2,14(0,61) * *$ & $0,76(0,23)$ & $0,66(0,13) *$ \\
\hline Abitur & $0,11(0,04) * * *$ & $0,50(0,14) *$ & $0,01(0,00) * * *$ \\
\hline Anteil Dienstleistungstätige & $1,15(0,22)$ & $1,52(0,30) *$ & $1,17(0,18)$ \\
\hline Interaktion Schulabschluss $\times$ Anteil & & & \\
\hline \multicolumn{4}{|l|}{ Dienstleistungstätige } \\
\hline Hauptschulabschluss & $1,00(0,04)$ & $1,02(0,05)$ & $1,03(0,03)$ \\
\hline Realschulabschluss & $0,95(0,04)$ & $0,95(0,04)$ & $1,02(0,03)$ \\
\hline Abitur & $1,01(0,04)$ & $1,02(0,04)$ & $1,00(0,03)$ \\
\hline Demografischer Anteil (unter 20-Jährige) & $0,72(0,09) * *$ & $0,68(0,08) * *$ & $0,72(0,07) * *$ \\
\hline Arbeitslosenquote & $1,04(0,08)$ & $0,87(0,06) *$ & $0,93(0,06)$ \\
\hline Schulenddatumsjahr & $0,83(0,14)$ & $0,65(0,11) *$ & $0,79(0,11)$ \\
\hline \multicolumn{4}{|l|}{ Soziale Herkunft - Bildung der Eltern } \\
\hline (Referenz: kein Abschluss / & & & \\
\hline \multicolumn{4}{|l|}{ Hauptschulabschluss ohne berufl. } \\
\hline \multicolumn{4}{|l|}{ Ausbildung (b. A.)) } \\
\hline Hauptschulabschluss mit b. A. & $1,55(0,38)$ & $0,92(0,27)$ & $1,74(0,36) * *$ \\
\hline Mittlere Reife ohne / mit b. A. & $1,08(0,30)$ & $0,89(0,28)$ & $1,98(0,46) * *$ \\
\hline Hochschulreife ohne/mit b. A. & $1,06(0,37)$ & $0,81(0,31)$ & $2,04(0,58) *$ \\
\hline FH-/ Universitätsabschluss & $0,67(0,21)$ & $0,75(0,24)$ & $2,42(0,60) * * *$ \\
\hline Geschlecht (weiblich) & $0,88(0,13)$ & $1,23(0,19)$ & $1,05(0,13)$ \\
\hline Konstante & $1,7 \mathrm{e}^{+163}\left(5,4 \mathrm{e}^{+165}\right)$ & $-(-) *$ & $4,0 \mathrm{e}^{+202}\left(1,1 \mathrm{e}^{+205}\right)$ \\
\hline $\mathrm{N}$ & & 3.651 & \\
\hline Log-Likelihood & & $-2927,99$ & \\
\hline McFadden-Pseudo-R ${ }^{2}$ & & 0,22 & \\
\hline $\begin{array}{l}\text { Quelle: Eigene Berechnungen basierend a } \\
\text { Koeffizienten sind die Odds Ratios sowie } \\
\text { bei den Stationskategorien: Stationen oh } \\
\text { Variablen im Modell wurden mittelwe } \\
\text { Kurs/Weiterbildung/Praktikum wurden be } \\
\mathrm{e}^{\mathrm{xy}} \text { steht für } 10^{\mathrm{xy}}(, 10 \text { hoch“). Legende für }\end{array}$ & $\begin{array}{l}\text { em NEPS. Multi } \\
\text { dazugehörigen } \\
\text { Weiterbildungscl } \\
\text { entriert (außer } \\
\text { r Konstante kein } \\
\text { Verte: } * * *=<0,0\end{array}$ & $\begin{array}{l}\text { iale logistische Regressi } \\
\text { dardfehler (in Klammerr } \\
\text { kter. Die metrisch skal } \\
\text { Schulenddatumsjahr). } \\
\text { ffizient und kein Standa } \\
*=<0,01 ; *=\leq 0,05 \text {. }\end{array}$ & $\begin{array}{l}\text { n. Die angegebenen } \\
\text { ). Referenzkategorie } \\
\text { erten unabhängigen } \\
\text { Für die Kategorie } \\
\text { dfehler ausgegeben. }\end{array}$ \\
\hline
\end{tabular}


Bei der in der vorliegenden Arbeit im Fokus stehenden Variante der Stationskategorien ohne FSJ- und Urlaubsepisoden und ohne unbestimmte Lückenepisoden zeigte sich, dass Hypothese 3d) nicht eindeutig verworfen werden konnte. Personen mit Realschulabschluss absolvierten seltener Kurse, Weiterbildungen oder Praktika als diejenigen ohne Abschluss (Odds Ratio: 0,76) und befanden sich somit häufiger in Stationen ohne Weiterbildungscharakter. Dieser Effekt war jedoch nicht signifikant. Der Anteil an Dienstleistungstätigen verstärkte diesen Effekt kaum (Odds Ratio: 0,95) und nicht signifikant. Aufgrund der nicht vorhandenen Signifikanz konnte dies auch rein zufällig zustande gekommen sein (siehe Tabelle 16).

Bei Ausschluss von FSJ- und Urlaubsepisoden sowie Berücksichtigung von unbestimmten Lückenepisoden zeigte sich, dass Hypothese 3d) verworfen werden musste. Jugendliche mit Realschulabschluss absolvierten signifikant seltener Kurse, Weiterbildungen oder Praktika als diejenigen ohne Abschluss und befanden sich somit eher in Stationen ohne Weiterbildungscharakter. Der Anteil an Dienstleistungstätigen verstärkte den Effekt derjenigen mit Realschulabschluss kaum und war nicht signifikant (siehe Anhang Tabelle 37). Das identische Ergebnis zeigte sich auch bei den beiden übrigen Analysevarianten, bei denen FSJ- und Urlaubsepisoden bei den Stationskategorien berücksichtigt und unbestimmte Lückenepisoden einmal ausgeschlossen und einmal eingeschlossen wurden (siehe Anhang Tabellen 35 und 39).

Bei den Stationskategorien ohne FSJ-, Urlaubsepisoden und ohne unbestimmte Lückenepisoden stellte sich heraus, dass Hypothese 3e) verworfen werden musste. Schulabgängerinnen und Schulabgänger mit Realschulabschluss befanden sich signifikant seltener in weiteren Schulepisoden als die Gruppe derjenigen ohne Schulabschluss (Odds Ratio: 0,66). Damit holten diejenigen ohne Abschluss signifikant mehr Bildungsabschlüsse nach als diejenigen mit mittlerer Reife. Der Anteil an Dienstleistungstätigen tangierte diesen Effekt kaum (Odds Ratio: 1,02) und nicht signifikant. Damit hatte der gestiegene Dienstleistungstätigenanteil keinen Einfluss auf den Effekt, dass diejenigen ohne Abschluss weitere Schulepisoden absolvierten (siehe Tabelle 16). Bei den drei anderen Analysevarianten wurde Hypothese 3e) ebenfalls verworfen. Dabei ergab sich jeweils lediglich der Unterschied, dass der Anteil der Dienstleistungstätigen den Effekt derjenigen mit Realschulabschluss signifikant beeinflusste. Jedoch waren die Koeffizienten ebenfalls sehr gering, wodurch der Einfluss nahezu nivelliert wurde (siehe Anhang Tabellen 35, 37 und 39). 
Bei Betrachtung der geschätzten Wahrscheinlichkeiten bezüglich der Zuordnung zu den vier Stationskategorien zeigte sich, dass Abiturientinnen und Abiturienten bei einem niedrigen Anteil an Dienstleistungstätigen eine deutlich höhere geschätzte Wahrscheinlichkeit hatten, Stationen ohne Weiterbildungscharakter $\mathrm{zu}$ durchlaufen als die drei anderen Schulabschlussgruppen (siehe Abbildung 27).

\section{Abbildung 27: Geschätzte Wahrscheinlichkeiten, Stationen ohne Weiterbildungscharakter zu durchlaufen, nach Schulabschluss in Abhängigkeit vom Anteil an Dienstleistungstätigen.}

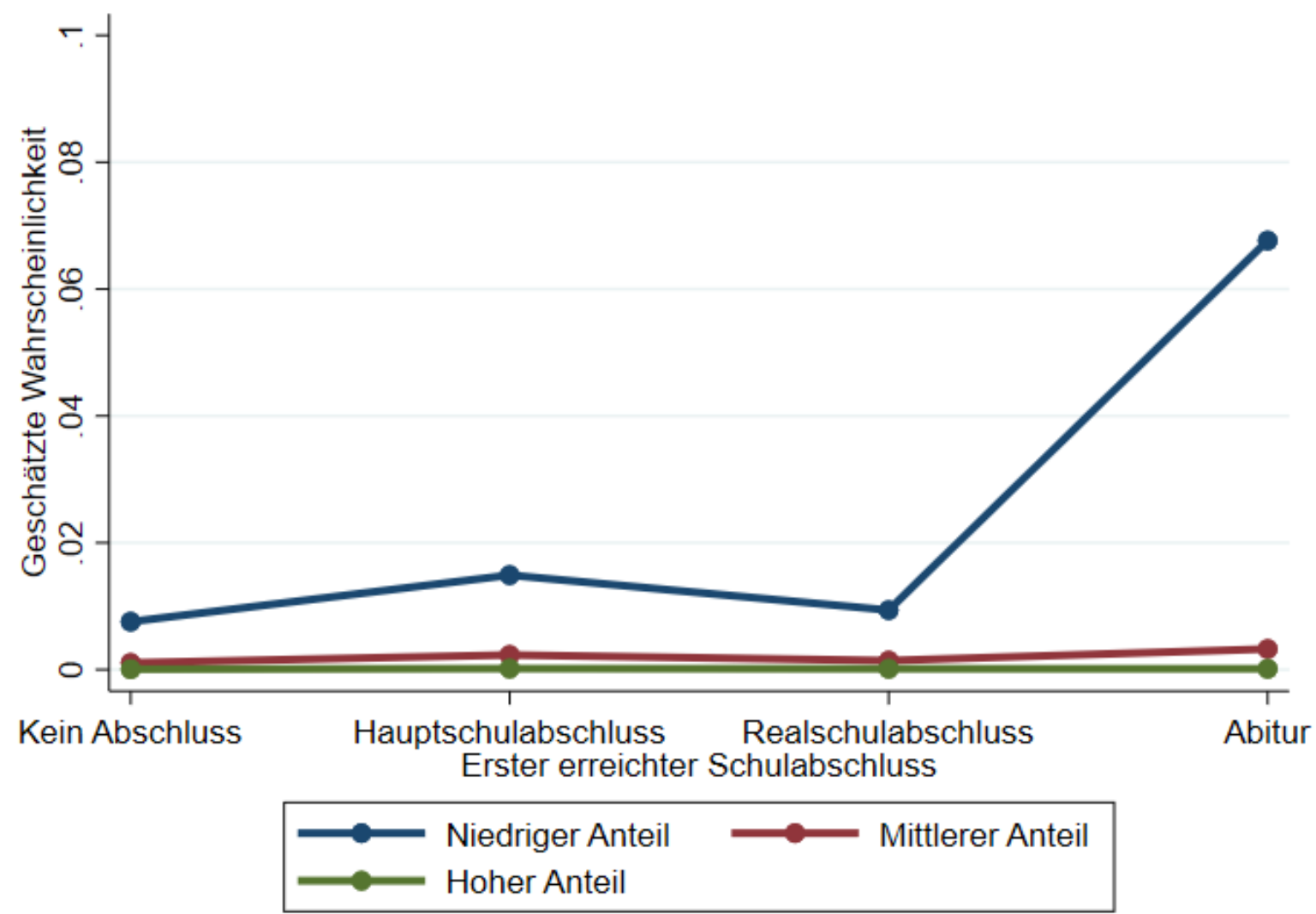

Quelle: Eigene Berechnungen basierend auf dem NEPS. Legende: Grün: Mittelwert des Dienstleistungstätigenanteils +1 Standardabweichung; Rot: Mittelwert des Dienstleistungstätigenanteils; Blau: Mittelwert des Dienstleistungstätigenanteils - 1 Standardabweichung. Fixierung der übrigen unabhängigen Variablen auf folgende Werte: Schulenddatumsjahr: 1971; Soz. Herkunft: kein Abschluss / Hauptschulabschluss ohne berufliche Ausbildung; Geschlecht: weiblich; übrige Variablen (Demografischer Anteil der unter 20Jährigen, Arbeitslosenquote) sind auf ihren jeweiligen Mittelwert fixiert. Um die Unterschiede zwischen den Anteilen besser erkennen zu können, wurde bei der geschätzten Wahrscheinlichkeit auf eine feinere Skalierung auf der Y-Achse $(0$ bis 0,1$)$ zurückgegriffen. $\mathrm{N}=586$.

Jugendliche mit einem Hauptschulabschluss hatten dagegen bei einem niedrigen Anteil an Dienstleistungstätigen die höchste geschätzte Wahrscheinlichkeit, berufsvorbereitende Maßnahmen zu absolvieren. Dies traf auch bei einem mittleren Anteil an Dienstleistungstätigen auf dem Arbeitsmarkt zu (siehe Abbildung 28). 
Abbildung 28: Geschätzte Wahrscheinlichkeiten, berufsvorbereitende Maßnahmen zu durchlaufen, nach Schulabschluss in Abhängigkeit vom Anteil an Dienstleistungstätigen.

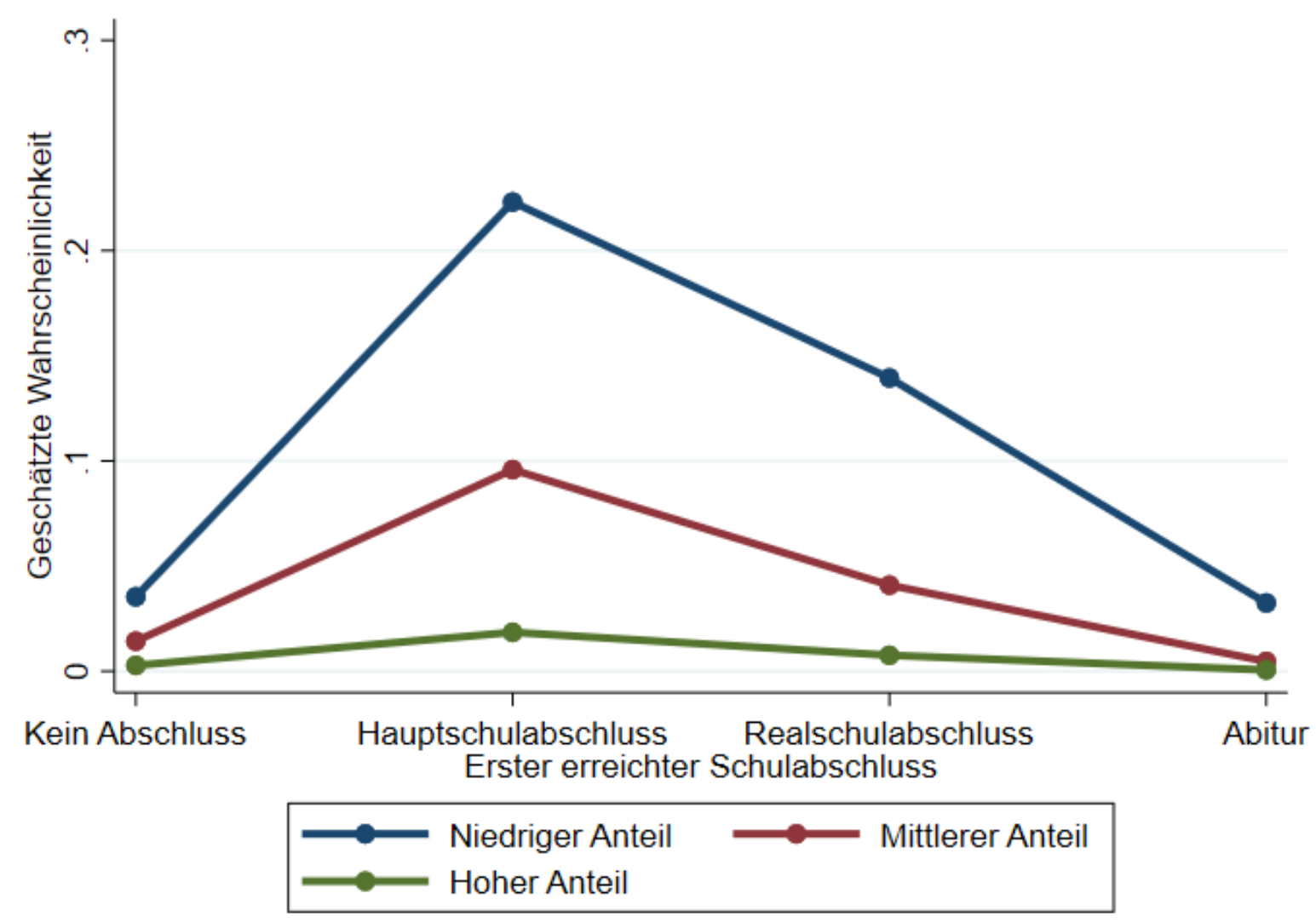

Quelle: Eigene Berechnungen basierend auf dem NEPS. Legende: Grün: Mittelwert des Dienstleistungstätigenanteils +1 Standardabweichung; Rot: Mittelwert des Dienstleistungstätigenanteils; Blau: Mittelwert des Dienstleistungstätigenanteils - 1 Standardabweichung. Fixierung der übrigen unabhängigen Variablen auf folgende Werte: Schulenddatumsjahr: 1971; Soz. Herkunft: kein Abschluss / Hauptschulabschluss ohne berufliche Ausbildung; Geschlecht: weiblich; übrige Variablen (Demografischer Anteil der unter 20Jährigen, Arbeitslosenquote) sind auf ihren jeweiligen Mittelwert fixiert. Um die Unterschiede zwischen den Anteilen besser erkennen zu können, wurde bei der geschätzten Wahrscheinlichkeit auf eine feinere Skalierung auf der Y-Achse (0 bis 0,3$)$ zurückgegriffen. $\mathrm{N}=459$.

Während der Anteil an Dienstleistungstätigen bei denjenigen mit Abitur keine Auswirkung auf die geschätzte Wahrscheinlichkeit hatte, Kurse, Weiterbildungen oder Praktika zu absolvieren, zeigten sich bei den drei anderen Schulabschlussgruppen zum Teil deutliche Unterschiede. Je höher der Anteil an Dienstleistungstätigen auf dem Arbeitsmarkt war, desto größer gestaltete sich die geschätzte Wahrscheinlichkeit, Kurse, Weiterbildungen oder Praktika zu durchlaufen für diejenigen ohne Abschluss, mit Hauptschulabschluss und mit mittlerer Reife. Am deutlichsten stellte sich dies bei denjenigen mit Hauptschulabschluss dar. Ein hoher Anteil an Dienstleistungstätigen führte bei dieser Gruppe zu einer sehr hohen geschätzten Wahrscheinlichkeit, Kurse, Weiterbildungen oder Praktika zu absolvieren. Bei einem mittleren und insbesondere bei einem niedrigen Anteil an Dienstleistungstätigen sank 
die geschätzte Wahrscheinlichkeit, Kurse, Weiterbildungen oder Praktika zu absolvieren, jedoch deutlich herab. Die gleiche Konstellation, wenn auch weniger deutlich, zeigte sich für diejenigen ohne Abschluss und mit mittlerer Reife (siehe Abbildung 29).

\section{Abbildung 29: Geschätzte Wahrscheinlichkeiten, Kurse, Weiterbildungen oder Praktika zu durchlaufen, nach Schulabschluss in Abhängigkeit vom Anteil an Dienstleistungstätigen.}

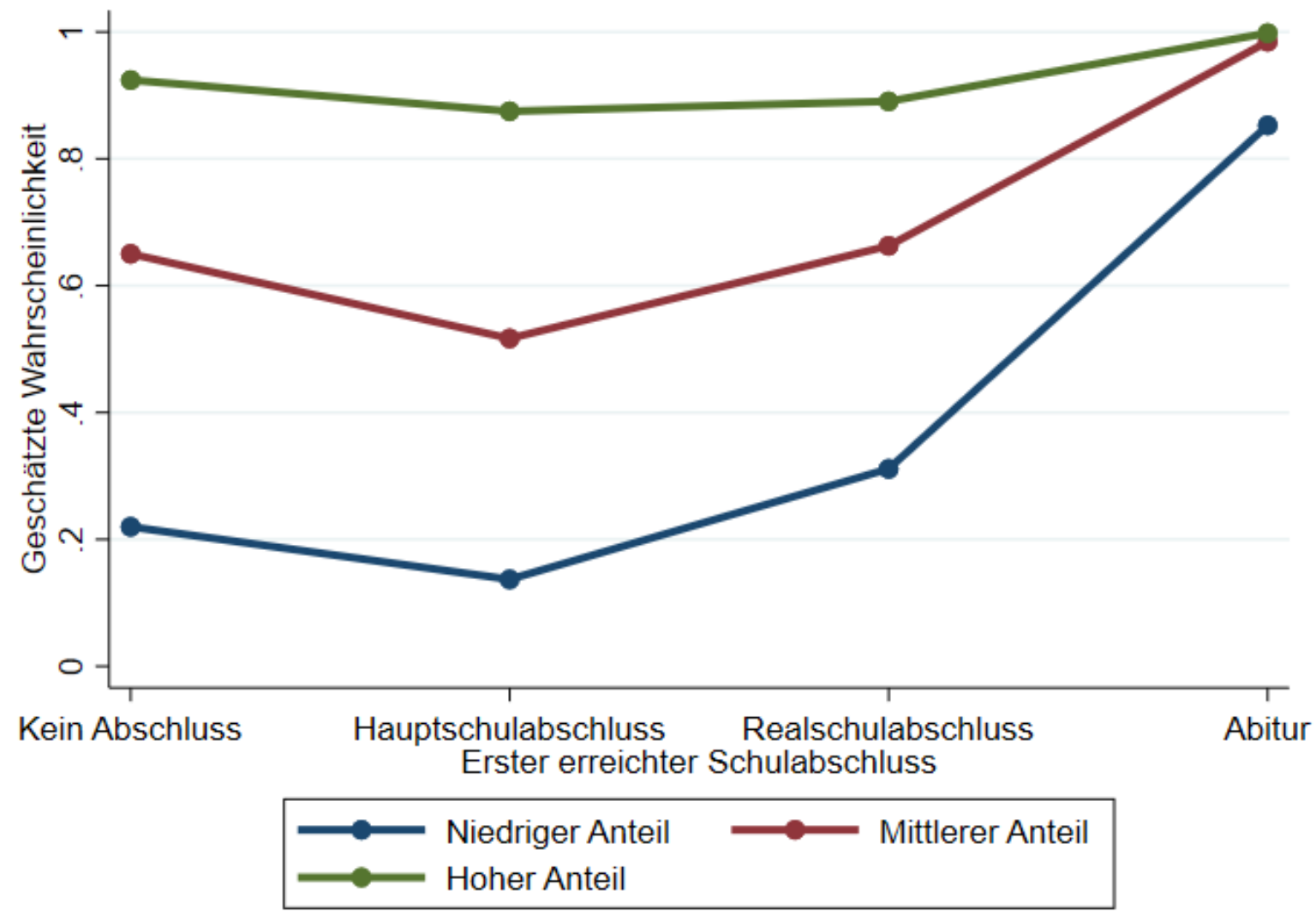

Quelle: Eigene Berechnungen basierend auf dem NEPS. Legende: Grün: Mittelwert des Dienstleistungstätigenanteils +1 Standardabweichung; Rot: Mittelwert des Dienstleistungstätigenanteils; Blau: Mittelwert des Dienstleistungstätigenanteils - 1 Standardabweichung. Fixierung der übrigen unabhängigen Variablen auf folgende Werte: Schulenddatumsjahr: 1971; Soz. Herkunft: kein Abschluss / Hauptschulabschluss ohne berufliche Ausbildung; Geschlecht: weiblich; übrige Variablen (Demografischer Anteil der unter 20Jährigen, Arbeitslosenquote) sind auf ihren jeweiligen Mittelwert fixiert. $\mathrm{N}=263$.

Bei einem niedrigen Anteil an Dienstleistungstätigen war die geschätzte Wahrscheinlichkeit derjenigen ohne Abschluss besonders hoch, weitere Schulepisoden zu durchlaufen. Auch bei denjenigen mit Haupt- oder Realschulabschluss war bei einem niedrigen Anteil an Dienstleistungstätigen auf dem Arbeitsmarkt die geschätzte Wahrscheinlichkeit, weiter die Schule zu besuchen deutlich erhöht, wenn auch nicht ganz so hoch wie bei denjenigen ohne Schulabschluss. Bei einem mittleren und einem hohen Anteil an Dienstleistungstätigen unterschieden sich diejenigen ohne Abschluss, mit Haupt- und mit Realschulabschluss kaum in ihren geschätzten Wahrscheinlichkeiten, weitere Schulepisoden zu durchlaufen. Dabei 
zeigte sich, dass sich bei einem mittleren und vor allem bei einem hohen Anteil an Dienstleistungstätigen die geschätzten Wahrscheinlichkeiten, weiter die Schule zu besuchen, im Vergleich zu einem niedrigen Anteil an Dienstleistungstätigen für diese drei Gruppen deutlich verringerten (siehe Abbildung 30).

Abbildung 30: Geschätzte Wahrscheinlichkeiten, zusätzliche Schulepisoden zu durchlaufen,
nach Schulabschluss in Abhängigkeit vom Anteil an Dienstleistungstätigen.

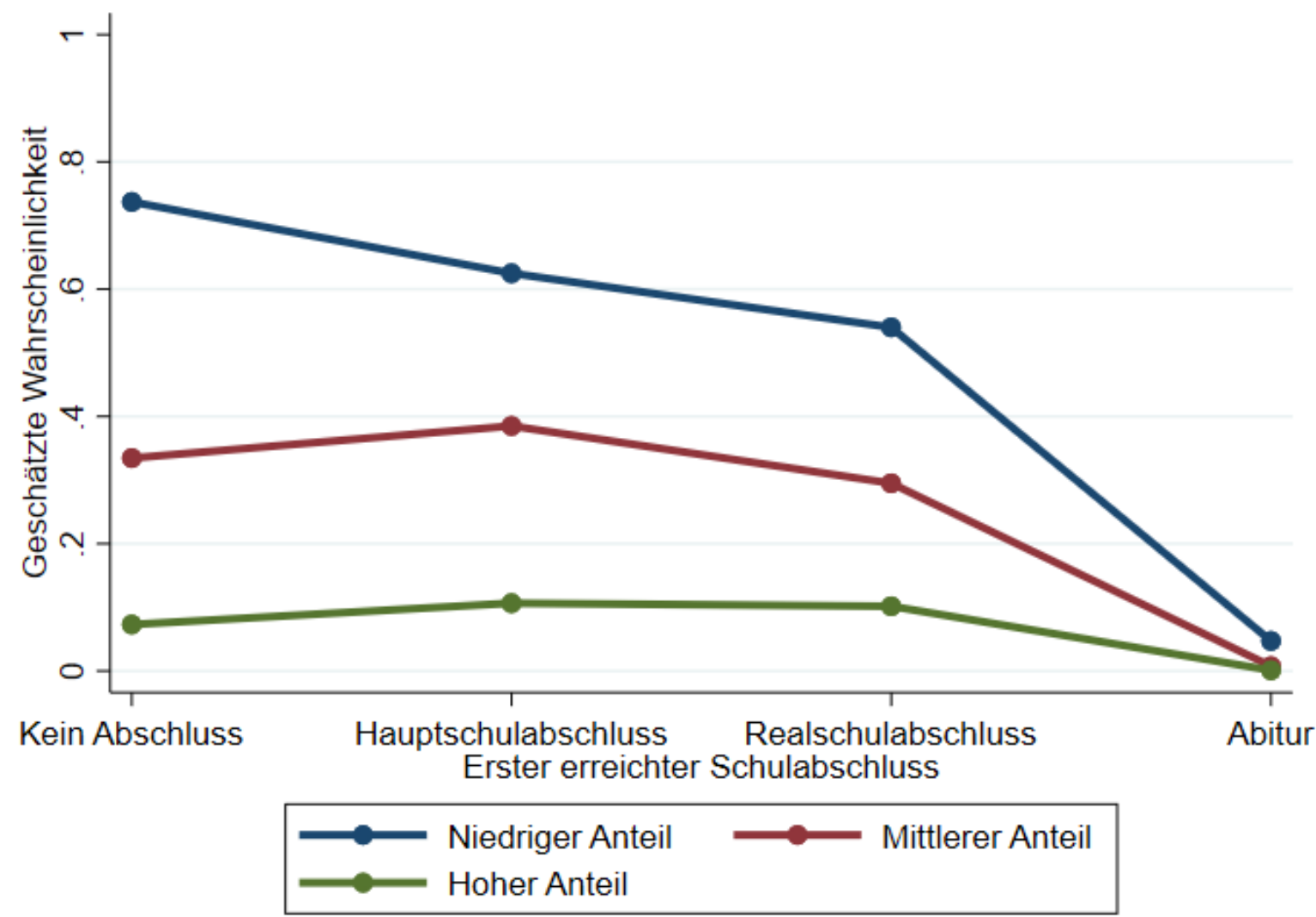

Quelle: Eigene Berechnungen basierend auf dem NEPS. Legende: Grün: Mittelwert des Dienstleistungstätigenanteils + 1 Standardabweichung; Rot: Mittelwert des Dienstleistungstätigenanteils; Blau: Mittelwert des Dienstleistungstätigenanteils - 1 Standardabweichung. Fixierung der übrigen unabhängigen Variablen auf folgende Werte: Schulenddatumsjahr: 1971; Soz. Herkunft: kein Abschluss / Hauptschulabschluss ohne berufliche Ausbildung; Geschlecht: weiblich; übrige Variablen (Demografischer Anteil der unter 20Jährigen, Arbeitslosenquote) sind auf ihren jeweiligen Mittelwert fixiert. N = 2.343.

Bei Betrachtung der geschätzten Wahrscheinlichkeiten der drei anderen Analysenvarianten in Bezug auf die Stationskategorien zeigten sich sehr ähnliche Ergebnisse. Bei allen drei Varianten hatten bei einem niedrigen Anteil an Dienstleistungstätigen diejenigen mit Abitur die höchste geschätzte Wahrscheinlichkeit, Stationen ohne Weiterbildungscharakter zu durchlaufen, während diejenigen mit Hauptschulabschluss die höchste geschätzte Wahrscheinlichkeit aufwiesen, berufsvorbereitende Maßnahmen zu absolvieren. Bei einem hohen Anteil an Dienstleistungstätigen hatten alle Schulabschlussgruppen eine ähnlich hohe 
geschätzte Wahrscheinlichkeit, Kurse, Weiterbildungen oder Praktika zu durchlaufen. Bei einem mittleren und niedrigen Anteil an Dienstleistungstätigen lagen die geschätzten Wahrscheinlichkeiten bei denjenigen ohne Abschluss, mit Haupt- und mit Realschulabschluss dagegen niedriger als bei den Abiturientinnen und Abiturienten. Bei einem niedrigen Anteil an Dienstleistungstätigen zeigte sich bei den drei weiteren Analysevarianten der Stationskategorien ebenfalls, dass diejenigen ohne Abschluss am wahrscheinlichsten weitere Schulepisoden durchliefen (siehe Anhang - Abbildungen 37 - 48).

\subsubsection{Negative binomiale Regressionsmodelle}

Da bei allen berechneten Poisson-Regressionsmodellen eine „Overdispersion“, also eine im Vergleich zum Mittelwert größere Varianz der abhängigen Zählvariablen vorlag, wurden grundsätzlich negative binomiale Regressionsmodelle bei den folgenden Hypothesen zur Untersuchung der Anzahl an Übergangsstationen verwendet. Bei den negativen binomialen Regressionsmodellen war eine Linearität der Logits nicht durchgehend gegeben, was bei der Interpretation der Modelle berücksichtigt werden musste. Bei der hier betrachteten Analysevariante wurden bei den Übergangsstationen sowohl FSJ- und Urlaubsepisoden als auch unbestimmte Lückenepisoden nicht einbezogen. FSJ- und Urlaubsepisoden waren dadurch auch bei der als Exposure-Variable verwendeten Übergangsdauer in dieser Analysevariante nicht berücksichtigt. Im ersten Modell (siehe Tabelle 17) wurden die Hypothesen 4a) und 4b) untersucht:

4a) Generell erhöht sich die Anzahl der Stationen vom Verlassen der Schule bis zum Beginn der Erstausbildung zwischen 1971 und 2012.

4b) Die Anzahl der Stationen ist bei denjenigen ohne Schulabschluss oder mit Hauptschulabschluss höher als bei denjenigen mit Abitur oder Realschulabschluss. 

Übergangsstationen.

IRR (SE)

\begin{tabular}{lc}
\hline Schulabschluss (Referenz: Kein Abschluss) & \\
$\quad$ Hauptschulabschluss & $1,17(0,05)^{* * *}$ \\
$\quad$ Realschulabschluss & $1,06(0,04)$ \\
$\quad$ Abitur & $1,68(0,08)^{* * *}$ \\
Schulenddatumsjahr & $1,01(0,00)^{* * *}$ \\
Soziale Herkunft - Bildung der Eltern (Referenz: kein Abschluss / & \\
Hauptschulabschluss ohne berufl. Ausbildung (b. A.)) & \\
$\quad$ Hauptschulabschluss mit b. A. & $1,09(0,05)$ \\
$\quad$ Mittlere Reife ohne / mit b. A. & $1,05(0,06)$ \\
$\quad$ Hochschulreife ohne/mit b. A. & $1,11(0,07)$ \\
$\quad$ FH-/ Universitätsabschluss & $1,11(0,06)$ \\
Geschlecht (weiblich) & $0,89(0,02)^{* * *}$ \\
Konstante & $1,14 \mathrm{e}^{-12}\left(3,29 \mathrm{e}^{-12}\right)^{* * *}$ \\
\hline Logarithmus(Übergangsdauer) & $1\left(\operatorname{exposure}^{*}\right)$ \\
\hline N & 3.639 \\
Log-Likelihood & $-5556,08$ \\
McFadden-Pseudo-R ${ }^{2}$ & 0,03
\end{tabular}

Quelle: Eigene Berechnungen basierend auf dem NEPS. Negatives binomiales Regressionsmodell. Die angegebenen Koeffizienten sind die Incident Rate Ratios (IRR) sowie die dazugehörigen Standardfehler (in Klammern). Sechs Personen belegen acht Stationen, vier Personen haben eine Anzahl von neun Stationen und zwei Personen eine Stationssumme von zwölf, weshalb sie beim negativen binomialen Modell ausgeschlossen werden. Dadurch werden bei diesen Modellen zwölf Fälle weniger gegenüber dem multinomial logistischen Modell einbezogen. $\mathrm{e}^{\mathrm{xy}}$ steht für $10^{\mathrm{xy}}(, 10$ hoch“). Legende für $\mathrm{p}$-Werte: $* * *=<0,001 ; * *=<0,01 ; *=\leq 0,05$.

Bei der ausführlich berichteten Variante der Stationssummen ohne FSJ-, Urlaubs- und unbestimmte Lückenepisoden stellte sich heraus, dass Hypothese 4a) verworfen werden musste. Durch ein Fortschreiten des Schulenddatumsjahres und damit im Zeitverlauf veränderte sich die Rate der absolvierten Übergangsstationen über alle Gruppen hinweg kaum $($ IRR $=1,01)$. Obwohl dieser Koeffizient signifikant war, bestand somit kein genereller Zeiteffekt auf die Rate der durchlaufenen Stationen (siehe Tabelle 17). Bei den drei weiteren Analysevarianten zu den Stationssummen wurde die Hypothese 4a) ebenfalls anhand des jeweils berechneten Modells falsifiziert. Bei allen drei Varianten änderte sich die Rate an Übergangsstationen mit fortlaufendem Schulenddatumsjahr nicht. Während dieser Effekt bei den Stationssummen ohne FSJ- und Urlaubsepisoden sowie mit unbestimmten Lückenepisoden und mit FSJ- und Urlaubsepisoden sowie ohne unbestimmte Lückenepisoden 
signifikant war, traf dies bei der Stationssumme mit FSJ-, Urlaubs- und unbestimmten Lückenepisoden nicht zu (siehe Anhang Tabellen 40, 41 und 42).

Die Hypothese 4b) wurde bei der Stationssumme ohne FSJ-, Urlaubs- und unbestimmte Lückenepisoden falsifiziert. Die Schulabgängerinnen und Schulabgänger mit Abitur wiesen eine signifikant höhere Rate an absolvierten Übergangsstationen auf als diejenigen, die keinen Abschluss erworben hatten (IRR $=1,68)$. Im Umkehrschluss durchliefen dementsprechend diejenigen ohne Schulabschluss signifikant weniger Stationen im Übergangszeitraum (siehe Tabelle 17). Bei den drei weiteren Analysevarianten bezüglich der Stationssummen zeigten sich ähnliche Ergebnisse. In allen drei Fällen wurde Hypothese 4b) verworfen. Diejenigen mit Realschulabschluss und mit Abitur hatten jeweils eine signifikant höhere Rate an Übergangsstationen als diejenigen ohne Abschluss. Der einzige Unterschied dieser drei Analysevarianten gegenüber der hier ausführlich vorgestellten Variante war eine deutlich höhere und signifikante Rate derjenigen mit Realschulabschluss (siehe Anhang Tabellen 40, 41 und 42).

Auch für die Überprüfung der Hypothese 4c) wurde ein negatives binomiales Regressionsmodell verwendet (siehe Tabelle 18). Die Hypothese 4c) wurde wie folgt formuliert:

4c) Vor dem Hintergrund eines gestiegenen Dienstleistungstätigenanteils ist eine höhere Anzahl der Stationen vom Verlassen der Schule bis zum Beginn der Erstausbildung zu beobachten. 

Übergangsstationen.

\begin{tabular}{|c|c|}
\hline & IRR (SE) \\
\hline Anteil Dienstleistungstätige & $0,96(0,03)$ \\
\hline Demografischer Anteil (unter 20-Jährige) & $1,04(0,02)$ \\
\hline Arbeitslosenquote & $1,03(0,01) *$ \\
\hline \multicolumn{2}{|l|}{ Schulabschluss (Referenz: Kein Abschluss) } \\
\hline Hauptschulabschluss & $1,18(0,05) * * *$ \\
\hline Realschulabschluss & $1,06(0,04)$ \\
\hline Abitur & $1,68(0,08) * * *$ \\
\hline Schulenddatumsjahr & $1,05(0,03)$ \\
\hline \multicolumn{2}{|c|}{ Soziale Herkunft - Bildung der Eltern (Referenz: kein Abschluss / } \\
\hline \multicolumn{2}{|c|}{ Hauptschulabschluss ohne berufl. Ausbildung (b. A.)) } \\
\hline Hauptschulabschluss mit b. A. & $1,09(0,05)$ \\
\hline Mittlere Reife ohne / mit b. A. & $1,05(0,06)$ \\
\hline Hochschulreife ohne/mit b. A. & $1,11(0,07)$ \\
\hline FH-/ Universitätsabschluss & $1,11(0,06)$ \\
\hline Geschlecht (weiblich) & $0,89(0,02) * * *$ \\
\hline Konstante & $1,13 \mathrm{e}^{-41}\left(6,64 \mathrm{e}^{-40}\right)$ \\
\hline Logarithmus(Übergangsdauer) & 1 (exposure) \\
\hline $\mathrm{N}$ & 3.639 \\
\hline Log-Likelihood & $-5553,16$ \\
\hline McFadden-Pseudo- $\mathrm{R}^{2}$ & 0,03 \\
\hline
\end{tabular}

Quelle: Eigene Berechnungen basierend auf dem NEPS. Negatives binomiales Regressionsmodell. Die angegebenen Koeffizienten sind die Incident Rate Ratios (IRR) sowie die dazugehörigen Standardfehler (in Klammern). Sechs Personen belegen acht Stationen, vier Personen haben eine Anzahl von neun Stationen und zwei Personen eine Stationssumme von zwölf, weshalb sie beim negativen binomialen Modell ausgeschlossen werden. Dadurch werden bei diesen Modellen zwölf Fälle weniger gegenüber dem multinomial logistischen Modell einbezogen. $\mathrm{e}^{\mathrm{xy}}$ steht für $10^{\mathrm{xy}}(, 10$ hoch“ $)$. Legende für $\mathrm{p}$-Werte: $* * *=<0,001 ; * *=<0,01 ; *=\leq 0,05$.

Basierend auf dem in Tabelle 18 dargestellten Modell stellte sich heraus, dass Hypothese 4c) falsifiziert werden musste. Bei Ausschluss von FSJ-, Urlaubs- und unbestimmten Lückenepisoden aus der Summe der Übergangsstationen führte der Anstieg des Anteils an Dienstleistungstätigen nicht zu einer höheren Rate an absolvierten Stationen. Der Koeffizient deutete auf eine kaum vorhandene Veränderung der Stationsrate hin (IRR $=0,96)$ und war des Weiteren auch nicht signifikant (siehe Tabelle 18). Auch bei den drei anderen Stationssummenvarianten war der Effekt des Dienstleistungstätigenanteils auf die Stationsrate jeweils kaum vorhanden und nicht signifikant (siehe Anhang Tabellen 43, 45 und 47). Dementsprechend wurde Hypothese 4c) auch bei diesen drei Summenvarianten verworfen. 
Im folgenden negativen binomialen Regressionsmodell wurde die Hypothese 4d) untersucht (siehe Tabelle 19). Zur grafischen Veranschaulichung diente die Abbildung 31.

4d) Vor dem Hintergrund eines gestiegenen Dienstleistungstätigenanteils ist eine höhere Anzahl der Stationen bei denjenigen ohne Schulabschluss oder mit Hauptschulabschluss als bei denjenigen mit Abitur oder Realschulabschluss zu beobachten. 
Tabelle 19: Effekt des gestiegenen Dienstleistungstätigenanteils auf die Anzahl der Übergangsstationen mit Interaktion zwischen Anteil Dienstleistungstätige und Schulabschluss.

\begin{tabular}{|c|c|}
\hline & IRR (SE) \\
\hline Anteil Dienstleistungstätige & $0,98(0,04)$ \\
\hline Demografischer Anteil (unter 20-Jährige) & $1,03(0,02)$ \\
\hline Arbeitslosenquote & $1,03(0,01) *$ \\
\hline \multicolumn{2}{|l|}{ Schulabschluss (Referenz: Abitur) } \\
\hline Kein Abschluss & $0,61(0,03) * * *$ \\
\hline Hauptschulabschluss & $0,72(0,04) * * *$ \\
\hline Realschulabschluss & $0,65(0,03) * * *$ \\
\hline Schulenddatumsjahr & $1,03(0,03)$ \\
\hline \multicolumn{2}{|c|}{ Soziale Herkunft - Bildung der Eltern (Referenz: kein Abschluss / } \\
\hline \multicolumn{2}{|l|}{ Hauptschulabschluss ohne berufl. Ausbildung (b. A.)) } \\
\hline Hauptschulabschluss mit b. A. & $1,09(0,05)$ \\
\hline Mittlere Reife ohne / mit b. A. & $1,05(0,06)$ \\
\hline Hochschulreife ohne/mit b. A. & $1,11(0,07)$ \\
\hline FH-/ Universitätsabschluss & $1,11(0,06)$ \\
\hline Geschlecht (weiblich) & $0,89(0,02) * * *$ \\
\hline \multicolumn{2}{|l|}{ Interaktion Anteil Dienstleistungstätige $\times$ Schulabschluss } \\
\hline Anteil Dienstleistungstätige $\times$ Kein Abschluss & $0,99(0,01)$ \\
\hline Anteil Dienstleistungstätige $\times$ Hauptschulabschluss & $0,99(0,01)$ \\
\hline Anteil Dienstleistungstätige $\times$ Realschulabschluss & $0,99(0,01)$ \\
\hline Konstante & $1,51 \mathrm{e}^{-30}\left(9,32 \mathrm{e}^{-29}\right)$ \\
\hline Logarithmus(Übergangsdauer) & 1 (exposure) \\
\hline $\mathrm{N}$ & 3.639 \\
\hline Log-Likelihood & $-5552,07$ \\
\hline McFadden-Pseudo-R ${ }^{2}$ & 0,03 \\
\hline
\end{tabular}

Quelle: Eigene Berechnungen basierend auf dem NEPS. Negatives binomiales Regressionsmodell. Die angegebenen Koeffizienten sind die Incident Rate Ratios (IRR) sowie die dazugehörigen Standardfehler (in Klammern). Sechs Personen belegen acht Stationen, vier Personen haben eine Anzahl von neun Stationen und zwei Personen eine Stationssumme von zwölf, weshalb sie beim negativen binomialen Modell ausgeschlossen werden. Dadurch werden bei diesen Modellen zwölf Fälle weniger gegenüber dem multinomial logistischen Modell einbezogen. $\mathrm{e}^{\mathrm{xy}}$ steht für $10^{\mathrm{xy}}\left(, 10\right.$ hoch“ $\left.^{“}\right)$. Legende für p-Werte: $* * *=<0,001 ; * *=<0,01 ; *=\leq 0,05$. 


\begin{abstract}
Abbildung 31: Geschätzte Anzahl der Übergangsstationen (ohne FSJ- und Urlaubsepisoden und ohne unbestimmte Lückenepisoden) nach Schulabschluss unter Einfluss des Anteils an Dienstleistungstätigen.
\end{abstract}

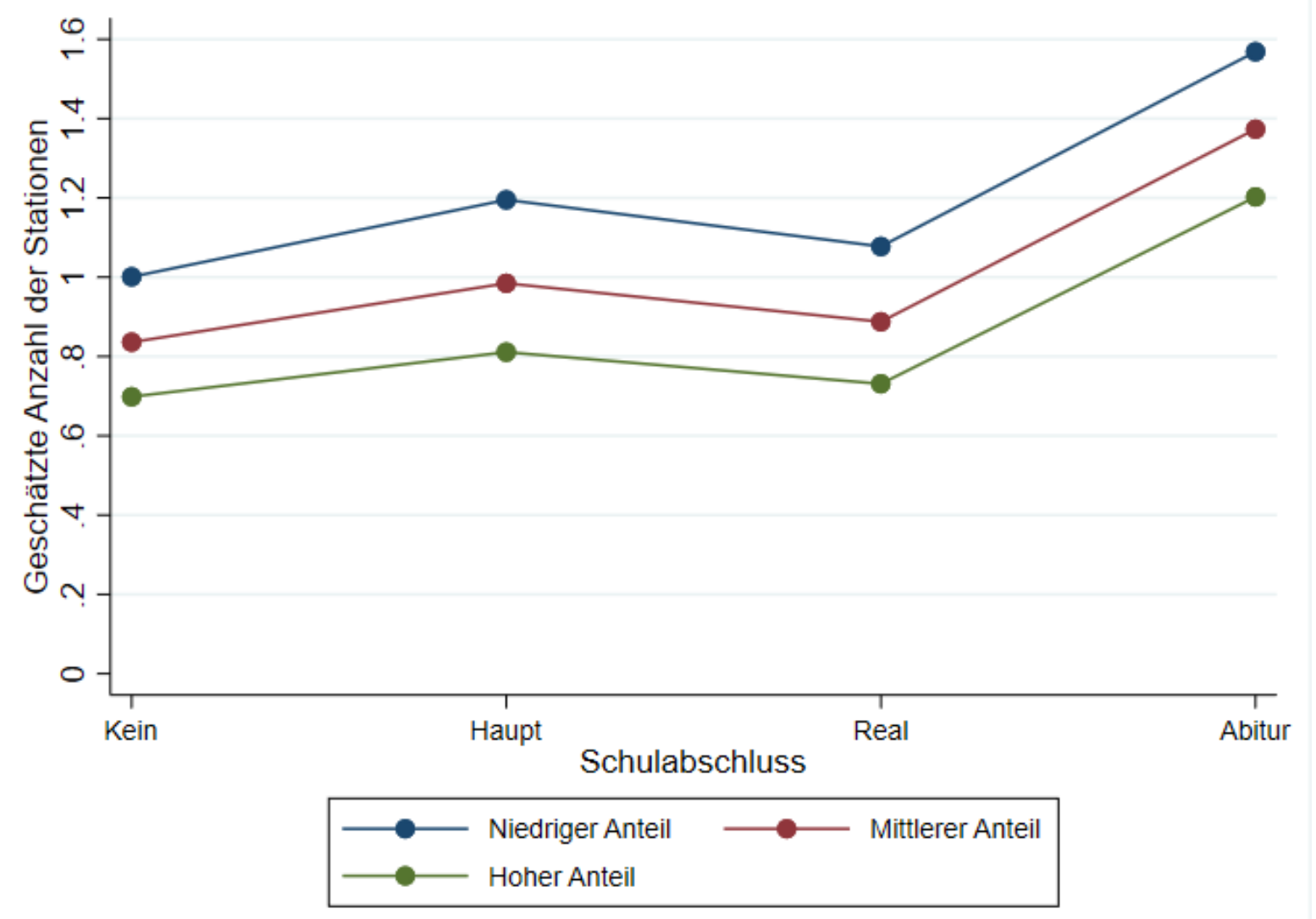

Quelle: Eigene Berechnungen basierend auf dem NEPS. Der Anteil der Dienstleistungstätigen wurde in drei Kategorien eingeteilt: Durchschnittlicher Anteil - 1 Standardabweichung (blau), durchschnittlicher Anteil (rot) und durchschnittlicher Anteil +1 Standardabweichung (grün). Die übrigen einbezogenen unabhängigen Variablen wurden wie folgt fixiert: Schulenddatumsjahr: 1971, Soziale Herkunft: Kein Abschluss/Hauptschulabschluss ohne berufliche Ausbildung, Geschlecht: männlich, demografischer Anteil der unter 20-Jährigen und Arbeitslosenquote jeweils mittelwertzentriert. $\mathrm{N}=3.639$.

Auf Basis des in Tabelle 19 dargestellten Regressionsmodells wurde Hypothese 4d) falsifiziert. Schulabgängerinnen und Schulabgänger ohne Schulabschluss (IRR =0,61) und mit Hauptschulabschluss (IRR $=0,72$ ) hatten jeweils eine signifikant geringere Rate an absolvierten Übergangsstationen als diejenigen mit Abitur. Der gestiegene Anteil an Dienstleistungstätigen hatte hierauf keinen Einfluss (IRR jeweils 0,99). Der Interaktionseffekt war des Weiteren jeweils nicht signifikant (siehe Tabelle 19). Bei den drei weiteren Stationssummenvarianten zeigte sich das gleiche Ergebnis. Auch bei diesen Analysevarianten wiesen diejenigen ohne Abschluss oder mit Hauptschulabschluss eine signifikant geringere Rate an Stationen auf als diejenigen mit Abitur. Ein gestiegener Anteil an Dienstleistungstätigen hatte darauf jeweils keinen Einfluss. Die Interaktionseffekte hierzu stellten sich jeweils als nicht signifikant heraus. Die einzige Ausnahme zeigte sich bei der 
Stationssumme mit FSJ-, Urlaubs- und unbestimmten Lückenepisoden. Hier bestand ein signifikanter Interaktionsterm zwischen dem Anteil der Dienstleistungstätigen und dem Hauptschulabschluss (siehe Anhang Tabellen 44, 46 und 48).

Unabhängig vom Anteil der Dienstleistungstätigen zeigte sich, dass diejenigen mit Abitur im Vergleich zu den drei anderen Schulabschlussgruppen die höchste geschätzte Anzahl an Stationen aufwiesen (siehe Abbildung 31). Dies bestätigte nochmals die Ablehnung von Hypothese 4d) bei der Stationssumme ohne FSJ-, Urlaubs- und unbestimmte Lückenepisoden. Auch bei den drei weiteren Stationssummen wurde unabhängig vom Anteil der Dienstleistungstätigen die mit Abstand höchste geschätzte Anzahl an absolvierten Übergangsstationen bei den Abiturientinnen und Abiturienten nachgewiesen (siehe Anhang Abbildung 49, 50 und 51). Bei diesen drei Varianten wurde die Ablehnung von Hypothese 4d) ebenfalls durch die grafische Veranschaulichung untermauert.

\subsubsection{Zusammenfassung der Sequenzdatenanalysen}

Zusammenfassend wurde im Rahmen der Sequenzdatenanalysen zunächst deskriptiv festgestellt, dass vor allem zusätzliche Schulepisoden als Übergangsstationen durchlaufen wurden. Dies manifestierte sich auch bei den Verlaufsmustern der Übergänge. Darin wurde deutlich, dass insbesondere Übergänge mit lediglich einer zusätzlichen Schulepisode absolviert wurden. Deutlich seltener wurden auch zwei Schulepisoden nacheinander bestritten. Insgesamt bestanden die Übergänge in den meisten Fällen aber nur aus einer Station. Diejenigen Personen, welche keinen allgemeinbildenden Schulabschluss erworben hatten, absolvierten durchschnittlich die meisten Übergangsstationen, während diejenigen mit Abitur durchschnittlich die wenigsten Übergangsstationen aufwiesen. Personen, die das Abitur erworben hatten, durchliefen seltener Kurse, Praktika oder Weiterbildungen als diejenigen Personen ohne Schulabschluss. Die Gruppe derjenigen mit Realschulabschluss absolvierte eher Stationen ohne Weiterbildungscharakter, wobei dieser Effekt durch einen gestiegenen Anteil an Dienstleistungstätigen kaum verändert wurde. Im Gegensatz dazu nahmen diejenigen ohne allgemeinbildenden Schulabschluss die Möglichkeit wahr, zusätzliche Schulepisoden zu absolvieren, um Bildungsabschlüsse nachzuholen. Dieser starke Effekt wurde von einem gestiegenen Dienstleistungstätigenanteil nicht tangiert. Die Gruppe derjenigen mit einem Hauptschulabschluss befand sich signifikant eher in Stationen ohne Weiterbildungscharakter als in zusätzlichen Schulepisoden, wobei dieser Effekt durch einen gestiegenen Anteil an Dienstleistungstätigen nicht beeinflusst wurde. Außerdem zeigte sich, 
dass sich durch einen gestiegenen Anteil an Dienstleistungstätigen die Rate an absolvierten Übergangsstationen nicht veränderte. Weiterhin wurde deutlich, dass Abiturientinnen und Abiturienten unabhängig vom Anteil an Dienstleistungstätigen eine höhere geschätzte Anzahl an durchlaufenen Übergangsstationen hatten als die übrigen drei Schulabschlussgruppen. 


\section{Zusammenfassung und Schlussfolgerungen}

Die vorliegende Arbeit fokussierte die Untersuchung der Entwicklung der Dauer und der absolvierten Stationen vom erstmaligen Verlassen der Schule bis zum Beginn einer dualen, vollzeitschulischen oder akademischen Erstausbildung. Dies wurde retrospektiv zwischen 1971 und 2012 und unter Einfluss eines gestiegenen Anteils an Dienstleistungstätigen auf dem Arbeitsmarkt untersucht. Die Untersuchung erfolgte auch differenziert für die vier Schulabschlussgruppen Abitur, Realschulabschluss, Hauptschulabschluss und kein Schulabschluss. Dabei wurde zunächst der Fragestellung nachgegangen, ob durch ein fortschreitendes Schulenddatumsjahr eine Verlängerung der Übergangsdauer eintritt. Des Weiteren wurde die Übergangsdauer getrennt nach Schulabschluss untersucht. Außerdem wurde überprüft, ob der gestiegene Anteil an Dienstleistungstätigen einen Einfluss auf die Übergangsdauer hat und darüber hinaus die Schulabschlusseffekte auf die Dauer tangiert. Des Weiteren wurden die vorwiegend durchlaufenen Stationskategorien auf einen möglichen Effekt der Schulabschlüsse und des Anteils an Dienstleistungstätigen untersucht. Abschließend wurde die Summe an absolvierten Stationen mit Hilfe des Schulenddatumsjahres auf einen möglichen Zeittrend untersucht und auch ein Effekt der Schulabschlüsse sowie des Anteils an Dienstleistungstätigen auf die Stationssumme überprüft. Die vorliegende Studie lieferte umfangreiche Erkenntnisse zur Entwicklung der Übergangsdauer und der Übergangsstationen in einer Retrospektivbetrachtung vor dem Hintergrund eines veränderten Arbeitsmarktes in Form des expandierenden Dienstleistungssektors.

\subsection{Zentrale Befunde der Studie}

Auf die beiden zentralen Fragestellungen der vorliegenden Arbeit kann anhand der durchgeführten Analysen eine tendenzielle Antwort gegeben werden. Die erste Fragestellung lautete:

„Inwieweit hat ein erhöhter Anteil von hochqualifizierten Arbeitskräften auf dem Arbeitsmarkt die Dauer des Übergangsprozesses vom erstmaligen Verlassen der Schule bis zum Beginn einer dualen, schulischen oder akademischen Erstausbildung generell für alle Jugendlichen und jungen Erwachsenen sowie differenziert für die einzelnen Schulabschlussgruppen im Zeitraum zwischen 1971 und 2012 verändert? “ 
Im Rahmen der Ergebnisse der vorliegenden Studie wurde deutlich, dass sich mit fortlaufendem Schulenddatumsjahr keine Veränderung bei der Übergangsdauer ergeben hatte und somit kein Zeittrend zwischen den Jahren 1971 und 2012 für die Übergangsdauer bestand. Darüber hinaus war die Übergangsdauer unabhängig von der Entwicklung des Dienstleistungssektors auf dem Arbeitsmarkt bei Jugendlichen ohne Schulabschluss deutlich länger gegenüber der Dauer derer mit Realschulabschluss oder mit Abitur. Dadurch hatten diejenigen mit Realschulabschluss und Abitur auch deutlich größere Chancen auf die Aufnahme einer Erstausbildung als die Gruppe derjenigen ohne Schulabschluss. Des Weiteren wurde ersichtlich, dass ein gestiegener Anteil an Dienstleistungstätigen auf dem Arbeitsmarkt schulabschlussübergreifend die Chance auf die Aufnahme einer Erstausbildung kaum beeinflusste und damit die Übergangsdauer vom erstmaligen Verlassen der allgemeinbildenden Schule bis zum Beginn der Erstausbildung nicht tangierte. Unabhängig vom erreichten Schulabschluss und vom Erstausbildungstyp hat sich somit die Übergangsdauer durch einen gestiegenen Dienstleistungstätigenanteil kaum verändert. Bei der Untersuchung eines möglichen Einflusses des Anteils an Dienstleistungstätigen auf die Effekte der Schulabschlüsse zeigte sich, dass diejenigen mit Realschulabschluss und mit Abitur im Vergleich zu denjenigen ohne Abschluss eine höhere Erstausbildungschance und eine kürzere Übergangsdauer hatten. Dieser Befund war jedoch unabhängig von einem gestiegenen Anteil an Dienstleistungstätigen. Der erhöhte Anteil im tertiären Sektor tangierte somit nicht die Erstausbildungschancen und die Übergangsdauer derjenigen mit Realschulabschluss und Abitur.

Auch bezüglich der zweiten Fragestellung kann eine Einschätzung anhand der Ergebnisse gegeben werden:

„Inwieweit hat ein erhöhter Anteil von hochqualifizierten Arbeitskräften auf dem Arbeitsmarkt das Absolvieren von Übergangsstationen, und dabei sowohl die Anzahl als auch die Kategorien der absolvierten Stationen generell für alle Jugendlichen und jungen Erwachsenen sowie differenziert für die einzelnen Schulabschlussgruppen im Zeitraum zwischen 1971 und 2012 verändert? “

Bei Betrachtung der Ergebnisse hinsichtlich der absolvierten Übergangsstationen zeigte sich, dass die untersuchten Abiturientinnen und Abiturienten während des Übergangs von der Schule in die Erstausbildung eher Stationen durchliefen, die keinen Weiterbildungscharakter 
besaßen. Diejenigen Schulabgängerinnen und Schulabgänger, welche keinen Schulabschluss erworben hatten, absolvierten dagegen im Übergangsprozess vorwiegend weitere Schulepisoden, was durch einen gestiegenen Anteil an Dienstleistungstätigen nicht beeinflusst wurde. Diejenigen mit Realschulabschluss durchliefen vorwiegend Stationen ohne Weiterbildungscharakter. Der gestiegene Anteil an Dienstleistungstätigen verstärkte diesen Trend kaum. Die Rate an absolvierten Übergangsstationen veränderte sich durch ein Fortlaufen des Schulenddatumsjahres zwischen 1971 und 2012 nicht. Abiturientinnen und Abiturienten wiesen eine signifikant höhere Rate an Übergangsstationen auf als diejenigen ohne Abschluss. Der erhöhte Anteil an Beschäftigten im tertiären Sektor hatte keinen Effekt auf die Rate an absolvierten Stationen. Jugendliche ohne Abschluss und mit Hauptschulabschluss wiesen eine signifikant geringere Rate an Übergangsstationen auf als diejenigen mit Abitur, was durch den Anteil an Arbeitskräften im Dienstleistungsbereich nicht beeinflusst wurde.

Zusammenfassend wurde deutlich, dass eine Expansion des Dienstleistungssektors insgesamt kaum einen Einfluss auf die Übergangsdauer und die absolvierten Übergangsstationen hatte. Jedoch zeigte sich, dass sich die Dauer umso mehr verlängerte, desto geringer der Schulabschluss war.

\subsection{Diskussion der Ergebnisse}

Bei einem Abgleich der vorliegenden Ergebnisse mit den Erkenntnissen der bisherigen Forschung wird deutlich, dass einige Übereinstimmungen, aber auch Unterschiede bestehen. Bei der deskriptiven Betrachtung der vorliegenden Daten wurde ersichtlich, dass sich im Untersuchungszeitraum der Anteil der Personen, die als Erstausbildung ein Studium aufnahmen, sukzessive erhöhte. Diese Entwicklung wurde auch für kürzere Zeiträume bestätigt (Konsortium Bildungsberichterstattung 2006: 21). Dagegen widerspricht der durch die deskriptiven Analysen gewonnene Befund, dass sich die durchschnittliche Übergangsdauer während des Untersuchungszeitraums nicht eindeutig veränderte, der bisherigen Forschung, dass viele Jugendliche größere Probleme beim Übergang in die Ausbildung haben und dafür mehr Zeit benötigen (vgl. u. a. Rukwid 2011; Palamidis und Schwarze 1989; Konsortium Bildungsberichterstattung 2006). Bei den genannten Studien wurden bestimmte Jugendliche, beispielsweise ohne Schulabschluss, betrachtet, weshalb bei diesen Teilgruppen größere Übergangsprobleme verbunden mit einer längeren Dauer festgestellt wurden. Der Befund der vorliegenden Arbeit, dass diejenigen ohne Schulabschluss 
die deutlich längste durchschnittliche Übergangsdauer unter allen Schulabschlussgruppen hatten, lässt sich im Rahmen der Ergebnisse der bisherigen Studien dahinhegend gut einordnen, dass einige Jugendliche vorübergehend oder dauerhaft von einer Berufsausbildung ausgeschlossen waren (Carl et al. 2002: 18; Seibert und Kleinert 2009: 1) und diejenigen ohne Schulabschluss große Probleme hatten, in eine duale Berufsausbildung zu gelangen (Stegmann und Holzbauer 1978: 148). Jugendliche mit einem niedrigen oder mittleren Bildungsniveau hatten schlechtere Übergangschancen (Kleinert und Jacob 2012: 229) sowie zum Teil auch längere Übergangsdauern (Eberhard et al. 2013: 8) als diejenigen mit einem höheren Bildungsniveau, was sich auch anhand der längeren durchschnittlichen Übergangsdauern derjenigen mit Haupt- und Realschulabschluss gegenüber denjenigen mit Abitur in den Ergebnissen bestätigte. Dieser Befund steht im Kontrast zu anderen Studienergebnissen, aus welchen hervorgeht, dass die Schulabgängerinnen und Schulabgänger schneller in eine schulische oder duale Berufsausbildung einmündeten als in ein Studium (Büchtemann et al. 1993: 509) und dass Jugendliche mit Hauptschulabschluss relativ schnell den Übergang in eine Berufsausbildung vollziehen (Solga et al. 2012: 4; Carl et al. 2002: 18). Diese Widersprüche erklären sich darin, dass unter anderem Hauptschülerinnen und Hauptschüler selektiv aus einer einzigen Stadt oder aus „Projektklassen“ untersucht wurden, welche einen frühzeitigen Kontakt zu den ausbildenden Betrieben hatten. Die in bisherigen Forschungsarbeiten herausgefundene Tendenz, dass diejenigen Jugendlichen mit einer mittleren Reife bessere Übergangschancen in die Ausbildung haben als diejenigen mit Hauptschulabschluss (Protsch 2014: 201; Beicht und Ulrich 2008b: 21; KJS Katholische Jugendsozialarbeit 2014; Steinmann 2000: 254) deckt sich nicht mit dem Ergebnis der Analysen, dass die durchschnittliche Übergangsdauer dieser beiden Schulabschlussgruppen im betrachteten Untersuchungszeitraum nahezu gleich war. Darüber hinaus wurde für diejenigen mit einem Hauptschulabschluss für den Zeitraum bis Mitte der 1980er Jahre sogar eine geringfügig kürzere durchschnittliche Übergangsdauer ermittelt als für die Gruppe mit mittlerer Reife. Auch der Befund, dass sich die Übergangschancen von schulisch niedrig qualifizierten Jugendlichen im Zeitverlauf verbessert haben (Solga 2004: 110), konnte durch die Analysen nicht bestätigt werden. Die durchschnittliche Übergangsdauer derjenigen mit einem Hauptschulabschluss erhöhte sich im Untersuchungszeitraum. Nachdem sich die Dauer bei denjenigen ohne Schulabschluss zunächst deutlich verkürzt hatte, verlängerte sie sich wieder zunehmend. Bei diesen Widersprüchen sind jeweils die Inkonsistenzen der 
zugrundeliegenden Rohdaten und die damit verbundenen Aufbereitungen zu berücksichtigen, welche im Rahmen der Limitationen genauer erörtert werden.

Auch bei den Ergebnissen zu den absolvierten Übergangsstationen fallen einige Diskrepanzen zu den Ergebnissen vorheriger Studien auf. Die Annahme, dass die Jugendlichen beim Übergang von der Schule in die Ausbildung sukzessive immer mehr Zwischenschritte und Stationen durchlaufen (Fink 2011: 92), konnte anhand der Ergebnisse der vorliegenden Arbeit dahingehend widerlegt werden, dass es im betrachteten Untersuchungszeitraum lediglich einen leichten Anstieg der durchschnittlichen Anzahl an Übergangsstationen zwischen 1990 und 2000 gab. Die durchschnittliche Anzahl an absolvierten Stationen blieb dagegen zwischen 1971 und 1990 relativ konstant. Im Rahmen der Datenaufbereitung wurden parallel verlaufende Stationen bereinigt und Stationen ausgeschlossen, welche vor dem bereinigten Schulenddatum oder nach dem bereinigten Erstausbildungsbeginn datiert waren. Dieser Umstand führte zu einer Verringerung der berücksichtigten Übergangsstationen.

Differenziert nach Schulabschluss zeigten sich anhand der Analysen zum Teil ähnliche Ergebnisse zur bisherigen Forschung. Während die beiden Gruppen ohne Schulabschluss und mit Hauptschulabschluss in der Regel mehrere Übergangsstationen, meistens im berufsvorbereitenden Übergangssystem, durchlaufen mussten (Lex und Geier 2010: 183), zeigte sich in den vorliegenden Ergebnissen für die Gruppe derer ohne erreichten allgemeinbildenden Schulabschluss über den gesamten Untersuchungszeitraum die durchschnittlich höchste Anzahl an Übergangsstationen. Im Gegensatz dazu stieg die durchschnittliche Anzahl an Stationen bei denjenigen Jugendlichen mit einem Hauptschulabschluss im Zeitverlauf kontinuierlich an. Interessant ist hierbei, dass die durchschnittliche Anzahl an Stationen von denjenigen mit Hauptschulabschluss bis Mitte der 1990er Jahre marginal unter der Anzahl derer mit mittlerer Reife lag. Erst ab Mitte der 1990er Jahre durchliefen Schulabgängerinnen und Schulabgänger mit Hauptschulabschluss durchschnittlich mehr Übergangsstationen als diejenigen mit einem Realschulabschluss. Hierbei ist $\mathrm{zu}$ beachten, dass auch die durchschnittliche Stationsanzahl derjenigen mit mittlerer Reife deutlich anstieg. Dieser nur sehr marginale Unterschied zwischen den beiden Gruppen mit Haupt- und mit Realschulabschluss steht durchaus im Kontrast zum Befund, dass insbesondere diejenigen ohne und mit Hauptschulabschluss viele Zwischenstationen durchlaufen müssen. Auch dieser Widerspruch könnte mit der bereits angesprochenen Bereinigung der Übergangsstationen zusammenhängen. 
Der durch die Analysen erhobene Befund, dass zusätzliche Schulepisoden die mit Abstand am häufigsten absolvierten Übergangsstationen sind, bestätigt sich an den bisherigen Erkenntnissen, dass viele Schulabgängerinnen und Schulabgänger mit Hauptschulabschluss (Gaupp und Geier 2008: 43), aber auch diejenigen mit einem Realschulabschluss weitere Schulepisoden absolvierten (Winkler 2017: 275). Jedoch betraf dies in der Gruppe der Hauptschülerinnen und Hauptschüler insbesondere Jugendliche mit guten schulischen Leistungen (Gaupp und Geier 2008: 43). Im Kontrast zum Befund der sehr starken Frequentierung von weiteren Schulepisoden steht dagegen die Erkenntnis, dass insbesondere Jugendliche ohne oder mit einem Hauptschulabschluss während des Übergangs an den Maßnahmen des berufsvorbereitenden Übergangssystems partizipieren (Beicht und Ulrich 2008b: 10, 19) und selbst ein beträchtlicher Anteil derjenigen mit einer mittleren Reife in das Übergangssystem einmündet (Baethge et al. 2007: 8). Die bisherigen Forschungsergebnisse stehen auch im Widerspruch $\mathrm{zu}$ der Tatsache, dass Arbeitslosigkeitsepisoden in der vorliegenden Studie als die am zweithäufigsten und berufsvorbereitende Maßnahmen als die am dritthäufigsten absolvierte Übergangsstation identifiziert wurden. Aufgrund der Zuweisung der Personen zu Stationskategorien und einer höheren Gewichtung von zusätzlichen Schulepisoden gegenüber berufsvorbereitenden Maßnahmen ist zu berücksichtigen, dass Personen in der vorliegenden Studie, welche beispielsweise sowohl eine berufsvorbereitende Maßnahme als auch eine zusätzliche Schulepisode durchlaufen hatten, zur Kategorie der zusätzlichen Schulepisode zugeordnet wurden. Zusätzliche Schulepisoden stellten dabei die am höchsten gewichtete Stationskategorie dar. Diese methodische Gewichtung kann dazu führen, dass berufsvorbereitende Maßnahmen oder Arbeitslosigkeit als Stationskategorien in der vorliegenden Arbeit seltener berücksichtigt wurden. Zudem beinhalteten die Häufigkeiten der Stationen in dieser Studie noch nicht die später zusammengefassten und gewichteten Stationskategorien.

Bei Einbezug weiterer Einflussfaktoren über den Schulabschluss hinaus zeigen sich ebenfalls einige Übereinstimmungen, aber auch Unterschiede zwischen den Ergebnissen der vorliegenden Studie und den Erkenntnissen vorheriger Studien. Während der Einfluss von demografischen und konjunkturellen Faktoren auf die Übergangschancen in die Ausbildung bei niedrigeren und mittleren Abschlüssen, jedoch nicht bei Abiturientinnen und Abiturienten nachgewiesen wurde (Kleinert und Jacob 2012: 229), zeigte sich in den durchgeführten Analysen, dass insgesamt demografische Veränderungen und die Arbeitslosenquote die Übergangsdauer nicht beeinflussten. Diese Diskrepanz ist möglicherweise damit zu erklären, 
dass Kleinert und Jacob lediglich das Gelingen des Übergangs selbst, jedoch nicht die Dauer und die Stationen des Übergangsprozesses untersuchten. Der gestiegene Anteil an Dienstleistungstätigen führte ebenfalls schulabschlussübergreifend zu keiner Veränderung der Übergangsdauer. Dies stand im Widerspruch zu bisherigen Erkenntnissen (Christe 2016: 11). Dabei ist zu berücksichtigen, dass Christe die Arbeitsmarktbedingungen als einen von mehreren Faktoren erwähnt, welche sowohl einen positiven als auch einen negativen Einfluss auf den Übergangsverlauf haben können. Auch die soziale Herkunft hatte meist keine Auswirkung auf die Übergangschancen und die Dauer. Eine Ausnahme bildete hierbei die Gruppe derjenigen, deren Eltern einen Hauptschulabschluss sowie eine berufliche Ausbildung hatten. Diese Gruppe hatte eine signifikant kürzere Übergangsdauer als diejenigen, deren Eltern keinen oder einen Hauptschulabschluss, jedoch keine Berufsausbildung besaßen. Die übrigen Herkunftskategorien hatten jedoch keinen signifikanten Effekt auf die Übergangsdauer. Insgesamt steht der Befund zur sozialen Herkunft somit im Widerspruch zu bisherigen Studien (Hillmert 2001: 27; Palamidis und Schwarze 1989: 114). Dabei ist zu berücksichtigen, dass in der vorliegenden Studie Übergangsverläufe von Personen über mehrere Jahrzehnte einbezogen wurden und die soziale Herkunft in Form der elterlichen Bildung sich in einzelnen Jahren oder Jahrzehnten anders auf die Übergangsprozesse der Jugendlichen ausgewirkt haben könnte als über den gesamten Untersuchungszeitraum hinweg.

Des Weiteren deckt sich das Ergebnis der vorliegenden Arbeit, dass Personen mit Abitur die deutlich kürzeste Übergangsdauer hatten, mit bisherigen Studien, welche beispielsweise einen zügigen Übergang in das Studium nachgewiesen hatten (Willich et al. 2011: 2). Auch diejenigen mit einer mittleren Reife oder einem Hauptschulabschluss schafften kurz nach dem Ende der Schulzeit den Übergang in die Ausbildung (Carl et al. 2002: 18; Beicht und Ulrich 2008b: 8). Dies stimmt mit den Ergebnissen der vorliegenden Studie überein, dass sowohl diejenigen mit einem Haupt- als auch diejenigen mit einem Realschulabschluss eine deutlich größere Übergangschance in die Ausbildung hatten als die Gruppe derjenigen ohne Schulabschluss. Für diese Gruppe wurde die deutlich geringste Übergangschance und damit längste Übergangsdauer festgestellt, was zum Teil mit den bisherigen Studien übereinstimmt, teilweise aber auch im Widerspruch dazu steht. Während einerseits nachgewiesen wurde, dass auch Jugendliche ohne Schulabschluss den Übergang in die Ausbildung zeitnah vollziehen (Solga 2002: 9), zeigten andere Arbeiten eine deutliche Verzögerung dieser Gruppe beim Übergang in die Ausbildung (Beicht und Ulrich 2008b: 10, 19; Christe 2016: 15; Seibert und 
Kleinert 2009: 1). Hierbei ist jedoch zu berücksichtigen, dass Solga den Übergang Jugendlicher ohne Schulabschluss nicht auf reguläre Ausbildungen bezog. Auch für die anderen Schulabschlussgruppen wurden deutlich verlängerte Übergangsprozesse festgestellt (Lex und Zimmermann 2011: 614 f.), was im Widerspruch zu den höheren Übergangschancen der drei Schulabschlussgruppen im Vergleich zu denjenigen ohne Abschluss in der vorliegenden Studie steht. Dieser Kontrast könnte damit erklärt werden, dass Lex und Zimmermann die Anteile einzelner Schulabschlussgruppen untersuchten, welche nicht im Jahr des Erwerbs des Abschlusses in eine Ausbildung übergegangen waren. Dabei ist zu vermuten, dass die Verzögerung derjenigen ohne Abschluss deutlich größer ausgefallen sein könnte als bei denjenigen mit Schulabschluss.

Auch unter Kontrolle eines gestiegenen Anteils an Dienstleistungstätigen zeigten die Ergebnisse, dass ein höherer Schulabschluss mit einer verkürzten Übergangsdauer einhergeht. Dieser Befund stimmt überein mit der bisherigen Forschung, dass ein hoher Schulabschluss und gute Abschlussnoten in Zusammenhang mit einer verkürzten Übergangsdauer standen (Eberhard et al. 2013: 8 f.). Der gestiegene Dienstleistungstätigenanteil hatte jedoch keinen Einfluss auf den Effekt des Abiturs und des Realschulabschlusses auf die Übergangsdauer. Auch auf die Rate an absolvierten Übergangsstationen hatte der gestiegene Anteil an Dienstleistungstätigen keinen Einfluss. Dies galt ebenfalls bezüglich eines möglichen Einflusses auf die Effekte der Schulabschlüsse auf die Rate der absolvierten Stationen. Bei vorherigen Forschungsarbeiten wurde ein verändertes Qualifikationsniveau auf dem Arbeitsmarkt, beispielsweise in Bezug auf die Entwicklung des tertiären Sektors, grundsätzlich nicht untersucht. Stattdessen wurde der elterliche Bildungs- und Erwerbsstatus als Einflussfaktor in Betracht gezogen. Dabei zeigte sich, dass bei einer fehlenden abgeschlossenen Berufsausbildung oder einer nicht vorhandenen Erwerbstätigkeit beider Elternteile die Übergangschancen deutlich geringer und die Übergangsdauer dadurch deutlich länger waren (Eberhard et al. 2013: 8 f.; Solga et al. 2012: 5).

Weiterhin konnten in Bezug auf die absolvierten Stationsinhalte getrennt nach Schulabschluss und unter Einfluss externer Faktoren einige Parallelen, aber auch Differenzen zu den bisherigen Studien ausgemacht werden. Die Analysen der vorliegenden Arbeit zeigten deutlich, dass die Gruppe derjenigen mit Abitur eher Stationen ohne einen Weiterbildungsbezug während des Übergangsprozesses durchlief. Dieses Ergebnis stimmt teilweise nicht mit den Erkenntnissen früherer Studien überein, durch welche herausgefunden wurde, dass viele junge Erwachsene mit einer Studienberechtigung an 
Weiterbildungsveranstaltungen teilnahmen, sich in Praktika oder einem freiwilligen sozialen Jahr befanden (Lewin und Schacher 1990: 1; Heine et al. 2010: 1; Beicht und Ulrich 2008b: 8). Dies ist jedoch mit dem Umstand zu erklären, dass diese Befunde in sehr begrenzten Zeiträumen erhoben wurden, wogegen der Befund der vorliegenden Studie mehrere Jahrzehnte abdeckt. Unter Stationen ohne Weiterbildungsbezug fallen beispielsweise auch Urlaube oder unbestimmte Phasen, an die sich die befragten Personen eventuell nicht mehr erinnern konnten. Diese Studien bestätigen jedoch auch zum Teil den Befund der vorliegenden Arbeit, dass die Gruppe der Hochschulzugangsberechtigten keine Stationen mit Weiterbildungscharakter absolvieren, da sie sich in Übergangstätigkeiten wie Jobben oder Au-Pair-Aufenthalten befanden oder den vorgeschriebenen Wehr- beziehungsweise Zivildienst leisteten (Heine et al. 2010: 1; Beicht und Ulrich 2008b: 8). Die Analysen brachten ebenfalls hervor, dass auch diejenigen mit einer mittleren Reife eher Stationen ohne einen Weiterbildungsbezug durchliefen. Dies widerspricht der bisherigen Erkenntnis, dass auch Jugendliche mit Realschulabschluss in das berufsvorbereitende Übergangssystem einmündeten (Baethge et al. 2007: 8), dessen Maßnahmen jedoch nicht durchgehend bessere Ausbildungschancen garantieren (Krone 2010: 23). Jedoch ist zu berïcksichtigen, dass es sich um etwas mehr als ein Viertel derjenigen mit mittlerer Reife handelte, welche zunächst in das Übergangssystem einmündeten, und dieser Anteil deutlich geringer ausfiel als bei denjenigen mit Hauptschulabschluss oder ohne Abschluss (Baethge et al. 2007: 8). Andere Studien widersprechen diesem Befund, da die Gruppe derer mit mittlerer Reife relativ häufig und schnell aus den Maßnahmen des berufsvorbereitenden Übergangssystems in eine vollqualifizierende Berufsausbildung übergehen konnte (Beicht und Ulrich 2008b: 19) oder direkt im Anschluss an den Realschulabschluss oder zu einem späteren Zeitpunkt das Abitur nachholte (Winkler 2017: 275).

Des Weiteren stellte sich heraus, dass die Gruppe der Jugendlichen ohne Schulabschluss sehr häufig weitere Schulepisoden im Anschluss an die erste allgemeinbildende Schulzeit durchlief, um zusätzliche Bildungsabschlüsse nachzuholen. Dieser Befund deckt sich nicht mit dem bisherigen Forschungsstand. Jugendliche ohne oder mit einem Hauptschulabschluss wurden als die Fokusgruppe für die Partizipation an berufsvorbereitenden Maßnahmen identifiziert (Beicht und Ulrich 2008b: 10, 19; Christe 2016: 15; Krüger-Charlé 2010: 1). Lediglich die Gruppe derer mit einem Hauptschulabschluss machte von einem weiteren Schulbesuch Gebrauch, um Schulabschlüsse nachzuholen und die eigenen Ausbildungschancen zu verbessern (Lex und Geier 2010: 184). Dies galt weiterhin auch für 
die Gruppe derjenigen mit einem Realschulabschluss in Form des Nachholens des Abiturs (Winkler 2017: 275). Dieser Widerspruch resultiert ebenfalls aus der stärkeren Gewichtung zusätzlicher Schulepisoden als Stationskategorie gegenüber berufsvorbereitenden Maßnahmen in der vorliegenden Studie. Die früheren Forschungsbefunde konnten jedoch zumindest im Rahmen der geschätzten Wahrscheinlichkeiten für die Gruppen mit Haupt- und mit Realschulabschluss, zusätzliche Schulepisoden zu absolvieren, in der vorliegenden Studie zum Teil bestätigt werden, wenngleich deren geschätzte Wahrscheinlichkeiten etwas geringer ausfielen als für die Gruppe ohne erreichten Abschluss. Während der gestiegene Anteil an Dienstleistungstätigen bei den vorliegenden Ergebnissen keinen Einfluss auf die Stationen der Schulabschlussgruppen hatte, wurden in den vorherigen Studien andere bedeutsame Einflussfaktoren ersichtlich. Hierzu zählten unter anderem der elterliche Sozialstatus (Gaupp et al. 2011: 182), die Unterstützungsmöglichkeiten der Herkunftsfamilie (Lex und Zimmermann 2011: 603) oder die Rahmenbedingungen des regionalen Arbeitsmarktes (Gaupp et al. 2008: 388), welche jeweils einen Einfluss darauf hatten, ob eine schulische Höherqualifizierung wahrgenommen wurde oder nicht.

Zusammenfassend kann festgehalten werden, dass sich einige in der bisherigen Forschung gefundene Trends zwar in den vorliegenden Ergebnissen bestätigten, jedoch auch einige Diskrepanzen zum Vorschein kamen. Beispielsweise zeigte sich bei der Entwicklung der Übergangsdauer, dass diejenigen ohne Abschluss die längste und diejenigen mit Abitur die mit Abstand kürzeste Übergangsdauer hatten. Dagegen gab es hierzu zwischen den beiden Gruppen mit Haupt- und mit Realschulabschluss kaum Unterschiede. Dies stellt einen Unterschied zu einigen bisherigen Forschungsarbeiten dar, welche für die Jugendlichen mit Realschulabschluss bessere Übergangschancen diagnostizierten als für diejenigen mit Hauptschulabschluss. Die gleiche Konstellation zeigte sich bei den Ergebnissen zur Anzahl der durchschnittlich absolvierten Übergangsstationen nach Schulabschluss. Dies lässt sich nicht zu der Erkenntnis zuordnen, dass diejenigen Jugendlichen mit einem Hauptschulabschluss neben der Gruppe ohne Schulabschluss am häufigsten im berufsvorbereitenden Übergangssystem verschiedene Maßnahmen und damit auch Stationen durchlaufen müssen. Dies konnte durch die bisherige Forschung nicht für die Gruppe derjenigen mit einem Realschulabschluss bestätigt werden.

Auch bei den absolvierten Stationen der einzelnen Schulabschlussgruppen selbst zeigten sich neben einigen Übereinstimmungen mit bisherigen Studien interessante Unterschiede. Während mit Hilfe der vorliegenden Ergebnisse bestätigt werden konnte, dass Jugendliche 
ohne Schulabschluss zusätzliche Schulepisoden absolvieren und Schulabschlüsse nachholen, zeigten bisherige Studien, dass Jugendliche ohne und mit Hauptschulabschluss als Zielgruppe für berufsvorbereitende Maßnahmen identifiziert wurden und sich viele derjenigen mit Hauptschulabschluss in weiteren Schulepisoden befanden. Auch die beiden Schulabschlussgruppen mit Abitur und mit Realschulabschluss wurden in den Ergebnissen der vorliegenden Studie tendenziell in nicht-weiterbildungsbezogenen Übergangsstationen verortet. Dies steht ebenfalls in Diskrepanz zu der bisherigen Forschung, welche für Abiturientinnen und Abiturienten ein Durchlaufen von weiterbildungsbezogenen Stationen wie beispielsweise Praktika attestierte und die Jugendlichem mit einer mittleren Reife sowohl in zusätzlichen Schulepisoden zum Nachholen des Abiturs oder in berufsvorbereitenden Maßnahmen sah. Bei vielen der Widersprüche zwischen früheren Forschungsbefunden und den Befunden der vorliegenden Studie sind jedoch die Aufbereitungsschritte und das methodische Vorgehen in dieser Arbeit zu berücksichtigen.

Im Folgenden werden die Ergebnisse der vorliegenden Studie anhand der beiden zentralen theoretischen Ansätze, der Humankapitaltheorie und der Verdrängungsthese, diskutiert und in diese eingeordnet. Bei Betrachtung der Humankapitaltheorie mit dem von Becker formulierten Ziel, sich Humankapital in Form von bildungs- und berufsrelevanten Qualifikationen anzueignen und dafür Kosten in Form von Zeit aufzuwenden (Becker 1982: 137) fanden sich in den Ergebnissen der vorliegenden Arbeit dazu einige passende Parallelen. Der Befund, dass vor allem die Gruppe derjenigen ohne einen erreichten allgemeinbildenden Schulabschluss weitere Schulepisoden durchlief und dabei versuchte, Schulabschlüsse nachzuholen, um die eigenen Ausbildungschancen zu verbessern, stimmt mit dem Gedanken des Humankapitalansatzes vollkommen überein. Hierfür muss die Gruppe derjenigen ohne Abschluss zwar ein weiteres Zeitfenster in Anspruch nehmen, was in Abhängigkeit mit dem anvisierten Schulabschluss steht. Jedoch können durch diese direkt nach der ersten allgemeinbildenden Schulzeit oder im späteren Verlauf nachgeholten Bildungsabschlüsse die eigenen Übergangschancen deutlich verbessert werden. Je mehr Bildungsabschlüsse die Gruppe der Jugendlichen ohne Schulabschluss nachholt, desto bessere Übergangs- und Ausbildungschancen haben sie im späteren Verlauf. Das bedeutet für diese Gruppe jedoch, dass sie umso mehr zusätzliche Zeit in weitere Schulepisoden stecken muss, je mehr Schulabschlüsse nachgeholt werden sollen. Dadurch können sie den Übergang in eine Ausbildung nicht direkt vollziehen, sondern müssen sich aufgrund der weiteren Schulepisoden hierzu noch gedulden. Dementsprechend hängt die Entscheidung, Zeit in 
zusätzliche Schulepisoden und Bildungsabschlüsse zu investieren von der Kosten-NutzenAbwägung ab, ob der zu erwartende Nutzen, nachgeholte Schulabschlüsse und daraus folgend bessere Ausbildungs- und Berufschancen, die aufzuwendenden Kosten vor allem in Form von Zeit oder Opportunitätskosten übersteigt (Becker 2011: 27; Rissiek 1998: 34; Becker und Hecken 2011: 373, 376; Persch 2008: 29). Die vorliegenden Ergebnisse zeigten deutlich, dass vor allem die Gruppe derjenigen, die am Ende der ersten allgemeinbildenden Schulzeit keinen Abschluss erworben hatten, den Nutzen von besseren Ausbildungs- und Arbeitsmarktchancen als hoch genug ansahen und dafür bereit waren, Kosten in Form von Zeit für weitere Schuljahre und Schulabschlüsse zu investieren. Mit den zusätzlichen Schulabschlüssen erweitern die Jugendlichen dabei ihr Allgemeinwissen, was ihnen später beim Zugang in die Ausbildung und in den Arbeitsmarkt zu Gute kommen kann. Dieses verbesserte schulische Allgemeinwissen ist somit als individuelles Humankapital $\mathrm{zu}$ betrachten, da es an ein Individuum gebunden ist und von diesem dem Arbeitsmarkt zur Verfügung gestellt wird (Rissiek 1998: 17). Diese Entscheidung kann als rational bedacht angesehen werden (Rissiek 1998: 29), zumal die Ergebnisse deutlich zeigten, dass sich viele derjenigen ohne erreichten Schulabschluss für weitere Schulepisoden entschieden. Dieses deutliche Resultat zeigte einerseits, dass die Entscheidung für das nachträgliche Erwerben weiterer allgemeinbildender Schulabschlüsse nicht rein zufällig von einigen wenigen der Jugendlichen ohne Abschluss getroffen wurde, sondern durchaus eine bewusste und wohlüberlegte Entscheidung gewesen sein kann. Andererseits zeigt sich aber auch, dass vielen Personen in dieser Schulabschlussgruppe oftmals keine andere Alternative übrig bleibt, als sich schulisch weiter zu qualifizieren. Ohne erworbenen Schulabschluss sind ihre Übergangschancen in eine vollqualifizierende Ausbildung sehr gering, was an den deutlich verzögerten Übergangsprozessen dieser Gruppe von der Schule in die Erstausbildung ersichtlich wird (Beicht und Ulrich 2008b: 10, 19; Christe 2016: 15).

Vor dem Hintergrund der Humankapitaltheorie wäre ein ähnlicher Trend zur schulischen Weiterqualifizierung auch bei der Gruppe mit einem Hauptschulabschluss denkbar. Auch diese Schulabschlussgruppe muss deutlich längere Übergangszeiten sowie Umwege in Kauf nehmen, um in eine vollqualifizierende Ausbildung zu gelangen (Lex und Zimmermann 2011: 614 f., 621 f.; Seibert und Kleinert 2009: 1). Jedoch zeigten die vorliegenden Ergebnisse, dass die Gruppe derjenigen mit Hauptschulabschluss vor allem berufsvorbereitende Maßnahmen während des Übergangs durchlief. Zwar war ihre geschätzte Wahrscheinlichkeit, weitere Schulepisoden zu durchlaufen und dadurch Bildungsabschlüsse nachzuholen, durchaus hoch, 
jedoch deutlich geringer als bei denjenigen Jugendlichen ohne Abschluss. Die höchste geschätzte Wahrscheinlichkeit hatten Jugendliche mit Hauptschulabschluss, eine berufsvorbereitende Maßnahme zu durchlaufen. In Bezug auf das Absolvieren weiterer Schulepisoden und das Nachholen von Schulabschlüssen konnte für die Schulabgängerinnen und Schulabgänger mit Hauptschulabschluss keine vollkommene Übereinstimmung mit der Humankapitaltheorie festgestellt werden. Bei dieser Gruppe war das Investieren in zusätzliche Schulabschlüsse schwächer ausgeprägt als bei denjenigen ohne Schulabschluss. Bei der Abwägung zwischen den Kosten und dem Nutzen bezüglich der Investition in Humankapital (Persch 2008: 29) entschieden sie sich damit deutlich seltener für den Zeitaufwand durch das Nachholen von Abschlüssen. Der hiermit verbundene Nutzen von verbesserten Ausbildungsund Arbeitsmarktchancen erschien ihnen als nicht so hoch wie denjenigen ohne Abschluss. Sie waren tendenziell bestrebt, beim Übergang in die Ausbildung möglichst keine Zeit zu verlieren und dadurch weniger bereit, die Kosten in Form der Zeit für einen weiteren Schulbesuch auf sich zu nehmen. Stattdessen landeten Jugendliche mit Hauptschulabschluss, wie durch die vorliegenden Ergebnisse nachgewiesen, meist in berufsvorbereitenden Maßnahmen. Bei diesen Maßnahmen bleibt unklar, ob diese sich als Humankapital im Sinne von Bildung beziehungsweise Aus- oder Weiterbildung zur Aneignung berufsrelevanter Qualifikationen (Becker 1982: 137) betrachten lassen. Jugendliche, die Maßnahmen des berufsvorbereitenden Übergangssystems absolvieren, können nicht immer einen schnellen Übergang in eine vollqualifizierende Ausbildung vollziehen (Beicht 2009: 1 ff.). Des Weiteren wird auch nur selten ein direkter Übergang in eine Berufsausbildung vollzogen (Beicht 2009: 10). Bei der Einschätzung der berufsvorbereitenden Maßnahmen ist zudem zu berücksichtigen, dass sich die betroffenen Jugendlichen in diesen Maßnahmen oftmals deutlich länger aufhalten müssen als beabsichtigt (Krone 2010: 23 ff.) und dass sie dies oftmals nicht freiwillig tun. In berufsvorbereitenden Maßnahmen befinden sich insbesondere Jugendliche, die zuvor keinen Ausbildungsplatz gefunden haben. Die Vielzahl an schulischen Bildungswegen und Maßnahmen, wie beispielsweise das berufsvorbereitende Jahr, führen nicht $\mathrm{zu}$ einem berufsqualifizierenden Abschluss. Darüber hinaus erweisen sich die vielfältigen Maßnahmen als intransparent und unübersichtlich für Jugendliche, welche zuvor beim Zugang zu einer vollqualifizierenden Berufsausbildung keinen Erfolg hatten (Krone 2010: 23). Das Ergebnis, dass Jugendliche mit Hauptschulabschluss vorwiegend berufsvorbereitende Maßnahmen durchliefen, steht somit im Kontrast zu der Idee der Humankapitaltheorie, nach einer Kosten-Nutzen-Abwägung und einer stärkeren Gewichtung 
des Nutzens in Humankapital zu investieren und damit die eigenen Ausbildungs- und Arbeitsmarktchancen zu verbessern. Stattdessen mündeten sie notgedrungen in die vielfältigen berufsvorbereitenden Maßnahmen ein, da sie den direkten Übergang von der Schule in die Berufsausbildung nicht bewerkstelligen konnten. Anstatt aus diesen Maßnahmen heraus schnell den Einstieg in eine Ausbildung zu erreichen, verbleiben sie in diesen teilweise länger als vorgesehen und können mit Hilfe der Maßnahmen keine Qualifikation im Sinne eines berufsqualifizierenden Abschlusses erreichen. Somit können die absolvierten berufsvorbereitenden Maßnahmen des Übergangssystems nicht als Humankapital zur Verbesserung der individuellen Ausbildungschancen angesehen werden.

Der Befund, dass Jugendliche mit Realschulabschluss tendenziell Stationen ohne Weiterbildungsbezug beim Übergang absolvierten, steht ebenfalls im Widerspruch mit der Annahme der Humankapitaltheorie. Diese Gruppe hätte die Möglichkeit, eine weitere Schulepisode $\mathrm{zu}$ durchlaufen und dadurch mit dem Abitur den höchstmöglichen Schulabschluss und gleichzeitig bessere Ausbildungs- und Arbeitsmarktchancen zu erreichen. Eine weitere Möglichkeit bestünde darin, während des Übergangszeitraums beispielsweise Praktika zu absolvieren. Von diesen Möglichkeiten machte die Gruppe derjenigen mit mittlerer Reife jedoch keinen Gebrauch. Für sie erschien der Nutzen von verbesserten Ausbildungs- und Arbeitsmarktchancen durch das Nachholen des Abiturs oder durch das Sammeln von berufsrelevanten Erfahrungen im Rahmen eines Praktikums als nicht sehr hoch. Dadurch wendeten sie hierfür eher keine Kosten in Form von Zeit auf und durchliefen, sofern sie nach Erlangen der mittleren Reife nicht direkt den Übergang in eine vollqualifizierende Ausbildung vollzogen, tendenziell Stationen ohne Weiterbildungscharakter.

Diejenigen mit Abitur absolvierten ebenfalls vorwiegend Stationen ohne Weiterbildungsbezug. Dieser Befund lässt sich dahingehend in die Humankapitaltheorie einordnen, dass diese Gruppe bereits über den höchsten Schulabschluss verfügte und daher keine Schulabschlüsse mehr nachholen konnte. Insofern lohnte sich für sie ein Kostenaufwand nicht, um in weitere Schulabschlüsse zu investieren. Die Tatsache, dass diejenigen mit Abitur, sofern sie nicht direkt den Übergang von der Schule in die Erstausbildung vollzogen, Stationen ohne Weiterbildungscharakter absolvierten, widerspricht jedoch der Grundannahme der Humankapitaltheorie. Diese Gruppe investierte Kosten in Form von Zeit für Stationen, welche nicht der Weiterbildung dienten und damit nicht als Humankapital zu betrachten sind. Dadurch hatten diese Personen einen Kostenaufwand, jedoch keinen erhöhten Nutzen, da Stationen ohne Weiterbildungsbezug die Ausbildungs- 
und Arbeitsmarktchancen nicht verbessern. Relativiert muss diese Tendenz jedoch dahingehend, dass Abiturientinnen und Abiturienten die durchschnittlich geringste Anzahl an Übergangsstationen sowie kürzeste Übergangsdauer aller Schulabschlussgruppen aufwiesen. Somit war die verwendete Zeit für nicht-weiterbildungsbezogene Stationen überschaubar. Gleichzeitig bestätigt dies wiederum die Humankapitaltheorie, da diejenigen mit Abitur bereits das meiste Humankapital mit dem höchsten Schulabschluss besitzen und damit am wenigsten Kosten in Form von Zeit aufwenden müssen, um ihre Ausbildungschancen zu verbessern.

Bezüglich der Einordnung der vorliegenden Ergebnisse in den zweiten zentralen theoretischen Ansatz, die Verdrängungsthese, wird deutlich, dass der gestiegene Anteil an Dienstleistungstätigen im tertiären Sektor als Indikator für ein höheres Qualifikationsniveau auf dem Arbeitsmarkt insgesamt keinen Einfluss auf die Übergangsdauer hatte. Bei denjenigen mit Abitur und mit Realschulabschluss hatte der gestiegene Dienstleistungstätigenanteil ebenfalls keinen Einfluss auf deren Übergangsdauer. Dies ist kompatibel zu der Annahme der Verdrängungsthese, dass sich die Zugangschancen in den Ausbildungs- und Arbeitsmarkt vor allem für die schulisch gering qualifizierten Jugendlichen verschlechtert haben (Hanesch 1990: 188), jedoch nicht für diejenigen, die mit dem Abitur den höchsten Schulabschluss erreicht haben. Durch den Bildungsexpansionsprozess und den Trend der Höherqualifizierung findet eine Verdrängung von schulisch gering qualifizierten Jugendlichen beziehungsweise Arbeitskräften aus dem Arbeitsmarkt durch schulisch höher Qualifizierte statt (Blossfeld 1990: 165). Der Trend zur Höherqualifizierung geht ebenfalls einher mit der Expansion des tertiären Sektors in Form des gestiegenen Anteils an Dienstleistungstätigen. Diese Entwicklungen führen dazu, dass sich der Anteil an hoch qualifizierten Arbeitskräften sukzessive erhöht und der schrumpfende Anteil der gering qualifizierten Arbeitskräfte immer größere Schwierigkeiten beim Übergang in den Ausbildungs- und Arbeitsmarkt zeigt (Solga 2011: 419 f.). Diese Entwicklung zeigte sich in den vorliegenden Ergebnissen anhand der verringerten geschätzten Chance und einer verlängerten Übergangsdauer durch den gestiegenen Anteil an Dienstleistungstätigen bei denjenigen mit Hauptschulabschluss. Im Rahmen der deskriptiven Ergebnisse zeigte sich, dass der Anteil derjenigen ohne Schulabschluss oder mit Hauptschulabschluss im Zeitverlauf deutlich abnahm, während der Anteil der Abiturientinnen und Abiturienten kontinuierlich anstieg. Dies spricht ebenfalls für die Verdrängungsthese in Form einer immer stärker 
werdenden Konkurrenz für den schrumpfenden Anteil der schulisch gering Qualifizierten durch den steigenden Anteil schulisch hoch qualifizierter Jugendlicher.

Unabhängig von externen Einflussfaktoren zeigten die Ergebnisse, dass die Gruppe derer ohne Abschluss die mit Abstand durchschnittlich längste Übergangsdauer aufwies, während sich die Dauer der Gruppe mit Realschulabschluss im Zeitverlauf im gleichen Ausmaß verlängerte wie bei denjenigen mit Hauptschulabschluss. Die deskriptiven Ergebnisse lassen vermuten, dass ein gestiegenes Qualifikationsniveau auf dem Arbeitsmarkt die Übergangsdauer aller Schulabschlussgruppen bis auf die Abiturientinnen und Abiturienten verlängert hat. Dies bestätigt die Verdrängungsthese. Der Befund, dass der gestiegene Anteil im tertiären Sektor generell die Übergangsdauer nicht beeinflusst hat, steht dagegen im Widerspruch zur Verdrängungsthese.

Bei der abschließenden Einordnung der Ergebnisse in die historischen Entwicklungen fällt bei den Anteilen der Schulabschlussgruppen auf, dass sich diese im Zeitverlauf mit dem Prozess der Bildungsexpansion decken. War zu Beginn des Untersuchungszeitraums noch der Hauptschlussabschluss der am stärksten frequentierte erste Schulabschluss, nahm der Anteil dieser Gruppe im weiteren Verlauf deutlich ab. Der Anteil derjenigen ohne Abschluss war zu Beginn eher gering, stieg im späteren Verlauf ganz leicht an und verringerte sich zum Ende deutlich. Während der Anteil derjenigen mit Realschulabschluss überwiegend konstant blieb, stieg der Anteil derjenigen mit Abitur im Zeitverlauf extrem stark an. Während diese Gruppe zu Beginn des Untersuchungszeitraums kaum repräsentiert war, stellte sie zum Ende die mit Abstand am stärksten frequentierte Schulabschlussgruppe dar. Diese Entwicklungen zeigten sich während des Prozesses der Bildungsexpansion dahingehend, dass der Anteil der Schülerschaft auf den Hauptschulen deutlich gesunken und der Anteil auf den Gymnasien deutlich gestiegen ist (Allmendinger et al. 2010: 52), wodurch das Gymnasium seit Mitte der 1990er Jahre die am häufigsten besuchte Schulform ist (Lauterbach 2012: 580). Der in den Ergebnissen über die Zeit konstant bleibende Anteil derjenigen mit mittlerer Reife steht jedoch im deutlichen Widerspruch zu der Erkenntnis der Bildungsexpansion, dass sich der Anteil der Schülerschaft auf den Realschulen enorm erhöht hat (Allmendinger et al. 2010: 52). Die im Rahmen der Bildungsexpansion konstatierte Zunahme des Anteil an Abiturientinnen und Abiturienten (Becker 2006: 32 f.) deckt sich dagegen mit dem Ergebnis des extrem gestiegenen Anteils der Gruppe mit Abitur im Untersuchungszeitraum.

Auch der Anstieg an hochqualifizierten Arbeitskräften auf dem Arbeitsmarkt durch eine zunehmende Anzahl an Personen mit höheren Schulabschlüssen bei gleichzeitig erhöhten 
Zugangsvoraussetzungen zum Arbeitsmarkt (Protsch 2014: 74) stimmt überein mit dem deutlich gestiegenen Anteil derjenigen mit Abitur sowie einer im Zeitverlauf verlängerten Übergangsdauer derjenigen mit Real-, Hauptschulabschluss und ohne Schulabschluss. Die Entwicklung, dass mehr Übergangsstationen vor allem zwischen dem Verlassen der Schule und dem Beginn der Erstausbildung unter anderem in Form von berufsvorbereitenden Maßnahmen absolviert werden (Pätzold 2008: 603), zeigte sich in den vorliegenden Ergebnissen darin, dass oftmals während des Übergangs berufsvorbereitende Maßnahmen absolviert wurden. Dies betraf in erster Linie diejenigen mit Hauptschulabschluss, bei denen sich die Übergangsdauer dadurch verlängerte.

Insgesamt sind die Ergebnisse der vorliegenden Studie zum Teil kompatibel mit den Ergebnissen vorheriger Studien. Jedoch bestehen auch einige Diskrepanzen. Gleiches trifft auf die Einordnung zur Humankapitaltheorie und Verdrängungsthese zu. Eine Übereinstimmung oder Abweichung zu bisherigen Studien und theoretischen Überlegungen hängt bei einer differenzierten Betrachtung der Ergebnisse unter anderem von der jeweiligen Schulabschlussgruppe ab.

\subsection{Limitationen der Studie}

Beim Blick auf die Limitationen der vorliegenden Studie fallen verschiedene Aspekte auf, welche insbesondere bei der Interpretation der Ergebnisse mit berücksichtigt werden müssen und auf welche im Folgenden ausführlich eingegangen wird. Zunächst ist zu beachten, dass die verwendeten Rohdaten der Erwachsenenkohorte des NEPS vielfältige nicht plausible Angaben und Inkonsistenzen aufwiesen, welche aufwändig bereinigt werden mussten. Diese umfangreiche Datenbereinigung stellte zunächst die Voraussetzung für die weitere Aufbereitung der Daten dar. Erst im Anschluss an diese Vorbereitungsschritte war eine sinnvolle Durchführung der Analysen möglich, welche den Erhalt von plausiblen Ergebnissen gewährleistete. Zunächst zeigten sich nicht plausible Angaben beim Alter der befragten Personen. Diese wurden an einer zeitlich viel zu geringen Differenz zwischen dem Geburtsjahr und dem Datum des erworbenen Schulabschlusses oder des Beginns der ersten Ausbildung deutlich. Manchmal entstanden auch Überschneidungen, wenn das Datum des Schulabschlusses oder des Beginns der Erstausbildung vor dem Geburtsjahr lag oder das Schulenddatum nach dem Erstausbildungsbeginn datiert war. Diese Inkonsistenzen wurden mit Hilfe einer Korrektur des jeweiligen Schulenddatums oder des Erstausbildungsbeginns basierend auf dem Geburtsjahr, dem erworbenen Schulabschluss sowie dem Enddatum einer 
darauf folgenden Schulepisode bereinigt. Anhand dieser Parameter konnte rekonstruiert werden, wann ein plausibler Zeitpunkt für das Enddatum der ersten Schulepisode oder den Beginn der Erstausbildung gewesen sein könnte. Aufgrund der benannten Korrekturen kann somit nicht sicher gewährleistet werden, ob die rekonstruierten und bereinigten Daten zu den Schulepisoden die Realität der schulischen Bildungsverläufe der befragten Personen besser abbilden als die teilweise nicht plausiblen Rohdaten der Erwachsenenkohorte des NEPS. Im Zweifel muss davon ausgegangen werden, dass diese Korrekturen eventuell mit einer Verzerrung oder Verfälschung der wirklichen Zeitpunkte für die Beendigung der Schulepisoden einhergehen.

Des Weiteren kann nicht mit Sicherheit davon ausgegangen werden, dass bei den aufgezählten Schulepisoden ein Anspruch auf Vollständigkeit besteht. Beispielsweise konnte nicht vollständig aufgeklärt werden, ob eine Person, welche nur eine Schulepisode mit dem erworbenen Abschluss Abitur angab, zuvor auf andere Abschlüsse hingearbeitet hatte. In dieser Konstellation war auch das Vorliegen der Schulform, wie in diesem Beispiel das Gymnasium, nicht von großer Hilfe. Die befragte Person könnte theoretisch auf dem Gymnasium zunächst auf den Hauptschulabschluss, dann auf die mittlere Reife und schließlich, nach dem Erwerb dieser beiden Abschlüsse, auf das Abitur hingearbeitet haben. Im Sinne der Humankapitaltheorie wäre der Erwerb des Realschulabschlusses und des Abiturs eine Investition in Humankapital zur Verbesserung der individuellen Ausbildungsund Erwerbschancen. Da die Schulepisoden jedoch in den Rohdaten des NEPS uneinheitlich erhoben wurden und einerseits einzeln und ausführlich, andererseits aber zum Beispiel auch zu einer Schulepisode zusammengefasst waren, kann nicht mit hundertprozentiger Sicherheit gewährleistet werden, dass alle einzelnen Schulepisoden, bei denen zusätzliche Schulabschlüsse erworben wurden, berücksichtigt sind. Dieser Umstand ist bei den Ergebnissen zu den zusätzlichen Schulepisoden als Übergangsstationen zu berücksichtigen. Bei der Anzahl der zusätzlichen Schulepisoden musste sich auf die in den NEPS-Rohdaten erhobenen Episoden verlassen werden, da keine Möglichkeit bestand, das Vorhandensein von eventuell zusätzlichen Schulepisoden zu überprüfen und diese nachträglich zu erheben. Die geschilderte Problematik gestaltete sich auch beim Startdatum der Erstausbildung. Dabei kann nicht ausgeschlossen werden, dass durch die Korrekturen der Erstausbildungsbeginn und auch der Erstausbildungstyp verzerrt sein könnte. Es bleibt unklar, warum zum Teil nicht plausible Angaben zum Erstausbildungsbeginn in den Rohdaten vorlagen. Aus diesem Grund musste auf die nach Plausibilitätskriterien korrigierten Daten zurückgegriffen werden. Dennoch sind 
die Korrekturen und die damit verbundenen Umstände sowohl bei den Schulepisoden als auch bei der Erstausbildung bezüglich der Interpretation der Übergangsdauer, der Schulabschlüsse und der Erstausbildungstypen zu berïcksichtigen. Hierbei muss von einer eingeschränkten Aussagekraft der Ergebnisse ausgegangen werden.

Inkonsistenzen zeigten sich auch bei den erhobenen Stationen zwischen Schulenddatum und Erstausbildungsbeginn. Hierbei lagen in den Rohdaten zahlreiche Dopplungen und Überschneidungen sowohl von identischen als auch von verschiedenen Stationen vor. Für die Bereinigung dieser Konstellationen war bei einer Dopplung oder Überschneidung zweier unterschiedlicher Stationen eine inhaltliche Gewichtung aller Stationskombinationen notwendig. Dadurch sollte zu einem bestimmten Zeitpunkt immer nur eine absolvierte Station angegeben sein, was Voraussetzung zur Durchführung der Sequenzdatenanalyse war. Somit konnte ein mögliches gleichzeitiges Absolvieren zweier Stationen wie beispielsweise eines Praktikums und einer Weiterbildung nicht abgedeckt werden. Aufgrund der aufgeführten Probleme und Unsicherheiten bei der Korrektur des Schulenddatums und Erstausbildungsstartdatums kann nicht ausgeschlossen werden, dass Übergangsstationen unberechtigt keine Berücksichtigung fanden, welche vor oder nach den beiden bereinigten Zeitpunkten datiert waren. Deshalb muss auf Basis der bereinigten Daten von einer begrenzten Aussagekraft der Ergebnisse zu den Übergangsstationen ausgegangen werden.

Insgesamt bleiben die Hintergründe für das Zustandekommen der nicht-plausiblen Angaben in den Rohdaten unklar. Dies könnte eventuell durch fehlerhafte Angaben oder auch durch Erinnerungslücken der befragten Personen entstanden sein. Sämtliche absolvierte bildungsund berufsbiografische Stationen wurden mit Hilfe von computergestützten Telefoninterviews erhoben, wobei die biografischen Daten aus dem Gedächtnis der Befragten geschildert wurden, da sie gegebenenfalls zeitlich weit zurück lagen und dadurch zum Befragungszeitpunkt eventuell verzerrt sein konnten. Jedoch bleiben die Begründungen rein spekulativ, da sich dies nicht aufklären lässt. Aufgrund der Vielzahl an nicht-plausiblen Angaben und Inkonsistenzen in den verwendeten Rohdaten und einer darauf folgenden Bereinigung und Korrektur kann nicht gewährleistet werden, dass die aufbereiteten Daten vollständig den realen Bildungs- und Übergangsverläufen der befragten Personen entsprechen. Die aufgeführten nicht-plausiblen Angaben und Inkonsistenzen traten jedoch nur bei einer Teilgruppe der verwendeten Stichprobe auf.

Eine weitere Limitation bei den verwendeten Daten bestand darin, dass die Zeiteinheit sämtlicher Episoden im Rahmen des NEPS in Monaten erhoben wurde. Dadurch gab es 
keinen Aufschluss darüber, ob eine Station oder Episode beispielsweise zum 1. August oder zum 15. August begonnen beziehungsweise geendet hat. Bei Vorliegen von Tagen als Zeiteinheit wären zeitlich noch präzisere Angaben möglich, wenngleich sich eine Bereinigung von Dopplungen und Überschneidungen bei Episoden auf der Basis von Tagen noch wesentlich umfangreicher und komplexer gestalten dürfte.

Weiterhin ist zu berücksichtigen, dass der Untersuchungszeitraum der vorliegenden Studie auf die Jahre 1971 bis 2012 eingegrenzt wurde. Beim Jahr 1971 handelt es sich um das erste einbezogene Schulenddatumsjahr, während das Jahr 2012 für das letzte berücksichtigte Jahr des Übergangs in eine Ausbildung steht. Das letzte berücksichtigte Schulenddatumsjahr ist dagegen das Jahr 2007, da die jüngste einbezogene Geburtskohorte des Jahrgangs 1986 in der Regel spätestens in diesem Jahr einen Schulabschluss erworben haben wird. Die weiteren Jahre bis 2012 finden deshalb Berücksichtigung in den Analysen, da somit auf zusätzlich erhobene Informationen der Panelteilnehmerinnen und -teilnehmer aus der letzten einbezogenen NEPS-Befragungswelle von 2011/2012 zurückgegriffen werden kann. Die Beschränkung auf diesen Zeitraum liegt insgesamt darin begründet, dass in der Zeit vor 1971 und nach 2012 die Anzahl an Abiturientinnen und Abiturienten zu gering (vor 1971) beziehungsweise zu hoch (nach 2012) war. Bei Einbezug der im NEPS zusätzlich akquirierten Personen der Geburtsjahrgänge 1944 bis 1955 könnten zwar ein längerer Untersuchungszeitraum und eine deutlich größere Stichprobe verwendet werden. Jedoch müsste mit vor allem in den früheren Jahrzehnten sehr ungleich verteilten Schulabschlussgruppen umgegangen werden, da insbesondere Personen mit Abitur in dieser Phase stark unterrepräsentiert waren.

Eine weitere inhaltliche beziehungsweise methodische Limitation bezieht sich auf die Auswertung der jeweiligen Stationsinhalte. Dabei ging es in der vorliegenden Studie darum, die absolvierten Übergangsstationen nicht nur hinsichtlich ihrer Anzahl zu analysieren, sondern auch den Fokus darauf zu legen, was für eine Station beziehungsweise Stationskategorie exakt absolviert wurde. Hierbei war die Überlegung zunächst, sich Typen von Stationssequenzen anzuschauen, welche besonders häufig durchlaufen werden, wie zum Beispiel erst eine berufsvorbereitende Maßnahme und im Anschluss eine Arbeitslosigkeit. Mit Hilfe der Bildung von Clustern an Sequenztypen sollten die am häufigsten auftretenden Sequenzverläufe an absolvierten Stationen extrahiert und diese für die weiteren Analysen verwendet werden. Jedoch ergaben sich hierbei kaum inhaltlich trennscharfe Cluster, was eine inhaltliche Differenzierung der Sequenztypen unmöglich machte. Dieser Umstand machte es 
notwendig, Stationskategorien zu bilden und jede der einbezogenen Personen einer Kategorie zuzuweisen. Beispielsweise wurden die Stationen Kurs, Weiterbildung und Praktikum zu einer Kategorie zusammengefasst. Hatte eine Person Stationen aus zwei verschiedenen Kategorien durchlaufen, wurde die Stationskategorie stärker gewichtet, welche den größeren Weiterbildungsbezug hatte. Als die Stationskategorie mit dem größten Weiterbildungscharakter wurden die zusätzlichen Schulepisoden gewichtet. Somit wurde jede Person einer Stationskategorie zugewiesen. Diese Vorgehensweise machte es unmöglich, aufgrund der Zuweisung zu einer Kategorie und der teilweisen Zusammenfassung von Stationen zu einer Kategorie einzelne Stationen zu identifizieren, die absolviert wurden. Dies betraf jedoch nicht die Stationskategorien „Berufsvorbereitende Maßnahmen“ und „Zusätzliche Schulepisoden“, welche jeweils eine eigene Stationskategorie bildeten. Außerdem konnten bei den Regressionsmodellen dadurch keine Stationsverläufe einzelner Personen berücksichtigt werden, das heißt, es konnte keine Aussage darüber getroffen werden, ob beispielsweise ein gestiegener Anteil an Dienstleistungstätigen einen Effekt darauf hatte, dass die einbezogenen Personen zunächst eher berufsvorbereitende Maßnahmen durchliefen und dann in eine Arbeitslosigkeit gerieten. Aussagen zu den Sequenzverläufen, welche Stationen beziehungsweise Kombinationen von Stationen nacheinander durchlaufen wurden, waren im Rahmen der vorliegenden Arbeit lediglich anhand von deskriptiven Verteilungen möglich. Des Weiteren konnten, sofern Stationen aus zwei oder mehr verschiedenen Kategorien durchlaufen wurden, die Stationen der geringer gewichteten Kategorien nicht berücksichtigt werden. Des Weiteren hing die Zuordnung auch davon ab, welche Stationskategorie einen größeren Weiterbildungsbezug hatte. In Bezug auf die Inhalte der absolvierten Stationen konnte somit aufgrund der geschilderten methodischen Probleme lediglich eine Kategorisierung der Stationen vorgenommen werden. Dabei spielte, sofern eine Person Stationen aus verschiedenen Kategorien absolviert hatte, lediglich die Stationskategorie mit dem größten Weiterbildungsbezug eine Rolle. Diese Kategorisierung konnte weder einem Verlauf mehrerer Stationen bei einzelnen Personen, noch einer genaueren Identifizierung einzelner Stationen sowie einer Berücksichtigung aller absolvierten Stationen gerecht werden. Durch eine bessere und inhaltlich trennschärfere Clusterbildung der Sequenzverläufe hätten diese Informationen für die Analysen im Rahmen der Regressionsmodelle zur Testung der Hypothesen verwendet werden können.

Weiterhin ist $\mathrm{zu}$ berücksichtigen, dass Stationen, welche keinen Bildungs- oder Arbeitsmarktbezug haben wie beispielweise der Wehrdienst oder eine Elternzeit, aus den 
Analysen ausgeschlossen wurden. Die Beschränkung auf rein bildungs- und arbeitsmarktrelevante Stationen und Episoden war der thematischen und vor allem theoretischen Ausrichtung der Studie geschuldet, wobei der Fokus vor allem auf der Investition in Humankapital zur Verbesserung der Ausbildungschancen lag. Diese Einschränkung hatte definitiv Auswirkungen auf die berechnete Übergangsdauer sowie die Anzahl an durchlaufenen Stationen. Bei einem Fokus auf einen generellen Übergangsverlauf mit Einbezug aller biografisch vorhandenen Zwischenstationen wäre dagegen eine Berücksichtigung aller Stationen unabhängig von deren Bezug zwingend notwendig. Dies würde bei der Betrachtung des gesamten Übergangs- beziehungsweise Lebensverlaufs ein vollständiges Bild aller vorhandenen Episoden liefern und auch uneingeschränkte Informationen zur Übergangsdauer und der Anzahl an Stationen bieten.

Eine zusätzliche methodische Limitation liegt darin vor, dass es sich bei den zusätzlich implementierten Daten zum Anteil an Dienstleistungstätigen, zur Arbeitslosenquote und zum demografischen Anteil der unter 20-jährigen Personen um Makrodaten handelt. Diese Daten liegen jeweils für die einzelnen Jahre vor und wurden anhand des Jahres des Schulenddatums (1971 - 2007) zu den Individualdaten der befragten Personen aus dem NEPS zugewiesen. Da $\mathrm{zu}$ den angesprochenen Variablen keine Individualdaten verfügbar waren, musste auf Makrodaten der einzelnen Jahre zurückgegriffen werden. Diese Zuweisung von Makrodaten zu Individualdaten führte im Rahmen der durchgeführten Regressionsanalysen zu teils sehr starken Multikollinearitäten zwischen den Makrovariablen und damit zu einer möglichen Verzerrung der jeweiligen Regressionseffekte beziehungsweise deren Standardfehlern.

Die jährlichen Anteile der Dienstleistungstätigen beziehen sich bis zum Jahr 1990 auf das frühere Bundesgebiet und damit Westdeutschland. Die Daten ab 1991 berücksichtigen zusätzlich die neuen Bundesländer und beziehen sich damit auf ganz Deutschland (Statistisches Bundesamt 2017a). Dadurch ist anhand der Datengrundlage des Statistischen Bundesamtes eine separate Betrachtung der Anteile für Westdeutschland ab dem Jahr 1991 nicht möglich, weshalb die Daten für ganz Deutschland verwendet werden müssen.

Durch Ausschluss der in Ostdeutschland geborenen Personen verringerte sich die Stichprobe, wobei dies wegen der Beschränkung auf Übergangsverläufe westdeutscher Jugendlicher erforderlich war. Ein weiterer Grund hierfür war die fehlende Vergleichbarkeit der Bildungsund Ausbildungssysteme der DDR beziehungsweise der neuen Bundesländer und der alten Bundesländer. Des Weiteren wurden im Ausland geborene Personen ausgeschlossen, welche zum Zeitpunkt ihrer Migration nach Deutschland älter als 14 Jahre waren. Diese Altersgrenze 
wurde gewählt, um sicherzustellen, dass diese Personengruppe sich zumindest ein Jahr lang im deutschen Schulsystem befindet. Dies wäre bei denjenigen Personen der Fall, welche einen Hauptschulabschluss erwerben. Das reguläre Alter hierfür beträgt 15 Jahre. Jedoch wurden auf diese Weise diejenigen Personen nicht berücksichtigt, welche mit 15 Jahren oder später nach Deutschland kamen, die Schule noch länger besuchten und eventuell auch höhere Bildungsabschlüsse erwarben.

\subsection{Schlussfolgerung}

Die vorliegende Arbeit untersuchte erstmals, ob sich bei westdeutschen Jugendlichen der Übergangsprozess vom Verlassen der Schule bis zum Beginn der Erstausbildung in Form der Dauer und der dabei absolvierten Stationen unter dem Einfluss einer externen Makrovariablen verändert hat. Vor dem Hintergrund der Bildungsexpansion und eines sich verändernden Arbeitsmarktes konnte der Einfluss des expandierenden tertiären Dienstleistungssektors auf den Übergangsprozess untersucht werden. Die Expansion des tertiären Wirtschaftssektors in Form eines gestiegenen Anteils an dienstleistungstätigen Erwerbstätigen wurde des Weiteren erstmals als zentrale Makrovariable einbezogen.

Dabei konnte nachgewiesen werden, dass der tertiäre Sektor den Übergangsprozess der westdeutschen Jugendlichen vom Verlassen der Schule bis zum Beginn der Erstausbildung nicht tangierte. Durch eine genauere Betrachtung sowohl der Übergangsdauer als auch der Übergangsstationen in Form der Anzahl und der Stationskategorien konnte nachgewiesen werden, dass der expandierende Dienstleistungssektor auf beide Indikatoren des Übergangsprozesses keinen Effekt hatte. Auch bei einer differenzierten Betrachtung nach Schulabschluss ergab sich die gleiche Erkenntnis.

\subsection{Ausblick}

Beim Ausblick auf die zukünftige Forschung ist festzustellen, dass durch die Verwendung von anderen Daten oder durch die Generierung einer noch größeren Stichprobe versucht werden kann, bezüglich der Sequenzverläufe der absolvierten Stationen inhaltlich trennscharfe Cluster zu bilden. Dadurch könnte im Rahmen von Regressionsmodellen auch ein möglicher Einfluss von externen Faktoren wie dem gestiegenen Anteil an Dienstleistungstätigen im tertiären Sektor auf das Absolvieren bestimmter Sequenzverläufe an Übergangsstationen überprüft werden. Diese Ergebnisse würden deutlich über die begrenzte 
Aussagekraft der gewichteten und zugewiesenen Stationskategorien der vorliegenden Studie hinausgehen.

Auch eine Ausweitung des Untersuchungszeitraums, welcher bereits vor 1971 beginnt und beziehungsweise oder nach 2012 endet, könnte einen noch weiteren und besseren Entwicklungsverlauf der Übergangsdauer und der Übergangsstationen unter Einfluss der Veränderungen im tertiären Sektor liefern. Der sehr ungleichen Schulabschluss-Verteilung in Form einer zu geringen Anzahl an Abiturientinnen und Abiturienten in früheren Jahrzehnten und einem zu großen Anteil dieser Schulabschlussgruppe in der jüngsten Vergangenheit könnte jedoch nur mit einer entsprechenden Gewichtung begegnet werden. Diese Vorgehensweise würde es jedoch ebenfalls ermöglichen, die zusätzlich im NEPS akquirierten Personen der Geburtskohorten 1944 bis 1955 mit einzubeziehen und dadurch die Stichprobe nochmals zu vergrößern.

Ein weiterer interessanter Forschungsansatz wäre, die gleiche Studie für die Übergangsverläufe zwischen Schule und Ausbildung für Personen in der DDR sowie in den neuen Bundesländern durchzuführen. Hierfür wäre es erforderlich, das Bildungs- und Ausbildungssystem der DDR und in den neuen Bundesländern ausführlich zu thematisieren, um die Ergebnisse einer solchen Untersuchung richtig einordnen zu können. Ein Vergleich zu den Ergebnissen der vorliegenden Untersuchung mit westdeutschen Jugendlichen wäre aber allein schon durch die unterschiedlichen Bildungs- und Ausbildungssysteme nicht möglich.

Des Weiteren wäre interessant, ob ein anderer Indikator für das gestiegene Qualifikationsniveau auf dem Arbeitsmarkt anstelle des gestiegenen Anteils an Dienstleistungstätigen zu ähnlichen Ergebnissen führt wie in der vorliegenden Studie. Beispielsweise könnte die Berufsgruppe der jeweiligen Ausbildung, in die die befragten Personen einmünden, dahingehend zugeordnet werden, ob es sich um einen Dienstleistungsberuf im tertiären Sektor handelt oder nicht. Diese Zuordnung des Ausbildungsberufs wäre dann ein möglicher zu untersuchender Prädiktor bezüglich der Übergangsdauer und der Stationen. Des Weiteren würde damit die Möglichkeit bestehen, auf der Ebene der Individualdaten zu verbleiben. Dadurch müssten keine externen Makrodaten implementiert werden, welche eine Multikollinearität bei Regressionsanalysen verursachen. 


\section{Anhang}

\section{Anhang A: Datenaufbereitung}

Tabelle 1: Übersicht zu Stichprobenziehung, Zielgruppe und Fallzahlen der ALWA-Studie.

\begin{tabular}{|c|c|c|c|}
\hline $\begin{array}{c}\text { Stichprobenziehung } \\
\text { / -selektion }\end{array}$ & Verfahren & Zielgruppe & Fallzahl n \\
\hline 1. Schritt & $\begin{array}{l}\text { Ermittlung von } 250 \text { Gemeinden aus } \\
\text { gemeindestatistischen Daten des } \\
\text { Statistischen Bundesamtes und der } \\
\text { Statistischen Landesämter }\end{array}$ & Berücksichtigung & Durchführung \\
\hline 2. Schritt & $\begin{array}{l}\text { - Zugang zu Adressliste von } \\
\text { Personen der Geburtsjahrgänge } \\
1956 \text { bis } 1988 \text { durch } \\
\text { randomisierte Auswahl der } \\
\text { Einwohnermeldeämter der } 250 \\
\text { Gemeinden } \\
\text { - Anschließend randomisiertes } \\
\text { Herausfiltern von insgesamt } \\
\text { 42.712 Adressen aus Bestand aller } \\
\text { Gemeinden; zu } 22.656 \text { Adressen } \\
\text { davon wurde eine Telefonnummer } \\
\text { recherchiert }\end{array}$ & $\begin{array}{l}\text { ausschließlich von Personen } \\
\text { (Geburtsjahrgänge } 1956 \text { bis } \\
\text { 1988) in erwerbsintensivem } \\
\text { Alter, bei denen Übergänge } \\
\text { zwischen Schule, } \\
\text { Ausbildung / Studium und } \\
\text { Erwerbstätigkeit } \\
\text { abgeschlossen sind / gerade } \\
\text { stattfinden }\end{array}$ & $\begin{array}{l}10.404 \\
\text { computer- } \\
\text { gestützten } \\
\text { Telefoninter- } \\
\text { views (offizielle } \\
\text { Stichproben- } \\
\text { größe der } \\
\text { ALWA- } \\
\text { Befragung) }\end{array}$ \\
\hline $\begin{array}{l}\text { Auswahl für Welle } \\
1 \text { im NEPS }\end{array}$ & $\begin{array}{l}\text { - Einbezug von Personen in NEPS } \\
\text { aus ursprünglicher ALWA- } \\
\text { Stichprobe, welche an einer / } \\
\text { mehreren NEPS-Befragungen } \\
\text { teilgenommen haben (N = } 6.855 \\
\text { Personen der Geburtsjahrgänge } \\
1956 \text { bis 1986) } \\
\text { - Rekrutierung von } 77 \text { Befragten } \\
\text { für ALWA-Befragung ohne } \\
\text { deutsche Sprachkenntnisse, } \\
\text { welche erst bei der ersten NEPS- } \\
\text { Befragungswelle } 2009 \text { / } 2010 \\
\text { befragt wurden (kein } \\
\text { fremdsprachiger Fragebogen bei } \\
\text { ALWA-Befragung) }\end{array}$ & $\begin{array}{l}\text { Personen der } \\
\text { Geburtsjahrgänge } 1956 \text { bis } \\
\text { 1986; Ausschluss von } \\
\text { Teilnehmenden der ALWA- } \\
\text { Studie der Jahrgänge } 1987 \\
\text { und 1988, die an keiner } \\
\text { NEPS-Befragungen } \\
\text { teilnahmen }\end{array}$ & $\begin{array}{l}\mathrm{N}=6.778 \\
\text { Personen }\end{array}$ \\
\hline
\end{tabular}

Quelle: Eigene Darstellung basierend auf Kleinert et al. 2008: 6, 29; Forschungsdatenzentrum der Bundesagentur für Arbeit im Institut für Arbeitsmarkt- und Berufsforschung a; Skopek 2013: 16 f.; NEPS Nationales Bildungspanel 2016a: 2; Aust et al. 2011: 18. 
Tabelle 2: Übersicht Ausprägungen Schulabschluss, Ausbildungstyp und soziale Herkunft sowie Zusammenfassung zu verwendeten Kategorien.

Variable

Ausprägungen

Zusammengefasste Kategorien

\begin{tabular}{|c|c|c|}
\hline \multirow{5}{*}{ Schulabschluss } & „Ohne Abschluss“ & Kein Abschluss \\
\hline & $\begin{array}{l}\text { „einfacher Hauptschulabschluss / Berufsbildungs- / } \\
\text { Berufsreife / Erster allgemeinbildender } \\
\text { Schulabschluss“ } \\
\text { „qualifizierender / qualifizierter } \\
\text { Hauptschulabschluss / Erweiterte } \\
\text { Berufsbildungsreife, Berufsreife mit } \\
\text { Leistungsfeststellung“ }\end{array}$ & Hauptschulabschluss \\
\hline & $\begin{array}{l}\text { „Mittlere Reife / Real- / Wirtschaftsschulabschluss / } \\
\text { Fachschul- / Fachoberschulreife / Mittlerer Schul- / } \\
\text { Mittlerer Bildungsabschluss“ }\end{array}$ & Realschulabschluss \\
\hline & $\begin{array}{l}\text { „Fachhochschulreife“ } \\
\text { „andere Hochschulreife (Abitur)“ }\end{array}$ & Abitur \\
\hline & $\begin{array}{l}\text { „Es war kein Abschluss vorgesehen“ } \\
\text { „Sonder- / Förderschulabschluss“" } \\
\text { „Anderer Abschluss“ }\end{array}$ & $\begin{array}{l}\text { Aus den Analysen } \\
\text { ausgeschlossen }\end{array}$ \\
\hline \multirow[b]{2}{*}{ Erstausbildungstyp } & $\begin{array}{l}\text { „Lehre (Facharbeiterausbildung, duale } \\
\text { Berufsausbildung)“ } \\
\text { „Berufsfachschulausbildung / Ausbildung an einer } \\
\text { Fachschule des Gesundheitswesens“ } \\
\text { „Studium an einer Fachhochschule“ } \\
\text { „Studium an einer Universität / Hochschule“ } \\
\text { „Studium an einer Berufsakademie“ }\end{array}$ & $\begin{array}{l}\text { Duale Berufsausbildung } \\
\text { Vollzeitschulische } \\
\text { Berufsfachschulausbildung }\end{array}$ \\
\hline & $\begin{array}{l}\text { „Studium an einer Verwaltungs- und } \\
\text { Wirtschaftsakademie“" } \\
\text { „Fachschulausbildung (auch } \\
\text { Fachlehrerausbildung)“ } \\
\text { „Meister- oder Technikerausbildung“ } \\
\text { „Promotion“ } \\
\text { „Habilitation“ } \\
\text { „Ausbildung zum Facharzt“ }\end{array}$ & $\begin{array}{l}\text { Aus den Analysen } \\
\text { ausgeschlossen }\end{array}$ \\
\hline
\end{tabular}


„Laufbahnprüfung im öffentlichen Dienst"

„Lehrgang bei Verband / Kammer (z.B. IHK-

Lehrgang)“

„Kurs zum Erwerb einer Lizenz (Schweißer-,

Berücksichtigung als

Stapler- oder Taxifahrerschein,

Netzwerkadministrator)“

Weiterbildung und damit als

Übergangsstationen

„anderer Kurs / Lehrgang““

„[1a] kein Abschluss“

,[1a] kein Abschluss / [1b]

,[1b] Hauptschulabschluss ohne berufliche

Hauptschulabschluss ohne

Ausbildung“"

berufliche Ausbildung"

\begin{tabular}{ll}
\hline,$[1 \mathrm{c}]$ Hauptschulabschluss mit beruflicher &,$[1 \mathrm{c}]$ Hauptschulabschluss mit \\
Ausbildung“ & beruflicher Ausbildung“
\end{tabular}

„[2b] Mittlere Reife ohne berufliche Ausbildung“ „[2b] Mittlere Reife ohne berufliche Ausbildung / [2a]

Soziale Herkunft

„[2a] Mittlere Reife mit beruflicher Ausbildung“

Mittlere Reife mit beruflicher Ausbildung“"

\begin{tabular}{ll}
$\begin{array}{l}\text { „[2c_gen] Hochschulreife ohne berufliche } \\
\text { Ausbildung“ }\end{array}$ & $\begin{array}{l}\text {,[2c_gen] Hochschulreife ohne } \\
\text { berufliche Ausbildung / } \\
{\left[2 \mathrm{c} \_ \text {voc] Hochschulreife mit }\right.} \\
\text { beruflicher Ausbildung“ }\end{array}$ \\
$\begin{array}{l}\text { „[2c_voc] Hochschulreife mit beruflicher } \\
\text { Ausbildung“ }\end{array}$ & \\
\hline$[3 \mathrm{a}]$ Fachhochschulabschluss“ &,$[3 \mathrm{a}]$ Fachhochschulabschluss / \\
,[3b] Universitätsabschluss“ & {$[3 \mathrm{~b}]$ Universitätsabschluss“ }
\end{tabular}

Quelle: Eigene Darstellung der ursprünglichen Ausprägungen zum Schulabschluss basierend auf NEPS Nationales Bildungspanel 2016b: 2292, der Ausbildungstypen basierend auf NEPS Nationales Bildungspanel 2016b: 2422 f. und den sozialen Herkunftskategorien basierend auf Brauns et al. 2003: 223. 
Tabelle 3: Aus ursprünglicher Stichprobe ausgeschlossene Gruppen und dazugehörige Fallzahlen.

$\begin{array}{ccc}\text { Ausgeschlossene Gruppe } & \text { Anzahl der } & \text { Übrige } \\ \text { ausgeschlossenen Fälle } & \text { Fallzahl n }\end{array}$

\begin{tabular}{lcc}
\hline Ursprüngliche Stichprobe (Teilnehmer an ALWA und den beiden & 12.611 \\
NEPS-Befragungen) & 2.381 & 10.230 \\
In Ostdeutschland / Ostberlin geborene Personen & 9.340 \\
Im Ausland geborenen Personen, welche bei der Migration nach & 890 & 9.337 \\
Deutschland älter als 14 Jahre waren & 3 & 9.330 \\
Personen, die nach 1986 geboren wurden & 7 & 9.254 \\
Personen ohne angegebenes Geburtsland & 76 & 9.254 \\
Personen mit Schulenddatumsjahr vor 1971 oder nach 2007 & & \\
\hline Verwendete Stichprobe für die Analysen & & \\
\hline Quelle: Eigene Darstellung basierend auf dem NEPS. & &
\end{tabular}

Quelle: Eigene Darstellung basierend auf dem NEPS. 
Tabelle 4: Übersicht über Gewichtungen der Übergangsstation für alle Kombinationen.

\begin{tabular}{|c|}
\hline Stationskombination mit Gewichtung $(</>)$ \\
\hline Kurs < berufsvorbereitende Maßnahme \\
\hline Kurs $<$ Elternzeit \\
\hline Kurs $<$ Wehrdienst \\
\hline Kurs $<$ Lücke \\
\hline Kurs $<$ zusätzliche Schulepisode \\
\hline Kurs $<$ Arbeitslosigkeit \\
\hline Kurs $<$ Praktikum / Volontariat \\
\hline Elternzeit $>$ Arbeitslosigkeit \\
\hline Arbeitslosigkeit < berufsvorbereitende Maßnahme \\
\hline Arbeitslosigkeit $<$ Wehrdienst \\
\hline Arbeitslosigkeit > Lücke \\
\hline Praktikum / Volontariat $>$ Arbeitslosigkeit \\
\hline Zusätzliche Schulepisode > Arbeitslosigkeit \\
\hline Elternzeit < berufsvorbereitende Maßnahme \\
\hline Wehrdienst $>$ Elternzeit \\
\hline Elternzeit $>$ Lücken \\
\hline Elternzeit $<$ Praktikum / Volontariat \\
\hline Zusätzliche Schulepisoden > Elternzeit \\
\hline Berufsvorbereitende Maßnahme $>$ Wehrdienst \\
\hline Lücken < berufsvorbereitende Maßnahme \\
\hline Berufsvorbereitende Maßnahme > Praktikum / Volontariat \\
\hline Zusätzliche Schulepisode < berufsvorbereitende Maßnahme \\
\hline Wehrdienst > Lücke \\
\hline Wehrdienst $<$ Praktikum / Volontariat \\
\hline Zusätzliche Schulepisode > Wehrdienst \\
\hline Lücken < Praktikum / Volontariat \\
\hline Zusätzliche Schulepisode > Lücke \\
\hline Praktikum / Volontariat < zusätzliche Schulepisode \\
\hline Weiterbildung > Kurs \\
\hline Weiterbildung $>$ Arbeitslosigkeit \\
\hline Weiterbildung > Lücke \\
\hline Weiterbildung > Elternzeit \\
\hline Weiterbildung $>$ Wehrdienst \\
\hline Berufsvorbereitende Maßnahme > Weiterbildung \\
\hline Weiterbildung > Praktikum / Volontariat \\
\hline Weiterbildung < zusätzliche Schulepisode \\
\hline
\end{tabular}

Quelle: Eigene Darstellung. Fett = stärker gewichtete Station. 
Tabelle 5: Zuordnung und Zusammenfassung der Ausprägungen beider Monatsvariablen in Rohdaten zu gemeinsamer Monatsvariablen.

\begin{tabular}{|c|c|c|}
\hline $\begin{array}{c}\text { Ausprägungen der edierten } \\
\text { Monatsvariable }\end{array}$ & $\begin{array}{c}\text { Ausprägungen der nicht-edierten } \\
\text { Monatsvariable }\end{array}$ & $\begin{array}{l}\text { Zuordnung in gemeinsamer } \\
\text { Monatsvariable für Analysen }\end{array}$ \\
\hline Januar & Januar & Januar \\
\hline Februar & Februar & Februar \\
\hline März & März & März \\
\hline April & April & April \\
\hline Mai & Mai & Mai \\
\hline Juni & Juni & Juni \\
\hline Juli & Juli & Juli \\
\hline August & August & August \\
\hline September & September & September \\
\hline Oktober & Oktober & Oktober \\
\hline November & November & November \\
\hline Dezember & Dezember & Dezember \\
\hline Keine Angabe & Keine Angabe & - \\
\hline \multirow[t]{6}{*}{ Monat nicht mehr gewusst } & Monat nicht mehr gewusst & - \\
\hline & Jahresanfang/Winter & Januar \\
\hline & Frühjahr/Ostern & April \\
\hline & Jahresmitte/Sommer & Juli \\
\hline & Herbst & Oktober \\
\hline & Jahresende & Dezember \\
\hline
\end{tabular}

Quelle: Eigene Darstellungen basierend auf dem NEPS. 
Tabelle 6: Abhängige und unabhängige Variablen und Fallzahl mit gültigen Werten.

\begin{tabular}{lcc}
\hline \multicolumn{2}{l}{ Variable } & $\begin{array}{c}\text { Fallzahl mit gültigen } \\
\text { Werten }\end{array}$ \\
\hline \multirow{2}{*}{ Abhängige Variablen } & Übergangsdauer & 9.254 \\
& Übergangsstationen & 9.254 \\
& 1 oder mehr Übergangsstationen & $3.755^{*}$ \\
\hline Unabhängige Variablen & Schulabschluss & 9.189 \\
& Anteil Dienstleistungstätige & 9.254 \\
& Demografischer Anteil der unter 20-Jährigen & 9.254 \\
Arbeitslosenquote & 9.254 \\
& Schulenddatumsjahr (fortlaufend) & 9.254 \\
& Soziale Herkunft & 9.094 \\
Geschlecht & 9.254 \\
\hline
\end{tabular}

Quelle: Eigene Darstellung basierend auf dem NEPS. *Die Fallzahl der Personen mit mindestens einer oder mehr Übergangsstationen bezieht sich auf die Analysevariante der Stationen ohne FSJ- und Urlaubsepisoden sowie ohne unbestimmte Lückenepisoden. Bei den drei Analysevarianten bezüglich der Stationen variiert die Fallzahl dementsprechend. 


\section{Anhang B: Deskriptive Ergebnisse und Mittelwerttests}

Tabelle 7: Deskriptive Statistik zu Übergangsdauer, Übergangsstationen, Anteil Dienstleistungstätige, demografischer Anteil und Arbeitslosenquote.

\begin{tabular}{|c|c|c|c|c|c|}
\hline & $\mathbf{N}$ & Minimum & Maximum & Mittelwert & Standardabweichung \\
\hline $\begin{array}{l}\text { Übergangsdauer (ohne } \\
\text { Wehrdienst, Elternzeit, } \\
\text { FSJ/Urlaub/Krankheit) }\end{array}$ & 9.254 & 0 & 445 & 20,83 & 35,14 \\
\hline $\begin{array}{l}\text { Übergangsdauer (ohne } \\
\text { Wehrdienst \& Elternzeit, } \\
\text { mit FSJ/Urlaub/Krankheit) }\end{array}$ & 9.254 & 0 & 445 & 21,66 & 35,35 \\
\hline $\begin{array}{l}\text { Summe Übergangsstationen } \\
\text { (ohne Wehrdienst, } \\
\text { Elternzeit, } \\
\text { FSJ/Urlaub/Krankheit) }\end{array}$ & 9.254 & 0 & 11 & 1,08 & 1,17 \\
\hline $\begin{array}{l}\text { Summe Übergangsstationen } \\
\text { (ohne Wehrdienst \& } \\
\text { Elternzeit, } \text { mit } \\
\text { FSJ/Urlaub/Krankheit) }\end{array}$ & 9.254 & 0 & 13 & 1,36 & 1,36 \\
\hline $\begin{array}{l}\text { Summe Übergangsstationen } \\
\text { (ohne Wehrdienst, } \\
\text { Elternzeit, } \\
\text { FSJ/Urlaub/Krankheit, ohne } \\
\text { unbestimmte Phasen) }\end{array}$ & 9.254 & 0 & 10 & 0,56 & 0,87 \\
\hline $\begin{array}{l}\text { Summe Übergangsstationen } \\
\text { (ohne Wehrdienst \& } \\
\text { Elternzeit, mit } \\
\text { FSJ/Urlaub/Krankheit, ohne } \\
\text { unbestimmte Phasen) }\end{array}$ & 9.254 & 0 & 12 & 0,84 & 1,07 \\
\hline $\begin{array}{l}\text { Prozentualer Anteil der } \\
\text { Dienstleistungstätigen im } \\
\text { tertiären Sektor }\end{array}$ & 9.254 & 46 & 73,2 & 58,23 & 7,04 \\
\hline $\begin{array}{l}\text { Prozentualer Anteil der } \\
\text { unter 20-Jährigen }\end{array}$ & 9.254 & 19,4 & 29,6 & 24,30 & 3,10 \\
\hline $\begin{array}{l}\text { Arbeitslosenquote am } \\
\text { Anteil aller abhängigen } \\
\text { zivilen Erwerbspersonen }\end{array}$ & 9.254 & 0,8 & 11 & 6,73 & 2,71 \\
\hline
\end{tabular}

Quelle: Eigene Berechnungen basierend auf dem NEPS und den Daten des Statistischen Bundesamts. 
Tabelle 8: Häufigkeiten und prozentuale Anteile zu Schulabschluss, Erstausbildungstyp, soziale Herkunft (Bildung der Eltern) und Geschlecht.

\begin{tabular}{|c|c|c|c|}
\hline Variable & Kategorie & Anzahl & $\%$ \\
\hline \multirow{5}{*}{ Schulabschluss } & Ohne Abschluss & 1.043 & 11,4 \\
\hline & Hauptschulabschluss & 2.523 & 27,5 \\
\hline & Realschulabschluss & 3.073 & 33,4 \\
\hline & Abitur & 2.550 & 27,8 \\
\hline & Gesamt & 9.189 & 100,0 \\
\hline \multirow{5}{*}{ Erstausbildungstyp } & Keine Ausbildung & 775 & 8,4 \\
\hline & Duale Berufsausbildung & 5.460 & 59,0 \\
\hline & Vollzeitschulische Berufsausbildung & 794 & 8,6 \\
\hline & Studium & 2.225 & 24,0 \\
\hline & Gesamt & 9.254 & 100,0 \\
\hline \multirow{6}{*}{$\begin{array}{l}\text { Bildung der Eltern - } \\
\text { Soziale Herkunft (Jeweils } \\
\text { höchste CASMIN- } \\
\text { Kategorie beider } \\
\text { Elternteile) }\end{array}$} & Kein Abschluss /Hauptschulabschluss ohne Berufsausbildung & 767 & 8,4 \\
\hline & Hauptschulabschluss mit Berufsausbildung & 4.306 & 47,3 \\
\hline & Realschulabschluss ohne / mit Berufsausbildung & 1.762 & 19,4 \\
\hline & Abitur ohne / mit Berufsausbildung & 668 & 7,3 \\
\hline & Fachhochschul-/Universitätsabschluss & 1.591 & 17,5 \\
\hline & Gesamt & 9.094 & 100,0 \\
\hline \multirow{3}{*}{ Geschlecht } & Männlich & 4.507 & 48,7 \\
\hline & Weiblich & 4.747 & 51,3 \\
\hline & Gesamt & 9.254 & 100,0 \\
\hline
\end{tabular}

Quelle: Eigene Berechnungen basierend auf dem NEPS. 
Tabelle 9: Mittelwerttest Übergangsdauer zwischen Schulabschlussgruppen.

\begin{tabular}{lcc}
\hline & $\begin{array}{c}\text { Ohne Wehrdienst \& Elternzeit, } \\
\text { FSJ/Urlaub/Krankheit }\end{array}$ & $\begin{array}{c}\text { Ohne Wehrdienst \& } \\
\text { Elternzeit; mit } \\
\text { FSJ/Urlaub/Krankheit }\end{array}$ \\
\hline Kein Abschluss vs. Hauptschulabschluss & $<0,001$ & $<0,001$ \\
Kein Abschluss vs. Realschulabschluss & $<0,001$ & $<0,001$ \\
Kein Abschluss vs. Abitur & $<0,001$ & $<0,001$ \\
Hauptschulabschluss vs. & 0,363 & 0,256 \\
Realschulabschluss & $<0,001$ & $<0,001$ \\
Hauptschulabschluss vs. Abitur & $<0,001$ & $<0,001$ \\
Realschulabschluss vs. Abitur &
\end{tabular}

Quelle: Eigene Berechnungen basierend auf dem NEPS. ANOVA mit Games-Howell-Test als Post-Hoc-Test. pWerte für die Vergleiche zwischen den einzelnen Schulabschlüssen. N =9.189.

Tabelle 10: Mittelwerttest Übergangsdauer zwischen Erstausbildungstypen.

\begin{tabular}{lcc}
\hline & $\begin{array}{c}\text { Ohne Wehrdienst \& } \\
\text { Elternzeit, } \\
\text { FSJ/Urlaub/Krankheit }\end{array}$ & $\begin{array}{c}\text { Ohne Wehrdienst \& } \\
\text { Elternzeit; mit } \\
\text { FSJ/Urlaub/ } \\
\text { Krankheit }\end{array}$ \\
\hline $\begin{array}{l}\text { Duale Berufsausbildung vs. Vollzeitschulische } \\
\text { Berufsausbildung }\end{array}$ & $<0,001$ & $<0,001$ \\
Duale Berufsausbildung vs. Studium & $<0,001$ & $<0,001$ \\
Duale Berufsausbildung vs. Keine Ausbildung & $<0,001$ & $<0,001$ \\
Vollzeitschulische Berufsausbildung vs. Studium & 0,584 & 0,729 \\
Vollzeitschulische Berufsausbildung vs. Keine & $<0,001$ & $<0,001$ \\
Ausbildung & $<0,001$ & $<0,001$ \\
Studium vs. Keine Ausbildung & & \\
\hline
\end{tabular}

Quelle: Eigene Berechnungen basierend auf dem NEPS. ANOVA mit Games-Howell-Test als Post-Hoc-Test. pWerte für die Vergleiche zwischen den einzelnen Erstausbildungstypen. $\mathrm{N}=9.254$. 
Tabelle 11: Mittelwerttest Summe Übergangsstationen zwischen Schulabschlussgruppen.

\begin{tabular}{|c|c|c|c|c|}
\hline & $\begin{array}{c}\text { Ohne } \\
\text { Wehrdienst, } \\
\text { Elternzeit, } \\
\text { FSJ/Urlaub }\end{array}$ & $\begin{array}{c}\text { Ohne } \\
\text { Wehrdienst und } \\
\text { Elternzeit, mit } \\
\text { FSJ/Urlaub }\end{array}$ & $\begin{array}{c}\text { Ohne } \\
\text { Wehrdienst, } \\
\text { Elternzeit, } \\
\text { FSJ/Urlaub, } \\
\text { unbest. Phasen }\end{array}$ & $\begin{array}{c}\text { Ohne } \\
\text { Wehrdienst, } \\
\text { Elternzeit, } \\
\text { unbest. Phasen, } \\
\text { mit FSJ/Urlaub, }\end{array}$ \\
\hline $\begin{array}{l}\text { Kein Abschluss vs. } \\
\text { Hauptschulabschluss }\end{array}$ & $<0,001$ & $<0,001$ & $<0,001$ & $<0,001$ \\
\hline $\begin{array}{l}\text { Kein Abschluss vs. } \\
\text { Realschulabschluss }\end{array}$ & $<0,001$ & $<0,001$ & $<0,001$ & $<0,001$ \\
\hline $\begin{array}{l}\text { Kein Abschluss vs. } \\
\text { Abitur }\end{array}$ & $<0,001$ & $<0,001$ & $<0,001$ & $<0,001$ \\
\hline $\begin{array}{l}\text { Hauptschulabschluss vs. } \\
\text { Realschulabschluss }\end{array}$ & 0,016 & $<0,001$ & 0,831 & 0,011 \\
\hline $\begin{array}{l}\text { Hauptschulabschluss vs. } \\
\text { Abitur }\end{array}$ & $<0,001$ & 0,911 & $<0,001$ & $<0,001$ \\
\hline $\begin{array}{l}\text { Realschulabschluss vs. } \\
\text { Abitur }\end{array}$ & $<0,001$ & $<0,001$ & $<0,001$ & $<0,001$ \\
\hline
\end{tabular}

Quelle: Eigene Berechnungen basierend auf dem NEPS. ANOVA mit Games-Howell-Test als Post-Hoc-Test. pWerte für die Vergleiche zwischen den einzelnen Schulabschlüssen. N = 9.189. 
Tabelle 12: Mittelwerttest Summe Übergangsstationen zwischen Erstausbildungstypen.

\begin{tabular}{|c|c|c|c|c|}
\hline & $\begin{array}{c}\text { Ohne } \\
\text { Wehrdienst, } \\
\text { Elternzeit, } \\
\text { FSJ/Urlaub }\end{array}$ & $\begin{array}{c}\text { Ohne } \\
\text { Wehrdienst und } \\
\text { Elternzeit, mit } \\
\text { FSJ/Urlaub }\end{array}$ & $\begin{array}{c}\text { Ohne } \\
\text { Wehrdienst, } \\
\text { Elternzeit, } \\
\text { FSJ/Urlaub, } \\
\text { unbest. Phasen }\end{array}$ & $\begin{array}{c}\text { Ohne } \\
\text { Wehrdienst, } \\
\text { Elternzeit, } \\
\text { unbest. Phasen, } \\
\text { mit FSJ/Urlaub, }\end{array}$ \\
\hline $\begin{array}{l}\text { Duale Berufsausbildung vs. } \\
\text { Vollzeitschulische } \\
\text { Berufsausbildung }\end{array}$ & $<0,001$ & $<0,001$ & 0,002 & $<0,001$ \\
\hline $\begin{array}{l}\text { Duale Berufsausbildung vs. } \\
\text { Studium }\end{array}$ & $<0,001$ & $<0,001$ & 0,117 & $<0,001$ \\
\hline $\begin{array}{l}\text { Duale Berufsausbildung vs. } \\
\text { Keine Ausbildung }\end{array}$ & $<0,001$ & $<0,001$ & $<0,001$ & $<0,001$ \\
\hline $\begin{array}{l}\text { Vollzeitschulische } \\
\text { Berufsausbildung vs. Studium }\end{array}$ & 0,119 & 0,001 & 0,179 & 0,808 \\
\hline $\begin{array}{l}\text { Vollzeitschulische } \\
\text { Berufsausbildung vs. Keine } \\
\text { Ausbildung }\end{array}$ & $<0,001$ & $<0,001$ & $<0,001$ & $<0,001$ \\
\hline Studium vs. Keine Ausbildung & $<0,001$ & $<0,001$ & $<0,001$ & $<0,001$ \\
\hline
\end{tabular}

Quelle: Eigene Berechnungen basierend auf dem NEPS. ANOVA mit Games-Howell-Test als Post-Hoc-Test. pWerte für die Vergleiche zwischen den einzelnen Erstausbildungstypen. $\mathrm{N}=9.254$. 


\section{Anhang C: Cox-Regressionsmodelle}

Tabelle 13: Effekte des Schulenddatumsjahres und des Schulabschlusses auf die Übergangsdauer (ohne Wehrdienst und Elternzeit; mit FSJ/Urlaub/Krankheit).

\begin{tabular}{lcccc}
\hline & Koeffizient & $\begin{array}{c}\text { Hazard- } \\
\text { Ratio }\end{array}$ & $\begin{array}{c}\text { Standard- } \\
\text { fehler }\end{array}$ & p-Wert \\
\hline $\begin{array}{l}\text { Schulabschluss } \\
\text { (Referenz: Kein Abschluss) }\end{array}$ & & & $<0,001^{\text {a }}$ \\
$\quad$ Hauptschulabschluss & $\mathbf{0 , 5 4}$ & $\mathbf{1 , 7 1}$ & 0,04 & $<0,001$ \\
$\quad$ Realschulabschluss & $\mathbf{0 , 4 6}$ & $\mathbf{1 , 5 9}$ & 0,04 & $<0,001$ \\
$\quad$ Abitur & $\mathbf{0 , 7 7}$ & $\mathbf{2 , 1 7}$ & 0,04 & $<0,001$ \\
Schulenddatumsjahr & 0,00 & 1,00 & 0,00 & 0,079 \\
Soziale Herkunft - Bildung der Eltern & & & & \\
(Referenzkategorie: kein Abschluss / & & & & $<0,001^{\mathrm{a}}$ \\
Hauptschulabschluss ohne berufliche Ausbildung & & & & \\
(b. A.)) & & & & 0,001 \\
$\quad$ Hauptschulabschluss mit b. A. & $\mathbf{0 , 1 5}$ & $\mathbf{1 , 1 6}$ & 0,04 & 0,493 \\
Mittlere Reife ohne / mit b. A. & 0,03 & 1,03 & 0,05 & 0,820 \\
$\quad$ Hochschulreife ohne / mit b. A. & 0,01 & 1,01 & 0,06 & 0,870 \\
FH-/ Universitätsabschluss & 0,01 & 1,01 & 0,05 & $<0,001$ \\
Geschlecht (weiblich) & $\mathbf{- 0 , 1 6}$ & $\mathbf{0 , 8 5}$ & 0,02 &
\end{tabular}

Quelle: Eigene Berechnungen basierend auf dem NEPS. ${ }^{a}=$ Omnibus-Tests für die Schulabschlüsse und die soziale Herkunft (Nullhypothese: Alle Koeffizienten sind gleich null). Statistisch signifikante Koeffizienten (p $\leq 0,05)$ sind fett gedruckt. $\mathrm{N}=9.040$. 
Tabelle 14: Effekt des Anteils an Dienstleistungstätigen auf die Übergangsdauer (ohne Wehrdienst und Elternzeit; mit FSJ/Urlaub/Krankheit).

\begin{tabular}{|c|c|c|c|c|}
\hline & Koeffizient & $\begin{array}{l}\text { Hazard- } \\
\text { Ratio }\end{array}$ & $\begin{array}{l}\text { Standard- } \\
\text { fehler }\end{array}$ & p-Wert \\
\hline Prozentualer Anteil der Dienstleistungstätigen & $-0,03$ & 0,97 & 0,03 & 0,281 \\
\hline Prozentualer Anteil der unter 20-Jährigen & $-0,01$ & 0,99 & 0,02 & 0,488 \\
\hline $\begin{array}{l}\text { Arbeitslosenquote am Anteil aller abhängigen } \\
\text { zivilen Erwerbspersonen }\end{array}$ & 0,02 & 1,02 & 0,01 & 0,180 \\
\hline $\begin{array}{l}\text { Schulabschluss } \\
\text { (Referenz: Kein Abschluss) }\end{array}$ & & & & $<0,001^{\mathrm{a}}$ \\
\hline Hauptschulabschluss & $\mathbf{0 , 5 4}$ & 1,72 & 0,04 & $<0,001$ \\
\hline Realschulabschluss & 0,46 & 1,59 & 0,04 & $<0,001$ \\
\hline Abitur & 0,77 & 2,15 & 0,04 & $<0,001$ \\
\hline Schulenddatumsjahr & 0,01 & 1,01 & 0,02 & 0,579 \\
\hline $\begin{array}{l}\text { Soziale Herkunft - Bildung der Eltern } \\
\text { (Referenzkategorie: kein Abschluss / } \\
\text { Hauptschulabschluss ohne berufliche Ausbildung } \\
\text { (b. A.)) }\end{array}$ & & & & $<0,001^{\mathrm{a}}$ \\
\hline Hauptschulabschluss mit b. A. & $\mathbf{0 , 1 4}$ & 1,15 & 0,04 & 0,001 \\
\hline Mittlere Reife ohne / mit b. A. & 0,03 & 1,03 & 0,05 & 0,522 \\
\hline Hochschulreife ohne / mit b. A. & 0,01 & 1,01 & 0,06 & 0,808 \\
\hline FH-/ Universitätsabschluss & 0,01 & 1,01 & 0,05 & 0,886 \\
\hline Geschlecht (weiblich) & $-0,16$ & $\mathbf{0 , 8 5}$ & 0,02 & $<0,001$ \\
\hline
\end{tabular}

Quelle: Eigene Berechnungen basierend auf dem NEPS. ${ }^{a}=$ Omnibus-Tests für die Schulabschlüsse und die soziale Herkunft (Nullhypothese: Alle Koeffizienten sind gleich null). Statistisch signifikante Koeffizienten (p $\leq 0,05)$ sind fett gedruckt. $\mathrm{N}=9.040$. 
Tabelle 15: Effekt des Schulabschlusses auf die Übergangsdauer (ohne Wehrdienst und Elternzeit; mit FSJ/Urlaub/Krankheit) mit Interaktion Schulabschluss und Anteil Dienstleistungstätige.

\begin{tabular}{|c|c|c|c|c|}
\hline & Koeffizient & $\begin{array}{l}\text { Hazard- } \\
\text { Ratio }\end{array}$ & $\begin{array}{l}\text { Standard- } \\
\text { fehler }\end{array}$ & $\mathrm{p}$-Wert \\
\hline Prozentualer Anteil der Dienstleistungstätigen & 0,01 & 1,01 & 0,03 & 0,743 \\
\hline Prozentualer Anteil der unter 20-Jährigen & $-0,03$ & 0,97 & 0,02 & 0,068 \\
\hline $\begin{array}{l}\text { Arbeitslosenquote am Anteil aller abhängigen } \\
\text { zivilen Erwerbspersonen }\end{array}$ & 0,01 & 1,01 & 0,01 & 0,313 \\
\hline $\begin{array}{l}\text { Schulabschluss } \\
\text { (Referenz: Kein Abschluss) }\end{array}$ & & & & $<0,001^{\mathrm{a}}$ \\
\hline Hauptschulabschluss & $\mathbf{0 , 5 2}$ & 1,68 & 0,04 & $<0,001$ \\
\hline Realschulabschluss & 0,46 & 1,59 & 0,04 & $<0,001$ \\
\hline Abitur & 0,75 & 2,11 & 0,04 & $<0,001$ \\
\hline Schulenddatumsjahr & $-0,02$ & 0,98 & 0,03 & 0,484 \\
\hline $\begin{array}{l}\text { Soziale Herkunft - Bildung der Eltern } \\
\text { (Referenzkategorie: kein Abschluss / } \\
\text { Hauptschulabschluss ohne berufliche Ausbildung } \\
\text { (b. A.)) }\end{array}$ & & & & $<0,001^{\mathrm{a}}$ \\
\hline Hauptschulabschluss mit b. A. & 0,14 & 1,15 & 0,04 & 0,001 \\
\hline Mittlere Reife ohne / mit b. A. & 0,04 & 1,04 & 0,05 & 0,472 \\
\hline Hochschulreife ohne / mit b. A. & 0,02 & 1,02 & 0,06 & 0,797 \\
\hline FH-/ Universitätsabschluss & 0,01 & 1,01 & 0,05 & 0,926 \\
\hline Geschlecht (weiblich) & $-0,16$ & 0,85 & 0,02 & $<0,001$ \\
\hline $\begin{array}{l}\text { Hauptschulabschluss } \times \text { Anteil } \\
\text { Dienstleistungstätige }\end{array}$ & $-0,02$ & 0,98 & 0,01 & $<0,001$ \\
\hline $\begin{array}{l}\text { Realschulabschluss } \times \text { Anteil } \\
\text { Dienstleistungstätige }\end{array}$ & $-0,01$ & 0,99 & 0,01 & 0,306 \\
\hline Abitur $\times$ Anteil Dienstleistungstätige & 0,00 & 1,00 & 0,01 & 0,754 \\
\hline
\end{tabular}


Anhang D: Sequenzdatenanalysen

Tabelle 16: Häufigkeit der Stationen, die von den Personen durchlaufen wurden (mit FSJ/Urlaub; ohne unbestimmte Lückenepisoden).

\begin{tabular}{lcc}
\hline $\begin{array}{l}\text { Übergangsstationen - Sequenzen (mit FSJ/Urlaub } \\
\text { und ohne Lücken) }\end{array}$ & Häufigkeit & Prozent \\
\hline Arbeitslosigkeit & 1.401 & 16,32 \\
Berufsvorbereitende Maßnahmen & 716 & 8,34 \\
Freiwilliges Soziales/Ökologisches Jahr & 176 & 2,05 \\
Kurse/Weiterbildungen/Praktika & 603 & 7,03 \\
Weitere Schulepisoden & 2.892 & 33,70 \\
Urlaub & 2.376 & 27,69 \\
Etwas Anderes gemacht & 317 & 3,69 \\
Hausfrau/-mann & 101 & 1,18 \\
\hline Gesamt & 8.582 & 100,00 \\
\hline
\end{tabular}

Quelle: Eigene Berechnungen basierend auf dem NEPS. N = 8.582 absolvierte Stationen insgesamt.

Tabelle 17: Verteilung der zehn häufigsten Übergangssequenzen beziehungsweise Übergangsmuster (mit FSJ/Urlaub; ohne unbestimmte Lückenepisoden).

\begin{tabular}{lcc}
\hline Sequenzmuster & Häufigkeit & Prozent \\
\hline Schulepisode & 1.163 & 32,92 \\
Urlaub & 1.092 & 30,91 \\
Berufsvorbereitende Maßnahme & 284 & 8,04 \\
Arbeitslosigkeit & 280 & 7,93 \\
Zwei Schulepisoden nacheinander & 183 & 5,18 \\
Schulepisode, danach Urlaub & 154 & 4,36 \\
Schulepisode, dann Arbeitslosigkeit & 107 & 3,03 \\
Urlaub, danach Schulepisode, danach Urlaub & 105 & 2,97 \\
Etwas Anderes gemacht & 95 & 2,69 \\
Kurs/Weiterbildung/Praktikum & 70 & 1,98 \\
\hline Gesamt & 3.533 & 100,00
\end{tabular}

Quelle: Eigene Berechnungen basierend auf dem NEPS. Die zehn häufigsten Übergangsverläufe wurden von N $=3.533$ Personen durchlaufen. 
Tabelle 18: Deskriptive Statistiken zur Länge der Übergangssequenzen (Anzahl der absolvierten Stationen insgesamt) und zur Anzahl an unterschiedlichen Stationen pro Übergangsverlauf (mit FSJ/Urlaub; ohne unbestimmte Lückenepisoden).

\begin{tabular}{lcccc}
\hline & Mittelwert & $\begin{array}{c}\text { Standard- } \\
\text { abweichung }\end{array}$ & Minimum & Maximum \\
\hline Länge der Sequenz & 1,72 & 1,20 & 1 & 12 \\
$\begin{array}{l}\text { Anzahl der Elemente } \\
\text { (unterschiedliche Stationen) pro }\end{array}$ & 1,40 & 0,68 & 1 & 5 \\
Sequenz & & & & \\
\hline
\end{tabular}

Quelle: Eigene Berechnungen basierend auf dem NEPS. N = 4.996 Personen mit Übergangsverläufen.

Tabelle 19: Häufigkeitsverteilung der Anzahl unterschiedlicher Übergangsstationen in den Übergangssequenzen (mit FSJ/Urlaub; ohne unbestimmte Lückenepisoden).

\begin{tabular}{lccc}
\hline Anzahl unterschiedlicher Stationen in Sequenzen & Häufigkeit & Prozent & Kumulierte Prozent \\
\hline 1 & 3.458 & 69,22 & 69,22 \\
2 & 1.189 & 23,80 & 93,01 \\
3 & 268 & 5,36 & 98,38 \\
4 & 69 & 1,38 & 100,00 \\
5 & 12 & 0,24 & 100,00 \\
\hline Gesamt & 4.996 & & \\
\hline
\end{tabular}

Quelle: Eigene Berechnungen basierend auf dem NEPS. N = 4.996 Personen mit Übergangsverläufen.

Tabelle 20: Deskriptive Statistik zur Anzahl an Übergangsstationen nach Schulabschluss (mit FSJ/Urlaub; ohne unbestimmte Lückenepisoden).

\begin{tabular}{lccc}
\hline $\begin{array}{l}\text { Erster erreichter } \\
\text { Schulabschluss }\end{array}$ & Mittelwert & Standardabweichung & Häufigkeit \\
\hline Kein Abschluss & 2,07 & 1,44 & 791 \\
Hauptschulabschluss & 1,79 & 1,32 & 1.222 \\
Realschulabschluss & 1,68 & 1,13 & 1.742 \\
Abitur & 1,46 & 0,89 & 1.208 \\
\hline Gesamt & 1,72 & 1,20 & 4.963
\end{tabular}

Quelle: Eigene Berechnungen basierend auf dem NEPS. N = 4.963 Personen, die einen Übergangsverlauf vorliegen hatten und für die ein Schulabschluss erhoben wurde. 
Tabelle 21: Deskriptive Statistik zur Anzahl an unterschiedlichen Übergangsstationen nach Schulabschluss (mit FSJ/Urlaub; ohne unbestimmte Lückenepisoden).

\begin{tabular}{lccc}
\hline $\begin{array}{l}\text { Erster erreichter } \\
\text { Schulabschluss }\end{array}$ & Mittelwert & Standardabweichung & Häufigkeit \\
\hline Kein Abschluss & 1,48 & 0,70 & 791 \\
Hauptschulabschluss & 1,43 & 0,73 & 1.222 \\
Realschulabschluss & 1,44 & 0,71 & 1.742 \\
Abitur & 1,25 & 0,52 & 1.208 \\
\hline Gesamt & 1,40 & 0,68 & 4.963
\end{tabular}

Quelle: Eigene Berechnungen basierend auf dem NEPS. $\mathrm{N}=4.963$ Personen, die einen Übergangsverlauf vorliegen hatten und für die ein Schulabschluss erhoben wurde.

Tabelle 22: Häufigkeit der Stationen, die von den Personen durchlaufen wurden (ohne FSJ/Urlaub; mit unbestimmten Lückenepisoden).

\begin{tabular}{lcc}
\hline $\begin{array}{l}\text { Übergangsstationen - Sequenzen (ohne FSJ/Urlaub } \\
\text { und ohne Lücken) }\end{array}$ & Häufigkeit & Prozent \\
\hline Arbeitslosigkeit & 1.401 & 13,85 \\
Berufsvorbereitende Maßnahmen & 716 & 7,08 \\
Kurse/Weiterbildungen/Praktika & 603 & 5,96 \\
Lückenepisoden (unbestimmte Phasen) & 4.082 & 40,37 \\
Weitere Schulepisoden & 2.892 & 28,60 \\
Etwas Anderes gemacht & 317 & 3,13 \\
Hausfrau/-mann & 101 & 1,00 \\
\hline Gesamt & 10.112 & 100,00 \\
\hline
\end{tabular}

Quelle: Eigene Berechnungen basierend auf dem NEPS. N = 10.112 absolvierte Stationen insgesamt. 
Tabelle 23: Verteilung der zehn häufigsten Übergangssequenzen beziehungsweise Übergangsmuster (ohne FSJ/Urlaub; mit unbestimmten Lückenepisoden).

\begin{tabular}{lcc}
\hline Sequenzmuster & Häufigkeit & Prozent \\
\hline Lückenepisode (unbestimmte Phase) & 1.640 & 38,78 \\
Schulepisode, dann Lücke & 1.143 & 27,03 \\
Schulepisode & 381 & 9,01 \\
Berufsvorbereitende Maßnahme, dann Lücke & 269 & 6,36 \\
Arbeitslosigkeit, dann Lücke & 224 & 5,30 \\
Zwei Schulepisoden nacheinander, dann Lücke & 189 & 4,47 \\
Berufsvorbereitende Maßnahme & 107 & 2,53 \\
Arbeitslosigkeit & 100 & 2,36 \\
Schulepisode, dann Arbeitslosigkeit, dann Lücke & 90 & 2,13 \\
Etwas anderes gemacht, dann Lücke & 86 & 2,03 \\
\hline Gesamt & 4.229 & 100,00 \\
\hline
\end{tabular}

Quelle: Eigene Berechnungen basierend auf dem NEPS. Die zehn häufigsten Übergangsverläufe wurden von N $=4.229$ Personen durchlaufen.

Tabelle 24: Deskriptive Statistiken zur Länge der Übergangssequenzen (Anzahl der absolvierten Stationen insgesamt) und zur Anzahl an unterschiedlichen Stationen pro Übergangsverlauf (ohne FSJ/Urlaub; mit unbestimmten Lückenepisoden).

\begin{tabular}{lcccc}
\hline & Mittelwert & $\begin{array}{c}\text { Standard- } \\
\text { abweichung }\end{array}$ & Minimum & Maximum \\
\hline Länge der Sequenz & 1,87 & 1,07 & 1 & 11 \\
$\begin{array}{l}\text { Anzahl der Elemente } \\
\text { (unterschiedliche Stationen) pro }\end{array}$ & 1,64 & 0,69 & 1 & 5 \\
Sequenz & & & \\
\hline Quelle: Eigene Berechnungen basierend auf dem NEPS. N = 5.388 Personen mit Übergangsverläufen.
\end{tabular}


Tabelle 25: Häufigkeitsverteilung der Anzahl unterschiedlicher Übergangsstationen in den Übergangssequenzen (ohne FSJ/Urlaub; mit unbestimmten Lückenepisoden).

\begin{tabular}{lccc}
\hline Anzahl unterschiedlicher Stationen in Sequenzen & Häufigkeit & Prozent & Kumulierte Prozent \\
\hline 1 & 2.506 & 46,51 & 46,51 \\
2 & 2.365 & 43,89 & 90,40 \\
3 & 447 & 8,30 & 98,70 \\
4 & 64 & 1,19 & 99,89 \\
5 & 6 & 0,11 & 100,00 \\
\hline Gesamt & 5.388 & 100,00 & \\
\hline
\end{tabular}

Quelle: Eigene Berechnungen basierend auf dem NEPS. N = 5.388 Personen mit Übergangsverläufen.

Tabelle 26: Deskriptive Statistik zur Anzahl an Übergangsstationen nach Schulabschluss (ohne FSJ/Urlaub; mit unbestimmten Lückenepisoden).

\begin{tabular}{lccc}
\hline $\begin{array}{l}\text { Erster erreichter } \\
\text { Schulabschluss }\end{array}$ & Mittelwert & Standardabweichung & Häufigkeit \\
\hline Kein Abschluss & 2,38 & 1,30 & 800 \\
Hauptschulabschluss & 2,01 & 1,14 & 1.235 \\
Realschulabschluss & 1,95 & 0,99 & 1.737 \\
Abitur & 1,40 & 0,73 & 1.581 \\
\hline Gesamt & 1,87 & 1,07 & 5.353
\end{tabular}

Quelle: Eigene Berechnungen basierend auf dem NEPS. $\mathrm{N}=5.353$ Personen, die einen Übergangsverlauf vorliegen hatten und für die ein Schulabschluss erhoben wurde.

Tabelle 27: Deskriptive Statistik zur Anzahl an unterschiedlichen Übergangsstationen nach Schulabschluss (ohne FSJ/Urlaub; mit unbestimmten Lückenepisoden).

\begin{tabular}{lccc}
\hline $\begin{array}{l}\text { Erster erreichter } \\
\text { Schulabschluss }\end{array}$ & Mittelwert & Standardabweichung & Häufigkeit \\
\hline Kein Abschluss & 1,87 & 0,67 & 800 \\
Hauptschulabschluss & 1,74 & 0,71 & 1.235 \\
Realschulabschluss & 1,78 & 0,71 & 1.737 \\
Abitur & 1,31 & 0,52 & 1.581 \\
\hline Gesamt & 1,64 & 0,69 & 5.353
\end{tabular}

Quelle: Eigene Berechnungen basierend auf dem NEPS. $\mathrm{N}=5.353$ Personen, die einen Übergangsverlauf vorliegen hatten und für die ein Schulabschluss erhoben wurde. 
Tabelle 28: Häufigkeit der Stationen, die von den Personen durchlaufen wurden (mit FSJ/Urlaub; mit unbestimmten Lückenepisoden).

\begin{tabular}{lcc}
\hline $\begin{array}{l}\text { Übergangsstationen - Sequenzen (ohne FSJ/Urlaub } \\
\text { und ohne Lücken) }\end{array}$ & Häufigkeit & Prozent \\
\hline Arbeitslosigkeit & 1.401 & 11,06 \\
Berufsvorbereitende Maßnahmen & 716 & 5,65 \\
Etwas Anderes gemacht & 317 & 2,50 \\
Freiwilliges Soziales/Ökologisches Jahr & 176 & 1,39 \\
Hausfrau/-mann & 101 & 0,80 \\
Kurse/Weiterbildungen/Praktika & 603 & 4,76 \\
Lücken (unbestimmte Phasen) & 4.082 & 32,23 \\
Weitere Schulepisoden & 2.892 & 22,84 \\
Urlaub & 2.376 & 18,76 \\
\hline Gesamt & 12.664 & 100,00 \\
\hline
\end{tabular}

Quelle: Eigene Berechnungen basierend auf dem NEPS. N = 12.664 absolvierte Stationen insgesamt.

Tabelle 29: Verteilung der zehn häufigsten Übergangssequenzen beziehungsweise Übergangsmuster (mit FSJ/Urlaub; mit unbestimmten Lückenepisoden).

\begin{tabular}{lcc}
\hline Sequenzmuster & Häufigkeit & Prozent \\
\hline Schulepisode, danach Lücke & 874 & 22,64 \\
Lückenepisode & 868 & 22,49 \\
Urlaub, danach Lücke & 657 & 17,02 \\
Urlaub & 434 & 11,24 \\
Schulepisode & 287 & 7,43 \\
Berufsvorb. Maßnahme, danach Lücke & 199 & 5,16 \\
Arbeitslosigkeit, danach Lücke & 187 & 4,84 \\
Zwei Schulepisoden nacheinander, danach Lücke & 140 & 3,63 \\
Schulepisode, danach Urlaub, danach Lücke & 121 & 3,13 \\
Arbeitslosigkeit & 93 & 2,41 \\
\hline Gesamt & 3.860 & 100,00
\end{tabular}

Quelle: Eigene Berechnungen basierend auf dem NEPS. Die zehn häufigsten Übergangsverläufe wurden von N $=3.860$ Personen durchlaufen. 
Tabelle 30: Deskriptive Statistiken zur Länge der Übergangssequenzen (Anzahl der absolvierten Stationen insgesamt) und zur Anzahl an unterschiedlichen Stationen pro Übergangsverlauf (mit FSJ/Urlaub; mit unbestimmten Lückenepisoden).

\begin{tabular}{lcccc}
\hline & Mittelwert & $\begin{array}{c}\text { Standard- } \\
\text { abweichung }\end{array}$ & Minimum & Maximum \\
\hline $\begin{array}{l}\text { Länge der Sequenz } \\
\text { Anzahl der Elemente }\end{array}$ & 2,16 & 1,21 & 1 & 12 \\
$\begin{array}{l}\text { (unterschiedliche Stationen) pro } \\
\text { Sequenz }\end{array}$ & 1,88 & 0,80 & 1 & 5 \\
\hline
\end{tabular}

Quelle: Eigene Berechnungen basierend auf dem NEPS. N = 5.854 Personen mit Übergangsverläufen.

Tabelle 31: Häufigkeitsverteilung der Anzahl unterschiedlicher Übergangsstationen in den Übergangssequenzen (mit FSJ/Urlaub; mit unbestimmten Lückenepisoden).

\begin{tabular}{lccc}
\hline $\begin{array}{l}\text { Anzahl unterschiedlicher } \\
\text { Stationen in Sequenzen }\end{array}$ & Häufigkeit & Prozent & Kumulierte Prozent \\
\hline 1 & 1.998 & 34,13 & 34,13 \\
2 & 2.763 & 47,20 & 81,33 \\
3 & 894 & 15,27 & 96,60 \\
4 & 170 & 2,90 & 99,50 \\
5 & 29 & 0,50 & 100,00 \\
\hline Gesamt & 5.854 & 100,00 & \\
\hline
\end{tabular}

Quelle: Eigene Berechnungen basierend auf dem NEPS. N = 5.854 Personen mit Übergangsverläufen.

Tabelle 32: Deskriptive Statistik zur Anzahl an Übergangsstationen nach Schulabschluss (mit FSJ/Urlaub; mit unbestimmten Lückenepisoden).

\begin{tabular}{lccc}
\hline Erster erreichter & Mittelwert & Standardabweichung & Häufigkeit \\
Schulabschluss & & 1,42 & 826 \\
\hline Kein Abschluss & 2,65 & 1,31 & 1.319 \\
Hauptschulabschluss & 2,27 & 1,16 & 1.885 \\
Realschulabschluss & 2,24 & 0,93 & 1.788 \\
Abitur & 1,75 & 1,21 & 5.818 \\
\hline Gesamt & 2,15 & .
\end{tabular}

Quelle: Eigene Berechnungen basierend auf dem NEPS. $\mathrm{N}=5.818$ Personen, die einen Übergangsverlauf vorliegen hatten und für die ein Schulabschluss erhoben wurde. 
Tabelle 33: Deskriptive Statistik zur Anzahl an unterschiedlichen Übergangsstationen nach Schulabschluss (mit FSJ/Urlaub; mit unbestimmten Lückenepisoden).

\begin{tabular}{lccc}
\hline Erster erreichter Schulabschluss & Mittelwert & Standardabweichung & Häufigkeit \\
\hline Kein Abschluss & 2,10 & 0,79 & 826 \\
Hauptschulabschluss & 1,94 & 0,82 & 1.319 \\
Realschulabschluss & 2,01 & 0,82 & 1.885 \\
Abitur & 1,61 & 0,69 & 1.788 \\
\hline Gesamt & 1,89 & 0,80 & 5.818
\end{tabular}

Quelle: Eigene Berechnungen basierend auf dem NEPS. $\mathrm{N}=5.818$ Personen, die einen Übergangsverlauf vorliegen hatten und für die ein Schulabschluss erhoben wurde. 
Tabelle 34: Effekt des Schulabschlusses auf die Zugehörigkeit zur jeweiligen Stationskategorie (mit FSJ/Urlaubsepisoden; ohne unbestimmte Lückenepisoden).

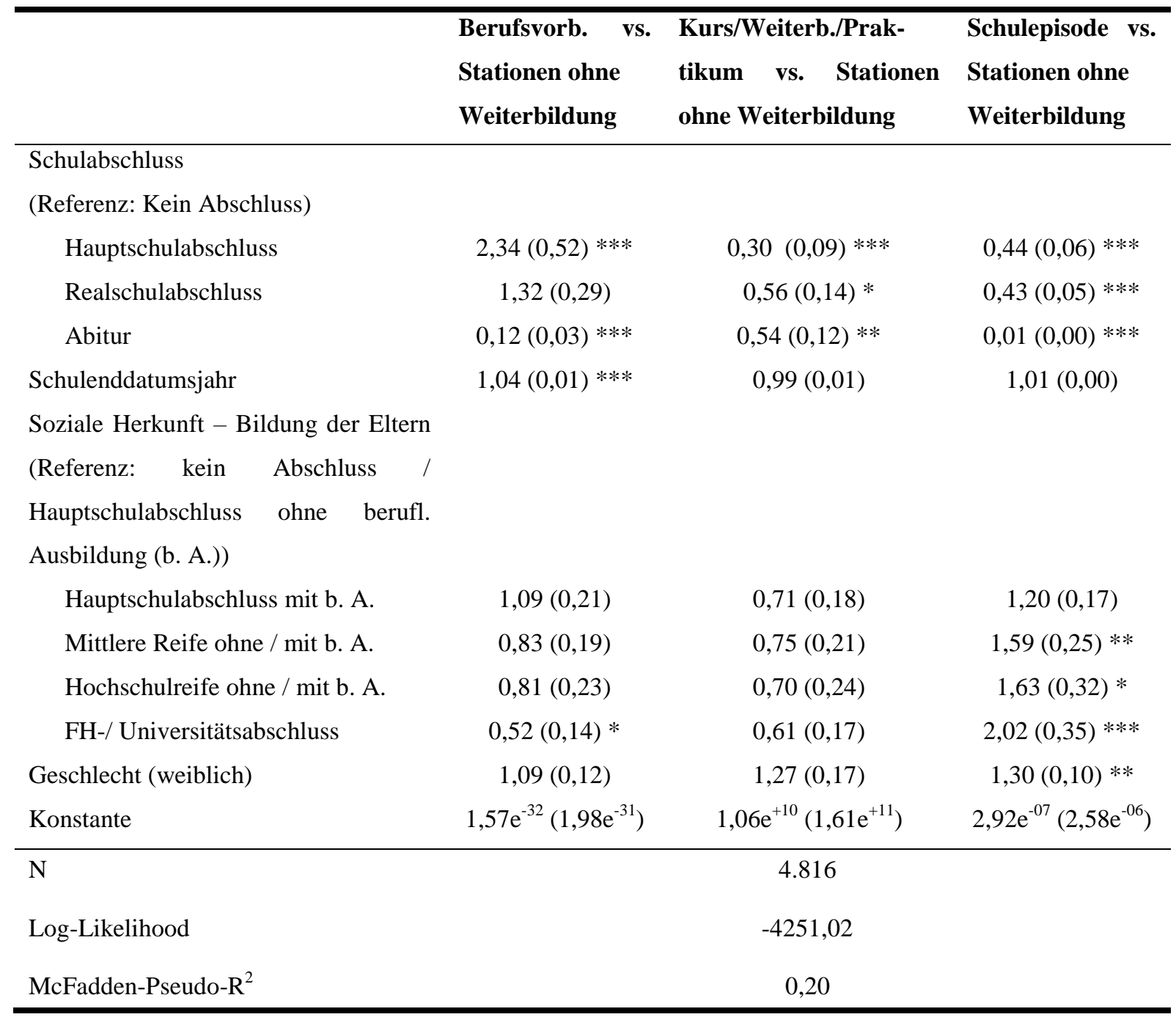

Quelle: Eigene Berechnungen basierend auf dem NEPS. Multinomiale logistische Regression. Die angegebenen Koeffizienten sind die Odds Ratios sowie die dazugehörigen Standardfehler (in Klammern). Referenzkategorie bei den Stationskategorien: Stationen ohne Weiterbildungscharakter. Die metrisch skalierten unabhängigen Variablen im Modell wurden mittelwertzentriert. Statt 4.875 Fällen sind 4.816 Fälle im Modell enthalten. 59 Fälle werden ausgeschlossen, welche als Station ein FSJ haben, welches kürzer ist als eine berufsvorbereitende Maßnahme (Dauer). Aufgrund der aufgestellten Bedingung bzgl. der Dauer werden diese Fälle bei den Kategorien nicht berücksichtigt. $\mathrm{e}^{\mathrm{xy}}$ steht für $10^{\mathrm{xy}}\left(, 10\right.$ hoch“ $\left.^{\prime}\right)$. Legende für p-Werte: $* * *=<0,001 ; * *=<0,01$; $*=\leq 0,05$. 
Tabelle 35: Effekt des Schulabschlusses und des Anteil an Dienstleistungstätigen auf die Zugehörigkeit zur jeweiligen Stationskategorie (mit FSJ/Urlaubsepisoden; ohne unbestimmte Lückenepisoden).

\begin{tabular}{|c|c|c|c|}
\hline & $\begin{array}{l}\text { Berufsvorb. vs. } \\
\text { Stationen ohne } \\
\text { Weiterbildung }\end{array}$ & $\begin{array}{l}\text { Kurs/Weiterb./Prak } \\
\text {-tikum vs. Stationen } \\
\text { ohne Weiterbildung }\end{array}$ & $\begin{array}{l}\text { Schulepisode vs } \\
\text { Stationen ohne } \\
\text { Weiterbildung }\end{array}$ \\
\hline \multicolumn{4}{|l|}{ Schulabschluss } \\
\hline \multicolumn{4}{|l|}{ (Referenz: Kein Abschluss) } \\
\hline Hauptschulabschluss & $2,73(0,64) * * *$ & $0,32(0,10) * * *$ & $0,46(0,06) * * *$ \\
\hline Realschulabschluss & $1,45(0,34)$ & $0,50(0,13) * *$ & $0,44(0,05) * * *$ \\
\hline Abitur & $0,12(0,04) * * *$ & $0,52(0,12) * *$ & $0,01(0,00) * * *$ \\
\hline Anteil Dienstleistungstätige & $1,00(0,16)$ & $1,31(0,23)$ & $0,99(0,10)$ \\
\hline Interaktion Schulabschluss $\times$ Anteil & & & \\
\hline \multicolumn{4}{|l|}{ Dienstleistungstätige } \\
\hline Hauptschulabschluss & $1,00(0,03)$ & $1,02(0,04)$ & $1,03(0,02)$ \\
\hline Realschulabschluss & $0,96(0,03)$ & $0,96(0,03)$ & $1,04(0,02) *$ \\
\hline Abitur & $0,99(0,04)$ & $1,01(0,03)$ & $0,99(0,03)$ \\
\hline Demografischer Anteil (unter 20-Jährige) & $0,89(0,09)$ & $0,80(0,09) *$ & $0,90(0,06)$ \\
\hline Arbeitslosenquote & $1,08(0,06)$ & $0,90(0,06)$ & $0,97(0,04)$ \\
\hline Schulenddatumsjahr & $1,00(0,14)$ & $0,77(0,16)$ & $0,97(0,09)$ \\
\hline $\begin{array}{l}\text { Soziale Herkunft }- \text { Bildung der Eltern } \\
\text { (Referenz: kein Abschluss / }\end{array}$ & & & \\
\hline Hauptschulabschluss ohne berufl. & & & \\
\hline \multicolumn{4}{|l|}{ Ausbildung (b. A.)) } \\
\hline Hauptschulabschluss mit b. A. & $1,04(0,20)$ & $0,70(0,18)$ & $1,18(0,17)$ \\
\hline Mittlere Reife ohne / mit b. A. & $0,81(0,18)$ & $0,75(0,21)$ & $1,57(0,25) * *$ \\
\hline Hochschulreife ohne / mit b. A. & $0,83(0,24)$ & $0,72(0,24)$ & $1,63(0,32) *$ \\
\hline FH-/ Universitätsabschluss & $0,52(0,14) *$ & $0,61(0,18)$ & $2,01(0,35) * * *$ \\
\hline Geschlecht (weiblich) & $1,07(0,12)$ & $1,25(0,17)$ & $1,31(0,10) * *$ \\
\hline Konstante & $1,92(517,80)$ & $6,3 \mathrm{e}^{+222}\left(1,9 \mathrm{e}^{+225}\right)$ & $3,82 \mathrm{e}^{+24}\left(6,85 \mathrm{e}^{+26}\right)$ \\
\hline $\mathrm{N}$ & & 4.816 & \\
\hline Log-Likelihood & & $-4226,21$ & \\
\hline McFadden-Pseudo- $\mathrm{R}^{2}$ & & 0,20 & \\
\hline
\end{tabular}

Quelle: Eigene Berechnungen basierend auf dem NEPS. Multinomiale logistische Regression. Die angegebenen Koeffizienten sind die Odds Ratios sowie die dazugehörigen Standardfehler (in Klammern). Referenzkategorie bei den Stationskategorien: Stationen ohne Weiterbildungscharakter. Die metrisch skalierten unabhängigen Variablen im Modell wurden mittelwertzentriert. Anmerkung: Statt 4.875 Fällen sind 4.816 Fälle im Modell enthalten. 59 Fälle werden ausgeschlossen. Diese haben als Station ein FSJ, welches aber kürzer ist als eine berufsvorbereitende Maßnahme (Dauer). Aufgrund der aufgestellten Bedingung bzgl. der Dauer werden diese Fälle bei den Kategorien nicht berücksichtigt. $\mathrm{e}^{\mathrm{xy}}$ steht für $10^{\mathrm{xy}}\left(, 10\right.$ hoch“ $^{\prime \prime}$. Legende für p-Werte: $* * *=<0,001$; $* *=<0,01 ; *=\leq 0,05$. 
Tabelle 36: Effekt des Schulabschlusses auf die Zugehörigkeit zur jeweiligen Stationskategorie (ohne FSJ/Urlaubsepisoden; mit unbestimmten Lückenepisoden).

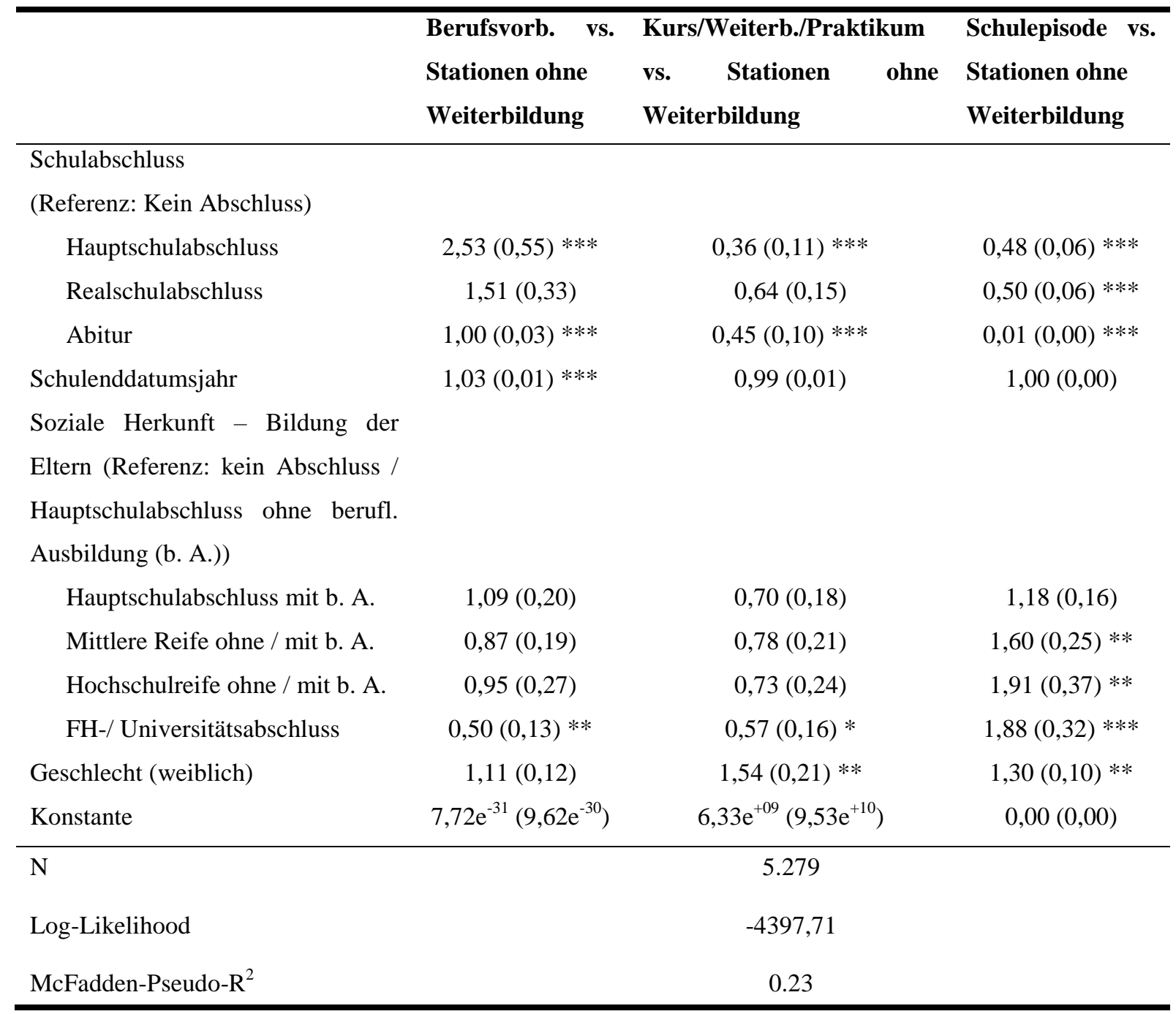

Quelle: Eigene Berechnungen basierend auf dem NEPS. Multinomiale logistische Regression. Die angegebenen Koeffizienten sind die Odds Ratios sowie die dazugehörigen Standardfehler (in Klammern). Referenzkategorie bei den Stationskategorien: Stationen ohne Weiterbildungscharakter. Die metrisch skalierten unabhängigen Variablen im Modell wurden mittelwertzentriert. $\mathrm{e}^{\mathrm{xy}}$ steht für $10^{\mathrm{xy}}\left(, 10\right.$ hoch“ $^{\text {) }}$. Legende für $\mathrm{p}$-Werte: *** = $<0,001 ; * *=<0,01 ; *=\leq 0,05$. 
Tabelle 37: Effekt des Schulabschlusses und des Anteil an Dienstleistungstätigen auf die Zugehörigkeit zur jeweiligen Stationskategorie (ohne FSJ/Urlaubsepisoden; mit unbestimmten Lückenepisoden).

\begin{tabular}{|c|c|c|c|}
\hline & $\begin{array}{l}\text { Berufsvorb. vs. } \\
\text { Stationen ohne } \\
\text { Weiterbildung }\end{array}$ & $\begin{array}{l}\text { Kurs/Weiterb./Prak- } \\
\text { tikum vs. Stationen } \\
\text { ohne Weiterbildung }\end{array}$ & $\begin{array}{l}\text { Schulepisode vs. } \\
\text { Stationen ohne } \\
\text { Weiterbildung }\end{array}$ \\
\hline \multicolumn{4}{|l|}{$\begin{array}{l}\text { Schulabschluss } \\
\text { (Referenz: Kein Abschluss) }\end{array}$} \\
\hline Hauptschulabschluss & $2,93(0,67) * * *$ & $0,35(0,10) * * *$ & $0,51(0,06) * * *$ \\
\hline Realschulabschluss & $1,61(0,37) *$ & $0,56(0,14) *$ & $0,50(0,06) * * *$ \\
\hline Abitur & $0,10(0,03) * * *$ & $0,43(0,10) * * *$ & $0,01(0,00) * * *$ \\
\hline Anteil Dienstleistungstätige & $1,01(0,16)$ & $1,27(0,22)$ & $1,00(0,00)$ \\
\hline $\begin{array}{l}\text { Interaktion Schulabschluss } \times \text { Anteil } \\
\text { Dienstleistungstätige }\end{array}$ & \multicolumn{2}{|c|}{ Dienstleistungstätige } & \\
\hline Hauptschulabschluss & $1,01(0,03)$ & $1,03(0,04)$ & $1,05(0,02) * *$ \\
\hline Realschulabschluss & $0,96(0,03)$ & $0,96(0,03)$ & $1,03(0,02) *$ \\
\hline Abitur & $1,00(0,04)$ & $1,02(0,03)$ & $1,00(0,03)$ \\
\hline $\begin{array}{l}\text { Demografischer Anteil (unter 20- } \\
\text { Jährige) }\end{array}$ & $0,90(0,09)$ & $0,82(0,09)$ & $0,92(0,06)$ \\
\hline Arbeitslosenquote & $1,07(0,06)$ & $0,90(0,06)$ & $0,96(0,04)$ \\
\hline Schulenddatumsjahr & $0,99(0,13)$ & $0,79(0,12)$ & $0,96(0,08)$ \\
\hline $\begin{array}{l}\text { Soziale Herkunft }- \text { Bildung der Eltern } \\
\text { (Referenz: kein Abschluss / }\end{array}$ & & & \\
\hline $\begin{array}{l}\text { Hauptschulabschluss ohne berufl. } \\
\text { Ausbildung (b. A.)) }\end{array}$ & & & \\
\hline Hauptschulabschluss mit b. A. & $1,04(0,20)$ & $0,69(0,18)$ & $1,16(0,16)$ \\
\hline Mittlere Reife ohne / mit b. A. & $0,85(0,19)$ & $0,77(0,21)$ & $1,59(0,25) * *$ \\
\hline Hochschulreife ohne / mit b. A. & $0,96(0,27)$ & $0,75(0,25)$ & $1,91(0,37) * *$ \\
\hline FH-/ Universitätsabschluss & $0,50(0,13) * *$ & $0,56(0,16) *$ & $1,87(0,32) * * *$ \\
\hline Geschlecht (weiblich) & $1,11(0,12)$ & $1,53(0,20) * *$ & $1,31(0,10) * * *$ \\
\hline Konstante & $8,54 \mathrm{e}^{+11}\left(2,29 \mathrm{e}^{+14}\right)$ & $1,6 \mathrm{e}^{+198}\left(4,9 \mathrm{e}^{+200}\right)$ & $3,53 \mathrm{e}^{+36}\left(6,21 \mathrm{e}^{+38}\right)$ \\
\hline $\mathrm{N}$ & & 5.279 & \\
\hline Log-Likelihood & & $-4374,44$ & \\
\hline McFadden-Pseudo- $\mathrm{R}^{2}$ & & 0.24 & \\
\hline
\end{tabular}

Quelle: Eigene Berechnungen basierend auf dem NEPS. Multinomiale logistische Regression. Die angegebenen Koeffizienten sind die Odds Ratios sowie die dazugehörigen Standardfehler (in Klammern). Referenzkategorie bei den Stationskategorien: Stationen ohne Weiterbildungscharakter. Die metrisch skalierten unabhängigen Variablen im Modell wurden mittelwertzentriert. $\mathrm{e}^{\mathrm{xy}}$ steht für $10^{\mathrm{xy}}$ (,10 hoch“). Legende für p-Werte: *** $=$ $<0,001 ; * *=<0,01 ; *=\leq 0,05$. 
Tabelle 38: Effekt des Schulabschlusses auf die Zugehörigkeit zur jeweiligen Stationskategorie (mit FSJ/Urlaubsepisoden; mit unbestimmten Lückenepisoden).

\begin{tabular}{|c|c|c|c|}
\hline & $\begin{array}{l}\text { Berufsvorb. } \\
\text { Stationen ohne } \\
\text { Weiterbildung }\end{array}$ & $\begin{array}{l}\text { Kurs/Weiterb./Prak- } \\
\text { tikum vs. Stationen } \\
\text { ohne Weiterbildung }\end{array}$ & $\begin{array}{l}\text { Schulepisode vs. } \\
\text { Stationen ohne } \\
\text { Weiterbildung }\end{array}$ \\
\hline \multicolumn{4}{|l|}{$\begin{array}{l}\text { Schulabschluss } \\
\text { (Referenz: Kein Abschluss) }\end{array}$} \\
\hline Hauptschulabschluss & $2,42(0,52) * * *$ & $0,31(0,09) * * *$ & $0,46(0,05) * * *$ \\
\hline Realschulabschluss & $1,35(0,29)$ & $0,57(0,13) *$ & $0,44(0,05) * * *$ \\
\hline Abitur & $0,10(0,03) * * *$ & $0,45(0,10) * * *$ & $0,01(0,00) * * *$ \\
\hline Schulenddatumsjahr & $1,04(0,01) * * *$ & $0,99(0,01)$ & $1,01(0,00) *$ \\
\hline \multicolumn{4}{|l|}{ Soziale Herkunft - Bildung der } \\
\hline \multicolumn{4}{|l|}{ Eltern (Referenz: kein Abschluss / } \\
\hline \multicolumn{4}{|l|}{ Hauptschulabschluss ohne berufl. } \\
\hline \multicolumn{4}{|l|}{ Ausbildung (b. A.)) } \\
\hline Hauptschulabschluss mit b. A. & $1,04(0,19)$ & $0,68(0,17)$ & $1,13(0,15)$ \\
\hline Mittlere Reife ohne / mit b. A. & $0,85(0,18)$ & $0,76(0,21)$ & $1,62(0,23) * *$ \\
\hline Hochschulreife ohne / mit b. A. & $0,86(0,24)$ & $0,70(0,23)$ & $1,73(0,31) * *$ \\
\hline FH-/ Universitätsabschluss & $0,50(0,13) * *$ & $0,57(0,16) *$ & $1,96(0,31) * * *$ \\
\hline Geschlecht (weiblich) & $1,15(0,13)$ & $1,50(0,20) * *$ & $1,35(0,10) * * *$ \\
\hline Konstante & $5,09 \mathrm{e}^{-34}\left(6,20 \mathrm{e}^{-33}\right) * * *$ & $9,22 \mathrm{e}^{+07}\left(1,38 \mathrm{e}^{+09}\right)$ & $1,33 \mathrm{e}^{-07}\left(1,07 \mathrm{e}^{-06}\right) *$ \\
\hline $\mathrm{N}$ & & 5.727 & \\
\hline Log-Likelihood & & $-4745,39$ & \\
\hline McFadden-Pseudo- $\mathrm{R}^{2}$ & & 0,22 & \\
\hline
\end{tabular}

Quelle: Eigene Berechnungen basierend auf dem NEPS. Multinomiale logistische Regression. Die angegebenen Koeffizienten sind die Odds Ratios sowie die dazugehörigen Standardfehler (in Klammern). Referenzkategorie bei den Stationskategorien: Stationen ohne Weiterbildungscharakter. Die metrisch skalierten unabhängigen Variablen im Modell wurden mittelwertzentriert. Anmerkung: Zwölf Fälle haben bei den Stationskategorien einen fehlenden Wert und werden ausgeschlossen. Diese Fälle können keiner Stationskategorie zugeordnet werden. Mit Ausnahme von einem Fall, welcher keine Stationen hat, handelt es sich dabei um Personen, welche ein FSJ absolviert haben, dessen Dauer kürzer als berufsvorbereitende Maßnahmen war. $\mathrm{e}^{\mathrm{xy}}$ steht für $10^{\mathrm{xy}}(, 10$ hoch“). Legende für p-Werte: $* * *=<0,001 ; * *=<0,01 ; *=\leq 0,05$. 
Tabelle 39: Effekt des Schulabschlusses und des Anteil an Dienstleistungstätigen auf die Zugehörigkeit zur jeweiligen Stationskategorie (mit FSJ/Urlaubsepisoden; mit unbestimmten Lückenepisoden).

\begin{tabular}{|c|c|c|c|}
\hline & $\begin{array}{l}\text { Berufsvorb. vs. } \\
\text { Stationen ohne } \\
\text { Weiterbildung }\end{array}$ & $\begin{array}{l}\text { Kurs/Weiterb./Prak- } \\
\text { tikum vs. Stationen } \\
\text { ohne Weiterbildung }\end{array}$ & $\begin{array}{l}\text { Schulepisode vs. } \\
\text { Stationen ohne } \\
\text { Weiterbildung }\end{array}$ \\
\hline \multicolumn{4}{|l|}{$\begin{array}{l}\text { Schulabschluss } \\
\text { (Referenz: Kein Abschluss) }\end{array}$} \\
\hline Hauptschulabschluss & $2,81(0,63) * * *$ & $0,34(0,10) * * *$ & $0,49(0,06) * * *$ \\
\hline Realschulabschluss & $1,46(0,33)$ & $0,51(0,12) * *$ & $0,45(0,05) * * *$ \\
\hline Abitur & $0,10(0,03) * * *$ & $0,44(0,10) * * *$ & $0,01(0,00) * * *$ \\
\hline Anteil Dienstleistungstätige & $0,99(0,15)$ & $1,25(0,21)$ & $0,98(0,09)$ \\
\hline $\begin{array}{l}\text { Interaktion Schulabschluss } \times \text { Anteil } \\
\text { Dienstleistungstätige }\end{array}$ & & & \\
\hline Hauptschulabschluss & $1,01(0,03)$ & $1,03(0,04)$ & $1,05(0,02) * *$ \\
\hline Realschulabschluss & $0,97(0,03)$ & $0,96(0,03)$ & $1,04(0,02) * *$ \\
\hline Abitur & $0,99(0,04)$ & $1,01(0,03)$ & $1,00(0,03)$ \\
\hline $\begin{array}{l}\text { Demografischer Anteil (unter 20- } \\
\text { Jährige) }\end{array}$ & $0,94(0,09)$ & $0,85(0,09)$ & $0,95(0,06)$ \\
\hline Arbeitslosenquote & $1,08(0,06)$ & $0,90(0,06)$ & $0,97(0,04)$ \\
\hline Schulenddatumsjahr & $1,02(0,13)$ & $0,81(0,12)$ & $0,99(0,08)$ \\
\hline $\begin{array}{l}\text { Soziale Herkunft }- \text { Bildung der Eltern } \\
\text { (Referenz: kein Abschluss / }\end{array}$ & & & \\
\hline $\begin{array}{l}\text { Hauptschulabschluss ohne berufl. } \\
\text { Ausbildung (b. A.)) }\end{array}$ & & & \\
\hline Hauptschulabschluss mit b. A. & $0,99(0,18)$ & $0,67(0,17)$ & $1,12(0,14)$ \\
\hline Mittlere Reife ohne / mit b. A. & $0,83(0,18)$ & $0,76(0,21)$ & $1,61(0,23) * *$ \\
\hline Hochschulreife ohne / mit b. A. & $0,87(0,24)$ & $0,72(0,24)$ & $1,73(0,31) * *$ \\
\hline FH-/ Universitätsabschluss & $0,50(0,13) * *$ & $0,57(0,16) *$ & $1,96(0,31) * * *$ \\
\hline Geschlecht (weiblich) & $1,14(0,13)$ & $1,48(0,20) * *$ & $1,37(0,10) * * *$ \\
\hline Konstante & $8,81 \mathrm{e}^{-15}\left(2,30 \mathrm{e}^{-12}\right)$ & $2,7 \mathrm{e}^{+182}\left(8,0 \mathrm{e}^{+184}\right)$ & $2,76 \mathrm{e}^{+12}\left(4,54 \mathrm{e}^{+14}\right)$ \\
\hline $\mathrm{N}$ & & 5.727 & \\
\hline Log-Likelihood & & $-4722,18$ & \\
\hline McFadden-Pseudo-R ${ }^{2}$ & & 0.23 & \\
\hline
\end{tabular}

Quelle: Eigene Berechnungen basierend auf dem NEPS. Multinomiale logistische Regression. Die angegebenen Koeffizienten sind die Odds Ratios sowie die dazugehörigen Standardfehler (in Klammern). Referenzkategorie bei den Stationskategorien: Stationen ohne Weiterbildungscharakter. Die metrisch skalierten unabhängigen Variablen im Modell wurden mittelwertzentriert. Anmerkung: Zwölf Fälle haben bei den Stationskategorien einen fehlenden Wert und werden ausgeschlossen, da sie keiner Stationskategorie zugeordnet werden können. Mit Ausnahme von einem Fall, welcher keine Stationen hat, handelt es sich dabei um Personen, welche ein FSJ absolviert haben, dessen Dauer kürzer als berufsvorbereitende Maßnahmen war. ${ }^{\mathrm{xy}}$ steht für $10^{\mathrm{xy}}(, 10$ hoch“). Legende für p-Werte: $* * *=<0,001 ; * *=<0,01 ; *=\leq 0,05$. 


\section{IRR (SE)}

\begin{tabular}{lc}
\hline Schulabschluss (Referenz: Kein Abschluss) & \\
$\quad$ Hauptschulabschluss & $1,30(0,05) * * *$ \\
$\quad$ Realschulabschluss & $1,25(0,05) * * *$ \\
$\quad$ Abitur & $2,62(0,12) * * *$ \\
Schulenddatumsjahr & $1,01(0,00) * * *$ \\
Soziale Herkunft - Bildung der Eltern (Referenz: kein Abschluss / & \\
Hauptschulabschluss ohne berufl. Ausbildung (b. A.)) & \\
$\quad$ Hauptschulabschluss mit b. A. & $1,11(0,06) *$ \\
$\quad$ Mittlere Reife ohne / mit b. A. & $1,08(0,06)$ \\
$\quad$ Hochschulreife ohne/mit b. A. & $1,13(0,07)$ \\
$\quad$ FH-/ Universitätsabschluss & $1,12(0,06)$ \\
Geschlecht (weiblich) & $0,89(0,02) * * *$ \\
Konstante & $1,11 \mathrm{e}^{-06}\left(3,21 \mathrm{e}^{-06}\right) * * *$ \\
\hline Logarithmus(Übergangsdauer) & $1(\mathrm{exposure})$ \\
\hline N & 4.867 \\
Log-Likelihood & $-8578,65$ \\
McFadden-Pseudo-R ${ }^{2}$ & 0.04
\end{tabular}

Quelle: Eigene Berechnungen basierend auf dem NEPS. Negatives binomiales Regressionsmodell. Die angegebenen Koeffizienten sind die Incident Rate Ratios (IRR) sowie die dazugehörigen Standardfehler (in Klammern). Anmerkung: Ausgangsstichprobe hier wieder $n=4.875$ (siehe Anmerkung zu Tabelle 34), da hier die 59 Fälle mit FSJ (kürzere Dauer als berufsvorbereitende Maßnahme) wieder drin sind. Acht Fälle werden aus dem Modell ausgeschlossen, da sie neun oder mehr Stationen haben und die Frequentierung zu gering auf diesen Stationssummen ist $(\mathrm{n}=4.867) . \mathrm{e}^{\mathrm{xy}}$ steht für $10^{\mathrm{xy}}\left(, 10\right.$ hoch“ $^{\prime \prime}$. Legende für $\mathrm{p}$-Werte: $* * *=<0,001 ; * *=<0,01 ; *$ $=\leq 0,05$. 


\begin{tabular}{lc}
\hline Schulabschluss (Referenz: Kein Abschluss) & \\
Hauptschulabschluss & $1,37(0,06)^{* * *}$ \\
$\quad$ Realschulabschluss & $1,29(0,05)^{* * *}$ \\
$\quad$ Abitur & $3,77(0,18)^{* * *}$ \\
Schulenddatumsjahr & $1,00(0,00)^{* *}$ \\
Soziale Herkunft - Bildung der Eltern (Referenz: kein Abschluss / & \\
Hauptschulabschluss ohne berufl. Ausbildung (b. A.)) & \\
$\quad$ Hauptschulabschluss mit b. A. & $1,16(0,06)^{* *}$ \\
$\quad$ Mittlere Reife ohne / mit b. A. & $1,07(0,06)$ \\
$\quad$ Hochschulreife ohne/mit b. A. & $1,12(0,08)$ \\
$\quad$ FH-/ Universitätsabschluss & $1,15(0,07) *$ \\
Geschlecht (weiblich) & $0,87(0,02)^{* * *}$ \\
Konstante & $3,26 \mathrm{e}^{-06}(0,00)^{* * *}$ \\
\hline Logarithmus(Übergangsdauer) & $1(\mathrm{exposure})$ \\
\hline N & 5.264 \\
Log-Likelihood & $-10423,54$ \\
McFadden-Pseudo-R ${ }^{2}$ & 0,05
\end{tabular}

Quelle: Eigene Berechnungen basierend auf dem NEPS. Negatives binomiales Regressionsmodell. Die angegebenen Koeffizienten sind die Incident Rate Ratios (IRR) sowie die dazugehörigen Standardfehler (in Klammern). Anmerkung: 15 Fälle belegen eine Stationssumme von acht oder mehr und werden aufgrund der geringen Frequentierung aus dem negativ binomialen Modell gegenüber dem multinomial logistischen Modell ausgeschlossen. $\mathrm{e}^{\mathrm{xy}}$ steht für $10^{\mathrm{xy}}(, 10$ hoch“ $)$. Legende für $\mathrm{p}$-Werte: $* * *=<0,001 ; * *=<0,01 ; *=\leq 0,05$. 


\section{IRR (SE)}

\begin{tabular}{lc}
\hline Schulabschluss (Referenz: Kein Abschluss) & \\
Hauptschulabschluss & $1,48(0,07) * * *$ \\
$\quad$ Realschulabschluss & $1,45(0,06)^{* * *}$ \\
$\quad$ Abitur & $3,79(0,16)^{* * *}$ \\
Schulenddatumsjahr & $1,00(0,00)$ \\
Soziale Herkunft - Bildung der Eltern (Referenz: kein Abschluss / & \\
Hauptschulabschluss ohne berufl. Ausbildung (b. A.)) & \\
$\quad$ Hauptschulabschluss mit b. A. & $1,14(0,06)^{*}$ \\
$\quad$ Mittlere Reife ohne / mit b. A. & $1,05(0,06)$ \\
$\quad$ Hochschulreife ohne/mit b. A. & $1,07(0,07)$ \\
$\quad$ FH-/ Universitätsabschluss & $1,08(0,07)$ \\
Geschlecht (weiblich) & $0,86(0,02) * * *$ \\
Konstante & $0,02(0,05)$ \\
\hline Logarithmus(Übergangsdauer) & $1($ exposure) \\
\hline N & 5.727 \\
Log-Likelihood & $-12189,97$ \\
McFadden-Pseudo-R ${ }^{2}$ & 0,05
\end{tabular}

Quelle: Eigene Berechnungen basierend auf dem NEPS. Negatives binomiales Regressionsmodell. Die angegebenen Koeffizienten sind die Incident Rate Ratios (IRR) sowie die dazugehörigen Standardfehler (in Klammern). Legende für p-Werte: $* * *=<0,001 ; * *=<0,01 ; *=\leq 0,05$. 
Tabelle 43: Effekt des gestiegenen Dienstleistungstätigenanteils auf die Anzahl der Übergangsstationen (mit FSJ- und Urlaubsepisoden; ohne unbestimmte Lückenepisoden).

\begin{tabular}{|c|c|}
\hline & IRR (SE) \\
\hline Anteil Dienstleistungstätige & $0,99(0,03)$ \\
\hline Demografischer Anteil (unter 20-Jährige) & $1,03(0,02)$ \\
\hline Arbeitslosenquote & $1,03(0,01) *$ \\
\hline \multicolumn{2}{|l|}{ Schulabschluss (Referenz: Kein Abschluss) } \\
\hline Hauptschulabschluss & $1,30(0,05) * * *$ \\
\hline Realschulabschluss & $1,26(0,05) * * *$ \\
\hline Abitur & $2,61(0,12) * * *$ \\
\hline Schulenddatumsjahr & $1,02(0,03)$ \\
\hline \multicolumn{2}{|c|}{ Soziale Herkunft - Bildung der Eltern (Referenz: kein Abschluss / } \\
\hline \multicolumn{2}{|c|}{ Hauptschulabschluss ohne berufl. Ausbildung (b. A.)) } \\
\hline Hauptschulabschluss mit b. A. & $1,11(0,06) *$ \\
\hline Mittlere Reife ohne / mit b. A. & $1,08(0,06)$ \\
\hline Hochschulreife ohne/mit b. A. & $1,12(0,07)$ \\
\hline FH-/ Universitätsabschluss & $1,11(0,06)$ \\
\hline Geschlecht (weiblich) & $0,89(0,02) * * *$ \\
\hline Konstante & $5,89 \mathrm{e}^{-18}\left(3,37 \mathrm{e}^{-16}\right)$ \\
\hline Logarithmus(Übergangsdauer) & 1 (exposure) \\
\hline $\mathrm{N}$ & 4.867 \\
\hline Log-Likelihood & $-8575,90$ \\
\hline McFadden-Pseudo- $\mathrm{R}^{2}$ & 0,04 \\
\hline
\end{tabular}

Quelle: Eigene Berechnungen basierend auf dem NEPS. Negatives binomiales Regressionsmodell. Die angegebenen Koeffizienten sind die Incident Rate Ratios (IRR) sowie die dazugehörigen Standardfehler (in Klammern). Anmerkung: Ausgangsstichprobe hier wieder $n=4.875$, da hier die 59 Fälle mit FSJ (kürzere Dauer als berufsvorbereitende Maßnahme) wieder drin sind. Acht Fälle werden aus dem Modell ausgeschlossen, da sie neun oder mehr Stationen haben und die Frequentierung zu gering auf diesen Stationssummen ist $(n=4.867)$. $e^{x y}$ steht für $10^{\mathrm{xy}}\left(, 10\right.$ hoch“) $^{*}$. Legende für p-Werte: $* * *=<0,001 ; * *=<0,01 ; *=\leq 0,05$. 
Tabelle 44: Effekt des gestiegenen Dienstleistungstätigenanteils auf die Anzahl der Übergangsstationen mit Interaktion zwischen Anteil Dienstleistungstätige und Schulabschluss (mit FSJ- und Urlaubsepisoden; ohne unbestimmte Lückenepisoden).

\begin{tabular}{|c|c|}
\hline & IRR (SE) \\
\hline Anteil Dienstleistungstätige & $1,00(0,04)$ \\
\hline Demografischer Anteil (unter 20-Jährige) & $1,02(0,02)$ \\
\hline Arbeitslosenquote & $1,02(0,01)$ \\
\hline \multicolumn{2}{|l|}{ Schulabschluss (Referenz: Abitur) } \\
\hline Kein Abschluss & $0,39(0,02) * * *$ \\
\hline Hauptschulabschluss & $0,50(0,02) * * *$ \\
\hline Realschulabschluss & $0,49(0,02) * * *$ \\
\hline Schulenddatumsjahr & $1,01(0,03)$ \\
\hline \multicolumn{2}{|c|}{ Soziale Herkunft - Bildung der Eltern (Referenz: kein Abschluss / } \\
\hline \multicolumn{2}{|l|}{ Hauptschulabschluss ohne berufl. Ausbildung (b. A.)) } \\
\hline Hauptschulabschluss mit b. A. & $1,11(0,06) *$ \\
\hline Mittlere Reife ohne / mit b. A. & $1,08(0,06)$ \\
\hline Hochschulreife ohne/mit b. A. & $1,13(0,07)$ \\
\hline FH-/ Universitätsabschluss & $1,11(0,06)$ \\
\hline Geschlecht (weiblich) & $0,89(0,02) * * *$ \\
\hline \multicolumn{2}{|l|}{ Interaktion Anteil Dienstleistungstätige $\times$ Schulabschluss } \\
\hline Anteil Dienstleistungstätige $\times$ Kein Abschluss & $1,00(0,01)$ \\
\hline Anteil Dienstleistungstätige $\times$ Hauptschulabschluss & $0,99(0,01)$ \\
\hline Anteil Dienstleistungstätige $\times$ Realschulabschluss & $0,99(0,01)$ \\
\hline Konstante & $4,30 \mathrm{e}^{-09}\left(2,59 \mathrm{e}^{-07}\right)$ \\
\hline Logarithmus(Übergangsdauer) & 1 (exposure) \\
\hline $\mathrm{N}$ & 4.867 \\
\hline Log-Likelihood & $-8574,19$ \\
\hline McFadden-Pseudo- $\mathrm{R}^{2}$ & 0,04 \\
\hline
\end{tabular}

Quelle: Eigene Berechnungen basierend auf dem NEPS. Negatives binomiales Regressionsmodell. Die angegebenen Koeffizienten sind die Incident Rate Ratios (IRR) sowie die dazugehörigen Standardfehler (in Klammern). Anmerkung: Ausgangsstichprobe hier wieder $n=4.875$, da hier die 59 Fälle mit FSJ (kürzere Dauer als berufsvorbereitende Maßnahme) wieder drin sind. Acht Fälle werden aus dem Modell ausgeschlossen, da sie neun oder mehr Stationen haben und die Frequentierung zu gering auf diesen Stationssummen ist $(n=4.867)$. $e^{x y}$ steht für $10^{\mathrm{xy}}\left(,, 10 \mathrm{hoch}^{“}\right)$. Legende für p-Werte: $* * *=<0,001 ; * *=<0,01 ; *=\leq 0,05$. 
Tabelle 45: Effekt des gestiegenen Dienstleistungstätigenanteils auf die Anzahl der Übergangsstationen (ohne FSJ- und Urlaubsepisoden; mit unbestimmten Lückenepisoden).

\begin{tabular}{|c|c|}
\hline & IRR (SE) \\
\hline Anteil Dienstleistungstätige & $0,98(0,03)$ \\
\hline Demografischer Anteil (unter 20-Jährige) & $1,02(0,02)$ \\
\hline Arbeitslosenquote & $1,03(0,01) *$ \\
\hline \multicolumn{2}{|l|}{ Schulabschluss (Referenz: Kein Abschluss) } \\
\hline Hauptschulabschluss & $1,38(0,06) * * *$ \\
\hline Realschulabschluss & $1,29(0,05) * * *$ \\
\hline Abitur & $3,74(0,17) * * *$ \\
\hline Schulenddatumsjahr & $1,02(0,03)$ \\
\hline \multicolumn{2}{|c|}{ Soziale Herkunft - Bildung der Eltern (Referenz: kein Abschluss / } \\
\hline \multicolumn{2}{|c|}{ Hauptschulabschluss ohne berufl. Ausbildung (b. A.)) } \\
\hline Hauptschulabschluss mit b. A. & $1,16(0,06) * *$ \\
\hline Mittlere Reife ohne / mit b. A. & $1,06(0,06)$ \\
\hline Hochschulreife ohne/mit b. A. & $1,11(0,08)$ \\
\hline FH-/ Universitätsabschluss & $1,15(0,07) *$ \\
\hline Geschlecht (weiblich) & $0,87(0,02) * * *$ \\
\hline Konstante & $1,27 \mathrm{e}^{-17}\left(7,73 \mathrm{e}^{-16}\right) * * *$ \\
\hline Logarithmus(Übergangsdauer) & 1 (exposure) \\
\hline $\mathrm{N}$ & 5.264 \\
\hline Log-Likelihood & $-10419,33$ \\
\hline McFadden-Pseudo- $\mathrm{R}^{2}$ & 0,05 \\
\hline
\end{tabular}

Quelle: Eigene Berechnungen basierend auf dem NEPS. Negatives binomiales Regressionsmodell. Die angegebenen Koeffizienten sind die Incident Rate Ratios (IRR) sowie die dazugehörigen Standardfehler (in Klammern). Anmerkung: 15 Fälle belegen eine Stationssumme von acht oder mehr und werden aufgrund der geringen Frequentierung aus dem negativ binomialen Modell ausgeschlossen. $\mathrm{e}^{\mathrm{xy}}$ steht für $10^{\mathrm{xy}}$ (,10 hoch“). Legende für p-Werte: $* * *=<0,001 ; * *=<0,01 ; *=\leq 0,05$. 
Tabelle 46: Effekt des gestiegenen Dienstleistungstätigenanteils auf die Anzahl der Übergangsstationen mit Interaktion zwischen Anteil Dienstleistungstätige und Schulabschluss (ohne FSJ- und Urlaubsepisoden; mit unbestimmten Lückenepisoden).

\begin{tabular}{|c|c|}
\hline & IRR (SE) \\
\hline Anteil Dienstleistungstätige & $0,98(0,04)$ \\
\hline Demografischer Anteil (unter 20-Jährige) & $1,02(0,02)$ \\
\hline Arbeitslosenquote & $1,04(0,01) *$ \\
\hline \multicolumn{2}{|l|}{ Schulabschluss (Referenz: Abitur) } \\
\hline Kein Abschluss & $0,27(0,01) * * *$ \\
\hline Hauptschulabschluss & $0,37(0,02) * * *$ \\
\hline Realschulabschluss & $0,34(0,01) * * *$ \\
\hline Schulenddatumsjahr & $1,02(0,03)$ \\
\hline \multicolumn{2}{|c|}{ Soziale Herkunft -Bildung der Eltern (Referenz: kein Abschluss / } \\
\hline \multicolumn{2}{|l|}{ Hauptschulabschluss ohne berufl. Ausbildung (b. A.)) } \\
\hline Hauptschulabschluss mit b. A. & $1,16(0,06) * *$ \\
\hline Mittlere Reife ohne / mit b. A. & $1,07(0,06)$ \\
\hline Hochschulreife ohne/mit b. A. & $1,11(0,08)$ \\
\hline FH-/ Universitätsabschluss & $1,15(0,07) *$ \\
\hline Geschlecht (weiblich) & $0,87(0,02) * * *$ \\
\hline \multicolumn{2}{|l|}{ Interaktion Anteil Dienstleistungstätige $\times$ Schulabschluss } \\
\hline Anteil Dienstleistungstätige $\times$ Kein Abschluss & $1,01(0,01)$ \\
\hline Anteil Dienstleistungstätige $\times$ Hauptschulabschluss & $1,00(0,01)$ \\
\hline Anteil Dienstleistungstätige $\times$ Realschulabschluss & $1,00(0,01)$ \\
\hline Konstante & $1,20 \mathrm{e}^{-18}\left(7,73 \mathrm{e}^{-17}\right)$ \\
\hline Logarithmus(Übergangsdauer) & 1 (exposure) \\
\hline $\mathrm{N}$ & 5.264 \\
\hline Log-Likelihood & $-10417,54$ \\
\hline McFadden-Pseudo- $\mathrm{R}^{2}$ & 0,05 \\
\hline
\end{tabular}

Quelle: Eigene Berechnungen basierend auf dem NEPS. Negatives binomiales Regressionsmodell. Die angegebenen Koeffizienten sind die Incident Rate Ratios (IRR) sowie die dazugehörigen Standardfehler (in Klammern). Anmerkung: 15 Fälle belegen eine Stationssumme von acht oder mehr und werden aufgrund der geringen Frequentierung aus dem negativ binomialen Modell ausgeschlossen. $\mathrm{e}^{\mathrm{xy}}$ steht für $10^{\mathrm{xy}}(, 10$ hoch“). Legende für p-Werte: $* * *=<0,001 ; * *=<0,01 ; *=\leq 0,05$. 
Tabelle 47: Effekt des gestiegenen Dienstleistungstätigenanteils auf die Anzahl der Übergangsstationen (mit FSJ- und Urlaubsepisoden; mit unbestimmten Lückenepisoden).

\begin{tabular}{|c|c|}
\hline & IRR (SE) \\
\hline Anteil Dienstleistungstätige & $1,00(0,03)$ \\
\hline Demografischer Anteil (unter 20-Jährige) & $1,02(0,02)$ \\
\hline Arbeitslosenquote & $1,03(0,01)$ \\
\hline \multicolumn{2}{|l|}{ Schulabschluss (Referenz: Kein Abschluss) } \\
\hline Hauptschulabschluss & $1,49(0,07) * * *$ \\
\hline Realschulabschluss & $1,45(0,06) * * *$ \\
\hline Abitur & $3,77(0,16) * * *$ \\
\hline Schulenddatumsjahr & $1,00(0,03)$ \\
\hline \multicolumn{2}{|c|}{ Soziale Herkunft - Bildung der Eltern (Referenz: kein Abschluss / } \\
\hline \multicolumn{2}{|c|}{ Hauptschulabschluss ohne berufl. Ausbildung (b. A.)) } \\
\hline Hauptschulabschluss mit b. A. & $1,14(0,06) *$ \\
\hline Mittlere Reife ohne / mit b. A. & $1,05(0,06)$ \\
\hline Hochschulreife ohne/mit b. A. & $1,07(0,07)$ \\
\hline FH-/ Universitätsabschluss & $1,08(0,07)$ \\
\hline Geschlecht (weiblich) & $0,86(0,02) * * *$ \\
\hline Konstante & $0,00(0,02)$ \\
\hline Logarithmus(Übergangsdauer) & 1 (exposure) \\
\hline $\mathrm{N}$ & 5.727 \\
\hline Log-Likelihood & -12187.23 \\
\hline McFadden-Pseudo- $\mathrm{R}^{2}$ & 0,05 \\
\hline
\end{tabular}

Quelle: Eigene Berechnungen basierend auf dem NEPS. Negatives binomiales Regressionsmodell. Die angegebenen Koeffizienten sind die Incident Rate Ratios (IRR) sowie die dazugehörigen Standardfehler (in Klammern). Legende für p-Werte: $* * *=<0,001 ; * *=<0,01 ; *=\leq 0,05$. 
Tabelle 48: Effekt des gestiegenen Dienstleistungstätigenanteils auf die Anzahl der Übergangsstationen mit Interaktion zwischen Anteil Dienstleistungstätige und Schulabschluss (mit FSJ- und Urlaubsepisoden; mit unbestimmten Lückenepisoden).

\begin{tabular}{|c|c|}
\hline & IRR (SE) \\
\hline Anteil Dienstleistungstätige & $1,01(0,04)$ \\
\hline Demografischer Anteil (unter 20-Jährige) & $1,01(0,02)$ \\
\hline Arbeitslosenquote & $1,02(0,01)$ \\
\hline \multicolumn{2}{|l|}{ Schulabschluss (Referenz: Abitur) } \\
\hline Kein Abschluss & $0,27(0,01) * * *$ \\
\hline Hauptschulabschluss & $0,39(0,02) * * *$ \\
\hline Realschulabschluss & $0,39(0,01) * * *$ \\
\hline Schulenddatumsjahr & $0,99(0,03)$ \\
\hline \multicolumn{2}{|c|}{ Soziale Herkunft - Bildung der Eltern (Referenz: kein Abschluss / } \\
\hline \multicolumn{2}{|l|}{ Hauptschulabschluss ohne berufl. Ausbildung (b. A.)) } \\
\hline Hauptschulabschluss mit b. A. & $1,14(0,06) *$ \\
\hline Mittlere Reife ohne / mit b. A. & $1,05(0,06)$ \\
\hline Hochschulreife ohne/mit b. A. & $1,07(0,07)$ \\
\hline FH-/ Universitätsabschluss & $1,08(0,07)$ \\
\hline Geschlecht (weiblich) & $0,85(0,02) * * *$ \\
\hline \multicolumn{2}{|l|}{ Interaktion Anteil Dienstleistungstätige $\times$ Schulabschluss } \\
\hline Anteil Dienstleistungstätige $\times$ Kein Abschluss & $1,00(0,01)$ \\
\hline Anteil Dienstleistungstätige $\times$ Hauptschulabschluss & $0,99(0,01) *$ \\
\hline Anteil Dienstleistungstätige $\times$ Realschulabschluss & $1,00(0,01)$ \\
\hline Konstante & $0,44\left(2,70 \mathrm{e}^{+08}\right)$ \\
\hline Logarithmus(Übergangsdauer) & 1 (exposure) \\
\hline $\mathrm{N}$ & 5.727 \\
\hline Log-Likelihood & $-12183,63$ \\
\hline McFadden-Pseudo- $\mathrm{R}^{2}$ & 0,05 \\
\hline
\end{tabular}

Quelle: Eigene Berechnungen basierend auf dem NEPS. Negatives binomiales Regressionsmodell. Die angegebenen Koeffizienten sind die Incident Rate Ratios (IRR) sowie die dazugehörigen Standardfehler (in

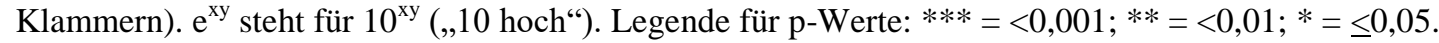




\section{Anhang E: Abbildungen zu Überschneidungskonstellationen von Stationen}

Abbildung 1: Mögliche Dauerkonstellationen einer Station während Übergangszeitraum.

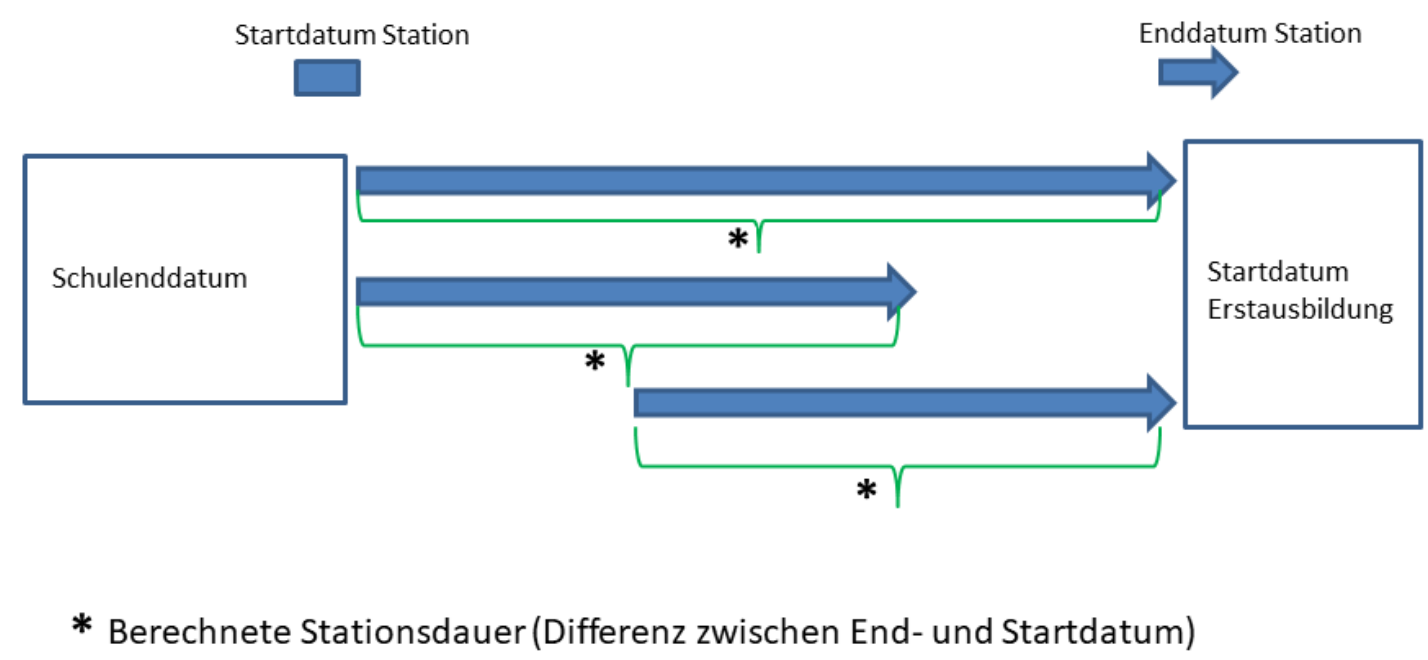

Quelle: Eigene Darstellung. Ohne Überschneidung mit dem Schulenddatum oder mit dem Startdatum der Erstausbildung.

Abbildung 2: Zeitliche Überschneidung einer Station mit Startdatum vor Schulenddatum.

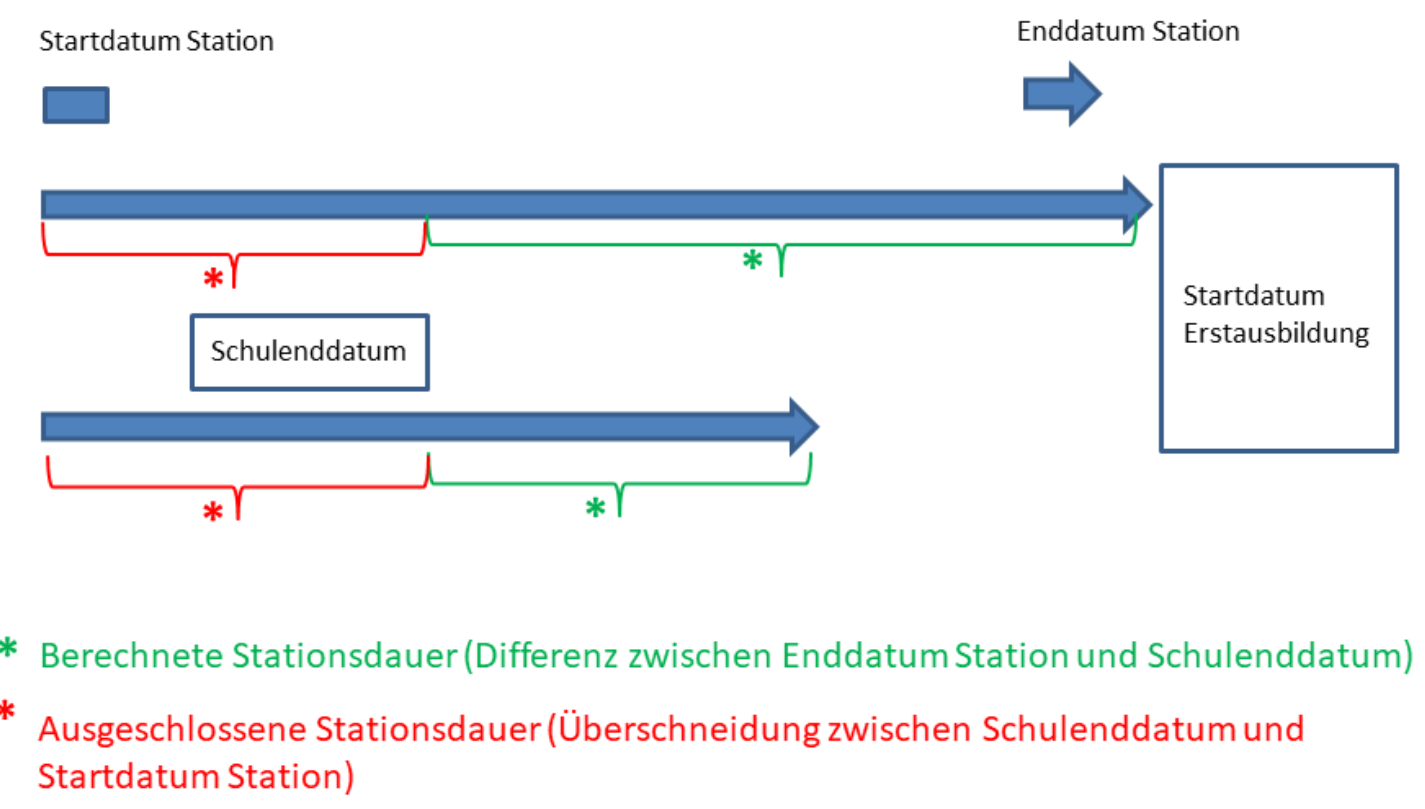

Quelle: Eigene Darstellung. 


\section{Abbildung 3: Zeitliche Überschneidung einer Station mit Enddatum nach Startdatum Erstausbildung.}

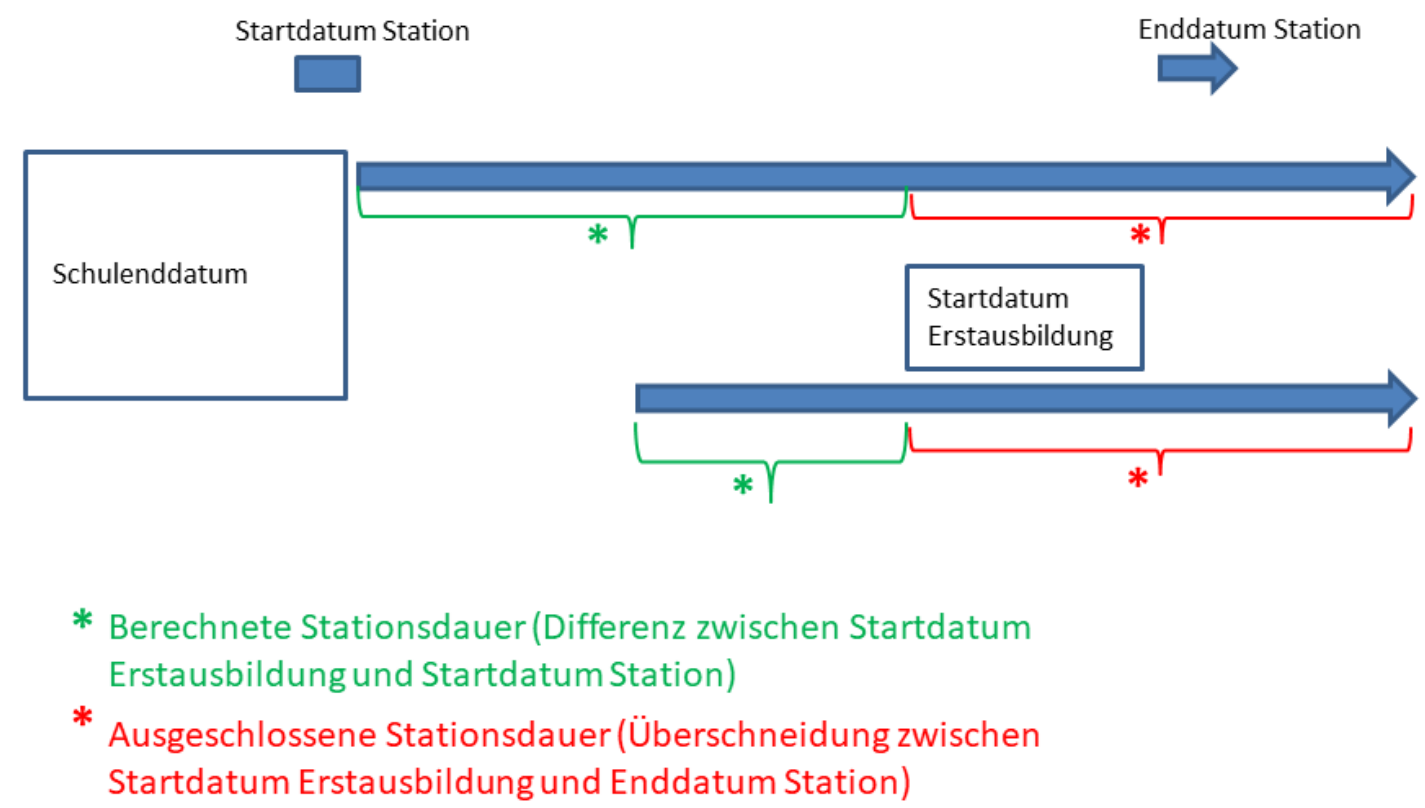

Quelle: Eigene Darstellung.

Abbildung 4: Zeitliche Überschneidung einer Station mit Startdatum vor Schulenddatum und Enddatum nach Startdatum Erstausbildung.

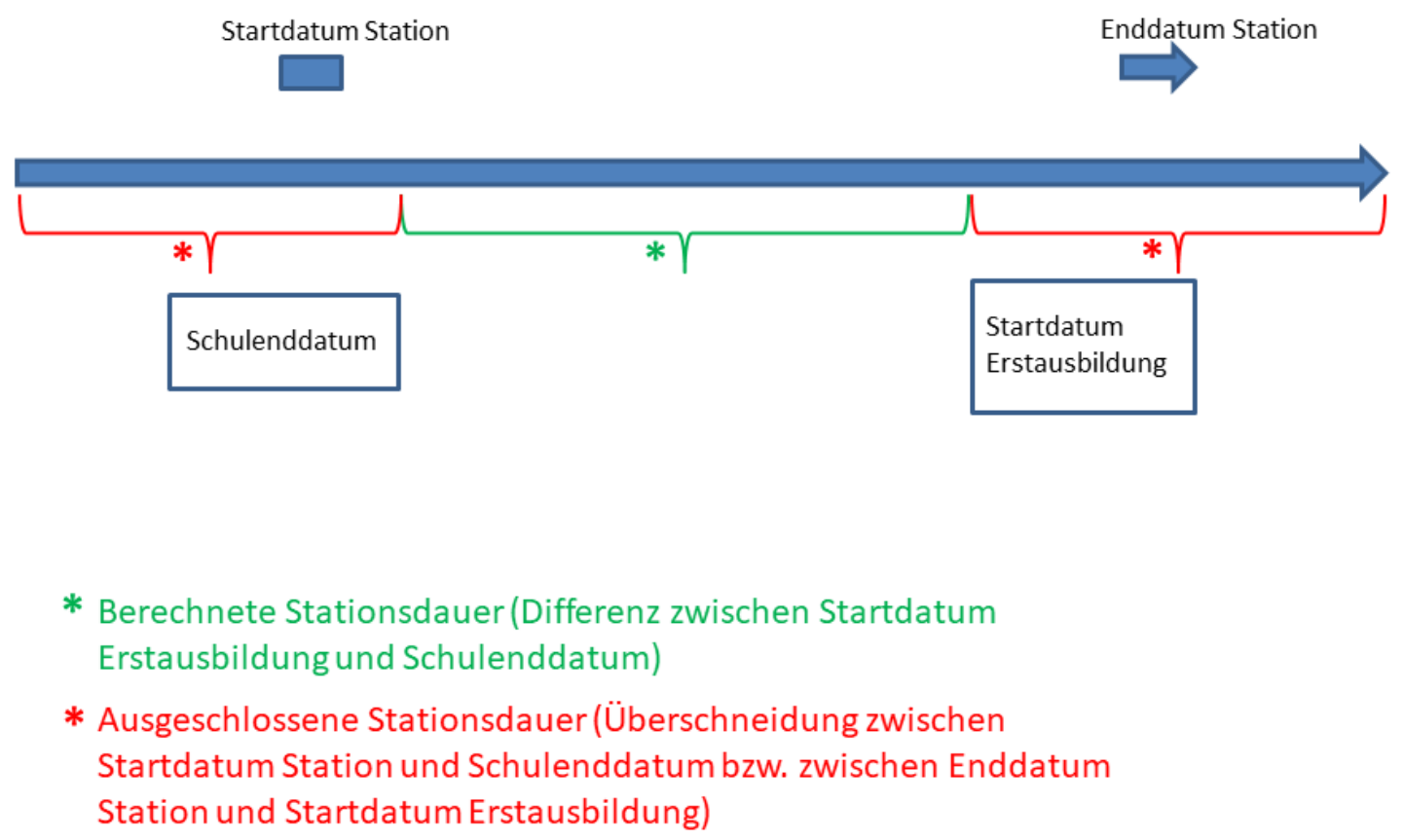

Quelle: Eigene Darstellung. 
Abbildung 5: Zeitliche Überschneidung zweier identischer Stationen mit identischem Enddatum.

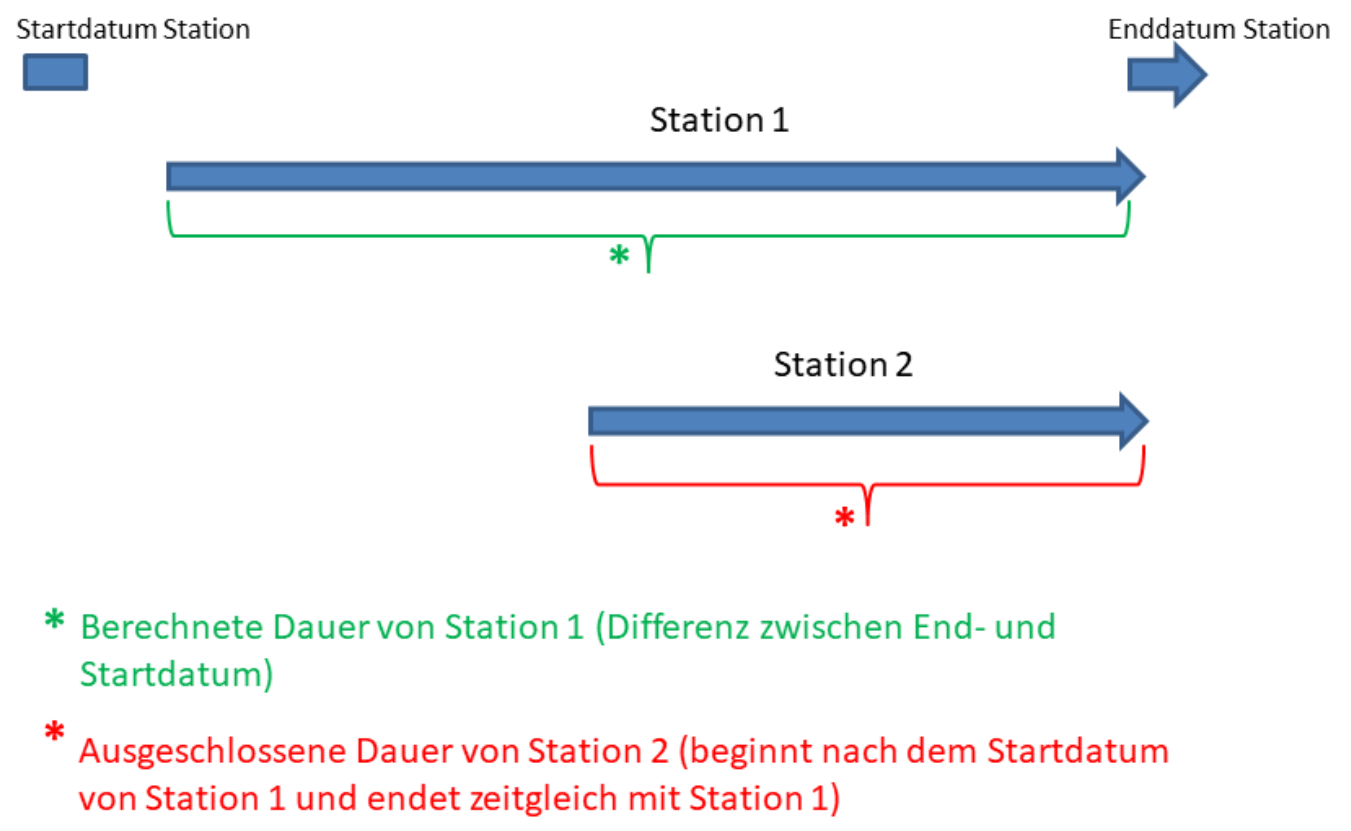

Quelle: Eigene Darstellung.

Abbildung 6: Zeitliche Überschneidung zweier identischer Stationen mit identischem Startdatum.

Startdatum Station Enddatum Station

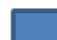

Station 1

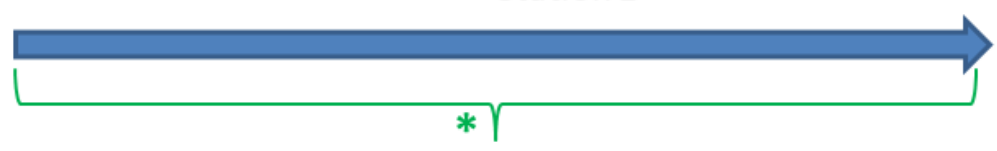

Station 2

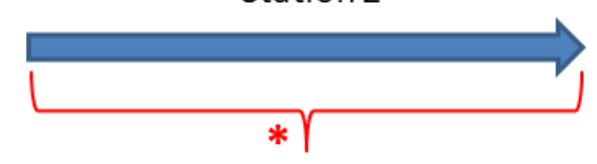

* Berechnete Dauer von Station 1 (Differenz zwischen End- und Startdatum)

* Ausgeschlossene Dauer von Station 2 (beginnt zeitgleich mit Station 1 und endet vor Station 1)

Quelle: Eigene Darstellung. 
Abbildung 7: Jeweils zeitliche Überschneidung des Start- und Enddatums zweier identischer Stationen.

Startdatum Station

Enddatum Station

$\square$

Station 1

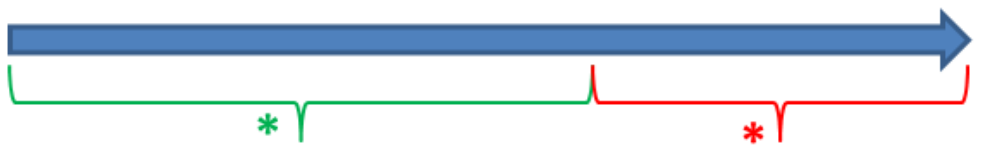

Station 2

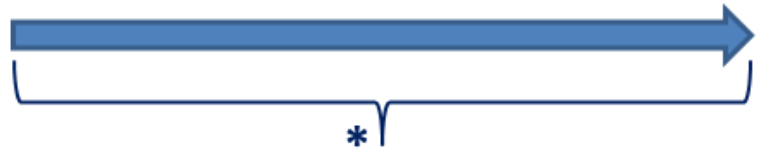

* Berechnete Dauer von Station 1 (Differenz zwischen Startdatum Station 2 und Startdatum Station 1)

* Ausgeschlossene Dauer von Station 1 (zeitgleich mit Startdatum Station 2 und Enddatum während Station 2)

* Berechnete Dauer von Station 2 (Differenz zwischen Enddatum Station 2 und Startdatum Station 2)

Quelle: Eigene Darstellung.

\section{Abbildung 8: Stationsdauer innerhalb Dauer einer identischen Station.}

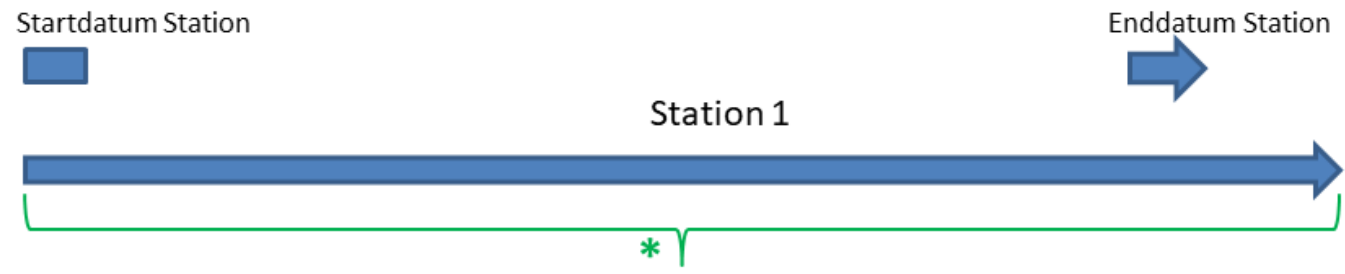

Station 2

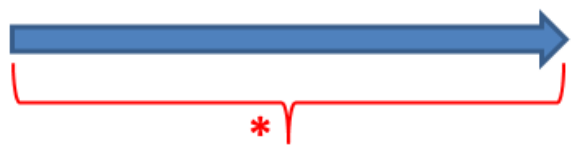

* Berechnete Dauer von Station 1 (Differenz zwischen End- und Startdatum)

* Ausgeschlossene Dauer von Station 2 (Start- und Enddatum befinden sich innerhalb der Dauer von Station 1)

Quelle: Eigene Darstellung. 
Abbildung 9: Zeitliche Überschneidung Startdatum zweier verschiedener Stationen mit identischem Enddatum.

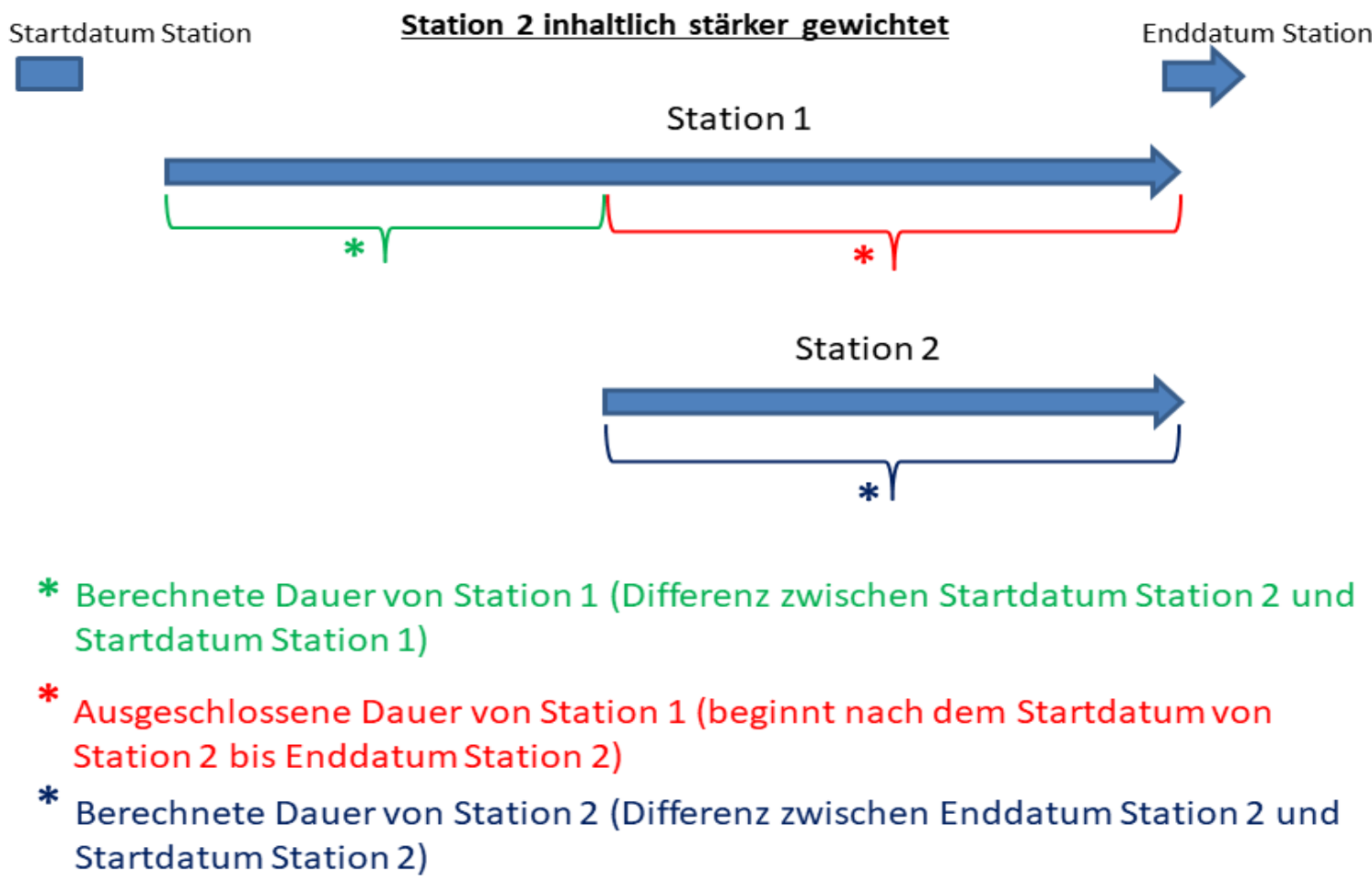

Quelle: Eigene Darstellung.

Abbildung 10: Zeitliche Überschneidung Enddatum zweier verschiedener Stationen mit identischem Startdatum und Ausschluss der gesamten Stationsdauer.

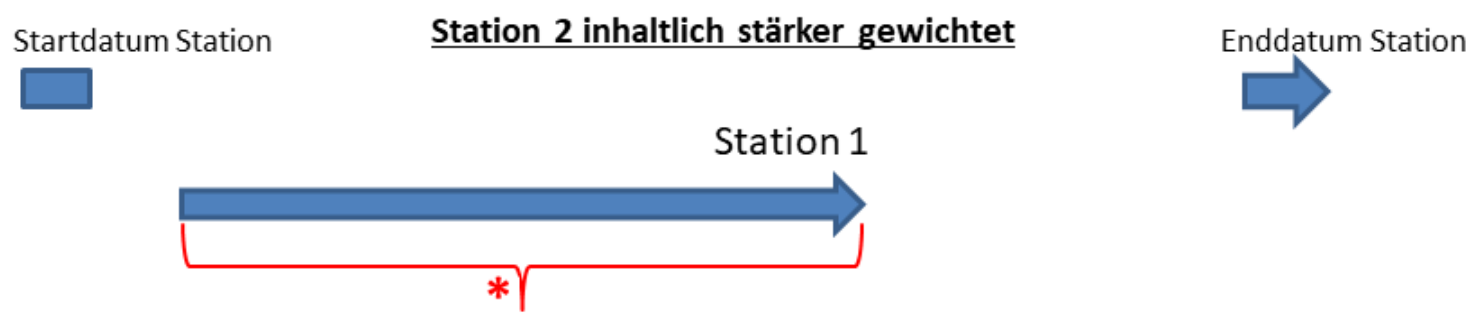

Station 2

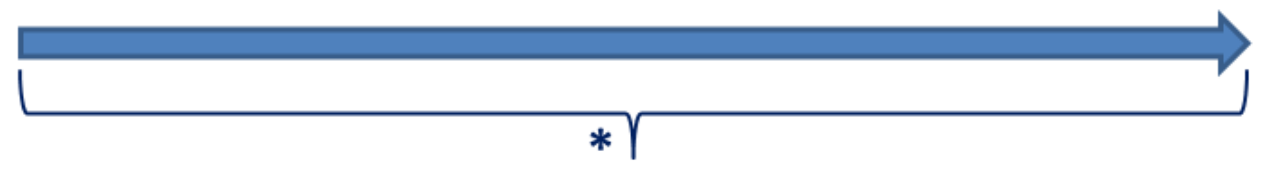

* Ausgeschlossene Dauer von Station 1 (beginnt zeitgleich mit Station 2 und endet vor Station 2)

* Berechnete Dauer von Station 2 (Differenz zwischen Enddatum Station 2 und Startdatum Station 2)

Quelle: Eigene Darstellung. 
Abbildung 11: Zeitliche Überschneidung Enddatum zweier verschiedener Stationen mit identischem Startdatum.
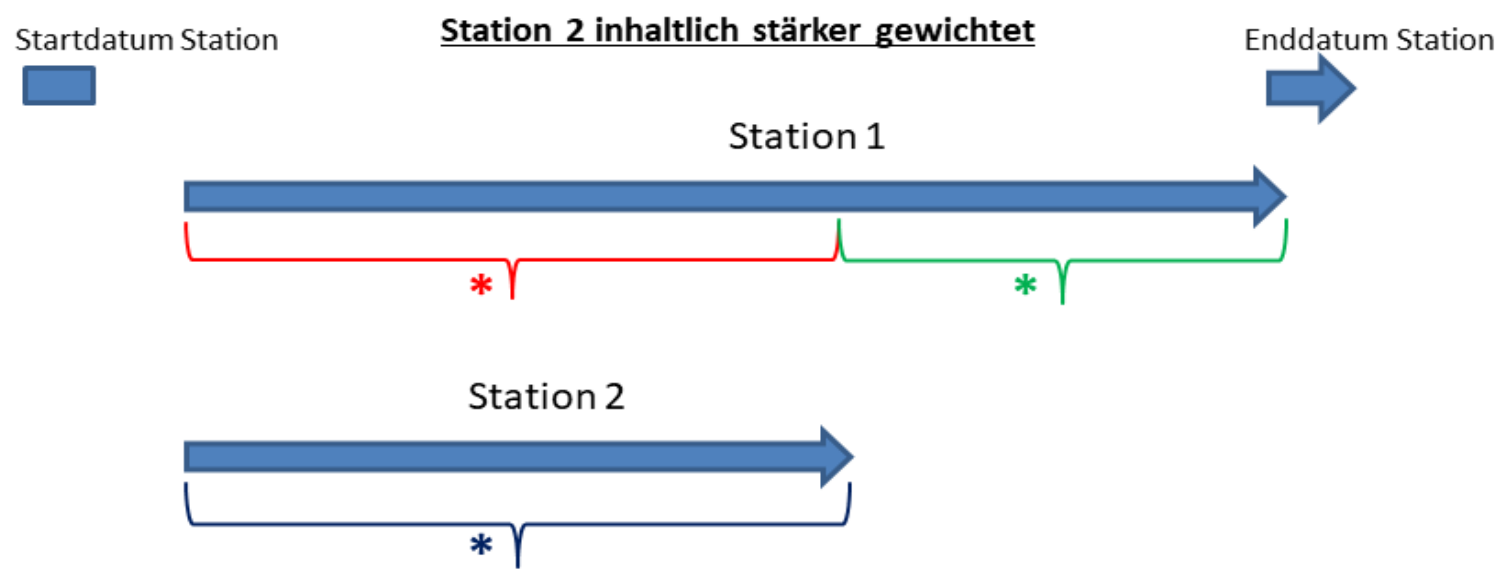

* Berechnete Dauer von Station 1 (Differenz zwischen Enddatum Station 1 und Enddatum Station 2)

* Ausgeschlossene Dauer von Station 1 (rot markierte Dauer liegt während der Dauer von Station 2)

* Berechnete Dauer von Station 2 (Differenz zwischen Enddatum Station 2 und Startdatum Station 2)

Quelle: Eigene Darstellung. 
Abbildung 12: Jeweils zeitliche Überschneidung des Start- und Enddatums zweier verschiedener Stationen.

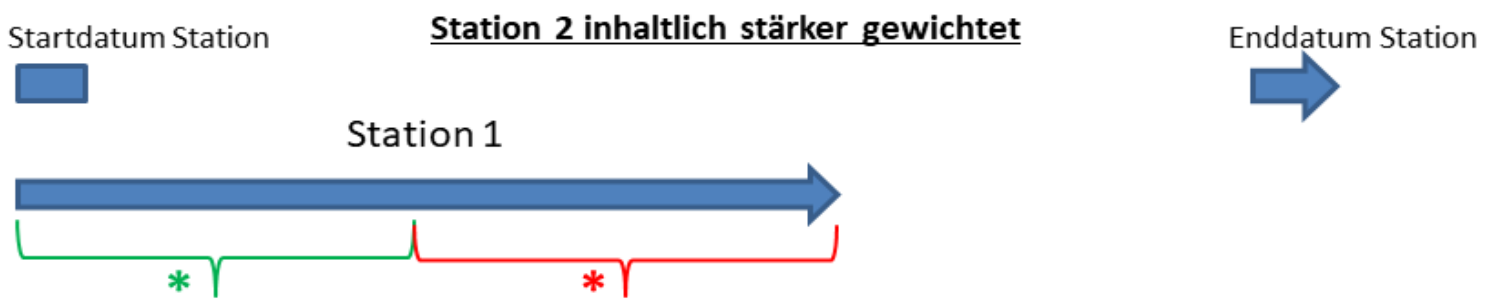

Station 2

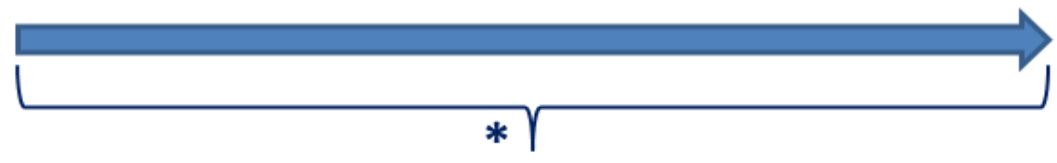

* Berechnete Dauer von Station 1 (Differenz zwischen Startdatum Station 2 und Startdatum Station 1)

* Ausgeschlossene Dauer von Station 1 (beginnt mit dem Startdatum von Station 2 bis Enddatum Station 1)

* Berechnete Dauer von Station 2 (Differenz zwischen Enddatum Station 2 und Startdatum Station 2)

Quelle: Eigene Darstellung. 
Abbildung 13: Dauer einer stärker gewichteten Station während einer weniger gewichteten Station.

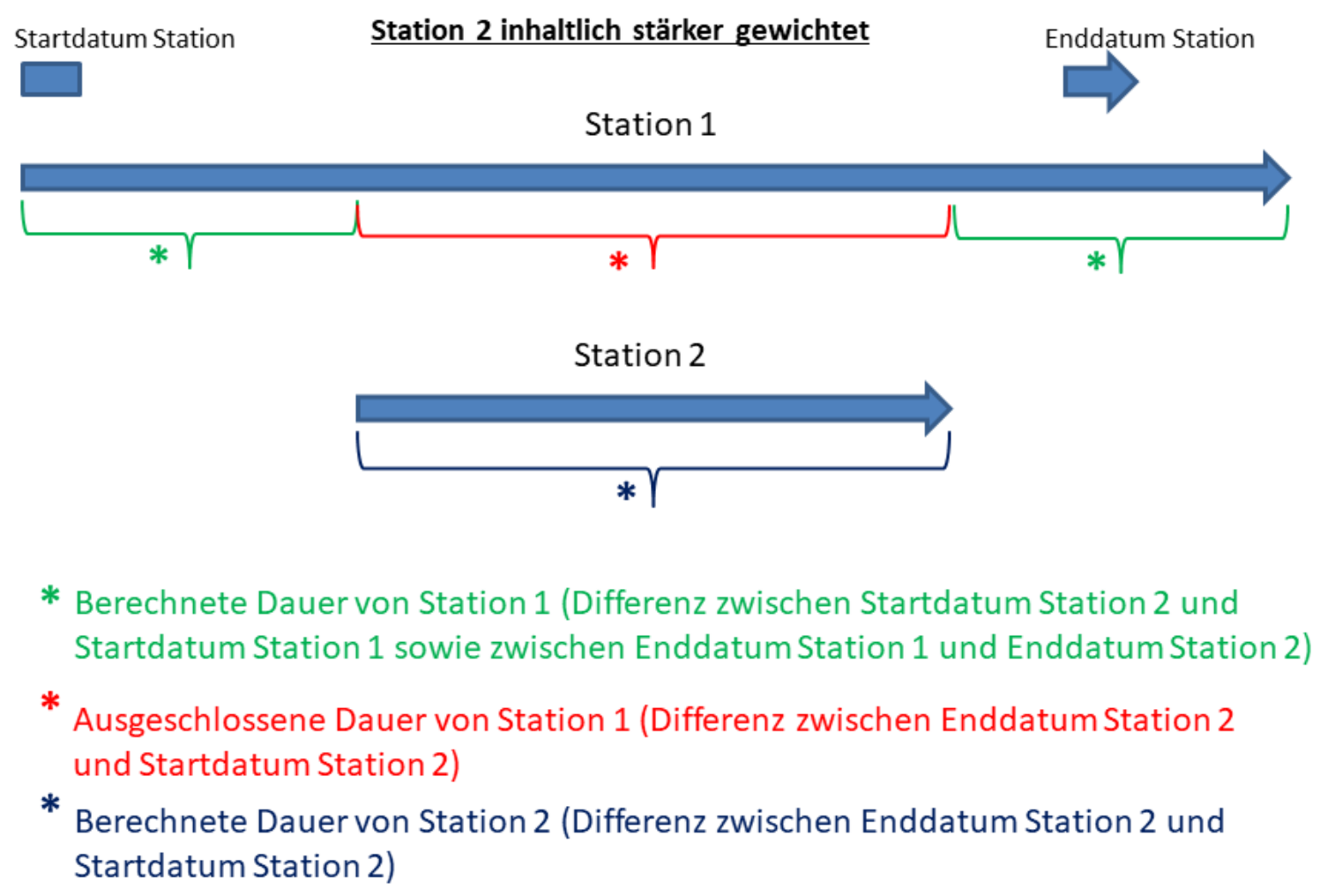

Quelle: Eigene Darstellung. 
Anhang F: Abbildungen zu deskriptiven Statistiken

Abbildung 14: Durchschnittliche Übergangsdauer im Zeitverlauf mit zusammengefassten Schulenddatumsjahren.

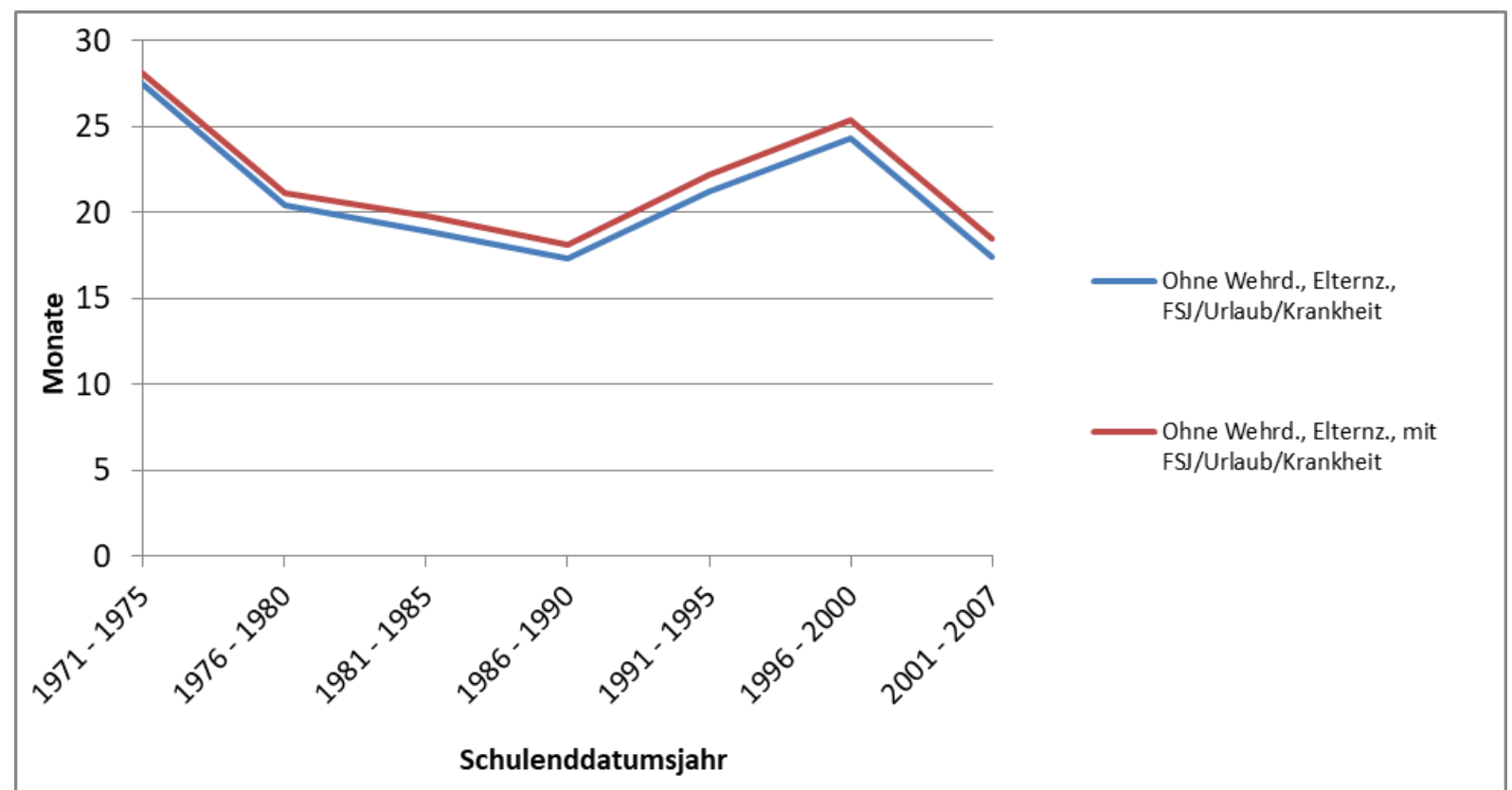

Quelle: Eigene Berechnungen basierend auf dem NEPS. Abkürzungen: Wehrd.: Wehrdienst, Elternz.: Elternzeit. Schulenddatumsjahre: Fünf-Jahres-Kategorien. $\mathrm{N}=9.254$.

Abbildung 15: Durchschnittliche Übergangsdauer ohne rechtszensierte Fälle im Zeitverlauf mit zusammengefassten Schulenddatumsjahren.

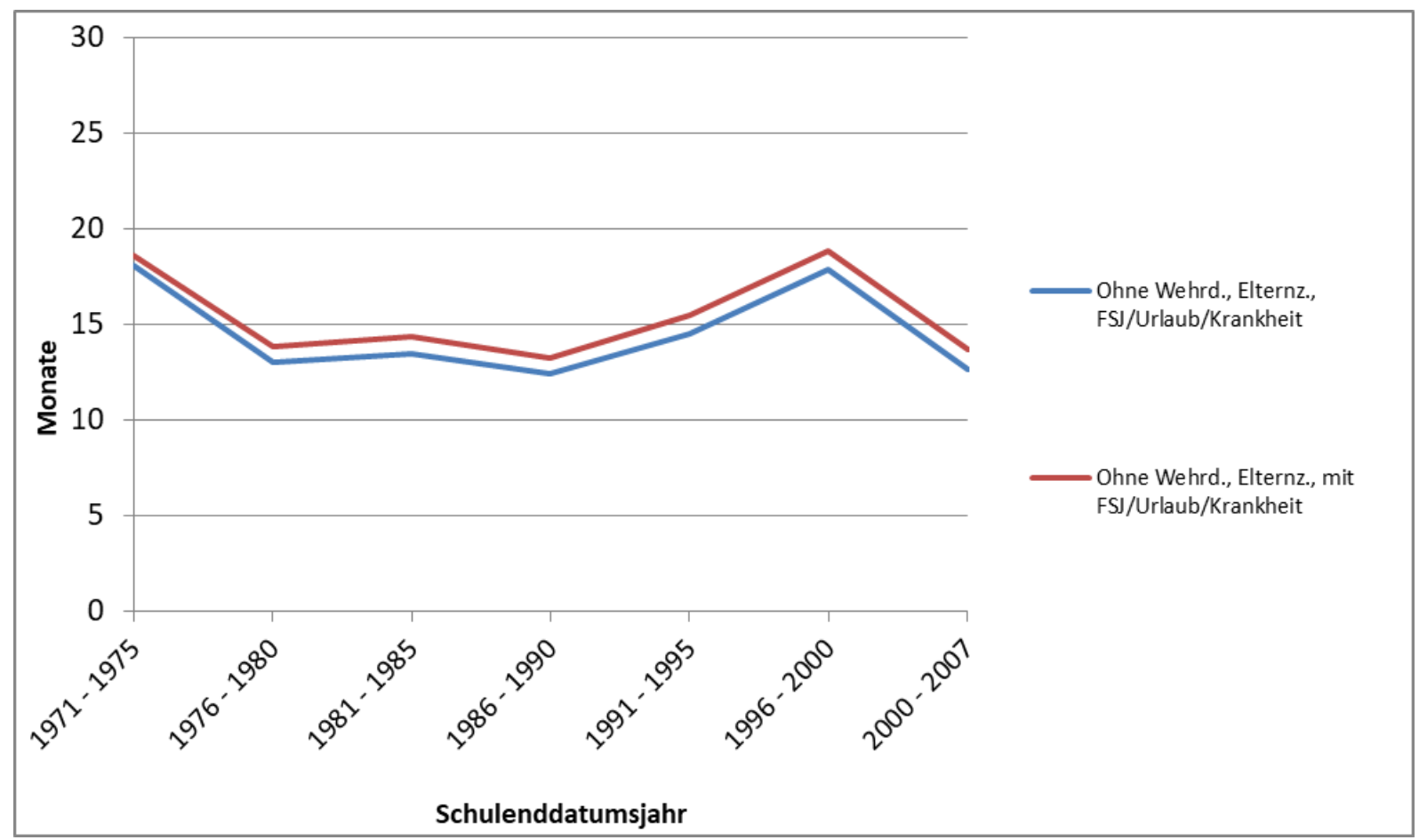

Quelle: Eigene Berechnungen basierend auf dem NEPS. Ohne rechtszensierte Fälle, die bis zum Ende des Untersuchungszeitraums keine Ausbildung begonnen haben. Schulenddatumsjahre: Fünf-Jahres-Kategorien. $\mathrm{N}=$ 8.479 . 
Abbildung 16: Durchschnittliche Übergangsdauer nach Schulabschluss im Zeitverlauf mit zusammengefassten Schulenddatumsjahren.

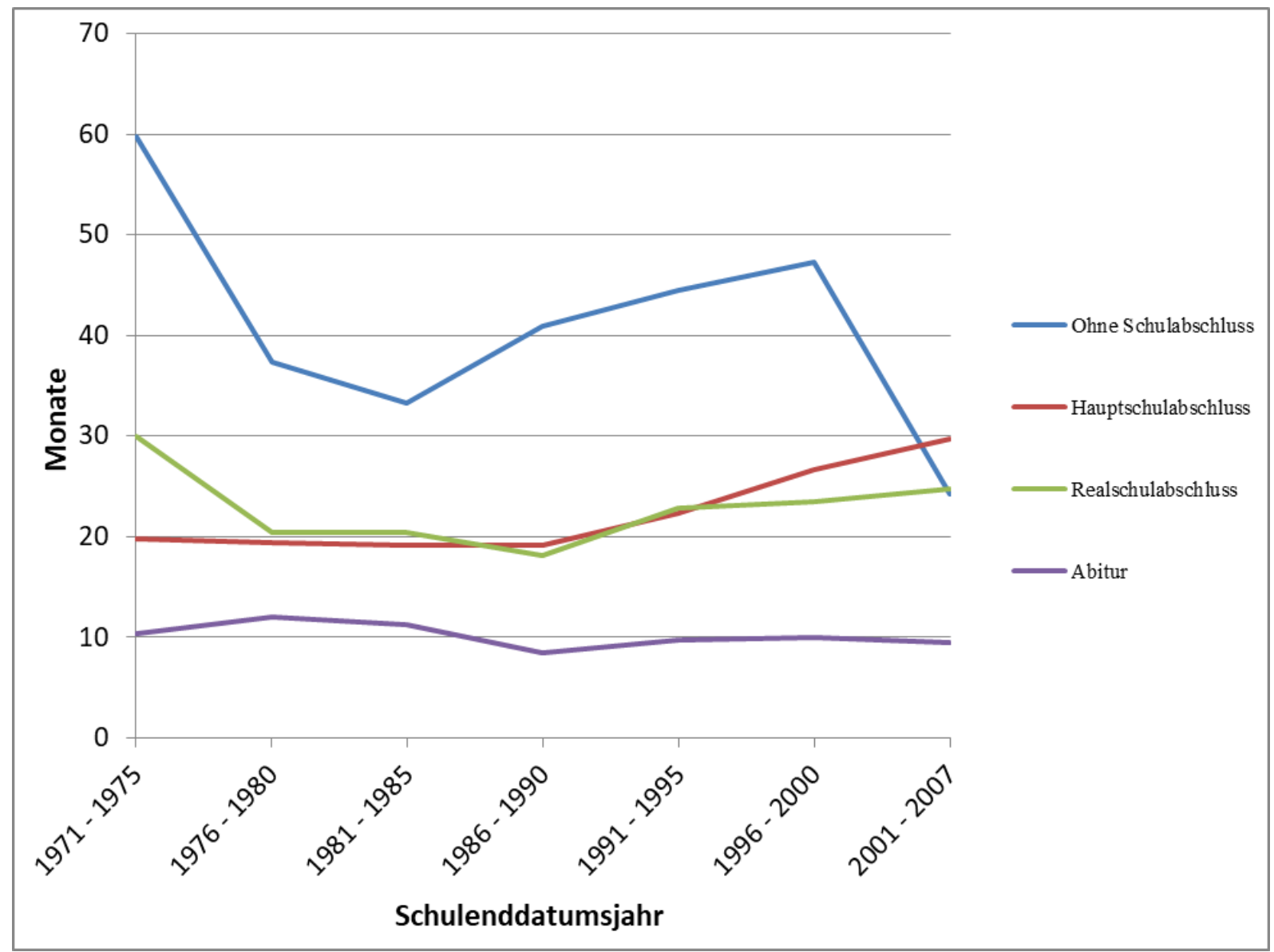

Quelle: Eigene Berechnungen basierend auf dem NEPS. Schulenddatumsjahre: Fünf-Jahres-Kategorien. $\mathrm{N}=$ 9.189 . 
Abbildung 17: Durchschnittliche Übergangsdauer (ohne Wehrdienst und Elternzeit, mit FSJ/Urlaub/Krankheit) nach Schulabschluss mit zusammengefassten Schulenddatumsjahren im Zeitverlauf.

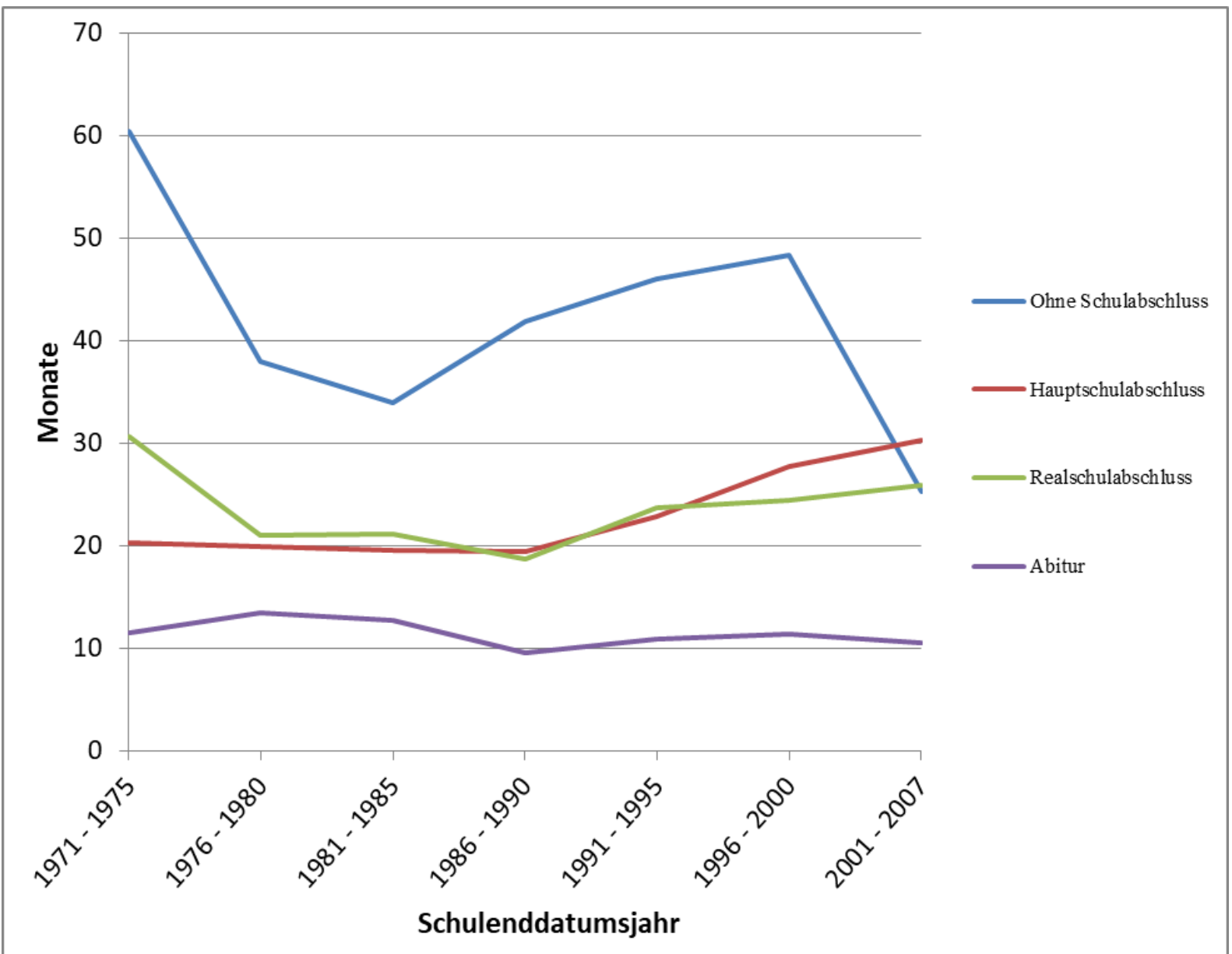

Quelle: Eigene Berechnungen basierend auf dem NEPS. Schulenddatumsjahre: Fünf-Jahres-Kategorien. $\mathrm{N}=$ 9.189. 
Abbildung 18: Durchschnittliche Anzahl Übergangsstationen im Zeitverlauf mit zusammengefassten Schulenddatumsjahren.

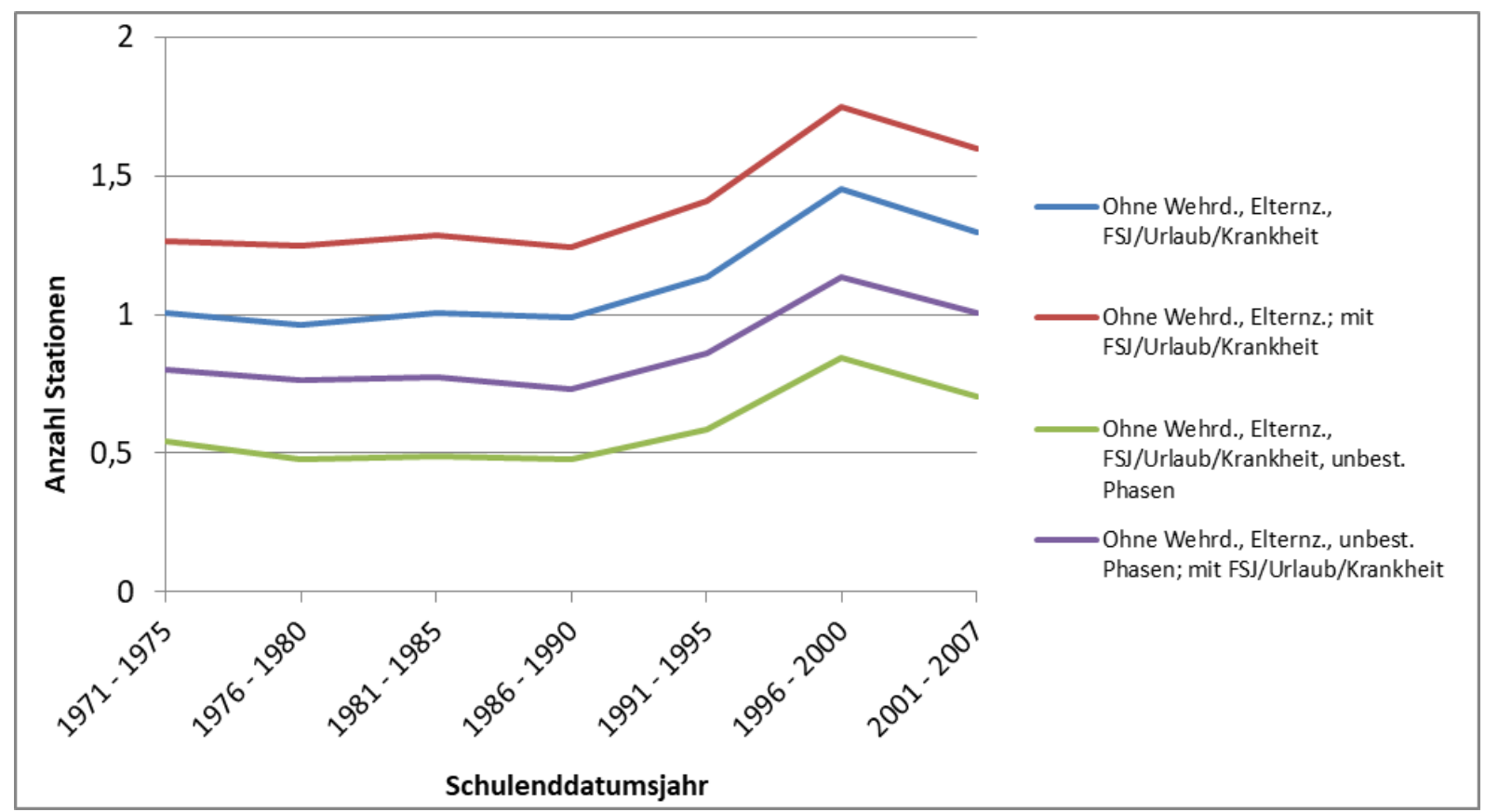

Quelle: Eigene Berechnungen basierend auf dem NEPS. Abkürzungen: Wehrd.: Wehrdienst, Elternz.: Elternzeit. Schulenddatumsjahre: Fünf-Jahres-Kategorien. N = 9.254.

Abbildung 19: Durchschnittliche Anzahl Übergangsstationen ohne rechtszensierte Fälle im Zeitverlauf mit zusammengefassten Schulenddatumsjahren.

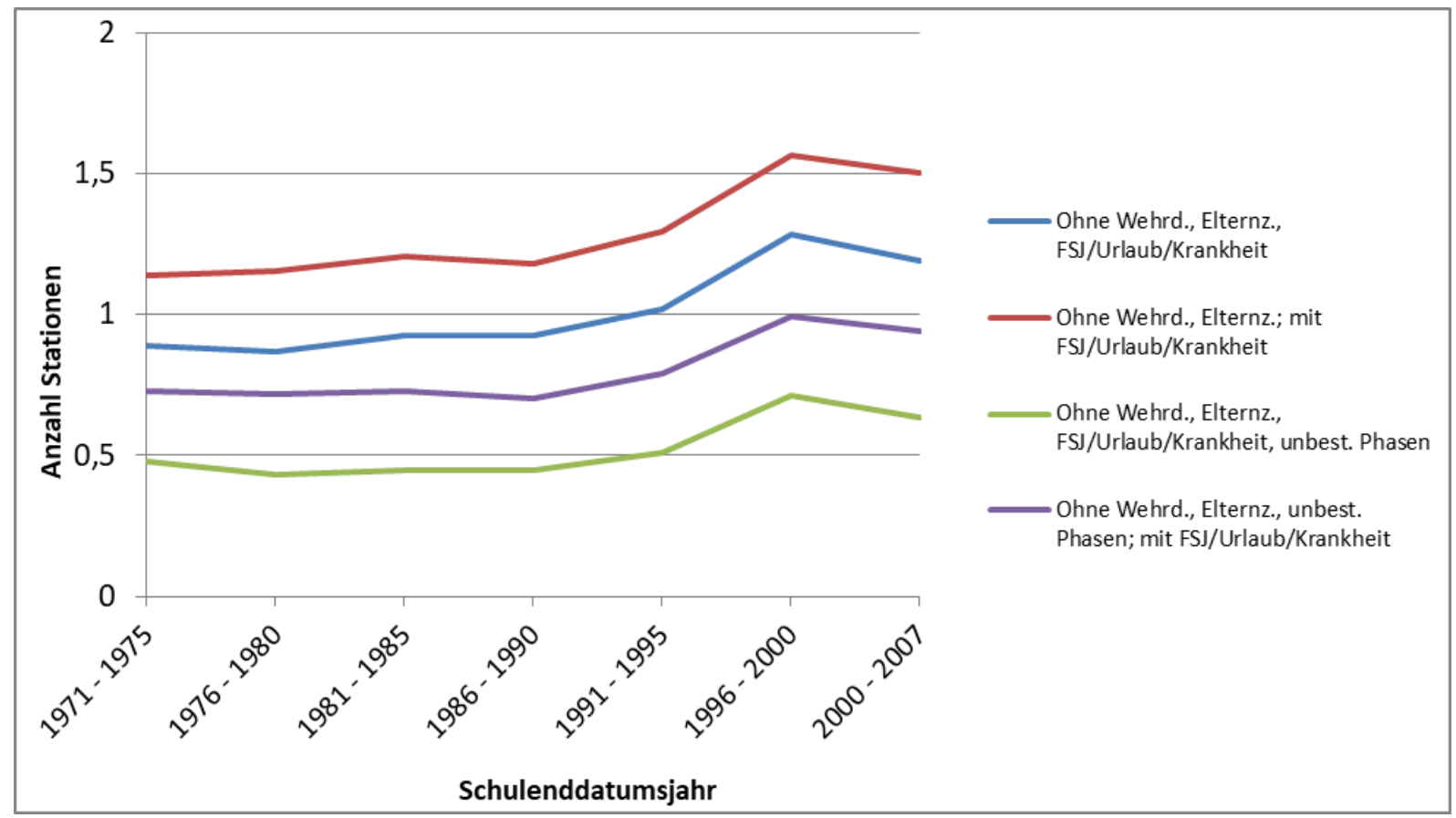

Quelle: Eigene Berechnungen basierend auf dem NEPS. Abkürzungen: Wehrd.: Wehrdienst, Elternz.: Elternzeit. Ohne rechtszensierte Fälle, die bis zum Ende des Untersuchungszeitraums keine Ausbildung begonnen haben. Schulenddatumsjahre: Fünf-Jahres-Kategorien. N = 8.479. 
Abbildung 20: Durchschnittliche Anzahl Übergangsstationen (ohne Wehrdienst, Elternzeit, FSJ/Urlaub/Krankheit) nach Schulabschluss im Zeitverlauf mit zusammengefassten Schulenddatumsjahren.

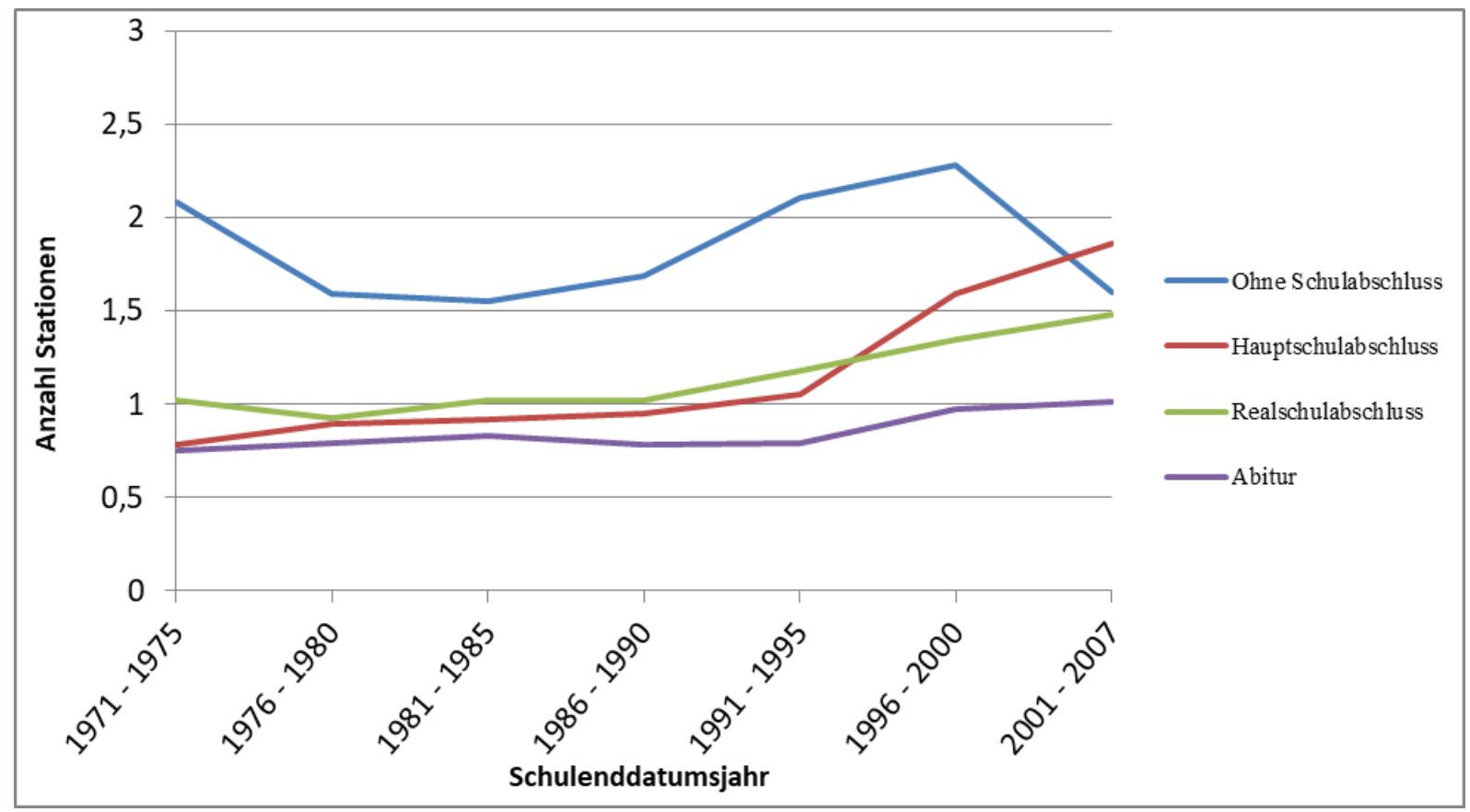

Quelle: Eigene Berechnungen basierend auf dem NEPS. Schulenddatumsjahre: Fünf-Jahres-Kategorien. $\mathrm{N}=$ 9.189 .

Abbildung 21: Durchschnittliche Anzahl Übergangsstationen (ohne Wehrdienst, Elternzeit; mit FSJ/Urlaub/Krankheit) nach Schulabschluss im Zeitverlauf mit zusammengefassten Schulenddatumsjahren.

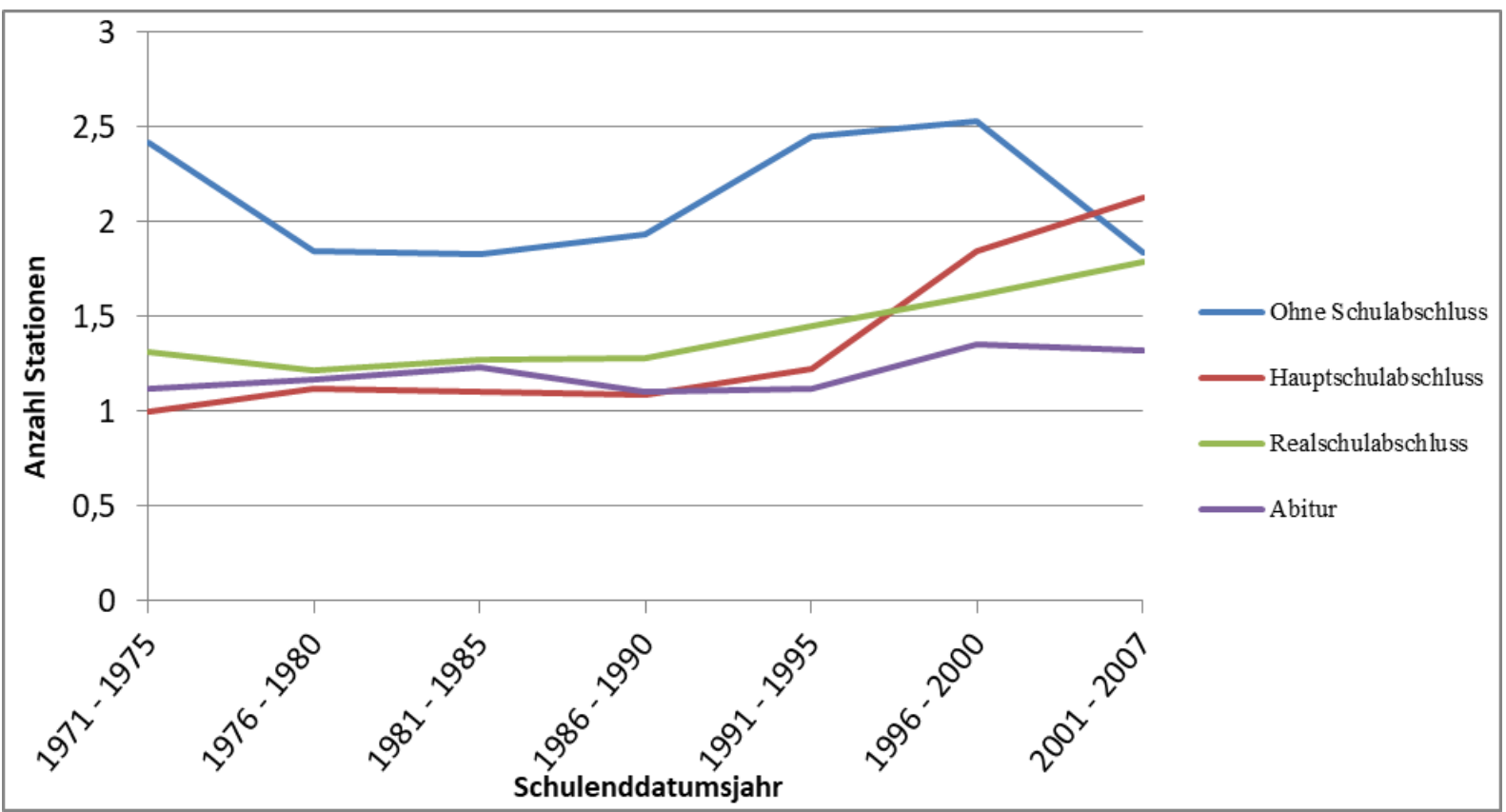

Quelle: Eigene Berechnungen basierend auf dem NEPS. Schulenddatumsjahre: Fünf-Jahres-Kategorien. N = 9.189 . 
Abbildung 22: Durchschnittliche Anzahl Übergangsstationen (ohne Wehrdienst, Elternzeit, FSJ/Urlaub/Krankheit, unbestimmte Phasen) nach Schulabschluss im Zeitverlauf mit zusammengefassten Schulenddatumsjahren.

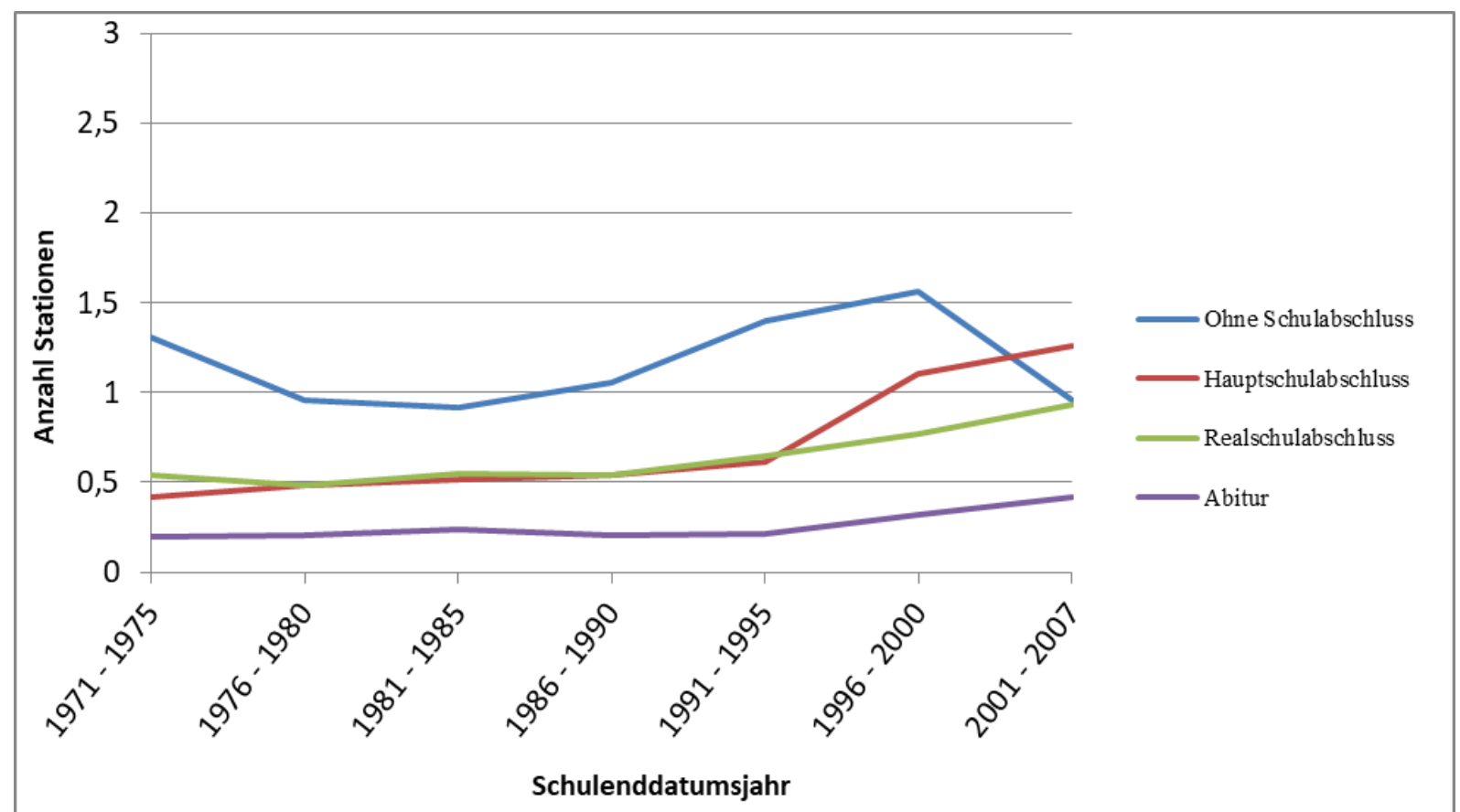

Quelle: Eigene Berechnungen basierend auf dem NEPS. Schulenddatumsjahre: Fünf-Jahres-Kategorien. N = 9.189.

Abbildung 23: Durchschnittliche Anzahl Übergangsstationen (ohne Wehrdienst, Elternzeit, unbestimmte Phasen; mit FSJ/Urlaub/Krankheit) nach Schulabschluss im Zeitverlauf mit zusammengefassten Schulenddatumsjahren.

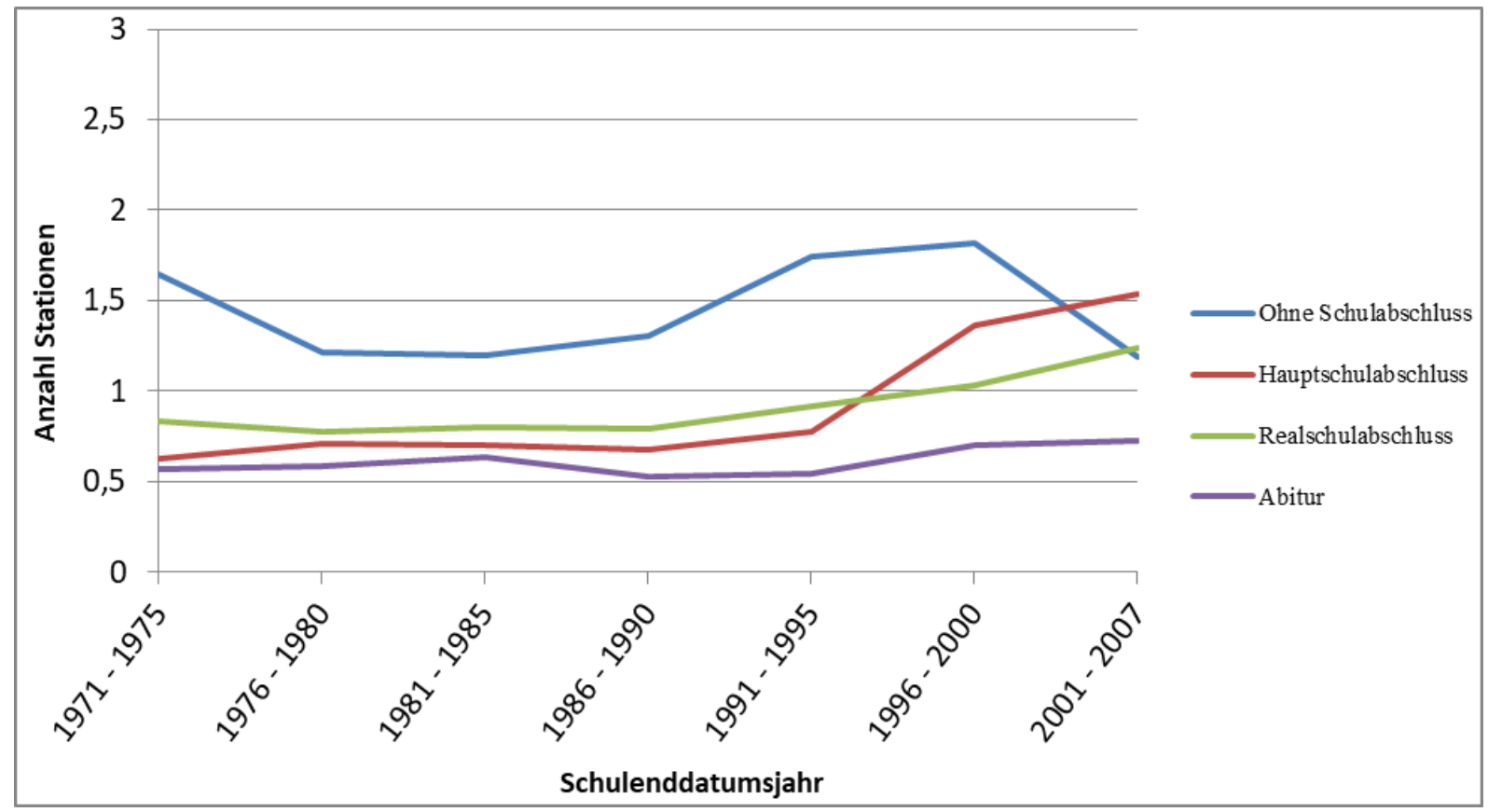

Quelle: Eigene Berechnungen basierend auf dem NEPS. Schulenddatumsjahre: Fünf-Jahres-Kategorien. N = 9.189. 


\section{Anhang G: Abbildungen zu Sterbetafeln (Überlebenswahrscheinlichkeiten)}

Abbildung 24: Kumulierte Überlebenswahrscheinlichkeit insgesamt beim Übergang (ohne Wehrdienst und Elternzeit, mit FSJ/Urlaub/Krankheit).

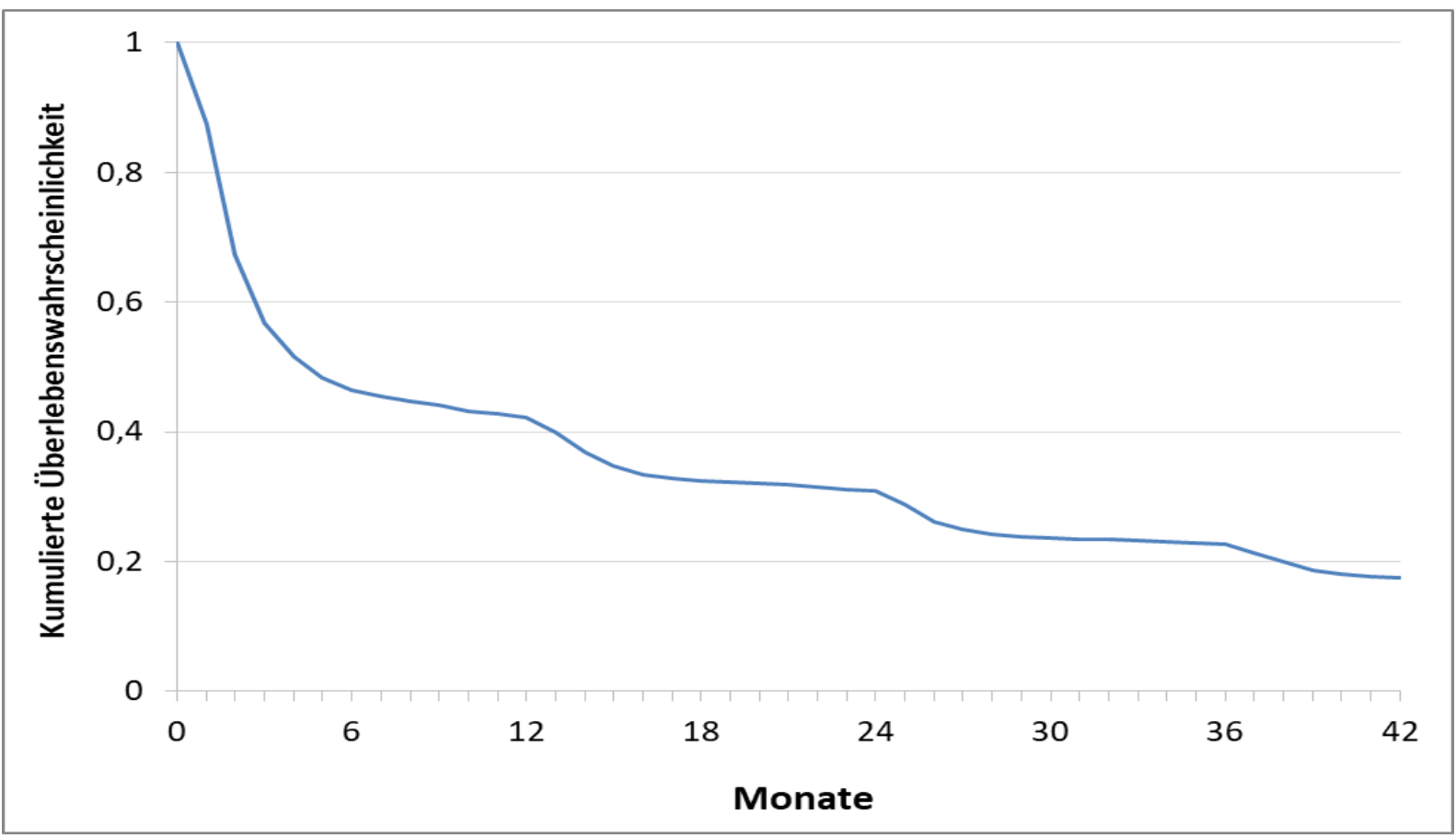

Quelle: Eigene Berechnungen basierend auf dem NEPS. Insgesamt über alle Schulabschluss- und Erstausbildungstypgruppen. Die Median der Überlebenszeit beträgt 4,5 Monate. $N=9.254$. Aus Platzgründen werden auf der X-Achse nicht alle Monate bis zum Ende des Untersuchungszeitraums angezeigt. 
Abbildung 25: Kumulierte Überlebenswahrscheinlichkeit getrennt nach Schulabschluss beim Übergang (ohne Wehrdienst und Elternzeit, mit FSJ/Urlaub/Krankheit).

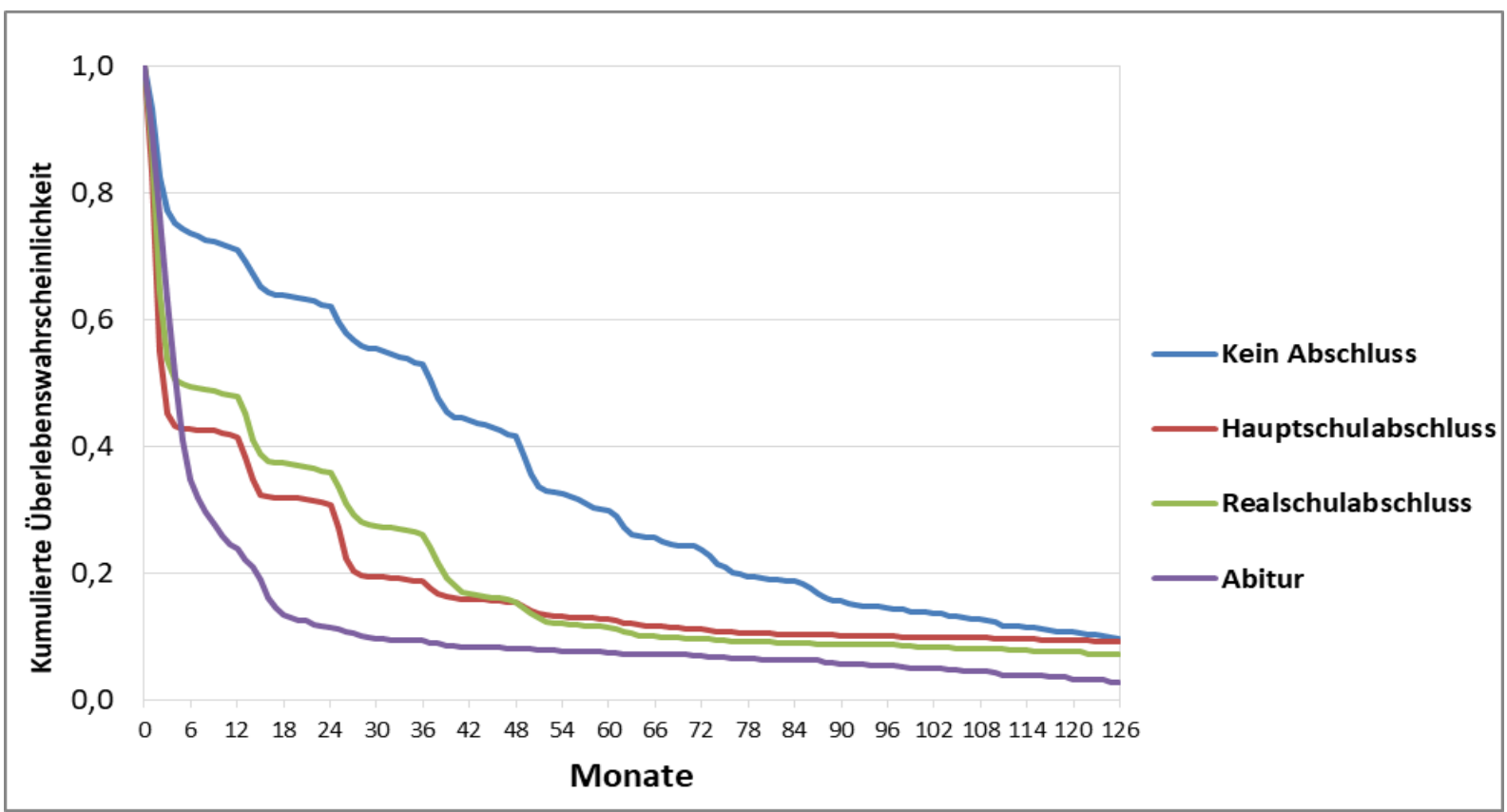

Quelle: Eigene Berechnungen basierend auf dem NEPS. Die Medianwerte der Überlebenszeit betragen für die einzelnen Schulabschlussgruppen: Kein Abschluss: 37,21 Monate, Hauptschulabschluss: 2,52 Monate, Realschulabschluss: 4,89 Monate und Abitur: 4,12 Monate. $\mathrm{N}=9.189$. Aus Platzgründen werden auf der $\mathrm{X}$ Achse nicht alle Monate bis zum Ende des Untersuchungszeitraums angezeigt. 
Abbildung 26: Kumulierte Überlebenswahrscheinlichkeit getrennt nach Erstausbildungstyp beim Übergang (ohne Wehrdienst und Elternzeit, mit FSJ/Urlaub/Krankheit).

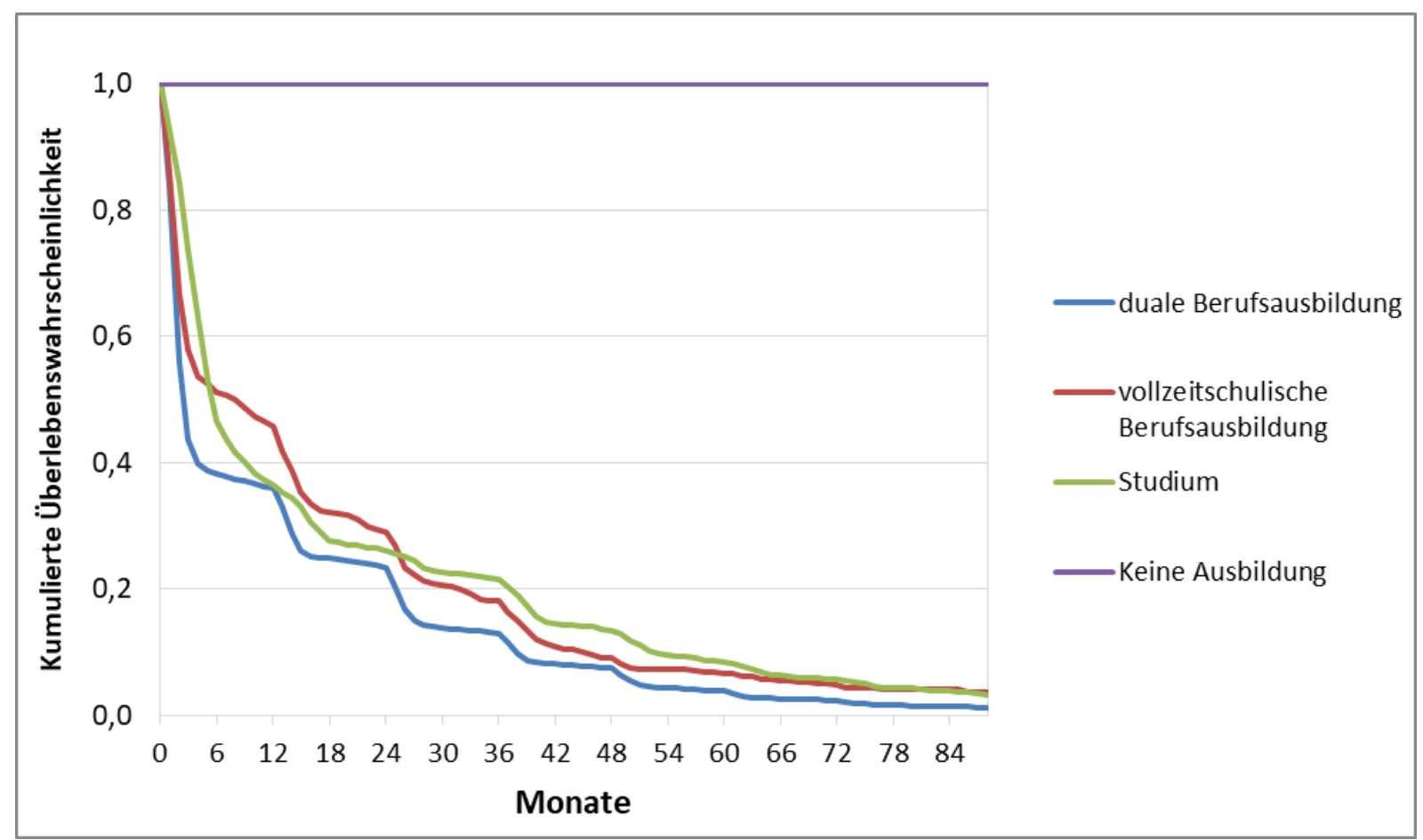

Quelle: Eigene Berechnungen basierend auf dem NEPS. Die Medianwerte der Überlebenszeit betragen für die einzelnen Erstausbildungstypgruppen: Duale Berufsausbildung: 2,48 Monate, Vollzeitschulische Berufsausbildung: 7,83 Monate, Studium: 5,48 Monate und Keine Ausbildung: 154,0 Monate. $\mathrm{N}=9.254$. Aus Platzgründen werden auf der X-Achse nicht alle Monate bis zum Ende des Untersuchungszeitraums angezeigt. 


\section{Anhang H: Abbildungen zu Cox-Regressionsmodellen}

Abbildung 27: Geschätzte Hazard-Ratio des Schulabschlusses über Anteil Dienstleistungstätige auf Übergangsdauer (mit FSJ/Urlaub/Krankheit).

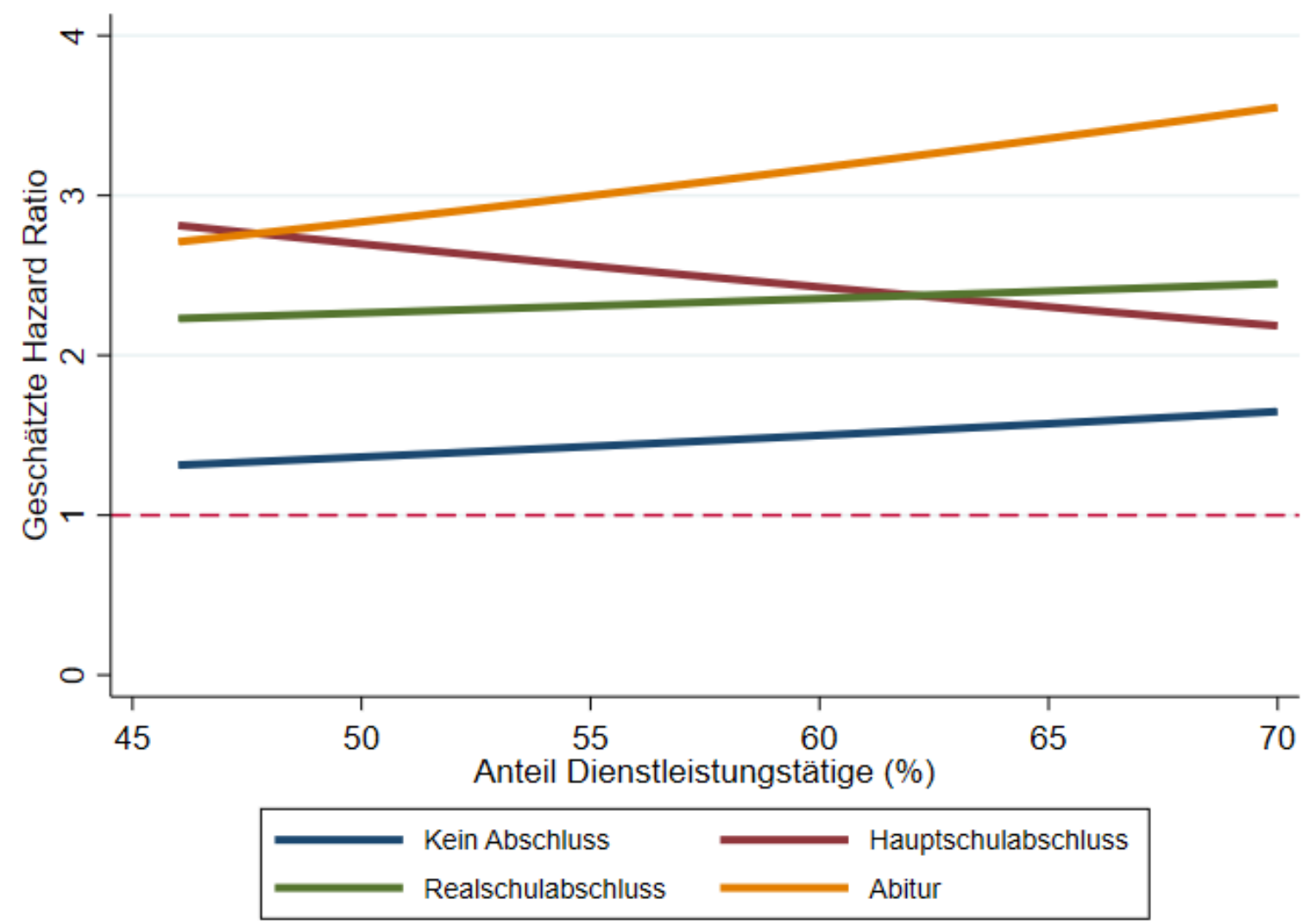

Quelle: Eigene Berechnungen basierend auf dem NEPS. Interaktionseffekt Anteil Dienstleistungstätige und Schulabschluss. $\mathrm{N}=9.040$. 


\section{Anhang I: Abbildungen zu Sequenzdatenanalysen}

Abbildung 28: Grafische Verteilung der Übergangsverläufe (mit FSJ/Urlaub, ohne unbestimmte Lückenepisoden).

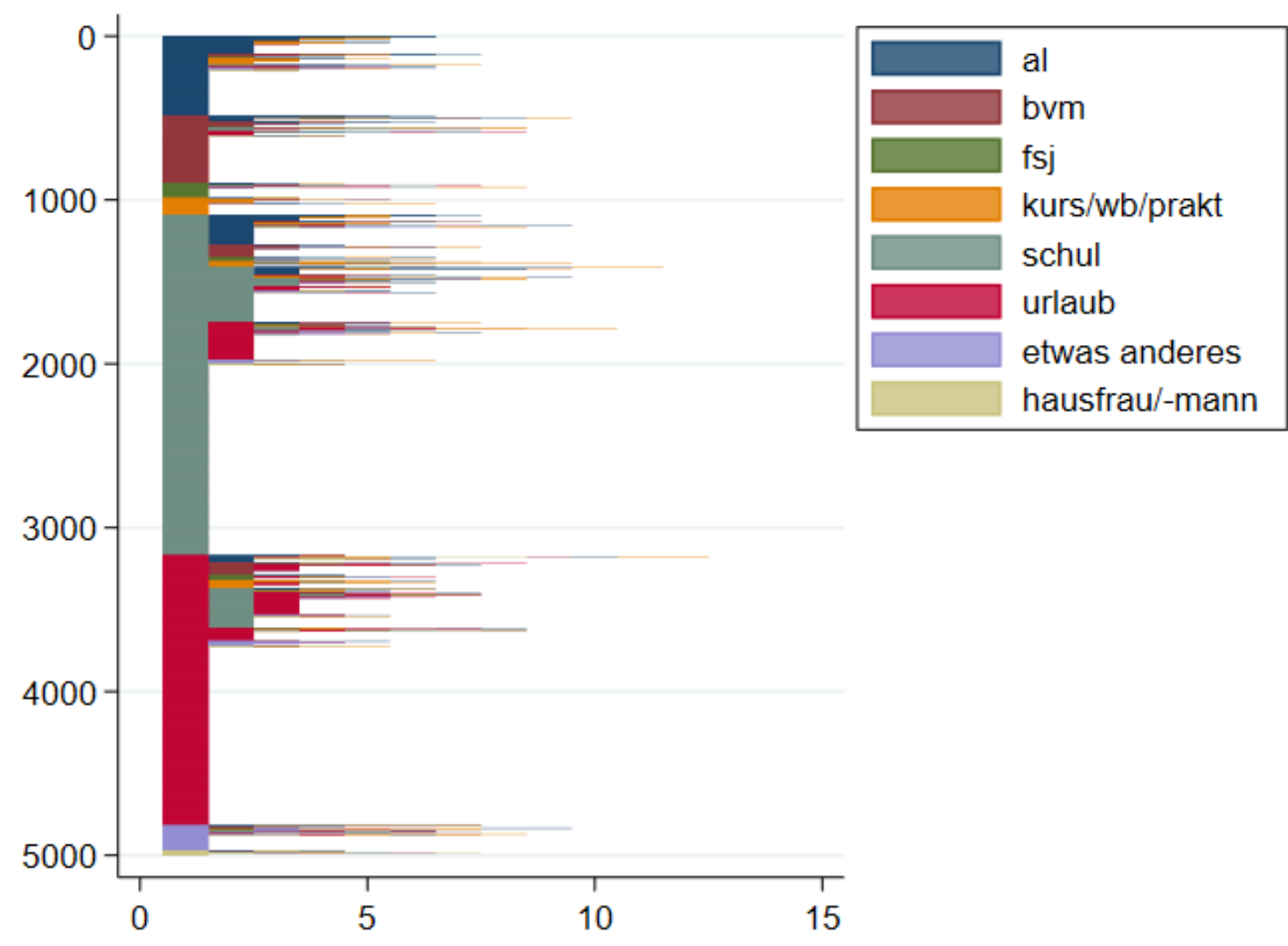

Quelle: Eigene Berechnungen basierend auf dem NEPS. Die Übergangsstationen sind in der Legende den Farben zugewiesen. Abkürzungen: al $=$ Arbeitslosigkeit, bvm $=$ Berufsvorbereitende Maßnahme, fsj $=$ Freiwilliges Soziales/Ökologisches Jahr, kurs/wb/prakt = Kurs/Weiterbildung/Praktikum, urlaub = Urlaub, hausfrau/-mann = Tätigkeit als Hausfrau/-mann, schul = Schulepisode, etwas anderes = Etwas anderes gemacht. X-Achse: Anzahl der absolvierten Stationen. Y-Achse: Häufigkeit der Übergangssequenzen, insgesamt: N = 4.996 Personen mit Übergangsverläufen. 
Abbildung 29: Grafischer Verlauf aller Übergangsverläufe (mit FSJ/Urlaub, ohne unbestimmte Lückenepisoden).

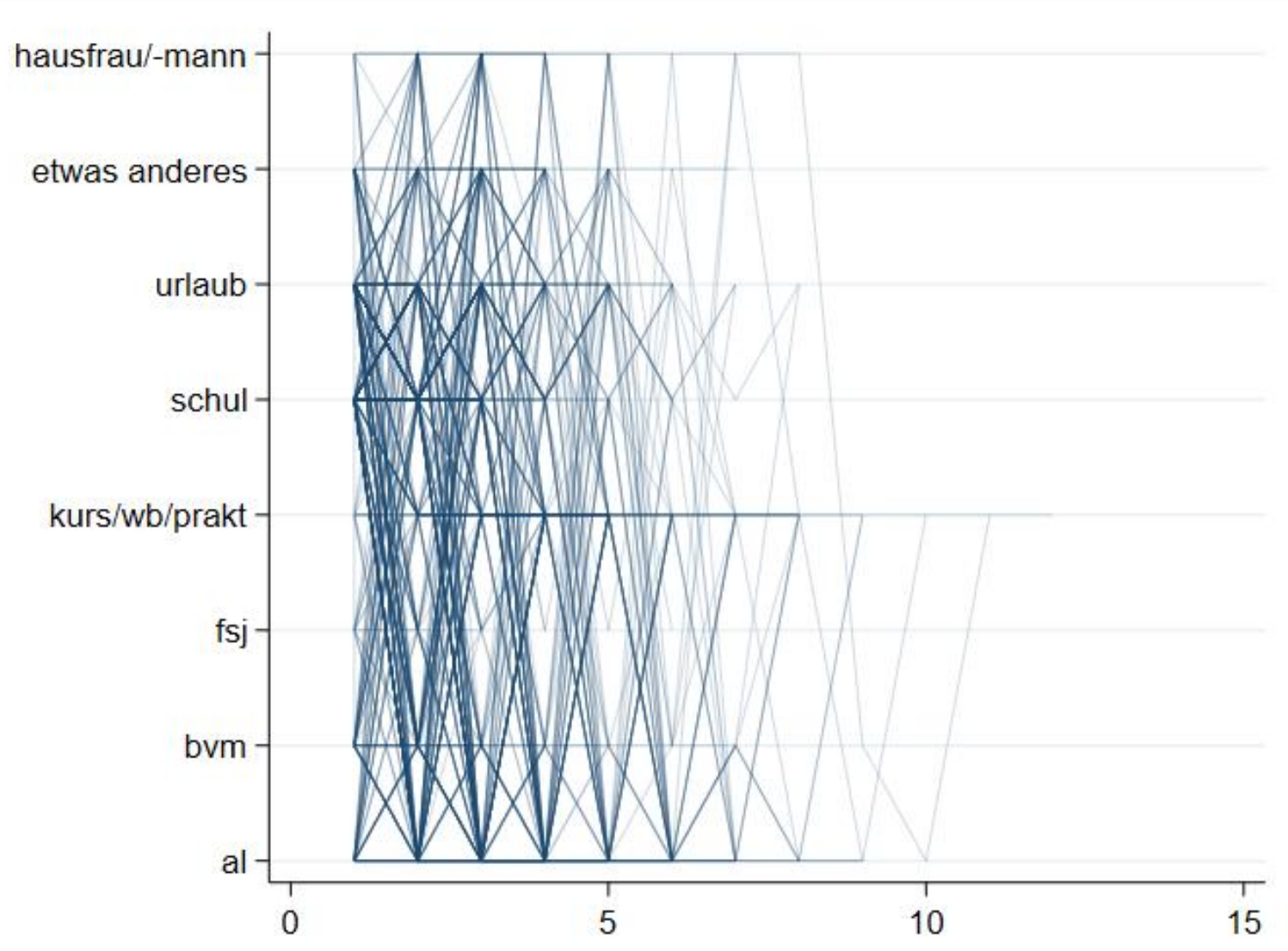

Quelle: Eigene Berechnungen basierend auf dem NEPS. Abkürzungen der Übergangsstationen: al = Arbeitslosigkeit, bvm = Berufsvorbereitende Maßnahme, fsj = Freiwilliges Soziales/Ökologisches Jahr, kurs/wb/prakt $=$ Kurs/Weiterbildung/Praktikum, urlaub $=$ Urlaub, hausfrau/-mann $=$ Tätigkeit als Hausfrau/mann, schul = Schulepisode, etwas anderes = Etwas anderes gemacht. X-Achse: Anzahl der absolvierten Übergangsstationen. $\mathrm{N}=4.996$ Personen mit Übergangsverläufen. 


\section{Abbildung 30: Grafischer Verlauf der zehn häufigsten Übergangsverläufe (mit FSJ/Urlaub, ohne unbestimmte Lückenepisoden).}

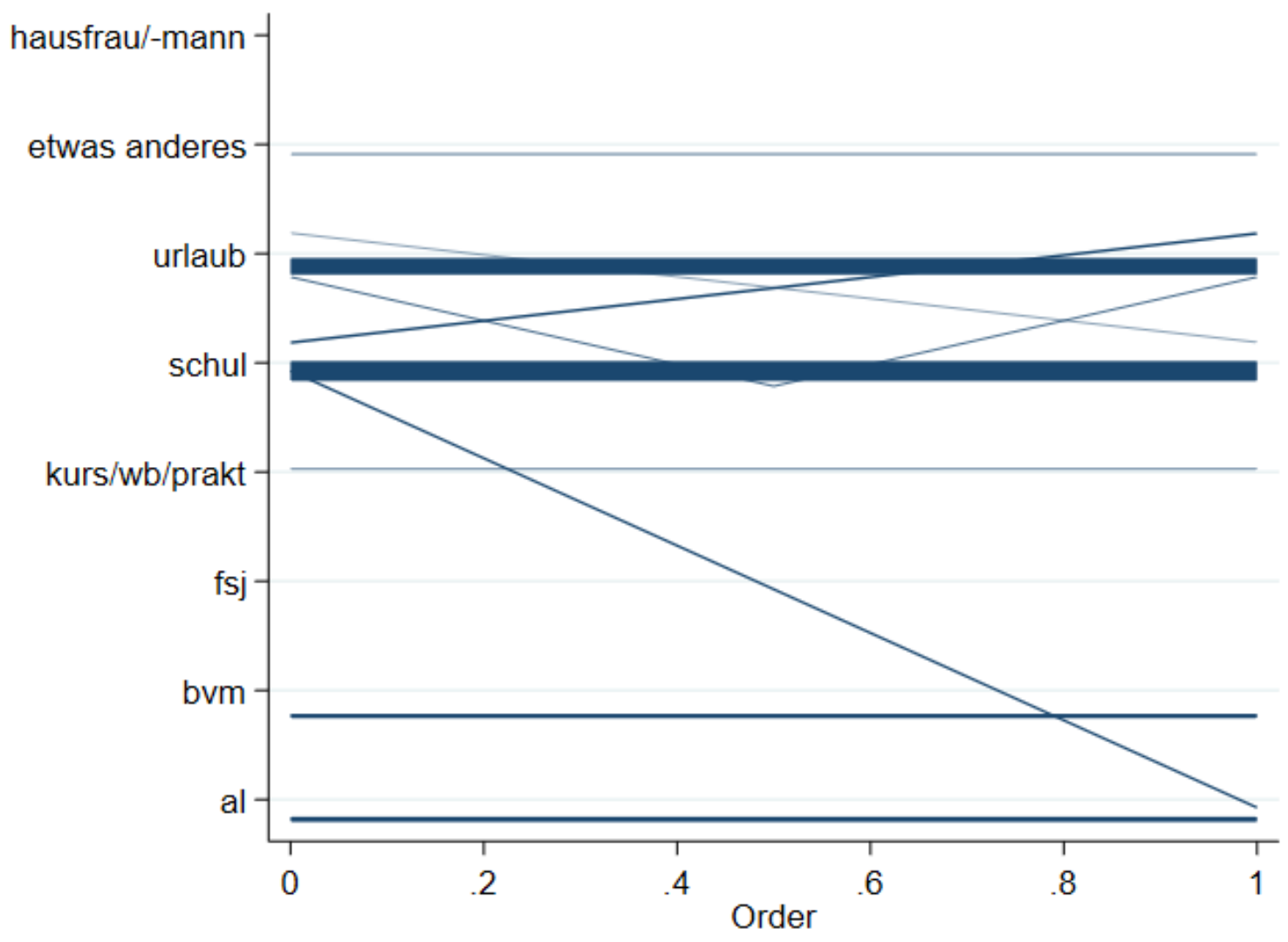

Quelle: Eigene Berechnungen basierend auf dem NEPS. Abkürzungen der Übergangsstationen: al = Arbeitslosigkeit, bvm = Berufsvorbereitende Maßnahme, fsj = Freiwilliges Soziales/Ökologisches Jahr, kurs/wb/prakt $=$ Kurs/Weiterbildung/Praktikum, urlaub $=$ Urlaub, hausfrau/-mann $=$ Tätigkeit als Hausfrau/mann, schul = Schulepisode, etwas anderes = Etwas anderes gemacht. Die zehn häufigsten Übergangsverläufe wurden von $\mathrm{N}=3.533$ Personen durchlaufen. 


\section{Abbildung 31: Grafische Verteilung der Übergangsverläufe (ohne FSJ/Urlaub, mit unbestimmten Lückenepisoden).}

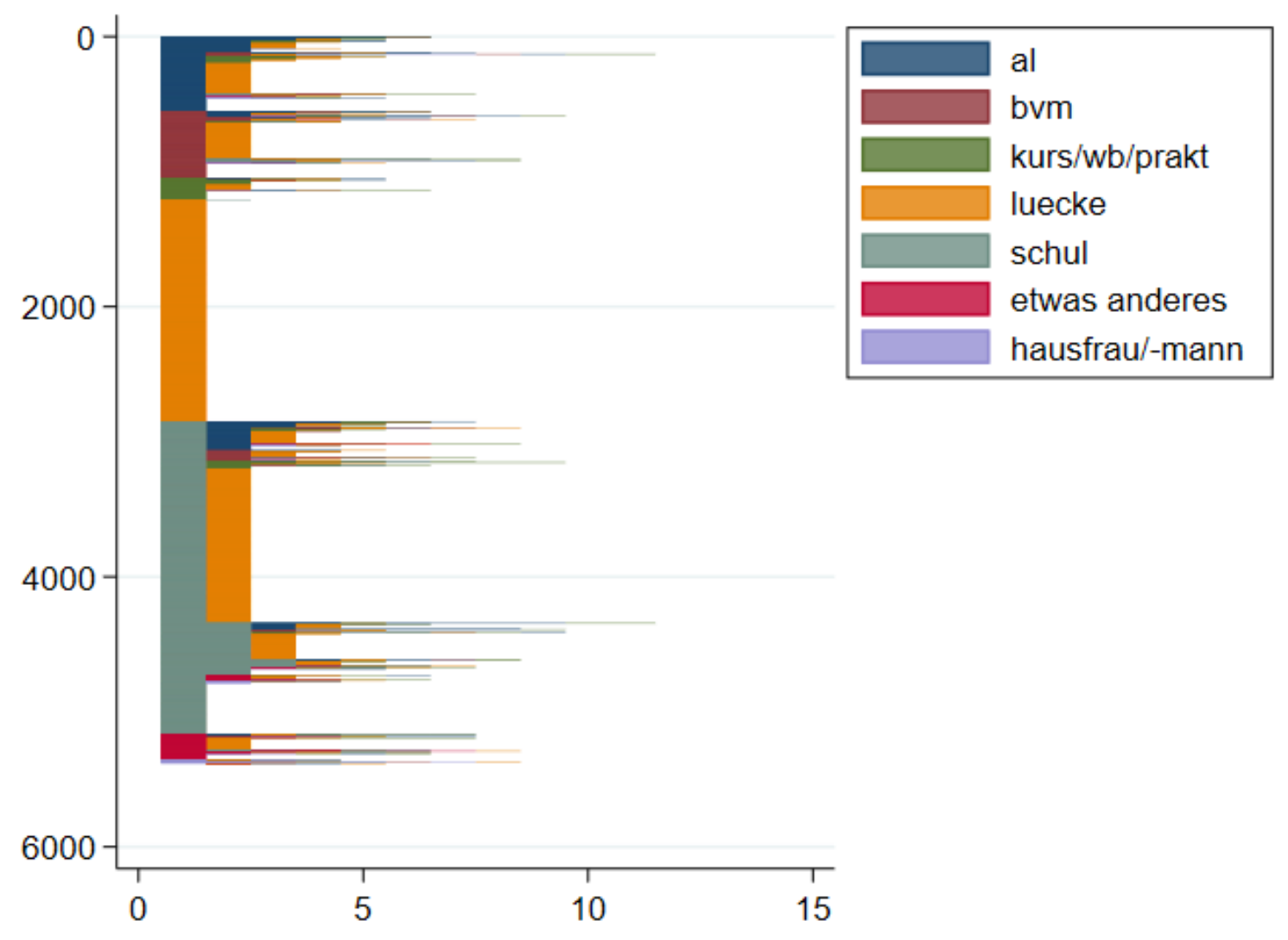

Quelle: Eigene Berechnungen basierend auf dem NEPS. Die Übergangsstationen sind in der Legende den Farben zugewiesen. Abkürzungen: al $=$ Arbeitslosigkeit, $\mathrm{bvm}=$ Berufsvorbereitende Maßnahme, $\mathrm{kurs} / \mathrm{wb} / \mathrm{prakt}=$ Kurs/Weiterbildung/Praktikum, luecke = Lücke (unbestimmte Phase), hausfrau/-mann = Tätigkeit als Hausfrau/mann, schul $=$ Schulepisode, etwas anderes $=$ Etwas anderes gemacht. X-Achse: Anzahl der absolvierten Stationen. Y-Achse: Häufigkeit der Übergangssequenzen, insgesamt: $\mathrm{N}=5.388$ Personen mit Übergangsverläufen. 


\section{Abbildung 32: Grafischer Verlauf aller Übergangsverläufe (ohne FSJ/Urlaub, mit unbestimmten Lückenepisoden).}

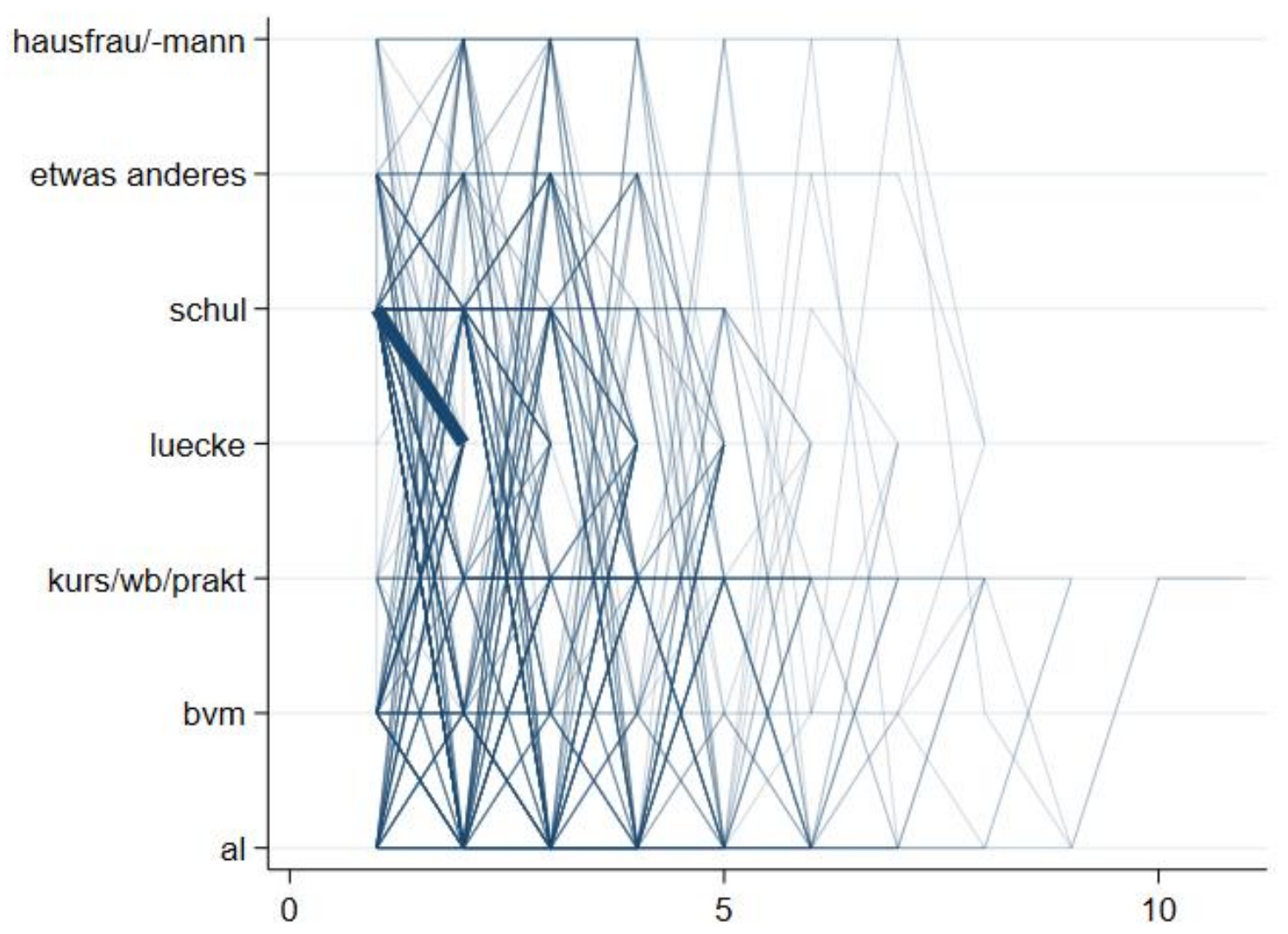

Quelle: Eigene Berechnungen basierend auf dem NEPS. Abkürzungen der Übergangsstationen: al = Arbeitslosigkeit, bvm = Berufsvorbereitende Maßnahme, kurs/wb/prakt = Kurs/Weiterbildung/Praktikum, luecke = Lücke (unbestimmte Phase), hausfrau/-mann = Tätigkeit als Hausfrau/-mann, schul = Schulepisode, etwas anderes $=$ Etwas anderes gemacht. X-Achse: Anzahl der absolvierten Übergangsstationen. $\mathrm{N}=5.388$ Personen mit Übergangsverläufen. 


\section{Abbildung 33: Grafischer Verlauf der zehn häufigsten Übergangsverläufe (ohne FSJ/Urlaub, mit unbestimmten Lückenepisoden).}

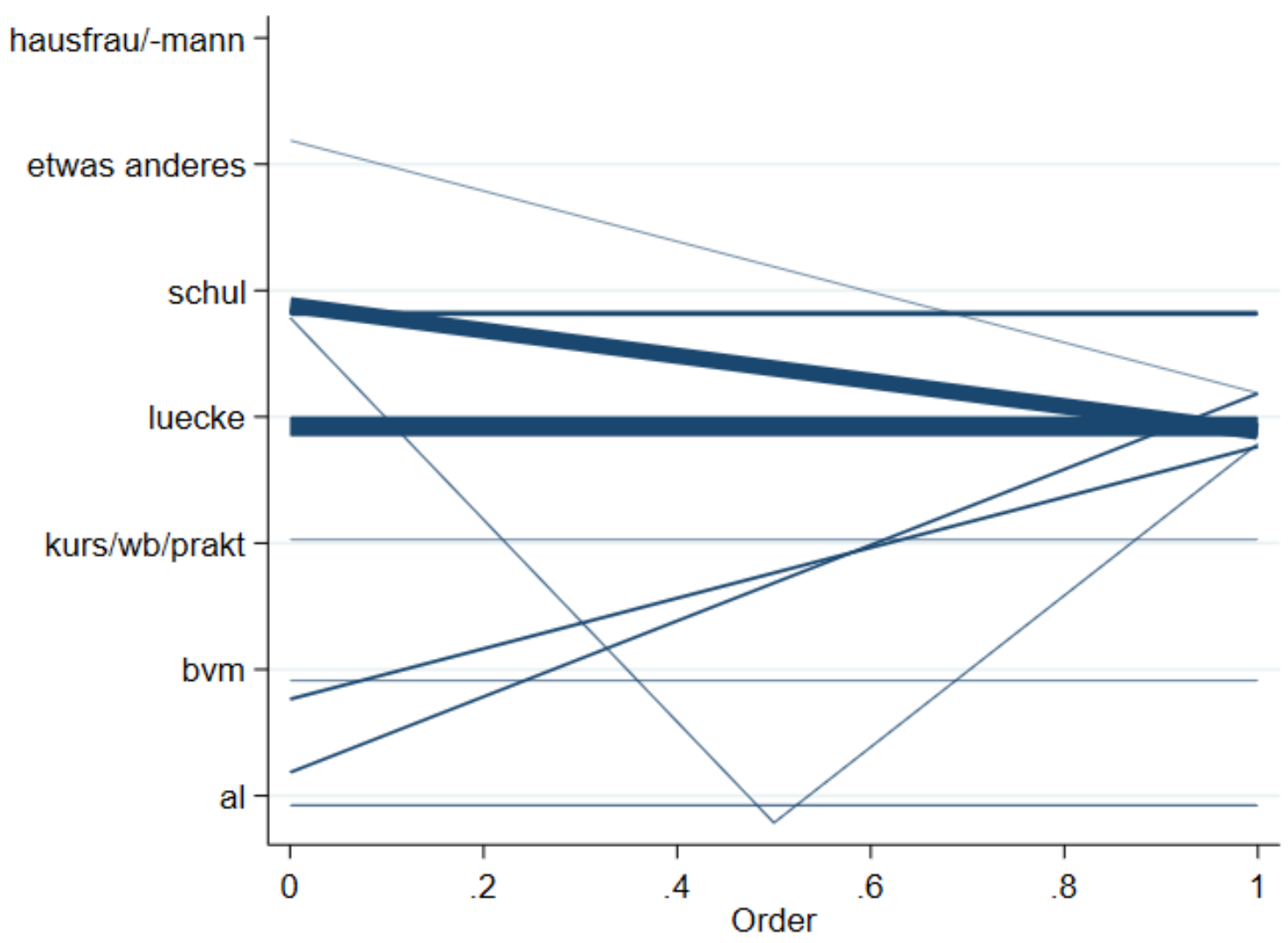

Quelle: Eigene Berechnungen basierend auf dem NEPS. Abkürzungen der Übergangsstationen: al = Arbeitslosigkeit, bvm = Berufsvorbereitende Maßnahme, kurs/wb/prakt = Kurs/Weiterbildung/Praktikum, luecke = Lücke (unbestimmte Phase), hausfrau/-mann = Tätigkeit als Hausfrau/-mann, schul = Schulepisode, etwas anderes = Etwas anderes gemacht. Die zehn häufigsten Übergangsverläufe wurden von $\mathrm{N}=4.229$ Personen durchlaufen. 


\section{Abbildung 34: Grafische Verteilung der Übergangsverläufe (mit FSJ/Urlaub, mit unbestimmten Lückenepisoden).}

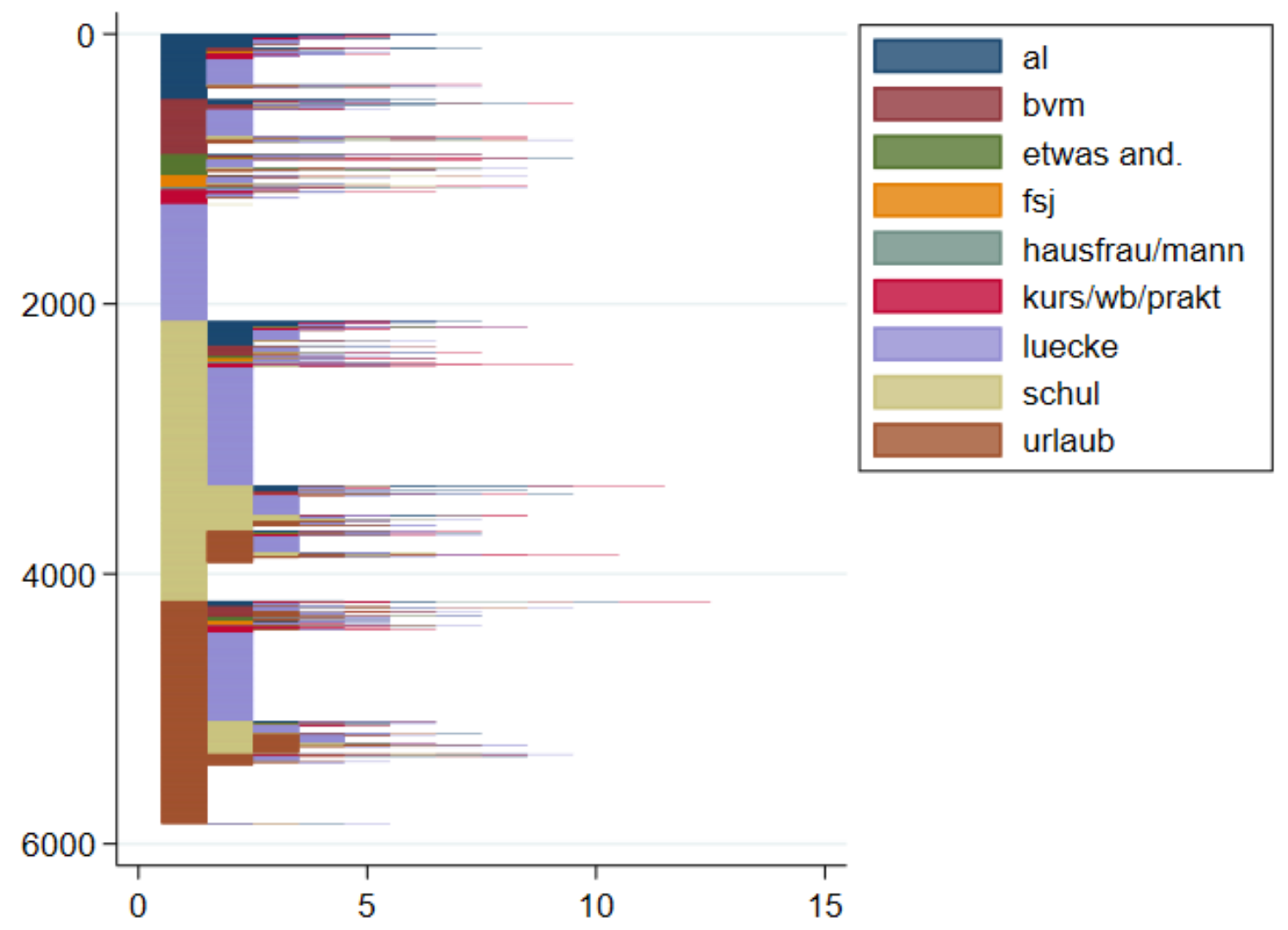

Quelle: Eigene Berechnungen basierend auf dem NEPS. Die Übergangsstationen sind in der Legende den Farben zugewiesen. Abkürzungen: $\mathrm{al}=$ Arbeitslosigkeit, $\mathrm{bvm}=$ Berufsvorbereitende Maßnahme, $\mathrm{kurs} / \mathrm{wb} / \mathrm{prakt}=$ Kurs/Weiterbildung/Praktikum, hausfrau/-mann = Tätigkeit als Hausfrau/-mann, schul = Schulepisode, etwas anderes $=$ Etwas anderes gemacht, fsj $=$ Freiwilliges Soziales/Ökologisches Jahr, luecke $=$ Lücke (unbestimmte Phase), urlaub $=$ Urlaub. X-Achse: Anzahl der absolvierten Stationen. Y-Achse: Häufigkeit der Übergangssequenzen, insgesamt: $\mathrm{N}=5.854$ Personen mit Übergangsverläufen. 
Abbildung 35: Grafischer Verlauf aller Übergangsverläufe (mit FSJ/Urlaub, mit unbestimmten Lückenepisoden).

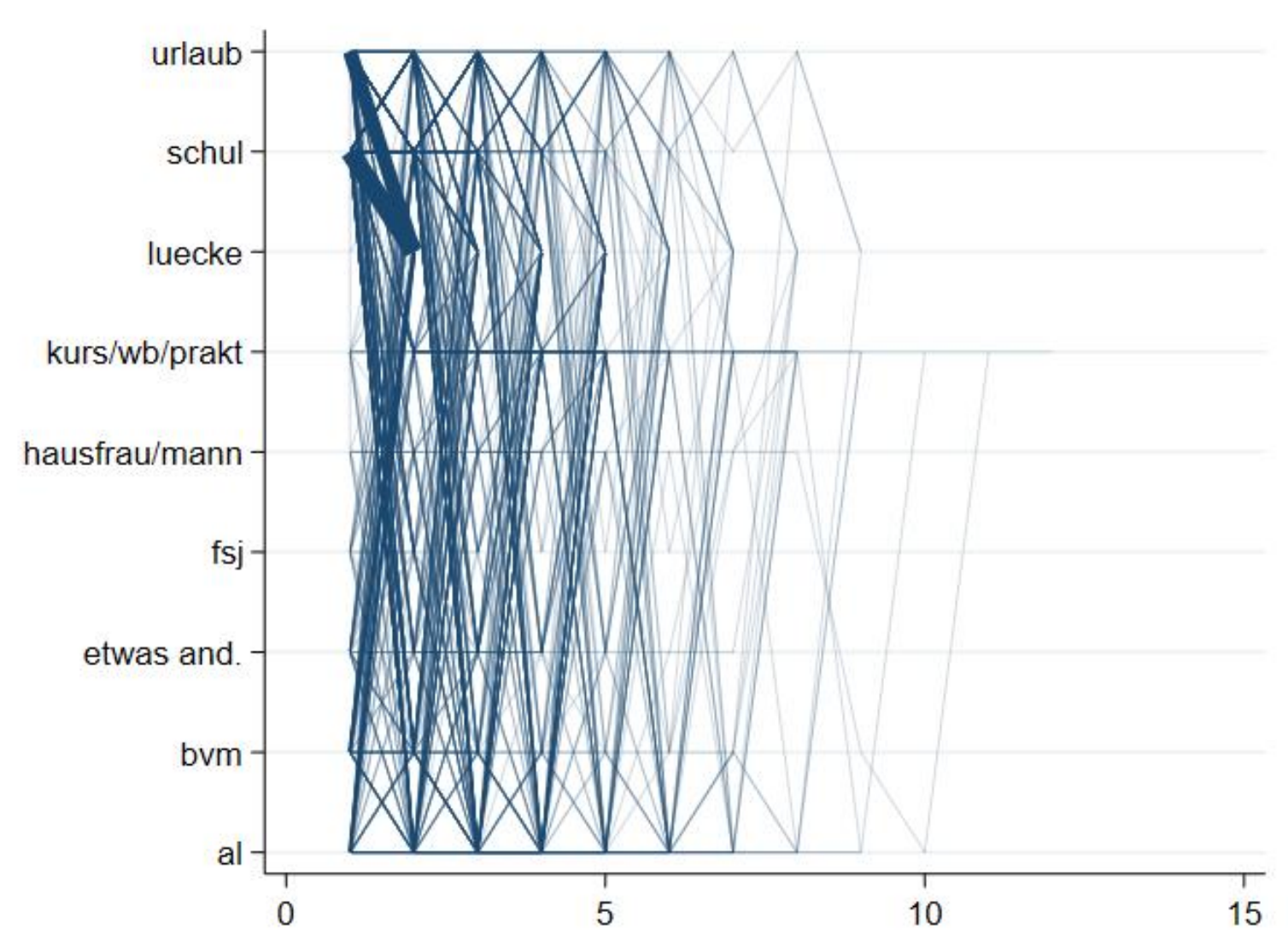

Quelle: Eigene Berechnungen basierend auf dem NEPS. Abkürzungen der Übergangsstationen: al = Arbeitslosigkeit, bvm = Berufsvorbereitende Maßnahme, kurs/wb/prakt = Kurs/Weiterbildung/Praktikum, hausfrau/-mann = Tätigkeit als Hausfrau/-mann, schul = Schulepisode, etwas anderes = Etwas anderes gemacht, $\mathrm{fsj}=$ Freiwilliges Soziales/Ökologisches Jahr, luecke = Lücke (unbestimmte Phase), urlaub = Urlaub. X-Achse: Anzahl der absolvierten Übergangsstationen. N = 5.854 Personen mit Übergangsverläufen. 


\section{Abbildung 36: Grafischer Verlauf der zehn häufigsten Übergangsverläufe (mit FSJ/Urlaub, mit unbestimmten Lückenepisoden).}

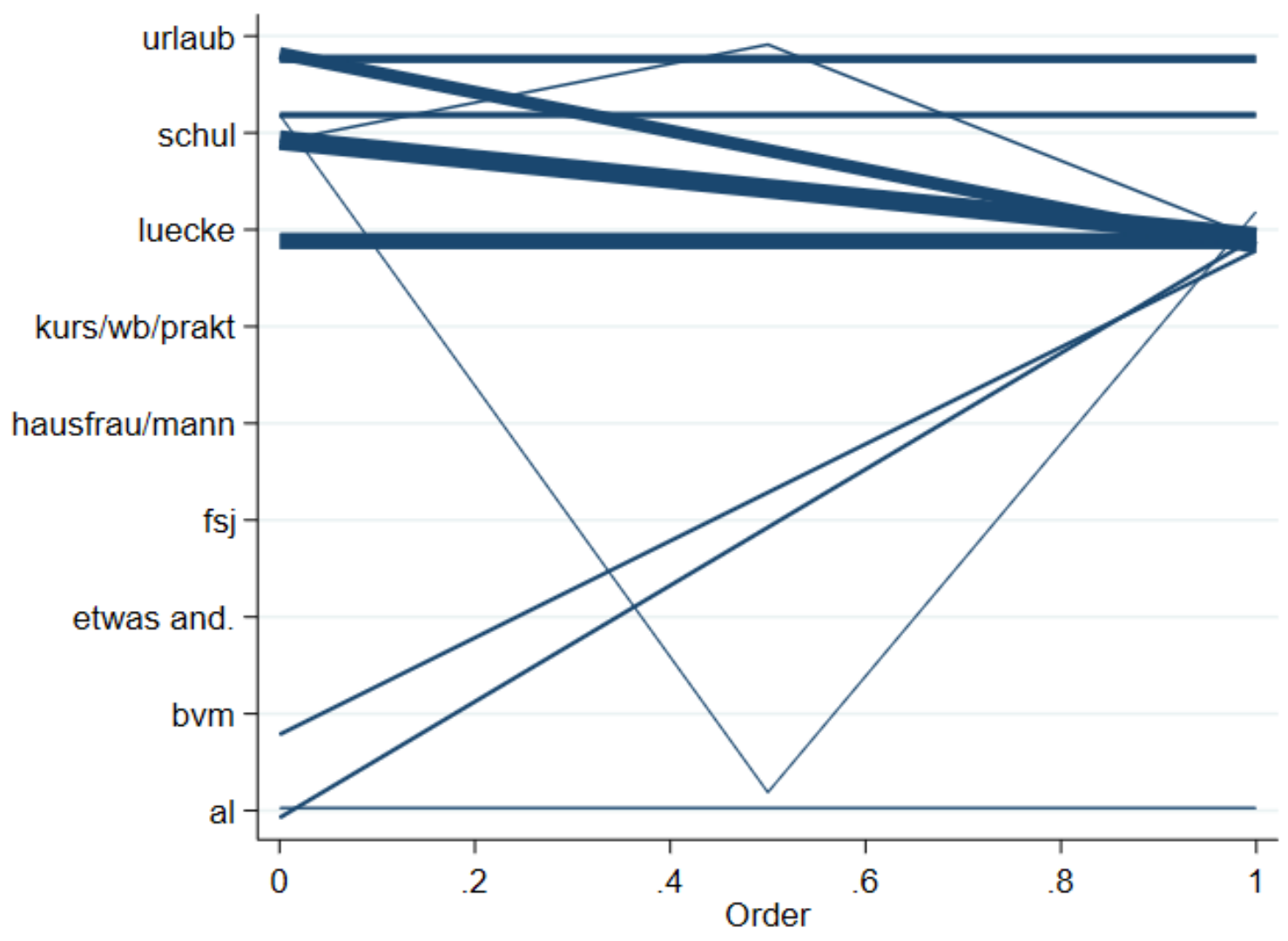

Quelle: Eigene Berechnungen basierend auf dem NEPS. Abkürzungen der Übergangsstationen: al = Arbeitslosigkeit, bvm = Berufsvorbereitende Maßnahme, kurs/wb/prakt $=$ Kurs/Weiterbildung/Praktikum, hausfrau/-mann = Tätigkeit als Hausfrau/-mann, schul $=$ Schulepisode, etwas anderes = Etwas anderes gemacht, fsj = Freiwilliges Soziales/Ökologisches Jahr, luecke $=$ Lücke (unbestimmte Phase), urlaub = Urlaub. Die zehn häufigsten Übergangsverläufe wurden von $\mathrm{N}=3.860$ Personen durchlaufen. 
Abbildung 37: Geschätzte Wahrscheinlichkeiten, Stationen ohne Weiterbildungscharakter zu durchlaufen, nach Schulabschluss in Abhängigkeit vom Anteil an Dienstleistungstätigen (mit FSJ/Urlaub, ohne unbestimmte Lückenepisoden).

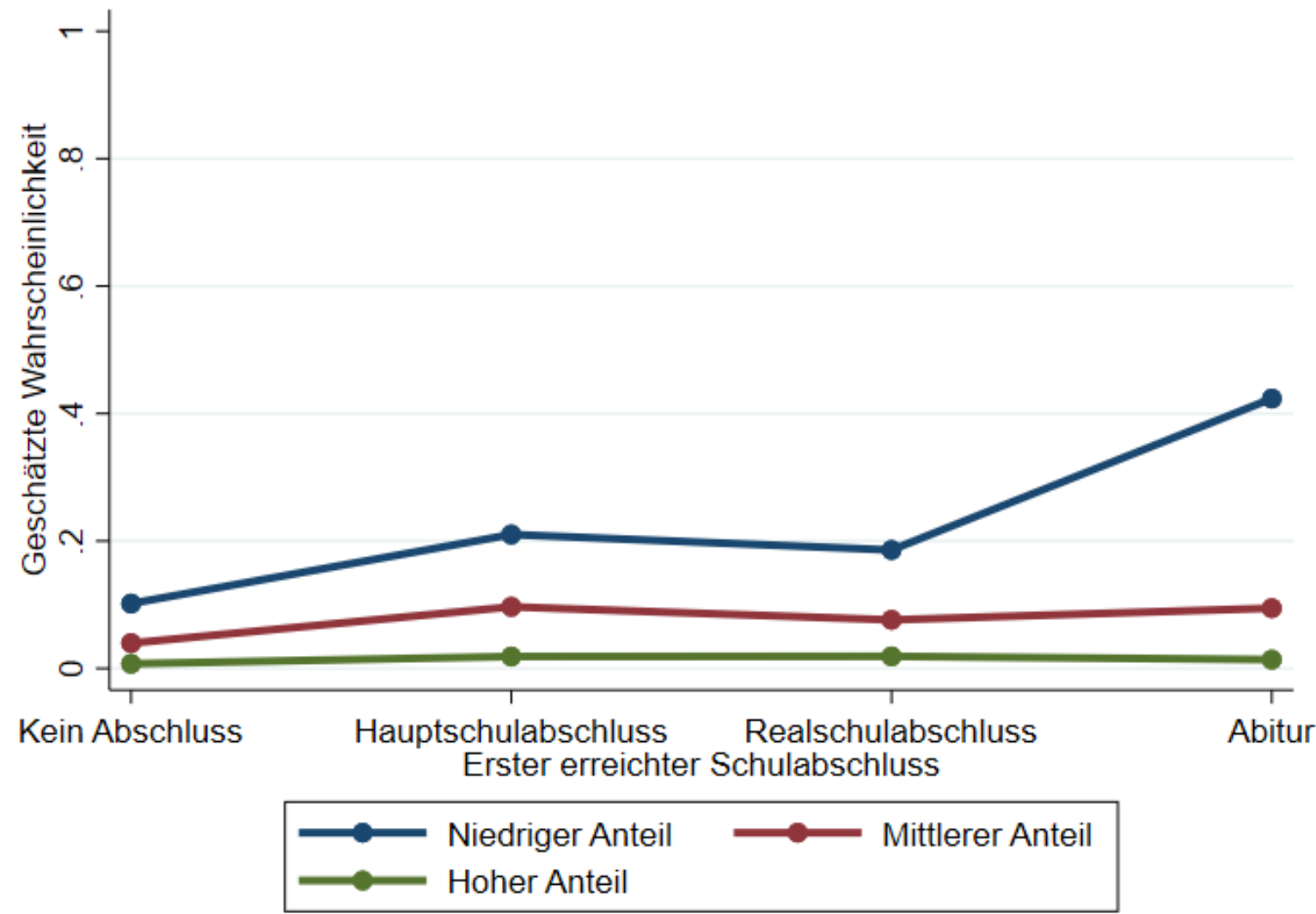

Quelle: Eigene Berechnungen basierend auf dem NEPS. Legende: Grün: Mittelwert des Dienstleistungstätigenanteils + 1 Standardabweichung; Rot: Mittelwert des Dienstleistungstätigenanteils; Blau: Mittelwert des Dienstleistungstätigenanteils - 1 Standardabweichung. Fixierung der übrigen unabhängigen Variablen auf folgende Werte: Schulenddatumsjahr: 1971; Soz. Herkunft: kein Abschluss / Hauptschulabschluss ohne berufliche Ausbildung; Geschlecht: weiblich; übrige Variablen (Demografischer Anteil der unter 20Jährigen, Arbeitslosenquote) sind auf ihren jeweiligen Mittelwert fixiert. N=1.751. 
Abbildung 38: Geschätzte Wahrscheinlichkeiten, berufsvorbereitende Maßnahmen zu durchlaufen, nach Schulabschluss in Abhängigkeit vom Anteil an Dienstleistungstätigen (mit FSJ/Urlaub, ohne unbestimmte Lückenepisoden).

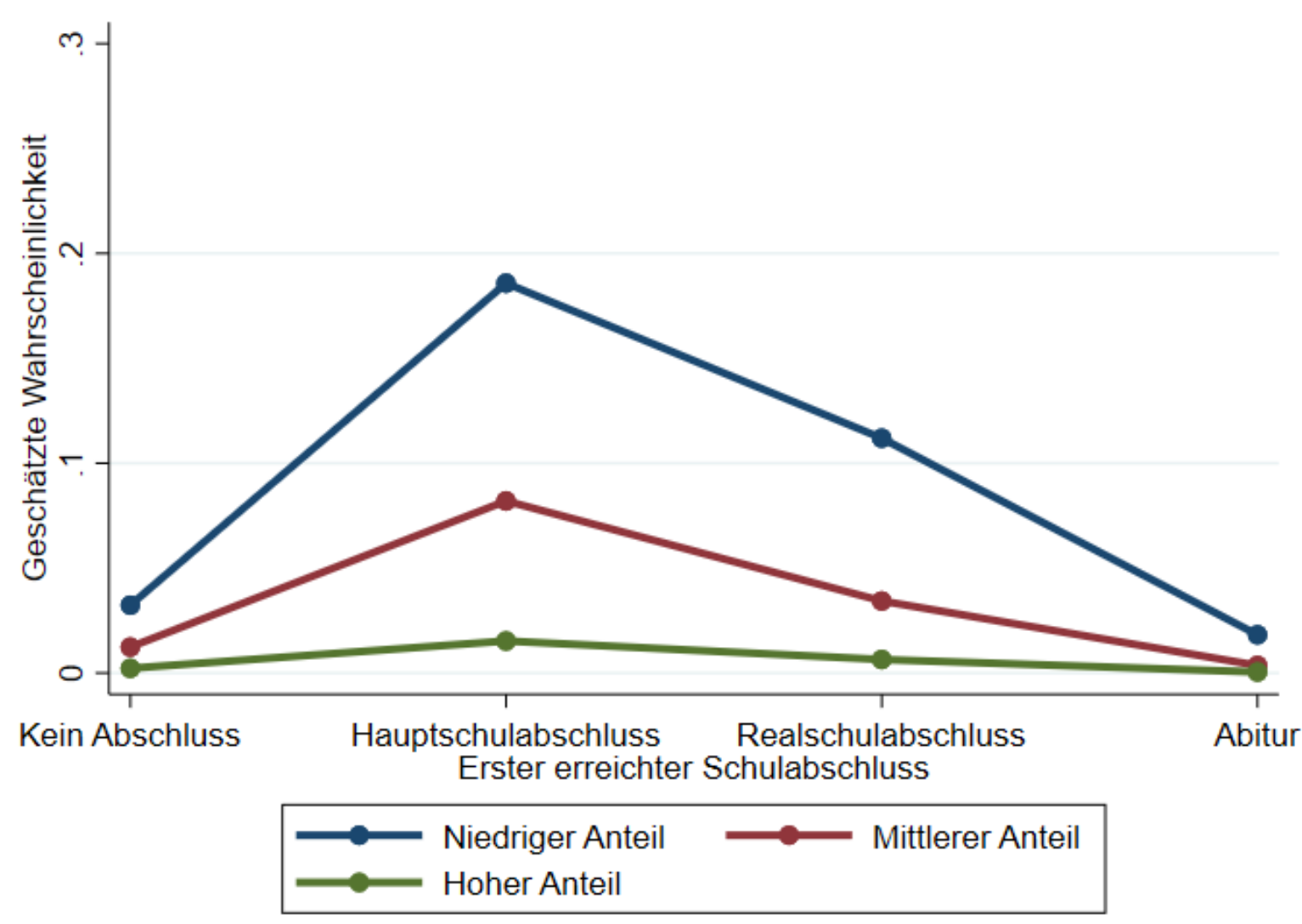

Quelle: Eigene Berechnungen basierend auf dem NEPS. Legende: Grün: Mittelwert des Dienstleistungstätigenanteils +1 Standardabweichung; Rot: Mittelwert des Dienstleistungstätigenanteils; Blau: Mittelwert des Dienstleistungstätigenanteils - 1 Standardabweichung. Fixierung der übrigen unabhängigen Variablen auf folgende Werte: Schulenddatumsjahr: 1971; Soz. Herkunft: kein Abschluss / Hauptschulabschluss ohne berufliche Ausbildung; Geschlecht: weiblich; übrige Variablen (Demografischer Anteil der unter 20Jährigen, Arbeitslosenquote) sind auf ihren jeweiligen Mittelwert fixiert. Um die Unterschiede zwischen den Anteilen besser erkennen zu können, wurde bei der geschätzten Wahrscheinlichkeit auf eine feinere Skalierung auf der Y-Achse (0 bis 0,3$)$ zurückgegriffen. $\mathrm{N}=459$. 
Abbildung 39: Geschätzte Wahrscheinlichkeiten, Kurse, Weiterbildungen oder Praktika zu durchlaufen, nach Schulabschluss in Abhängigkeit vom Anteil an Dienstleistungstätigen (mit FSJ/Urlaub, ohne unbestimmte Lückenepisoden).

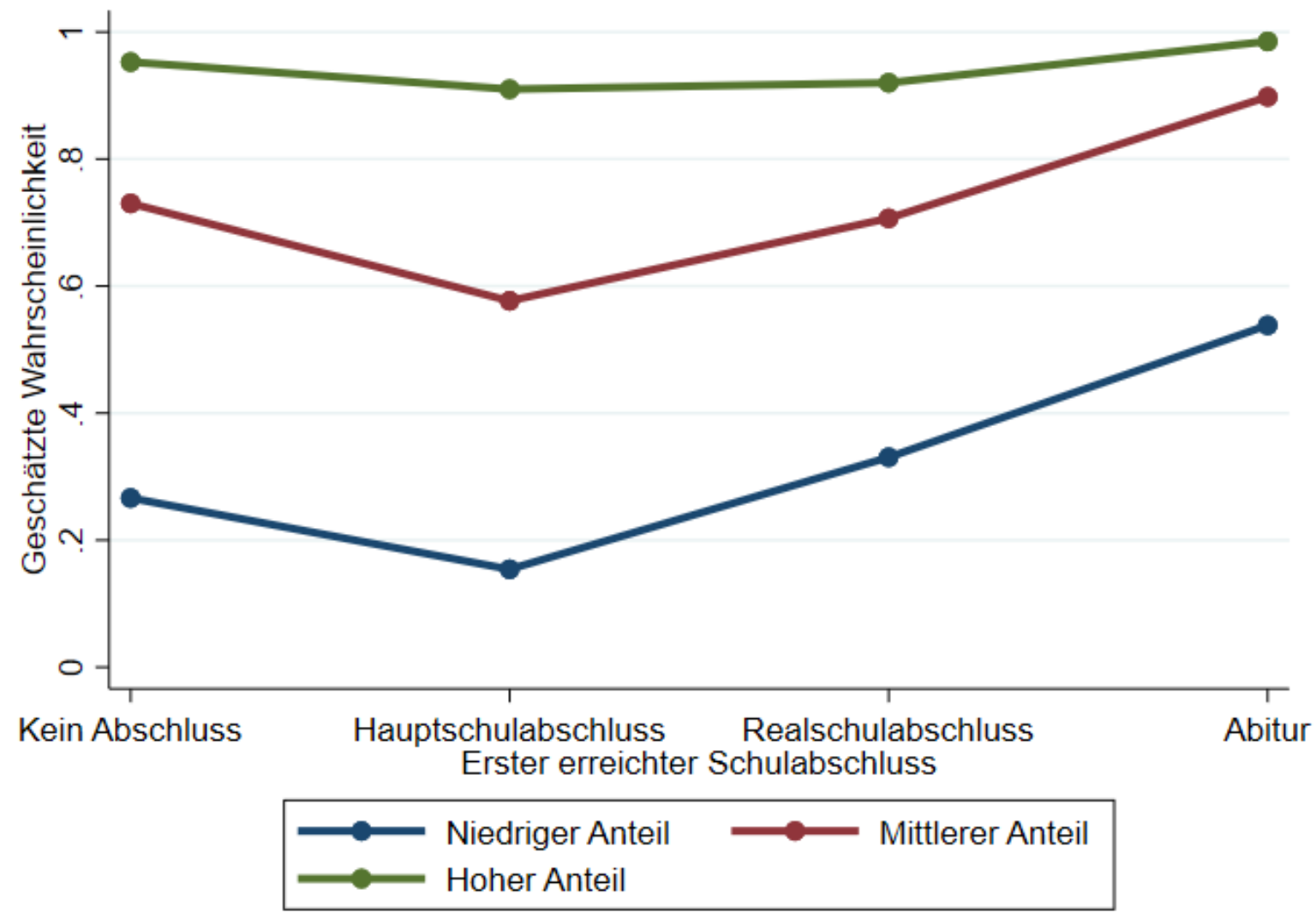

Quelle: Eigene Berechnungen basierend auf dem NEPS. Legende: Grün: Mittelwert des Dienstleistungstätigenanteils +1 Standardabweichung; Rot: Mittelwert des Dienstleistungstätigenanteils; Blau: Mittelwert des Dienstleistungstätigenanteils - 1 Standardabweichung. Fixierung der übrigen unabhängigen Variablen auf folgende Werte: Schulenddatumsjahr: 1971; Soz. Herkunft: kein Abschluss / Hauptschulabschluss ohne berufliche Ausbildung; Geschlecht: weiblich; übrige Variablen (Demografischer Anteil der unter 20Jährigen, Arbeitslosenquote) sind auf ihren jeweiligen Mittelwert fixiert. N = 263. 


\begin{abstract}
Abbildung 40: Geschätzte Wahrscheinlichkeiten, zusätzliche Schulepisoden zu durchlaufen, nach Schulabschluss in Abhängigkeit vom Anteil an Dienstleistungstätigen (mit FSJ/Urlaub, ohne unbestimmte Lückenepisoden).
\end{abstract}

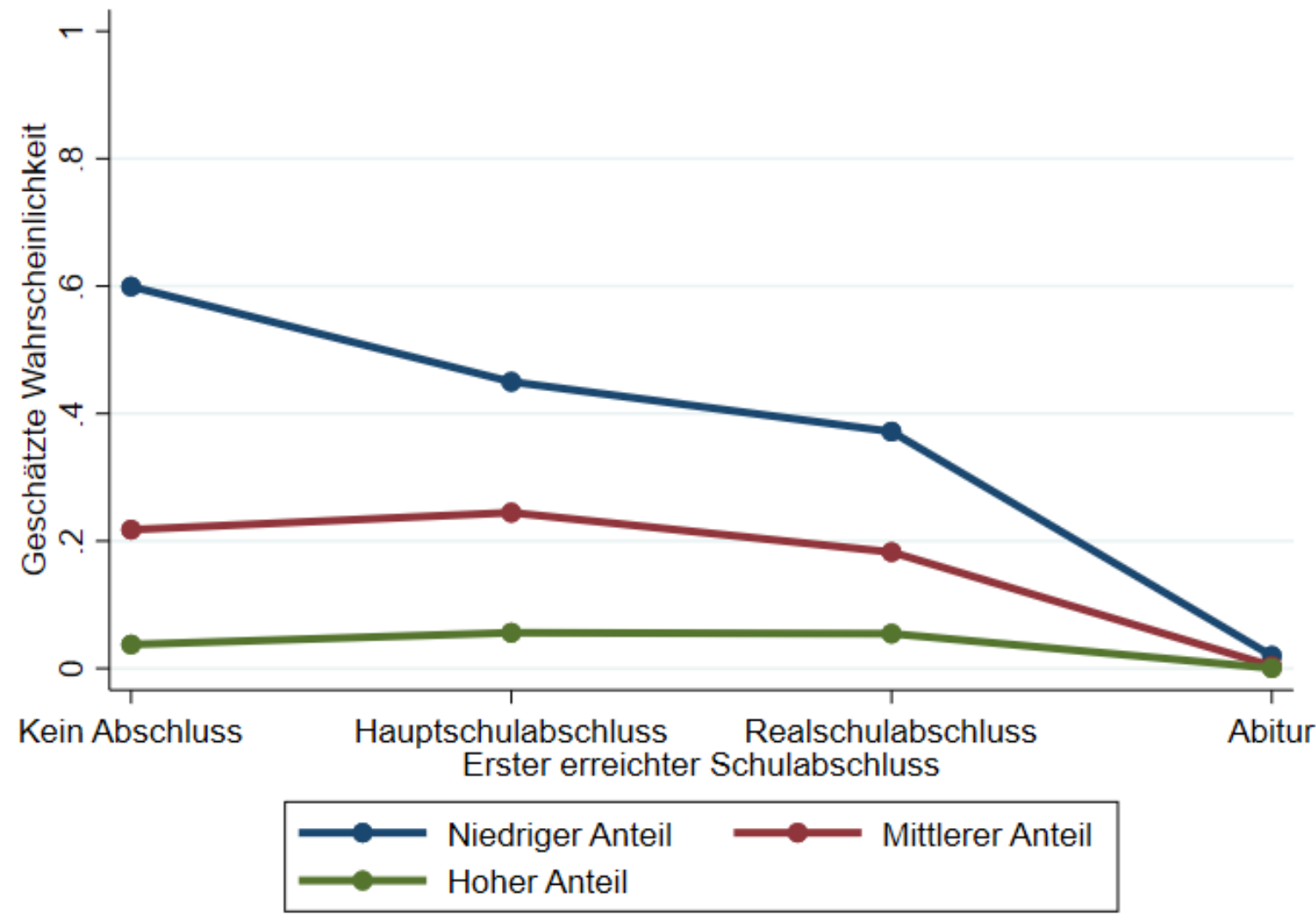

Quelle: Eigene Berechnungen basierend auf dem NEPS. Legende: Grün: Mittelwert des Dienstleistungstätigenanteils + 1 Standardabweichung; Rot: Mittelwert des Dienstleistungstätigenanteils; Blau: Mittelwert des Dienstleistungstätigenanteils - 1 Standardabweichung. Fixierung der übrigen unabhängigen Variablen auf folgende Werte: Schulenddatumsjahr: 1971; Soz. Herkunft: kein Abschluss / Hauptschulabschluss ohne berufliche Ausbildung; Geschlecht: weiblich; übrige Variablen (Demografischer Anteil der unter 20Jährigen, Arbeitslosenquote) sind auf ihren jeweiligen Mittelwert fixiert. N = 2.343. 
Abbildung 41: Geschätzte Wahrscheinlichkeiten, Stationen ohne Weiterbildungscharakter zu durchlaufen, nach Schulabschluss in Abhängigkeit vom Anteil an Dienstleistungstätigen (ohne FSJ/Urlaub, mit unbestimmten Lückenepisoden).

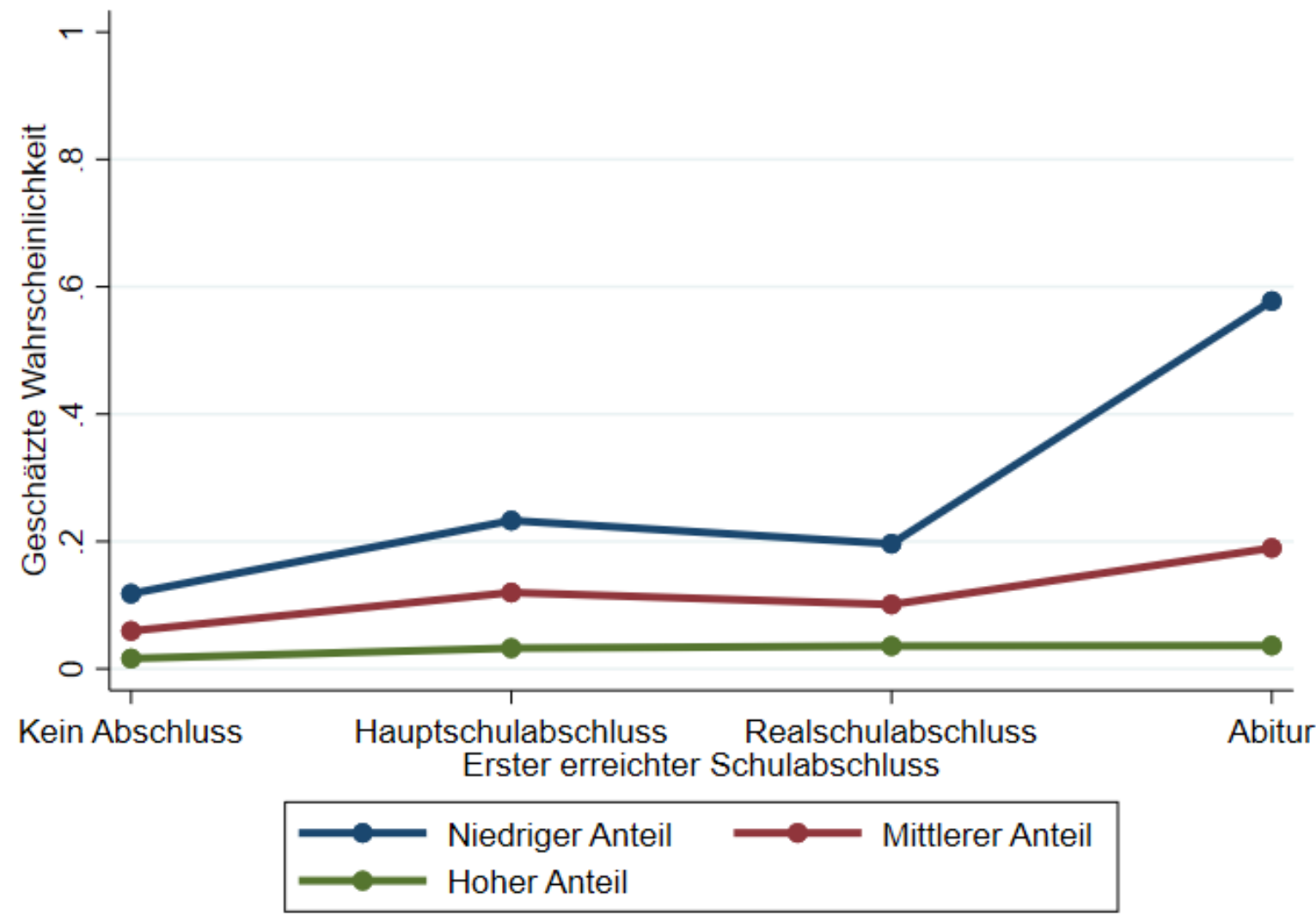

Quelle: Eigene Berechnungen basierend auf dem NEPS. Legende: Grün: Mittelwert des Dienstleistungstätigenanteils + 1 Standardabweichung; Rot: Mittelwert des Dienstleistungstätigenanteils; Blau: Mittelwert des Dienstleistungstätigenanteils - 1 Standardabweichung. Fixierung der übrigen unabhängigen Variablen auf folgende Werte: Schulenddatumsjahr: 1971; Soz. Herkunft: kein Abschluss / Hauptschulabschluss ohne berufliche Ausbildung; Geschlecht: weiblich; übrige Variablen (Demografischer Anteil der unter 20Jährigen, Arbeitslosenquote) sind auf ihren jeweiligen Mittelwert fixiert. N = 2.214. 
Abbildung 42: Geschätzte Wahrscheinlichkeiten, berufsvorbereitende Maßnahmen zu durchlaufen, nach Schulabschluss in Abhängigkeit vom Anteil an Dienstleistungstätigen (ohne FSJ/Urlaub, mit unbestimmten Lückenepisoden).

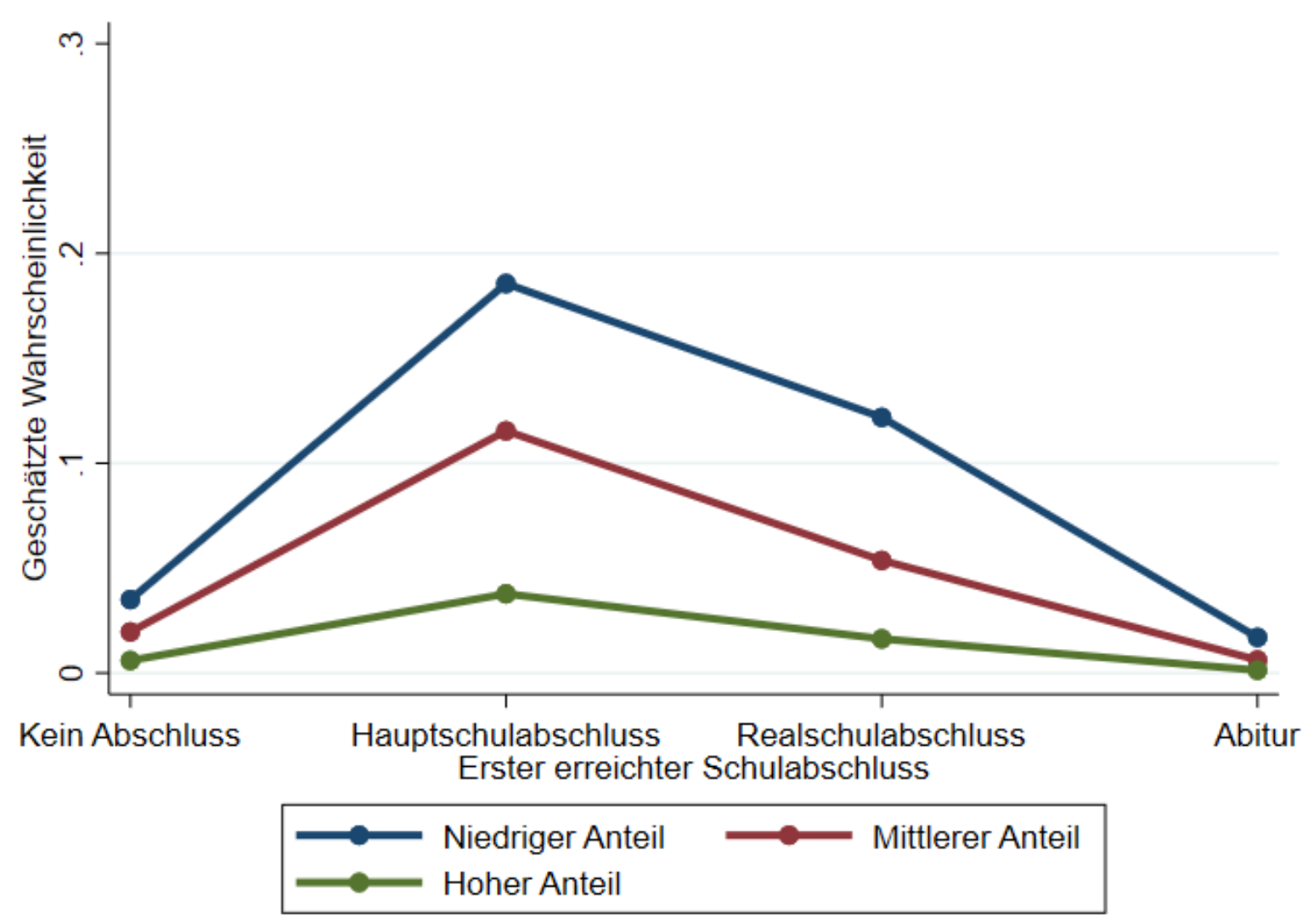

Quelle: Eigene Berechnungen basierend auf dem NEPS. Legende: Grün: Mittelwert des Dienstleistungstätigenanteils +1 Standardabweichung; Rot: Mittelwert des Dienstleistungstätigenanteils; Blau: Mittelwert des Dienstleistungstätigenanteils - 1 Standardabweichung. Fixierung der übrigen unabhängigen Variablen auf folgende Werte: Schulenddatumsjahr: 1971; Soz. Herkunft: kein Abschluss / Hauptschulabschluss ohne berufliche Ausbildung; Geschlecht: weiblich; übrige Variablen (Demografischer Anteil der unter 20Jährigen, Arbeitslosenquote) sind auf ihren jeweiligen Mittelwert fixiert. Um die Unterschiede zwischen den Anteilen besser erkennen zu können, wurde bei der geschätzten Wahrscheinlichkeit auf eine feinere Skalierung auf der Y-Achse (0 bis 0,3$)$ zurückgegriffen. $\mathrm{N}=459$. 
Abbildung 43: Geschätzte Wahrscheinlichkeiten, Kurse, Weiterbildungen oder Praktika zu durchlaufen, nach Schulabschluss in Abhängigkeit vom Anteil an Dienstleistungstätigen (ohne FSJ/Urlaub, mit unbestimmten Lückenepisoden).

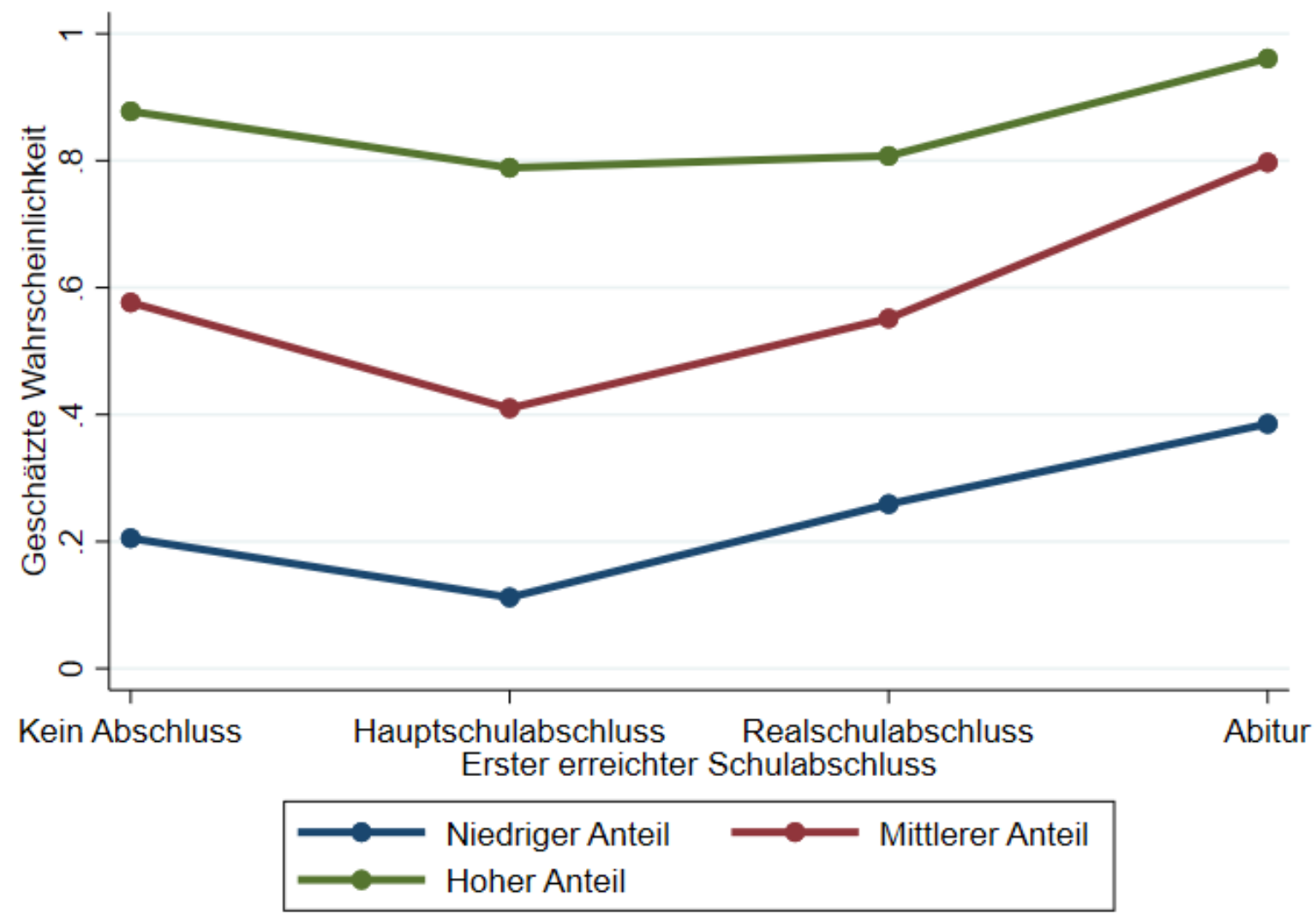

Quelle: Eigene Berechnungen basierend auf dem NEPS. Legende: Grün: Mittelwert des Dienstleistungstätigenanteils + 1 Standardabweichung; Rot: Mittelwert des Dienstleistungstätigenanteils; Blau: Mittelwert des Dienstleistungstätigenanteils - 1 Standardabweichung. Fixierung der übrigen unabhängigen Variablen auf folgende Werte: Schulenddatumsjahr: 1971; Soz. Herkunft: kein Abschluss / Hauptschulabschluss ohne berufliche Ausbildung; Geschlecht: weiblich; übrige Variablen (Demografischer Anteil der unter 20Jährigen, Arbeitslosenquote) sind auf ihren jeweiligen Mittelwert fixiert. N = 263. 


\begin{abstract}
Abbildung 44: Geschätzte Wahrscheinlichkeiten, zusätzliche Schulepisoden zu durchlaufen, nach Schulabschluss in Abhängigkeit vom Anteil an Dienstleistungstätigen (ohne FSJ/Urlaub, mit unbestimmten Lückenepisoden).
\end{abstract}

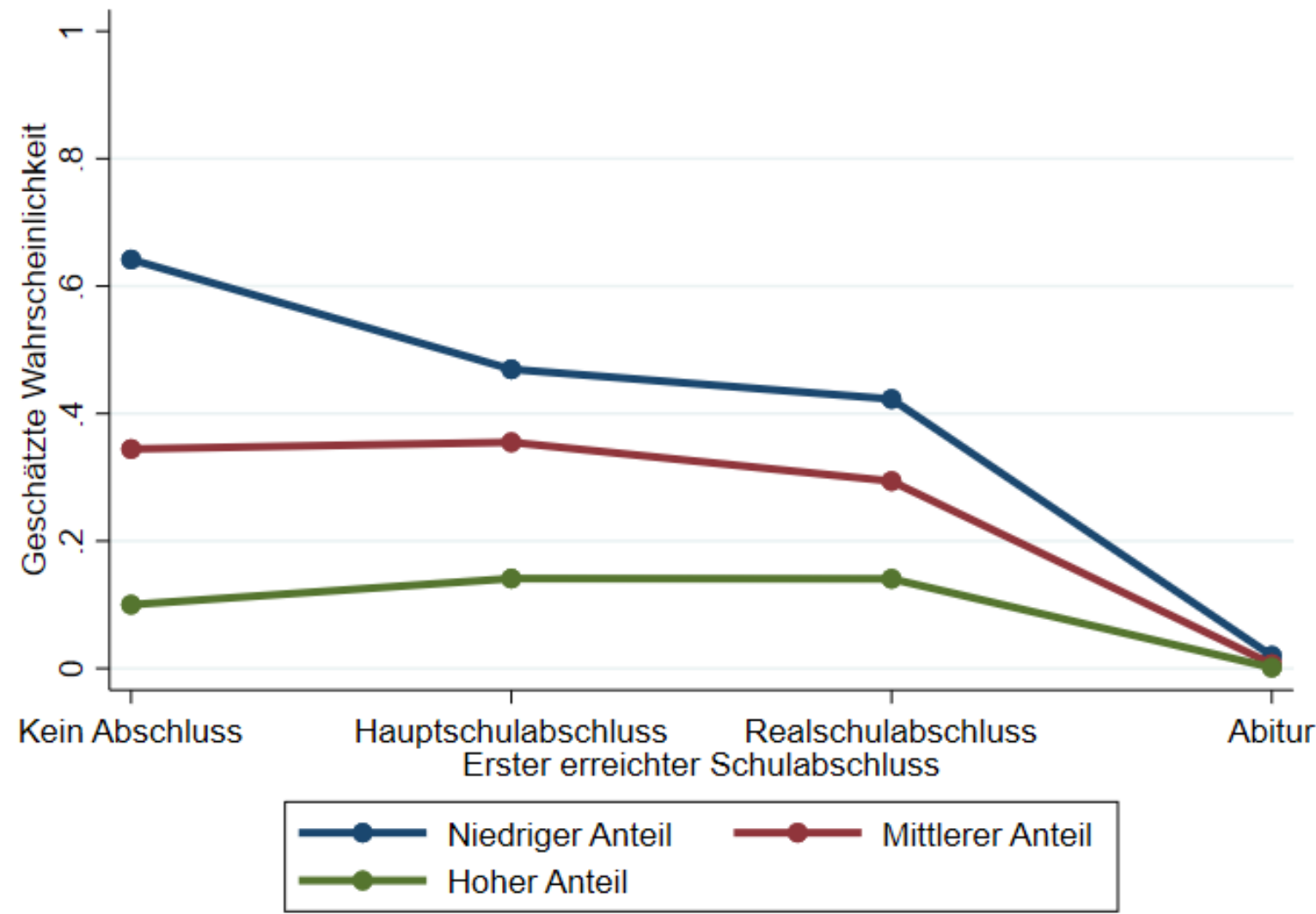

Quelle: Eigene Berechnungen basierend auf dem NEPS. Legende: Grün: Mittelwert des Dienstleistungstätigenanteils + 1 Standardabweichung; Rot: Mittelwert des Dienstleistungstätigenanteils; Blau: Mittelwert des Dienstleistungstätigenanteils - 1 Standardabweichung. Fixierung der übrigen unabhängigen Variablen auf folgende Werte: Schulenddatumsjahr: 1971; Soz. Herkunft: kein Abschluss / Hauptschulabschluss ohne berufliche Ausbildung; Geschlecht: weiblich; übrige Variablen (Demografischer Anteil der unter 20Jährigen, Arbeitslosenquote) sind auf ihren jeweiligen Mittelwert fixiert. N = 2.343. 
Abbildung 45: Geschätzte Wahrscheinlichkeiten, Stationen ohne Weiterbildungscharakter zu durchlaufen, nach Schulabschluss in Abhängigkeit vom Anteil an Dienstleistungstätigen (mit FSJ/Urlaub, mit unbestimmten Lückenepisoden).

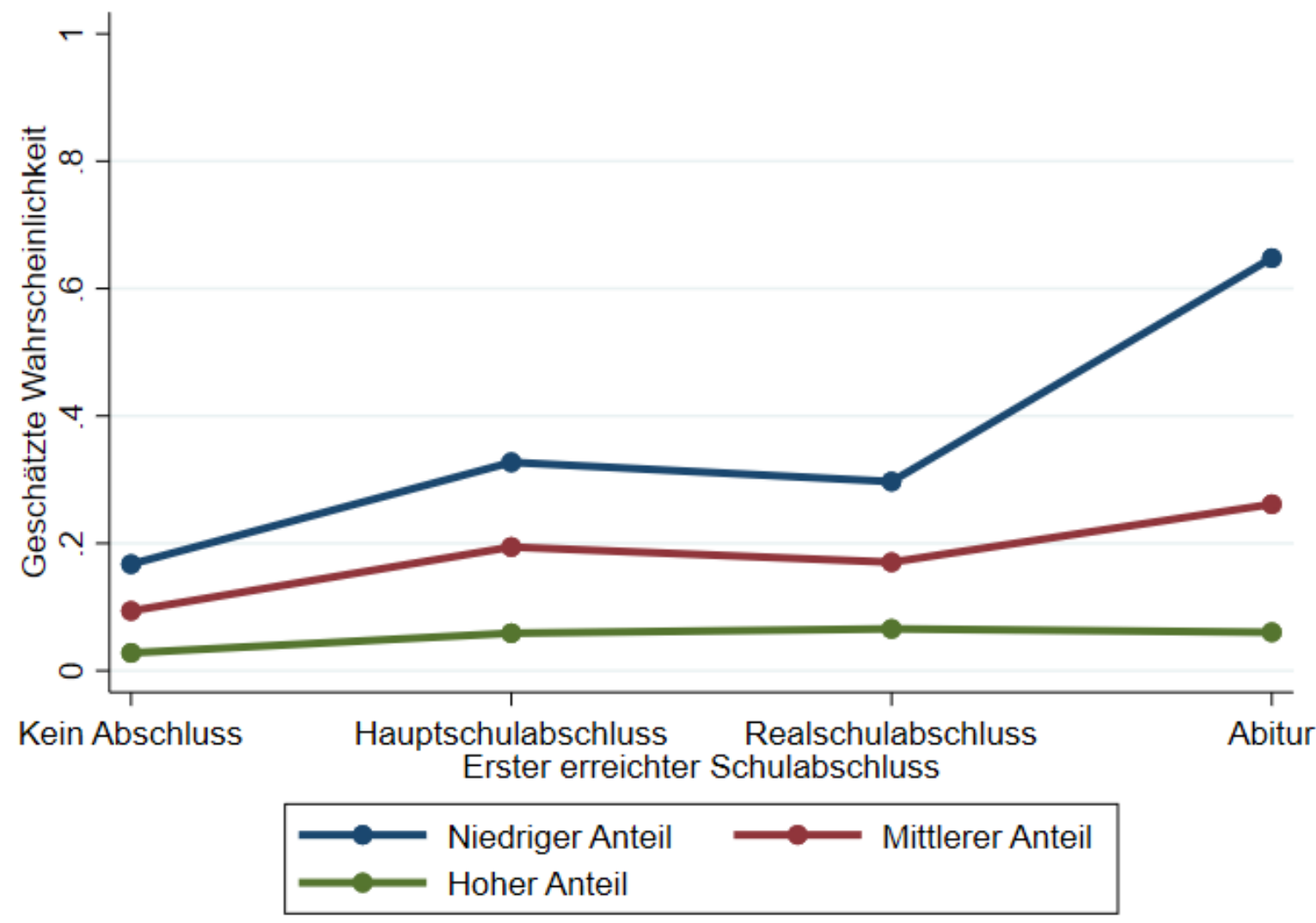

Quelle: Eigene Berechnungen basierend auf dem NEPS. Legende: Grün: Mittelwert des Dienstleistungstätigenanteils + 1 Standardabweichung; Rot: Mittelwert des Dienstleistungstätigenanteils; Blau: Mittelwert des Dienstleistungstätigenanteils - 1 Standardabweichung. Fixierung der übrigen unabhängigen Variablen auf folgende Werte: Schulenddatumsjahr: 1971; Soz. Herkunft: kein Abschluss / Hauptschulabschluss ohne berufliche Ausbildung; Geschlecht: weiblich; übrige Variablen (Demografischer Anteil der unter 20Jährigen, Arbeitslosenquote) sind auf ihren jeweiligen Mittelwert fixiert. N = 2.662. 
Abbildung 46: Geschätzte Wahrscheinlichkeiten, berufsvorbereitende Maßnahmen zu durchlaufen, nach Schulabschluss in Abhängigkeit vom Anteil an Dienstleistungstätigen (mit FSJ/Urlaub, mit unbestimmten Lückenepisoden).

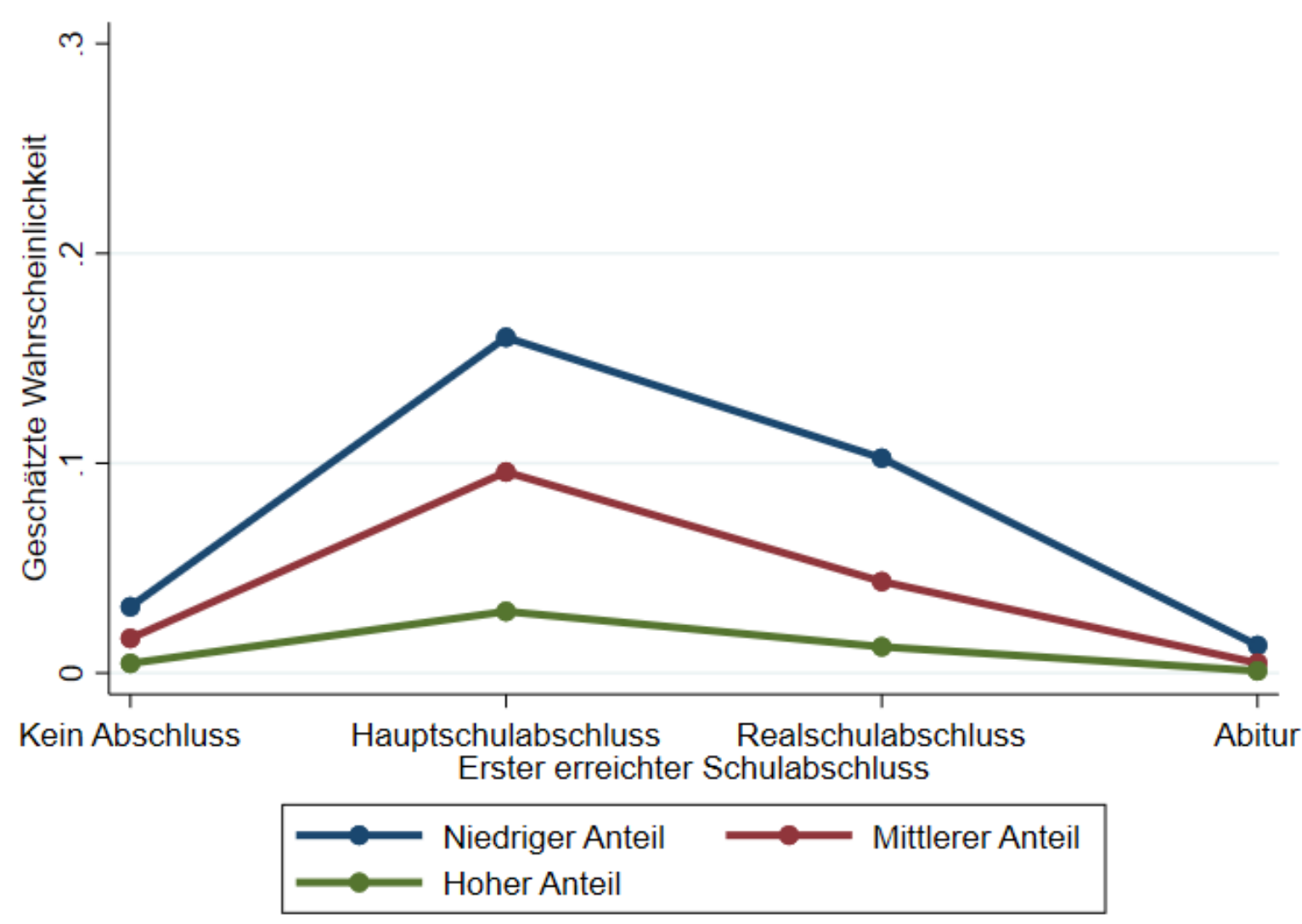

Quelle: Eigene Berechnungen basierend auf dem NEPS. Legende: Grün: Mittelwert des Dienstleistungstätigenanteils +1 Standardabweichung; Rot: Mittelwert des Dienstleistungstätigenanteils; Blau: Mittelwert des Dienstleistungstätigenanteils - 1 Standardabweichung. Fixierung der übrigen unabhängigen Variablen auf folgende Werte: Schulenddatumsjahr: 1971; Soz. Herkunft: kein Abschluss / Hauptschulabschluss ohne berufliche Ausbildung; Geschlecht: weiblich; übrige Variablen (Demografischer Anteil der unter 20Jährigen, Arbeitslosenquote) sind auf ihren jeweiligen Mittelwert fixiert. Um die Unterschiede zwischen den Anteilen besser erkennen zu können, wurde bei der geschätzten Wahrscheinlichkeit auf eine feinere Skalierung auf der Y-Achse (0 bis 0,3$)$ zurückgegriffen. $\mathrm{N}=459$. 
Abbildung 47: Geschätzte Wahrscheinlichkeiten, Kurse, Weiterbildungen, Praktika oder FSJEpisoden zu durchlaufen, nach Schulabschluss in Abhängigkeit vom Anteil an Dienstleistungstätigen (mit FSJ/Urlaub, mit unbestimmten Lückenepisoden).

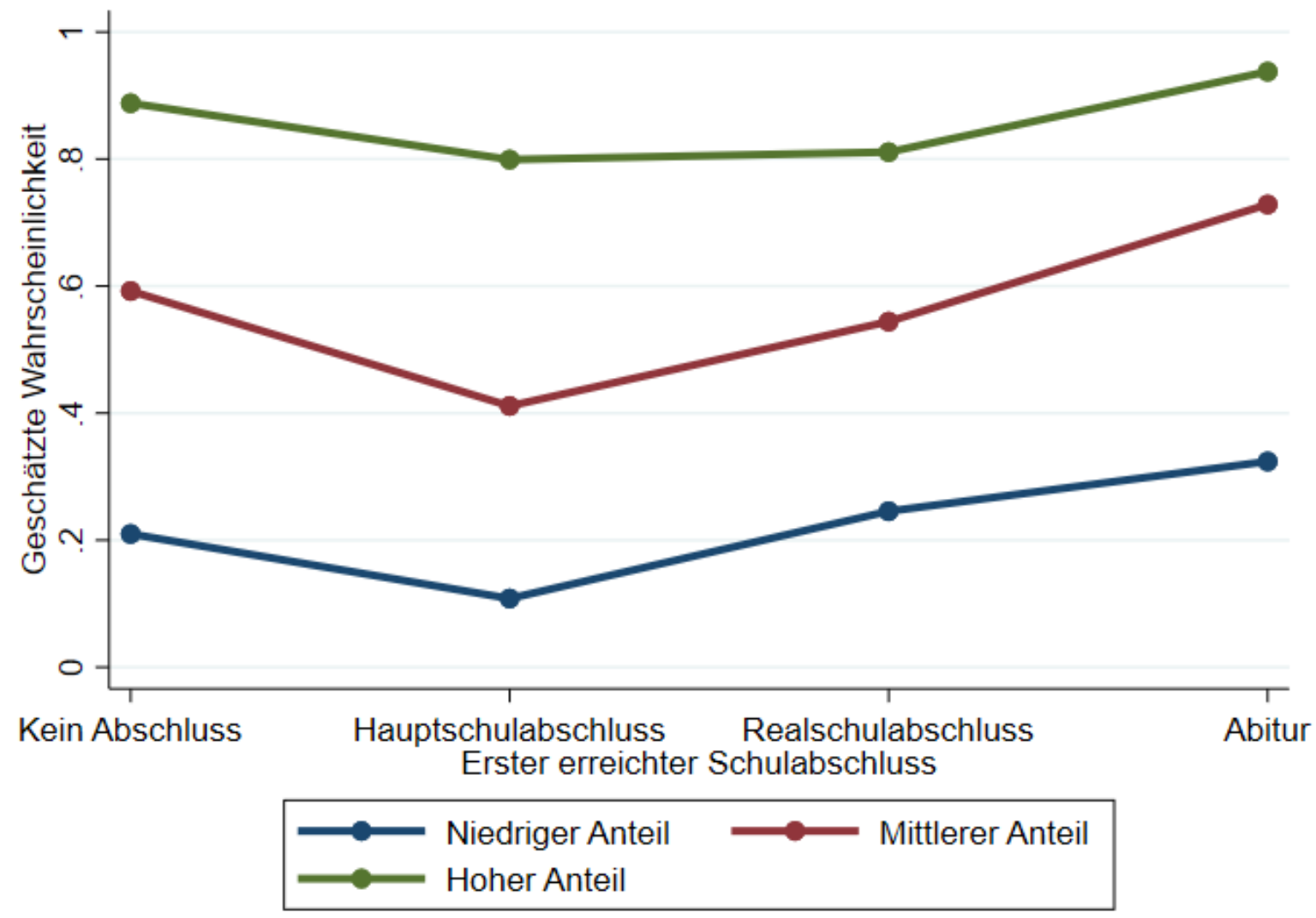

Quelle: Eigene Berechnungen basierend auf dem NEPS. Legende: Grün: Mittelwert des Dienstleistungstätigenanteils + 1 Standardabweichung; Rot: Mittelwert des Dienstleistungstätigenanteils; Blau: Mittelwert des Dienstleistungstätigenanteils - 1 Standardabweichung. Fixierung der übrigen unabhängigen Variablen auf folgende Werte: Schulenddatumsjahr: 1971; Soz. Herkunft: kein Abschluss / Hauptschulabschluss ohne berufliche Ausbildung; Geschlecht: weiblich; übrige Variablen (Demografischer Anteil der unter 20Jährigen, Arbeitslosenquote) sind auf ihren jeweiligen Mittelwert fixiert. N = 263. 


\begin{abstract}
Abbildung 48: Geschätzte Wahrscheinlichkeiten, zusätzliche Schulepisoden zu durchlaufen, nach Schulabschluss in Abhängigkeit vom Anteil an Dienstleistungstätigen (mit FSJ/Urlaub, mit unbestimmten Lückenepisoden).
\end{abstract}

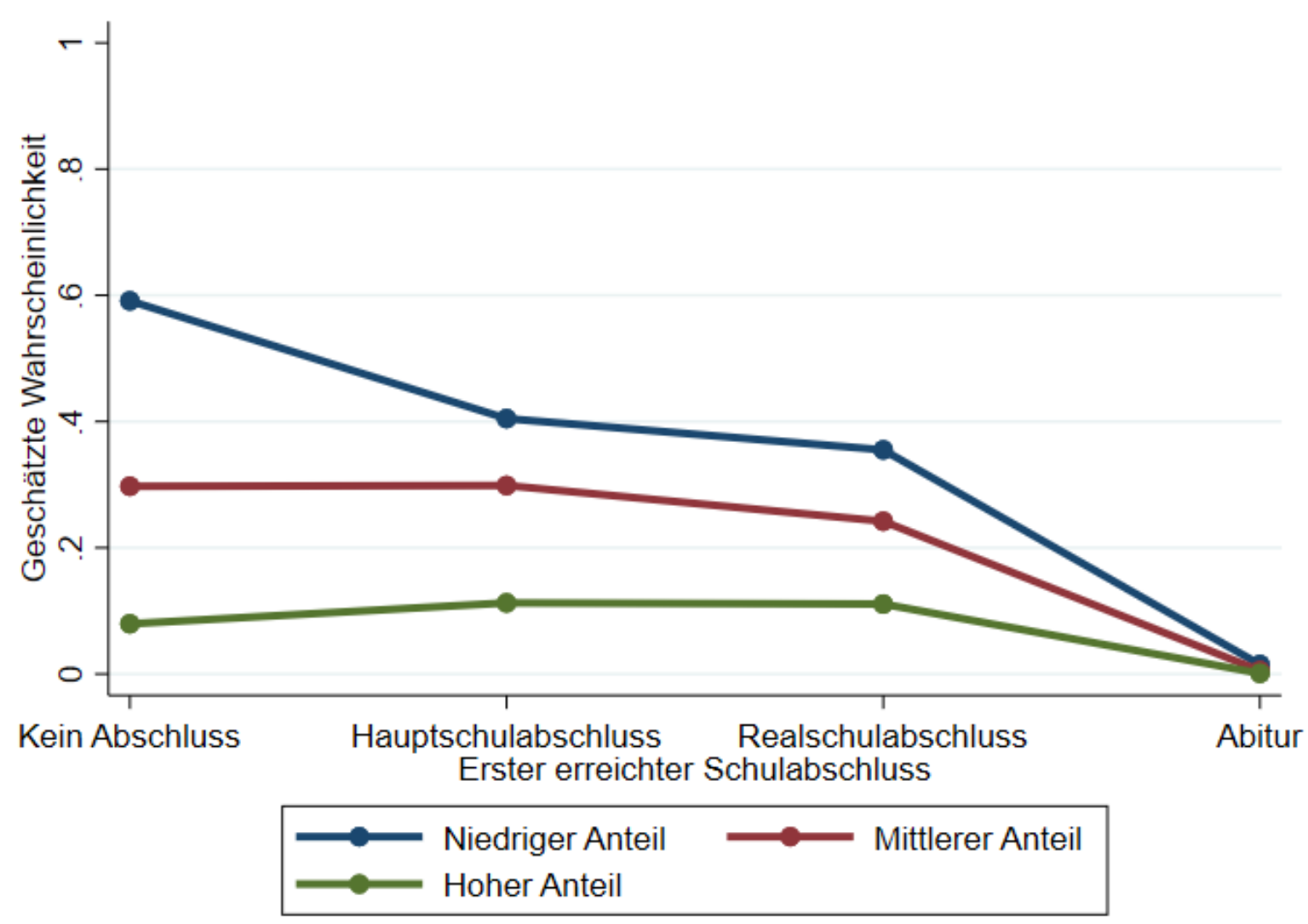

Quelle: Eigene Berechnungen basierend auf dem NEPS. Legende: Grün: Mittelwert des Dienstleistungstätigenanteils + 1 Standardabweichung; Rot: Mittelwert des Dienstleistungstätigenanteils; Blau: Mittelwert des Dienstleistungstätigenanteils - 1 Standardabweichung. Fixierung der übrigen unabhängigen Variablen auf folgende Werte: Schulenddatumsjahr: 1971; Soz. Herkunft: kein Abschluss / Hauptschulabschluss ohne berufliche Ausbildung; Geschlecht: weiblich; übrige Variablen (Demografischer Anteil der unter 20Jährigen, Arbeitslosenquote) sind auf ihren jeweiligen Mittelwert fixiert. N = 2.343. 


\begin{abstract}
Abbildung 49: Geschätzte Anzahl der Übergangsstationen (mit FSJ- und Urlaubsepisoden; ohne unbestimmte Lückenepisoden) nach Schulabschluss unter Einfluss des Anteils an Dienstleistungstätigen.
\end{abstract}

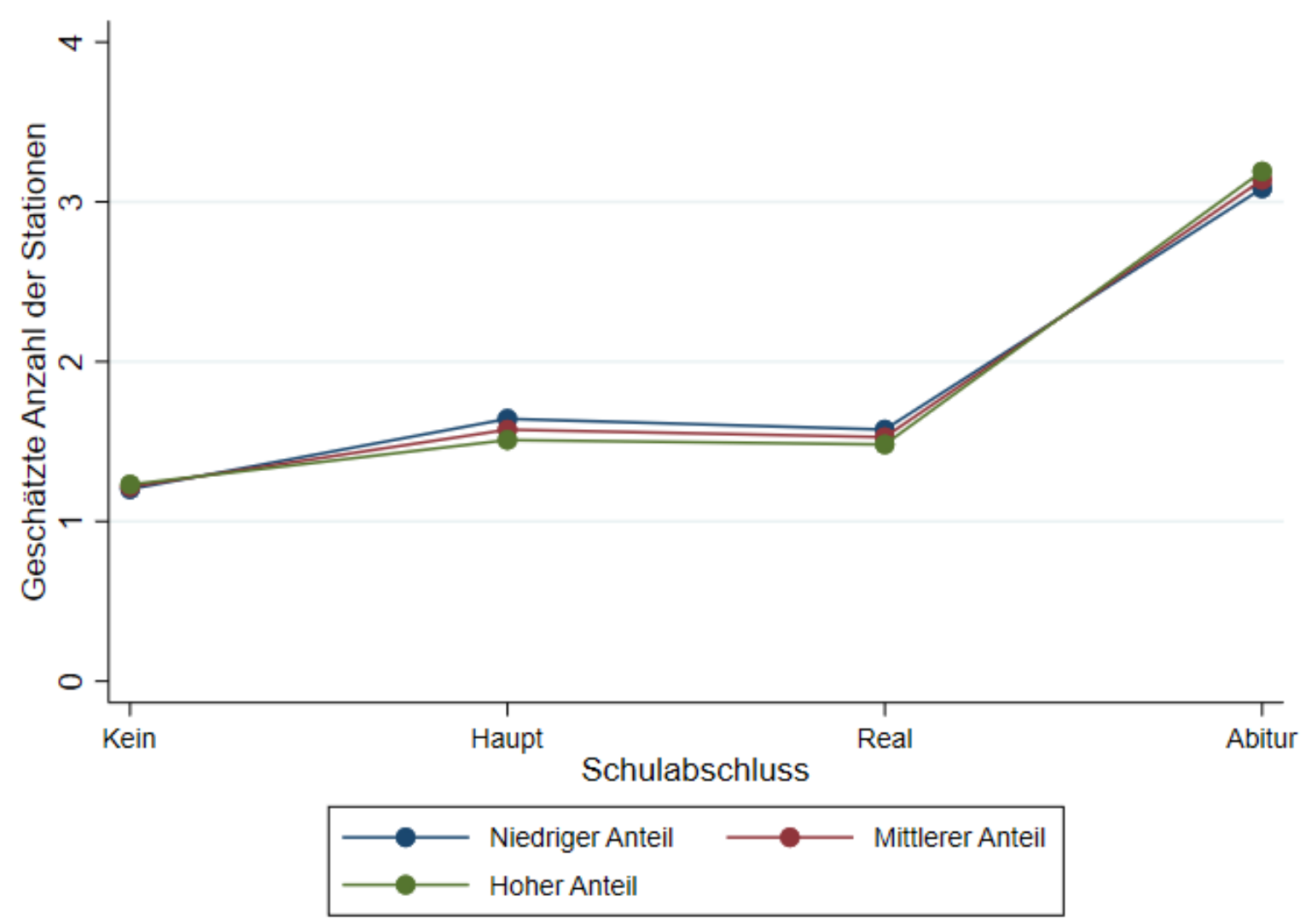

Quelle: Eigene Berechnungen basierend auf dem NEPS. Interaktion Anteil Dienstleistungstätige und Schulabschluss. Der Anteil der Dienstleistungstätigen wurde in drei Kategorien eingeteilt: Durchschnittlicher Anteil - 1 Standardabweichung (blau), durchschnittlicher Anteil (rot) und durchschnittlicher Anteil +1 Standardabweichung (grün). Die übrigen einbezogenen unabhängigen Variablen wurden wie folgt fixiert: Schulenddatumsjahr: 1971, Soziale Herkunft: Kein Abschluss/Hauptschulabschluss ohne berufliche Ausbildung, Geschlecht: männlich, demografischer Anteil der unter 20-Jährigen und Arbeitslosenquote jeweils mittelwertzentriert. $\mathrm{N}=4.867$. 


\begin{abstract}
Abbildung 50: Geschätzte Anzahl der Übergangsstationen (ohne FSJ- und Urlaubsepisoden; mit unbestimmten Lückenepisoden) nach Schulabschluss unter Einfluss des Anteils an Dienstleistungstätigen.
\end{abstract}

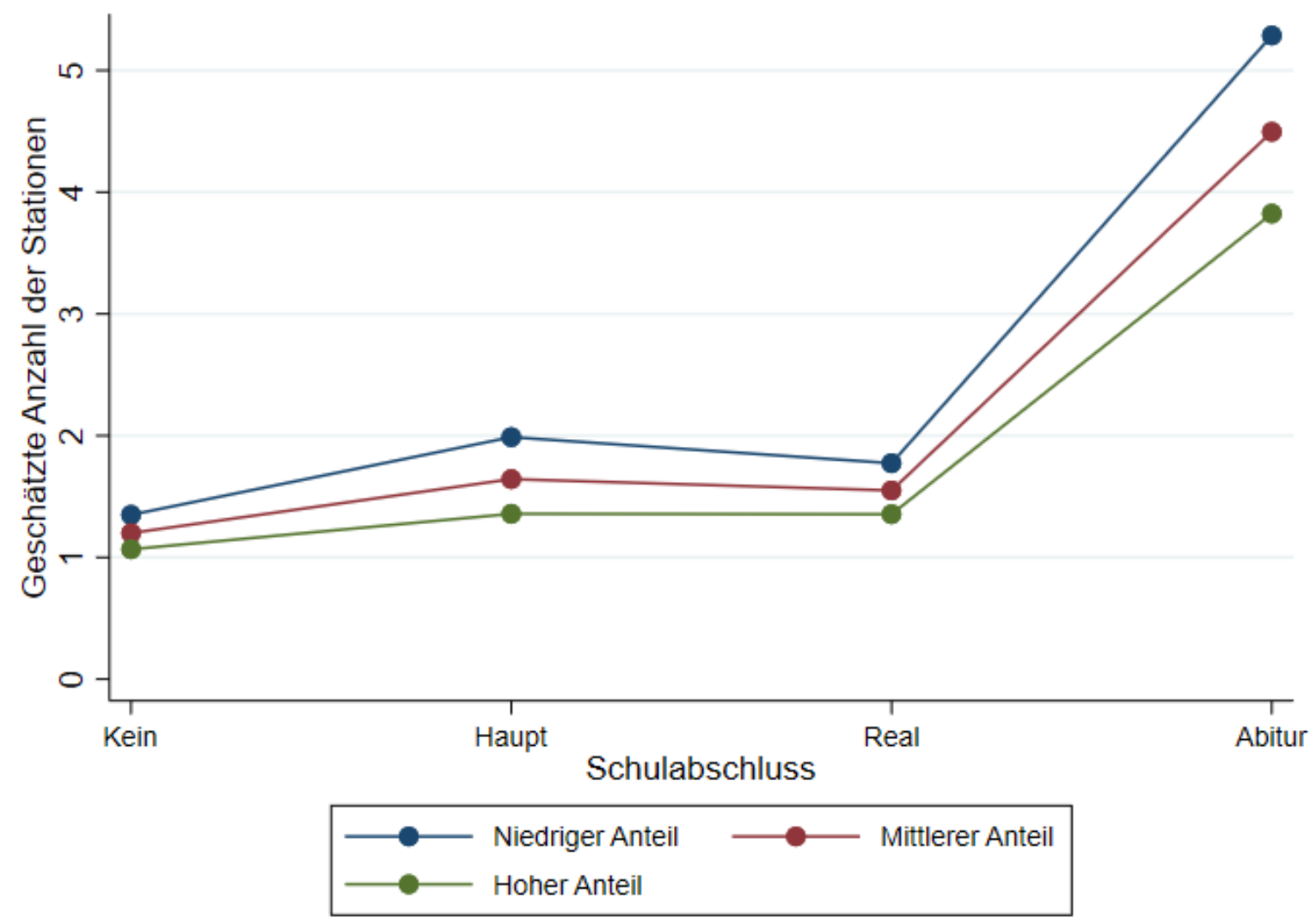

Quelle: Eigene Berechnungen basierend auf dem NEPS. Interaktion Anteil Dienstleistungstätige und Schulabschluss. Der Anteil der Dienstleistungstätigen wurde in drei Kategorien eingeteilt: Durchschnittlicher Anteil - 1 Standardabweichung (blau), durchschnittlicher Anteil (rot) und durchschnittlicher Anteil +1 Standardabweichung (grün). Die übrigen einbezogenen unabhängigen Variablen wurden wie folgt fixiert: Schulenddatumsjahr: 1971, Soziale Herkunft: Kein Abschluss/Hauptschulabschluss ohne berufliche Ausbildung, Geschlecht: männlich, demografischer Anteil der unter 20-Jährigen und Arbeitslosenquote jeweils mittelwertzentriert. $\mathrm{N}=5.264$. 
Abbildung 51: Geschätzte Anzahl der Übergangsstationen (mit FSJ- und Urlaubsepisoden; mit unbestimmten Lückenepisoden) nach Schulabschluss unter Einfluss des Anteils an Dienstleistungstätigen.

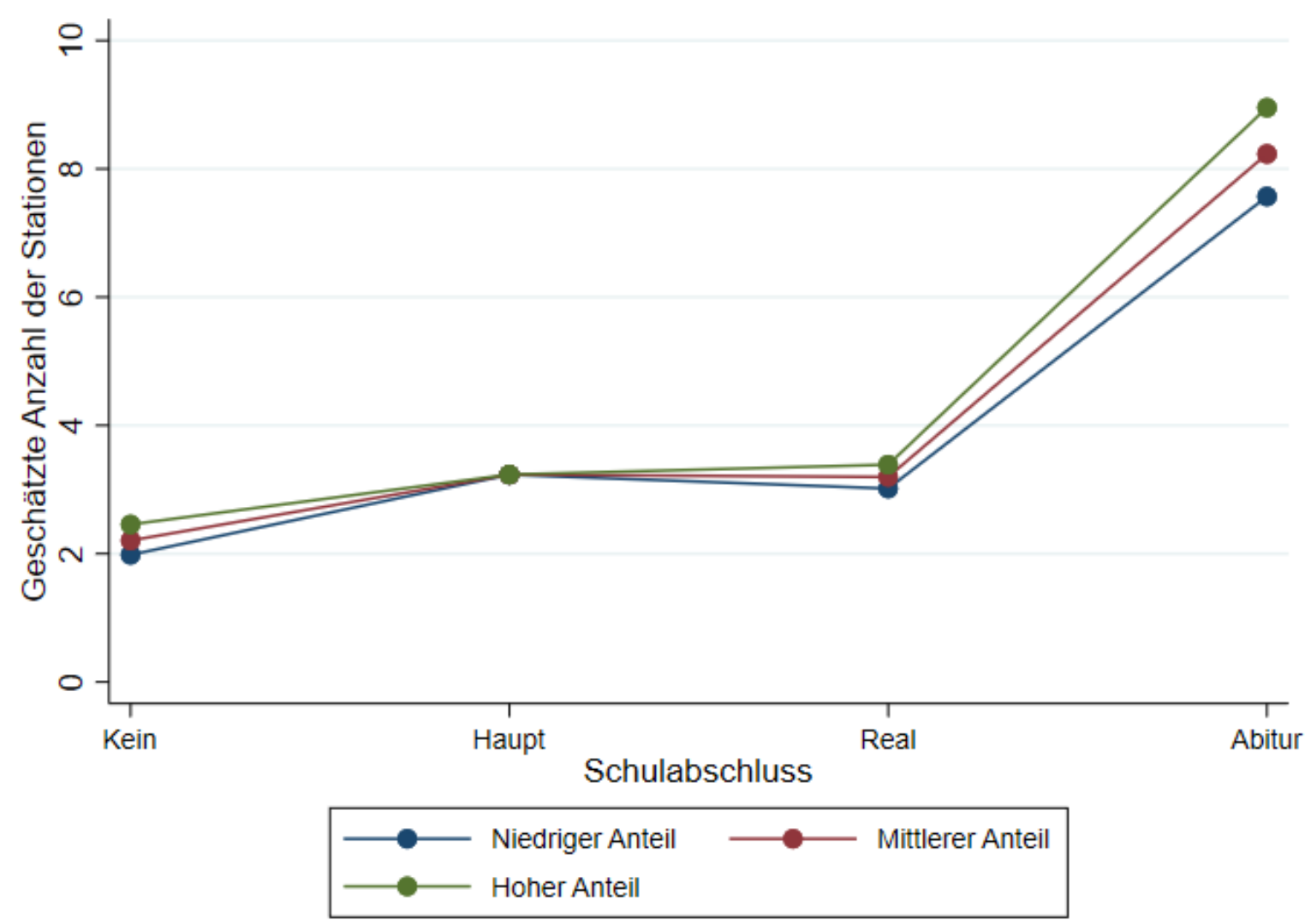

Quelle: Eigene Berechnungen basierend auf dem NEPS. Interaktion Anteil Dienstleistungstätige und Schulabschluss. Der Anteil der Dienstleistungstätigen wurde in drei Kategorien eingeteilt: Durchschnittlicher Anteil - 1 Standardabweichung (blau), durchschnittlicher Anteil (rot) und durchschnittlicher Anteil +1 Standardabweichung (grün). Die übrigen einbezogenen unabhängigen Variablen wurden wie folgt fixiert: Schulenddatumsjahr: 1971, Soziale Herkunft: Kein Abschluss/Hauptschulabschluss ohne berufliche Ausbildung, Geschlecht: männlich, demografischer Anteil der unter 20-Jährigen und Arbeitslosenquote jeweils mittelwertzentriert. $\mathrm{N}=5.727$. 


\section{Literaturverzeichnis}

Allmendinger, Jutta, Ebner, Christian, und Nikolai, Rita. 2010. Soziologische Bildungsforschung. In Handbuch Bildungsforschung, Hrsg. Rudolf Tippelt, und Bernhard Schmidt, 47 - 70. Wiesbaden: VS Verlag für Sozialwissenschaften.

Arrow, Kenneth J. 1994. Methodological Individualism and Social Knowledge. In American Economic Review, 84: Heft 2: 1 - 9. Nashville: American Economic Association.

Aust, Folkert, Gilberg, Reiner, Hess, Doris, Kleudgen, Martin, und Steinwede, Angelika. 2011. Methodenbericht. NEPS Etappe 8. Befragung von Erwachsenen. Haupterhebung 1. Welle 2009/2010. Bonn: infas Institut für angewandte Sozialwissenschaft GmbH. https://www.nepsdata.de/Portals/0/NEPS/Datenzentrum/Studienuebersicht/Methodenbericht_B72.pdf (Zugegriffen: 26.03.2021).

Autorengruppe Bildungsberichterstattung. 2008. Bildung in Deutschland 2008. Ein indikatorengestützter Bericht mit einer Analyse zu Übergängen im Anschluss an den Sekundarbereich I. Bielefeld: Bertelsmann. https://web.archive.org/web/20181123180800/http://ev-stadtakademiegoe.de/downl/bildungsbericht_bb_2008.pdf (Zugegriffen: 26.03.2021).

Baas, Maike, und Philipps, Veronika. 2017. Über Ausbildung in Arbeit? Verläufe gering gebildeter Jugendlicher. In Berichterstattung zur sozioökonomischen Entwicklung in Deutschland. Exklusive Teilhabe - ungenutzte Chancen; 3. Bericht: 1-36. Bielefeld: W. Bertelsmann Verlag. https://www.ssoar.info/ssoar/bitstream/handle/document/66107/ssoar-2017-baas_et_alUber_Ausbildung_in_Arbeit_Verlaufe.pdf?sequence $=1 \&$ isAllowed $=y \& \operatorname{lnkname}=$ ssoar2017-baas_et_al-Uber_Ausbildung_in_Arbeit_Verlaufe.pdf (Zugegriffen: 26.03.2021). Baethge, Martin, Solga, Heike, und Wieck, Markus. 2007. Berufsbildung im Umbruch. Signale eines überfälligen Aufbruchs. Berlin: Friedrich-Ebert-Stiftung. http://library.fes.de/pdf-files/stabsabteilung/04258/studie.pdf (Zugegriffen: 26.03.2021).

Bauer, Ullrich, Bolder, Axel, Bremer, Helmut, Dobischat, Rolf, und Kutscha, Günter. 2014. Bildungsexpansion zwischen Emanzipationsanspruch, staatlicher Reformpolitik und Hegemonie des Marktes. Widersprüche im Prozess der Re-Strukturierung der Klassengesellschaft. In Expansive Bildungspolitik - Expansive Bildung?, Hrsg. Ullrich Bauer, Axel Bolder, Helmut Bremer, Rolf Dobischat, und Günter Kutscha, 9 - 36. Wiesbaden: Springer VS. 
Becker, Gary Stanley. 1993. Human Capital. A Theoretical and Empirical Analysis with Special Reference to Education. Chicago und London: University of Chicago Press. 3. Auflage.

Becker, Gary Stanley. 1982. Der ökonomische Ansatz zur Erklärung menschlichen Verhaltens. Tübingen: Mohr.

Becker, Gary Stanley. 1978. The Economic Approach to Human Behavior. Chicago und London: University of Chicago Press.

Becker, Rolf. 2011. Bildungssoziologie - Was sie ist, was sie will, was sie kann. In Lehrbuch der Bildungssoziologie, Hrsg. Rolf Becker, 9 - 36. Wiesbaden: VS Verlag für Sozialwissenschaften.

Becker, Rolf, und Hecken, Anna Etta. 2011. Berufliche Weiterbildung - theoretische Perspektiven und empirische Befunde. In Lehrbuch der Bildungssoziologie, Hrsg. Rolf Becker, 367 - 410. Wiesbaden: VS Verlag für Sozialwissenschaften.

Becker, Rolf. 2006. Dauerhafte Bildungsungleichheiten als unerwartete Folge der Bildungsexpansion? In Die Bildungsexpansion. Erwartete und unerwartete Folgen, Hrsg. Andreas Hadjar, und Rolf Becker, 27 - 61. Wiesbaden: VS Verlag für Sozialwissenschaften.

Beicht, Ursula, und Walden, Günter. 2018. Neue Bildungsexpansion und Verdrängungseffekte in der betrieblichen Ausbildung. Sozialer Fortschritt, 67: Heft 3, 141 - 172. Berlin: Duncker und Humblot.

Beicht, Ursula. 2009. Verbesserung der Ausbildungschancen oder sinnlose Warteschleife? Zur Bedeutung und Wirksamkeit von Bildungsgängen am Übergang Schule Berufsausbildung. BIBB-REPORT $11 / 09$.

http://www.bollettinoadapt.it/old/files/document/3762GER_VERBESSERUNG.pdf (Zugegriffen: 26.03.2021).

Beicht, Ursula, und Ulrich, Joachim Gerd. 2008a. Welche Jugendlichen bleiben ohne Berufsausbildung? Analyse wichtiger Einflussfaktoren unter besonderer Berücksichtigung der Bildungsbiografie. BIBB-Report 6/2008. Forschungs- und Arbeitsergebnisse aus dem Bundesinstitut für Berufsbildung. Bonn: Bundesinstitut für Berufsbildung. $\quad$ https://www.bibb.de/dokumente/pdf/a12_bibbreport_2008_06.pdf (Zugegriffen: 26.03.2021).

Beicht, Ursula, und Ulrich, Joachim Gerd. 2008b. Übergänge von der allgemeinbildenden Schule in eine vollqualifizierende Ausbildung. Ergänzende Analysen für den zweiten 
nationalen Bildungsbericht zum Schwerpunktthema „Übergänge im Bildungssystem und zwischen Bildungssystem und Arbeitsmarkt“" auf Basis der BIBB-Übergangsstudie 2006. Arbeitspapier. Bonn: Bundesinstitut für Berufsbildung. https://web.archive.org/web/20131026193108/http://www.bildungsbericht.de/daten2008/ arbeitspapier_bibb.pdf (Zugegriffen: 26.03.2021).

Beicht, Ursula, Krekel, Elisabeth M., und Walden, Günter. 2004. Berufliche Weiterbildung welchen Nutzen haben die Teilnehmer? BWP 05/2004. Bundesinstitut für Berufsbildung. http://d-nb.info/104986168X/34 (Zugegriffen: 26.03.2021).

Bertelsmann-Stiftung. 2015. Ländermonitor berufliche Bildung 2015. Zusammenfassung der Ergebnisse.

https://www.bertelsmannstiftung.de/fileadmin/files/BSt/Publikationen/GrauePublikationen/LL_GP_Laendermonit orberuflicheBildung_Zusammf_2015_final.pdf (Zugegriffen: 26.03.2021).

Best, Henning, und Wolf, Christof. 2010. Logistische Regression. In Handbuch der sozialwissenschaftlichen Datenanalyse, Hrsg. Christof Wolf, und Henning Best, 827 854. Wiesbaden: VS-Verlag für Sozialwissenschaften.

Blossfeld, Hans-Peter, Roßbach, Hans-Günther, und von Maurice, Jutta. 2011. Editorial. In Education as a Lifelong Process. The German National Educational Panel Study (NEPS), Hrsg. Hans-Peter Blossfeld, Hans-Günther Roßbach, und Jutta von Maurice, 1 - 4. Wiesbaden: VS-Verlag für Sozialwissenschaften. Zeitschrift für Erziehungswissenschaft, Sonderheft 14.

Blossfeld, Hans-Peter. 2010. Survival- und Ereignisanalyse. In Handbuch der sozialwissenschaftlichen Datenanalyse, Hrsg. Christof Wolf, und Henning Best, 995 1016. Wiesbaden: VS-Verlag für Sozialwissenschaften.

Blossfeld, Hans-Peter. 1990. Changes in Educational Careers in the Federal Republic of Germany. In Sociology of Education, 63: Heft 3, 165 - 177.

Blossfeld, Hans-Peter. 1983. Höherqualifizierung und Verdrängung - Konsequenzen der Bildungsexpansion in den Siebziger Jahren. In Beschäftigungssystem im gesellschaftlichen Wandel, Hrsg. Max Haller, und Walter Müller, 184 - 240. Frankfurt am Main: Campus.

BMBF Bundesministerium für Bildung und Forschung. 2005. Berufsbildungsbericht 2005. https://web.archive.org/web/20140828193323/http://www.bmbf.de/pub/bbb_2005.pdf (Zugegriffen: 26.03.2021). 
Brandes, Harald, Brosi, Walter H., und Menk, Angela. 1986. Wege in die berufliche Bildung. Befragung von Lehrlingen in fünf Bundesländern 1983 und 1984. In Mitteilungen aus der Arbeitsmarkt- und Berufsforschung, 19: Heft 2, 287 - 297. http://doku.iab.de/mittab/1986/1986_2_MittAB_Brandes_Brosi_Menk.pdf (Zugegriffen: 26.03.2021).

Brauns, Hildegard, Scherer, Stefani, und Steinmann, Susanne. 2003. The CASMIN Educational Classification in International Comparative Research. In Advances in CrossNational Comparison. An European Working Book for Demographic and SocioEconomic Variables, Hrsg. Jürgen H. P. Hoffmeyer-Zlotnik, und Christof Wolf, 221-244. New York, Boston, Dordrecht, London, Moscow: Kluwer Academic / Plenum Publishers. Bröckling, Ulrich. 2003. Menschenökonomie, Humankapital. Eine Kritik der biopolitischen Ökonomie. $\quad$ Mittelweg, $\quad 1, \quad 3 \quad-\quad 22 . \quad \underline{\text { http://www.his- }}$ online.de/fileadmin/verlag/leseproben/9783936096064.pdf (Zugegriffen: 16.01.2016).

Brzinsky-Fay, Christian, Kohler, Ulrich, und Luniak, Magdalena. 2006. Sequence analysis with Stata. In The Stata Journal, 6: Heft 4, 435-460. College Station, Texas.

Büchtemann, Christoph F., Schupp, Jürgen, und Soloff, Dana J. 1993. Übergänge von der Schule in den Beruf. Deutschland und USA im Vergleich. In Mitteilungen aus der Arbeitsmarkt- und Berufsforschung, 26: Heft 4, 507 - 520. https://web.archive.org/web/20170809081250/http://doku.iab.de/mittab/1993/1993_4_mit tab_buechtemann_schupp_soloff.pdf (Zugegriffen: 26.03.2021).

Bühner, Markus, und Ziegler, Matthias. 2017. Statistik für Psychologen und Sozialwissenschaftler. Hallbergmoos: Pearson. 2. aktualisierte und erweiterte Auflage.

Bühl, Achim. 2014. SPSS 22. Einführung in die moderne Datenanalyse. Hallbergmoos: Pearson.

Bühl, Achim. 2008. SPSS 16. Einführung in die moderne Datenanalyse. München: Pearson Studium.

Bundesamt für Familie und zivilgesellschaftliche Aufgaben. o.J. Das Bundesamt. Chronik. Köln: Bundesamt für Familie und zivilgesellschaftliche Aufgaben. https://web.archive.org/web/20150709011349/http://www.bafza.de/dasbundesamt/chronik.html (Zugegriffen: 26.03.2021).
Bundesamt für Familie un
zivilgesellschaftliche
Aufgaben. o.J. Der Bundesfreiwilligendienst. Über
den BFD. 
https://web.archive.org/web/20180202041440/http://www.bundesfreiwilligendienst.de/de r-bundesfreiwilligendienst/ueber-den-bfd.html (Zugegriffen: 26.03.2021).

Bundesversicherungsamt. 2015. Dritte Bestimmung des Bundesversicherungsamtes nach $\S$ 273 Abs. 2 Satz 5 SGB V zur kassenübergreifenden Auffälligkeitsprüfung im Rahmen der Sicherung der Datengrundlagen für den Risikostrukturausgleich (RSA) in der $\begin{array}{lll}\text { Fassung } & \text { 10.06.2015. }\end{array}$ https://www.bundesversicherungsamt.de/fileadmin/redaktion/Risikostrukturausgleich/Pru efungen/20150610_Bestimmung_273Auff_Pruefung_BJ2010.pdf (Zugegriffen: 26.03.2021).

Bundeswehr.de. 2013. Streitkräfte. Wehrpflicht und Wehrdienst. Geschichte und Statistik. Die Geschichte der Wehrpflicht. Stand vom 03.12.2013. https://web.archive.org/web/20151128185741/http://www.bundeswehr.de/portal/a/bwde/! ut/p/c4/FcbBDYAgDAXQWVygvXtzC_VW8AMNBAk0sr6ad3188qfKo1FM7yqFdz68r m6SmxdoWIda7oJgoInUWyjqk1HE8OkfuOVteQFmm3g_/ (Zugegriffen: 26.03.2021).

Bundeszentrale für politische Bildung. 2016. Dienstleistungssektor. Duden Wirtschaft von A bis Z. Grundlagenwissen für Schule und Studium, Beruf und Alltag. 6. Auflage. Mannheim: Bibliographisches Institut 2016. Lizenzausgabe Bonn: Bundeszentrale für politische Bildung 2016. http://www.bpb.de/nachschlagen/lexika/lexikon-derwirtschaft/19052/dienstleistungssektor (Zugegriffen: 26.03.2021).

Bundeszentrale für politische Bildung. 2011. Wehrpflicht und Zivildienst. Zur Geschichte der Wehrpflicht - Essay. http://www.bpb.de/apuz/59653/zur-geschichte-der-wehrpflichtessay?p=all (Zugegriffen: 26.03.2021).

Bundeszentrale für politische Bildung. 2010. Die Entwicklung der Arbeitslosigkeit in Deutschland. Dossier: Lange Wege der deutschen Einheit. https://web.archive.org/web/20150624133027/http://www.bpb.de/geschichte/deutscheeinheit/lange-wege-der-deutschen-einheit/47242/arbeitslosigkeit?p=all (Zugegriffen: 26.03.2021).

Buttler, Friedrich, und Tessaring, Manfred. 1993. Humankapital als Standortfaktor. Argumente zur Bildungsdiskussion aus arbeitsmarktpolitischer Sicht. In Mitteilungen aus der Arbeitsmarkt- und Berufsforschung, 26: Heft 4, 467 - 476. http://doku.iab.de/mittab/1993/1993_4_MittAB_Buttler_Tessaring.pdf (Zugegriffen: 26.03.2021). 
Carl, Wolfgang, Pohl, Axel, und Schneider, Sabine. 2002. Ehemalige Sindelfinger HauptschülerInnen im Übergang in die Arbeitswelt. Ergebnisse der Längsschnittuntersuchung zum beruflichen Verbleib von HauptschulabsolventInnen in Sindelfingen. IRIS e. V. http://www.neccessaire.com/capaconsult/docs/19972002\%20SAFRAN-Untersuchung.doc (Zugegriffen: 26.03.2021).

CESifo Group Munich. 2017. ifo Institut. Center for Economic Studies (CES). CESifo

GmbH. Fakten. Glossar zum ifo Wirtschaftskompass. Bildung. CASMINBildungsklassifikation. München: ifo-Institut. https://web.archive.org/web/20130709142021/https://www.cesifogroup.de/de/ifoHome/facts/Glossar/05-Bildung/CASMIN-Bildungsklassifikation.html (Zugegriffen: 26.03.2021).

Christe, Gerhard. 2016. Neue Konzepte für den Übergang in Ausbildung. Ausbildungschancen für alle. Gute Gesellschaft - gute Demokratie. \#2017 plus. Bonn: Friedrich-Ebert-Stiftung. https://library.fes.de/pdf-files/wiso/12497.pdf (Zugegriffen: 26.03.2021).

Dahrendorf, Ralf. 1965. Bildung ist Bürgerrecht. Plädoyer für eine aktive Bildungspolitik. Hamburg: Nannen-Verlag.

Diaz-Bone, Rainer, und Weischer. Christoph. 2015. Methoden-Lexikon für die Sozialwissenschaften. Wiesbaden: Springer-VS.

Dobischat, Rolf. 2010. Schulische Berufsbildung im Gesamtsystem der beruflichen Bildung. Herausforderungen an der Übergangspassage von der Schule in den Beruf. In Das Berufsbildungssystem in Deutschland. Aktuelle Entwicklungen und Standpunkte, Hrsg. Gerhard Bosch, Sirikit Krone, und Dirk Langer, 101 - 131. Wiesbaden: VS-Verlag für Sozialwissenschaften.

Döring, Nicola, und Bortz, Jürgen. 2016. Datenanalyse. In Forschungsmethoden und Evaluation in den Sozial- und Humanwissenschaften, Hrsg. Nicola Döring, und Jürgen Bortz, 597 - 784. Berlin, Heidelberg: Springer. 5. vollständig überarbeitete, aktualisierte und erweiterte Auflage.

Druwe, Ulrich. 2011. Wilhelm v. Humboldts „Idee der Universität“. Pädagogische Hochschule Freiburg. Hochschuldidaktischer Tag am 14.10.2011. Präsentation. https://www.ph-

freiburg.de/fileadmin/dateien/zentral/zwh/hochschuldidaktik/WS2011_2012/Praesentatio n-Druwe.pdf (abgerufen am 18.11.2020). 
Duales Studium Hessen. o.J. Karriere und Zukunft. Lass deine Chancen wachsen! Heute im Hörsaal. Morgen im Betrieb. Zukunft in Hessen. http://www.dualesstudium-hessen.de/ (Zugegriffen: 26.03.2021).

Eberhard, Verena, Beicht, Ursula, Krewerth, Andreas, und Ulrich, Joachim Gerd. 2013. Perspektiven beim Übergang Schule - Berufsausbildung. Methodik und erste Ergebnisse aus der BIBB-Übergangsstudie 2011. Bundesinstitut für Berufsbildung. Wissenschaftliche Diskussionspapiere. Heft 142. Bonn: Bundesinstitut für Berufsbildung. https://web.archive.org/web/20150702052230/http://www.fachkraeftebuero.de/fileadmin/ user_upload/Daten_und_Fakten/BIBB-UEbergangsstudie_2011.pdf

(Zugegriffen: 26.03.2021).

Eberhard, Verena, und Ulrich, Joachim Gerd. 2010. Übergänge zwischen Schule und Berufsausbildung. In Das Berufsbildungssystem in Deutschland. Aktuelle Entwicklungen und Standpunkte, Hrsg. Gerhard Bosch, Sirikit Krone, und Dirk Langer, 133 - 164. Wiesbaden: VS-Verlag für Sozialwissenschaften.

Eberhard, Verena, und Ulrich, Joachim Gerd. 2009. „Ausbildungsreif“ und dennoch ein Fall für das Übergangssystem? Determinanten der Einmündung von Ausbildungsstellenbewerbern in teilqualifizierende Bildungsgänge. Bonn: Bundesinstitut für Berufsbildung. Neue Jugend? Neue Ausbildung? BIBB-Fachtagung 28./29. Oktober 2009 im Gustav-Stresemann-Institut Bonn. https://web.archive.org/web/20121115092439/http://www.bibb.de/dokumente/pdf/a12pr veranstaltung_2009_10_28_neue_jugend_neue_ausbildung_eberhard_ulrich.pdf (Zugegriffen: 26.03.2021).

Eckert, Thomas. 2010. Bildungsstatistik. In Handbuch Bildungsforschung, Hrsg. Rudolf Tippelt, und Bernhard Schmidt, 589 - 606. Wiesbaden: VS-Verlag für Sozialwissenschaften. 3. durchgesehene Auflage.

Elliott, Robert F. 1991. Labor Economics. A comparative text. London: McGraw-Hill College.

Erban, Tanja Alexandra. 2010. Das Berufsvorbereitungsjahr als Übergang von der Schule zum Beruf. Eine Längsschnittuntersuchung zum Verbleib eines Absolventenjahrgangs und zur Wirksamkeit des Berufsvorbereitungsjahres. München: Technische Universität.

erzieherin-ausbildung.de. o.J. Anerkennungsjahr und Berufspraktikum. http://www.erzieherin-ausbildung.de/content/anerkennungsjahr-und-berufspraktikum (Zugegriffen: 26.03.2021). 
Fachhochschule für öffentliche Verwaltung NRW. 2018a. Studium. Studieren an der FHöV. Übersicht.

https://web.archive.org/web/20190812141846/https://www.fhoev.nrw.de/studium/studier en-an-der-fhoev/uebersicht/ (Zugegriffen: 26.03.2021).

Fachhochschule für öffentliche Verwaltung NRW. 2018b. Studium. Studieren an der FHöV. $\begin{array}{lllll}\text { Zugang } \quad \& \quad \text { Zulassung. Zugang } & \text { zum }\end{array}$ https://web.archive.org/web/20190812084528/https://www.fhoev.nrw.de/studium/studier en-an-der-fhoev/zugang-zulassung/zugang-zum-studium/ (Zugegriffen: 26.03.2021).

Fahrmeir, Ludwig. 2007. Lebensdauer- und Ereignisanalyse. Vorlesungsskript. Institut für Statistik. Ludwig-Maximilians-Universität $\quad$ München. $\quad$ SS http://www.lichtundgesundheit.de/Lichtundgesundheit/Blog/Entries/2014/8/29_Wie_bere chnet_man_Lebensdauer_files/Survival.pdf (Zugegriffen: 26.03.2021).

Fink, Christina. 2011. Der Übergang von der Schule in die berufliche Ausbildung. Perspektiven für die kommunale Bildungslandschaft. Wiesbaden: VS-Verlag für Sozialwissenschaften.

Friebertshäuser, Barbara. 2008. Statuspassage von der Schule ins Studium. In Handbuch der Schulforschung, Hrsg. Werner Helsper, und Jeanette Böhme, 611 - 627. Wiesbaden: VSVerlag für Sozialwissenschaften. 2. durchgesehene und erweitere Auflage.

Forschungsdatenzentrum der Bundesagentur für Arbeit im Institut für Arbeitsmarkt- und Berufsforschung. o.J. Personendaten/Haushaltsdaten. Arbeiten und Lernen im Wandel (ALWA). $\quad$ http://fdz.iab.de/de/FDZ_Individual_Data/ALWA.aspx 26.03.2021).

Fürstenberg, Friedrich. 1978. Struktureller Qualifikationsüberhang und seine Folgen. In Ausbildungsgrad und Beschäftigung, Hrsg. Gerhard Brinkmann, 83 - 95. Berlin: Duncker \& Humblot.

Gaupp, Nora, Geier, Boris, Lex, Tilly, und Reißig, Birgit. 2011. Wege in Ausbildungslosigkeit. Determinanten misslingender Übergänge in Ausbildung von Jugendlichen mit Hauptschulbildung. In Zeitschrift für Pädagogik, 57: Heft 2, 173 - 186. Frankfurt am Main: Deutsches Institut für Pädagogische Forschung (DIPF). http://www.pedocs.de/volltexte/2014/8713/pdf/ZfPaed_2_2011_Gaupp_et_al_Wege_in_ Ausbildungslosigkeit.pdf (Zugegriffen: 26.03.2021).

Gaupp, Nora, Lex, Tilly, und Reißig, Birgit. 2008. Ohne Schulabschluss in die Berufsausbildung. Ergebnisse einer Längsschnittuntersuchung. In Zeitschrift für 
Erziehungswissenschaft, 11: Heft 3, 388-405. Berlin; Heidelberg: Springer; Wiesbaden: VS Verl. für Sozialwissenschaften.

Gaupp, Nora, und Geier, Boris. 2008. Stuttgarter Haupt- und Förderschüler/innen auf dem Weg von der Schule in die Berufsausbildung. Bericht zur zweiten Erhebung der Stuttgarter Schulabsolventenstudie. Stuttgart. Deutsches Jugendinstitut. September 2008. https://web.archive.org/web/20200922134656/http://www.perspektive-

berufsabschluss.de/downloads/Downloads_Projekte_Uebergangsmanagement/Uebergang smanagement_Stuttgart_folgeerhebung2008.pdf (Zugegriffen: 26.03.2021).

Gaupp, Nora, 2008. Schule - und dann? Hauptschülerinnen und Hauptschüler auf dem Weg von der Schule in die Arbeitswelt. Ergebnisse aus dem DJI-Übergangspanel. München: Deutsches Jugendinstitut. Forschungsschwerpunkt „Übergänge in Arbeit“. http://www2.ibw.uni-heidelberg.de/wisskoll/pdf/gaupp.pdf (Zugegriffen: 26.03.2021).

Gaupp, Nora, Hofmann-Lun, Irene, Lex, Tilly, und Reißig, Birgit. 2005. Jugendliche in bayerischen Praxisklassen. Reelle Chancen auch ohne Schulabschluss? In Chancen für Schulmüde. Reader zur Abschlusstagung des Netzwerks Prävention von Schulmüdigkeit und Schulverweigerung am Deutschen Jugendinstitut e. V. Forschungsschwerpunkt Übergänge in Arbeit. 16. September 2005 in der GaraGe - Technologiezentrum für Jugendliche gGmbH Leipzig, Hrsg. Deutsches Jugendinstitut, 132 - 149. München und Halle: Deutsches Jugendinstitut e. V. https://www.dji.de/fileadmin/user_upload/bibs/229_6264_Reader_Chancen_fuer_Schulm uede.pdf\#page=134 (Zugegriffen: 26.03.2021).

Gerlach, Irene, und Laß, Inga. 2012. Gesellschaftliche Rahmenbedingungen betrieblicher Familienpolitik. In Betriebliche Familienpolitik. Kontexte, Messungen und Effekte, Hrsg. Irene Gerlach, und Helmut Schneider, 29 - 44. Wiesbaden: VS-Verlag für Sozialwissenschaften.

Grace-Martin, Karen. 2019a. The Analysis Factor. The Exposure Variable in Poisson Regression Models. https://www.theanalysisfactor.com/the-exposure-variable-inpoission-regression-models/ (Zugegriffen: 26.03.2021).

Grace-Martin, Karen. 2019b. The Analysis Factor. Poisson Regression Analysis for Count Data. https://www.theanalysisfactor.com/poisson-regression-analysis-for-count-data/ (Zugegriffen: 26.03.2021). 
Hadjar, Andreas, und Becker, Rolf. 2006. Bildungsexpansion - erwartete und unerwartete Folgen. In Die Bildungsexpansion. Erwartete und unerwartete Folgen, Hrsg. Andreas Hadjar, und Rolf Becker, 11 - 24. Wiesbaden: VS Verlag für Sozialwissenschaften.

Hammon, Angelina, Zinn, Sabine, Aßmann, Christian, und Würbach, Ariane. 2016. Samples, weights, and nonresponse. The adult cohort of the National Educational Panel Study (Wave 2 to 6). NEPS Survey Paper No. 7. November 2016. Bamberg: Leibniz Institute for educational trajectories. https://www.nepsdata.de/Portals/0/NEPS/Datenzentrum/Forschungsdaten/SC6/9-0-0/SC6_6-0-0_W.pdf (Zugegriffen: 26.03.2021).

Hanesch, Walter. 1990. Unterversorgung im Bildungssystem. Das Beispiel berufliche Bildung. In Armut im Wohlstand, Hrsg. Diether Döring, Walter Hanesch, und ErnstUlrich Huster, 185 - 205. Frankfurt am Main: Suhrkamp-Verlag.

Heine, Christoph, Quast, Heiko, und Beuße, Mareike. 2010. Studienberechtigte 2008 ein halbes Jahr nach Schulabschluss. Übergang in Studium, Beruf und Ausbildung. Hannover: HIS: Forum Hochschule. 3 | 2010. http://www.wissenschaftsmanagementonline.de/sites/www.wissenschaftsmanagement-online.de/files/migrated_wimoarticle/fh201003.pdf (Zugegriffen: 26.03.2021).

Hillmert, Steffen, und Jacob, Marita. 2003. Social inequality in higher education. Is vocational training a pathway leading to or a away from university? In European Sociological Review, 19: Heft 3, 319 - 334. Published by: Oxford University Press. Issue Stable URL: http://www.jstor.org/stable/i369569. (Zugegriffen: 26.03.2021).

Hillmert, Steffen. 2001. Kohortendynamik und Konkurrenz an den zwei Schwellen des dualen Ausbildungssystems. Übergänge zwischen Schule und Arbeitsmarkt im Kontext ökonomischen und demographischen Wandels. Arbeitspapier Nr. 2 des Projekts Ausbildungs- und Berufsverläufe der Geburtskohorten 1964 und 1971 in Westdeutschland. Berlin: Max-Planck-Institut für Bildungsforschung. http://library.mpib-berlin.mpg.de/ft/shi/SHI_Kohortendynamik_2001.pdf (Zugegriffen: 26.03.2021).

Hochschulrektorenkonferenz: Hochschulkompass. o.J. Hochschularten in Deutschland. http://www.hochschulkompass.de/studium/hilfe-bei-der-studienwahl/hochschularten.html (Zugegriffen: 26.03.2021). 
Holtmann, Anne Christine, Menze, Laura, und Solga, Heike. 2019. Schulabgänger und -abgängerinnen mit maximal Hauptschulabschluss. In Handbuch Bildungsarmut, Hrsg. Gudrun Quenzel, und Klaus Hurrelmann, 365 - 388. Wiesbaden: Springer.

Holtmann, Anne Christine, Menze, Laura, und Solga, Heike. 2018. Unentdeckte Kompetenzen. Jugendliche ohne Mittleren Schulabschluss finden schwer einen Ausbildungsplatz. WZBrief Bildung, 36, 1-7. Berlin: Wissenschaftszentrum Berlin für Sozialforschung. https://www.econstor.eu/bitstream/10419/182537/1/1031642013.pdf (Zugegriffen: 26.03.2021).

Horstkemper, Marianne, und Tillmann, Klaus-Jürgen. 2008. Schulformvergleiche und Studien zu Einzelschulen. In Handbuch der Schulforschung, Hrsg. Werner Helsper, und Jeanette Böhme, 285 - 320. Wiesbaden: VS-Verlag für Sozialwissenschaften. 2. durchgesehene und erweiterte Auflage.

IBM Knowledge Center. o.J. IBM Home. SPSS Statistics 23.0.0. Hilfe. Option „Statistics base". Bivariate

Korrelationen. https://www.ibm.com/support/knowledgecenter/de/SSLVMB_23.0.0/spss/base/idh_corr. html (Zugegriffen: 26.03.2021).

idre Institute for Digital Research and Education. 2021. UCLA: Statistical Consulting. Testing the proportional hazard assumption in Cox models. https://stats.idre.ucla.edu/other/examples/asa2/testing-the-proportional-hazardassumption-in-cox-models/ (Zugegriffen: 26.03.2021).

idre Institute for Digital Research and Education. 2020. UCLA: Statistical Consulting. \begin{tabular}{ll|lll} 
Poisson & Regression & Stata ata
\end{tabular} https://stats.idre.ucla.edu/stata/dae/poisson-regression/ (Zugegriffen: 26.03.2021).

Jacob, Marita, und Weiss, Felix. 2010. Soziale Selektivität beim Hochschulzugang Veränderungen der Zugangssequenzen zur Hochschule im Kohortenvergleich. In Vom Kindergarten bis zur Hochschule. Die Generierung von ethnischen und sozialen Disparitäten in der Bildungsbiographie, Hrsg. Birgit Becker, und David Reimer, 285 312. Wiesbaden: VS Verlag für Sozialwissenschaften.

Jacob, Marita, und Tieben, Nicole. 2010. Wer nutzt die Durchlässigkeit zwischen verschiedenen Schulformen? Soziale Selektivität bei Schulformwechseln und nachgeholten Schulabschlüssen. In Vom Kindergarten bis zur Hochschule. Die Generierung von ethnischen und sozialen Disparitäten in der Bildungsbiographie, Hrsg. 
Birgit Becker, und David Reimer, 145 - 178. Wiesbaden: VS-Verlag für Sozialwissenschaften.

Kamm, Ruth. 2014. Hochschulreformen in Deutschland. Hochschulen zwischen staatlicher Steuerung und Wettbewerb. Bamberg: University of Bamberg Press. Serie: Schriften aus der Fakultät Sozial- und Wirtschaftswissenschaften der Otto-Friedrich-Universität Bamberg; 18.

KJS Katholische Jugendsozialarbeit. 2014. Ausbildung. Verdrängen Abiturienten $\begin{array}{llll}\text { Hauptschüler vom } \quad \text { Ausbildungsmarkt? } & \text { News-Archiv. }\end{array}$ http://www.bagkjs.de/ausbildung_werden_hauptschueler_verdraengt (Zugegriffen: 29.08.2014).

Kleinert, Corinna, und Jacob, Marita. 2012. Strukturwandel des Übergangs in eine berufliche Ausbildung. In Soziologische Bildungsforschung, Hrsg. Rolf Becker, und Heike Solga, 211 - 233. Wiesbaden: Imprint: Springer VS, 2012. Serie: Kölner Zeitschrift für Soziologie und Sozialpsychologie Sonderhefte ; 52.

Kleinert, Corinna, Matthes, Britta, und Jacob, Marita. 2008. Die Befragung „Arbeiten und Lernen im Wandel“. Theoretischer Hintergrund und Konzeption. IAB-Forschungsbericht 5/2008. Nürnberg: Institut für Arbeitsmarkt- und Berufsforschung. http://doku.iab.de/forschungsbericht/2008/fb0508.pdf (Zugegriffen: 26.03.2021).

Knauf, Helen, und Rosowski, Elke. 2009. Wie tragfähig ist die Studien- und Berufswahl? Biographische Verläufe und Orientierungsprozesse nach dem Abitur. In Abitur und was dann? Berufsorientierung und Lebensplanung junger Frauen und Männer und der Einfluss von Schule und Eltern, Hrsg. Mechtild Oechsle, Helen Knauf, Christiane Maschetzke, und Elke Rosowski, 283 - 324. Wiesbaden: VS-Verlag für Sozialwissenschaften.

Kock, Klaus. 2008. Auf Umwegen in den Beruf. Destandardisierte und prekäre Beschäftigung von Jugendlichen an der zweiten Schwelle - eine Auswertung empirischer Befunde. Hans-Böckler-Stiftung. Dortmund: Technische Universität. Sozialforschungsstelle Dortmund. http://www.kowa.sfs.tudortmund.de/cms/Medienpool/files_veroeffentlichungen/Auf-Umwegen-in-den-Beruf.pdf (Zugegriffen: 26.03.2021).

Konietzka, Dirk. 1999. Beruf und Ausbildung im Generationenvergleich. In Beruf und Berufsbildung. Situation, Reformperspektiven, Gestaltungsmöglichkeiten, Hrsg. Klaus Harney, und Heinz-Elmar Tenorth, 289-320. Weinheim u.a. : Beltz. - (Zeitschrift für 
Pädagogik, Beiheft; 40) - URN: urn:nbn:de:0111-opus-85224. http://www.pedocs.de/volltexte/2014/8522/pdf/Konietzka_1999_Beruf_und_Ausbildung _im_Generationenvergleich.pdf (Zugegriffen: 26.03.2021).

Konsortium Bildungsberichterstattung. 2006. Bildung in Deutschland. Ein indikatorengestützter Bericht mit einer Analyse zu Bildung und Migration. Frankfurt am Main.

https://web.archive.org/web/20160402090155/http://www.bildungsbericht.de/daten/press emitteilung_bb06.pdf (Zugegriffen: 26.03.2021).

Kristen, Cornelia. 1999. Bildungsentscheidungen und Bildungsungleichheit - ein Überblick über den Forschungsstand. Mannheim: Arbeitspapier des Mannheimer Zentrums für Europäische Sozialforschung (MZES), Nr. 5.

Krone, Sirikit. 2010. Aktuelle Problemfelder der Berufsbildung in Deutschland. In Das Berufsbildungssystem in Deutschland. Aktuelle Entwicklungen und Standpunkte, Hrsg. Gerhard Bosch, Sirikit Krone, und Dirk Langer, 19 - 36. Wiesbaden: VS-Verlag für Sozialwissenschaften.

Krüger-Charlé, Michael. 2010. Übergänge zwischen Schule, Ausbildung und Beruf. Strukturen, Einschätzungen und Gestaltungsperspektiven. Forschung aktuell. Gelsenkirchen: Institut Arbeit und Technik der Fachhochschule. 2. korrigierte Fassung vom 14.10.2010. http://www.iatge.de/forschung-aktuell/2010/fa2010-11.pdf (Zugegriffen: 26.03.2021).

Kühnel, Steffen M., und Krebs, Dagmar. 2010. Multinomiale und ordinale Regression. In Handbuch der sozialwissenschaftlichen Datenanalyse, Hrsg. Christof Wolf, und Henning Best, 855 - 886. Wiesbaden: VS-Verlag für Sozialwissenschaften.

Kultusministerkonferenz. o.J. Themen. Berufliche Schulen. Berufliche Weiterbildung. Berufliche Weiterbildung an Fachschulen - ein Karrieresprungbrett. https://www.kmk.org/themen/berufliche-schulen/berufliche-weiterbildung.html (Zugegriffen: 26.03.2021).

Landesapothekerkammer Hessen. 2017. Pharmazie. Ausbildung. Apotheker/in. Pharmazeuten im Praktikum. https://web.archive.org/web/20170425040615/http://www.apothekerkammer.de/pharmazi e/ausbildung/apotheker+in/pharmazeuten+im+praktikum/ (Zugegriffen: 26.03.2021). 
Lauterbach, Wolfgang. 2012. Sekundäre Bildung in Deutschland. In Handbuch Bildungs- und Erziehungssoziologie, Hrsg. Ullrich Bauer, Uwe H. Bittlingmayer, und Albert Scherr, 573 - 593. Wiesbaden: VS-Verlag für Sozialwissenschaften.

Lewin, Karl, Minks, Karl-Heinz, und Uhde, Sönke. 1996. Abitur - Berufsausbildung Studium. Zur Strategie der Doppelqualifizierung von Abiturienten. In Mitteilungen aus der Arbeitsmarkt- und Berufsforschung, 29: Heft 3, 431 - 454. http://213.241.152.197/mittab/1996/1996_3_MittAB_Lewin_Minks_Uhde.pdf (Zugegriffen: 26.03.2021).

Lewin, Karl, und Schacher, Martin. 1990. Studienberechtigte des Jahres 1976 auf dem Weg in den Beruf bis 1988. Erwartungen alles in allem erfüllt. Hannover: HIS HochschulInformations-System GmbH. Hochschulplanung Band 84.

Lex, Tilly, und Zimmermann, Julia. 2011. Wege in Ausbildung. Befunde aus einer schrittweisen Betrachtung des Übergangsprozesses. In Zeitschrift für Erziehungswissenschaft, 14: Heft 4: 603 - 627. München: Deutsches Jugendinstitut. Online publiziert: 15.11.2011.

Lex, Tilly, und Geier, Boris. 2010. Übergangssystem in der beruflichen Bildung: Wahrnehmung einer zweiten Chance oder Risiken des Ausstiegs? In Das Berufsbildungssystem in Deutschland. Aktuelle Entwicklungen und Standpunkte, Hrsg. Gerhard Bosch, Sirikit Krone, und Dirk Langer, 165 - 187. Wiesbaden: VS-Verlag für Sozialwissenschaften.

Lohmann, Henning. 2010. Nicht-Linearität und Nicht-Additivität in der multiplen Regression: Interaktionseffekte, Polynome und Splines. In Handbuch der sozialwissenschaftlichen Datenanalyse, Hrsg. Christof Wolf, und Henning Best, 677 - 706. Wiesbaden: VS-Verlag für Sozialwissenschaften.

Maaz, Kai. 2006. Soziale Herkunft und Hochschulzugang. Effekte institutioneller Öffnung im Bildungssystem. Wiesbaden: VS Verlag für Sozialwissenschaften. 1. Aufl.

Machin, David, Cheung, Yin Bun, und Parmar, Mahesh K. B. 2006. Survival Analysis. A Practical Approach. Second Edition. Chichester, West Sussex: John Wiley \& Sons.

Matheus, Sylvia. 1995. Wechselmöglichkeiten aus industriellen Berufen in Dienstleistungsberufe. Eine Analyse mit Daten des Sozioökonomischen Panels. In Mitteilungen aus der Arbeitsmarkt- und Berufsforschung, 28: Heft 2, 224 - 238. https://web.archive.org/web/20060929072609/http://doku.iab.de/mittab/1995/1995_2_mit tab_matheus.pdf (Zugegriffen: 26.03.2021). 
Matthes, Britta, und Trahms, Annette. 2012. Arbeiten und Lernen im Wandel. Teil II Codebuch. Korrigierte Version. In FDZ Datenreport. Dokumentation zu Arbeitsmarktdaten. 02/2010. Forschungsdatenzentrum der Bundesagentur für Arbeit am $\begin{array}{llll}\text { Institut für } \quad \text { Arbeitsmarkt- } & \text { Bnd }\end{array}$ http://doku.iab.de/fdz/reporte/2010/DR_02-10.pdf (Zugegriffen: 26.03.2021).

Menze, Laura, und Holtmann, Anne Christine. 2019. Was können Schulabgängerinnen und Schulabgänger ohne Mittleren Schulabschluss aus Übergangsmaßnahmen mitnehmen? Entwicklungen und Übergangschancen in Ausbildung. In Zeitschrift für Erziehungswissenschaft, 22: Heft 3: 509 - 533. Berlin, Heidelberg: Springer; Wiesbaden: VS-Verlag für Sozialwissenschaften.

Mollwo, Ingrid. 1980. Der Übergang der Absolventen eines Berufsgrundbildungsjahres in eine weitere Berufsausbildung bzw. in die Erwerbstätigkeit. Aus der Untersuchung des IAB: Jugendliche beim Übergang vom Bildungs- in das Beschäftigungssystem (Projekt 3-213). In Mitteilungen aus der Arbeitsmarkt- und Berufsforschung, 13: Heft 2, $242-$ 254. http://doku.iab.de/mittab/1980/1980_2_MittAB_Mollwo.pdf (Zugegriffen: 26.03.2021).

NEPS National Education Panel Study. 2020. NEPS Starting Cohort 6-Adults. Adult Education and Lifelong Learning. Data Manual. Scientific Use File Version 11.0.0. FDZ-LIfBi. Bamberg: Leibniz Institute for Educational Trajectories (LIfBi). https://www.neps-data.de/Portals/0/NEPS/Datenzentrum/Forschungsdaten/SC6/11-00/SC6_11-0-0_Datamanual.pdf (Zugegriffen: 26.03.2021).

NEPS Nationales Bildungspanel. 2020. Codebook. NEPS Startkohorte 6 - Erwachsene. Bildung im Erwachsenenalter und lebenslanges Lernen. Scientific Use File Version 11.0.0. Forschungsdatenzentrum Leibniz-Institut für Bildungsverläufe. https://www.nepsdata.de/Portals/0/NEPS/Datenzentrum/Forschungsdaten/SC6/11-0-0/SC6_11-0-

0_Codebook_de.pdf (Zugegriffen: 26.03.2021).

NEPS Nationales Bildungspanel. 2016a. Startkohorte 6: Erwachsene (SC 6). Studienübersicht. Wellen 1 bis 7. Bamberg: Leibniz-Institut für Bildungsverläufe e. V. https://www.nepsdata.de/Portals/0/NEPS/Datenzentrum/Forschungsdaten/SC6/SC6_Studien_W1-7.pdf (Zugegriffen: 26.03.2021).

NEPS Nationales Bildungspanel. 2016b. Startkohorte 6: Erwachsene (SC6). SUF-Version 7.0.0. Codebook (de). Bamberg: Leibniz-Institut für Bildungsverläufe e. V. 
https://www.neps-data.de/Portals/0/NEPS/Datenzentrum/Forschungsdaten/SC6/7-00/SC6_7-0-0_Codebook_de.pdf (Zugegriffen: 26.03.2021).

NEPS Nationales Bildungspanel. 2009. Erwachsene. Befragung von Erwachsenen. Interviewerhandbuch. Bonn: infas Institut für angewandte Sozialwissenschaft. GmbH. Hauptstudie November 2009. infas 4335 - 11/2009. https://www.nepsdata.de/Portals/0/Neps/Datenzentrum/Forschungsdaten/SC6/1-0-

0/Interviewerhandbuch_Erst_B72.pdf (Zugegriffen: 26.03.2021).

NEPS-Datenzentrum. o.J. a. Bamberg: Leibniz-Institut für Bildungsverläufe e. V. (LIfBi). Startkohorte Erwachsene. Bildung im Erwachsenenalter und lebenslanges Lernen. https://web.archive.org/web/20160811112229/https://www.neps-data.de/dede/datenzentrum/datenunddokumentation/startkohorteerwachsene.aspx (Zugegriffen: 26.03.2021).

NEPS-Datenzentrum. o.J. b. Bamberg: Leibniz-Institut für Bildungsverläufe e. V. (LIfBi). Übersichten und Hilfen. Übersicht über das Datenangebot. https://web.archive.org/web/20160810112157/https://www.neps-data.de/dede/tabid/2658/language/de-DE/Default.aspx (Zugegriffen: 26.03.2021).

NEPS-Datenzentrum. o.J. c. Bamberg: Leibniz-Institut für Bildungsverläufe e. V. (LIfBi). Daten und Dokumentation. Starting Cohort Adults. doi: 10.5157/NEPS:SC6:5.1.0. https://www.neps-data.de/tabid/2665/language/en-US/ (Zugegriffen: 26.03.2021).

NEPS-Datenzentrum. o.J. d. Bamberg: Leibniz-Institut für Bildungsverläufe e. V. (LIfBi). Daten und Dokumentation. Die Panelstudien des NEPS. https://web.archive.org/web/20160811111420/https://www.neps-data.de/dede/datenzentrum/datenunddokumentation.aspx (Zugegriffen: 26.03.2021).

Nuissl, Ekkehard, und Brandt, Peter. 2009. Porträt Weiterbildung Deutschland. Bielefeld: W. Bertelsmann-Verlag. 2. vollständig überarbeitete Auflage. Herausgebende Institution: Deutsches Institut für Erwachsenenbildung (DIE). http://www.die-bonn.de/doks/2009weiterbildungsforschung-deutschland-01.pdf (Zugegriffen: 26.03.2021).

Palamidis, Helene, und Schwarze, Johannes. 1989. Jugendliche beim Übergang in eine betriebliche Berufsausbildung und in die Erwerbstätigkeit. Literaturüberblick und neuere empirische Ergebnisse. In Mitteilungen aus der Arbeitsmarkt- und Berufsforschung, 22: Heft 1, $114 \quad-\quad$ 124. Nürnberg: Bundesagentur für Arbeit. https://web.archive.org/web/20190713183536/http://doku.iab.de/mittab/1989/1989_1_mit tab_palamidis_schwarze.pdf (Zugegriffen: 26.03.2021). 
Pätzold, Günter. 2008. Übergang Schule - Berufsausbildung. In Handbuch der Schulforschung, Hrsg. Werner Helsper, und Jeanette Böhme, 593 - 610. Wiesbaden: VSVerlag für Sozialwissenschaften. 2. durchgesehene und erweitere Auflage.

Persch, Peter-Roman. 2008. Die Bewertung von Humankapital - eine kritische Analyse. Mering: Rainer Hampp Verlag.

Picht, Georg. 1965. Die deutsche Bildungskatastrophe. München: Deutscher TaschenbuchVerlag.

Plicht, Hannelore. 2016. Die ersten fünf Jahre nach einer berufsvorbereitenden Bildungsmaßnahme $(B v B)$ - Befunde zum Übergang in Ausbildung und Beschäftigung. In Sozialer Fortschritt, 65, Heft 6, 142 - 151. Berlin: Duncker und Humblot.

Protsch, Paula. 2014. Segmentierte Ausbildungsmärkte. Berufliche Chancen von Hauptschülerinnen und Hauptschülern im Wandel. Opladen [u. a.]: Budrich UniPress. https://library.oapen.org/bitstream/handle/20.500.12657/25666/1004425.pdf?sequence=1 \&isAllowed=y (Zugegriffen: 26.03.2021).

Rehadat Institut der deutschen Wirtschaft Köln e. V. 2017. Rehadat Bildung. Bildungsmaßnahmen. Berufsvorbereitende Bildungsmaßnahme der Agentur für Arbeit (BvB). $\quad$ http://www.rehadat-bildung.de/de/angebote/BvB-berufsvorbereitendebildungsmassnahme/ (Zugegriffen: 26.03.2021).

Reißig, Birgit, und Gaupp, Nora. 2018. Übergänge Jugendlicher von Schule in Ausbildung aus soziologischer Perspektive. In Handbuch Kindheits- und Jugendsoziologie, Hrsg. Andreas Lange, Herwig Reiter, Sabina Schutter, und Christine Steiner, 191 - 202. Wiesbaden: Springer Fachmedien.

Rissiek, Jörg. 1998. Investitionen in Humankapital. Wiesbaden: Deutscher UniversitätsVerlag.

Rohrbach-Schmidt, Daniela. 2010. BIBB-Übergangsstudie 2006. BIBB-FDZ Daten- und Methodenberichte. Nr. 1/2010. Bonn: Bundesinstitut für Berufsbildung Forschungsdatenzentrum.

https://web.archive.org/web/20160506144041/http://www.bibb.de/dokumente/pdf/Ueber gangsstudie_2006_Datenhandbuch_1.0.pdf (Zugegriffen: 26.03.2021).

Rukwid, Ralf. 2011. Der Übergang von der Schule in die Berufsausbildung - eine Analyse für Baden-Württemberg. JEL No. I2, J7, R1. Oktober 2011. http://www.bildung-undbeschaeftigung.de/aktuelles/kritik-an-selektion/studie-duales-system-rukwid.pdf (Zugegriffen: 19.05.2015). 
Schendera, Christian FG. 2014. Regressionsanalyse mit SPSS. München: De Gruyter Oldenbourg.

Scherer, Stefani, und Brüderl, Josef. 2010. Sequenzdatenanalyse. In Handbuch der sozialwissenschaftlichen Datenanalyse, Hrsg. Christof Wolf, und Henning Best, 1031 1051. Wiesbaden: VS-Verlag für Sozialwissenschaften.

Schindler, Steffen. 2012. Aufstiegsangst? Eine Studie zur sozialen Ungleichheit beim Hochschulzugang im historischen Zeitverlauf. Düsseldorf: Vodafone Stiftung Deutschland. http://www.uniheidelberg.de/md/journal/2012/11/studie_hochschulzugang_web.pdf (Zugegriffen: 26.03.2021).

Schober, Karen. 1985. Jugend im Wartestand. Zur aktuellen Situation der Jugendlichen auf dem Arbeits- und Ausbildungsstellenmarkt. In Mitteilungen aus der Arbeitsmarkt- und $\begin{array}{lllllll}\text { Berufsforschung, } & \text { 18: } & \text { Heft } & 2, & 247 & - & 264\end{array}$ https://web.archive.org/web/20181103045946/http://doku.iab.de/mittab/1985/1985_2_mit tab_schober.pdf (Zugegriffen: 26.03.2021).

Schultz, Theodore William. 1961. Education and Economic Growth. In Social Forces Influencing American Education, Hrsg. Henry B. Nelson, 46 - 88. Chicago: University of Chicago Press.

Schulze-Bentrop, Carsten. 2014. Management von Markentransfers. Der Einfluss des Kaufentscheidungsverhaltens auf die Erfolgsfaktoren. Wiesbaden: Springer Gabler. Zugleich: Dissertation Westfälische Wilhelms-Universität Münster, 2013.

Seibert, Holger, und Kleinert, Corinna. 2009. Duale Berufsausbildung. Ungelöste Probleme trotz Entspannung. IAB-Kurzbericht 10/2009. Nürnberg: Institut für Arbeitsmarkt- und Berufsforschung. http://doku.iab.de/kurzber/2009/kb1009.pdf (Zugegriffen: 26.03.2021). Severing, Eckart. 2010. Berufsausbildung in Deutschland - $\mathrm{Zu}$ wenige Fachkräfte für die Wirtschaft und $\mathrm{zu}$ viele Jugendliche ohne Ausbildungsperspektive. In Das Berufsbildungssystem in Deutschland. Aktuelle Entwicklungen und Standpunkte, Hrsg. Gerhard Bosch, Sirikit Krone, und Dirk Langer, 91 - 99. Wiesbaden: VS-Verlag für Sozialwissenschaften.

Skopek, Jan. 2013. Data Manual. Starting Cohort 6 - Adult Education and Lifelong Learning. Release 3.0.1. Bamberg: NEPS Data Center. August 20, 2013. https://www.nepsdata.de/Portals/0/NEPS/Datenzentrum/Forschungsdaten/SC6/3-0-1/SC6_3-0-

1_DataManual_en.pdf (Zugegriffen: 26.03.2021). 
Solga, Heike, Baas, Meike, und Kohlrausch, Bettina. 2012. Mangelnde Ausbildungsreife. Hemmnis bei der Lehrstellensuche von Jugendlichen mit Hauptschulabschluss? In WZBrief Bildung, 19, 1 - 7. Berlin: Wissenschaftszentrum Berlin für Sozialforschung. http://bibliothek.wzb.eu/wzbrief-

bildung/WZBriefBildung192011_solga_baas_kohlrausch.pdf (Zugegriffen: 26.03.2021).

Solga, Heike. 2011. Bildungsarmut und Ausbildungslosigkeit in der Bildungs- und Wissensgesellschaft. In Lehrbuch der Bildungssoziologie, Hrsg. Rolf Becker, 411 - 448. Wiesbaden: VS Verlag für Sozialwissenschaften.

Solga, Heike. 2004. Increasing risks of stigmatization. Changes in school-to-work transitions of less-educated West Germans. In Yale Journal of Sociology, 4, 99 - 129. New Haven, Connecticut: Yale University: Department of Sociology. https://web.archive.org/web/20160802060632/http://www.yale.edu/sociology/yjs/yjs_fall 2004.pdf (Zugegriffen: 26.03.2021).

Solga, Heike. 2002. Ohne Schulabschluss - und was dann? Bildungs- und Berufsbiographien westdeutscher Jugendlicher ohne Schulabschluss, geboren zwischen 1930 und 1971. Selbständige Nachwuchsgruppe „Ausbildungslosigkeit. Bedingungen und Folgen mangelnder Berufsausbildung“. Working Paper 2/2002. Berlin: Max-Planck-Institut für Bildungsforschung. https://web.archive.org/web/20110512144503/https://www.mpibberlin.mpg.de/volltexte/institut/dok/full/nwg/NWG_solga_WP2_2002.pdf (Zugegriffen: 26.03.2021).

Solga, Heike, und Wagner, Sandra. 2001. Paradoxie der Bildungsexpansion. Die doppelte Benachteiligung von Hauptschülern. In Zeitschrift für Erziehungswissenschaft, 14: Heft 1: 107-127.

Spiegel Online. 2004. Leben und Lernen. Abschaffung des AiP. Aus Praktikanten werden Assistenzärzte. Samstag, $\quad$ 08.05.2004. $14: 35$ Uhr. http://www.spiegel.de/lebenundlernen/job/abschaffung-des-aip-aus-praktikanten-werdenassistenzaerzte-a-298827.html (Zugegriffen: 26.03.2021).

SPSS Inc. 2007. SPSS Advanced Models 16.0. Chicago, IL: SPSS Inc. https://www.unimuenster.de/imperia/md/content/ziv/service/software/spss/handbuecher/deutsch/spss_adv anced_models_16.0.pdf (Zugegriffen: 26.03.2021).

Statista Research Department 2012. Anteil der Schüler nach Schulart in Deutschland von 1952 bis 2005. Veröffentlicht von Statista Research Department, 21.03.2012. 
https://de.statista.com/statistik/daten/studie/76115/umfrage/anteil-der-schueler-nachschulart-in-deutschland-seit-1952/ (Zugegriffen am: 02.01.2020).

Statistik der Bundesagentur für Arbeit, Arbeitslosigkeit im Zeitverlauf. 2018. Statistik nach Themen. Arbeitslose, Unterbeschäftigung und Arbeitsstellen. Arbeitslose und Unterbeschäftigung. Arbeitslose und Arbeitslosenquoten - Deutschland und West/Ost (Zeitreihe Monats- und Jahreszahlen ab 1950). Tabelle 1.1: Entwicklung der Arbeitslosigkeit im Bundesgebiet (West) nach Strukturmerkmalen - Jahreszahlen. Tabelle 2.1.1: Arbeitslose insgesamt - Jahreszahlen. Datenstand: Februar 2018 (DZ/AM). https://statistik.arbeitsagentur.de/Statistikdaten/Detail/Aktuell/iiia4/alo-zeitreihe-dwo/alozeitreihe-dwo-b-0-xlsx.xlsx (Zugegriffen: 26.03.2021).

Statistisches Bundesamt. 2017a. Zahlen \& Fakten. Gesamtwirtschaft \& Umwelt. Arbeitsmarkt. Arbeitsmarkt. Erwerbstätige im Inland nach Wirtschaftssektoren. https://web.archive.org/web/20170313003610/https://www.destatis.de/DE/ZahlenFakten/ Indikatoren/LangeReihen/Arbeitsmarkt/lrerw013.html (Zugegriffen: 26.03.2021).

Statistisches Bundesamt. 2017b. Zahlen \& Fakten. Gesamtwirtschaft \& Umwelt. Arbeitsmarkt. Erwerbstätigkeit. Arbeitsmarkt. Bevölkerung und Erwerbstätigkeit $\begin{array}{lllll}\text { (Inländer) } \quad \text { Deutschland } & \text { in } & 1000 . & \text { In } & 1000 .\end{array}$ https://web.archive.org/web/20171228225557/https://www.destatis.de/DE/ZahlenFakten/ GesamtwirtschaftUmwelt/Arbeitsmarkt/Erwerbstaetigkeit/TabellenErwerbstaetigenrechn ung/BevoelkerungErwerbstaetigkeit.html (Zugegriffen: 26.03.2021).

Statistisches Bundesamt. 2017c. Zahlen \& Fakten. Gesellschaft \& Staat. Bevölkerung. Bevölkerungsstand. $\quad$ Bevölkerung nach https://web.archive.org/web/20170103162742/https://www.destatis.de/DE/ZahlenFakten/ GesellschaftStaat/Bevoelkerung/Bevoelkerungsstand/Tabellen_/lrbev01.html (Zugegriffen: 26.03.2021).

Statistisches Bundesamt. 2017d. Zahlen \& Fakten. Gesamtwirtschaft \& Umwelt. Arbeitsmarkt. Arbeitsmarkt. Registrierte Arbeitslose, Arbeitslosenquote nach Gebietsstand.

https://web.archive.org/web/20171129001141/https://www.destatis.de/DE/ZahlenFakten/ Indikatoren/LangeReihen/Arbeitsmarkt/lrarb003.html (Zugegriffen: 26.03.2021).

Statistisches Bundesamt. 2016a. Zahlen \& Fakten. Gesellschaft \& Staat. Bevölkerung. Bevölkerungsstand. Bevölkerung nach Altersgruppen. Früheres Bundesgebiet. https://web.archive.org/web/20161116123932/https://www.destatis.de/DE/ZahlenFakten/ 
GesellschaftStaat/Bevoelkerung/Bevoelkerungsstand/Tabellen_/rbev01.html?cms_gtp=1 51914_list\%253D3\&https=1 (Zugegriffen: 26.03.2021).

Statistisches Bundesamt. 2016b. Publikationen. STATmagazin. Arbeitsmarkt. Was sind Erwerbstätige?

https://web.archive.org/web/20161115084316/https://www.destatis.de/DE/Publikationen/ STATmagazin/Arbeitsmarkt/2009_03/ErwerbstaetigeVGR.html (Zugegriffen: 26.03.2021).

Statistisches Bundesamt. 2015a. Zahlen \& Fakten. Gesamtwirtschaft \& Umwelt. Arbeitsmarkt.

Arbeitsmarkt. https://web.archive.org/web/20151113185118/https://www.destatis.de/DE/ZahlenFakten/ Indikatoren/LangeReihen/Arbeitsmarkt/lrerw013.html (Zugegriffen: 26.03.2021).

Statistisches Bundesamt. 2015d. Zahlen \& Fakten. Gesellschaft \& Staat. Bevölkerung. Was ist der

Mikrozensus?

https://www.destatis.de/DE/ZahlenFakten/GesellschaftStaat/Bevoelkerung/Mikrozensus. html (Zugegriffen: 26.03.2021).

Statistisches Bundesamt. 2008. Klassifikation der Wirtschaftszweige. Mit Erläuterungen. 2008. Wiesbaden: Statistisches Bundesamt. https://www.destatis.de/static/DE/dokumente/klassifikation-wz-20083100100089004.pdf (Zugegriffen: 26.03.2021).

Statistisches Bundesamt. 2003. Klassifikation der Wirtschaftszweige mit Erläuterungen. Ausgabe 2003. Wiesbaden: Statistisches https://web.archive.org/web/20161115104712/https://www.destatis.de/DE/Methoden/Kla ssifikationen/GueterWirtschaftklassifikationen/klassifikationwz2003_erl.pdf? blob=pub licationFile (Zugegriffen: 26.03.2021).

Statistisches Bundesamt. 1992. Unternehmens- und Betriebssystematik. Systematik der Wirtschaftszweige mit Erläuterungen. Ausgabe 1979. Statistisches Bundesamt Wiesbaden. 5. unveränderte Auflage. Stuttgart: Metzler-Poeschel. https://web.archive.org/web/20161115104927/https://www.destatis.de/DE/Methoden/Kla ssifikationen/GueterWirtschaftklassifikationen/klassifikationenwz1979.pdf? blob=publi cationFile (Zugegriffen: 26.03.2021).

Stegmann, Heinz, und Holzbauer, Irmgard. 1978. Die Nachfrage nach Ausbildungsplätzen und ihre Realisierung bei Absolventen und Abgängern allgemeinbildender Schulen. Aus der Untersuchung des IAB: Jugendliche beim Übergang vom Bildungs- in das 
Beschäftigungssystem. In Mitteilungen aus der Arbeitsmarkt- und Berufsforschung, 11: Heft 2 , 148 170. http://doku.iab.de/mittab/1978/1978_2_MittAB_Stegmann_Holzbauer.pdf (Zugegriffen: 26.03.2021).

Steinmann, Susanne. 2000. Bildung, Ausbildung und Arbeitsmarktchancen in Deutschland.

Eine Studie zum Wandel der Übergänge von der Schule in das Erwerbsleben. Opladen: Leske und Budrich.

Straubhaar, Thomas. 1994. Wissen als strategische Grundlage der internationalen Wettbewerbsfähigkeit. In Die Weltwirtschaft vor neuen Herausforderungen. Strategischer Handel, Protektion und Wettbewerb, Hrsg. Rolf H. Hasse, und Wolf Schäfer, 111 - 131. Göttingen: Vandenhoeck \& Ruprecht.

Trainee-Geflüster. 2017. Karriere-Tipps. Volontariat. Berufseinstieg: Trainee-Programm vs. $\begin{array}{llllll}\text { Volontariat. } & \text { Aktualisiert } & \text { am } & \text { Mai } & \end{array}$ https://web.archive.org/web/20170712084237/https://www.traineegefluester.de/tipps/volontariat/trainee-vs-volontariat\# (Zugegriffen: 26.03.2021).

Universität Innsbruck. 2017. Kapitel 2. Nichtlineare Funktionsformen. Angewandte Ökonometrie. https://www.uibk.ac.at/econometrics/einf/kap02_log.pdf (Zugegriffen: 26.03.2021).

Universität Zürich. 2018. Methodenberatung. Einfaktorielle Varianzanalyse (ohne Messwiederholung). Startseite Methodenportal. Datenanalyse. Unterschiede. Zentrale $\begin{array}{llll}\text { Tendenz. } & \text { Einfaktorielle } & \text { 29.01.2018. }\end{array}$ https://web.archive.org/web/20180404173853/http://www.methodenberatung.uzh.ch/de/d atenanalyse/unterschiede/zentral/evarianz.html (Zugegriffen: 26.03.2021).

Völkl, Kerstin, und Korb, Christoph. 2018. Deskriptive Statistik. Eine Einführung für Politikwissenschaftlerinnen und Politikwissenschaftler. Wiesbaden: Springer Fachmedien.

Voos, Dunja. 2014. IRR: Incidence Rate Ratio, Inzidenzrate. Medizin im Text. Startseite. Begriffe. Statistik. IRR: Incidence Rate Ratio, Inzidenzrate. 12.03.2014. https://www.medizin-im-text.de/blog/2014/26464/irr-incidence-rate-ratio-inzidenzrate/ (Zugegriffen: 26.03.2021).

VWA. 2018. VWA-Studium. Frankfurt am Main: Bundesverband Deutscher Verwaltungsund Wirtschafts-Akademien e. V. 
https://web.archive.org/web/20160327120258/https://www.vwa.de/vwa-studium

(Zugegriffen: 26.03.2021).

Wagner, Sandra J. 2005. Jugendliche ohne Berufsausbildung. Eine Längsschnittstudie zum Einfluss von Schule, Herkunft und Geschlecht auf ihre Bildungschancen. Aachen: Shaker Verlag.

Walter, Thomas. 2006. Der Bologna- Prozess. Ein Wendepunkt europäischer Hochschulpolitik? Wiesbaden: VS Verlag für Sozialwissenschaften. 1. Auflage.

Weil, Mareike, und Lauterbach, Wolfgang. 2011. Von der Schule in den Beruf. In Lehrbuch der Bildungssoziologie, Hrsg. Rolf Becker, 329 - 366. Wiesbaden: VS-Verlag für Sozialwissenschaften. 2. überarbeitete und erweiterte Auflage.

Wenzelburger, Georg, Jäckle, Sebastian, und König, Pascal. 2014. Weiterführende statistische Methoden für Politikwissenschaftler. Eine anwendungsbezogene Einführung mit Stata. München: Oldenbourg.

Willich, Julia, Buck, Daniel, Heine, Christoph, und Sommer, Dieter. 2011. Studienanfänger im Wintersemester 2009/10. Wege zum Studium, Studien- und Hochschulwahl, Situation bei Studienbeginn. HIS: Forum Hochschule 06/2011. Deutsches Zentrum für Hochschulund Wissenschaftsforschung. Hannover: Hochschul-Informations-System GmbH. http://www.dzhw.eu/pdf/pub_fh/fh-201106.pdf (Zugegriffen: 26.03.2021).

Winkler, Oliver. 2017. Aufstiege und Abstiege im Bildungsverlauf. Eine empirische Untersuchung zur Öffnung von Bildungswegen. Wiesbaden: Springer Fachmedien Wiesbaden.

Ziegler, Andreas, Lange, Stefan, und Bender, Ralf. 2007. Überlebenszeitanalyse. Die CoxRegression. Artikel Nr. 17 der Statistik-Serie in der DMW. In DMW - Deutsche Medizinische Wochenschrift, Januar 2007;132, Ausgabe S 01, e42-e44. · Stuttgart; New York: Georg Thieme Verlag KG. ·ISSN 0012-0472. DOI 10.1055/s-2007-959039. https://d-nb.info/1184820953/34 (Zugegriffen: 26.03.2021).

Zwiener, Isabella, Bettner, Maria, und Hommel, Gerhard. 2011. Überlebenszeitanalyse. Teil 15 der Serie zur Bewertung wissenschaftlicher Publikationen. Deutsches Ärzteblatt. Jg. 108. Heft 10. 11. März 2011. Seiten 163 - 169. https://www.aerzteblatt.de/pdf.asp?id=81171 (Zugegriffen: 26.03.2021). 


\section{Erklärung}

Hiermit erkläre ich,

$\bigotimes$ dass ich die Dissertation selbständig verfasst und alle in Anspruch genommenen Hilfsmittel in der Dissertation angegeben habe,

$\bigotimes$ dass ich nicht die Hilfe einer kommerziellen Promotionsvermittlung in Anspruch genommen habe,

$\bigotimes$ dass meine Arbeit noch in keinem Prüfungsverfahren vorgelegen hat,

$\bigotimes$ dass ich nicht schon einmal einen Antrag auf Eröffnung eines Promotionsverfahrens gestellt habe und dass ich noch in keinem früheren Promotionsverfahren erfolglos geblieben bin,

$\bigotimes$ dass mir die Promotionsordnung bekannt ist.

$\bigotimes$ dass die Arbeit weder ganz, noch in Auszügen veröffentlicht ist.

Ort, Datum

Unterschrift 